AIII02 6 33833

NBS

PUBLICATIONS
NATL INST OF STANDARDS \& TECH R.I.C.

. I.|.

A11102633833 fFederal laboratory directory, 1982
QC100 U57 NO.646 1983 Vig3 C.2 NBS-PUB

\section{U.S. DEPARTMENT OF COMMERCE}

National Bureau of Standards Washington, DC 20234

\title{
FEDERAL LABORATORY DIRECTORY 1982
}




\section{NATIONAL BUREAU OF STANDARDS}

The National Bureau of Standards' was established by an act of Congress on March 3, 1901 . The Bureau's overall goal is to strengthen and advance the Nation's science and technology and facilitate their effective application for public benefit. To this end, the Bureau conducts research and provides: (1) a basis for the Nation's physical measurement system, (2) scientific and technological services for industry and government, (3) a technical basis for equity in trade, and (4) technical services to promote public safety. The Bureau's technical work is performed by the National Measurement Laboratory, the National Engineering Laboratory, and the Institute for Computer Sciences and Technology.

THE NATIONAL MEASUREMENT LABORATORY provides the national system of physical and chemical and materials measurement; coordinates the system with measurement systems of other nations and furnishes essential services leading to accurate and uniform physical and chemical measurement throughout the Nation's scientific community, industry, and commerce; conducts materials research leading to improved methods of measurement, standards, and data on the properties of materials needed by industry, commerce, educational institutions, and Government; provides advisory and research services to other Government agencies; develops, produces, and distributes Standard Reference Materials; and provides calibration services. The Laboratory consists of the following centers:

\section{Absolute Physical Quantities ${ }^{2}$ - Radiation Research - Chemical Physics - Analytical Chemistry - Materials Science}

THE NATIONAL ENGINEERING LABORATORY provides technology and technical services to the public and private sectors to address national needs and to solve national problems; conducts research in engineering and applied science in support of these efforts; builds and maintains competence in the necessary disciplines required to carry out this research and technical service; develops engineering data and measurement capabilities; provides engineering measurement traceability services; develops test methods and proposes engineering standards and code changes; develops and proposes new engineering practices; and develops and improves mechanisms to transfer results of its research to the ultimate user. The Laboratory consists of the following centers:

Applied Mathematics - Electronics and Electrical Engineering ${ }^{2}$ - Manufacturing Engineering - Building Technology - Fire Research - Chemical Engineering ${ }^{2}$

THE INSTITUTE FOR COMPUTER SCIENCES AND TECHNOLOGY conducts research and provides scientific and technical services to aid Federal agencies in the selection, acquisition, application, and use of computer technology to improve effectiveness and economy in Government operations in accordance with Public Law 89-306 (40 U.S.C. 759), relevant Executive Orders, and other directives; carries out this mission by managing the Federal Information Processing Standards Program, developing Federal ADP standards guidelines, and managing Federal participation in ADP voluntary standardization activities; provides scientific and technological advisory services and assistance to Federal agencies; and provides the technical foundation for computer-related policies of the Federal Government. The Institute consists of the following centers:

Programming Science and Technology - Computer Systems Engineering.

'Headquarters and Laboralories at Gailhersburg, MD, unless otherwise noted; mailing address Washingıon, DC 20234.

${ }^{2}$ Some divisions within the cenler are located at Boulder, CO 80303. 


\section{NBS Special Publication 646 \\ FEDERAL LABORATORY DIRECTORY 1982}

James M. Wyckoff, Editor

Office of Research and

Technology Applications

National Bureau of Standards

Washington, DC 20234

Prepared in cooperation with the

Federal Laboratory

Consortium for

Technology Transfer

TECHNOLOGY TRANSFER

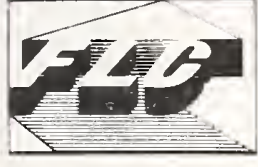

Issued February 1983

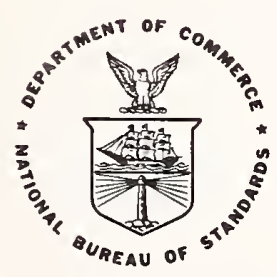

U.S. DEPARTMENT OF COMMERCE, Malcolm Baldrige, Secretary NATIONAL BUREAU OF STANDARDS, Emest Ambler, Director 
Library of Congress Catalog Card Number: $82-600663$

National Bureau of Standards Special Publication 646

Natt. Bur. Stand. (U.S.), Spec. Publ. 646, 263 pages (Feb. 1983)

CODEN: XNBSAV

U.S. GOVERNMENT PRINTING OFFICE

WASHINGTON: 1981

\footnotetext{
For sale by the Superintendent of Documents, U.S. Government Printing Office, Washington, D.C. 20402 Price $\$ 8.00$

(Add 25 percent for other than U.S. mailing)
} 
With the tremendous investment of the Federal Government in research and development, the need to survey the laboratories where much of the work is done is a recurring requirement. The Stevenson-Wydler Technology Innovations Act of 1980 focused attention on the potential for Federal laboratories to serve users outside of the Federal agencies that manage the laboratories. This Federal Laboratory Directory has been prepared with the need for effective transfer of technology much in mind.

Over the past decade, the Federal Laboratory Consortium for Technology Transfer (FLC) has taken a growing lead in facilitating the sharing of information, products, processes and services between laboratories, the private sector, and governments at the State and local level. It was due to the requests of the FLC that we undertook, at the National Bureau of Standards, the gathering of this data and through the cooperation of the agencies represented we have been able to complete it. At the Bureau, we are pleased to be able to support this process, as like many laboratories, we draw on the strengths of sister organizations to achieve our goals. We also make every effort to serve those Federal agencies, the private sector, and State and local governments with our programs. We hope this document will help in achieving that goal.

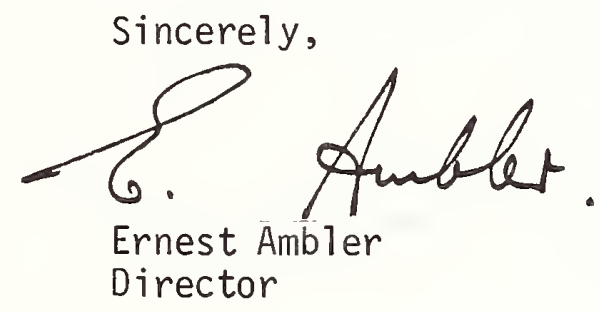




\section{ABSTRACT}

This Directory provides limited information about some 388 Federal laboratories with ten or more full-time professionals engaged in research and development. Summary data arranged by Federal agency and by State provide a broad overview of the Federal laboratory system. Laboratory lists by staff size, by State and by agency provide a cross reference. For each laboratory, a contact for obtaining technical information is given by name, address, and phone number. Major mission and major scientific or testing equipment is 7 isted for each laboratory.

Key words: contacts; directory; Federal laboratory; research and development; technology transfer. 


\section{Table of Contents}

1. Introduction . . . . . . . . . . . . . 1

2. Discussion of Data . . . . . . . . . . . . 2

3. Credits. . . . . . . . . . . . . . . 4

4. References . . . . . . . . . . . . . . 5

5. Tables . . . . . . . . . . . . . . 6

(1) Federal Laboratories by Agency, Summary Data . . . 6

(2) Federal Laboratories by State, Summary Data. . . . 7

(3) Largest Federal Laboratory Facilities. . . . . . 8

(4) Federal Laboratories Listed in Order of Total . . . . 11 Staff Size

(5) Federal Laboratories Listed by State . . . . . . 19

(6) Federal Laboratories Listed by Agency and . . . . 27 Staff Size

(7) Federal Laboratory Contacts Listed by Agency . . . . 35 and Staff Size

6. Federal Laboratory Information Sheets . . . . . . . . . 71

Department of Commerce . . . . . . . . . . . 73

Department of Defense. . . . . . . . . . . . 93

Department of Energy . . . . . . . . . . . 141

Department of Interior . . . . . . . . . . 163

Department of Justice. . . . . . . . . . . . 177

Department of Transportation . . . . . . . . . 181

Environmental Protection Agency . . . . . . . . 187

Heal th and Human Services . . . . . . . . . . 197

National Aeronautics and Space Administration. . . . . 213

National Science Foundation. . . . . . . . . . . 221

Smithsonian Institution . . . . . . . . . . . . 227

Tennessee Valley Authority . . . . . . . . . . . 233

U.S. Department of Agriculture. . . . . . . . . . 237

Veterans Administration . . . . . . . . . . . 273

7. Appendix A - VA Letter . . . . . . . . . . . . 275 



\section{INTRODUCTION}

The Federal Laboratory Directory, 1982 edition, has been compiled to meet the needs of several audiences. Planners and policymakers, in government and out, need to have a broad view of Federal research and development laboratory resources. For their purposes, summary data on laboratory size and geographical and agency distribution may be particularly useful.

For those who would use the technology from Federal laboratories, this Directory includes individual data sheets on each laboratory including, where available, the name, address, and telephone number of an initial contact for research and technology applications information. The information on the major mission of the laboratory and any major scientific or testing equipment will give some indication of the resources to be expected.

These data may be particularly useful to those in intermediary organizations such as the Department of Commerce's Center for the Utilization of Federal Technology, or the Federal Laboratory Consortium for Technology Transfer which have mandated responsibilities for providing access to Federal laboratory resources to users from State or local governments, or from the private sector.

With minor modifications, the data sheet for each laboratory is patterned after earlier work. Names and addresses are included in spite of the recognition that rather frequent updates will be required. An attempt at showing the organizational relationships of the laboratories has been included although other documents may provide better guidance to such interactions.

The sources of data for this Directory were the individual information sheets which are included, as submitted by each agency, in Section 6 . These data have been summarized and organized in several tables in Section 5 by staff size, then by State, and by agency. A list of Research and Technology Application contacts includes data on laboratory staff size. A11 summary data are stored in a computer database for periodic updating for future editions of the Directory. 


\section{DISCUSSION OF DATA}

For this Directory, data have been gathered only for laboratories with ten or more full-time professionals engaged in research and development. This is a significant departure from earlier directories and accounts-in part--for the sharp drop in the total number of laboratories listed. The definition of the term "professional" has been left to each laboratory. For civil service employees, it will have a common meaning, but for other employees, it may vary. For this reason, comparisons between laboratories should be made with caution. The value of "percent research and development" was subjective for those who prepared the data. Defense laboratories provided data with research, development, and testing combined. Once more, these data must be used with caution.

The total staff size is probably one of the most reliable numbers as of the date reported. As that date preceded the change in Administration, it will change in subsequent editions. For some laboratories, large changes may be expected. Current staff counting procedures provide "full-time equivalent" data reflecting an averaging process over the fiscal year and proper weighting for part-time employees. However, not all laboratories have used that procedure so some variations are expected and the totals must be viewed as approximates.

As mentioned, a computer has been used to store some of the data and sort it by laboratory characteristics. One set of data was obtained by a handsorting--the listing of facilities rather than laboratories, in order of size. For purposes of technology transfer, that sorting may have more meaning. A facility is defined as a group of laboratories at a single geographical location. In some cases these laboratories have closely related missions but in other cases they simply share common services.

The term laboratory is used in a generic sense in this Directory. A glance at the listings will show that a number of other terms are used: bureau, center, facility, division, institute, activity, office, museum, station, research unit or observatory.

The data on some laboratory description sheets are incomplete. In future editions, every attempt will be made to correct that. Numerical data reflecting staff size are complete and so allow ordering of laboratories by size and summary computations.

Many agencies found it difficult to decide if their facilities could properly be included in this Directory. They have grown in a wide variety of patterns to meet the needs of their constituents and their agencies. The Veterans Administration, for example, conducts research in the clinical setting of many of its hospitals, but proper treatment of the patient is always its primary aim. A letter giving some details of that system is included in Appendix $A$ and some 60 medical centers are listed without detailed staff statistics. The U.S. Geological Survey is another case where extensive 
field offices are necessary to gather and apply data related to their mission. Research for that group is very unlike the work centered around large complex facilities in many defense or space laboratories.

Networks of small facilities characterize the U.S. Department of Agriculture's Agricultural Research Service, and U.S. Forest Service. They have been reported as regional facilities by the Forest Service but listed separately by the Agricultural Research Service. The Department of Commerce's National Oceanographic and Atmospheric Administration similarly has a large number of rather small laboratories. For each of these organizations, some laboratories were too small to be included in this Directory.

It is important to point out that many laboratories provide routine, but important, measurement services in the fields of air and water quality, weather, health and safety, clinical, and forensic sciences. They frequently do not meet the "research and development" criteria for inclusion in this Directory.

Another category of activity excluded are the very essential information processing offices that often serve as intermediary organizations for those seeking technical assistance.

Some laboratories serving regulatory agencies, such as the Food and Drug Administration, have not been 7 isted since they are conceived as providing only measurement services related to agency functions.

\section{A Final Word}

Errors creep into any document of this size and the editor would appreciate having such errors called to his attention so that corrections can be made in future editions. Any suggestions for making this document more useful would be most welcome. 


\section{CREDITS}

The task of gathering these data has been coordinated through the Federal Laboratory Consortium for Technology Transfer and the diligence of many individuals is very much appreciated.

\begin{tabular}{|c|c|c|}
\hline USDA & Agriculture & $\begin{array}{l}\text { Harold G. Marx and } \\
\text { Maurice LeBourhis }\end{array}$ \\
\hline USDoC & Commerce & Ferris Webster for NOAA \\
\hline USDoD & Defense & $\begin{array}{l}\text { George F. Linsteadt and } \\
\text { Michael Palomar }\end{array}$ \\
\hline DoE & Energy & Charles F. Miller \\
\hline EPA & Environmental Protection Agency & $\begin{array}{l}\text { Chris L. West and } \\
\text { Michael L. Mastracci }\end{array}$ \\
\hline HHS & Health and Human Services & Samue] D. Korper, Ph.D. \\
\hline DoI & Interior & $\begin{array}{l}\text { Don Ralston, BOM } \\
\text { Dr. Ethan T. Smith, USGS }\end{array}$ \\
\hline DoJ & Justice & James H. Geer \\
\hline NASA & $\begin{array}{l}\text { National Aeronautics and Space } \\
\text { Administration }\end{array}$ & Dr. Paul R. Brockman \\
\hline NSF & National Science Foundation & Dr. Francis S. Johnson \\
\hline Smith & Smithsonian & Dr. David Challinor \\
\hline TVA & Tennessee Valley Authority & Shirley Ray \\
\hline Dot & Transportation & Alphonso B. Linhares \\
\hline VA & Veterans Administration & Dr. Earl X. Freed \\
\hline
\end{tabular}

The continued interest of Dr. Eugene L. Stark, Chairman of the FLC, and Dr. Howard E. Sorrows, Director of the NBS Office of Research and Technology Applications, was essential to the completion of this task. Betty Joyce Molino, co-author of the Omnidata computer program and patient teacher of the system, made the data handling task a manageable one. The dedicated assistance of Linda Chester in each step of preparing this document for publication was indispensable. 
The last generally available listing of all "Federal R\&D Installations" was published by the National Science Foundation in 1970. ${ }^{1}$ Since that time, two listings of many Federal laboratories have appeared as part of Congressional committee proceedings ${ }^{2,3}$ in 1974 and 1979. Two other works, Directory of Federal Technology Transfer ${ }^{4,5} 1975$ and 1977 provided information submitted by each agency with descriptive material on the type of resources available and mechanisms for obtaining assistance. More detailed and up-to-date information on the organization of all Federal agencies can be obtained from the annual "United States Government Manua ${ }^{6}$." Individual agencies such as the Departments of Energy and Defense have prepared more detailed 1istings of their research and development facilities and organization ${ }^{7,8}, 9$. The National Aeronautics and Space Administration (NASA) publishes an annual report, Spinoff ${ }^{10}$, which 7 ists both outstanding accomplishments and contact organizations separated somewhat from the laboratory structure.

1. Directory of Federal R\&D Installations, Zola Bronson, Ed. National Science Foundation, Washington, D.C. 1970 NBS-70-23

2. Utilization of Federal Laboratories Agriculture-Environment and Consumer Protection Appropriations for 1975-Part 7 Investigative Report. Subcommittee of the Committee on Appropriations, U.S. House of Representatives

3. Investigative Report on the Utilization of Federal Laboratories, Part II Hearings before the Subcommittee on Agriculture and Related Agencies of the Committee on Appropriations of the U.S. House of Representatives of the 95th Congress, Second Session, 95th Congress on Appropriations for 1979.

4. Directory of Federal Technology Transfer A.B. Linhares Ed., EOP U.S. GPO Stock No. 052003-00376-7 Washington, D.C. 20402 (1975)

5. Directory of Federal Technology Transfer 1971 A.B. Linhares, Ed., U.S. GPO, Washington, D.C. 20402

6. The United States Government Manual 1981/82 Office of the Federal Register, National Archives and Record Service, GSA, Washington, D.C. 20408, U.S. GPO, Washington, D.C. 20402

7. DOE Research and Development and Field Facilities DOE/ER-0029, June 1979 U.S. GPO, Washington, D.C. 20402 Stock No. 061-00000318-5

8. Capsule Review of the DoE Research and Development and Field Facilities, DoE/ER 0092 September 1980. NTIS U.S. Department of Commerce, 5285 Port Royal Road, Springfield, Virginia 22161

9. Department of Defense In-House RDT\&E Activities, Harold F. Davidson, October 1980, U.S. Army Materiel Development and Readiness Command, 5001 Eisenhower Avenue, Alexandria, Virginia 22333

10. Spinoff 1981, An Annual Report, NASA, James J. Haggerty, Superintendent of Documents, U.S. GPO, Washington, D.C. 20402 
Table 1

Federal Laboratories by Agency,

Summary Data

\begin{tabular}{|c|c|c|c|c|}
\hline Agency & $\begin{array}{l}\text { Number } \\
\text { of Labs }\end{array}$ & $\begin{array}{l}\text { Total } \\
\text { Staff }\end{array}$ & $\begin{array}{c}\text { Average } \\
\text { Lab. Staff }\end{array}$ & $\begin{array}{r}F Y 81 \text { R\&D } \\
\$ \text { Millions } \\
\end{array}$ \\
\hline DOD & 92 & 89076 & 968 & 16821 \\
\hline DOE & 39 & 64544 & 1655 & 5017 \\
\hline NASA & 11 & 24885 & 2262 & 5317 \\
\hline DOI & 24 & 13482 & 562 & 413 \\
\hline *HHS & 21 & 8540 & 407 & 3879 \\
\hline USDA & 67 & 7786 & 116 & 758 \\
\hline DOC & 36 & 5077 & 141 & 377 \\
\hline DOT & 7 & 2625 & 375 & 379 \\
\hline NSF & 6 & 1641 & 274 & 994 \\
\hline EPA & 14 & 1565 & 112 & 469 \\
\hline TVA & 4 & 1404 & 351 & 20 \\
\hline VA & 60 & $600+$ & $10+$ & 130 \\
\hline DOJ & 1 & 429 & 429 & 42 \\
\hline \multirow[t]{2}{*}{ Smith } & 6 & 291 & 48 & 45 \\
\hline & 388 & 221885 & 572 & 34661 \\
\hline${ }^{*} \operatorname{Repc}$ & Incomple & & & \\
\hline $\begin{array}{c}* \star F i g u r \\
\text { HHS } 1\end{array}$ & $\begin{array}{l}\text { as of } \\
1 / 81\end{array}$ & 100 & & $79, N$ \\
\hline
\end{tabular}


Table 2

Federal Laboratories by State,

Summary Data

\begin{tabular}{|c|c|c|c|c|c|c|c|}
\hline & $\begin{array}{l}\text { Total } \\
\text { Staff }\end{array}$ & $\begin{array}{l}\text { No. of } \\
\text { Labs }\end{array}$ & $\begin{array}{c}\text { Average } \\
\text { Size }\end{array}$ & & $\begin{array}{l}\text { Total } \\
\text { Staff }\end{array}$ & $\begin{array}{l}\text { No. of } \\
\text { Labs }\end{array}$ & $\begin{array}{c}\text { Average } \\
\text { Size }\end{array}$ \\
\hline$C A$ & 42734 & 36 & 1187 & SC & 1067 & 4 & 267 \\
\hline MD & 30891 & 41 & 753 & IA & 821 & 3 & 274 \\
\hline NM & 20739 & 10 & 2074 & WV & 643 & 4 & 161 \\
\hline $\mathrm{NJ}$ & 10280 & 15 & 685 & MN & 636 & 5 & 127 \\
\hline $\mathrm{FL}$ & 10178 & 17 & 599 & LA & 560 & 9 & 62 \\
\hline VA & 9552 & 11 & 868 & $O R$ & 538 & 4 & 135 \\
\hline NY & 9110 & 14 & 651 & OK & 510 & 6 & 85 \\
\hline IL & 7750 & 9 & 861 & WI & 448 & 6 & 75 \\
\hline $\mathrm{CO}$ & 7491 & 16 & 468 & $\mathrm{NH}$ & 277 & 1 & 277 \\
\hline PA & 7106 & 8 & 888 & ND & 271 & 4 & 68 \\
\hline$D C$ & 6658 & 10 & 666 & NV & 234 & 3 & 78 \\
\hline TN & 6311 & 8 & 789 & MO & 217 & 6 & 36 \\
\hline$A L$ & 6094 & 8 & 762 & $C Z$ & 173 & 2 & 87 \\
\hline $\mathrm{OH}$ & 5975 & 13 & 460 & RI & 160 & 3 & 53 \\
\hline ID & 5147 & 2 & 2574 & WY & 148 & 1 & 148 \\
\hline TX & 5002 & 13 & 385 & $\mathrm{CH}$ & 144 & 1 & 144 \\
\hline WA & 4770 & 10 & 477 & MT & 128 & 1 & 128 \\
\hline MA & 3494 & 12 & 291 & NE & 99 & 3 & 33 \\
\hline CT & 3153 & 6 & 526 & $\mathrm{HI}$ & 87 & 2 & 44 \\
\hline IN & 2257 & 4 & 564 & $E G$ & 81 & 1 & 81 \\
\hline $\mathrm{AZ}$ & 2182 & 7 & 312 & KS & 64 & 1 & 64 \\
\hline MS & 1994 & 9 & 222 & AK & 62 & 2 & 31 \\
\hline NC & 1633 & 10 & 163 & PR & 50 & 1 & 50 \\
\hline GA & 1579 & 11 & 144 & SD & 28 & $i$ & 28 \\
\hline UT & 1252 & 6 & 209 & $A R$ & 10 & 1 & 10 \\
\hline MI & 1087 & 6 & 181 & KY & 10 & 1 & 10 \\
\hline
\end{tabular}

*Includes overseas laboratories in

Puerto Rico (PR), Canal Zone (CZ), Egypt (EG), Chile (CH) 
Table 3

LARGEST FEDERAL LABORATORY FACILITIES

(1isted in order of total FY 80 staff size)

\begin{tabular}{|c|c|c|c|c|}
\hline No. & Agency & Facility & Total & $\mathrm{FLC}^{*}$ \\
\hline 1 & DOE & Sandia National Laboratories & 7639 & FLC \\
\hline 2 & DOE & $\begin{array}{l}\text { Hanford, WA } \\
\text { Hanford Engineering Development Lab }\end{array}$ & 7202 & FLC \\
\hline 3 & DOE & Lawrence Livermore National Lab & 7102 & FLC \\
\hline 4 & DOE & Los Alamos National Lab & 6937 & FLC \\
\hline 5 & DOD & Armament Division (Elgin) & 6389 & FLC \\
\hline 6 & $\mathrm{DOD}$ & $\begin{array}{l}\text { Edwards AFB, CA } \\
\text { Flight Test Center } \\
\text { Rocket Propulsion Lab } \\
\text { Aviation Engineering Flight Activity }\end{array}$ & 5733 & FLC \\
\hline 7 & DOE & $\begin{array}{l}\text { Oak Ridge, TN } \\
\text { Oak Ridge National Lab } \\
\text { Oak Ridge Associated Universities }\end{array}$ & 5583 & FLC \\
\hline 8 & DOE & Argonne National Lab & 5118 & FLC \\
\hline 9 & DOE & Idaho National Engineering Lab & 5100 & FLC \\
\hline 10 & HHS & $\begin{array}{l}\text { National Institutes of Health, Bethesda, Md. } \\
\text { National Cancer Institute } \\
\text { National Institute of Arthritis, Diabetes, } \\
\text { and Digestive and Kidney Diseases } \\
\text { Neurology and Communicative Disorders } \\
\text { Research Lab } \\
\text { National Institute of Allergy and } \\
\text { Infectious Diseases } \\
\text { National Institute of Mental Health, } \\
\text { Intramural Research Program } \\
\text { National Institute of Child Health and } \\
\text { Human Development } \\
\text { National Heart, Lung, and Blood Institute } \\
\text { Division of Computer Research and } \\
\text { Technology } \\
\text { National Institute of Dental Research, } \\
\text { Intramural Research Program } \\
\text { National Eye Institute, Intramural } \\
\text { Research Program }\end{array}$ & 5082 & \\
\hline 11 & DOD/NAVY & Pacific Missile Test Center & 4803 & \\
\hline 12 & DOD/NAVY & Naval Surface Weapons Center & 4732 & FLC \\
\hline 13 & DOD/NAVY & Naval Weapons Center & 4540 & FLC \\
\hline 14 & DOI/USGS & USGS Central Region & 4500 & \\
\hline 15 & DOD/ARMY & $\begin{array}{l}\text { White Sands, NM } \\
\text { White Sands Missile Range } \\
\text { Atmospheric Sciences Lab } \\
\text { Ordnance Missile Test Facility }\end{array}$ & 4345 & FLC \\
\hline
\end{tabular}

Footnote: Groups of labs located at a single facility have been lumped together for this analysis

*Federal Laboratory Consortium for Technology Transfer (FLC) members 


\begin{tabular}{ll} 
No. & Agency \\
\hline & \\
16 & NASA \\
17 & DOI/USGS \\
18 & DOD/AF \\
19 & NASA \\
20 & NASA \\
21 & DOE \\
22 & NASA \\
23 & DOD
\end{tabular}

$\begin{array}{ll}24 & \text { DOD/NAVY } \\ 25 & \text { DOE } \\ 26 & \text { DOE } \\ 27 & \text { DOC } \\ 28 & \text { DOI/USGS } \\ 29 & \text { NASA } \\ 30 & \text { DOE } \\ 31 & \text { DOD/NAVY } \\ & \\ 32 & \text { NASA } \\ 33 & \text { DOD/NAVY } \\ 34 & \text { DOD/NAVY } \\ 35 & \text { DOD/AF }\end{array}$

Jet Propulsion Laboratory

Total

FLC

$$
\text { Western Region }
$$

Naval Air Test Center

3788

3676

3566

George C. Marshall Space Flight Center 3563

Lyndon B. Johnson Space Center

3508

Brookhaven National Lab

3502

Goddard Space Flight Center

3436

Aberdeen Proving Ground, Md.

3407

Materials Testing Directorate

Chemical Systems Lab

Ballistics Research Lab

Material Systems Analysis Activity

Medical Research Institute of Chemical Defense

Human Engineering Lab

Naval Research Lab

3394

Bettis Atomic Power Lab

Lawrence Berkeley Lab

National Bureau of Standards

National Center

Langley Research Center

Knolls Atomic Power Lab

Newport, RI

Naval Underwater Systems Center

Underwater Range Department

Lewis Research Center

Ocean Systems Center

David W. Taylor Ship R\&D Center

Wright Patterson AFB, $\mathrm{OH}$

FLC

FLC

FLC

FLC

FLC

FLC

Avionics Lab

Flight Dynamics Lab

Materials Lab

Aerospace Medical Research Lab

36 DOD/NAVY

Naval Air Development Center

Dover, NJ

2399

2370

FLC

FLC

\section{FLC}

FLC

FLC

3158

FLC

3072

3000

2966

2913

2882

Large Caliber Weapons Systems Lab

Fire Control and Small Caliber Systems Lab

$38 \quad$ NASA

39 DOD/NAVY

John F. Kennedy Space Center

2201

2150

Naval Avionics Center

2822

2791

2567

2566

FLC

FLC

FLC

\section{FLC}

FLC

FLC

FLC

FLC

Fort Belvoir, VA

Mobility Equipment R\&D Command

Night Vision \& Electro-Optics Lab

2067

Engineer Topographic Lab

$\begin{array}{ll}41 & \text { DOE } \\ 42 & \text { DOD/NAVY } \\ 43 & \text { NASA } \\ 44 & \text { DOD/ARMY }\end{array}$

Fermi National Accelerator Lab

Naval Air Engineering Center

Ames Research Center

Fort Monmouth, $\mathrm{NJ}$

Electronic Warfare Lab

Communications R\&D Command

Electronics Technology \& Devices Lab

Avionics R\&D Activity

Combat Surveillance \& Target Ag. Lab

45 DOD/ARMY

Natick, MA

FLC

FLC

Natick R\&D Labs

Research Inst. of Environmental Meas.

FLC Clothing \& Textile Research Facility

FLC

FLC

FLC

FLC

FLC

FLC

FLC

FLC

FLC 


$\begin{array}{ll}46 & \text { DOE } \\ 47 & \text { DOE } \\ 48 & \text { DOD/NAVY } \\ 49 & \text { DOD/AF } \\ 50 & \text { DOD/NAVY } \\ 51 & \text { USDA/ARS } \\ 52 & \text { DOD/ARMY } \\ 53 & \text { DOD/AF } \\ 54 & \text { DPE }\end{array}$

55 DOT

56 HHS

57 DOD/AF

$58 \quad$ HHS

Princeton Plasma Physics Lab

1406

Stanford Linear Accelerator Center

Engineer Waterways Experiment Station

1399

Rome Air Development Center

FLC

1323

Naval Weapons Station Earle

1300

Beltsville Agricultural Research Center 1247

Harry Diamond Lab

1154

Air Force Weapons Lab

Savannah River

1111

Savannah River Lab, SC

Savannah River Ecology Lab, GA

FAA Technical Center

1062

Frederick Cancer Research Facility

1043

1043

Brooks AFB, TX

School of Aerospace Medicine

1031

1026

Human Resources Lab

Centers for Disease Control, Atlanta

1026

Center for Infectious Diseases

Clinical Chemistry Division

$\begin{array}{ll}59 & \text { DOD/ARMY } \\ 60 & \text { DOD/ARMY } \\ 61 & \text { TVA } \\ 62 & \text { DOD/ARMY } \\ 63 & \text { DOD/ARMY } \\ 64 & \text { DOD/ARMY } \\ 65 & \text { DOD/ARMY }\end{array}$

Missile R\&D Command

1007

986

Yuma Proving

FLC

National Fertilizer Development Center

969

Walter Reed Army Institute of Research

913

Tank-Automotive Command

835

Electronic Proving Ground

800

Fort Detrick, MD

Medical Research Inst. of Infectious Diseases

792

FLC

FLC

Medical R\&D Command

Medical Bioengineering R\&D Lab

66 DOD/NAVY

Dugway Proving Grounds

Naval Coastal Systems Center

Solar Energy Research Institute

Research Triangle Park, NC

Health Effects Research Lab

Industrial Environmental Research Lab

Environmental Science Research Lab

Environmental Monitoring Systems Lab

$70 \quad$ NSF

71 DOD/ARMY

National Center for Atmospheric Research

778

732

725

719

FLC

Materials and Mechanics Research Center

Geophyics Laboratory

Transportation Systems Center

Air Propulsion Center

Aviation Research and Technology Lab

DOT

DOD/NAVY

DOD/ARMY 
TABLE 4

FEDERAL LABORATORIES LISTED IN ORDER OF TOTAL STAFF SIZE

\begin{tabular}{|c|c|c|c|}
\hline $\begin{array}{l}\mathrm{SEQH1} \\
=====\end{array}$ & $\begin{aligned} & \text { AGENCY } \\
&=========\end{aligned}$ & 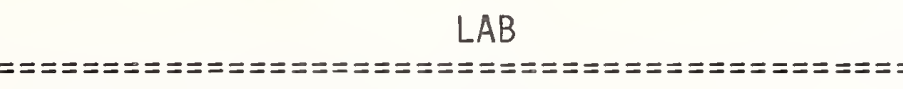 & $\begin{aligned} & \text { TOTAL } \\
&======\end{aligned}$ \\
\hline 1 & $\mathrm{DOE}$ & SANDIA NATIONAL LABORATORIES & 7639 \\
\hline 2 & DOE & LAWRENCE LIVERMORE NATIONAL LABORATORY & 7102 \\
\hline 3 & $\mathrm{DOE}$ & LOS ALAMOS NATIONAL LABORATORY & 6937 \\
\hline 4 & $\mathrm{DOD} / \mathrm{AF}$ & ARMAMENT DIVISION & 6389 \\
\hline 5 & $\mathrm{DOE}$ & OAK RIDGE NATIONAL LABORATORY & 5175 \\
\hline 6 & $\mathrm{DOD} / \mathrm{AF}$ & FLIGHT TEST CENTER & 5136 \\
\hline 7 & DOE & ARGONNE NATIONAL LABORATORY & 5118 \\
\hline 8 & DOE & IDAHO NATIONAL ENGINEER ING LABORATORY & 5100 \\
\hline 9 & DOD/NAVY & PACIFIC MISSILE TEST CENTER & 4803 \\
\hline 10 & DOD/NAVY & SURFACE WEAPONS CENTER & 4732 \\
\hline 11 & DOD/NAVY & NAVAL WEAPONS CENTER & 4540 \\
\hline 12 & DOI/USGS & USGS CENTRAL REGION & 4500 \\
\hline 13 & DOE & BROOKHAVEN NATIONAL LABORATORY & 4102 \\
\hline 14 & NASA & JET PROPULSION LABORATORY & 3788 \\
\hline 15 & DOI/USGS & USGS WESTERN REGION & 3676 \\
\hline 16 & DOD/ARMY & WHITE SANDS MISSILE RANGE & 3672 \\
\hline 17 & DOD/NAVY & AIR TEST CENTER & 3566 \\
\hline 18 & NASA & GEORGE C. MARSHALL SPACE FLIGHT CENTER & 3563 \\
\hline 19 & NASA & LYNDON B.JOHNSON SPACE CENTER & 3508 \\
\hline 20 & NASA & GODDARD SPACE FLIGHT CENTER & 3436 \\
\hline 21 & DOD/NAVY & RESEARCH LABORATORY & 3394 \\
\hline 22 & DOE & BETTIS ATOMIC POWER LABORATORY & 3358 \\
\hline 23 & DOE & LAWRENCE BERKELEY LABORATORY & 3158 \\
\hline 24 & $\mathrm{DOC}$ & NATIONAL BUREAU OF STANDARDS & 3072 \\
\hline 25 & DOI /USGS & USGS NATIONAL CENTER & 3000 \\
\hline 26 & NASA & LANGLEY RESEARCH CENTER & 2966 \\
\hline 27 & DOE & KNOLLS ATOMIC POWER LABORATORY & 2913 \\
\hline 28 & NASA & LEWIS RESEARCH CENTER & 2822 \\
\hline 29 & DOD/NAVY & NAVAL UNDERWATER SYSTEMS C.ENTER & 2800 \\
\hline 30 & $\mathrm{DOD} / \mathrm{NAVY}$ & OCEAN SYSTEMS CENTER & 2791 \\
\hline 31 & DOD/NAVY & DAVID TAYLOR NAVAL SHIP R AND D CENTER & 2567 \\
\hline 32 & DOD/NAVY & NAVAL AIR DEVELOPMENT CENTER & 2399 \\
\hline 33 & NASA & JOHN F. KENNEDY SPACE CENTER & 2201 \\
\hline 34 & DOD/NAVY & NAVAL AVIONIC CENTER & 2150 \\
\hline 35 & DOE & PACIF IC NORTHWEST LABORATORY & 2084 \\
\hline 36 & DOE & FERMI NATIONAL ACCELERATOR LABORATORY & 1982 \\
\hline 37 & DOE & HANFORD ENGINEERING DEVELOPMENT LABORATORY & 1926 \\
\hline 38 & DOD/ARMY & LARGE CALIBER WEAPONS SYSTEM LABORATORY & 1791 \\
\hline 39 & DOD/NAVY & NAVAL AIR ENGINEERING CENTER & 1776 \\
\hline 40 & NASA & AMES RESEARCH CENTER & 1651 \\
\hline 41 & HHS & NATIONAL CANCER INSTITUTE & 1606 \\
\hline 42 & DOE & PRINCETON PLASMA PHYSICS LABORATORY & 1406 \\
\hline 43 & $\mathrm{DOE}$ & STANFORD LINEAR ACCELERATOR CENTER & 1402 \\
\hline 44 & DOD/ARMY & ENGINEER WATERWAYS EXPERIMENT STATION & 1399 \\
\hline 45 & $\mathrm{DOD} / \mathrm{AF}$ & ROME AIR DEVELOPMENT CENTER & 1323 \\
\hline 46 & DOD/NAVY & NAVAL WEAPONS STATION EARLE & 1300 \\
\hline 47 & DOD/ARMY & MOBILITY EQUIPMENT R\&D COMMAND & 1262 \\
\hline 48 & USDA/ARS & BELTSVILLE AGRICULTURAL RESEARCH CENTER & 1247 \\
\hline 49 & DOD/ARMY & NATICK R\&D COMMAND & 1187 \\
\hline 50 & DOD/ARMY & HARRY DIAMOND LABORATORIES & 1154 \\
\hline
\end{tabular}


FEDERAL LABORATORIES LISTED IN ORDER OF TOTAL STAFF SIZE(CONT)

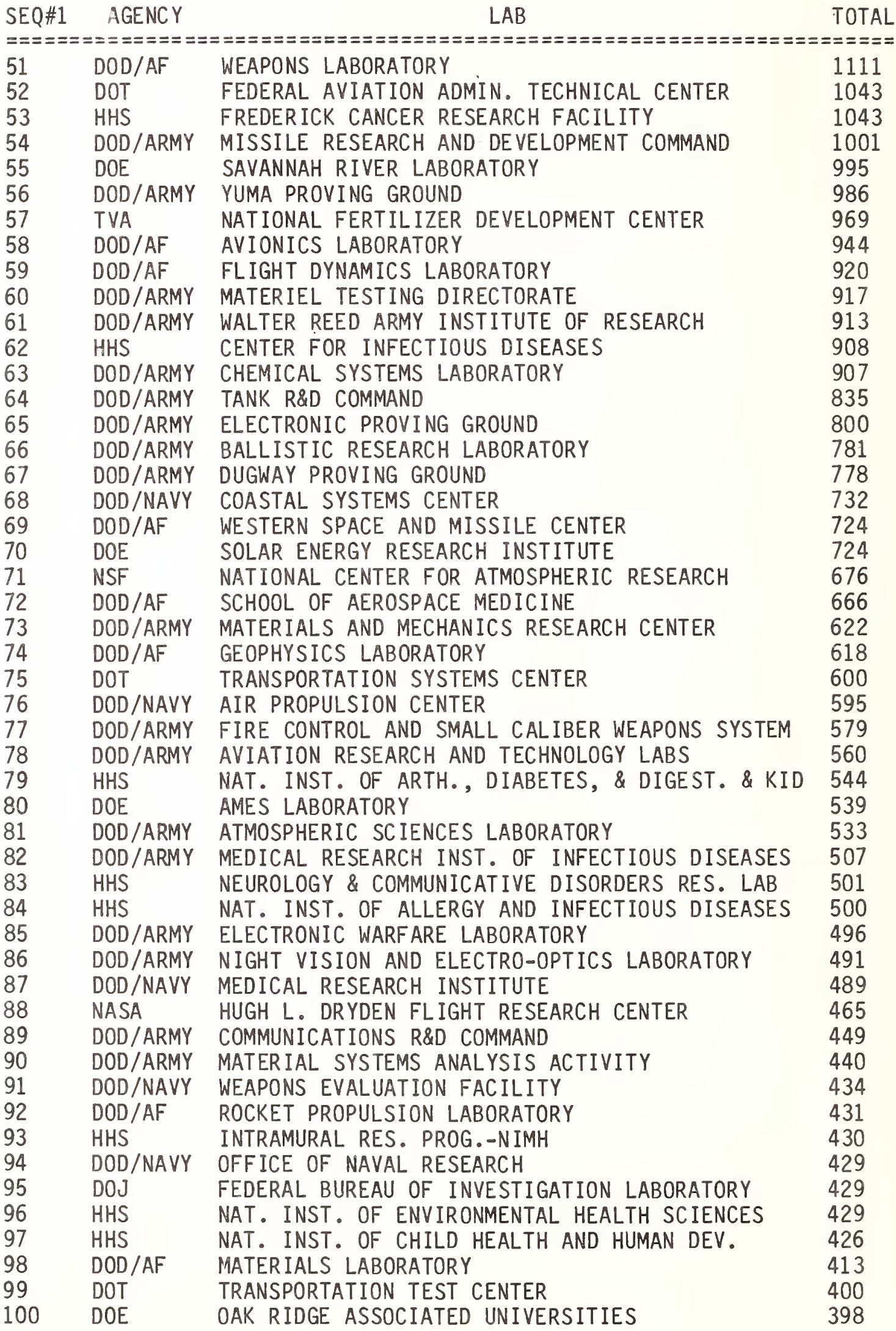


FEDERAL LABORATORIES LISTED IN ORDER OF TOTAL STAFF SIZE(CONT)

\begin{tabular}{|c|c|c|c|}
\hline $\begin{array}{l}\text { SEQ\# } 1 \\
======\end{array}$ & $\begin{array}{l}\text { AGENCY } \\
=======\end{array}$ & 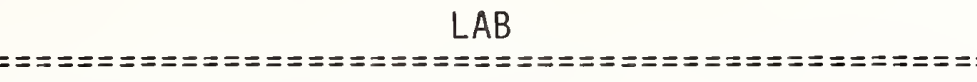 & \\
\hline 101 & USDA/FS & NORTHEASTERN FOREST EXPERIMENT STATION & 396 \\
\hline 102 & NASA & WALLOPS FLIGHT CENTER & 382 \\
\hline 103 & DOD/AF & HUMAN RESOURCES LABORATORY & 365 \\
\hline 104 & HHS & NATIONAL HEART, LUNG, AND BLOOD INSTITUTE & 362 \\
\hline 105 & DOD/ARMY & RES. INST. FOR BEHAVIORAL AND SOCIAL SCIENCES & 358 \\
\hline 106 & DOE & PITTSBURGH ENERGY TECHNOLOGY CENTER & 352 \\
\hline 107 & USDA/FS & NORTH CENTRAL EXPERIMENT STATION & 346 \\
\hline 108 & USDA/FS & FOREST PRODUCTS LABORATORY & 339 \\
\hline 109 & USDA/ARS & PLUM ISLAND ANIMAL DISEASE CENTER & 337 \\
\hline 110 & NSF & NATIONAL RADIO ASTRONOMY OBSERVATORY & 332 \\
\hline 111 & DOE & ENERGY TECHNOLOGY ENGINEERING CENTER & 330 \\
\hline 112 & EPA & HEALTH EFFECTS RESEARCH LABORATORY & 328 \\
\hline 113 & DOD/NAVY & CIVIL ENGINEERING LABORATORY & 325 \\
\hline 114 & DGD /ARMY & AVIATION DEVELOPMENT TEST ACTIVITY & 324 \\
\hline 115 & USDA/ARS & WESTERN REGIONAL RESEARCH CENTER & 322 \\
\hline 116 & USDA/ARS & EASTERN REGIONAL RESEARCH CENTER & 322 \\
\hline 117 & USDA/FS & SOUTHERN FOREST EXPERIMENT STATION & 322 \\
\hline 118 & TVA & ENERGY DEMONSTRATIONS AND TECHNOLOGY & 321 \\
\hline 119 & DOD/ARMY & ENGINEER TOPOGRAPHIC LABORATORIES & 314 \\
\hline 120 & USDA/ARS & NORTHERN REGIONAL RESEARCH CENTER & 306 \\
\hline 121 & DOD/ARMY & COLD REGIONS TEST CENTER & 304 \\
\hline 122 & DOD/NAVY & PERSONNEL RESEARCH AND DEVELOPMENT CENTER & 294 \\
\hline 123 & $\mathrm{DOD} / \mathrm{AF}$ & AEROSPACE MEDICAL RESEARCH LABORATORY & 289 \\
\hline 124 & NSF & KITT PEAK NATIONAL OBSERVATORY & 288 \\
\hline 125 & USDA/FS & SOUTHEASTERN FOREST EXPERIMENT STATION & 287 \\
\hline 126 & USDA/FS & PACIFIC NORTHWEST EXPERIMENT STATION & 282 \\
\hline 127 & DOD/ARMY & LETTERMAN ARMY INSTITUTE OF RESEARCH & 278 \\
\hline 128 & DOD/ARMY & ELECTRONICS TECHNOLOGY AND DEVICES LABORATORY & 278 \\
\hline 129 & DOD/ARMY & COLD REGIONS R\&D LABORATORY & 277 \\
\hline 130 & HHS & DIVISION OF COMPUTER RESEARCH AND TECHNOLOGY & 274 \\
\hline 131 & USDA/ARS & NATIONAL ANIMAL DISEASE CENTER & 272 \\
\hline 132 & DOD/NAVY & OCEAN R AND D ACTIVITY & 271 \\
\hline 133 & HHS & INTRAMURAL RES.PROG.-NIDR & 266 \\
\hline 134 & DOI/BOM & PITTSBURGH MINING AND SAFETY RES. CENTER & 259 \\
\hline 135 & USDA/FS & PACIFIC SOUTHWEST FOREST EXPERIMENT STATION & 255 \\
\hline 136 & DOE & MORGANTOWN ENERGY TECHNOLOGY CENTER & 251 \\
\hline 137 & USDA/FS & INTERMOUNTAIN FOREST AND RANGE EXP. STA. & 240 \\
\hline 138 & HHS & APPALACHIAN LAB FOR OCC. SAFETY AND HEALTH & 239 \\
\hline 139 & HHS & GERENTOLOGY RES CENTER-NAT. INST. ON AGING & 238 \\
\hline 140 & DOD/ARMY & SIGNALS WARFARE LABORATORY & 237 \\
\hline 141 & DOI & ENGINEERING AND RESEARCH LABORATORY & 230 \\
\hline 142 & USDA/FS & ROCKY MOUNTAIN FORESTRY AND RANGE EXP. STATION & 230 \\
\hline 143 & DOD/ARMY & INSTITUTE OF SURGICAL RESEARCH & 229 \\
\hline 144 & $\mathrm{DOD} / \mathrm{AF}$ & ARNOLD ENGINEERING DEVELOPMENT CENTER & 223 \\
\hline 145 & DOD/ARMY & AVIONICS R\&D ACTIVITY & 223 \\
\hline 146 & DOD & ARMED FORCES RADIOBIOLOGY RESEARCH LAB & 216 \\
\hline 147 & DOE & INHALATION TOXICOLOGY RESEARCH INSTITUTE & 212 \\
\hline 148 & DOI/FWS & DENVER WILDLIFE RESEARCH CENTER & 208 \\
\hline 149 & DOD/ARMY & CONSTRUCTION ENGINEERING RESEARCH LABORATORY & 207 \\
\hline 150 & DOD/NAVY & EXPLOSIVE ORDNANCE DISPOSAL FACILITY & 201 \\
\hline
\end{tabular}


FEDERAL LABORATORIES LISTED IN ORDER OF TOTAL STAFF SIZE(CONT)

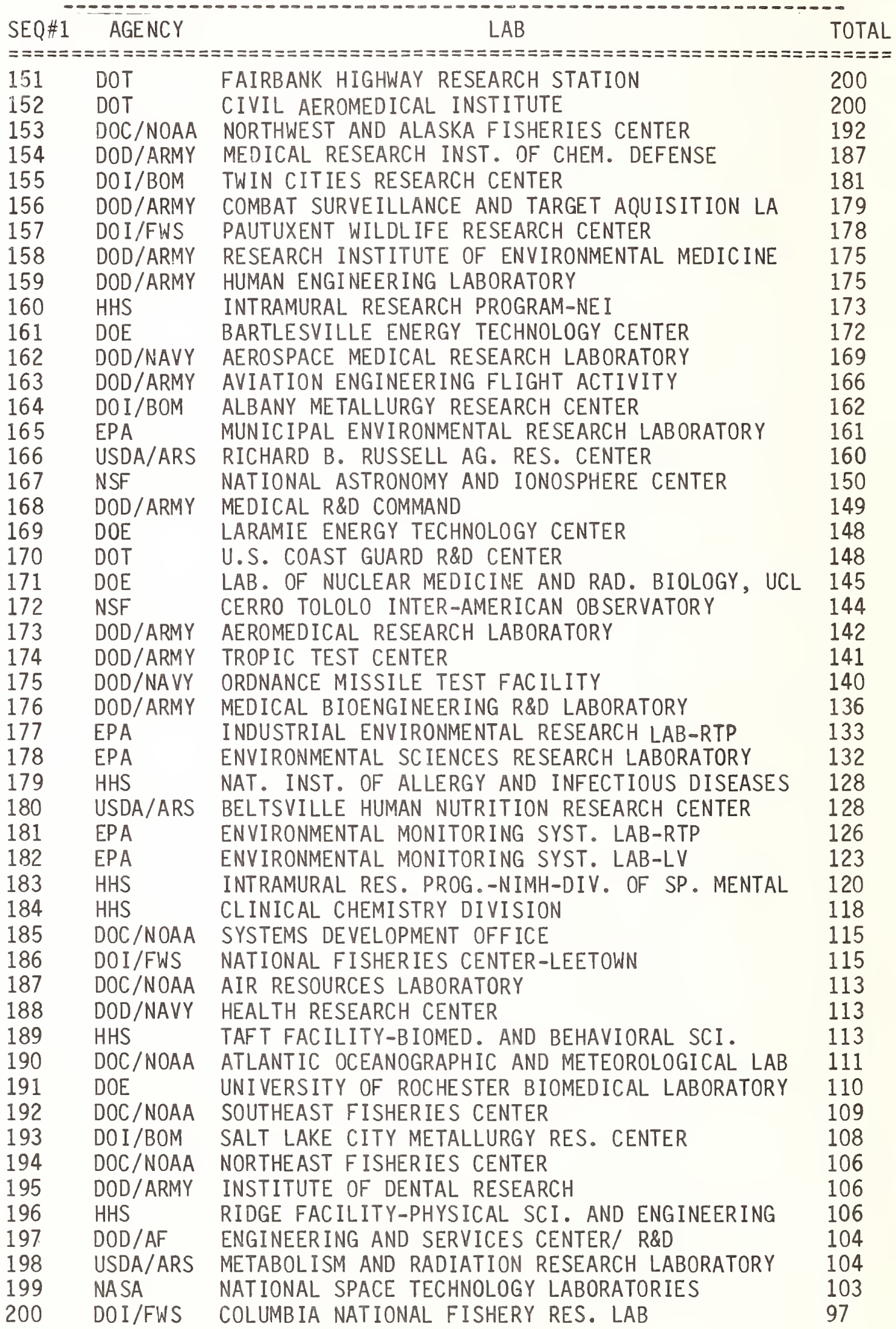


FEDERAL LABORATORIES LISTED IN ORDER OF TOTAL STAFF SIZE(CONT)

\begin{tabular}{|c|c|c|c|}
\hline $\begin{array}{l}\text { SEQ\#1 } \\
=====\end{array}$ & $\begin{array}{c}\text { AGENCY } \\
=======\end{array}$ & 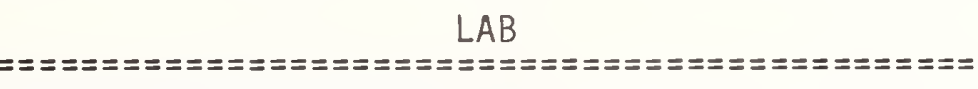 & $\begin{array}{l}\text { TOTAL } \\
======\end{array}$ \\
\hline 201 & DOD/ARMY & ARMY RESEARCH OFFICE & 96 \\
\hline 202 & DOE & ENVIRONMENTAL MEASUREMENT LABORATORY & 95 \\
\hline 203 & $\mathrm{DOC}$ & INSTITUTE FOR TELECOMMUNICATION SCIENCES & 94 \\
\hline 204 & DOD/NAVY & SUBMARINE MEDICAL RESEARCH LABORATORY & 94 \\
\hline 205 & $\mathrm{DOI} / \mathrm{BOM}$ & DENVER MINING RESEARCH CENTER & 93 \\
\hline 206 & DOE & COMPARATIVE ANIMAL RESEARCH LAB & 92 \\
\hline 207 & $\mathrm{DOI} / \mathrm{BOM}$ & SPOKANE MINING RESEARCH CENTER & 90 \\
\hline 208 & DO I/FWS & GREAT LAKES FISHERY LAB & 89 \\
\hline 209 & USDA/ARS & SOUTHEAST WATERSHED RESEARCH LABORATORY & 87 \\
\hline 210 & $\mathrm{DOI} / \mathrm{BOM}$ & AVONDALE METALLURGY RESEARCH CENTER & 86 \\
\hline 211 & SMITHSON & NATIONAL MUSEUM OF NATURAL HISTORY & 85 \\
\hline 212 & EPA & ENVIRONMENTAL RESEARCH LAB-CORVALLIS & 84 \\
\hline 213 & EPA & ENVIRONMENTAL RESEARCH LAB-DULUTH & 83 \\
\hline 214 & DOD/NAVY & UNDERSEA RANGES DEPARTMENT & 82 \\
\hline 215 & TVA & ENGINEER ING LABORATORY & 82 \\
\hline 216 & DOD/NAVY & MEDICAL RESEARCH UNIT NO. 3 & 81 \\
\hline 217 & DOC/NOAA & GEOPHYSICAL FLUID DYNAMICS LABORATORY & 80 \\
\hline 218 & EPA & ENVIRONMENTAL MONITORING SUPPORT LABORATORY & 79 \\
\hline 219 & EPA & ENVIRONMENTAL RESEARCH LABORATORY -ATHENS & 78 \\
\hline 220 & USDA/ARS & INSECT ATTRACTANTS, BEHAVIOR \& BASIC BIO. RES. & 78 \\
\hline 221 & $\mathrm{DOI} / \mathrm{BOM}$ & RENO METALLURGY RESEARCH CENTER & 76 \\
\hline 222 & DOC/NOAA & SANDY HOOK LABORATORY & 75 \\
\hline 223 & DOE & BATES LINEAR ACCELERATOR FACILITY & 75 \\
\hline 224 & EPA & INDUSTRIAL ENVIRONMENTAL RESEARCH LAB-CIN. & 74 \\
\hline 225 & $\mathrm{DOC} / \mathrm{NOAA}$ & SOUTHWEST FISHERIES CENTER & 73 \\
\hline 226 & SMITHSON & SMITHSONIAN ASTROPHYSICAL OBSERVATORY & 72 \\
\hline 227 & DOE & NOTRE DAME RADIATION LABORATORY & 71 \\
\hline 228 & DOC/NOAA & WAVE PROPAGATION LABORATORY & 70 \\
\hline 229 & DOE & SAVANNAH RIVER ECOLOGY LABORATORY & 69 \\
\hline 230 & DOC/NOAA & SPACE ENVIRONMENT LABORATORY & 68 \\
\hline 231 & DOE & GRAND FORKS ENERGY TECHNOLOGY CENTER & 68 \\
\hline 232 & DOC/NOAA & OFFICE OF WEATHER RESEARCH AND MODIFICATION & 66 \\
\hline 233 & $\mathrm{DOI} / \mathrm{BOM}$ & ROLLA METALLURGY RESEARCH CENTER & 65 \\
\hline 234 & USDA/ARS & U.S. GRAIN MARKETING RESEARCH CENTER & 64 \\
\hline 235 & USDA/ARS & U.S. HORTICULTURAL LABORATORY & 62 \\
\hline 236 & USDA/ARS & CROPS RESEARCH LABORATORY & 62 \\
\hline 237 & DOE & NEW BRUNSWICK LABORATORY & 60 \\
\hline 238 & SMITHSON & RADIATION BIOLOGY LABORATORY & 60 \\
\hline 239 & DOI/FWS & NORTHERN PRAIRIE WILDLIFE RESEARCH CENTER & 57 \\
\hline 240 & DOC/NOAA & BEAUFORT LABORATORY & 56 \\
\hline 241 & DOD/NAVY & CLOTHING AND TEXTILE RESEARCH FACILITY & 56 \\
\hline 242 & USDA/ARS & REGIONAL POULTRY RESEARCH LABORATORY & 56 \\
\hline 243 & USDA/ARS & ROMAN L. HRUSKA U.S. MEAT ANIMAL RES. LAB & 56 \\
\hline 244 & EPA & ROBERT S. KERR ENVIRONMENTAL RESEARCH LAB & 55 \\
\hline 245 & EPA & ENVIRONMENTAL RESEARCH LAB-RI & 55 \\
\hline 246 & DOE & RADIOBIOLOGY LABORATORY, UNIV. OF UTAH & 54 \\
\hline 247 & EPA & ENVIRONMENTAL RESEARCH LAB-SABINE & 54 \\
\hline 248 & USDA/ARS & INSECTS AFFECTING MAN AND ANIMALS RES. LAB & 54 \\
\hline 249 & DOC/NOAA & PACIF IC MARINE ENVIRONMENTAL LABORATORY & 53 \\
\hline 250 & $\mathrm{DOI} / \mathrm{BOM}$ & TUSCALOOSA METALLURGY RESEARCH CENTER & 53 \\
\hline
\end{tabular}


FEDERAL LABORATORIES LISTED IN ORDER OF TOTAL STAFF SIZE(CONT)

\begin{tabular}{|c|c|c|c|}
\hline $\begin{array}{l}\text { SEQ\#1 } \\
=====\end{array}$ & $\begin{aligned} & \text { AGENCY } \\
======= & =\end{aligned}$ & 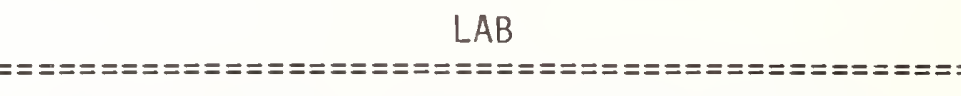 & $\begin{aligned} & \text { TOTAL } \\
&======\end{aligned}$ \\
\hline 251 & DOI/FWS & SEATTLE NATIONAL FISHERY RESEARCH CENTER & 53 \\
\hline 252 & USDA/ARS & STORED-PRODUCTS INSECTS RES. \& DEV. LAB & 52 \\
\hline 253 & NSF & SACRAMENTO PEAK OBSERVATORY & 51 \\
\hline 254 & DOC/NOAA & RESEARCH FACILITIES CENTER & 50 \\
\hline 255 & DOE & MICH STATE UNIV./DOE PLANT RESEARCH LAB & 50 \\
\hline 256 & DOE & CENTER FOR ENERGY AND ENVIRONMENTAL RESEARCH & 50 \\
\hline 257 & USDA/ARS & VETERINARY TOXICOLOGY AND ENTOMOLOGY LAB & 48 \\
\hline 258 & DOC/NOAA & AUKE BAY LABORATORY & 47 \\
\hline 259 & DOC/NOAA & GREAT LAKES ENVIRONMENTAL RESEARCH LAB & 47 \\
\hline 260 & DOD/NAVY & DENTAL RESEARCH INSTITUTE NTC & 47 \\
\hline 261 & USDA/ARS & SNAKE RIVER CONSERVATION RESEARCH CENTER & 47 \\
\hline 262 & USDA/ARS & TROPICAL FRUIT AND VEGETABLE RES. FACILITY & 46 \\
\hline 263 & USDA/ARS & ARTHROPOD-BORNE ANIMAL DISEASES RES. LAB & 45 \\
\hline 264 & $\mathrm{DOC} / \mathrm{NOAA}$ & AERONOMY LABORATORY & 44 \\
\hline 265 & USDA/ARS & OILSEED AND FOOD LABORATORY & 44 \\
\hline 266 & USDA/ARS & SEDIMENTATION LABORATORY & 43 \\
\hline 267 & USDA/ARS & U. S. LIVESTOCK INSECTS LABORATORY & 43 \\
\hline 268 & DOE & LAB OF RADIOBIOLOGY AND ENV. HEALTH/ UC & 42 \\
\hline 269 & USDA/ARS & WESTERN COTTON RESEARCH LABORATORY & 42 \\
\hline 270 & USDA/ARS & NORTHERN GREAT PLAINS RESEARCH CENTER & 42 \\
\hline 271 & DOC/NOAA & HONOLULU LABORATORY & 41 \\
\hline 272 & DOC/NOAA & NATIONAL SEVERE STORMS LABORATORY & 41 \\
\hline 273 & DOI/FWS & NATIONAL FISHERY RESEARCH LAB-LA CROSSE & 41 \\
\hline 274 & USDA/ARS & BOLL WEEVIL RESEARCH LABORATORY & 41 \\
\hline 275 & DOE & LAB. FOR ENERGY-RELATED HEALTH RES., UC/DAVIS & 40 \\
\hline 276 & USDA/ARS & U.S.WATER CONSERVATION LABORATORY & 40 \\
\hline 277 & USDA/ARS & APPALACHIAN FRUIT RESEARCH STATION & 38 \\
\hline 278 & DOC/NOAA & SATELLITE EXPER IMENT LABORATORY & 37 \\
\hline 279 & USDA/ARS & COTTON TEXTILE PROCESSING LABORATORY & 37 \\
\hline 280 & DOC/NOAA & MISSISSIPPI LABORATORIES & 36 \\
\hline 281 & USDA/ARS & TOBACCO RESEARCH LABORATORY & 36 \\
\hline 282 & DOI/BOM & BOULDER CITY METALLURGY ENGINEER ING LAB & 35 \\
\hline 283 & USDA/ARS & SOUTHEAST POULTRY RESEARCH LABORATORY & 35 \\
\hline 284 & USDA/ARS & BIOENVIRONMENTAL INSECTS CONTROL LABORATORY & 35 \\
\hline 285 & $\mathrm{DOC} / \mathrm{NOAA}$ & CHARLESTON LABORATORY & 34 \\
\hline 286 & DOT & VEHICLE RESEARCH AND TEST CENTER & 34 \\
\hline 287 & USDA/ARS & COMPOSITION AND PROPERTIES LABORATORY & 34 \\
\hline 288 & USDA/ARS & SOUTHERN WEED SCIENCE LABORATORY & 34 \\
\hline 289 & $\mathrm{DOD} / \mathrm{AF}$ & FRANK J. SEIBLER RESEARCH LABORATORY & 33 \\
\hline 29 & USDA/ARS & LIVESTOCK INSECTS RESEARCH LABORATORY & 33 \\
\hline 291 & SMITHSON & SMITHSONIAN TROPICAL RESEARCH INST. & 32 \\
\hline 292 & TYA & AIR RESOURCES PROGRAM-RESEARCH SECTION & 32 \\
\hline 293 & USDA/ARS & SOUTHEASTERN FRUIT \& TREE NUT RES. LAB & 32 \\
\hline 294 & USDA/ARS & CROP SCIENCE AND ENGINEERING RES. LABORATORY & 32 \\
\hline & USDA/ARS & WATER QUAL ITY MANAGEMENT LABORATORY & 32 \\
\hline & USDA/ARS & GRASSLAND, SOIL AND WATER RESEARCH LABORATORY & 32 \\
\hline 297 & USDA/ARS & YAKIMA AGRICULTURAL RESEARCH LABORATORY & 31 \\
\hline 298 & DOC/NOAA & GALVESTON LABORATORY & 30 \\
\hline & DOI/FWS & NATIONAL WILDL IFE HEALTH LABORATORY & 30 \\
\hline & USDA/ARS & SOUTHERN PIEDMONT CONSERVATION LABORATORY & 30 \\
\hline
\end{tabular}


FEDERAL LABORATORIES LISTED IN ORDER OF TOTAL STAFF SIZE(CONT)

\begin{tabular}{|c|c|c|c|}
\hline $\begin{array}{l}\text { SEQ\#1 } \\
=====\end{array}$ & $\begin{array}{l}\text { AGENCY } \\
:=======\end{array}$ & 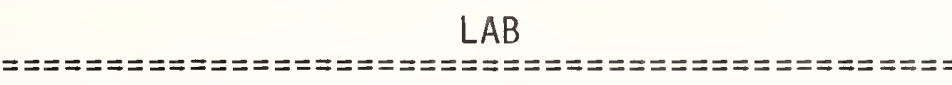 & $\underset{:=====}{\text { TOTAL }}$ \\
\hline 301 & USDA/ARS & U.S. SUGARCANE FIELD LABORATORY & 30 \\
\hline 302 & USDA/ARS & NATURAL POLYMERS LABORATORY & 30 \\
\hline 303 & USDA/ARS & ENGINEERING AND DEVELOPMENT LABORATORY & 29 \\
\hline $\begin{array}{l}304 \\
305\end{array}$ & DOC/NOAA & GLOUCE STER LABORATORY & $\begin{array}{l}28 \\
28\end{array}$ \\
\hline $\begin{array}{l}305 \\
306\end{array}$ & $\begin{array}{l}\text { USDA/ARS } \\
\text { USDA/ARS }\end{array}$ & $\begin{array}{l}\text { U.S. VEGETABLE LABORATORY } \\
\text { NORTHERN GRAIN INSECTS RESEARCH LABORATORY }\end{array}$ & $\begin{array}{l}28 \\
28\end{array}$ \\
\hline 307 & USDA/ARS & CONSERVATION AND PRODUCTION RESEARCH LAB & 28 \\
\hline 308 & DOC/NOAA & ENG INEER ING DEVELOPMENT OFFICE & 27 \\
\hline 309 & $\mathrm{DOC} / \mathrm{NOAA}$ & NATIONAL MARINE MAMMAL LABORATORY & 27 \\
\hline 310 & DOC/NOAA & MILFORD LABORATORY & 26 \\
\hline 311 & USDA/ARS & FRUIT AND VEGETABLE CHEM. RES. UNIT & 26 \\
\hline 312 & USDA/ARS & SOIL EROSION LABORATORY & 26 \\
\hline 313 & USDA/ARS & BIOLOGICAL CONTROL OF INSECTS RESEARCH LAB & 25 \\
\hline 314 & SMITHSON & CHESAPEAKE BAY CENTER FOR ENV. STUDIES & 24 \\
\hline 315 & USDA/ARS & COTTON TEXTILE CHEMISTRY LABORATORY & 24 \\
\hline 316 & DOC/NOAA & NARRAGANSETT LABORATORY & 23 \\
\hline 317 & USDA/ARS & COTTON RESEARCH LABORATORY & 23 \\
\hline 318 & DOC/NOAA & HYDROLOGICAL RESEARCH LABORATORY & 20 \\
\hline 319 & USDA/ARS & U.S. PLANT, SOIL, AND NUTRITION LABORATORY & 20 \\
\hline 320 & DOC/NOAA & TIBURON LABORATORY & 18 \\
\hline 321 & DOC/NOAA & PANAMA CITY LABORATORY & 18 \\
\hline 322 & SMITHSON & NATIONAL ZOOLOGICAL PARK AND CONS. CENTER & 18 \\
\hline 323 & USDA/ARS & DAIRY FORAGE RESEARCH CENTER & 18 \\
\hline 324 & USDA/ARS & CITRUS AND SUB-TROPICAL PRODUCTS LABORATORY & 17 \\
\hline 325 & HHS & CLINICAL RES. SEC. \& S.W. FIELD STUDIES SEC. & 16 \\
\hline 26 & USDA/ARS & WEED RESEARCH LABORATORY & \\
\hline 327 & DOC/NOAA & KODIAK LABORATORY & 15 \\
\hline 328 & DOC/NOAA & GEODETIC RESEARCH AND DEVELOPMENT LABORATORY & 15 \\
\hline 329 & VA & BIRMINGHAM VA MEDICAL CENTER & $10+$ \\
\hline 330 & VA & LITTLE ROCK VA MEDICAL CENTER & $10+$ \\
\hline 331 & VA & TUCSON VA MEDICAL CENTER & $10+$ \\
\hline 332 & VA & LONG BEACH VA MEDICAL CENTER & $10+$ \\
\hline 333 & VA & LOS ANGELES BRENTWOOD VA MEDICAL CENTER & $10+$ \\
\hline 334 & VA & LOS ANGELES WADSWORTH DIV. VA MEDICAL CENTER & $10+$ \\
\hline 35 & VA & MARTINEZ VA MEDICAL CENTER & $10+$ \\
\hline 36 & VA & PALO ALTO VA MEDICAL CENTER & $10+$ \\
\hline 37 & VA & SAN DIEGO VA MEDICAL CENTER & $10+$ \\
\hline 38 & VA & SAN FRANCISCO VA MEDICAL CENTER & $10+$ \\
\hline 339 & VA & SEPULVEDA VA MEDICAL CENTER & $10+$ \\
\hline 340 & VA & DENVER VA MEDICAL CENTER & $10+$ \\
\hline 341 & VA & WEST HAVEN VA MEDICAL CENTER & $10+$ \\
\hline 342 & VA & WASHINGTON VA MEDICAL CENTER & $10+$ \\
\hline 43 & VA & GAINESVILLE VA MEDICAL CENTER & $10+$ \\
\hline 344 & VA & MIAMI VA MEDICAL CENTER & $10+$ \\
\hline 345 & VA & TAMPA VA MEDICAL CENTER & $10+$ \\
\hline 346 & VA & AUGUST VA MEDICAL CENTER & $10+$ \\
\hline & VA & IOWA CITY VA MEDICAL CENTER & $10+$ \\
\hline & VA & CHICAGO LAKESIDE VA MEDICAL CENTER & $10+$ \\
\hline s & VA & CHICAGO WEST SIDE VA MEDICAL CENTER & $10+$ \\
\hline & VA & HINES VA MEDICAL CENTER & $10+$ \\
\hline
\end{tabular}


FEDERAL LABORATORIES LISTED IN ORDER OF TOTAL STAFF SIZE(CONT)

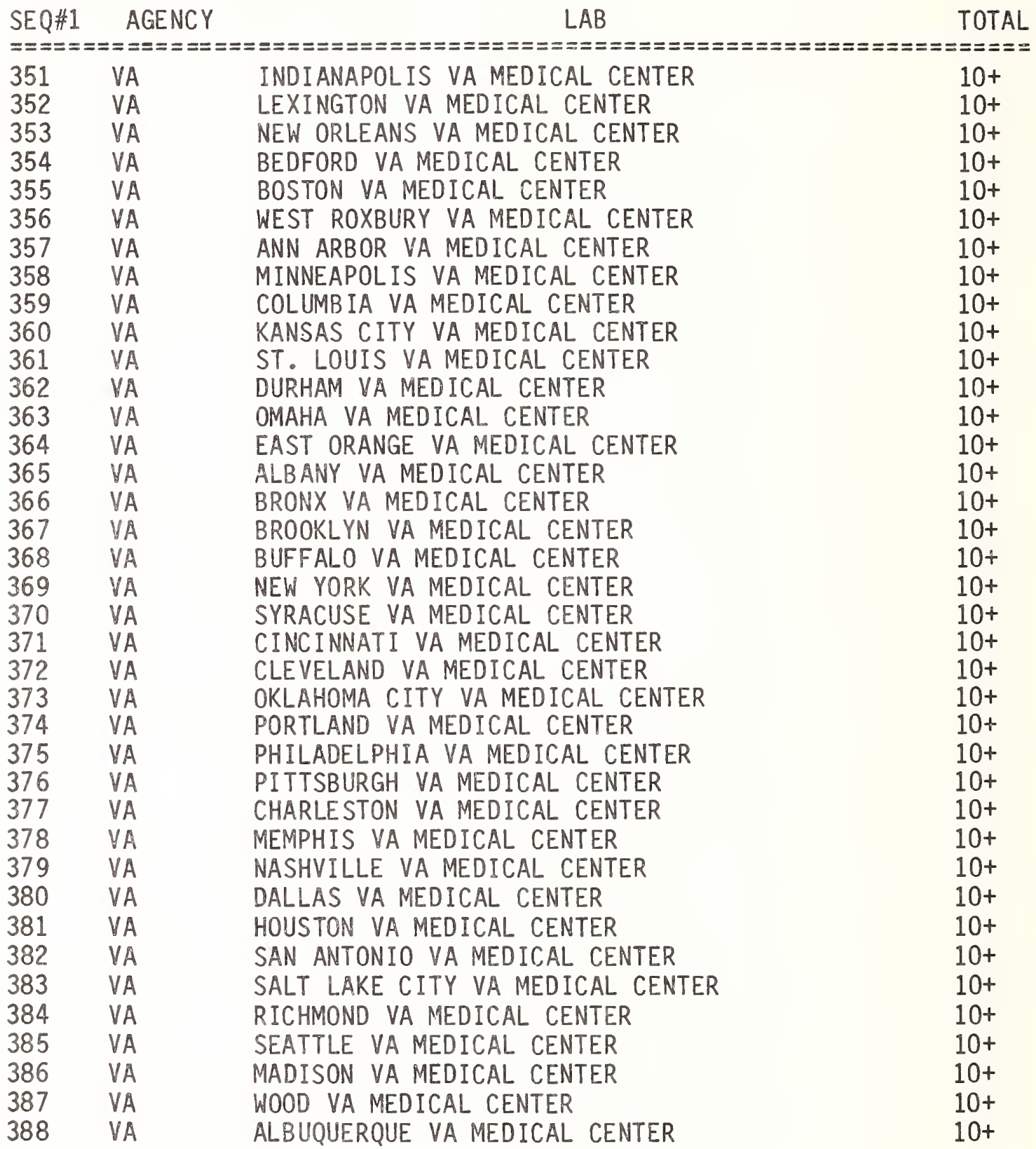


TABLE 5

FEDERAL LABORATORIES LISTED BY STATE

ST AGENCY

$L A B$

TOTAL

$===========$

AK DOC/NOAA

AK DOC/NOAA

AL NASA

AUKE BAY LABORATORY

47

KODIAK LABORATORY

15

AL DOD/ARMY

GEORGE C. MARSHALL SPACE FLIGHT CENTER

3563

MISSILE RESEARCH AND DEVELOPMENT COMMAND

1001

AL TVA

NATIONAL FERTILIZER DEVELOPMENT CENTER

969

AL DOD/ARMY

AVIATION DEVELOPMENT TEST ACTIVITY

324

AL DOD/ARMY

AEROMEDICAL RESEARCH LABORATORY

142

AL DOI/BOM

TUSCALOOSA METALLURGY RESEARCH CENTER

53

AL TVA

AIR RESOURCES PROGRAM-RESEARCH SECTION

32

BIRM INGHAM VA MEDICAL CENTER

$10+$

LITTLE ROCK VA MEDICAL CENTER

$10+$

AR VA

YUMA PROVING GROUND

AZ DOD/ARMY

ELECTRONIC PROVING GROUND

986

KITT PEAK NATIONAL OBSERVATORY

AZ NSF

800

WESTERN COTTON RESEARCH LABORATORY 42

AZ USDA/ARS

U.S. WATER CONSERVATION LABORATORY

40

CLINICAL RES. SEC. \& S.W. FIELD STUDIES SEC. 16

AZ HHS

$A Z \quad V A$

CA DOE

CA DOD /AF

TUCSON VA MEDICAL CENTER

LAWRENCE LIVERMORE NATIONAL LABORATORY 7102

FLIGHT TEST CENTER

CA DOD/NAVY

PACIFIC MISSILE TEST CENTER

NAVAL WEAPONS CENTER

JET PROPULSION LABORATORY

5136

4803

4540

3788

CA NASA

USGS WESTERN REGION

3676

LAWRENCE BERKELEY LABORATORY 3158

CA DOE

CA DOD/NAVY

CA NASA

OCEAN SYSTEMS CENTER

2791

AMES RESEARCH CENTER

1651

STANFORD LINEAR ACCELERATOR CENTER 1402

CA DOE

CA DOD/AF

WESTERN SPACE AND MISSILE CENTER

724

560

CA NASA

AVIATION RESEARCH AND TECHNOLOGY LABS

465

ROCKET PROPULSION LABORATORY 431

CA DOD/AF

ENERGY TECHNOLOGY ENGINEERING CENTER

330

CA DOD/NAVY

CIVIL ENGINEERING LABORATORY

325

CA USDA/ARS

WESTERN REGIONAL RESEARCH CENTER

322

PERSONNEL RESEARCH AND DEVELOPMENT CENTER 294

CA DOD/NAVY

LETTERMAN ARMY INSTITUTE OF RESEARCH

278

CA USDA/FS

PACIFIC SOUTHWEST FOREST EXPERIMENT STATION

255

AVIATION ENGINEERING FLIGHT ACTIVITY 166

CA DOD/ARMY

CA DOE

CA DOD/NAVY

LAB. OF NUCLEAR MEDICINE AND RAD. BIOLOGY, UCL

145

HEALTH RESEARCH CENTER

113

SOUTHWEST FISHERIES CENTER

73

LAB OF RADIOBIOLOGY AND ENV. HEALTH/ UC 42

CA DOE

LAB. FOR ENERGY-RELATED HEALTH RES., UC/DAVIS

40

FRUIT AND VEGETABLE CHEM. RES. UNIT 26

CA USDA/ARS

CA DOC/NOAA

TIBURON LABORATORY

18

LONG BEACH VA MEDICAL CENTER

$10+$

CA VA

LOS ANGELES BRENTWOOD VA MEDICAL CENTER

$10+$

CA VA

LOS ANGELES WADSWORTH DIV. VA MEDICAL CENTER

$10+$

CA VA

MARTINEZ VA MEDICAL CENTER

$10+$ 
FEDERAL LABORATORIES LISTED BY STATE(CONT)

\begin{tabular}{|c|c|c|c|}
\hline \multicolumn{4}{|c|}{ 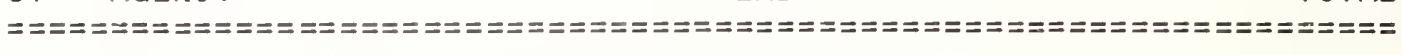 } \\
\hline CA & VA & PALO ALTO VA MEDICAL CENTER & $10+$ \\
\hline CA & VA & SAN DIEGO VA MEDICAL CENTER & $10+$ \\
\hline CA & VA & SAN FRANCISCO VA MEDICAL CENTER & $10+$ \\
\hline$C A$ & VA & SEPULVEDA VA MEDICAL CENTER & $10+$ \\
\hline $\mathrm{CH}$ & NSF & CERRO TOLOLO INTER-AMERICAN OBSERVATORY & 144 \\
\hline $\mathrm{CO}$ & DOI/USGS & USGS CENTRAL REGION & 4500 \\
\hline $\mathrm{CO}$ & DOE & SOLAR ENERGY RESEARCH INSTITUTE & 724 \\
\hline $\mathrm{CO}$ & NSF & NATIONAL CENTER FOR ATMOSPHERIC RESEARCH & 676 \\
\hline $\mathrm{CO}$ & DOT & TRANSPORTATION TEST CENTER & 400 \\
\hline $\mathrm{CO}$ & DOI & ENGINEER ING AND RESEARCH LABORATORY & 230 \\
\hline $\mathrm{CO}$ & USDA/FS & ROCKY MOUNTAIN FORESTRY AND RANGE EXP. STATION & 230 \\
\hline $\mathrm{CO}$ & DOI /FWS & DENVER WILDLIFE RESEARCH CENTER & 208 \\
\hline $\mathrm{CO}$ & $\mathrm{DOC}$ & INSTITUTE FOR TELECOMMUNICATION SCIENCES & 94 \\
\hline $\mathrm{CO}$ & $\mathrm{DOI} / \mathrm{BOM}$ & DENVER MINING RESEARCH CENTER & 93 \\
\hline $\mathrm{CO}$ & $\mathrm{DOC} / \mathrm{NOAA}$ & WAVE PROPAGATION LABORATORY & 70 \\
\hline $\mathrm{CO}$ & DOC/NOAA & SPACE ENVIRONMENT LABORATORY & 68 \\
\hline $\mathrm{CO}$ & DOC/NOAA & OFFICE OF WEATHER RESEARCH AND MODIFICATION & 66 \\
\hline $\mathrm{CO}$ & USDA/ARS & ARTHROPOD-BORNE ANIMAL DISEASES RES. LAB & 45 \\
\hline $\mathrm{CO}$ & DOC/NOAA & AERONOMY LABORATORY & 44 \\
\hline $\mathrm{CO}$ & $\mathrm{DOD} / \mathrm{AF}$ & FRANK J. SEIBLER RESEARCH LABORATORY & 33 \\
\hline $\mathrm{CO}$ & VA & DENVER VA MEDICAL CENTER & $10+$ \\
\hline CT & DOD/NAVY & NAVAL UNDERWATER SYSTEMS CENTER & 2800 \\
\hline CT & DOT & U.S. COAST GUARD R\&D CENTER & 148 \\
\hline $\mathrm{CT}$ & DOD / NAVY & SUBMARINE MEDICAL RESEARCH LABORATORY & 94 \\
\hline CT & DOE & BATES LINEAR ACCELERATOR FACILITY & 75 \\
\hline CT & DOC/NOAA & MILFORD LABORATORY & 26 \\
\hline CT & VA & WEST HAVEN VA MEDICAL CENTER & $10+$ \\
\hline $\mathrm{CZ}$ & DOD/ARMY & TROPIC TEST CENTER & 141 \\
\hline CZ & SMITHSON & SMITHSONIAN TROPICAL RESEARCH INST. & 32 \\
\hline DC & DOD/NAVY & RESEARCH LABORATORY & 3394 \\
\hline DC & DOD / ARMY & HARRY DIAMOND LABORATORIES & 1154 \\
\hline DC & DOD / ARMY & WALTER REED ARMY INSTITUTE OF RESEARCH & 913 \\
\hline DC & DOD / NAVY & OFFICE OF NAVAL RESEARCH & 429 \\
\hline DC & DOJ & FEDERAL BUREAU OF INVESTIGATION LABORATORY & 429 \\
\hline DC & HHS & INTRAMURAL RES. PROG.-NIMH-DIV. OF SP. MENTAL & 120 \\
\hline DC & DOD/ARMY & INSTITUTE OF DENTAL RESEARCH & 106 \\
\hline DC & SMITHSON & NATIONAL MUSEUM OF NATURAL HISTORY & 85 \\
\hline DC & SMITHSON & NATIONAL ZOOLOGICAL PARK AND CONS. CENTER & 18 \\
\hline DC & VA & WASHINGTON VA MEDICAL CENTER & $10+$ \\
\hline$E G$ & DOD / NAVY & MEDICAL RESEARCH UNIT NO. 3 & 81 \\
\hline $\mathrm{FL}$ & $\mathrm{DOD} / \mathrm{AF}$ & ARMAMENT DIVISION & 6389 \\
\hline $\mathrm{FL}$ & NASA & JOHN F. KENNEDY SPACE CENTER & 2201 \\
\hline $\mathrm{FL}$ & DOD/NAVY & COASTAL SYSTEMS CENTER & 732 \\
\hline $\mathrm{FL}$ & DOD/NAVY & AEROSPACE MEDICAL RESEARCH LABORATORY & 169 \\
\hline $\mathrm{FL}$ & DOC/NOAA & ATLANTIC OCEANOGRAPHIC AND METEOROLOGICAL LAB & 111 \\
\hline FL & DOC/NOAA & SOUTHEAST FISHERIES CENTER & 109 \\
\hline FL & $\mathrm{DOD} / \mathrm{AF}$ & ENGINEERING AND SERVICES CENTER/ R\&D & 104 \\
\hline $\mathrm{FL}$ & USDA/ARS & INSECT ATTRACTANTS, BEHAVIOR \& BASIC BIO. RES. & 78 \\
\hline $\mathrm{FL}$ & USDA/ARS & U.S. HORTICULTURAL LABORATORY & 62 \\
\hline $\mathrm{FL}$ & EPA & ENVIRONMENTAL RESEARCH LAB-SABINE & 54 \\
\hline
\end{tabular}


FEDERAL LABORATORIES LISTED BY STATE(CONT)

ST AGENCY

$\mathrm{LAB}$

TOTAL

$=========2.0=0$

INSECTS AFFECTING MAN AND ANIMALS RES. LAB

54

FL DOC/NOAA

RESEARCH FACILITIES CENTER

50

FL DOC/NOAA

PANAMA CITY LABORATORY

FL USDA/ARS

CITRUS AND SUB-TROPICAL PRODUCTS LABORATORY

17

FL VA

FL VA

GAINESVILLE VA MEDICAL CENTER

MIAMI VA MEDICAL CENTER

FL VA

GA HHS

TAMPA VA MEDICAL CENTER

CENTER FOR INFECTIOUS DISEASES

$10+$

$10+$

$10+$

908

GA USDA/ARS

GA HHS

RICHARD B. RUSSELL AG. RES. CENTER

160

CLINICAL CHEMISTRY DIVISION

118

SOUTHEAST WATERSHED RESEARCH LABORATORY

87

ENVIRONMENTAL RESEARCH LABORATORY -ATHENS

78

SAVANNAH RIVER ECOLOGY LABORATORY

69

DOE

STORED-PRODUCTS INSECTS RES \& \& DEV. LAB

52

GA USDA/ARS

SOUTHEAST POULTRY RESEARCH LABORATORY

35

GA USDA/ARS

SOUTHEASTERN FRUIT \& TREE NUT RES. LAB

32

SOUTHERN PIEDMONT CONSERVATION LABORATORY

30

$10+$

GA VA

AUGUST VA MEDICAL CENTER

HI USDA/ARS

TROPICAL FRUIT AND VEGETABLE RES. FACILITY

46

HI DOC/NOAA

IA DOE

HONOLULU LABORATORY

41

AMES LABORATORY

539

272

NATIONAL ANIMAL DISEASE CENTER

$10+$

IDAHO NATIONAL ENGINEERING LABORATORY

5100

ID DOE

SNAKE RIVER CONSERVATION RESEARCH CENTER

47

ARGONNE NATIONAL LABORATORY

5118

FERMI NATIONAL ACCELERATOR LABORATORY

1982

306

IL USDA/ARS

NORTHERN REGIONAL RESEARCH CENTER

207

NEW BRUNSWICK LABORATORY

60

IL DOE

IL DOD/NAVY

DENTAL RESEARCH INSTITUTE NTC

47

CHICAGO LAKESIDE VA MEDICAL CENTER

$10+$

CHICAGO WEST SIDE VA MEDICAL CENTER

$10+$

HINES VA MEDICAL CENTER

$10+$

NAVAL AVIONIC CENTER

2150

NOTRE DAME RADIATION LABORATORY

71

SOIL EROSION LABORATORY

26

INDIANAPOL IS VA MEDICAL CENTER

$10+$

64

KS USDA/ARS

U.S. GRAIN MARKETING RESEARCH CENTER

$10+$

LEXINGTON VA MEDICAL CENTER

322

SOUTHERN FOREST EXPERIMENT STATION

OILSEED AND FOOD LABORATORY

44

37

COTTON TEXTILE PROCESSING LABORATORY

COMPOSITION AND PROPERTIES LABORATORY

30

30
29

NATURAL POLYMERS LABORATORY

24

$10+$

LA USDA/ARS

ENGINEERING AND DEVELOPMENT LABORATORY

COTTON TEXTILE CHEMISTRY LABORATORY

NEW ORLEANS VA MEDICAL CENTER

1187

MA DOD/ARMY NATICK R\&D COMMAND 
FEDERAL LABORATORIES LISTED BY STATE(CONT)

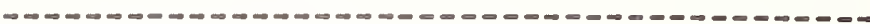

$$
\text { ST }
$$

AGENCY

$L A B$

TOTAL

$====\approx=======$
MA DOD/ARMY

MATERIALS AND MECHANICS RESEARCH CENTER

622

MA DOD/AF

GEOPHYSICS LABORATORY

618

MA DOT

TRANSPORTATION SYSTEMS CENTER

600

MA DOD/ARMY

RESEARCH INSTITUTE OF ENVIRONMENTAL MEDICINE

175

MA DOC/NOAA

NORTHEAST FISHERIES CENTER

106

MA SMITHSON

SMITHSONIAN ASTROPHYSICAL OBSERVATORY

72

MA DOD/NAVY

CLOTHING AND TEXTILE RESEARCH FACILITY

56

MA DOC/NOAA

GLOUCESTER LABORATORY

28

BEDFORD VA MEDICAL CENTER

$10+$

MA VA

BOSTON VA MEDICAL CENTER

WEST ROXBURY VA MEDICAL CENTER

$10+$

MA VA

SURFACE WEAPONS CENTER

$10+$

MD DOD/NAVY

MD DOD/NAVY

MD NASA

MD DOC

AIR TEST CENTER

GODDARD SPACE FLIGHT CENTER

4732

3566

NATIONAL BUREAU OF STANDARDS

3436

MD DOD/NAVY

MD HHS

DAVID TAYLOR NAVAL SHIP R AND D CENTER

3072

NATIONAL CANCER INSTITUTE

2567

MD USDA/ARS

BELTSVILLE AGRICULTURAL RESEARCH CENTER

1606

FREDERICK CANCER RESEARCH FACILITY

1247

MATERIEL TESTING DIRECTORATE

1043

MD DOD/ARMY

MD DOD/ARMY

MD DOD/ARMY

MD HHS

MD DOD/ARMY

MD HHS

MD HHS

MD DOD/NAVY

MD DOD/ARMY

MD HHS

MD HHS

MD HHS

MD HHS

MD HHS

MD HHS

MD DOD

MD DOD/NAVY

CHEMICAL SYSTEMS LABORATORY

917

BALLISTIC RESEARCH LABORATORY

907

NAT. INST. OF ARTH., DIABETES, \& DIGEST. \& KID

781

MEDICAL RESEARCH INST. OF INFECTIOUS DISEASES

544

NEUROLOGY \& COMMUNICATIVE DISORDERS RES. LAB

NAT. INST. OF ALLERGY AND INFECTIOUS DISEASES

MEDICAL RESEARCH INSTITUTE

MATERIAL SYSTEMS ANALYSIS ACTIVITY

INTRAMURAL RES. PROG.-NIMH

507

501

500

489

440

NAT. INST. OF CHILD HEALTH AND HUMAN DEV.

NATIONAL HEART, LUNG, AND BLOOD INSTITUTE

430

426

362

DIVISION OF COMPUTER RESEARCH AND TECHNOLOGY 274

INTRAMURAL RES. PROG.-NIDR

266

GERENTOLOGY RES CENTER-NAT. INST. ON AGING 238

ARMED FORCES RADIOBIOLOGY RESEARCH LAB

216

EXPLOSIVE ORDNANCE DISPOSAL FACILITY

201

MD DOD/ARMY

MD DOI/FWS

MD DOD/ARMY

MEDTCAL RESEARCH INST. OF CHEM. DEFENSE

187

PAUTUXENT WILDLIFE RESEARCH CENTER

178

HUMAN ENGINEERING LABORATORY

175

INTRAMURAL RESEARCH PROGRAM-NEI

173

149

136

128

115

113

86

MD DOI/BOM

MD SMITHSON

MD DOC/NOAA

MD DOC/NOAA

MD SMITHSON

MEDICAL BIOENGINEERING R\&D LABORATORY

BELTSVILLE HUMAN NUTRITION RESEARCH CENTER

SYSTEMS DEVELOPMENT OFF ICE

AIR RESOURCES LABORATORY

60

RADIATION BIOLOGY LABORATORY

SATELLITE EXPERIMENT LABORATORY

ENGINEER ING DEVELOPMENT OFFICE 
FEDERAL LABORATORIES LISTED BY STATE(CONT)

\begin{tabular}{|c|c|c|c|}
\hline ST & AGENCY & LAB & TOTAL \\
\hline MD & DOC/NOAA & HYDROLOGICAL RESEARCH LABORATORY & 20 \\
\hline MD & DOC/NOAA & GEODETIC RESEARCH AND DEVELOPMENT LABORATORY & 15 \\
\hline MI & DOD/ARMY & TANK R\&D COMMAND & 835 \\
\hline MI & DOI/FWS & GREAT LAKES FISHERY LAB & 89 \\
\hline MI & USDA/ARS & REGIONAL POULTRY RESEARCH LABORATORY & 56 \\
\hline MI & DOE & MICH STATE UNIV./DOE PLANT RESEARCH LAB & 50 \\
\hline MI & DOC/NOAA & GREAT LAKES ENVIRONMENTAL RESEARCH LAB & 47 \\
\hline MI & VA & ANN ARBOR VA MEDICAL CENTER & $10+$ \\
\hline MN & USDA/FS & NORTH CENTRAL EXPERIMENT STATION & 346 \\
\hline MN & $\mathrm{DOI} / \mathrm{BOM}$ & TWIN CITIES RESEARCH CENTER & 181 \\
\hline MN & EPA & ENVIRONMENTAL RESEARCH LAB-DULUTH & 83 \\
\hline MN & USDA/ARS & WEED RESEARCH LABORATORY & 16 \\
\hline MN & VA & MINNEAPOL IS VA MEDICAL CENTER & $10+$ \\
\hline MO & DOI/FIS & COLUMBIA NATIONAL FISHERY RES. LAB & 97 \\
\hline MO & DOI/BOM & ROLLA METALLURGY RESEARCH CENTER & 65 \\
\hline MO & USDA/ARS & B IOLOGICAL CONTROL OF INSECTS RESEARCH LAB & 25 \\
\hline MO & VA & COLUMBIA VA MEDICAL CENTER & $10+$ \\
\hline MO & VA & KANSAS CITY VA MEDICAL CENTER & $10+$ \\
\hline MO & VA & ST. LOUIS VA MEDICAL CENTER & $10+$ \\
\hline MS & DOD/ARMY & ENGINEER WATERWAYS EXPERIMENT STATION & 1399 \\
\hline MS & DOD/NAVY & OCEAN R AND D ACTIVITY & 271 \\
\hline MS & NASA & NATIONAL SPACE TECHNOLOGY LABORATORIES & 103 \\
\hline MS & USDA/ARS & SEDIMENTATION LABORATORY & 43 \\
\hline MS & USDA/ARS & BOLL WEEVIL RESEARCH LABORATORY & 41 \\
\hline MS & DOC/NOAA & MISSISSIPPI LABORATORIES & 36 \\
\hline MS & USDA/ARS & B IOENVIRONMENTAL INSECTS CONTROL LABORATORY & 35 \\
\hline MS & USDA/ARS & SOUTHERN WEED SCIENCE LABORATORY & 34 \\
\hline MS & USDA/ARS & CROP SCIENCE AND ENGINEERING RES. LABORATORY & 32 \\
\hline MT & HHS & NAT. INST. OF ALLERGY AND INFECTIOUS DISEASES & 128 \\
\hline NC & HHS & NAT. INST. OF ENVIRONMENTAL HEALTH SC IENCES & 429 \\
\hline NC & EPA & HEALTH EFFECTS RESEARCH LABORATORY & 328 \\
\hline NC & USDA/FS & SOUTHEASTERN FOREST EXPERIMENT STATION & 287 \\
\hline NC & & INDUSTR IAL ENVIRONMENTAL RESEARCH LAB-RTP & 133 \\
\hline NC & EPA & ENVIRONMENTAL SC IENCES RESEARCH LABORATORY & 132 \\
\hline NC & EPA & ENVIRONMENTAL MONITORING SYST.LAB-RTP & 126 \\
\hline NC & DOD/ARMY & ARMY RESEARCH OFFICE & 96 \\
\hline NC & DOC/NOAA & BEAUFORT LABORATORY & 56 \\
\hline NC & USDA/ARS & TOBACCO RESEARCH LABORATORY & 36 \\
\hline NC & VA & DURHAM VA MEDICAL CENTER & $10+$ \\
\hline ND & USDA/ARS & METABOLISM AND RADIATION RESEARCH LABORATORY & 104 \\
\hline ND & DOE & GRAND FORKS ENERGY TECHNOLOGY CENTER & 68 \\
\hline ND & DOI/FWS & NORTHERN PRAIRIE WILDLIFE RESEARCH CENTER & 57 \\
\hline ND & USDA/ARS & NORTHERN GREAT PLAINS RESEARCH CENTER & 42 \\
\hline NE & USDA/ARS & ROMAN L. HRUSKA U.S. MEAT ANIMAL RES. LAB & 56 \\
\hline NE & USDA/ARS & LIVESTOCK INSECTS RESEARCH LABORATORY & 33 \\
\hline NE & & OMAHA VA MEDICAL CENTER & $10+$ \\
\hline NH & DOD/ARMY & COLD REGIONS R\&D LABORATORY & 277 \\
\hline NJ & DOD/ARMY & LARGE CALIBER WEAPONS SYSTEM LABORATORY & 1791 \\
\hline NJ & DOD/NAVY & NAVAL AIR ENGINEER ING CENTER & 1776 \\
\hline NJ & DOE & PRINCETON PLASMA PHYSICS LABORATORY & 1406 \\
\hline
\end{tabular}


FEDERAL LABORATOR IES LISTED BY STATE(CONT)

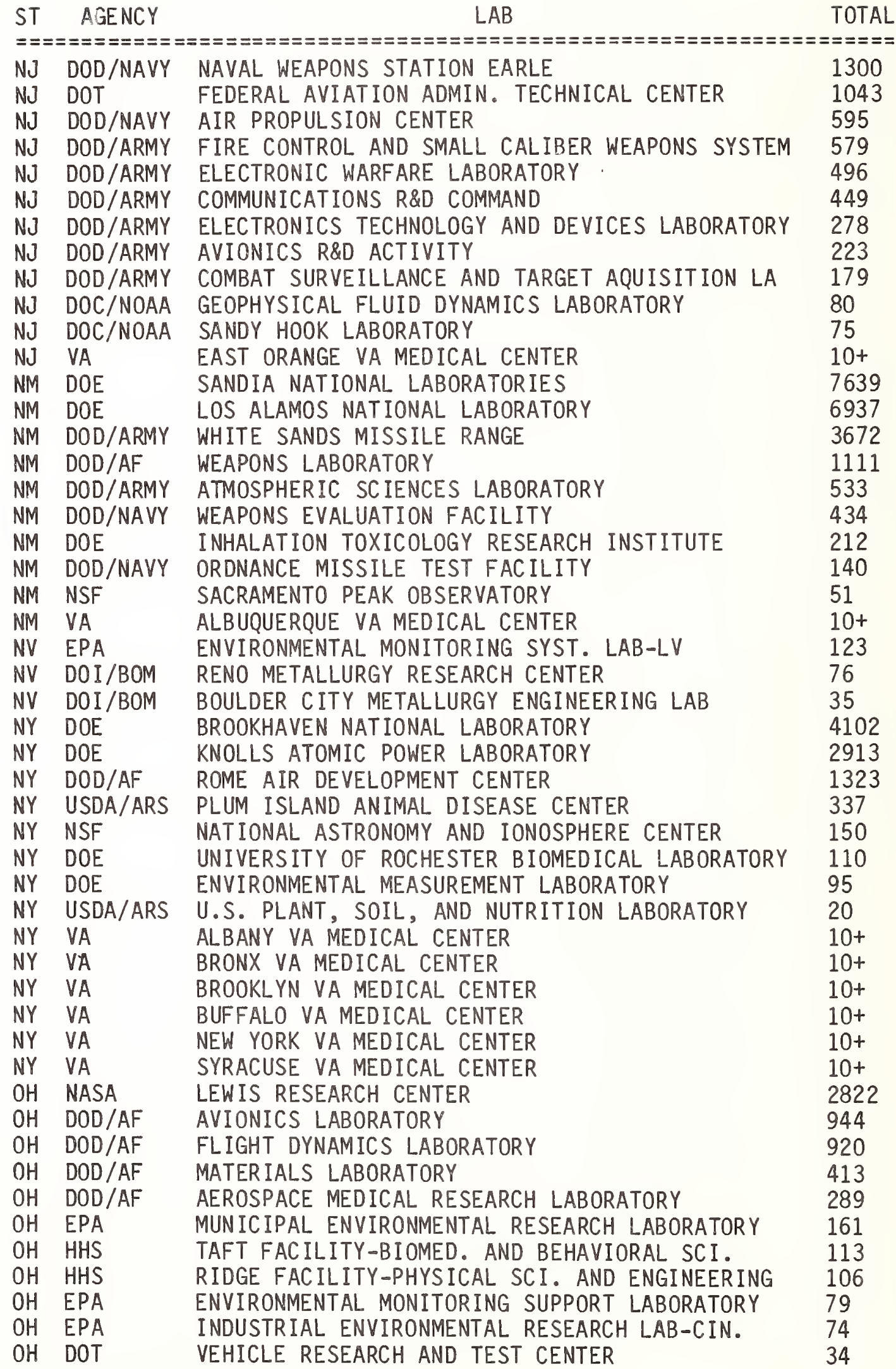


FEDERAL LABORATORIES LISTED BY STATE(CONT)

\begin{tabular}{|c|c|c|}
\hline $\begin{array}{l}\text { ST AGENCY } \\
===========\end{array}$ & $\begin{aligned} & L A B \\
==== & \end{aligned}$ & \\
\hline $\mathrm{OH} \quad \mathrm{VA}$ & CINCINNATI VA MEDICAL CENTER & $10+$ \\
\hline VA & CLEVELAND VA MEDICAL CENTER & $10+$ \\
\hline DOT & CIVIL AEROMEDICAL INSTITUTE & 200 \\
\hline DOE & BARTLESVILLE ENERGY TECHNOLOGY CENTER & 172 \\
\hline EPA & ROBERT S. KERR ENVIRONMENTAL RESEARCH LAB & 55 \\
\hline DOC/NOAA & NATIONAL SEVERE STORMS LABORATORY & 41 \\
\hline USDA/ARS & WATER QUAL ITY MANAGEMENT LABORATORY & 32 \\
\hline VA & OKLAHOMA CITY VA MEDICAL CENTER & $10+$ \\
\hline USDA/FS & PACIF IC NORTHWEST EXPERIMENT STATION & 282 \\
\hline $\mathrm{DOI} / \mathrm{BOM}$ & ALBANY METALLURGY RESEARCH CENTER & 162 \\
\hline EPA & ENVIRONMENTAL RESEARCH LAB-CORVALLIS & 84 \\
\hline VA & PORTLAND VA MEDICAL CENTER & $10+$ \\
\hline DOE & BETTIS ATOMIC POWER LABORATORY & 3358 \\
\hline DOD/NAVY & NAVAL AIR DEVELOPMENT CENTER & 2399 \\
\hline USDA/FS & NORTHEASTERN FOREST EXPERIMENT STATION & 396 \\
\hline DOE & PITTSBURGH ENERGY TECHNOLOGY CENTER & 352 \\
\hline USDA/ARS & EASTERN REGIONAL RESEARCH CENTER & 322 \\
\hline $\mathrm{DOI} / \mathrm{BOM}$ & PITTSBURGH MINING AND SAFETY RES. CENTER & 259 \\
\hline VA & PHILADELPHIA VA MEDICAL CENTER & $10+$ \\
\hline VA & PITTSBURGH VA MEDICAL CENTER & $10+$ \\
\hline DOE & CENTER FOR ENERGY AND ENVIRONMENTAL RESEARCH & 50 \\
\hline DOD/NAVY & UNDERSEA RANGES DEPARTMENT & 82 \\
\hline EPA & ENVIRONMENTAL RESEARCH LAB-RI & 55 \\
\hline DOC/NOAA & NARRAGANSETT LABORATORY & 23 \\
\hline DOE & SAVANNAH RIVER LABORATORY & 995 \\
\hline DOC/NOAA & CHARLESTON LABORATORY & 34 \\
\hline USDA/ARS & U.S. VEGETABLE LABORATORY & 28 \\
\hline VA & CHARLESTON VA MEDICAL CENTER & $10+$ \\
\hline USDA/ARS & NORTHERN GRAIN INSECTS RESEARCH LABORATORY & 28 \\
\hline DOE & OAK RIDGE NATIONAL LABORATORY & 5175 \\
\hline DOE & OAK RIDGE ASSOCIATED UNIVERSITIES & 398 \\
\hline TVA & ENERGY DEMONSTRATIONS AND TECHNOLOGY & 321 \\
\hline $\mathrm{DOD} / \mathrm{AF}$ & ARNOLD ENGINEERING DEVELOPMENT CENTER & 223 \\
\hline DOE & COMPARATIVE ANIMAL RESEARCH LAB & 92 \\
\hline TVA & ENGINEER ING LABORATORY & 82 \\
\hline VA & MEMPHIS VA MEDICAL CENTER & $10+$ \\
\hline VA & NASHVILLE VA MEDICAL CENTER & $10+$ \\
\hline NASA & LYNDON B. JOHNSON SPACE CENTER & 3508 \\
\hline $\mathrm{DOD} / \mathrm{AF}$ & SCHOOL OF AEROSPACE MEDICINE & 666 \\
\hline $\mathrm{DOD} / \mathrm{AF}$ & HUMAN RESOURCES LABORATORY & 365 \\
\hline DOD/ARMY & INSTITUTE OF SURGICAL RESEARCH & 229 \\
\hline USDA/ARS & VETER INARY TOXICOLOGY AND ENTOMOLOGY LAB & 48 \\
\hline USDA/ARS & U. S. LIVESTOCK INSECTS LABORATORY & .43 \\
\hline USDA / ARS & GRASSLAND, SOIL AND WATER RESEARCH LABORATORY & 32 \\
\hline DOC/NOAA & GALVESTON LABORATORY & 30 \\
\hline USDA/ARS & CONSERVATION AND PRODUCTION RESEARCH LAB & 28 \\
\hline USDA/ARS & COTTON RESEARCH LABORATORY & 23 \\
\hline VA & DALLAS VA MEDICAL CENTER & $10+$ \\
\hline VA & HOUSTON VA MEDICAL CENTER & $10+$ \\
\hline VA & SAN ANTONIO VA MEDICAL CENTER & $10+$ \\
\hline
\end{tabular}


FEDERAL LABORATORIES LISTED BY STATE(CONT)

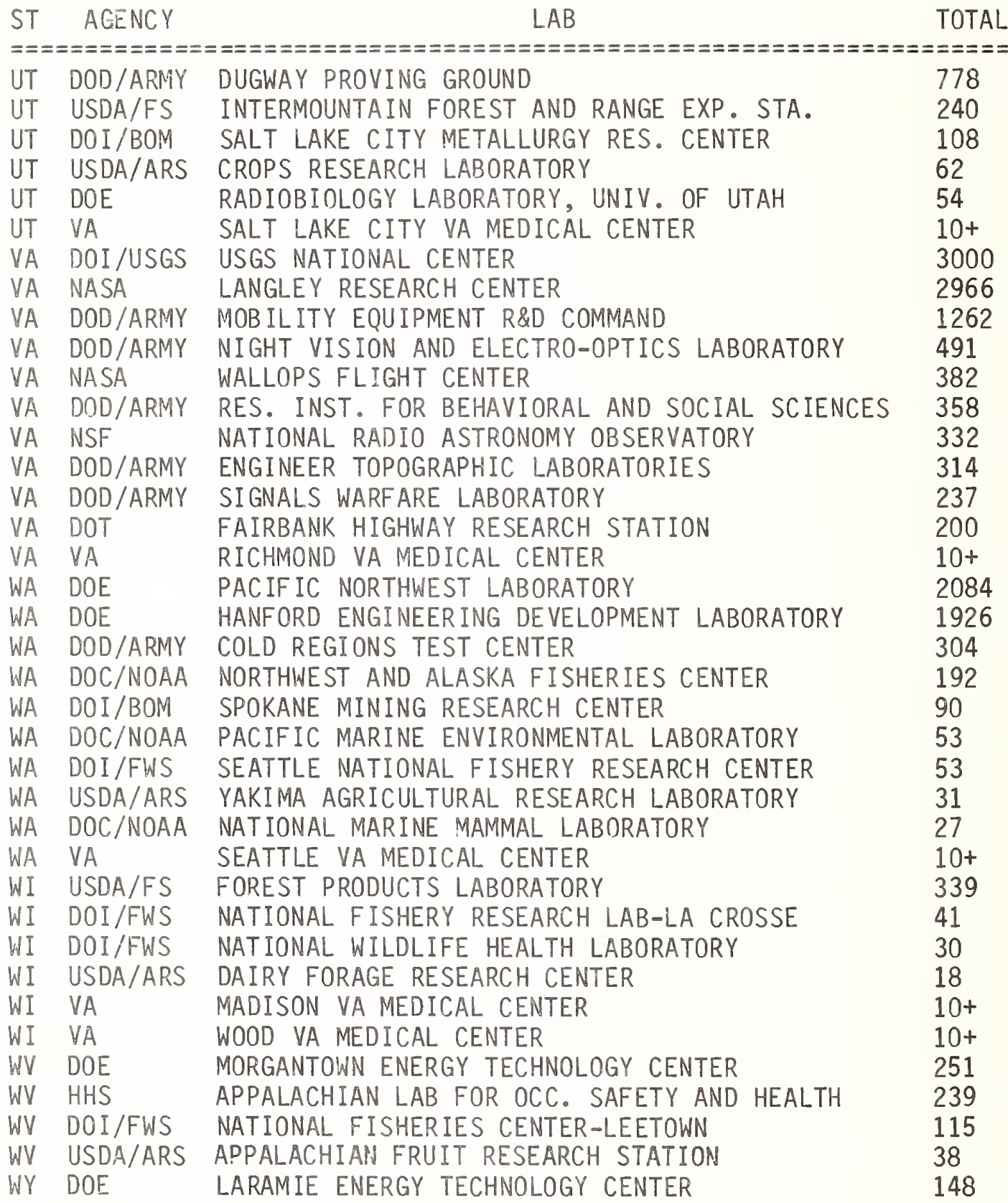


TABLE 6

FEDERAL LABORATORIES LISTED BY AGENCY AND STAFF SIZE

\begin{tabular}{|c|c|c|}
\hline $\begin{array}{l}\text { AGENCY } \\
=======\end{array}$ & 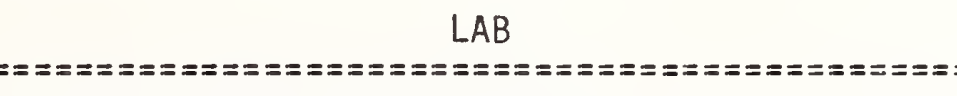 & $\begin{array}{l}\text { TOTAL } \\
======\end{array}$ \\
\hline $\mathrm{DOC}$ & NATIONAL BUREAU OF STANDARDS & 3072 \\
\hline DOC & INSTITUTE FOR TELECOMMUNICATION SCIENCES & 94 \\
\hline DOC/NOAA & NORTHWEST AND ALASKA FISHERIES CENTER & 192 \\
\hline DOC/NOAA & SYSTEMS DEVELOPMENT OFF ICE & 115 \\
\hline DOC/NOAA & AIR RESOURCES LABORATORY & 113 \\
\hline DOC/NOAA & ATLANTIC OCEANOGRAPHIC AND METEOROLOGICAL LAB & 111 \\
\hline DOC/NOAA & SOUTHEAST FISHERIES CENTER & 109 \\
\hline DOC/NOAA & NORTHEAST F ISHER IES CENTER & 106 \\
\hline DOC/NOAA & GEOPHYSICAL FLUID DYNAMICS LABORATORY & 80 \\
\hline DOC/NOAA & SANDY HOOK LABORATORY & 75 \\
\hline DOC/NOAA & SOUTHWEST FISHERIES CENTER & 73 \\
\hline DOC/NOAA & WAVE PROPAGATION LABORATORY & 70 \\
\hline$D O C / N O A A$ & SPACE ENVIRONMENT LABORATORY & 68 \\
\hline DOC/NOAA & OFFICE OF WEATHER RESEARCH AND MODIFICATION & 66 \\
\hline DOC/NOAA & BEAUFORT LABORATORY & 56 \\
\hline$D O C / N O A A$ & PACIF IC MARINE ENVIRONMENTAL LABORATORY & 53 \\
\hline DOC/NOAA & RESEARCH FACILITIES CENTER & 50 \\
\hline DOC/NOAA & AUKE BAY LABORATORY & 47 \\
\hline DOC/NOAA & GREAT LAKES ENVIRONMENTAL RESEARCH LAB & 47 \\
\hline$D O C / N O A A$ & AERONOMY LABORATORY & 44 \\
\hline$D O C / N O A A$ & HONOLULU LABORATORY & 41 \\
\hline$D O C / N O A A$ & NATIONAL SEVERE STORMS LABORATORY & 41 \\
\hline DOC/NOAA & SATELLITE EXPERIMENT LABORATORY & 37 \\
\hline$D O C / N O A A$ & MISSISSIPPI LABORATORIES & 36 \\
\hline DOC/NOAA & CHARLESTON LABORATORY & 34 \\
\hline$D O C / N O A A$ & GALVESTON LABORATORY & 30 \\
\hline DOC/NOAA & GLOUCESTER LABORATORY & 28 \\
\hline DOC/NOAA & ENGINEER ING DEVELOPMENT OFFICE & 27 \\
\hline$D O C / N O A A$ & NATIONAL MARINE MAMMAL LABORATORY & 27 \\
\hline DOC/NOAA & MILFORD LABORATORY & 26 \\
\hline DOC/NOAA & NARRAGANSETT LABORATORY & 23 \\
\hline DOC/NOAA & HYDROLOGICAL RESEARCH LABORATORY & 20 \\
\hline DOC/NOAA & TIBURON LABORATORY & 18 \\
\hline DOC/NOAA & PANAMA CITY LABORATORY & 18 \\
\hline DOC/NOAA & KODIAK LABORATORY & 15 \\
\hline DOC/NOAA & GEODETIC RESEARCH AND DEVELOPMENT LABORATORY & 15 \\
\hline DOD & ARMED FORCES RADIOBIOLOGY RESEARCH LAB & 216 \\
\hline $\mathrm{DOD} / \mathrm{AF}$ & ARMAMENT DIVISION & 6389 \\
\hline $\mathrm{DOD} / \mathrm{AF}$ & FLIGHT TEST CENTER & 5136 \\
\hline $\mathrm{DOD} / \mathrm{AF}$ & ROME AIR DEVELOPMENT CENTER & 1323 \\
\hline DOD / AF & WEAPONS LABORATORY & 1111 \\
\hline DOD/AF & AVIONICS LABORATORY & 944 \\
\hline $\mathrm{DOD} / \mathrm{AF}$ & FLIGHT DYNAMICS LABORATORY & 920 \\
\hline $\mathrm{DOD} / \mathrm{AF}$ & WESTERN SPACE AND MISSILE CENTER & 724 \\
\hline $\mathrm{DOD} / \mathrm{AF}$ & SCHOOL OF AEROSPACE MEDICINE & 666 \\
\hline $\mathrm{DOD} / \mathrm{AF}$ & GEOPHYSICS LABORATORY & 618 \\
\hline $\mathrm{DOD} / \mathrm{AF}$ & ROCKET PROPULSION LABORATORY & 431 \\
\hline $\mathrm{DOD} / \mathrm{AF}$ & MATERIALS LABORATORY & 413 \\
\hline $\mathrm{DOD} / \mathrm{AF}$ & HUMAN RESOURCES LABORATORY & 365 \\
\hline $\mathrm{DOD} / \mathrm{AF}$ & AEROSPACE MEDICAL RESEARCH LABORATORY & 289 \\
\hline
\end{tabular}


FEDERAL LABORATORIES LISTED BY AGENCY AND STAFF SIZE(CONT)

AGENCY

$L A B$

TOTAL

$======$
$D O D / A F$

ARNOLD ENGINEER ING DEVELOPMENT CENTER

223

DOD / AF

ENGINEERING AND SERVICES CENTER/ R\&D

DOD/AF

FRANK J. SEIBLER RESEARCH LABORATORY

104

DOD/ARMY

WHITE SANDS MISSILE RANGE

33

DOD/ARMY

LARGE CAL IBER WEAPONS SYSTEM LABORATORY

3672

DOD/ARMY

ENGINEER WATERWAYS EXPERIMENT STATION

1791

DOD/ARMY

MOBILITY EQUIPMENT R\&D COMMAND

1399

DOD/ARMY

NATICK R\&D COMMAND

1262

DOD /ARMY

HARRY DIAMOND LABORATORIES

1187

DOD/ARMY

MISSILE RESEARCH ANO DEVELOP

1154

DOD/ARMY

DOD/ARMY

DOD / ARMY

DOD/ARMY

DOD/ARMY

DOD / ARMY

DOD/ARMY

DOD/ARMY

DOD/ARMY

DOD / ARMY

DOD / ARMY

DOD / ARMY

DOD/ARMY

DOD/ARMY

YUMA PROVING GROUND

1001

MATERIEL TESTING DIRECTORATE

986

WALTER REED ARMY INSTITUTE OF RESEARCH

CHEMICAL SYSTEMS LABORATORY RESEARCH

CHEMICAL SYSTEMS LABORATORY

917

TANK R\&D COMMAND

907

ELECTRONIC PROVING GROUND

835

BALLISTIC RESEARCH LABORATORY

800

DUGWAY PROVING GROUND

781

MATERIALS AND MECHANICS RESEARCH CENTER

778

FIRE CONTROL AND SMALL CALIBER WEAPONS SYSTEM

622

AVIATION RESEARCH AND TECHNOLOGY LABS

ATMOSPHERIC SCIENCES LABORATORY

579

560

MEDICAL RESEARCH INST. OF INFECTIOUS DISEASES

533

ELECTRONIC WARFARE LABORATORY

DOD/ARMY

DOD/ARMY

DOD / ARMY

NIGHT VISION AND ELECTRO-OPTICS LABORATORY

COMMUNICATIONS R\&D COMMAND

MATERIAL SYSTEMS ANALYSIS ACTIVITY

507

496

491

449

DOD/ARMY

RES. INST. FOR BEHAVIORAL AND SOCIAL SCIENCES

440

DOD/ARMY

AVIATION DEVELOPMENT TEST ACTIVITY

358

DOD/ARMY

DOD/ARMY

ENGINEER TOPOGRAPHIC LABORATORIES

324

COLD REGIONS TEST CENTER

314

LETTERMAN ARMY INSTITUTE OF RESEARCH

304

DOD/ARMY

ELECTRONICS TECHNOLOGY AND DEVICES LABORATORY

278

DOD/ARMY

COLD REGIONS R\&D LABORATORY

278

277

DOD/ARMY

SIGNALS WARFARE LABORATORY

237

INSTITUTE OF SURGICAL RESEARCH

229

DOD/ARMY

AVIDNICS R\&D ACTIVITY

223

DOD/ARMY

CONSTRUCTION ENGINEERING RESEARCH LABORATORY

207

DOD/ARMY

MEDICAL RESEARCH INST. OF CHEM. DEFENSE

187

DOD/ARMY

COMBAT SURVEILLANCE AND TARGET AQUISITION LA

179

RESEARCH INSTITUTE OF ENVIRONMENTAL MEDICINE 175

HUMAN ENGINEER ING LABORATORY

175

AVIATION ENGINEER ING FLIGHT ACTIVITY 166

DOD/ARMY

MEDICAL R\&D COMMAND

149

AEROMEDICAL RESEARCH LABORATORY 142

DOD/ARMY

DOD/ARMY

TROPIC TEST CENTER

141

MEDICAL BIOENGINEERING R\&D LABORATORY 136

DOD/ARIY

INSTITUTE OF DENTAL RESEARCH

105

DOD/ARMY

ARMY RESEARCH OFFICE

96

DOD/NAVY

PACIFIC MISSILE TEST CENTER

4803 


\begin{tabular}{|c|c|c|}
\hline DOD/NAVY & SURFACE WEAPONS CENTER & 4732 \\
\hline DOD/NAVY & NAVAL WEAPONS CENTER & 4540 \\
\hline DOD/NAVY & AIR TEST CENTER & 3566 \\
\hline DOD/NAVY & RESEARCH LABORATORY & 3394 \\
\hline DOD/NAVY & NAVAL UNDERWATER SYSTEMS CENTER & 2800 \\
\hline DOD/NAVY & OCEAN SYSTEMS CENTER & 2791 \\
\hline DOD/NAVY & DAVID TAYLOR NAVAL SHIP R AND D CENTER & 2567 \\
\hline DOD/NAVY & NAVAL AIR DEVELOPMENT CENTER & 2399 \\
\hline DOD/NAVY & NAVAL AVIONIC CENTER & 2150 \\
\hline DOD/NAVY & NAVAL AIR ENGINEERING CENTER & 1776 \\
\hline DOD/NAVY & NAVAL WEAPONS STATION EARLE & 1300 \\
\hline DOD/NAVY & COASTAL SYSTEMS CENTER & 732 \\
\hline DOD/NAVY & AIR PROPULSION CENTER & 595 \\
\hline DOD/NAVY & MEDICAL RESEARCH INSTITUTE & 489 \\
\hline DOD/NAVY & WEAPONS EVALUATION FACILITY & 434 \\
\hline DOD/NAVY & OFFICE OF NAVAL RESEARCH & 429 \\
\hline DOD/NAVY & CIVIL ENGINEERING LABORATORY & 325 \\
\hline DOD/NAVY & PERSONNEL RESEARCH AND DEVELOPMENT CENTER & 294 \\
\hline DOD/NAVY & OCEAN R AND D ACTIVITY & 271 \\
\hline DOD/NAVY & EXPLOSIVE ORDNANCE DISPOSAL FACILITY & 201 \\
\hline DOD/NAVY & AEROSPACE MEDICAL RESEARCH LABORATORY & 169 \\
\hline DOD/NAVY & ORDNANCE MISSILE TEST FACILITY & 140 \\
\hline DOD/NAVY & HEALTH RESEARCH CENTER & 113 \\
\hline DOD/NAVY & SUBMARINE MEDICAL RESEARCH LABORATORY & 94 \\
\hline DOD/NAVY & UNDERSEA RANGES DEPARTMENT & 82 \\
\hline DOD/NAVY & MEDICAL RESEARCH UNIT NO. 3 & 81 \\
\hline DOD/NAVY & CLOTHING AND TEXTILE RESEARCH FACILITY & 56 \\
\hline DOD/NAVY & DENTAL RESEARCH INSTITUTE NTC & 47 \\
\hline $\mathrm{DOE}$ & SANDIA NATIONAL LABORATORIES & 7639 \\
\hline DOE & LAWRENCE LIVERMORE NATIONAL LABORATORY & 7102 \\
\hline DOE & LOS ALAMOS NATIONAL LABORATORY & 6937 \\
\hline DOE & OAK RIDGE NATIONAL LABORATORY & 5175 \\
\hline DOE & ARGONNE NATIONAL LABORATORY & 5118 \\
\hline DOE & IDAHO NATIONAL ENGINEER ING LABORATORY & 5100 \\
\hline DOE & BROOKHAVEN NATIONAL LABORATORY & 4102 \\
\hline DOE & BETTIS ATOMIC POWER LABORATORY & 3358 \\
\hline $\mathrm{DOE}$ & LAWRENCE BERKELEY LABORATORY & 3158 \\
\hline DOE & KNOLLS ATOMIC POWER LABORATORY & 2913 \\
\hline DOE & PACIF IC NORTHWEST LABORATORY & 2084 \\
\hline DOE & FERMI NATIONAL ACCELERATOR LABORATORY & 1982 \\
\hline DOE & HANFORD ENGINEER ING DEVELOPMENT LABORATORY & 1926 \\
\hline DOE & PRINCETON PLASMA PHYSICS LABORATORY & 1406 \\
\hline DOE & STANFORD LINEAR ACCELERATOR CENTER & 1402 \\
\hline DOE & SAVANNAH RIVER LABORATORY & 995 \\
\hline DOE & SOLAR ENERGY RESEARCH INSTITUTE & 724 \\
\hline DOE & AMES LABORATORY & 539 \\
\hline DOE & OAK RIDGE ASSOCIATED UNIVERSITIES & 398 \\
\hline DOE & PITTSBURGH ENERGY TECHNOLOGY CENTER & 352 \\
\hline & ENERGY TECHNOLOGY ENGINEER ING CENTER & 330 \\
\hline DOE & MORGANTOWN ENERGY TECHNOLOGY CENTER & 251 \\
\hline
\end{tabular}




\begin{tabular}{|c|c|c|}
\hline $\begin{array}{l}\text { AGENCY } \\
========\end{array}$ & 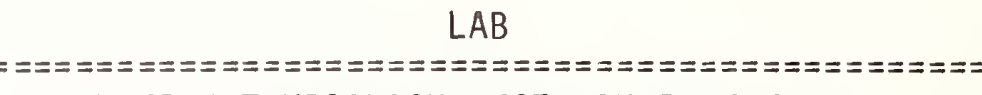 & $\begin{array}{l}\text { TOTAL } \\
======\end{array}$ \\
\hline DOE & INHALATION TOXICOLOGY RESEARCH INSTITUTE & 212 \\
\hline DOE & BARTLESVILLE ENERGY TECHNOLOGY CENTER & 172 \\
\hline DOE & LARAMIE ENERGY TECHNOLOGY CENTER & 148 \\
\hline DOE & LAB. OF NUCLEAR MEDICINE AND RAD. BIOLOGY, UCL & 145 \\
\hline DOE & UNIVERSITY OF ROCHESTER BIOMEDICAL LABORATORY & 110 \\
\hline DOE & ENVIRONMENTAL MEASUREMENT LABORATORY & 95 \\
\hline DOE & COMPARATIVE ANIMAL RESEARCH LAB & 92 \\
\hline DOE & BATES LINEAR ACCELERATOR FACILITY & 75 \\
\hline DOE & NOTRE DAME RADIATION LABORATORY & 71 \\
\hline DOE & SAVANNAH RIVER ECOLOGY LABORATORY & 69 \\
\hline DOE & GRAND FORKS ENERGY TECHNOLOGY CENTER & 68 \\
\hline DOE & NEW BRUNSWICK LABORATORY & 60 \\
\hline DOE & RADIOBIOLOGY LABORATORY, UNIV. OF UTAH & 54 \\
\hline DOE & MICH STATE UNIV./DOE PLANT RESEARCH LAB & 50 \\
\hline DOE & CENTER FOR ENERGY AND ENVIRONMENTAL RESEARCH & 50 \\
\hline DOE & LAB OF RADIOBIOLOGY AND ENV. HEALTH/ UC & 42 \\
\hline DOE & LAB. FOR ENERGY-RELATED HEALTH RES., UC/DAVIS & 40 \\
\hline DOI & ENGINEER ING AND RESEARCH LABORATORY & 230 \\
\hline $\mathrm{DOI} / \mathrm{BOM}$ & PITTSBURGH MINING AND SAFETY RES. CENTER & 259 \\
\hline $\mathrm{DOI} / \mathrm{BOM}$ & TWIN CITIES RESEARCH CENTER & 181 \\
\hline DOI /BOM & ALBANY METALLURGY RESEARCH CENTER & 162 \\
\hline DO I / BOM & SALT LAKE CITY METALLURGY RES. CENTER & 108 \\
\hline DO I / BOM & DENVER MINING RESEARCH CENTER & 93 \\
\hline DOI/BOM & SPOKANE MINING RESEARCH CENTER & 90 \\
\hline $\mathrm{DOI} / \mathrm{BOM}$ & AVONDALE METALLURGY RESEARCH CENTER & 86 \\
\hline DO I / BOM & RENO METALLURGY RESEARCH CENTER & 76 \\
\hline DOI / BOM & ROLLA METALLURGY RESEARCH CENTER & 65 \\
\hline DOI / BOM & TUSCALOOSA METALLURGY RESEARCH CENTER & 53 \\
\hline $\mathrm{DOI} / \mathrm{BOM}$ & BOULDER CITY METALLURGY ENGINEERING LAB & 35 \\
\hline DO I/FWS & DENVER WILDLIFE RESEARCH CENTER & 208 \\
\hline DOI/FWS & PAUTUXENT WILDLIFE RESEARCH CENTER & 178 \\
\hline DOI/FWS & NATIONAL FISHER IES CENTER-LEETOWN & 115 \\
\hline DOI /FWS & COLUMBIA NATIONAL FISHERY RES. LAB & 97 \\
\hline DOI/FWS & GREAT LAKES FISHERY LAB & 89 \\
\hline DOI/FWS & NORTHERN PRAIRIE WILDLIFE RESEARCH CENTER & 57 \\
\hline DOI/FWS & SEATTLE NATIONAL F ISHERY RESEARCH CENTER & 53 \\
\hline DOI/FWS & NATIONAL FISHERY RESEARCH LAB-LA CROSSE & 41 \\
\hline DOI/FWS & NATIONAL WILDLIFE HEALTH LABORATORY & 30 . \\
\hline DOI/USGS & USGS CENTRAL REGION & 4500 \\
\hline DOI/USGS & USGS WESTERN REGION & 3676 \\
\hline DOI/USGS & USGS NATIONAL CENTER & 3000 \\
\hline DOJ & FEDERAL BUREAU OF INVESTIGATION LABORATORY & 429 \\
\hline DOT & FEDERAL AVIATION ADMIN. TECHNICAL CENTER & 1043 \\
\hline DOT & TRANSPORTATION SYSTEMS CENTER & 600 \\
\hline DOT & TRANSPORTATION TEST CENTER & 400 \\
\hline DOT & FAIRBANK HIGHWAY RESEARCH STATION & 200 \\
\hline DOT & CIVIL AEROMED ICAL INSTITUTE & 200 \\
\hline DOT & U.S. COAST GUARD R\&D CENTER & 148 \\
\hline DOT & VEHICLE RESEARCH AND TEST CENTER & 34 \\
\hline EPA & HEALTH EFFECTS RESEARCH LABORATORY & 328 \\
\hline
\end{tabular}


FEDERAL LABORATORIES LISTED BY AGENCY AND STAFF SIZE (CONT)

AGENCY

$L A B$

TOTAL

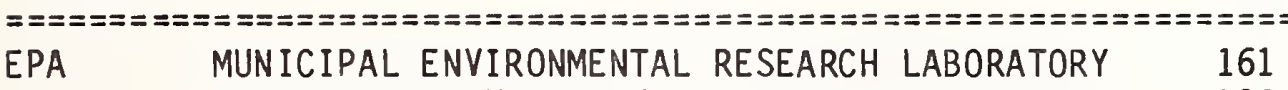

EPA

161

EPA

EPA

EPA

EPA

EPA

EPA

EPA

EPA

EPA

EPA

EPA

HHS

HHS

INDUSTRIAL ENVIRONMENTAL RESEARCH LAB-RTP

133

ENVIRONMENTAL SCIENCES RESEARCH LABORATORY

132

ENVIRONMENTAL MONITORING SYST. LAB-RTP

126

ENVIRONMENTAL MONITORING SYST. LAB-LV

123

ENVIRONMENTAL RESEARCH LAB-CORVALLIS

84

ENVIRONMENTAL RESEARCH LAB-DULUTH

83

ENVIRONMENTAL MONITORING SUPPORT LABORATORY

79

ENVIRONMENTAL RESEARCH LABORATORY -ATHENS

78

INDUSTRIAL ENVIRONMENTAL RESEARCH LAB-CIN.

74

ROBERT S. KERR ENVIRONMENTAL RESEARCH LAB

55

ENVIRONMENTAL RESEARCH LAB-RI

55

ENVIRONMENTAL RESEARCH LAB-SABINE

54

NATIONAL CANCER INSTITUTE

1606

FREDERICK CANCER RESEARCH FACILITY

1043

CENTER FOR INFECTIOUS DISEASES

908

HHS

NAT. INST. OF ARTH., DIABETES, \& DIGEST. \& KID

544

NEUROLOGY \& COMMUNICATIVE DISORDERS RES. LAB 501

NAT. INST. OF ALLERGY AND INFECTIOUS DISEASES 500

INTRAMURAL RES. PROG.-NIMH

430

NAT. INST. OF ENVIRONMENTAL HEALTH SCIENCES 429

NAT. INST. OF CHILD HEALTH AND HUMAN DEV. 426

NATIONAL HEART, LUNG, AND BLOOD INSTITUTE 362

DIVISION OF COMPUTER RESEARCH AND TECHNOLOGY 274

INTRAMURAL RES. PROG.-NIDR

266

APPALACHIAN LAB FOR OCC. SAFETY AND HEALTH 239

GERENTOLOGY RES CENTER-NAT.INST. ON AGING 238

INTRAMURAL RESEARCH PROGRAM-NEI

173

NAT. INST. OF ALLERGY AND INFECTIOUS DISEASES 128

INTRAMURAL RES. PROG.-NIMH-DIV. OF SP. MENTAL 120

CLINICAL CHEMISTRY DIVISION

118

TAFT FAC ILITY-BIOMED. AND BEHAVIORAL SCI. 113

RIDGE FACILITY-PHYSICAL SCI. AND ENGINEERING 106

HHS

CLINICAL RES. SEC. \& S.W. FIELD STUDIES SEC.

16

NASA

JET PROPULSION LABORATORY

3788

3563

GEORGE C. MARSHALL SPACE FLIGHT CENTER

3508

LYNDON B. JOHNSON SPACE CENTER

3436

GODDARD SPACE FLIGHT CENTER

2966

LANGLEY RESEARCH CENTER

2822

LEWIS RESEARCH CENTER

2201

JOHN F. KENNEDY SPACE CENTER

1651

AMES RESEARCH CENTER

465

HUGH L. DRYDEN FLIGHT RESEARCH CENTER

WALLOPS FLIGHT CENTER

382

NATIONAL SPACE TECHNOLOGY LABORATORIES

103

NATIONAL CENTER FOR ATMOSPHERIC RESEARCH

676

NATIONAL RADIO ASTRONOMY OBSERVATORY 332

KITT PEAK NATIONAL OBSERVATORY 288

NAT IONAL ASTRONOMY AND IONOSPHERE CENTER

150

CERRO TOLOLO INTER-AMERICAN OBSERVATORY

144 
FEDERAL LABORATORIES LISTED BY AGENCY AND STAFF SIZE(CONT)

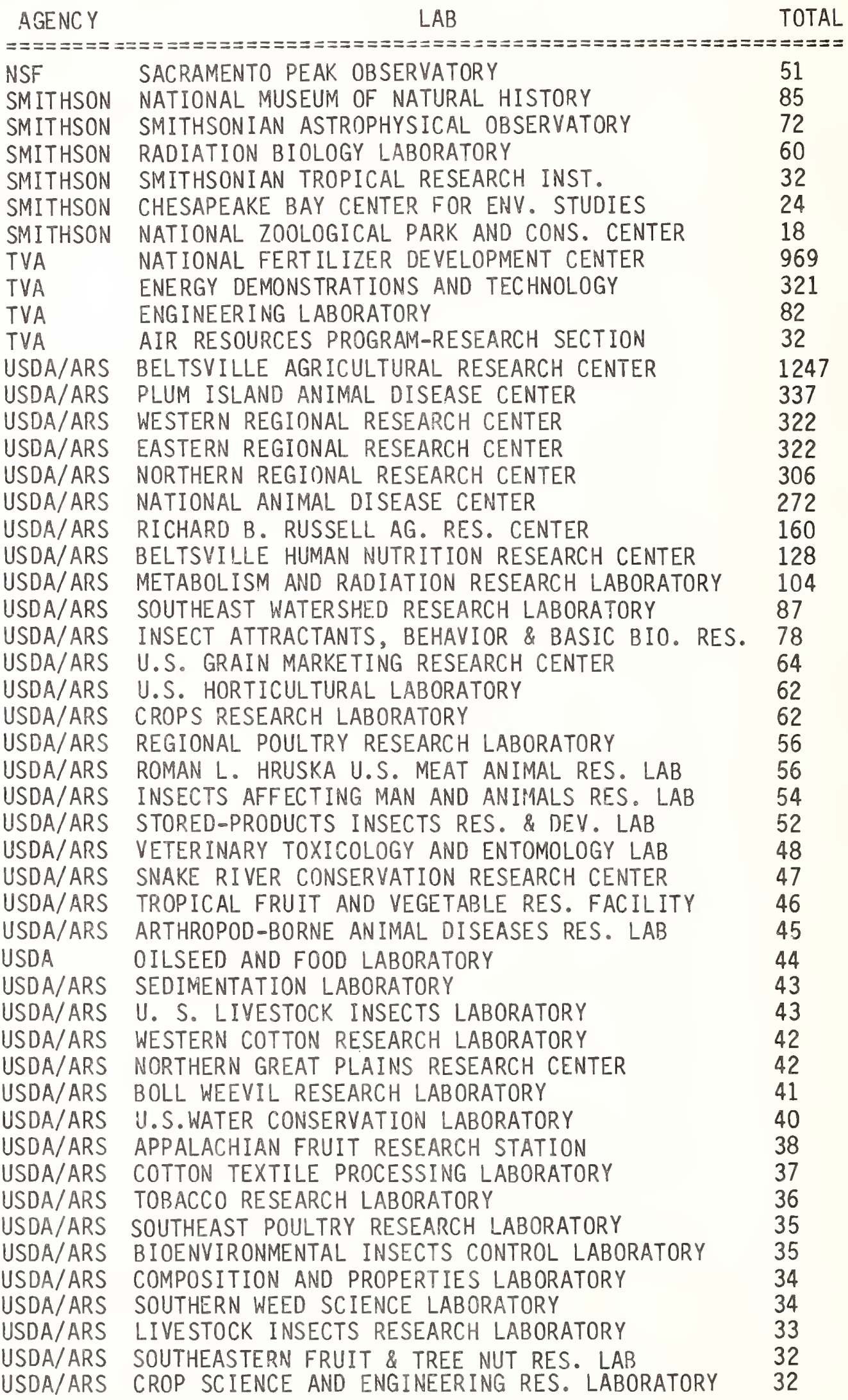




\begin{tabular}{|c|c|c|}
\hline USDA/ARS & WATER QUALITY MANAGEMENT LABORATORY & 32 \\
\hline USDA/ARS & GRASSLAND, SOIL AND WATER RESEARCH LABORATORY & 32 \\
\hline USDA/ARS & YAKIMA AGRICULTURAL RESEARCH LABORATORY & 31 \\
\hline USDA/ARS & SOUTHERN PIEDMONT CONSERVATION LABORATORY & 30 \\
\hline USDA/ARS & U.S. SUGARCANE FIELD LABORATORY & 30 \\
\hline USDA/ARS & NATURAL POLYMERS LABORATORY & 30 \\
\hline USDA/ARS & ENGINEER ING AND DEVELOPMENT LABORATORY & 29 \\
\hline USDA/ARS & U.S. VEGETABLE LABORATORY & 28 \\
\hline USDA/ARS & NORTHERN GRAIN INSECTS RESEARCH LABORATORY & 28 \\
\hline USDA/ARS & CONSERVATION AND PRODUCTION RESEARCH LAB & 28 \\
\hline USDA/ARS & FRUIT AND VEGETABLE CHEM.RES. UNIT & 26 \\
\hline USDA/ARS & SOIL EROSION LABORATORY & 26 \\
\hline USDA/ARS & B IOLOGICAL CONTROL OF INSECTS RESEARCH LAB & 25 \\
\hline USDA/ARS & COTTON TEXTILE CHEMISTRY LABORATORY & 24 \\
\hline USDA/ARS & COTTON RESEARCH LABORATORY & 23 \\
\hline USDA/ARS & U.S. PLANT, SOIL, AND NUTRITION LABORATORY & 20 \\
\hline USDA/ARS & DAIRY FORAGE RESEARCH CENTER & 18 \\
\hline USDA/ARS & CITRUS AND SUB-TROPICAL PRODUCTS LABORATORY & 17 \\
\hline USDA/ARS & WEED RESEARCH LABORATORY & 16 \\
\hline USDA/FS & NORTHEASTERN FOREST EXPERIMENT STATION & 396 \\
\hline USDA/FS & NORTH CENTRAL EXPERIMENT STATION & 346 \\
\hline USDA/FS & FOREST PRODUCTS LABORATORY & 339 \\
\hline USDA/FS & SOUTHERN FOREST EXPERIMENT STATION & 322 \\
\hline USDA/FS & SOUTHEASTERN FOREST EXPERIMENT STATION & 287 \\
\hline USDA/FS & PACIFIC NORTHWEST EXPERIMENT STATION & 282 \\
\hline USDA/FS & PACIFIC SOUTHWEST FOREST EXPERIMENT STATION & 255 \\
\hline USDA/FS & INTERMOUNTAIN FOREST AND RANGE EXP. STA. & 240 \\
\hline USDA/FS & ROCKY MOUNTAIN FORESTRY AND RANGE EXP. STATION & 230 \\
\hline VA & BIRM INGHAM VA MEDICAL CENTER & $10+$ \\
\hline VA & LITTLE ROCK VA MEDICAL CENTER & $10+$ \\
\hline VA & TUCSON VA MEDICAL CENTER & $10+$ \\
\hline VA & LONG BEACH VA MEDICAL CENTER & $10+$ \\
\hline VA & LOS ANGELES BRENTWOOD VA MEDICAL CENTER & $10+$ \\
\hline VA & LOS ANGELES WADSWORTH DIV. VA MEDICAL CENTER & $10+$ \\
\hline VA & MARTINEZ VA MEDICAL CENTER & $10+$ \\
\hline VA & PALO ALTO VA MEDICAL CENTER & $10+$ \\
\hline VA & SAN DIEGO VA MEDICAL CENTER & $10+$ \\
\hline VA & SAN FRANCISCO VA MEDICAL CENTER & $10+$ \\
\hline VA & SEPULVEDA VA MEDICAL CENTER & $10+$ \\
\hline VA & DENVER VA MEDICAL CENTER & $10+$ \\
\hline VA & WEST HAVEN VA MEDICAL CENTER & $10+$ \\
\hline VA & WASHINGTON VA MEDICAL CENTER & $10+$ \\
\hline VA & GAINESVILLE VA MEDICAL CENTER & $10+$ \\
\hline VA & MIAMI VA MEDICAL CENTER & $10+$ \\
\hline VA & TAMPA VA MEDICAL CENTER & $10+$ \\
\hline VA & AUGUST VA MEDICAL CENTER & $10+$ \\
\hline VA & IOWA CITY VA MEDICAL CENTER & $10+$ \\
\hline VA & CHICAGO LAKESIDE VA MEDICAL CENTER & $10+$ \\
\hline VA & CHICAGO WEST SIDE VA MEDICAL CENTER & $10+$ \\
\hline VA & HINES VA MEDICAL CENTER & $10+$ \\
\hline
\end{tabular}


FEDERAL LABORATORIES LISTED BY AGENCY AND STAFF SIZE(CONT)

AGENCY

$L A B$

TOTAL

VA

INDIANAPOLIS VA MEDICAL CENTER

$=====$

VA LEXINGTON VA MEDICAL CENTER

VA NEW ORLEANS VA MEDICAL CENTER

VA

BEDFORD VA MEDICAL CENTER

BOSTON VA MEDICAL CENTER

WEST ROXBURY VA MEDICAL CENTER

ANN ARBOR VA MEDICAL CENTER

MINNEAPOL IS VA MEDICAL CENTER

COLUMBIA VA MEDICAL CENTER

KANSAS CITY VA MEDICAL CENTER

$10+$

$10+$

$10+$

$10+$

$10+$

$10+$

$10+$

$10+$

$10+$

$10+$

ST. LOUIS VA MEDICAL CENTER

DURHAM VA MEDICAL CENTER

OMAHA VA MEDICAL CENTER

EAST ORANGE VA MEDICAL CENTER

$10+$

$10+$

$10+$

$10+$

ALBANY VA MEDICAL CENTER

BRONX VA MEDICAL CENTER

BROOKLYN VA MEDICAL CENTER

BUFFALO VA MEDICAL CENTER

NEW YORK VA MEDICAL CENTER

SYRACUSE VA MEDICAL CENTER

CINCINNAT I VA MEDICAL CENTER

$10+$

$10+$

$10+$

$10+$

$10+$

$10+$

$10+$

CLEVELAND VA MEDICAL CENTER

$10+$

OKLAHOMA CITY VA MEDICAL CENTER

$10+$

PORTLAND VA MEDICAL CENTER

$10+$

PHILADELPHIA VA MEDICAL CENTER

$10+$

PITTSBURGH VA MEDICAL CENTER

$10+$

CHARLESTON VA MEDICAL CENTER

$10+$

MEMPHIS VA MEDICAL CENTER

$10+$

NASHVILLE VA MEDICAL CENTER

DALLAS VA MEDICAL CENTER

HOUSTON VA MEDICAL CENTER

$10+$

$10+$

$10+$

SAN ANTONIO VA MEDICAL CENTER

$10+$

SALT LAKE CITY VA MEDICAL CENTER

$10+$

RICHMOND VA MEDICAL CENTER

$10+$

SEATTLE VA MEDICAL CENTER

$10+$

MADISON VA MEDICAL CENTER

$10+$

WOOD VA MEDICAL CENTER

$10+$

VA

ALBUQUERQUE VA MEDICAL CENTER

$10+$ 
TABLE 7

FEDERAL LABORATORY CONTACTS LISTED BY AGENCY AND STAFF SIZE(CONT)

HOWARD E. SORROWS 'DIRECTOR' ${ }^{*}$ DR. ERNEST AMBLER

DOC NATIONAL BUREAU OF STANDARDS STAFF:TOT 3072

ROOM A402, ADMINISTRATION BUILDING R\&D 1996

WASHINGTON DC 20234 PHONE: 301-921-2226 FTS 921-2226

DR. HARVEY M. GATES 'DIRECTOR': DR. WILLIAM E. UTLAUT

DOC INSTITUTE FOR TELECOMMUNICATION SCIENCES STAFF:TOT 94 R\&D 94

BOULDER CO 80302 PHONE: 303-497-3589 FTS 302-3589

DR. WILLIAM ARON 'DIRECTOR': DR. WILLIAM ARON

DOC/NOAA NORTHWEST AND ALASKA FISHERIES CENTER STAFF:TOT 192

2725 MONRL AKE BLVD., EAST R\&D 192

SEATTLE WA 98112 PHONE: 206-442-4760 FTS 399-4760

'DIRECTOR': DR. DOUGLAS H. SARGEANT

DOC/NOAA SYSTEMS DEVELOPMENT OFFICE

STAFF :TOT 115

SILVER SPRING MD PHONE:

R\&D 86

'DIRECTOR': DR. LESTER MACHTA

DOC/NOAA AIR RESOURCES LABORATORY

STAFF :TOT 113

SILVER SPRING

MD

PHONE :

R\&D 113

'DIRECTOR' : DR. HUGO BEZDEK

DOC/NOAA ATLANTIC OCEANOGRAPHIC AND METEOROLOGICAL LAB STAFF:TOT 111

MIAMI FL PHONE:

R\&D 111

DR. WILLIAM W. FOX, JR. 'DIRECTOR': DR. WILLIAM W. FOX, JR. DOC/NOAA SOUTHEAST FISHERIES CENTER STAFF:TOT 109 75 VIRGINIA BEACH DRIVE R\&D 109 MIAMI

FL 33149 PHONE: 305-361-4284 FTS 305-1284

RICHARD E. HENNEMUTH DOC/NOAA NORTHEAST FISHERIES CENTER

'DIRECTOR': RICHARD E. HENNEMUTH

WOODS HOLE

MA 02543 PHONE: 617-548-5123 FTS 840-1233

'DIRECTOR' : DR. JOSEPH SMAGORINSKY

DOC/NOAA GEOPHYSICAL FLUID DYNAMICS LABORATORY STAFF:TOT 80

PRINCETON NJ PHONE:

R\&D 80

CARL J. SINDERMAN

'DIRECTOR': CARL J. SINDERMAN

DOC/NOAA SANDY HOOK LABORATORY

STAFF:TOT 75

R\&D 71

HIGHLANDS

NJ 07732

PHONE: 201-782-0200 FTS 392-8200

* The title 'Director' is used in a generic sense. The actual title used varies from Agency to Agency. In DOD laboratories there are usually separate positions of Commander and Technical Director. In those cases, the Commander has been 7 isted in this table. 
FEDERAL LABORATORY CONTACTS LISTED BY AGENCY AND STAFF SIZE (CONT)

IZADORE BARRETT

'DIRECTOR': IZADORE BARRETT

DOC/NOAA SOUTHWEST FISHERIES CENTER

8604 LA JOLLA SHORES DRIVE, P.0. BOX 271

STAFF:TOT 73

LA JOLLA

CA 92038

R\&D 73

DOC/NOAA WAVE PROPAGATION LABORATORY

BOULDER CO PHONE:

$\begin{array}{rrrr} & \text { 'DIRECTOR': } & \text { DR. DONALD WILLIAMS } \\ \text { DOC/NOAA SPACE ENVIRONMENT LABORATORY } & \text { STAFF:TOT } & 68 \\ & \text { R\&D } & 41\end{array}$

BOULDER CO PHONE:

'DIRECTOR': DR. CHARLES CHAPPELL

DOC/NOAA OFFICE OF WEATHER RESEARCH AND MODIFIC.ATION STAFF:TOT 66

BOULDER CO PHONE:

R\&D 63

DR. THEODORE RICE

DOC/NOAA BEAUFORT LABORATORY

P. O. BOX 570

BEAUFORT

'DIRECTOR': DR. THEODORE RICE

STAFF :TOT 56

R\&D 56

NC 26516 PHONE: 919-728-4594 FTS 670-2451

'DIRECTOR': DR. JOHN APEL

DOC/NOAA PACIFIC MARINE ENVIRONMENTAL LABORATORY STAFF:TOT 53

SEATTLE WA PHONE:

R\&D 53

'DIRECTOR': DR.C.B. EMMANUEL

DOC/NOAA RESEARCH FACILITIES CENTER

STAFF:TOT 50

MIAM I

FL

PHONE :

R\&D 47

'DIRECTOR'： DR. WILLIAM A. SMOKER

DOC/NOAA AUKE BAY LABORATORY

STAFF:TOT 47

P.0. BOX 155

AUKE BAY

AK 39180 PHONE:

R\&D 47

EXT. 2533

'DIRECTOR': DR. EUGENE AUBERT

DOC/NOAA GREAT LAKES ENVIRONMENTAL RESEARCH LAB

ANN ARBOR MI 0 PHONE:

R\&D 47

'DIRECTOR': DR. ELDON FERGUSON

DOC/NOAA AERONOMY LABORATORY

STAFF :TOT

R\&D 44

BOULDER

DO

PHONE :

RICHARD S. SHOMURA

DOC/NOAA HONOLULU LABORATORY

'DIRECTOR': RICHARD S. SHOMURA

P.O. BOX 3830

STAFF:TOT 41

R\&D 41

HONOLULU

HI 96812 PHONE: 808-946-2181 FTS 556-0220 
FEDERAL LABORATORY CONTACTS LISTED BY AGENCY AND STAFF SIZE(CONT)

'DIRECTOR' : DR. EDWIN KESSLER

DOC/NOAA NATIONAL SEVERE STORMS LABORATORY

STAFF :TOT 41

R\&D $\quad 41$

NORMAN

OK

PHONE :

MR. HARVEY KLASSEN 'DIRECTOR': DR. WARREN A. HOVIS

DOC/NOAA SATELLITE EXPERIMENT LABORATORY STAFF:TOT 37

FB4, ROOM 0215

SUITLAND

MD 20233 PHONE : 203-447-4590 A/V:636-4590

DR. ANDREW J. KEMMERER 'DIRECTOR': DR. ANDREW J. KEMMERER DOC/NOAA MISSISSIPPI LABORATORIES STAFF:TOT 36 3209 FREDERICK ST., P.0. DRAWER $1207 \quad$ R\&D 36 PASCAGOULA MS 39567 PHONE: 601-688-4850 FTS 499-3650

DR. HARRY L. SEAGRAN 'DIRECTOR': DR. HARRY L. SEAGRAN DOC/NOAA CHARLESTON LABORATORY STAFF:TOT 34 $\begin{array}{lll}\text { P.0. BOX } 1206 & \text { R\&D } 34\end{array}$ CHARLESTON SC 29412 PHONE: 803-724-4770 FTS 677-4770

DR. EDWARD J. KLIMA 'DIRECTOR': DR. EDWARD J. KLIMA DOC/NOAA GALVESTON LABORATORY STAFF:TOT 30 4700 AVENUE U GALVESTON

TX 77550 PHONE: $713-763-1211$ FTS 527-6501

LOUIS J. RONSIVALLI

'DIRECTOR': LOUIS J. RONSIVALLI

DOC/NOAA GLOUCESTER LABORATORY STAFF:TOT 28

EMERSON AVENUE

GLOUCESTER

MA 01930 PHONE: $\quad 617-281-3600$ FTS 837-9276

'DIRECTOR': M. E. RINGENBACH

DOC/NOAA ENGINEER ING DEVELOPMENT OFFICE

RIVERDALE

MD

PHONE :

STAFF:TOT 27 R\&D 21

DR. WILLIAM ARON

'DIRECTOR': DR. MICHAEL TILLMAN

DOC/NOAA NAT IONAL MARINE MAMMAL LABORATORY STAFF:TOT 27

2725 MONTLAKE BLVD., EAST

R\&D 27

SEATTLE WA 98112 PHONE: 206-442-4760 FTS 399-4760

DR. JAMES HANKF

DOC/NOAA MILFORD LABORATORY

MILFORD

CT 06460

DR. KENNETH SHERMAN

'DIRECTOR': DR. JAMES HANKF

STAFF:TOT 26

R\&D 23

DOC/NOAA NARRAGANSETT LABORATORY

ROUTE 7A, P.0. BOX 522A

NARRAGANSETT

RI 02882 PHONE: 401-789-9326 FTS 838-7174

'DIRECTOR': DR. MICHAEL D. HUDLOW DOC/NOAA HYDROLOGICAL RESEARCH LABORATORY STAFF:TOT 20 R\&D 19

PHONE: 
FEDERAL LABORATORY CONTACTS LISTED BY AGENCY AND STAFF SIZE(CONT)

NORMAN ABRAMSON

DOC/NOAA TIBURON LABORATORY

3150 PARADISE DRIVE

TIBURON
'DIRECTOR': NORMAN ABRAMSON

STAFF:TOT 18

R\&D 18

CA 94920 PHONE: 415-435-3149 FTS 556-0565
EUGENE L. NAKAMURA DOC/NOAA PANAMA CITY LABORATORY 3500 DELWOOD BEACH ROAD PANAMA CITY

FL 32407
'DIRECTOR' : EUGENE L. NAKAMURA STAFF:TOT 18 R\&D 18
ROBERT WOLOTIRA DOC/NOAA KODIAK LABORATORY P.0. BOX 1638 KODIAK

AK 99615 PHONE: 907-487-4961

'DIRECTOR' : ROBERT WOLOTIRA

STAFF :TOT 15 R\&D 15

'DIRECTOR': BERNARD H. CHOVITZ

DOC/NOAA GEODETIC RESEARCH AND DEVELOPMENT LABORATORY STAFF:TOT 15 ROCKVILLE MD PHONE:

'DIRECTOR': CAPT. PAUL E. TYLER

DOD ARMED FORCES RADIOBIOLOGY RESEARCH LAB STAFF:TOT 216

BETHE SDA

MD 0

PHONE:

R\&D 168

MS. SANDRA M. LEFSTRAND

DOD/AF ARMAMENT DIVISION

AFATL/DLOD

ELGIN AFB
$\mathrm{FL} \quad 32542$

'DIRECTOR': MAJ. ROBERT M. BONE

STAFF :TOT 6389

R\&D 3756

PHONE: $904-822-5344 \quad 904-822-4740$

'DIRECTOR': CDR BG.P.J. CONLEY JR. STAFF :TOT 5136 R\&D 3185

EDWARDS AFB

CA

PHONE:

'DIRECTOR' : COL.P.O. BOUCHARD

DAVID C. PIERCE

DOD/AF ROME AIR DEVELOPMENT CENTER

STAFF :TOT 1323

RADC/DOT

NY 13441 PHONE: 315-330-3047 A/V: 587-3047

MS. M. PUTNAM

'DIRECTOR': DR. W. L. LEHMANN

DOD/AF WEAPONS LABORATORY

STAFF :TOT 1111

AFWL/CA

R\&D 1033

KIRTLAND AFB

NM 87117 PHONE: 505-844-4293 A/V:244-4293

MR. JAMES JOHNSON

'DIRECTOR': COL.R. F. LOPINA
STAFF:TOT DOD/AF AVIONICS LABORATORY

STAFF:TOT 944

AFWAL/TST

R\&D 916

WRIGHT PATTERSON AFB OH 45433 PHONE: 513-255-5804

MR. JAMES JOHNSON 'DIRECTOR': COL.R. C. BARLOW

DOD/AF FLIGHT DYNAMICS LABORATORY

AFWAL/TST

WRIGHT PATTERSON AFB

$\mathrm{OH} \quad 45433$

STAFF:TOT 920

R\&D 810 
FEDERAL LABORATORY CONTACTS LISTED BY AGENCY AND STAFF SIZE(CONT)

'DIRECTOR': COL.W. M. BURKETT

DOD/AF WESTERN SPACE AND MISSILE CENTER

STAFF:TOT 724

R\&D 66

VANDENBERG AFB

$C A$

PHONE:

MAJOR MICHAEL J. MAC DONALD 'DIRECTOR': DR. BILLY E. WELCH

DOD/AF SCHOOL OF AEROSPACE MEDICINE

STAFF:TOT 666

TECHNICAL PLANS AND ANALYSIS DIVISION

BROOKS AFB

TX 78235 PHONE: $512-536-3406$

R\&D 486

DR. J.W. HOWARD

'DIRECTOR': COL. JAMES E. BAKER

DOD/AF GEOPHYSICS LABORATORY

STAFF:TOT 618

AFGL/XOP

HANSCOM AFB

MA 01730 PHONE: 617-861-3606

R\&D 519

MR. GERALD SAYLES

DOD/AF ROCKET PROPULSION LABORATORY

'DIRECTOR': COL. WILLIAM F. MORRIS

AFRPL/SR, MS 24

EDWARDS AFB

CA

PHONE: 805-277-5342 A/V:350-5342

MR. JAMES JOHNSON

DOD/AF MATERIALS LABORATORY

'DIRECTOR':

AFWAL/TST

WRIGHT PATTERSON AFB OH 45433 PHONE: 513-255-5804

STAFF:TOT 413

R\&D 206

COL. WILLIAM C. DE BOE 'DIRECTOR': COL. RONALD W. TERRY

DOD/AF HUMAN RESOURCES LABORATORY STAFF:TOT 365

AFHL/TSZ

BROOKS AFB

TX 78235 PHONE: 512-536-3426 A/V:240-3426

MR. W.R. BLADOS 'DIRECTOR': COL. ROY H. DE HART

DOD/AF AEROSPACE MEDICAL RESEARCH LABORATORY STAFF:TOT 289 HDQS. AFSC

ANDREWS AFB

20334 PHONE: $301-981-4493 \quad \mathrm{~A} / \mathrm{V}: 858-4493$

R\&D 289

'DIRECTOR': CDR CO. MICHAEL H. ALEXAN'D

DOD/AF ARNOLD ENGINEERING DEVELOPMENT CENTER STAFF:TOT 223

TULLAHOMA TN PHONE:

R\&D 208

CAPT. NORMAN FLEMING 'DIRECTOR': COL. FRANCIS CROWLEY, III DOD/AF . ENGINEERING AND SERVICES CENTER/ R\&D STAFF:TOT 104 HQAFESC/RDXX

R\&D 85

TYNDALL AFB

FL 32401 PHONE: 904-283-6493 904-283-6309

MAJOR T. DALE BAXTER 'DIRECTOR': LT. COL. W. D. SIORO

DOD/AF FRANK J. SEIBLER RESEARCH LABORATORY STAFF:TOT 33

FJSRL/XO

USAF ACADEMY

CO 80840 PHONE: $303-472-3120$

R\&D 16

'DIRECTOR': D. BALL

DOD/ARMY WHITE SANDS MISSILE RANGE

STAFF:TOT 3672

WHITE SANDS

NM

PHONE : 
FEDERAL LABORATORY CONTACTS LISTED BY AGENCY AND STAFF SIZE(CONT)

DR. WILLIAM FISCO

'DIRECTOR': COL. D.P. WHALFN

DOD/ARMY LARGE CALIBER WEAPONS SYSTEM LABORATORY

STAFF:TOT 1791

LCWSL-DRDAR-LCE-C

DOVER

NJ 07801 PHONE: 201-328-2561 A/V:880-2561

MR. AL SHERLOCK

'DIRECTOR': MR. F.R. BROWN

DOD/ARMY ENGINEER WATERWAYS EXPERIMENT STATION

WESTV, P. O. BOX 631

VICKSBURG

MS 39180 PHONE: 601-636-3111 EXT. 2533

R\&D 504

DR. KARL H. STEINBACH 'DIRECTOR': COL. ALBERT F. DORRISE

DOD/ARMY MOBILITY EQUIPMENT R\&D COMMAND STAFF:TOT 1262

ATTN : DRDME-ZK

FORT BELVOIR

VA

PHONE: $703-664-4970 \quad \mathrm{~A} / \mathrm{V}: 354-4970$

DR. R. DECAREAU

DOD/ARMY NATICK R\&D COMMAND

'DIRECTOR': COL.R.J. CUTHBERTSON

FOOD SCIENCES LABORATORY

NATICK

MA 01760 PHONE: 617-633-5172 A/V:256-5172

MR. CL IFFORD E. LANHAM 'DIRECTOR': COL. CLIFTON R. GOODWIN

DOD/ARMY HARRY DIAMOND LABORATORIES

STAFF:TOT 1154

CODE DELHD-TT, 2800 POWDER MILL RD.

ADELPHI

DC 20783

R\&D 623

DR. VIC RUWE

'DIRECTOR': CDR LOUIS RACHMELER

DOD/ARMY MISSILE RESEARCH AND DEVELOPMENT COMMAND STAFF:TOT 1001 DRSMI-RST

REDSTONE ARSENAL

AL 35898 PHONE： 205-876-8650

R\&D 590

PHONE : 202-394-2296 A/V:290-2296

'DIRECTOR': COL. JAMES M. DAVIS, JR.

DOD/ARMY YUMA PROVING GROUND

STAFF:TOT 986

YUMA

$\mathrm{AZ}$

PHONE :

R\&D 760

'DIRECTOR': DONALD RESCH

DOD/ARMY MATERIEL TESTING DIRECTORATE STAFF:TOT 917

R\&D 624

ABERDEEN PROVING GROUND MD

PHONE :

MR. L. WARE

'DIRECTOR': COL. PHOLLIPS K. RUSSELL DDD/ARMY WALTER REED ARMY INSTITUTE OF RESEARCH STAFF:TOT 913 R\&D 611 WASHINGTON

$\mathrm{DC}$

PHONE: $\quad 301-663-7325 \quad \mathrm{~A} / \mathrm{V}: 343-7325$

WILLIAM A. BARR DOD/ARMY CHEMICAL SYSTEMS LABORATORY DRDAR-CLY - L

ABERDEEN PROVING GROUND MD 21010 PHONE: 301-671-2031 A/V:584-2031
MR. S.W. ARGO

DOD/ARMY TANK R\&D COMMAND

DRDTA-RG

WARREN
'DIRECTOR': A. H. ANDERSON

STAFF:TOT 835

$R \& D \quad 435$

MI 48090 PHONE: $313-574-6372$ A/V:786-6372 
FEDERAL LABORATORY CONTACTS LISTED BY AGENCY AND STAFF SIZE(CONT)

'DIRECTOR': COL. JAMES H. PHILLIPS

DOD/ARMY ELECTRONIC PROVING GROUND

STAFF :TOT 800

FORT HUACHUCHUA

$A Z$

PHONE :

R\&D 328

MR. A.D. COATES

'DIRECTOR' : DR. ROBERT J. EICHELBERGER

DOD/ARMY BALLISTIC RESEARCH LABORATORY

STAFF :TOT 781

R\&D 734

ABERDEEN PROVING GROUND MD 21005 PHONE: 301-278-6954 A/V:283-6954

DR. MORTIMER ROTHENBUR

'DIRECTOR' : COL. CHARLES H. BAY

DOD/ARMY DUGWAY PROVING GROUND STAFF:TOT 778

STEDP-C

R\&D 599

DUGWAY

UT 84022 PHONE: 801-522-3314 A/V:789-3314

MR. D.W. SEITZ

'DIRECTOR' : CO.W.R. BENOIT

DOD/ARMY MATERIALS AND MECHANICS RESEARCH CENTER STAFF:TOT 622

CODE DRXME-PMT

WATERTOWN

MA 02172 PHONE: 617-923-3522

R\&D 435

DR. WILLIAM FISCO

'DIRECTOR': COL. AARON J. LARKINGS

DOD/ARMY FIRE CONTROL AND SMALL CALIBER WEAPONS SYSTEM STAFF:TOT 579 LCWSL-DRDAR-LCE-C

DOVER

NJ 07801 PHONE: 201-328-2561 A/V:880-2561

'DIRECTOR': DR. RICHARD M. CARLSON

DOD/ARMY AVIATION RESEARCH AND TECHNOLOGY LABS STAFF:TOT 560

MOFFETT FIELD

CA

PHONE :

R\&D 515

ROBERT E. NORTHRUP, JR. 'DIRECTOR': COL. WILLIAM F. RAWLINSON DOD/ARMY ATMOSPHERIC SCIENCES LABORATORY STAFF:TOT 533

DELAS-DP

WHITE SANDS

NM 88002 PHONE: 505-678-5236 A/V:789-5236

MR. L. WARE 'DIRECTOR': COL. RICHARD F. BARQUIST

DOD/ARMY MEDICAL RESEARCH INST. OF INFECTIDUS DISEASES STAFF:TOT 507

BUILDING 521

FT. DETRICK

MD 21701 PHONE: 301-663-7325 A/V:343-7325

DR. W.S. MCAFEE 'DIRECTOR': MR. MAX ADLER

DOD/ARMY ELECTRONIC WARFARE LABORATORY STAFF:TOT 496

DRDEL-SA

FORT MONMOUTH

NJ 07703 PHONE: 201-544-4131 A/V:992-4131

MR. RICHARD V. FULTON

'DIRECTOR' : DR. LOIJIS CAMERON

DOD/ARMY NIGHT VISION AND ELECTRO-OPTICS LABORATORY STAFF:TOT 491

ATTN: DELNV-D

R\&D 93

FORT BELVOIR

VA 22060 PHONE: 703-664-5605

A/V:454-3923

'DIRECTOR': MR. EMMETT PAIGE, JR.

DOD/ARMY COMMUNICATIONS R\&D COMMAND STAFF:TOT 449

FORT MONMOUTH

NJ

PHONE :

R\&D 400 
FEDERAL LABORATORY CONTACTS LISTED BY AGENCY AND STAFF SIZE(CONT)

'DIRECTOR': CO. ALBERT DEPROSPERO

DOD/ARMY MATERIAL SYSTEMS ANALYSIS ACTIVITY STAFF:TOT 440

ABERDEEN PROVING GROUND MD PHONE:

R\&D $\quad 375$

DR. JACK MATTICKS

'DIRECTOR': COL. FRANKLIN A. HART

DOD/ARMY RES. INST. FOR BEHAVIORAL AND SOCIAL SCIENCES STAFF:TOT 358 PERI - P AMC BLDG.

ALEXANDRIA

VA

R\&D 248

PHONE: $202-274-8275 \quad \mathrm{~A} / \mathrm{V}: 284-8275$

'DIRECTOR': COL. WILLIAM E. CROUCH, JR DOD/ARMY AVIATION DEVELOPMENT TEST ACTIVITY STAFF:TOT 324 FORT RUCKER

$\mathrm{AL}$

PHONE :

R\&D 214

DR. KENNETH R. KOTHE

'DIRECTOR': COL. DANIEL L. LYCAN

DOD/ARMY ENGINEER TOPOGRAPHIC LABORATORIES

STAFF:TOT 314

BUILDING 2592

R\&D 288

FT. BELVOIR

VA 22060 PHONE: 703-664-5828

A/V $354-5828$

'DIRECTOR': COL. JAMES F. HENRIONNET

DOD/ARMY COLD REGIONS TEST CENTER

STAFF :TOT 304

APO SEATTLE

WA

PHONE :

R\&D 165

MR. L. WARE

'DIRECTOR': CO. JOHN D. MARSHALL, JR.

DOD/ARMY
PRESIDIO

SAN FRANCISC 0

LETTERMAN ARMY INSTITUTE OF RESEARCH

STAFF:TOT 278

R\&D 178

DR. W.S. MCAFEE

CA

PHONE : 301-663-7325

$A / V: 343-7325$

DOD/ARMY ELECTRONICS TECHNOLOGY AND DEVICES LABORATORY STAFF:TOT 278 DRDEL-SA

'DIRECTOR': DR. C. G. THORNTON

FORT MONMOUTH

NJ 07703 PHONE: 201-544-4131 A/V:992-4131

DR. ANDREW ASSUR

'DIRECTOR': COL.W.A. HANSON

DOD/ARMY COLD REGIONS R\&D LABORATORY STAFF:TOT 277

72 LYME ROAD

HANOVER

NH 03755

R\&D 122

DOD/ARMY SIGNALS WARFARE LABORATORY

'DIRECTOR': HERBERT S. HOVEY, JR.

PHONE: $603-646-4237 \quad A / V: 684-4237$

VINT HILL

VA

PHONE :

STAFF:TOT 237

R\&D 36

MR. L. WARE

'DIRECTOR': COL. BASIL A. PRUITT, JR

DOD/ARMY INSTITUTE OF SURGICAL RESEARCH

FORT SAM HOUSTON

TX

PHONE:

STAFF:TOT 229

R\&D 108

DR. W.S. MCAFEE

'DIRECTOR': COL. D. PETERSON

DOD/ARMY AVIONICS R\&D ACTIVITY

STAFF:TOT 223

DRDEL-SA

NJ 07703 PHONE: 201-544-4131

FORT MONMOUTH

A/V: 992-4131 
FEDERAL LABORATORY CONTACTS LISTED BY AGENCY AND STAFF SIZE(CONT)

G. R. WILLIAMSON

'DIRECTOR': COL.L. J. CIRCEO

DOD/ARMY CONSTRUCTION ENGINEERING RESEARCH LABORATORY STAFF:TOT 207 P. 0. BOX 4005

CHAMPAIGN

IL 61820 PHONE: 217-352-6511

R\&D 129

MS. SUSAN LUCKAN 'DIRECTOR': COL. FRANKLIN H. TDP, JR. DOD/ARMY MEDICAL RESEARCH INST. OF CHEM. DEFENSE STAFF:TOT 187 SORD-IJV-ZA

ABERDEEN PROVING GROUND MD 21010 PHONE: 301-671-3653 A/V:584-3653

DR. W.S. MCAFEE

'DIRECTOR': COL. J.G. MIKULA

DOD/ARMY COMBAT SURVEILLANCE AND TARGET AQUISITION LA STAFF:TOT 179

DRDEL-SA

R\&D 88

FORT MONMOUTH

NJ 07703 PHONE: 201-544-4131 A/V:992-4131

MR. L. WARE

'DIRECTOR' : COL. HARRY G. DANGERFIELD

DOD/ARMY RESEARCH INSTITUTE OF ENVIRONMENTAL MEDICINE STAFF:TOT 175

NAT ICK

MA

PHONE :

R\&D 140

DR. DONALD EGNER

'DIRECTOR' : DR. JOHN D. WEISZ

DOD/ARMY HUMAN ENGINEERING LABORATORY

STAFF:TOT 175

R\&D 159

ABERDEEN PROVING GROUND MD 21005 PHONE: 301-278-4567 A/V:283-4567

'DIRECTOR' : COL. LEWIS J. MC CONNELL

DOD/ARMY AVIATION ENGINEERING FLIGHT ACTIVITY STAFF:TOT 166

EDWARDS AFB

CA

PHONE :

R\&D 134

MR. LAWRENCE WARE

DOD/ARMY MEDICAL R\&D COMMAND

'DIRECTOR': CDR BG G. PAPMUND

BUILDING 521

FORT DETRICK

MD 21701 PHONE: 301-663-7325 A/V:343-7325

MS. JUNE GREER

'DIRECTOR': COL. STANLEY C. KNAPP

DOD/ARMY AEROMEDICAL RESEARCH LABORATORY

P. O. BOX 577

FORT RUCKER

AL 36362 PHONE: 205-255-5107

STAFF :TOT 142

R\&D 113

'DIRECTOR': CDR COL. RICHARD P. VARRER

DOD/ARMY TROPIC TEST CENTER

STAFF :TOT 141

R\&D 109

FT. CLAYTON

$C Z$

PHONE:

'DIRECTOR': COL. JOHN N. ALBERTSON, JR

MR. L. WARE

R\&D LABORATORY STAFF:TOT 136

DOD/ARMY MEDI

BUILDING 521

FORT DETRICK

MD 21701 PHONE： 301-663-7325

R\&D 116

MR. L. WARE

'DIRECTOR': COL. DUANE E. CUTRIGHT

DOD/ARMY INSTITUTE OF DENTAL RESEARCH

STAFF :TOT 106

WASH INGTON

$D C$

PHONE :

R\&D 64 
FEDERAL LABORATORY CONTACTS LISTED BY AGENCY AND STAFF SIZE(CONT)

'DIRECTOR': COL. ANTHONY P. SIMKUS

DOD/ARMY ARMY RESEARCH OFFICE

STAFF :TOT 96

DURHAM NC PHONE:

R\&D 95

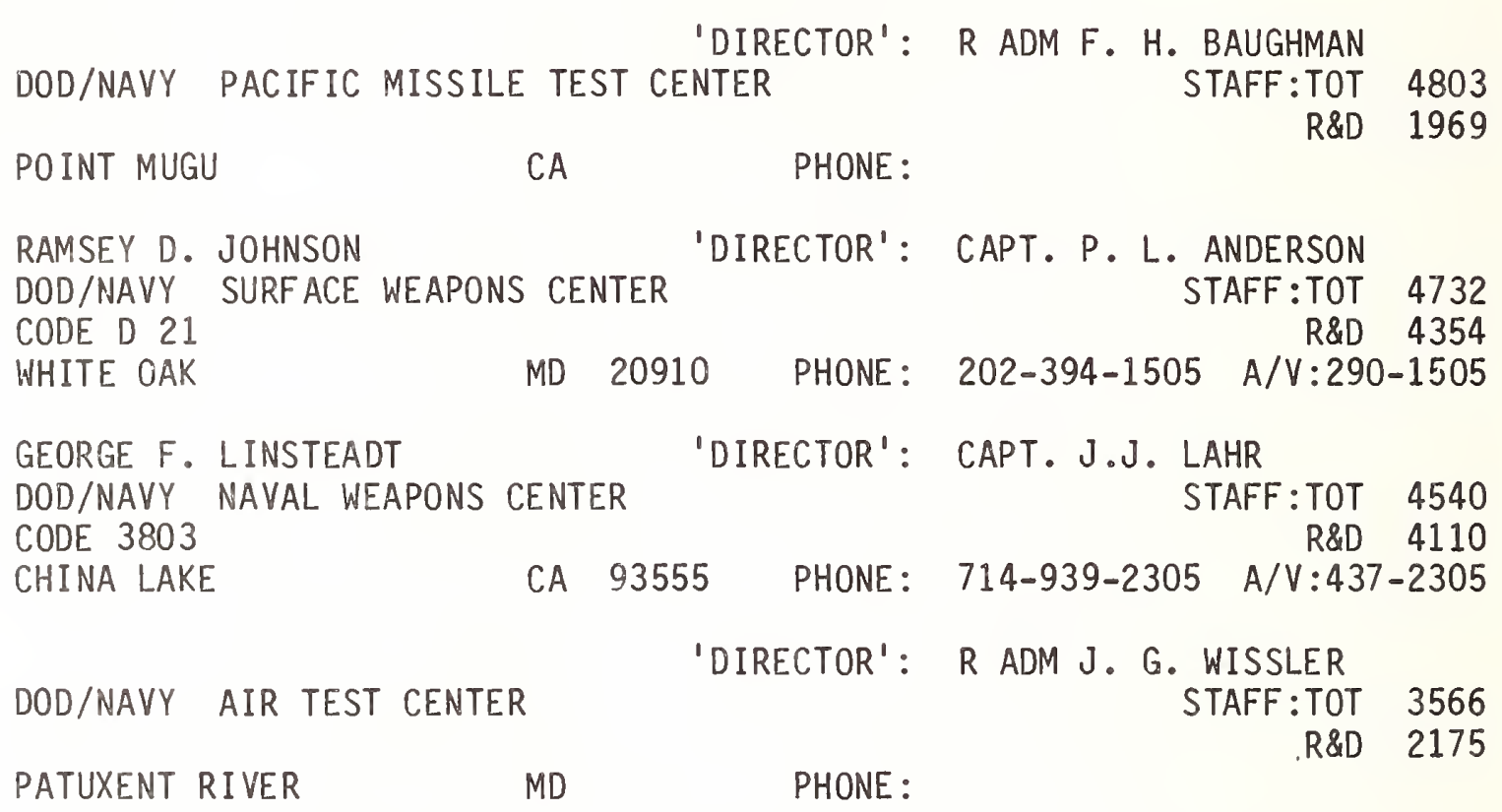

MR. RICHARD FULPER

DOD/NAVY RESEARCH LABORATORY

'DIRECTOR': CAPT.E. E. HENIFIN

CODE 1434

WASHINGTON

DC 20375 PHONE: 202-767-3726 A/V:297-3744

R\&D 2919

MS. M.M. MCNAMARA 'DIRECTOR': DR. C. NICHOLAS PRYOR

DOD/NAVY NAVAL UNDERWATER SYSTEMS CENTER STAFF:TOT 2800

CODE 0702, BLDG。80T

NEW LONDON

CT 06320 PHONE: 203-447-4590 A/V:636-4590

EUGENE COOPER

'DIRECTOR': CAPT. J.M. PATTON

DOD/NAVY OCEAN SYSTEMS CENTER STAFF:TOT 2791

CODE 013(B)

SAN DIEGO

CA 92152 PHONE: 714-225-7455 A/V:933-7455

DR. BASIL NAKONECHNY 'DIRECTOR': CAPT. BARRICK TIBBITTS

DOD/NAVY DAVID TAYLOR NAVAL SHIP R AND D CENTER STAFF:TOT 2567

CODE 012.2

R\&D 1899

BETHESDA MD 20084 PHONE: 202-227-1037 A/V:287-1037

MR. JEROME BORTMAN 'DIRECTOR': DR. P. K. LOBB

DOD/NAVY NAVAL AIR DEVELOPMENT CENTER STAFF:TOT 2399

CODE 7012

WARMINSTER

PA 18974 PHONE: 215-441-3100

R\&D 1631

TERRY ISING

DOD/NAVY NAVAL AVIONIC CENTER

'DIRECTOR': JOHN HAGUE

6000 21ST STREET

INDI ANAPOLIS

IN 46218

PHONE: $317-353-3220$ 
FEDERAL LABORATORY CONTACTS LISTED BY AGENCY AND STAFF SIZE(CONT)

MICHAEL PALAMAR

'DIRECTOR':

DOD/NAVY NAVAL AIR ENGINEERING CENTER

CODE 9011 , BLDG. \#26

LAKEHURST

NJ 08733 PHONE: 201-323-2391

STAFF:TOT 1776

R\&D 533

M. GRAY

'DIRECTOR': J. KELLY

DOD/NAVY NAVAL WEAPONS STATION EARLE

CODE 80A

COLTS NECK

NJ 07722 PHONE: 201-462-7688

STAFF:TOT 1300

R\&D 390

MR. W.H. WILLIAMS

'DIRECTOR': CAPT. RAYMEND D. BENNETT

DOD/NAVY COASTAL SYSTEMS CENTER

STAFF:TOT 732

CODE 120 NCSC

PANAMA CITY

FL 32407 PHONE: 904-234-4113 A/V:436-4113

DOD/NAVY AIR PROPULSION CENTER

'DIRECTOR': CAPT. B. T. ALLGOOD, JR. R\&D 542

TRENTON

NJ

PHONE :

STAFF:TOT 595

R\&D 523

'DIRECTOR': CAPT. WALTER F. MINER

DOD/NAVY MEDICAL RESEARCH INSTITUTE

STAFF:TOT 489

R\&D 392

BETHESDA

MD

PHONE :

'DIRECTOR': CAPT. DR. R. WEICHMANN

DOD/NAVY WEAPONS EVALUATION FACILITY

STAFF :TOT 434

PHONE :

R\&D $\quad 430$

'DIRECTOR': R ADM A. J. BACIOCCO, JR.

DOD/NAVY OFFICE OF NAVAL RESEARCH

STAFF:TOT 429

R\&D 309

WASHINGTON

$D C$

PHONE:

MR. JAMES F. JENKINS 'DIRECTOR': CAPT. RONALD P. COPE

DOD/NAVY CIVIL ENGINEERING LABORATORY STAFF:TOT 325

LO3C

PORT HUENEME

$C A$

PHONE: $805-982-4070$

R\&D 279

MR. HAROLD H. ROSEN

'DIRECTOR': CAPT. D. F. PARKER

DOD/NAVY PERSONNEL RESEARCH AND DEVELOPMENT CENTER STAFF:TOT 294 CODE 303

SAN DIEGO

CA 92152 PHONE: 714-225-7109

R\&D 273

$A / V: 933-7109$

MR. GEORGE E. STANFORD

DOD/NAVY OCEAN R AND D ACTIVITY

'DIRECTOR': CAPT.C.G. DARRELL

NSTL STATION

BAY ST. LOUIS

MS 39529 PHONE: $601-688-4790$

STAFF:TOT 271 R\&D 249

MR. LIONEL DICKINSON 'DIRECTOR': CDR. J. T. KENNEDY

DOD/NAVY EXPLOSIVE ORDNANCE DISPOSAL FACILITY STAFF:TOT 201

INDI AN HEAD

MD 20640 PHONE: $301-743-4439$ 
FEDERAL LABORATORY CONTACTS LISTED BY AGENCY AND STAFF SIZE(CONT)

'DIRECTOR': CAPT.R. E. MITCHELL

DOD/NAVY AEROSPACE MEDICAL RESEARCH LABORATORY STAFF:TOT 169

PENSACOLA FL PHONE:

R\&D 140

$\begin{array}{rrr}\text { DOIRECTOR': } & \text { CAPT.M. A. PETERSON, JR. } \\ \text { DOD/NAVY ORDNANCE MISSILE TEST FACILITY } & \text { STAFF:TOT } & 140 \\ \text { R\&D } & 105\end{array}$

WHITE SANDS NM PHONE:

LCDR J.C. FERGUSON 'DIRECTOR': CAPT. RICHARD H. RAHE

DOD/NAVY HEALTH RESEARCH CENTER STAFF:TOT 113

R\&D 90

SAN DIEGO CA 92138 PHONE: 714-225-2061 A/V:933-2061

'DIRECTOR': CDR. R. A. MARQULIES

DOD/NAVY SUBMARINE MEDICAL RESEARCH LABORATORY STAFF:TOT 94

GROTON CT PHONE:

R\&D 76

'DIRECTOR' : CAPT. A. S. MC LARSEN

DOD/NAVY UNDERSEA RANGES DEPARTMENT STAFF:TOT 82

NEWPORT

RI

PHONE :

R\&D 65

'DIRECTOR': CO. CAPT. RAYMOND H. WATTE DOD/NAVY MEDICAL RESEARCH UNIT NO. 3 STAFF:TOT 81

CAIRO

$E G$

R\&D 61

$\begin{array}{lcccc} & & \text { 'DIRECTOR' : CDR DONALD S. PARSONS } \\ \text { DOD/NAVY } & \text { CLOTHING AND TEXTILE RESEARCH FACILITY } & \text { STAFF:TOT } & 56 \\ \text { NATICK } & M A & \text { PHONE: } & \text { R\&D } & 29\end{array}$

DOD/NAVY DENTAL RESEARCH INSTITUTE NTC

'DIRECTOR': CAPT. M. R. WIRTHLIN, JR. GREAT LAKES

IL

PHONE :

STAFF:TOT 47

R\&D 28

R. P. STROMBERG

DOE SANDIA NATIONAL LABORATORIES

'DIRECTOR': GEORGE C. DACEY

ORG. 400

ALBUQUERQUE

NM 87185 PHONE: $505-844-5535$ FTS $844-5535$

GERALD T. RICHARDS 'DIRECTOR': R.E. BATZEL

DOE LAWRENCE LIVERMORE NATIONAL LABORATORY STAFF:TOT 7102

UNIV. OF CAL., P.0. BOX 808, L404

R\&D

LIVERMORE

CA 94550 PHONE: 415-422-6416 FTS 532-6416

DR. EUGENE STARK 'DIRECTOR': D.M. KERR

DOE LOS ALAMOS NATIONAL LABORATORY STAFF:TOT 6937

MS 185

LOS ALAMOS

NM 87545 PHONE: $505-667-4858$ FTS $843-4548$ 
FEDERAL LABORATORY CONTACTS LISTED BY AGENCY AND STAFF SIZE(CONT)

DONALD W. JARED 'DIRECTOR': H. POSTMA

DOE OAK RIDGE NATIONAL LABORATORY

STAFF :TOT 5175

R\&D

TV/C, P.O. BOX X

TN 37830 PHONE: 615-574-4193 FTS 624-4193

OAK RIDGE

BRUCE C. HUGUELET 'DIRECTOR': DR. WALTER E. MASSEY

DOE ARGONNE NATIONAL LABORATORY STAFF:TOT 5118

BLDG. 362, 9700 S. CASS AVE.

R\&D 5118

ARGONNE

IL 60439 PHONE: $312-972-3064$

STUART B. MILAM 'DIRECTOR': W.E. MCCULLOUGH

DOE IDAHO NATIONAL ENGINEERING LABORATORY STAFF:TOT 5100

IDAHO FALLS ID 83401 PHONE: 208-526-1618

$R \& D$

MS. MARGARET BOGOSIAN 'DIRECTOR': G.H. VINYARD

DOE BROOKHAVEN NATIONAL LABORATORY STAFF:TOT 4102

TECH. UTILIZATION OFFICER, BLDG. 460

R\&D

UPTON NY 11973 PHONE: 516-282-7338 FTS 666-7338

'DIRECTOR': A.C. DAVIS

DOE BETTIS ATOMIC POWER LABORATORY STAFF:TOT 3358

P.O. BOX 79

WEST MIFFLIN PA 15122 PHONE: 412-462-5000

R\&D

ROBERT J. MORRIS 'DIRECTOR': D.A. SHIRLEY

DOE LAWRENCE BERKELEY LABORATORY STAFF:TOT 3158

OFFICE OF TECH. AP., UNIV. OF CAL., BLDG. 930,RM.309 R\&D

BERKELEY CA 94720 PHONE: 415-843-2740 EXT 5111

CARL H. SCHMITT 'DIRECTOR': A.E.KAKRETZ

DOE KNOLLS ATOMIC POWER LABORATORY STAFF:TOT 2913 P.0. BOX 1072

SCHENECTADY

NY 12301 PHONE: 202-252-5564

R\&D

LOREN SCHMIDT 'DIRECTOR': D.E. OLESEN

DOE PACIFIC NORTHWEST LABORATORY STAFF:TOT 2084 P. O. BOX 999

RICHLAND

WA 99352 PHONE: 509-357-2559

R\&D 1250

JOHN MCCOOK

'DIRECTOR': R.R. WILSON

DOE FERMI NATIONAL ACCELERATOR LABORATORY STAFF:TOT 1982

C/O DIR. OF SPECIAL PROJECTS, P.0. BOX 500 R\&D

BATAVIA IL 60510 PHONE: 312-840-3211 FTS 370-3211

RON BLISS 'DIRECTOR': J.E. NOLAN

DOE HANFORD ENGINEERING DEVELOPMENT LABORATORY STAFF:TOT 1926 P.O. BOX 1970

RICHLAND WA 99352 PHONE: 509-376-3998

R\&D

ROBERT C. MILLS 'DIRECTOR': M.B. GOTTLIEB

DOE PRINCETON PLASMA PHYSICS LABORATORY STAFF:TOT 1406

PRINCETON UNIV., P.O. BOX 451

PRINCETON

NJ 08540 RHONE: 609-683-2469 
FEDERAL LABORATORY CONTACTS LISTED BY AGENCY AND STAFF SIZE(CONT)

EUGENE B. RICKANSRUD

'DIRECTOR': W.K.H. PANOFSKY

DOE STANFORD LINEAR ACCELERATOR CENTER

STANFORD UNIV. , P.0. BOX 4349

STAFF :TOT 1402

$R \& D$

$\begin{array}{lllll}\text { STANFORD CA } 94305 & \text { PHONE: } & 415-854-3300 & \text { EXT } 2217\end{array}$

A.H. PETERS

'DIRECTOR': A.H. PETERS

DOE SAVANNAH RIVER LABORATORY

STAFF:TOT 995

AIKEN SC 29801 PHONE: 803-725-2277

R\&D

H. DANA MORAN

'DIRECTOR' : DENIS HAYES

DOE SOLAR ENERGY RESEARCH INSTITUTE

1536 COLE BOULEVARD

GOLDEN

CO 80401 PHONE: $303-231-7115$ FTS $327-7115$

DANIEL E. WILLIAMS

'DIRECTOR': R. SITTANSEN

DOE AMES LABORATORY

STAFF :TOT 539

OFFICE AND LAB. BLDG., RM. 119, IOWA STATE U.

R\&D

AMES

IA 50011 PHONE: 515-294-2635 FTS 865-2635

PHILLIP L. JOHNSON 'DIRECTOR': PHILLIP L. JOHNSON

DOE OAK RIDGE ASSOCIATED UNIVERSITIES STAFF:TOT

398

ATTN: R\&D LAB. TT OFFICER, P.0. BOX 117

OAK RIDGE TN 37630 PHONE: 615-576-3300

WILLIAM PETERS 'DIRECTOR': S.W.CHUN

DOE PITTSBURGH ENERGY TECHNOLOGY CENTER STAFF:TOT 352

P.0. BOX 10940

PITTSBURGH

PA 15236 PHONE: 412-675-6251

R\&D

GUY ERVIN, III 'DIRECTOR': H.C. WEISENECH

DOE ENERGY TECHNOLOGY ENGINEERING CENTER STAFF:TOT 330

P.0. BOX 1449

CANOGA PARK

CA 91304 PHONE: 213-341-1000 EXT 6474

AUGUSTINE A. PITROLO 'DIRECTOR': AUGUSTINE A. PITROLO

DOE MORGANTOWN ENERGY TECHNOLOGY CENTER STAFF:TOT

R\&D

P.0. BOX 880

MORGANTOWN

WV 26505 PHONE: 303-599-7511

251

ROBERT JONES 'DIRECTOR': R.R.WILSON

DOE INHALATION TOXICOLOGY RESEARCH INSTITUTE STAFF:TOT 212

LOVELACE BIOMED. AND ENVIRON. RES. INST.,P.O.BOX 5890

ALBUQUERQUE NM 87115 PHONE: 505-844-2502

R\&D

C.C. LINVILLE 'DIRECTOR': H.R. JOHNSON

DOE BARTLESVILLE ENERGY TECHNOLOGY CENTER STAFF:TOT 172

P.0. BOX 1398

BARTLESVILLE OK 74003 PHONE: $918-336-2400$

$R \& D$

DR. R.J. JIACOLETTE 'DIRECTOR': J.P. WATSON

DOE LARAMIE ENERGY TECHNOLOGY CENTER STAFF:TOT 148

OFF. OF TECH. PROG. MGMT., P.0. BOX 3395, UNIV. STA. R\&D

LARAMIE WY 82071 PHONE: 307-721-2212 
FEDERAL LABORATORY CONTACTS LISTED BY AGENCY AND STAFF SIZE(CONT)

OWEN R. LUNT

'DIRECTOR' : OWEN R. LUNT

DOE LAB. OF NUCLEAR MEDICINE AND RAD. BIOLOGY, UC STAFF:TOT 145

900 VETERAN AVENUE

LOS ANGELES

CA 90024 PHONE: 213-825-9431

R\&D

PAUL L. LACELLE 'DIRECTOR': PAUL L. LACELLE

DOE UNIVERSITY OF ROCHESTER BIOMEDICAL LABORATORY STAFF:TOT 110 SCHOOL OF MEDICINE AND DENTISTRY

ROCHESTER

NY 14642 PHONE: $716-275-3723$

R\&D

HERBERT L. VOLCHOK 'DIRECTOR': HERBERT L. VOLCHOK

DOE ENVIRONMENTAL MEASUREMENT LABORATORY STAFF:TOT 95

376 HUDSON STREET R\&D

NEW YORK NY 10014 PHONE: 212-620-3616

'DIRECTOR': H. E. WALBURG

DOE COMPARATIVE ANIMAL RESEARCH LAB STAFF:TOT

R\&D

PHONE :

DR. PETER DEMOS 'DIRECTOR': PETER DEMOS

DOE BATES LINEAR ACCELERATOR FACILITY STAFF:TOT 75

MASS. INST. OF TECH., P.0. BOX $95 \quad$ R\&D

MIDDLETOWN MA 01949 PHONE: 617-245-6600

ROBERT H. SCHULER 'DIRECTOR': ROBERT H. SCHULER

DOE NOTRE DAME RADIATION LABORATORY STAFF:TOT 71

UNIVERSITY OF NOTRE DAME

NOTRE DAME

IN 45556 PHONE: 219-283-7502

$R \& D$

MICHAEL H. SMITH

'DIRECTOR': MICHAEL H. SMITH

DOE SAVANNAH RIVER ECOLOGY LABORATORY STAFF:TOT

DRAWER E

AIKEN

SC 28901 PHONE: $803-725-2472$

R\&D

69

PHILLIP G. FREEMAN 'DIRECTOR': G.H. GRONHORD

DOE GRAND FORKS ENERGY TECHNOLOGY CENTER STAFF:TOT 68 P.0. BOX 8213, UNIVERSITY STATION

GRAND FORKS ND 58202 PHONE: 701-759-8109

$R \& D$

CARLETON D. BINGHAM 'DIRECTOR': CARLETON D. BINGHAM

DOE NEW BRUNSWICK LABORATORY STAFF:TOT 60

9800 CASS AVENUE

ARGONNE

IL 60439 PHONE: 312-972-2446

R\&D

MCDONALD E. WRENN 'DIRECTOR': W.S.S. JEE

DOE RADIOBIOLOGY LABORATORY, UNIV. OF UTAH STAFF:TOT 54

UNIV. OF UTAH, BLDG. $351 \quad$ R\&D

SALT LAKE CITY UT 84112 PHONE: 901-581-6600

GARY W. WATSON 'DIRECTOR': H. KENDE

DOE MICH STATE UNIV./DOE PLANT RESEARCH LAB STAFF:TOT 50

MSU-DOE, PLANT RESEARCH LABORATORY

EAST LANSING

MI 48824 PHONE: 517-353-2270 
FEEERAL LABORATORY CONTACTS LISTED BY AGENCY AND STAFF SIZE(CONT) WILL IAM OCASION

DOE CENTER FOR ENERGY AND ENVIRONMENTAL RESEARCH STAFF:TOT 50 UNIV. OF PUERTO RICO, CAPORRA HEIGHTS STATION R\&D SAN JUAN PR 00935 PHONE: 809-767-0321

HARVEY M. PATT 'DIRECTOR': HARVEY M. PATT

DOE LAB OF RADIOBIOLOGY AND ENV. HEALTH/ UC STAFF:TOT 42 SCHOOL OF MEDICINE

SAN FRANCISCO CA 94143 PHONE: 415-666-1636

MOSHE SHIFRINE 'DIRECTOR': MOSHE SHIFRINE

DOE LAB. FOR ENERGY-RELATED HEALTH RES., UC/DAVIS STAFF:TOT 40 UNIVERSITY OF CALIFORNIA

DAVIS

CA 95616 PHONE: $916-752-1341$

B.J. BRINK

'DIRECTOR': DR. FRANCIS G. MC LEAN

DOI ENGINEERING AND RESEARCH LABORATORY

DIV。OF RES., CODE D-1500B, DENVER FED. CENTER

STAFF :TOT 230

DENVER

CO 80225 PHONE: 303-234-3549 FTS 234-3549

WALT EVANS 'DIRECTOR': JOHN MURPHY

DOI/BOM PITTSBURGH MINING AND SAFETY RES。CENTER STAFF:TOT 259

P.O.BOX 18070

PITTSBURGH

PA 15236 PHONE: 412-675-6698

R\&D

RICHARD DICK

'DIRECTOR': JOHN CORWIN

DOI/BOM TWIN CITIES RESEARCH CENTER

5629 MINNEHAHA AVE. SOUTH

TWIN CITIES MN 55417 PHONE: 612-725-4562

STAFF:TOT 181

R\&D

STEPHEN OHARE 'DIRECTOR': FRANK BLOCK

DOI/BOM ALBANY METALLURGY RESEARCH CENTER

1450 QUEEN AVE. SW, P.0. BOX 70

ALBANY OR 97321 PHONE:

STAFF:TOT 162

$R \& D$

BILL NISSEN 'DIRECTOR': WILLIAM MCKINNEY

DOI/BOM SALT LAKE CITY METALLURGY RES. CENTER STAFF:TOT 108

729 ARAPEEN DRIVE

SALT LAKE CITY UT 84108 PHONE: $801-524-6146$

$R \& D$

STEVE UTTER 'DIRECTOR': GALEN WADDELL

DOI/BOM DENVER MINING RESEARCH CENTER STAFF :TOT

DENVER FEDERAL CENTER, BLDG. \#20

DENVER CO 80225 PHONE: 303-234-3754

R\&D

TOM BRADY 'DIRECTOR': DOUG BOLSTAD

DOI/BOM SPOKANE MINING RESEARCH CENTER STAFF:TOT 90

315 MONTGOMERY AVENUE R\&D

SPOKANE WA 99207 PHONE: 509-484-1610

STEPHEN LAW 'DIRECTOR': WILLIAM CAMPBELL

DOI/BOM AVONDALE METALLURGY RESEARCH CENTER STAFF:TOT 4900 LASALLE ROAD

AVONDALE

MD 20782 PHONE: 301-436-7542 
FEDERAL LABORATORY CONTACTS LISTED BY AGENCY AND STAFF SIZE(CONT)

BOB LEWIS

DOI/BOM RENO METALLURGY RESEARCH CENTER STAFF:TOT 76

1605 EVANS AVENUE

RENO

NV 89512 PHONE: 702-784-5363

$R \& D$

JIM STEPHENSON

'DIRECTOR'： L. NEUMEIER

DOI/BOM ROLLA METALLURGY RESEARCH CENTER

STAFF:TOT 65

P.0. BOX 280, 1300 BISHOP AVE.

ROLLA

MO 65401 PHONE: 314-364-3169

R\&D

IGNATZ FELD 'DIRECTOR': MARTIN STANCZYK

DOI/BOM TUSCALOOSA METALLURGY RESEARCH CENTER STAFF:TOT 53 P.O. BOX L, UNIVERSITY

TUSCALOSSA AL PHONE: 205-758-0491

R\&D

BOB LEWIS 'DIRECTOR': BILL STEPHENS

DOI/BOM BOULDER CITY METALLURGY ENGINEERING LAB STAFF:TOT 35 RENO RES. CENTER, 1605 EVANS AVE.

RENO NV 89512 PHONE: 702-784-5363 R\&D

RICHARD D. CURNOW 'DIRECTOR':

DOI/FWS DENVER WILDLIFE RESEARCH CENTER STAFF:TOT 208

FEDERAL CENTER, BLDG。 16

DENVER

CO 80225 PHONE: $303-234-2283$ FTS 234-2283

JOHN G. ROGERS 'DIRECTOR': JOHN G. ROGERS

DOI/FWS PAUTUXENT WILDLIFE RESEARCH CENTER STAFF:TOT 178

R\&D 178

LAUREL $\quad$ MD 20811 PHONE: 301-937-7211 FTS 937-7211

JAMES E. WEAVER 'DIRECTOR':

DOI/FWS NATIONAL FISHERIES CENTER-LEETOWN

RT. 3, BOX 40

KEARNE YSVILLE

WV 25430 PHONE :

STAFF:TOT 115

R\&D

FTS $925-5218$

RICHARD J. GRAHAM 'DIRECTOR':

DOI/FWS COLUMBIA NATIONAL FISHERY RES. LAB

RT. 1

COLUMBIA MO 65201 PHONE:

STAFF :TOT 97

R\&D 87

FTS 276-5399

JOSEPH H. KUTKUHN

DOI/FWS GREAT LAKES FISHERY LAB

1451 GREEN ROAD

ANN ARBOR

MI 48105 PHONE :

'DIRECTOR': JOSEPH H. KUTKUHN

REY STENDALL 'DIRECTOR':

DOI/FWS NORTHERN PRAIRIE WILDLIFE RESEARCH CENTER STAFF:TOT 57

P.O. BOX 1747

JAMESTOWN

ND 58401 PHONE:

STAFF:TOT 89

R\&D 91

FTS $378-1200$

ALFRED C. FOX

'DIRECTOR': ALFRED C. FOX

DOI/FWS SEATTLE NATIONAL FISHERY RESEARCH CENTER

BLDG. 204, NAVAL SUPPORT ACTIVITY

R\&D 43

FTS 783-6152

SEATTLE

WA 98115 PHONE :

STAFF :TOT 53

R\&D 50

FTS $399-5960$ 
FEDERAL LABORATORY CONTACTS LISTED BY AGENCY AND STAFF SIZE(CONT)

DONALD C. HALES 'DIRECTOR':

DOI/FWS NATIONAL FISHERY RESEARCH LAB-LA CROSSE

P.O. BOX 818

LA CROSSE

WI 54601 PHONE :

STAFF :TOT 41

R\&D 39

FTS $364-3212$

JAMES J. KENNELLY 'DIRECTOR':

DOI/FWS NATIONAL WILDLIFE HEALTH LABORATORY

1655 LINDEN DR., U. OF $W$.

MADISON WI 53706 PHONE:

STAFF :TOT 30

R\&D

FTS $364-5411$

JOHN MC LAURIN

'DIRECTOR': JOHN MC LAURIN

DOI/USGS USGS CENTRAL REGION

MAIL STOP 511, BOX 25046, DENVER FED.

STAFF :TOT 4500

$\begin{array}{lll}\text { CENTER } & \text { R\&D } 900 \\ \text { PHONE: } & 303-234-2351 & \text { FTS } 234-2351\end{array}$

DENVER

CO 80225 PHONE:

GEORGE GRYC

DOI/USGS USGS WESTERN REGION

'DIRECTOR': DR. DALLAS L. PECK

345 MIDDLEFIELD ROAD

MENLO PARK

CA 94025 PHONE: $415-323-8111$ FTS $467-2917$

STAFF:TOT 3676

R\&D 2206

DR. ETHAN T. SMITH

'DIRECTOR': DR. DALLAS PECK

DOI/USGS USGS NATIONAL CENTER

STAFF : TOT 3000

CODE 105

RESTON

VA 22092 PHONE: 703-860-7811 FTS 860-7811

CECIL YATES

'DIRECTOR': JAMES H. GREER

DOJ FEDERAL BUREAU OF INVESTIGATION LABORATORY STAFF:TOT 429

FORENSIC SCI. RES. \& TNG. UNIT, FBI

QUANTICO

VA 22135 PHONE: 703-640-6131

R\&D 43

LARRY WILL IAMS

'DIRECTOR': EDMUND J. DOENKE

DOT FEDERAL AVIATION ADMIN. TECHNICAL CENTER STAFF:TOT 1043

FAA TECHNICAL CENTER

R\&D 751

ATLANTIC CITY AIRPORT NJ 08405 PHONE: 609-641-8200 EXT. 3653

R.V. GIANGRANDE

'DIRECTOR': DR. JAMES COSTANTINO

DOT TRANSPORTATION SYSTEMS CENTER

OFFICE OF TECH. SHARING, DTS-31, KENDALL SQ.

STAFF:TOT 600

CAMBRIDGE

MA 02142 PHONE:

R\&D 480

DR. DONALD L. SPANTON 'DIRECTOR': DR. DONALD L. SPANTON

DOT TRANSPORTATION TEST CENTER

400 SEVENTH STREET, S.W.

WASHINGTON

DC 20590 PHONE: 202-426-9601

R\&D 40

R.J. BETSOLD 'DIRECTOR': E.M. WOOD

DOT FAIRBANK HIGHWAY RESEARCH STATION

OFFICE OF IMPLEMENTATION (HTR-1)

WASHINGTON

DC 20590 PHONE: 703-285-2034

STAFF :TOT 200

R\&D 160

J. ROBERT DILLE, M.D. 'DIRECTOR': J. ROBERT DILLE, M.D. DOT CIVIL AEROMEDICAL INSTITUTE

AERONAUTICAL CENTER

OKLAHOMA CITY

OK

PHONE :

STAFF:TOT 200 R\&D 100 
FEDERAL LABORATORY CONTACTS LISTED BY AGENCY AND STAFF SIZE(CONT)

M.J. D ANGELO 'DIRECTOR': CAPT. K.D. URFER

DOT U.S. COAST GUARD R\&D CENTER STAFF:TOT 148 AVERY POINT

GROTON

CT 06340 PHONE : 203-445-8501

R\&D 74

JAMES E. HOFFERBERTH 'DIRECTOR': ROBERT L. CARTER

DOT VEHICLE RESEARCH AND TEST CENTER STAFF:TOT 34

P.0. BOX 37

EAST LIBERTY $\quad \mathrm{OH} 43319$ PHONE:

R\&D 27

MR. R. BOKSLEITNER 'DIRECTOR': DR. F. GORDON HUETER

EPA HEALTH EFFECTS RESEARCH LABORATORY STAFF:TOT 328

USEPA

R\&D 311

RESEARCH TRIANGLE PARK NC 27711 PHONE: 919-541-2611 FTS 629-2611

MR. M. MASTRACCI 'DIRECTOR': MR. FRANCIS T. MAYO

EPA MUNICIPAL ENVIRONMENTAL RESEARCH LABORATORY STAFF:TOT 161 USEPA/MERL

CINCINNAT I

OH 45268 PHONE: 513-684-7951

R\&D 161

MR. R. BOKSLEITNER 'DIRECTOR': MR. FRANK T. PRINCIOTTA

EPA INDUSTRIAL ENVIRONMENTAL RESEARCH LABORATORY STAFF:TOT 133

USEPA

R\&D 161

RESEARCH TRIANGLE PARK NC 27711 PHONE: 919-541-2611 FTS 629-2611

MR. R. BOKSLEITNER

'DIRECTOR' : DR. ALFRED H. ELLISON

EPA ENVIRONMENTAL SCIENCES RESEARCH LABORATORY STAFF:TOT 132

USEPA

R\&D 127

RESEARCH TRIANGLE PARK NC 27711 PHONE: 919-541-2611 FTS 629-2611

MR. R. BOKSLEITNER 'DIRECTOR': DR. THOMAS R. HAUSER

EPA ENVIRONMENTAL MONITORING SYST. LAB-RTP STAFF:TOT 126

USEPA

R\&D 122

RESEARCH TRIANGLE PARK NC 27711 PHONE: 919-541-2611 FTS 629-2611

MRS. GENEVA DOUGLAS 'DIRECTOR': MR. GLENN E. SCHWEITZER

EPA ENVIRONMENTAL MONITORING SYST. LAB-LV STAFF:TOT 123

USEPA/EMSL, P.0. BOX 15027

LAS VEGAS NV 89114 PHONE: 702-736-2969

R\&D 123

MS. KAREN RANDOLPH 'DIRECTOR': DR. THOMAS A. MURPHY

EPA ENVIRONMENTAL RESEARCH LAB-CORVALLIS STAFF:TOT 84

USEPA/ERL, 200 S.W. 35TH STREET

CORVALLIS OR 97330 PHONE: 503-757-4637

R\&D 84

MR. M. MASTRACCI 'DIRECTOR': DR. NORBERT A. JAWORSKI

EPA ENVIRONMENTAL RESEARCH LAB-DULUTH STAFF:TOT 83

USEPA/ERL, 6201 CONGDON BLVD. R\&D 83

DULUTH MN 55804 PHONE: 218-727-6692

MR. M. MASTRACCI 'DIRECTOR': MR. ROBERT L. BOOTH

EPA ENVIRONMENTAL MONITORING SUPPORT LABORATORY STAFF:TOT 79 USEPA/EMSL

CINCINNATI OH 45268 PHONE: 513-684-7301

R\&D 79 
FEDERAL LABORATORY CONTACTS LISTED BY AGENCY AND STAFF SIZE (CONT)

MR. M. MASTRACCI

'DIRECTOR': DR. DAVID W. DUTTWËILEER

EPA ENVIRONMENTAL RESEARCH LABORATORY STAFF:TOT 78

USEPA/ERL, COLLEGE STATION ROAD

ATHENS GA 30605 PHONE : 404-546-3134

R\&D 78

MR. M. MASTRACCI

'DIRECTOR': DR. DAVID G. STEPHAN

EPA INDUSTRIAL ENVIRONMENTAL RESEARCH LABORATORY STAFF:TOT

USEPA/IERL

CINC INNATI

OH 45268 PHONE: $513-684-4247$

R\&D 74

MR. M. MASTRACCI 'DIRECTOR': MR. CLINTON HALL

EPA ROBERT S. KERR ENVIRONMENTAL RESEARCH LAB STAFF:TOT 55

USEPA/RSKERL, P. O. BOX 1198

ADA OK 74820 PHONE: 405-332-8800

$R \& D \quad 55$

MR. M. MASTRACCI 'DIRECTOR': DR. TUDOR T. DAVIES

EPA ENVIRONMENTAL RESEARCH LAB-RI STAFF:TOT 55

USEPA/ERL, SOUTH FERRY ROAD

$\begin{array}{llll}\text { NARRAGANSETT } & \text { RI } 02882 \text { PHONE: 401-789-1071 }\end{array}$

R\&D 55

'DIRECTOR': DR. HENRY F. ENOS

EPA ENVIRONMENTAL RESEARCH LAB-SABINE STAFF:TOT 54

USEPA/ERL

SABINE IS., GULF BREEZE FL 35261 PHONE: 904-932-5311

R\&D 54

DR. IHOR MASNYK 'DIRECTOR': DR. VINCENT T. DEVITA

HHS NATIONAL CANCER INSTITUTE STAFF:TOT 1606

BUILDING 32, ROOM 3A04

BETHESDA

MD 20205 PHONE: 301-496-3251

R\&D 1590

DR. BERGE HAMPAR 'DIRECTOR': DR. PETER FISCHINGER

HHS FREDERICK CANCER RESEARCH FACILITY STAFF:TOT 1043

FT. DETRICK

FREDERICK

MD 21701 PHONE: 301-965-1108

R\&D 1043

ALBERT BALOWS 'DIRECTOR': WALTER R. DOWDLE, PH.D.

HHS CENTER FOR INFECTIOUS DISEASES STAFF:TOT 908

CENTER FOR DISEASE CONTROL

ATLANTA GA 30333 PHONE: 404-329-3967

R\&D 363

ELIZABETH.SINGER 'DIRECTOR': DR. LESTER B. SALANS

HHS NAT. INST. OF ARTH., DIABETES, \& DIGEST. \& KI STAFF:TOT 544 NIADDK, NIH

BETHESDA MD 20205 PHONE： 301-496-3583

R\&D 544

MS. SYLVIA W. SHAFFER 'DIRECTOR': DR. THOMAS N. CHASE

HHS NEUROLOGY \& COMMUNICATIVE DISORDERS RES. LAB STAFF:TOT 501

BLDG. 31, RM. 8A06, NIH

BETHESDA MD 20205 PHONE: 301-496-5751

R\&D 501

KENNETH W. SELL, M.D., PH.D. 'DIRECTOR': KENNETH W. SELL, M.D. , PH. HHS NAT. INST. OF ALLERGY AND INFECTIOUS DISEASES STAFF:TOT 500 NIH, NIAID, BLDG. 10, RM. $11 \mathrm{C} 103$ BE THE SDA

MD 20205

PHONE : 301-496-3006

R\&D 490 
FEDERAL LABORATORY CONTACTS LISTED BY AGENCY AND STAFF SIZE(CONT)

DR. FREDERICK K. GOODWIN 'DIRECTOR': DR. FREDERICK K. GOODWIN HHS INTRAMURAL RES.PROG.-NIMH STAFF:TOT 430 9000 ROCKVILLE PIKE BETHESDA

MD 20205 PHONE: 301-496-3501

R\&D 430

DR. CHARLES E. CARTER 'DIRECTOR': DR. DAVID P. RALL

HHS NAT. INST. OF ENVIRONMENTAL HEALTH SCIENCES STAFF:TOT 429 $\begin{array}{ll}\text { BOX } 12233 & \text { R\&D } 240\end{array}$ RESEARCH TRIANGLE PARK NC 27709 PHONE: 919-541-3205

BENJAMIN E. FULTON 'DIRECTOR': BETTY H. PICKETT, PH. D. HHS NAT. INST. OF CHILD HEALTH AND HUMAN DEV. STAFF:TOT 426 BLDG. 31, ROOM 2A11, NIH R\&D 405 BETHESDA MD 20205 PHONE: 301-496-4121

LARRY BLASER 'DIRECTOR': DR. JACK ORLOFF

HHS NATIONAL HEART, LUNG, AND BLOOD INSTITUTE STAFF:TOT 362 BUILDING 31, ROOM 4A21 BETHESDA

MD 20205 PHONE: $301-496-4236$

R\&D 362

L. LEE MANUEL 'DIRECTOR': ARNOLD W. PRATT, M.D. HHS DIVISION OF COMPUTER RESEARCH AND TECHNOLOGY STAFF:TOT 274 ROOM 2023, BUILDING 12A, NIH R\&D 164 BETHESDA MD 20205 PHONE: 301-496-5206

DR. KENNETH LYNN 'DIRECTOR': MARIE U. NYLEN

HHS INTRAMURAL RES. PROG.-NIDR STAFF:TOT 266 OSHR, NIDR, BLDG. 31, RM. 2C34, NIH R\&D 266 BETHESDA MD 20205 PHONE: 301-496-4261

ROBERT GLENN 'DIRECTOR': DR. J. DONALD MILLAR HHS APPALACHIAN LAB FOR OCC. SAFETY AND HEALTH STAFF:TOT 239 944 CHESTNUT RIDGE ROAD R\&D 179 MORGANTOWN WV 26505 PHONE: 304-291-4474 FTS 923-4474

DANIEL ROGERS 'DIRECTOR': RICHARD C. GREULICH, PH.D. HHS GERENTOLOGY RES CENTER-NAT. INST. ON AGING STAFF:TOT 238 BALTIMORE CITY HOSPITALS R\&D 202 BALTIMORE MD 21224 PHONE: 301-396-9421

JIN H. KINOSHITA 'DIRECTOR': JIN H. KINOSHITA, PH. D. HHS INTRAMURAL RESEARCH PROGRAM-NEI STAFF:TOT 173 BLDG. 41, 6A04, 9000 ROCKVILLE PIKE BETHESDA MD 20205 PHONE: 301-496-3552 R\&D 173

KENNETH W. SELL, M.D., PH.D. 'DIRECTOR': DR. KENNETH W. SELL HHS NAT. INST. OF ALLERGY AND INFECTIOUS DISEASES STAFF:TOT 128 NIH, NIAID, BLDG. 10, RM. 11C103 BETHESDA MD 20205 PHONE: 301-496-3006

DR. RICHARD WYATT

'DIRECTOR': DR. RICHARD WYATT

HHS INTRAMURAL RES. PROG.-NIMH-DIV. OF SP. MENTAL STAFF:TOT 120 WAW-RM. 536, ST. ELIZABETHS HOSPITAL WASHINGTON DC 20032 PHONE: 202-574-757- 
FEDERAL LABORATORY CONTACTS LISTED BY AGENCY AND STAFF SIZE(CONT)

$\begin{array}{lll}\text { DAVID D. BAYSE, PH.D. } & \text { 'DIRECTOR': } \\ \text { HHS } & \text { CLINICAL CHEMISTRY DIVISION } & \text { D. BAYSE, PH.D. } \\ \text { STAFF:TOT } 118\end{array}$ CENTERS FOR DISEASE CONTROL

R\&D 59

ATLANTA GA 30333 PHONE: 404-452-4152 FTS 236-4152

DR. BARRY JOHNSON 'DIRECTOR': DR. J. DONALD MILLAR

HHS TAFT FACILITY-BIOMED. AND BEHAVIORAL SCI. STAFF:TOT 113

4676 COLUMBIA PARKWAY

CINCINNATI

OH 45226 PHONE: 513-684-8465 FTS $684-8465$

WALTER HAAG

'DIRECTOR': DR. J. DONALD MILLAR

HHS RIDGE FACILITY-PHYSICAL SCI. AND ENGINEERING STAFF:TOT 106

5555 RIDGE AVENUE

CINCINNAT I

OH 45213 PHONE: 513-684-4321 FTS 684-4321

ELIZABETH SINGER

'DIRECTOR' : LESTER B. SALANS, M.D.

HHS CLINICAL RES. SEC. \& S.W. FIELD STUDIES SEC. STAFF:TOT 16 NIADDK, NIH

BE THE SDA

MD 20205 PHONE: 301-496-3583 301-496-5877

MR. A.D. SMITH

'DIRECTOR' : MR. BRUCE C. MURRAY

NASA JET PROPULSION LABORATORY STAFF:TOT 3788

JPL-MAIL STOP 180-801

PASADENA

CA 91109 PHONE: 213-354-4849 792-4849

R\&D 3788

MR. I. AKBAY

'DIRECTOR': DR. WILLIAM R. LUCAS

NASA GEORGE C. MARSHALL SPACE FLIGHT CENTER STAFF:TOT 3563

MSFC, MAIL CODE AT01

R\&D 3563

MARSHALL SPACE FL. CENT AL 35812 PHONE: 205-453-2224 FTS 872-2224

JOHN T. WHEELER 'DIRECTOR': DR. CHRISTOPHER C. KRAFT,

NASA LYNDON B. JOHNSON SPACE CENTER STAFF:TOT 3508

JSC, MAIL CODE AT3

HOUSTON

TX 77058 PHONE:

R\&D 3508

FTS 525-3809

DONALD S. FRIEDMAN 'DIRECTOR': MR. A. THOMAS YOUNG

NASA GODDARD SPACE FLIGHT CENTER STAFF:TOT 3436

GSFC, MAIL CODE 702.1

GREENBELT

MD 20771 PHONE:

R\&D 3436

JOHN SAMOS 'DIRECTOR': DR. DONALD P. HEARTH

NASA LANGLEY RESEARCH CENTER STAFF:TOT 2966

LA RC, MAIL STOP 139A

HAMPTON

VA 23665 PHONE:

R\&D 2966

FTS $928-3281$

HARRISON ALLEN, JR. 'DIRECTOR': DR. JOHN F. MC CARTHY, JR.

NASA LEWIS RESEARCH CENTER STAFF:TOT 2822

LE RC, MAIL STOP 7-2

CLEVELAND

OH 44135 PHONE:

R\&D 2822

FTS 294-6422

U. REED BARNETT

'DIRECTOR': MR. RICHARD G. SMITH

NASA JOHN F. KENNEDY SPACE CENTER STAFF:TOT 2201

KSC, MAIL CODE PT-SPD

KENNEDY SPACE CENTER

FL

PHONE :

R\&D 2201

FTS 823-3017 
FEDERAL LABORATORY CONTACTS LISTED BY AGENCY AND STAFF SIZE(CONT)

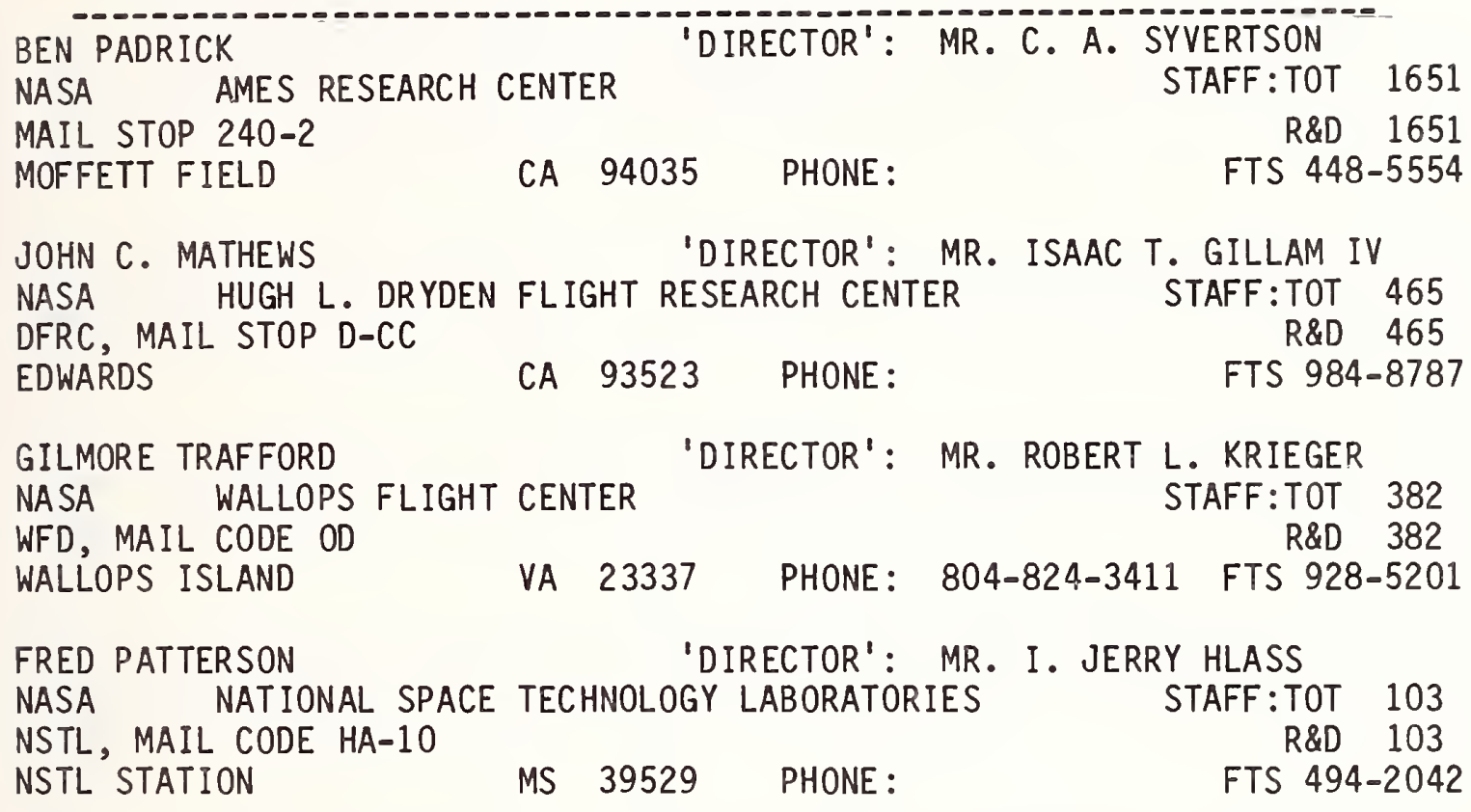

DR. HAROLD W. BAYNTON 'DIRECTOR': DR. WILMOT N. HESS

NSF NATIONAL CENTER FOR ATMOSPHERIC RESEARCH STAFF:TOT 676

$\begin{array}{lll}\text { P.0. BOX } 3000 & \text { R\&D } 594\end{array}$

BOULDER CO 80307 PHONE: $303-484-5151$

DR. ROBERT HAVLEN 'DIRECTOR': DR. MORTON S. ROBERTS

NSF NATIONAL RADIO ASTRONOMY OBSERVATORY STAFF:TOT 332

EDGEMONT ROAD

CHARLOTTESVILLE

VA PHONE: 804-296-0211

R\&D 332

JAMES F. KATTINGE 'DIRECTOR': DR. GEOFFREY R. BURBIDGE

NSF KITT PEAK NATIONAL OBSERVATORY STAFF:TOT 288

$\begin{array}{ll}\text { P.0. BOX } 26732 & \text { R\&D } 288\end{array}$

TUCSON AZ 85726 PHONE: 602-325-9366

EUGENE BARTEL 'DIRECTOR': DR. TOR HAGFORS

NSF NATIONAL ASTRONOMY AND IONOSPHERE CENTER STAFF:TOT 150

CORNELL UNIVERSITY

ITHACA NY PHONE: 607-256-3734

R\&D 150

JAMES B. WAY, JR. 'DIRECTOR': DR. PATRICK S. OSMER

NSF CERRO TOLOLO INTER-AMERICAN OBSERVATORY STAFF:TOT 144 CASILLA 603

LA SERENA $\quad$ CH $\quad$ PHONE: 1353

R\&D 144

FRANK A. HEGWER 'DIRECTOR': DR. JACK B. ZIRKER

NSF SACRAMENTO PEAK OBSERVATORY STAFF:TOT 51

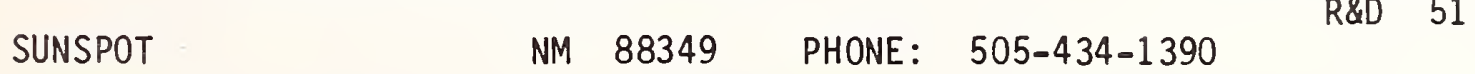

DR. JAMES F. MELLO 'DIRECTOR': DR. RICHARD FISKE

SMITHSON NATIONAL MUSEUM OF NATURAL HISTORY STAFF:TOT 85

NHB $106,10 T H \&$ CONSTITUTION AVE.

WASHINGTON DC 20560 PHONE: 202-357-2661 
FEDERAL LABORATORY CONTACTS LISTED BY AGENCY AND STAFF SIZE(CONT)

JOHN G. GREGORY

'DIRECTOR': DR. GEORGE B. FIELD

SMITHSON SMITHSONIAN ASTROPHYSICAL OBSERVATORY

STAFF:TOT 72

SAO, 60 GARDEN STREET

CAMBRIDGE

MA 02138 PHONE: $617-495-7102$

$R \& D \quad 24$

DR. WILLIAM H. KLEIN 'DIRECTOR': DR. WILLIAM H. KLEIN

SMITHSON RADIATION BIOLOGY LABORATORY

RBL, 12441 PARKLAWN DRIVE

ROCKVILLE

MD 20852 PHONE: 301-443-2329

STAFF:TOT 60

R\&D 60

DR. FRANK MORRIS

'DIRECTOR.': DR. IRA RUBINOFF

SMITHSON SMITHSONIAN TROPICAL RESEARCH INST. STAFF:TOT 32

APO

MIAMI

FL 34002 PHONE:

R\&D 32

DR. DAVID L. CORRELL

'DIRECTOR': DR. J. KEVIN SULLIVAN

SMITHSON CHESAPEAKE BAY CENTER FOR ENV. STUDIES STAFF:TOT 24

CBCES, P. 0. BOX 28

EDGEWATER

MD 20137 PHONE: $301-789-4424$

R\&D 24

DR. JOHN EISENBERG

'DIRECTOR': DR. THEODORE H. REED

SMITHSON NATIONAL ZOOLOGICAL PARK AND CONS. CENTER STAFF:TOT

NATIONAL ZOOLOGICAL PARK

WASHINGTON

DC 20008 PHONE: 202-673-4705

R\&D 18

J. HARLOLD PARKER

'DIRECTOR': DR. BILLY J. BOND

TVA NATIONAL FERTILIZER DEVELOPMENT CENTER STAFF:TOT 969

MUSCLE SHOALS

AL 35660 PHONE: 205-386-2593

R\&D 678

SHIRLEY S. RAY

'DIRECTOR': M. D. HIGH

TVA ENERGY DEMONSTRATIONS AND TECHNOLOGY STAFF:TOT 321

1340 COMMERCE UNION BANK BLDG.

R\&D 164

CHATTANOOGA

TN 37401 PHONE: $615-755-2771$

WILLIAM R. WALDROP 'DIRECTOR': E. ELY DRIVER

TVA ENGINEERING LABORATORY STAFF:TOT 82

P. O. BOX DRAWER E

NORRIS

TN 37828 PHONE: $\quad 615-632-4460$

R\&D 49

HERBERT C。 JONES 'DIRECTOR': JOHN P. BLACKWELL

TVA AIR RESOURCES PROGRAM-RESEARCH SECTION STAFF:TOT 32

TVA, 51 RIVER OAKS BUILDING MUSCLE SHOALS AL

PHONE: 205-386-2341 FTS 872-8342

DR. PAUL A. PUTNAM 'DIRECTOR': DR. PAUL A. PUTNAM

USDA/ARS BELTSVILLE AGRICULTURAL RESEARCH CENTER STAFF:TOT 1247

USDA-S\&E-ARS-NER, ROOM 333, BLDG.003, BARC-W

BELTSVILLE MD 20705 PHONE: 301-344-3078

R\&D 1185

DR. JERRY J.CALLIS

'DIRECTOR': DR. JERRY J. CALLIS

USDA/ARS PLUM ISLAND ANIMAL DISEASE CENTER

STAFF:TOT 337

USDA-S\&E-ARS-NER, P O O BOX 848

GREENPORT

NY 11944 PHONE: 516-323-2500 
.FEDERAL LABORATORY CONTACTS LISTED BY AGENCY AND STAFF SIZE(CONT)

DR. ARTHUR I. MORGAN, JR.

'DIRECTOR': DR. ARTHUR I. MORGAN, JR. USDA/ARS WESTERN REGIONAL RESEARCH CENTER

STAFF : TOT 322

USDA-S\&E-ARS-WRRC, 800 BUCHANAN STREET

BERKELEY CA 94710 PHONE: 415-486-3506

R\&D 322

DR. H. L. ROTHBART 'DIRECTOR': DR. H. L. ROTHBART

USDA/ARS EASTERN REGIONAL RESEARCH CENTER STAFF:TOT 322

USDA-S\&E-ARS-NER, 600 EAST MERMAID LANE

PHILADELPHIA PA 19118 PHONE: 215-233-6593

R\&D 322

DR. A.M. COWAN 'DIRECTOR': DR. WILLIAM H. TALLENT

USDA/ARS NORTHERN REGIONAL RESEARCH CENTER STAFF:TOT 306

USDA-S\&E-ARS, NRC, $1815 \mathrm{~N}$. UNIVERSITY ST.

R\&D 306

PEORIA IL 61604 PHONE: 309-685-4011 FTS 306-4269

DR. PHILLIP A. O'BERRY 'DIRECTOR': DR. PHILLIP A. O'BERRY

USDA/ARS NATIONAL ANIMAL DISEASE CENTER

STAFF :TOT 272

USDA-S\&E-ARS-NCR, P.O. BOX 70

R\&D 272

AMES

IA 50010 PHONE:

FTS $682-8201$

DR. DAVID ZIMMER 'DIRECTOR': DR. DAVID ZIMMER

USDA/ARS RICHARD B. RUSSELL AG. RES. CENTER STAFF:TOT 160

USDA-S\&E-ARS-SR, P.O.BOX 5677

R\&D 160

ATHENS

GA 30613 PHONE: 404-546-3328 FTS 250-3328

DR. WALTER MERTZ 'DIRECTOR': DR. WALTER MERTZ

USDA/ARS BELTSVILLE HUMAN NUTRITION RESEARCH CENTER STAFF:TOT 128

USDA-S\&E-ARS-NER, ROOM 223, BLDG. 308, BARC-E R\&D 128

BELTSVILLE MD 20705 PHONE: 301-344-2157

DR. D. S. FREAR 'DIRECTOR': DR. D. S. FREAR

USDA/ARS METABOLISM AND RADIATION RESEARCH LABORATORY STAFF:TOT 104

USDA-S\&E-ARS-NCR, P.0.BOX 5674,STATE UNIV. STATION R\&D 104

FARGO ND 58105 PHONE: 701-237-5771 FTS 783-5445

DR. LORIS ASMUSSEN 'DIRECTOR': DR. LORIS ASMUSSEN

USDA/ARS SOUTHEAST WATERSHED RESEARCH LABORATORY STAFF:TDT 87

USDA-S\&E-ARS-SR, P.0.BOX 946

TIFTON GA 31793 PHONE: 912-386-3462

R\&D 87

DR. DERRELL L. CHAMBERS 'DIRECTOR': DR. DERRELL L. CHAMBERS USDA/ARS INSECT ATTRACTANTS, BEHAVIOR \& BASIC BIO. RES STAFF:TOT 78 USDA-S\&E-ARS-SR, 1700 S.W.23RD DR., P.0. BOX 14565

GAINESVILLE $\quad$ FL 32604 PHONE:

R\&D 78

FTS $947-7205$

DR. YESHAJAHU POMERANZ

'DIRECTOR': DR. YESHAJAHU POMERANZ

USDA/ARS U.S. GRAIN MARKETING RESEARCH CENTER

USDA-S\&E-ARS-NCR, 1515 COLLEGE AVE.

STAFF:TOT 64

R\&D 64

MANHATTAN

KS 66502 PHONE:

FTS 752-4201

DR. ROGER YOUNG

'DIRECTOR': DR. ROGER YOUNG

USDA/ARS U.S. HORTICULTURAL LABORATORY

USDA-S\&E-ARS-SR, 2120 CAMDEN RD.

ORLANDO

FL 32803 PHONE:

STAFF:TOT 62

R\&D 62

FTS 820-6051 
FEDERAL LABORATORY CONTACTS LISTED BY AGENCY AND STAFF SIZE(CONT) DR. R. DEAN PLOWMAN USDA/ARS CROPS RESEARCH LABORATORY STAFF:TOT 62 USDA-S\&E-ARS-WR, UT. STATE UNIV.,UMC 48 AG.SCI.BLDG. R\&D 54 LOGAN UT 84322 PHONE: 801-750-3453

DR. RICHARD L. WITTER 'DIRECTOR': DR. RICHARD L. WITTER USDA/ARS REGIONAL POULTRY RESEARCH LABORATORY STAFF:TOT 56 USDA-S\&E-ARS-NCR, 3606 EAST MT. HOPE ROAD EAST LANSING MI 48823 PHONE : R\&D 56 FTS $374-6828$

DR. ROBERT R. OLTJEN 'DIRECTOR': DR. ROBERT R. OLTJEN USDA/ARS ROMAN L. HRUSKA U.S. MEAT ANIMAL RES. LAB STAFF:TOT 56 USDA-S\&E-ARS-NCR, P.0. BOX 166, STATE SPUR 18D CLAY CENTER NE 68933 PHONE: 402-762-3241

R\&D 56

DR. DONALD E. WEIDHAAS 'DIRECTOR': DR. DONALD E. WEIDHAAS USDA/ARS INSECTS AFFECTING MAN AND ANIMALS RES. LAB STAFF:TOT 54 USDA-S\&E-ARS-SR, 1600 S.W. 23RD DR., P.0. BOX 14565 R\&D 54 GAINESVILLE $\quad F L 32604$ PHONE: FTS $947-7290$

DR. ROBERT DAVIS 'DIRECTOR': DR. ROBERT DAVIS USDA/ARS STORED-PRODUCTS INSECTS RES. \& DEV. LAB STAFF:TOT 52 USDA-S\&E-ARS-SR, 3401 EDWIN AVE., P.0. BOX $22909 \quad$ R\&D 52 SAVANNAH GA 31403 PHONE: FTS 248-4397

DR. DONALD A. WITZEL

'DIRECTOR': DR. DONALD A. WITZEL USDA/ARS VETERINARY TOXICOLOGY AND ENTOMOLOGY LAB STAFF:TOT 48 USDA-S\&E-ARS, P.0. DRAWER GE COLLEGE STATION TX 77841 PHONE:

DR. W. DORAL KEMPER 'DIRECTOR': DR. W. DORAL KEMPER USDA/ARS SNAKE RIVER CONSERVATION RESEARCH CENTER STAFF:TOT 47 USDA-S\&E-ARS-WR, ROUTE 1, BOX 186 KIMBERLY ID 83341

DR. D. LEROY WILLIAMSON 'DIRECTOR': DR. D. LEROY WILLIAMSON USDA/ARS TROPICAL FRUIT AND VEGETABLE RES. FACILITY STAFF:TOT 46 USDA-S\&E-ARS-WR, 2727 WOODLAWN DR., P.0. BOX $2280 \quad$ R\&D 46 HONOLULU $\quad H I 96804$ PHONE: 808-988-2158 FTS 556-0220

DR. THOMAS E. WALTON 'DIRECTOR': DR. THOMAS E. WALTON USDA/ARS ARTHROPOD-BORNE ANIMAL DISEASES RES. LAB STAFF:TOT USDA-S\&E-ARS-WR, P.0. BOX 25327, DENVER FED. CENTER DENVER CO 80225 PHONE: 303-234-2474 R\&D 45

\section{'DIRECTOR':}

USDA/ARS OILSEED AND FOOD LABORATORY USDA-S\&E-ARS-SRRC, 1100 ROBERT E. LEE BLVD.,PO BOX 19687 NEW ORLEANS

LA 70179 PHONE:

STAFF:TOT 44 R\&D 44

FTS $682-7557$ 
FEDERAL LABORATORY CONTACTS LISTED BY AGENCY AND STAFF SIZE(CONT)

DR. ROGER O. DRUMMOND

'DIRECTOR' : DR. ROGER 0. DRUMMOND

USDA/ARS U. S. LIVESTOCK INSECTS LABORATORY

STAFF :TOT 43

USDA-S\&E-ARS-SR, P.0. BOX 232

KERRV ILLE

TX

PHONE: $\quad 512-257-3566$

R\&D 43

DR. THOMAS J. HENNEBERRY

'DIRECTOR' :

USDA/ARS WESTERN COTTON RESEARCH LABORATORY

DR. THOMAS J. HENNEBERRY

USDA-S\&E-ARS-WR, 4135 EAST BROADWAY

STAFF : TOT 42

PHOENIX

AZ 85040

PHONE: - 602-261-3524 FTS 261-3524

DR. ALFRED L. BLACK

'DIRECTOR' : DR. ALFRED L. BLACK

USDA/ARS NORTHERN GREAT PLAINS RESEARCH CENTER STAFF:TOT 42

USDA-S\&E-ARS-NCR, P.0.BOX 459

R\&D 42

MANDAN

ND 58554 PHONE: 701-663-6445 FTS 783-4011

DR. T. B. DAVICH

'DIRECTOR': DR. T.B. DAVICH

USDA/ARS BOLL WEEVIL RESEARCH LABORATORY

STAFF :TOT 41

USDA-S\&E-ARS-SR, P.O. BOX 5367

R\&D 41

MISSISSIPPI STATE

MS 3976

PHONE : $601-323-2230$ FTS 490-4676

DR. HERMAN BOUWER

'DIRECTOR' :

USDA/ARS U.S.WATER CONSERVATION LABORATORY

DR. HERMAN BOUWER

USDA-S\&E-ARS-WR, 4331 EAST BROADWAY

STAFF : TOT 40

R\&D

PHOENIX

AZ 85040

PHONE: 602-261-4356 FTS 261-4356

MR. BILL A. BUTT

'DIRECTOR': MR. BILL A. BUTT

USDA/ARS APPALACHIAN FRUIT RESEARCH STATION

USDA-S\&E-ARS-NER, ROUTE 2, BOX 45

KEARNEYSVILLE

WV 25430

PHONE : $\quad 304-725-3451$

STAFF:TOT 38

R\&D 38

DR. ALBERT BARIL

'DIRECTOR' : DR. ALBERT BARIL

USDA/ARS COTTON TEXTILE PROCESSING LABORATORY

STAFF:TOT 37

USDA-S\&E-ARS-SRRC, 1100 ROBERT E. LEE BLVD., PO BOX 19687

R\&D 37

NEW ORLEANS

LA 70179 PHONE:

FTS $682-7582$

DR. JAMES F. CHAPLIN

'DIRECTOR': DR. JAMES F. CHAPLIN

USDA/ARS TOBACCO RESEARCH LABORATORY

USDA-S\&E-ARS-SR, ROUTE 2, BOX 16G

OXFORD

NC 27565

PHONE: $919-693-5151$

STAFF :TOT 36

R\&D 36

DR. CHARLES W. BEARD

'DIRECTOR' : DR. CHARLES W. BEARD

USDA/ARS SOUTHEAST POULTRY RESEARCH LABORATORY

USDA-S\&E-ARS-SR, 934 COLLEGE STATION RD.

STAFF:TOT 35

ATHENS

GA 30613 PHONE:

R\&D 35

250-3432

DR. F. I. PROSHOLD

'DIRECTOR': DR. F. I. PROSHOLD

USDA/ARS BIOENVIRONMENTAL INSECTS CONTROL LABORATORY STAFF:TOT 35

USDA-S\&E-ARS-SR, P. O. BOX 225

STONEVILLE

MS 38776 PHONE:

DR.`RALPH BERNI 'DIRECTOR': DR. RALPH BERNI

USDA/ARS COMPOSITION AND PROPERTIES LABORATORY STAFF:TOT 34

USDA-S\&E-ARS-SRRC, 1100 ROBERT E. LEE BLVD., PO BOX $19687 \quad$ R\&D 34

NEW ORLEANS

LA 70179 PHONE:

FTS $682-7547$ 
FEDERAL LABORATORY CONTACTS LISTED BY AGENCY AND STAFF SIZE(CONT)

DR. CHESTER G. MC WHORTER

'DIRECTOR': DR. CHESTER G. MC WHORTER

USDA/ARS SOUTHERN WEED SCIENCE LABORATORY

STAFF:TOT 34

USDA-S\&E-ARS-SR, P. 0. BOX 225

STONEVILLE MS 38776 PHONE:

R\&D 34

FTS $497-2221$

DR. IVAN L. BERRY 'DIRECTOR': DR. IVAN L. BERRY

USDA/ARS LIVESTOCK INSECTS RESEARCH LABORATORY STAFF:TOT 33

USDA, UNIV. OF NE, E. CAMPUS, 305A PLANT INDUSTRY BLDG. R\&D 33

LINCOLN NE 68533 PHONE: 402-472-2918 FTS 541-5267

DR. JAMES $W$. SNOW 'DIRECTOR': DR. JAMES $W$. SNOW

USDA/ARS SOUTHEASTERN FRUIT \& TREE NUT RES. LAB STAFF:TOT 32

USDA-S\&E-ARS-SR, P.0.BOX $87 \quad$ R\&D 32

BYRON GA 31008 PHONE: FTS 238-0422

DR. JOHNIE N. JENKINS 'DIRECTOR': DR. JOHNIE N. JENKINS

USDA/ARS CROP SCIENCE AND ENGINEERING RES. LABORATORY STAFF:TOT 32

USDA-S\&E-ARS-SR, P.0. BOX 5465

MISSISSIPPI STATE MS 39762 PHONE:

R\&D 32

FTS $490-4676$

DR. J. ROGER MC HENRY 'DIRECTOR': DR. J. ROGER MC HENRY

USDA/ARS WATER QUALITY MANAGEMENT LABORATORY STAFF:TOT 32

USDA-S\&E-ARS-SR, 801 WILSON ST., P.0. BOX $1430 \quad$ R\&D 32

DURANT OK 74701 PHONE:

FTS $736-5401$

DR. EARL BURNETT 'DIRECTOR': DR. EARL BURNETT

USDA/ARS GRASSLAND, SOIL AND WATER RESEARCH LABORATORY STAFF:TOT 32

USDA-S\&E-ARS-SR, P.O.BOX $748 \quad$ R\&D 32

$\begin{array}{llll}\text { TEMPLE } & \text { TX } 76501 \text { PHONE: } & \text { FTS 736-1201 }\end{array}$

DR. ARTHUR K. BURDITT, JR。 'DIRECTDR': DR, ARTHUR K. BURDITT, JR. USDA/ARS YAKIMA AGRICULTURAL RESEARCH LABORATORY STAFF:TOT 31 USDA-S\&E-ARS-WR, 3706 WEST NOB HILL ROAD

YAKIMA WA 98902 PHONE: 509-575-5877

R\&D 31

DR. JAMES E. BOS, JR. 'DIRECTOR': DR. JAMES E. BOX, JR.

USDA/ARS SOUTHERN PIEDMONT CONSERVATION LABORATORY STAFF:TOT 30

USDA-S\&E-ARS-SR, HIGHWAY 53, P.0. BOX 555

R\&D 30

WATKINSVILLE GA 30677 PHONE:

FTS $250-2425$

DR. JAMES E. IRVINE 'DIRECTOR': DR. JAMES E. IRVINE

USDA/ARS U.S. SUGARCANE FIELD LABORATORY STAFF:TOT 30

USDA-S\&E-ARS-SR, P.0.BOX 470

R\&D 30

HOUMA

LA 70526 PHONE:

FTS $682-2611$

'DIRECTOR' :

USDA/ARS NATURAL POLYMERS LABORATORY

USDA-S\&E-ARS-SRRC, 1100 ROBERT E. LEE BLVD.,PO BOX 1.9687

NEW ORLEANS LA 70179 PHONE:

STAFF :TOT 30

R\&D 30

FTS $682-7076$

DR. ANGELO V. GRACI, JR. 'DIRECTOR': DR. ANGELO V. GRACI, JR. USDA/ARS ENGINEERING AND DEVELOPMENT LABORATORY STAFF:TOT 29 USDA-S\&E-ARS-SRRC, 1100 ROBERT E. LEE BLVD., PO BOX 19687 R\&D 29 NEW ORLEANS LA 70179 PHONE:

FTS $682-7521$ 
FEDERAL LABORATORY CONTACTS LISTED BY AGENCY AND STAFF SIZE(CONT)

DR. ELBERT $V$. WANN

'DIRECTOR': DR. ELBERT $V$. WANN

USDA/ARS U.S. VEGETABLE LABORATORY

STAFF:TOT 28

USDA-S\&E-ARS-SR, 2875 SAVANNAH HIGHWAY

CHARLESTON SC 29407 PHONE:

FTS $677-4396$

DR. GERALD R. SUTTER

'DIRECTOR' :

R. SUTTER

USDA/ARS NORTHERN GRAIN INSECTS RESEARCH LABORATORY

USDA-S\&E-ARS-NCR，R. R. \#3

SD 57006 PHONE:

STAFF:TOT 28

R\&D 28

BROOKINGS

'DIRECTOR' : DR. BOBBY STEWART

DR. BOBBY STEWART

USDA/ARS CONSERVATION AND PRODUCTION
USDA-S\&E-ARS-SR,P.O. DRAWER BOX 10

STAFF :TOT 28

R\&D 28

BUSHLAND

TX 79012 PHONE:

FTS $734-2524$

DR. VINCENT P. MAIER

'DIRECTOR': DR. VINCENT P. MAIER

USDA/ARS FRUIT AND VEGETABLE CHEM. RES. UNIT

STAFF:TOT 26

USDA-S\&E-ARS-WR, 263 S. CHESTER AVENUE

R\&D 26

PASADENA

CA 9110

PHONE : 213-681-7294 FTS 681-7294

DR. WILLIAM C. MOLDENHAUER 'DIRECTOR': DR. WILLIAM C. MOLDENHAUER

USDA/ARS SOIL EROSION LABORATORY

USDA-S\&E-ARS-NCR, 2700 KENT AVE.

WEST LAFAYETTE

IN 47906 PHONE:

STAFF : TOT 26

R\&D 26

DR. CAROLO M. IGNOFFO

'DIRECTOR' : DR. CAROLO M. IGNOFFO

USDA/ARS BIOLOGICAL CONTROL OF INSECTS RESEARCH LAB STAFF:TOT 25

USDA-S\&E-ARS-NCR, P. O. BOX A

R\&D 25

COLUMBIA

MO 65205 PHONE:

FTS 276-3194

DR. SIDNEY VAIL

'DIRECTOR': DR. SIDNEY VAIL

USDA/ARS COTTON TEXTILE CHEMISTRY LABORATORY

USDA-S\&E-ARS-SRRC, 1100 ROBERT E. LEE BLVD., PO BOX 19687

NEW ORLEANS

LA 70179 PHONE:

STAFF :TOT 24

R\&D 24

FTS $682-7064$

DR. JERRY QUISENBERRY

'DIRECTOR': DR. JERRY QUISENBERRY

USDA/ARS COTTON RESEARCH LABORATORY

STAFF:TOT 23

USDA-S\&E-ARS-SR, ROUTE 3

LUBBOCK

TX 79401 PHONE:

R\&D 23

FTS $738-7413$

DR. D. R. VAN CAMPEN

'DIRECTOR': DR. D.R. VAN CAMPEN

USDA/ARS U.S. PLANT, SOIL, AND NUTRITION LABORATORY STAFF:TOT 20

USDA-S\&E-ARS-NER, CORNELL UNIV., TOWER ROAD

R\&D 20

ITHACA

NY 14853 PHONE: 607-256-5480 FTS $882-4280$

DR. RAMOND J. BULA

'DIRECTOR': DR. RAYMEND J. BULA

USDA/ARS DAIRY FORAGE RESEARCH CENTER

USDA, U. OF WI, DEPT. OF AGRONOMY, RM. 338 MOORE HALL

STAFF:TOT 18

R\&D 18

MADISON

WI 53706

PHONE: 608-262-0377

FTS $364-5242$

DR. ROBERT E. BERRY

'DIRECTOR': DR. ROBERT E. BERRY

USDA/ARS CITRUS AND SUB-TROPICAL PRODUCTS LABORATORY STAFF:TOT 17

USDA-S\&E-ARS-SR, P.0.BOX 1909

WINTER HAVEN

FL 33880

PHONE: 813-293-4133

R\&D 17 
EEDERAL LABORATORY CONTACTS LISTED BY AGENCY AND STAFF SIZE(CONT)

DR. ROBERT N. ANDERSON

USDA/ARS WEED RESEARCH LABORATORY

'DIRECTOR': DR. ROBERT N. ANDERSON

USDA-S\&E-ARS-NCR，U. OF MN, AGRONOMY, 1590 GORTNER AVE. R\&D 16

ST. PAUL

MN 55108 PHONE: 612-373-0877

FTS 786-0855

RON GLASS

'DIRECTOR' : DAVID B. THORUD

USDA/FS NORTHEASTERN FOREST EXPERIMENT STATION

$P \& A$ AD, 370 REED ROAD

BROOMALL

PA

STAFF :TOT 396

R\&D 396

RODGER LEONARD

'DIRECTOR': ROBERT A. HANN

USDA/FS NORTH CENTRAL EXPERIMENT STATION

PHONE: $814-863-1936$

P\&A 1992 FOWELL AVENUE

ST. PAUL

MN

PHONE : $\quad 612-645-5251$

STAFF :TOT 346

R\&D 346

R. STEIDEMANN 'DIRECTOR': ROBERT YOUNGS

USDA/FS FOREST PRODUCTS LABORATORY
P\&A, AD, NORTH WALNUT ST., BOX 5130

MADISON WI 53705 PHONE: 608-264-5745

STAFF :TOT 339

R\&D 305

JOHN HENLEY

'DIRECTOR' : LAURENCE E.LASSEN

USDA/FS SOUTHERN FOREST EXPERIMENT STATION

STAFF :TOT 322

P\&A AD, T-10210, U.S. POSTAL SV. BLDG., 701 LOYOLA AVE.

R\&D 322

NEW ORLEANS

LA PHONE: 504-589-6712

DR. G. LEWIS 'DIRECTOR': ELDON ROSS

USDA/FS SOUTHEASTERN FOREST EXPERIMENT STATION STAFF:TOT 287

P\&A AD, POST OFFICE BLDG., BOX 2570

ASHEVILLE NC 28804 PHONE: 704-258-2850

R\&D 287

ELDON ESTEP 'DIRECTOR': ROBERT L. ETHINGTON

USDA/FS PACIFIC NORTHWEST EXPERIMENT STATION STAFF:TOT 282 809 NE 6TH AVE.

PORTLAND

OR PHONE: 503-231-2059

R\&D 282

RICHARD HUBBERD

'DIRECTOR': ROBERT Z. CALLAHAM

USDA/FS PACIFIC SOUTHWEST FOREST EXPERIMENT STATION STAFF:TOT 255

P\&A AD, 1960 ADDISON ST., BOX 245

BERKELEY

CA 0

PHONE: $\quad 415-486-3286$

R\&D 255

KEITH EVENS

'DIRECTOR' : ROGER R. BAY

USDA/FS INTERMOUNTAIN FOREST AND RANGE EXP. STA.

P\&A AD, 507 25TH ST.

OGDEN

UT

PHONE: $\quad 801-626-3361$

STAFF :TOT 240

R\&D 240

JAY KRAMMES

'DIRECTOR': DIXIE SMITH

USDA/FS ROCKY MOUNTAIN FORESTRY AND RANGE EXP. STATIO STAFF:TOT 230

$P \& A$ AD, 240 W. PROSPECT ST.

R\&D 230

FT. COLLINS

$\mathrm{CO}$

PHONE : $\quad 303-221-4390$

E.E. EDDLEMAN, JR., M.D. 'DIRECTDR':

VA BIRMINGHAM VA MEDICAL CENTER

700 SOUTH 19TH STREET

B IRM INGHAM

AL 35233 PHONE: $534-6323$

STAFF :TOT $10+$

R\&D 
FEDERAL LABORATORY CONTACTS LISTED BY AGENCY AND STAFF SIZE(CONT) KARL D. STRAUB, M.D. 'DIRECTOR':

VA LITTLE ROCK VA MEDICAL CENTER

STAFF :TOT $10+$

300 ROOSEVELT ROAD

LITTLE ROCK

AR 72206 PHONE: $740-2455$

R\&D

STANTON G. AXLINE, M.D. 'DIRECTOR':

VA TUCSON VA MEDICAL CENTER

STAFF :TOT $10+$

$R \& D$

TUCSON

AZ 85723 PHONE: $765-6424$

ROBERT W. PORTER, M.D., PH.D. 'DIRECTOR':

VA LONG BEACH VA MEDICAL CENTER

5901 EAST 7TH STREET

LONG BEACH

CA 90822 PHONE: 799-9711

STAFF :TOT $10+$

$R \& D$

EUSTACE A. SERAFETINIDES, M.D. 'DIRECTOR':

VA LOS ANGELES BRENTWOOD VA MEDICAL CENTER

11301 WILSHIRE BLVD.

LOS ANGELES

CA 90073 PHONE: 794-2460

STAFF :TOT $10+$

$R \& D$

LUCIAN B. GUZE, M.D. 'DIRECTOR':

VA LOS ANGELES WADSWORTH DIV. VA MEDICAL CENTER WADSWORTH DIVISION

LOS ANGELES

CA 90073 PHONE: 794-2347

ROBERT EFRON, M.D.

'DIRECTOR':

VA MARTINEZ VA MEDICAL CENTER

150 MUIR ROAD

MARTINEZ

CA 94553 PHONE: 450-0596

FRANKLIN G. EBAUGH, M.D. 'DIRECTOR':

VA PALO ALTO VA MEDICAL CENTER

3801 MIRANDA AVENUE

PALO ALTO

CA 94304 PHONE: 449-5410

STAFF :TOT $10+$ $R \& D$

STAFF : TOT $10+$ $R \& D$

STAFF : TOT $10+$ $R \& D$

LEE W. HENDERSON, M.D.

'DIRECTOR':

VA SAN DIEGO VA MEDICAL CENTER

3350 LAJOLLA VILLAGE DR.

SAN DIEGO

CA 92161 PHONE: $897-3657$

STAFF:TOT $10+$

R\&D

THOMAS B. BRADLEY, JR., M.D. 'DIRECTOR':

VA SAN FRANCISCO VA MEDICAL CENTER

4150 CLEMENT STREET

SAN FRANCISCO

CA 94121 PHONE: $470-4446$

STAFF :TOT $10+$

$R \& D$

ISADORE MICHAEL SAMLOFF, M.D. 'DIRECTOR':

VA SEPULVEDA VA MEDICAL CENTER

SUPULVEDA

CA 91343 PHONE: 960-9481

STAFF:TOT $10+$

R\&D

JOHN J. FRANKS, M.D.

VA DENVER VA MEDICAL CENTER

'DIRECTOR' :

1055 CLERMONT STREET

DENVER

CO 80220 PHONE: $322-3447$

STAFF:TOT $10+$ $R \& D$ 
FEDERAL LABORATORY CONTACTS LISTED BY AGENCY AND STAFF SIZE(CONT) PHILIP KRAMER BONDY, M.D.

VA WEST HAVEN VA MEDICAL CENTER

STAFF:TOT $10+$

WEST SPRING STREET

WEST HAVEN

CT 06516 PHONE: 641-7608

R\&D

RICHARD F. LEVINE, M.D. 'DIRECTOR':

VA WASHINGTON VA MEDICAL CENTER

50 IRVING STREET, N.W.

WASHINGTON

DC 20422 PHONE: $\quad 389-7277$

STAFF:TOT $10+$

R\&D

STAFF :TOT $10+$

$R \& D$

ARCHER ROAD

GAINESVILLE FL 32602 PHONE: 947-6069

LAWRENCE M. FISHMEN, M.D. 'DIRECTOR':

VA MIAMI VA MEDICAL CENTER

1201 NORTHWEST 16TH ST.

MIAM I

FL $33125 \quad$ PHONE: $350-3211$

ROBERT V. FARESE, M.D. 'DIRECTOR':

VA TAMPA VA MEDICAL CENTER

1300 NORTH 30TH STREET

TAMPA

FL 33612 PHONE: 826-6566

JAMES W. HOLSINGER, JR. M.D. 'DIRECTOR' :

VA AUGUST YA MEDICAL CENTER

GA 30904 PHONE: 251-2443

AUGUST

PHILIP GEORGE SCHMID, JR.,M.D. 'DIRECTOR':

VA IOWA CITY VA MEDICAL CENTER

STAFF:TOT $10+$

R\&D

IOWA CITY

IA 52240 PHONE: 863-6246

THEODORE N. PULLMAN, M.D. 'DIRECTOR':

VA CHICAGO LAKESIDE VA MEDICAL CENTER

333 EAST HURON STREET

CHICAGO

IL 60611 PHONE: 384-8511

FRANK A. DELEON-JONES, M.D. 'DIRECTOR':

VA CHICAGO WEST SIDE VA MEDICAL CENTER

820 SOUTH DAMEN AVENUE

CHICAGO

IL 60680 PHONE: $388-8744$

STAFF :TOT $10+$

$R \& D$

$R \& D$

STAFF :TOT $10+$

$R \& D$

R\&D

STAFF : TOT $10+$

R\&D

STAFF:TOT $10+$

$R \& D$

STAFF :TOT $10+$

R\&D

STAFF :TOT $10+$ $R \& D$

EDWARD PALOYAN, M.D.

VA HINES VA MEDICAL
EDWARD HINES, JR. HOSPITAL

HINES

IL 60141 PHONE: 387-3291

TING KAI LI, M.D. 'DIRECTOR' :

VA INDIANAPOLIS VA MEDICAL CENTER

1481 WEST 10TH STREET

INDIANAPOSIS

IN 46202 PHONE: 332-2525 
FEDERAL LABORATORY CONTACTS LISTED BY AGENCY AND STAFF SIZE(CONT)

RUSSELL G. MCALLISTER, JR. 'DIRECTOR':

VA LEXINGTON VA MEDICAL CENTER

STAFF :TOT $10+$

R\&D

LEXINGTON

KY 40507 PHONE: $355-2635$

EXT. 517

ROBERT E. BURCH, M.D. 'DIRECTOR':

VA NEW ORLEANS VA MEDICAL CENTER

1601 PERDIDO STREET

NEW ORLEANS

LA 70146 PHONE: $682-5279$

STAFF: TOT $10+$

R\&D

IRA SHERWIN, M.D. 'DIRECTOR':

VA BEDFORD VA MEDICAL CENTER

200 SPRINGS ROAD

BEDFORD

MA 01730 PHONE: $840-0530$

STAFF :TOT $10+$

$R \& D$

OSCAR C. BING, M.D.

'DIRECTOR':

VA BOSTON VA MEDICAL CENTER

150 SOUTH HUNTINGTON AVE.

BOSTON

MA 02130 PHONE: 839-0771

STAFF :TOT $10+$

R\&D

AMICO BIGNAMI, M.D. 'DIRECTOR':

VA WEST ROXBURY VA MEDICAL CENTER

1400 VETERANS OF FOREIGN WARS PKWY.

WEST ROXBURY MA 02132 PHONE: $837-5430$

STAFF :TOT $10+$

R\&D

WILLIAM 0. DOBBINS, III, M.D. 'DIRECTOR':

VA ANN ARBOR VA MEDICAL CENTER

2215 FULLER RD.

ANN ARBOR

MI 48105 PHONE: $374-5333$

STAFF :TOT $10+$

$R \& D$

MICHAEL DAVID LEVITT, M.D. 'DIRECTOR':

VA MINNEAPOLIS VA MEDICAL CENTER

54TH ST. \& 48TH AVE., SOUTH

MINNEAPOLIS

MN 55417 PHONE: 784-6561

STAFF:TOT $10+$

$R \& D$

TRAVIS SOLOMON, M.D.

'DIRECTOR':

VA COLUMBIA VA MEDICAL CENTER

800 STADIUM ROAD

COLUMBIA

MO 65201 PHONE: 276-6453

STAFF : TOT $10+$

R\&D

DAVID V. COHN, PH.D.

'DIRECTOR':

VA KANSAS CITY VA MEDICAL CENTER

4801 LINWOOD BOULEVARD

KANSAS CITY

MO 64128 PHONE: 754-1501

STAFF :TOT $10+$

R\&D

'DIRECTOR':

FRANCIS J. CAREY, M.D. 'DIREC
VA ST. LOUIS VA MEDICAL CENTER

ST. LOUIS

MO 63125 PHONE: $276-4356$

STAFF :TOT $10+$

$R \& D$

GERALD LOGIJE, M.D.

'DIRECTOR' :

VA DURHAM VA MEDICAL CENTER

508 FULTON STREET

DURHAM

NC 27705 PHONE: 629-6500

STAFF :TOT $10+$

R\&D 
FEDERAL LABORATORY CONTACTS LISTED BY AGENCY AND STAFF SIZE(CONT)

M.F. SORELL, M.D. 'DIRECTOR':

VA OMAHA VA MEDICAL CENTER

4101 WOOLWORTH AVENUE

OMAHA

NE 68105 PHONE: 864-0404

STAFF :TOT $10+$

R\&D

RICHARD PETER WEDEEN, M.D. 'DIRECTOR':

VA EAST ORANGE VA MEDICAL CENTER

STAFF :TOT $10+$

R\&D

EAST ORANGE

NJ 07019 PHONE: 342-1269

CHARLES BRUCE TAYLOR, M.D. 'DIRECTOR':

VA ALBANY VA MEDICAL CENTER

STAFF:TOT $10+$

$R \& D$

END NY 12208 PHONE: 563-9374

HERBERT ROSE, M.D. 'DIRECTOR':

VA BRONX VA MEDICAL CENTER STAFF:TOT 10+

130 WEST KINGSBRIDGE RD.

BRONX

NY 10468 PHONE: 212-579-1630

$R \& D$

ALBERT B. EISENSTEIN, M.D. 'DIRECTOR':

VA BROOKLYN VA MEDICAL CENTER

800POLY PLACE

BROOKLYN

NY 11209 PHONE: 265-6351

STAFF : TOT $10+$

R\&D

JACK K. GOLDMAN, M.D.

'DIRECTOR' :

VA BUFFALO VA MEDICAL CENTER

3495 BAILEY AVENUE

BUFFALO

NY 14215 PHONE: $432-9502$

STAFF :TOT $10+$

R\&D

VINCENT J. FISHER, M.D. 'DIRECTOR':

VA NEW YORK VA MEDICAL CENTER

FIRST AVE. AT EAST 24TH ST.

NEW YORK NY 10010 PHONE: 660-9667

STAFF :TOT $10+$

$R \& D$

STEPHEN A. LANDAW, M.D.

'DIRECTOR' :

VA SYRACUSE VA MEDICAL CENTER

IRVING AVENUE AND UNIVERSITY PLACE

$\begin{array}{llll}\text { SYRACUSE } & \text { NY } 13210 \text { PHONE: 953-7401 }\end{array}$

STAFF:TOT $10+$

$R \& D$

RONALD E. MYERS, M.D. 'DIRECTOR' :

VA CINCINNATI VA MEDICAL CENTER

3200 VINE STREET

CINC INNATI .

OH 45220 PHONE: $773-4318$

STAFF:TOT $10+$

R\&D

CHARLES L. HOPPEL, M.D.

'DIRECTOR' :

VA CLEVELAND VA MEDICAL CENTER

10701 EAST BOULEVARD

CLEVELAND

OH $44106 \quad$ PHONE: $294-7521$

STAFF:TOT $10+$

R\&D

MICHAEL F. WILSON, M.D. 'DIRECTOR':

VA OKLAHOMA CITY VA MEDICAL CENTER

921 NORTHEAST 13TH STREET

OKLAHOMA CITY

OK 73104 PHONE: 743-3582

STAFF :TOT $10+$

R\&D 
FEDERAL LABORATORY CONTACTS LISTED BY AGENCY AND STAFF SIZE(CONT)

JOHN W. KENDELL 'DIRECTOR':

VA PORTLAND VA MEDICAL CENTER

STAFF : TOT $10+$

3710 S.W. U.S. VETERANS HOSPITAL RD.

PORTLAND OR 97201 PHONE: 424-9221

R\&D

DAVID M. CAPUZZI, M.D.

'DIRECTOR' :

VA PHILADELPHIA VA MEDICAL CENTER

UNIVERSITY \& WOODLAND AVES.

$\begin{array}{lrll}\text { PHILADELPHIA } & \text { PA } 19104 & \text { PHONE: } & 488-9549\end{array}$

STAFF : TOT $10+$

R\&D

MARTIN SAX, PH.D.

'DIRECTOR' :

VA PITTSBURGH VA MEDICAL CENTER

(U.D.) UNIVERSITY DR. C

PITTSBURGH

PA 15240 PHONE: $726-3426$

STAFF :TOT $10+$

R\&D

JOHN A. COLWELL, M.D.

'DIRECTOR' :

VA CHARLESTON VA MEDICAL CENTER

STAFF : TOT $10+$

109 BEE STREET

CHARLESTON

NC 92403 PHONE: $677-7346$

$R \& D$

ANDREW H. KANG, M.D.

'DIRECTOR' :

VA MEMPHIS VA MEDICAL CENTER

1030 JEFFERSON AVENUE

MEMPHIS

TN 38104 PHONE: 222-7266

STAFF :TOT $10+$

R\&D

CONRAD WAGNER, PH.D. 'DIRECTOR' :

VA NASHVILLE VA MEDICAL CENTER

1310 24TH AVENUE, SOUTH

NASHVILLE

TN 37203 PHONE: 852-9771

STAFF :TOT $10+$

R\&D

MARK FELDMAN, M.D. 'DIRECTOR' :

VA DALLAS VA MEDICAL CENTER

4500 SOUTH LANCASTER RD.

DALLAS

TX 75216 PHONE: 749-5382

STAFF :TOT $10+$

R\&D

ISMET KARACAN, M.D. 'DIRECTOR' :

VA HOUSTON VA MEDICAL CENTER

2002 HOLCOMBE BOULEVARD

HOUSTON

TX 77211 PHONE: 527-7469

STAFF :TOT $10+$

R\&D

FREDERIC C. BARTTER, M.D. 'DIRECTOR':

VA SAN ANTONIO VA MEDICAL CENTER

7400 MERTON MINTER BLVD.

SAN ANTONIO

TX 78284 PHONE: 738-0463

STAFF :TOT $10+$

R\&D

E. ANDREW DEISS, JR., M.D. 'DIRECTOR' :

VA SALT LAKE CITY VA MEDICAL CENTER

500 FOOTHILL BOULEVARD

SALT LAKE CITY

UT 84148 PHONE: 588-1514

STAFF :TOT $10+$

R\&D

FRANKLIN J. ZIEVE, M.D.

'DIRECTOR' :

VA RICHMOND VA MEDICAL CENTER

1201 BROAD ROCK ROAD

RICHMOND

VA 23249 PHONE: 925-9421

STAFF :TOT $10+$

R\&D 
FEDERAL LABORATORY CONTACTS LISTED BY AGENCY AND STAFF SIZE(CONT)

DANIEL PORTE, JR., M.D.

'DIRECTOR' :

VA SEATTLE VA MEDICAL CENTER

STAFF:TOT $10+$

4435 BEACON AVE., SOUTH

SEATTLE

WA 98108 PHONE: 396-1493

$R \& D$

THEODORE L. GOODFRIEND, M.D. 'DIRECTOR':

VA MADISON VA MEDICAL CENTER

2500 OVERLOOK TERRACE

MADISON

WI 53705 PHONE: 364-1277

STAFF:TOT $10+$

$R \& D$

JORDAN N. FINK, M.D. 'DIRECTOR':

VA WOOD VA MEDICAL CENTER

5000 WEST NATIONAL AVE.

WI 53193 PHONE: 362-7611

STAFF :TOT $10+$ WOOD (MILWAUKEE)

WILLIAM P. REED, M.D. 'DIRECTOR':

VA ALBUQUERQUE VA MEDICAL CENTER

$R \& D$

2100 RIDGECREST DR. S.E.

ALBUQUERQUE

NM

PHONE : $\quad 572-9319$

STAFF :TOT $10+$

$R \& D$ 
Federal Laboratory Information DEPARTMENT OF COMMERCE Shoots

These sheets, as provided by Federal agencies, have been arranged by agency, by major subelement, and in order of staff size as listed in Table 6 and Table 7. 

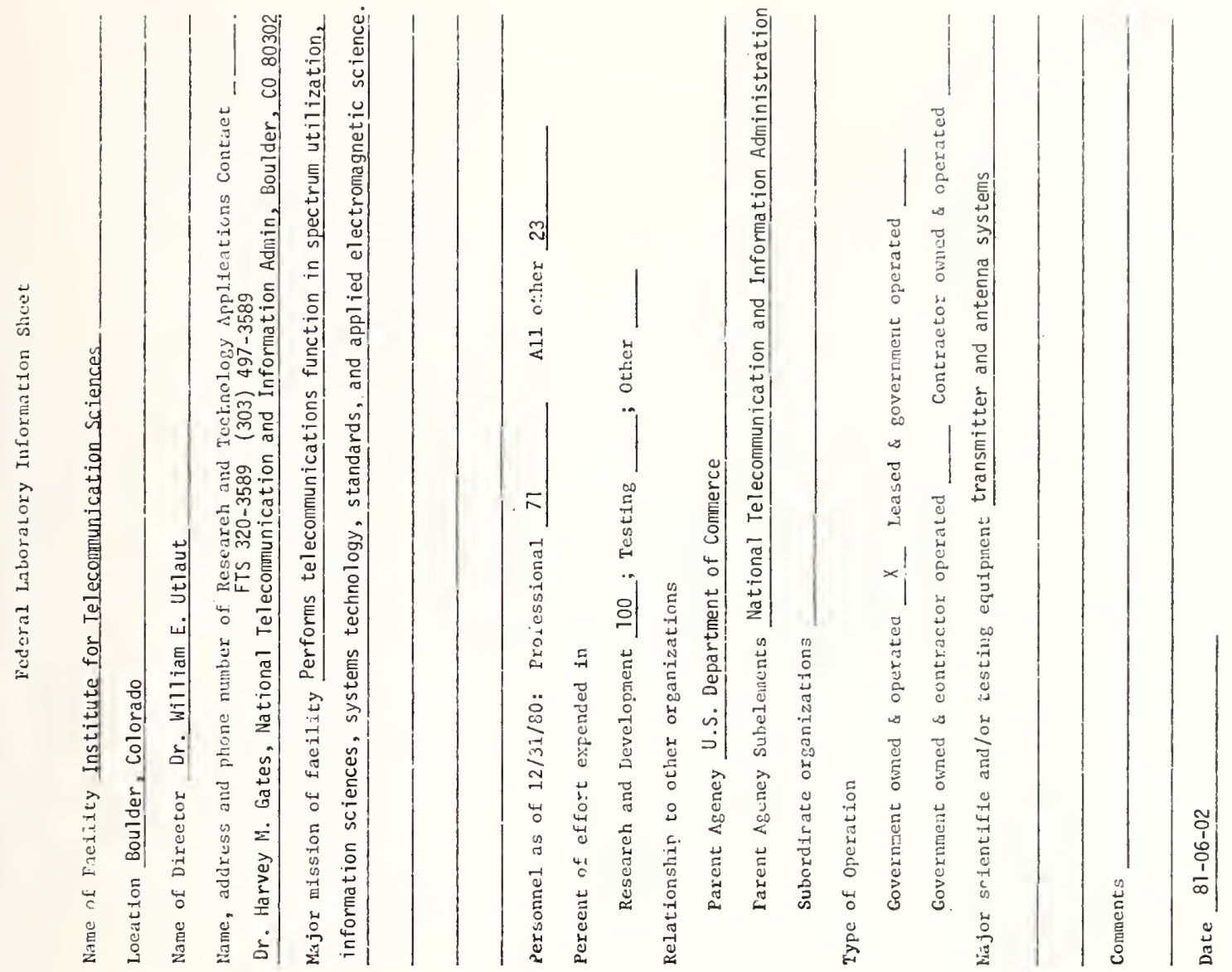

온
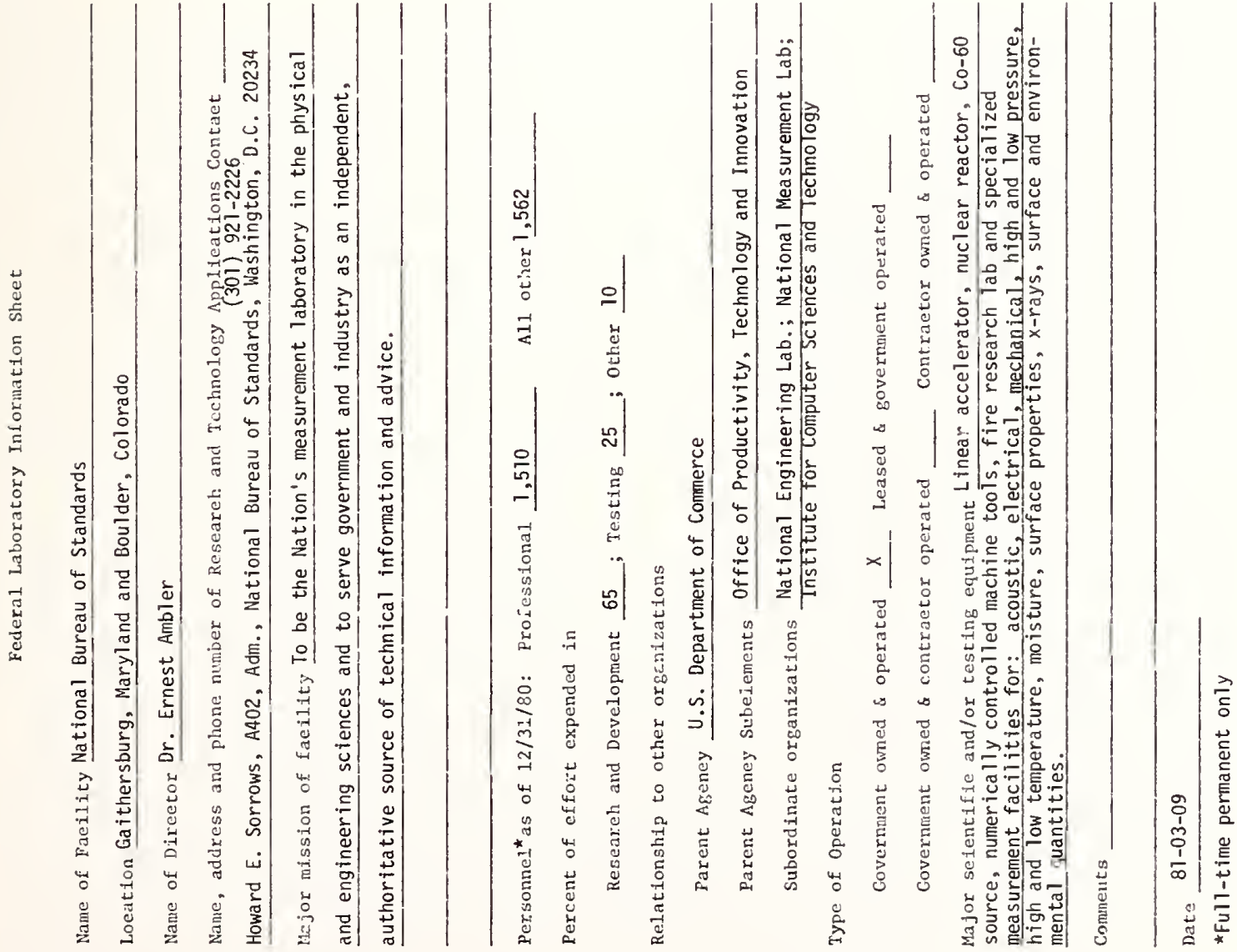

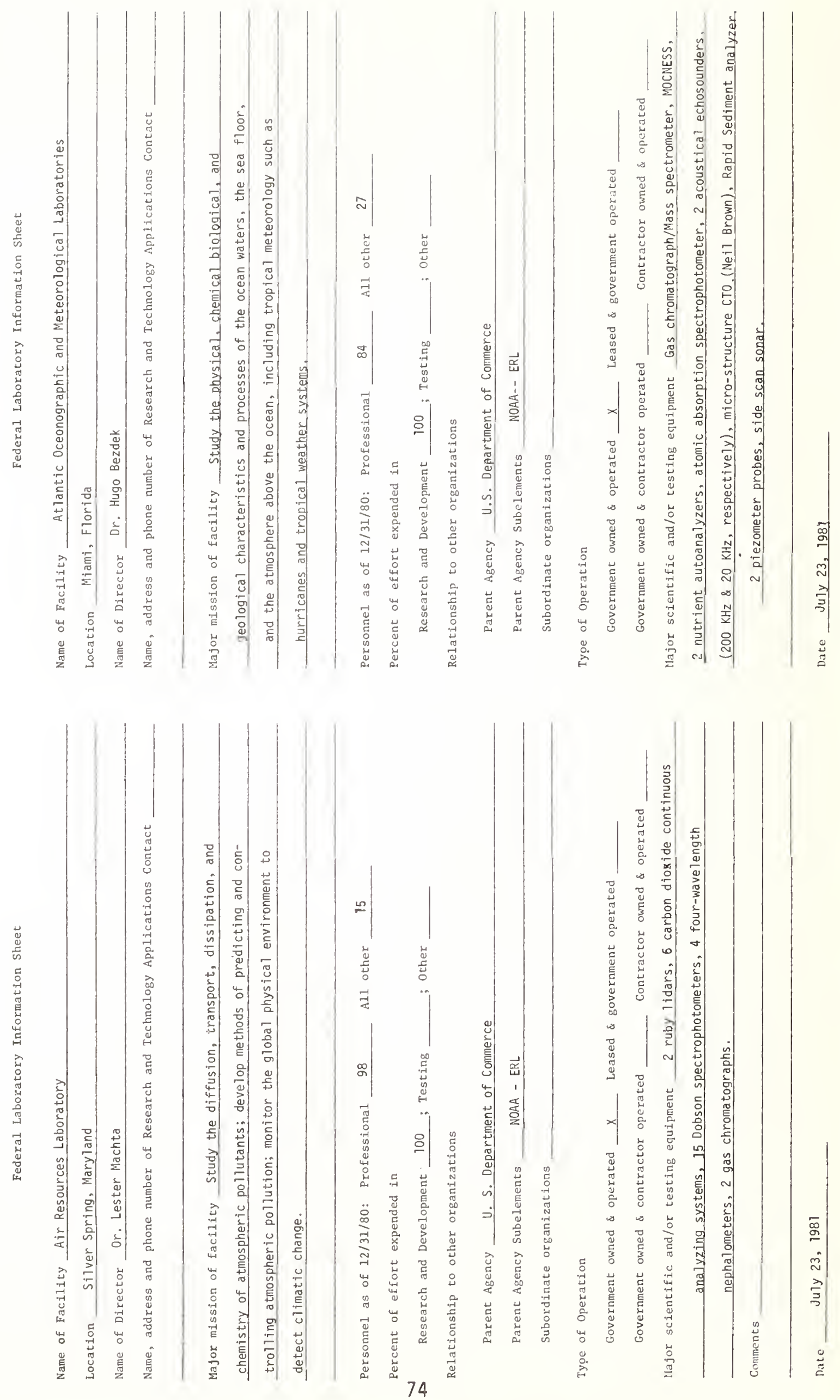

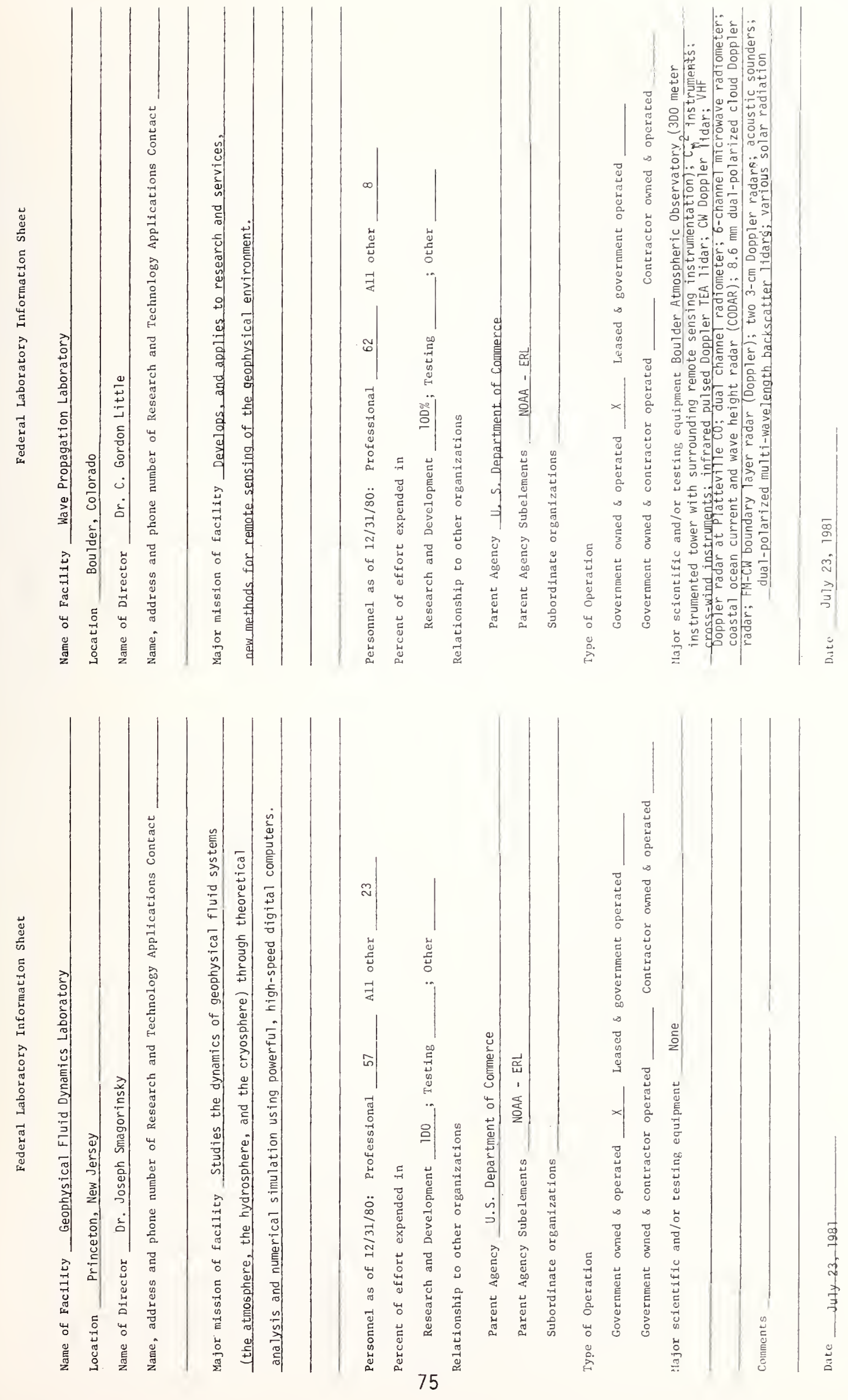

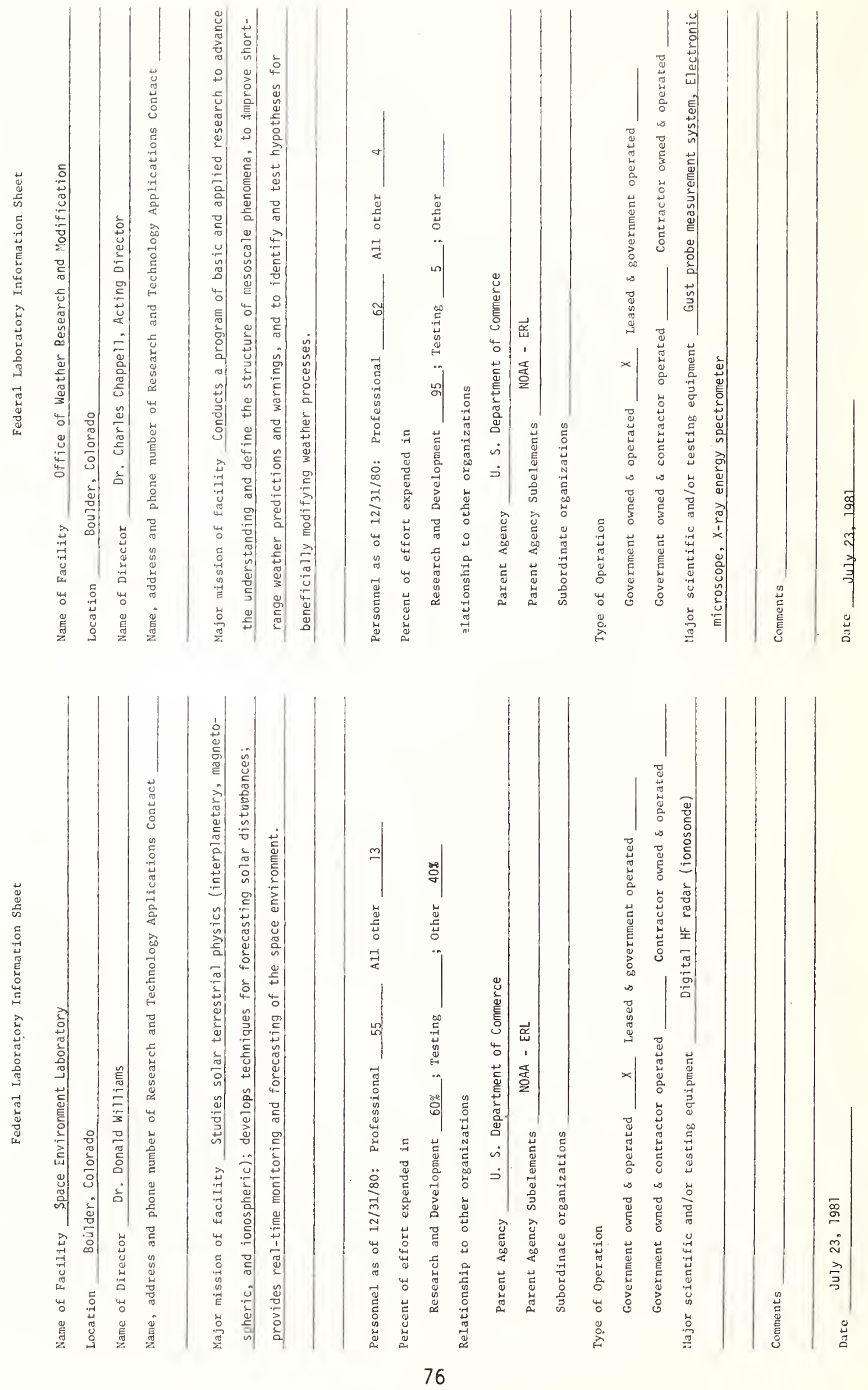

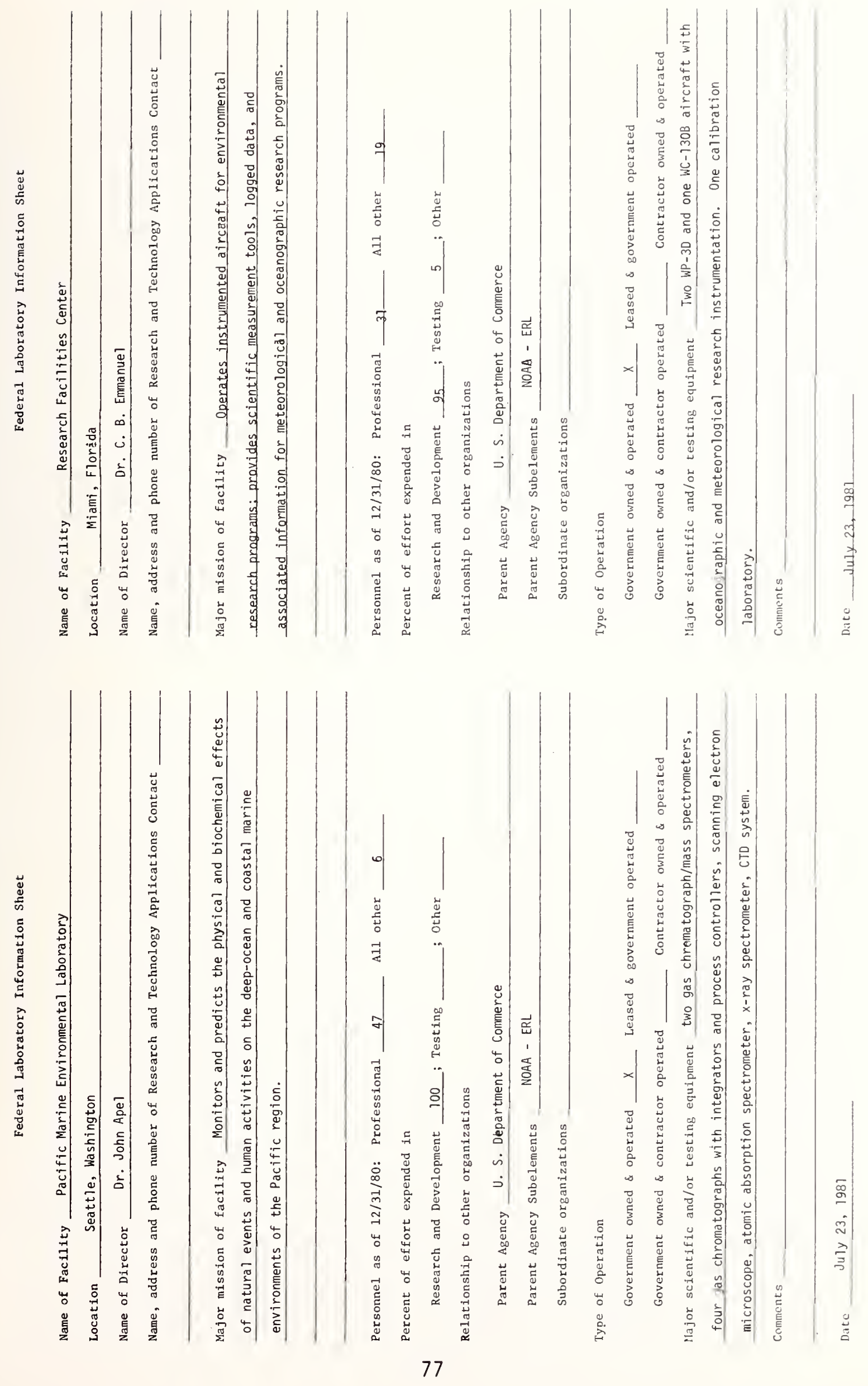

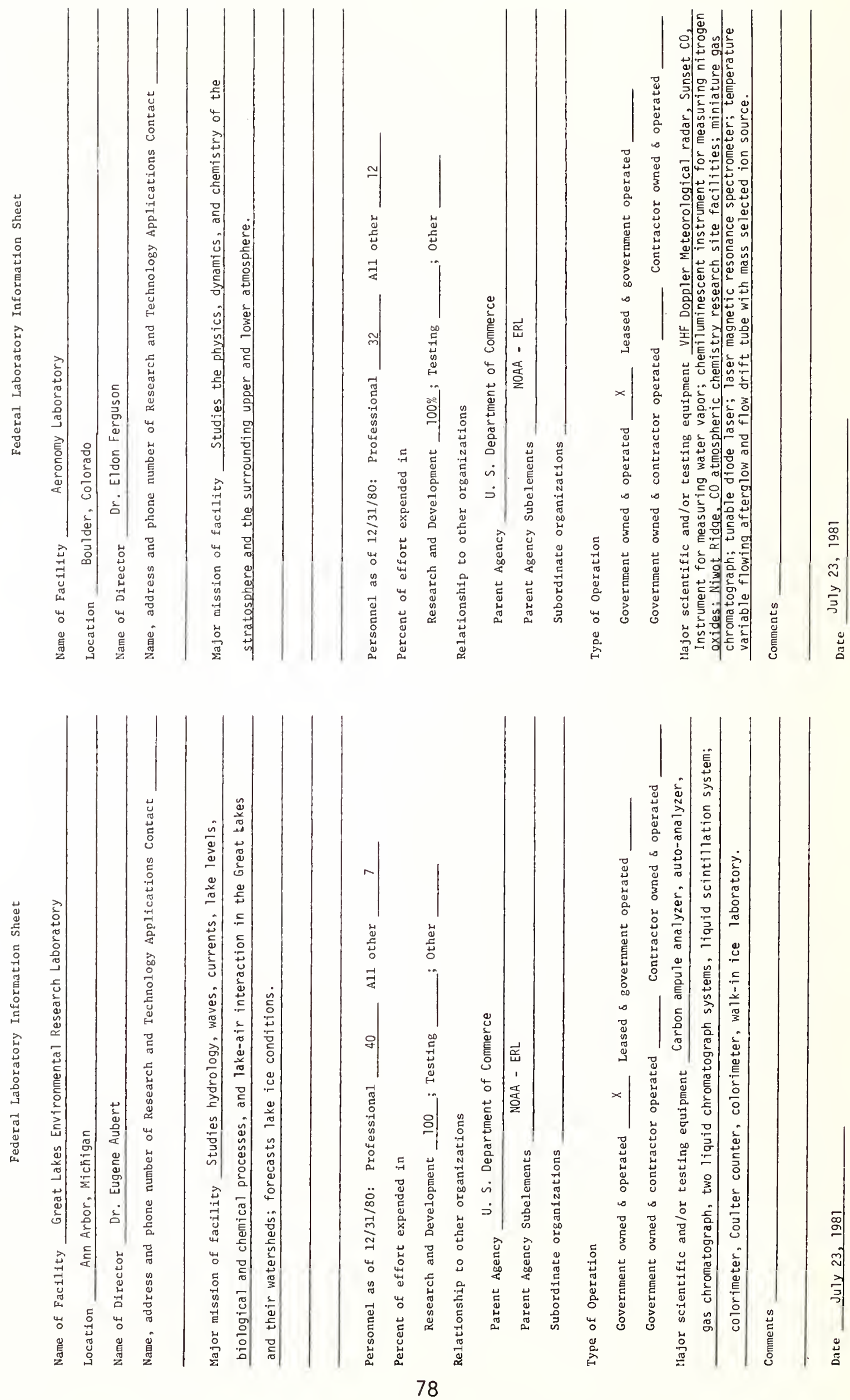

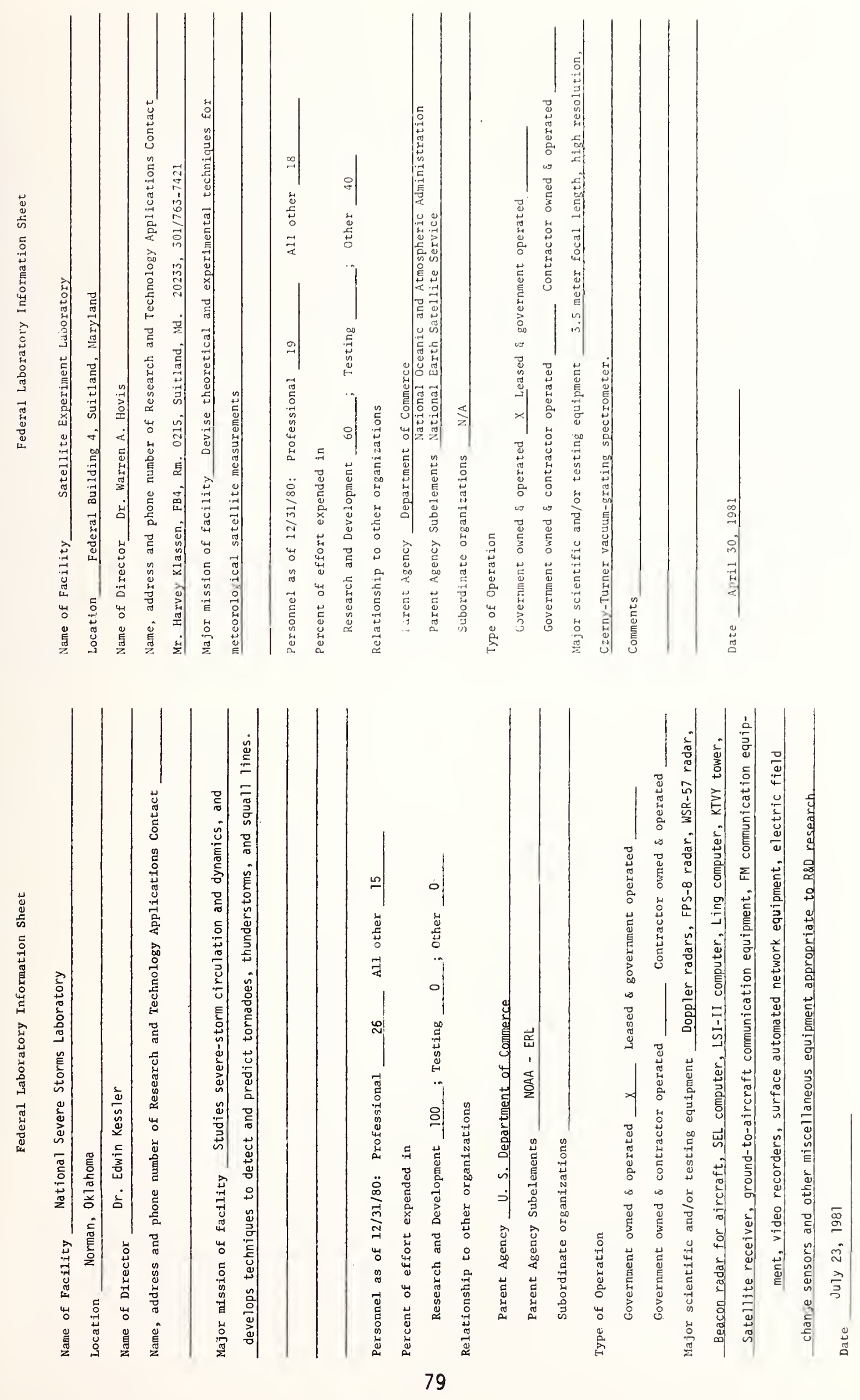

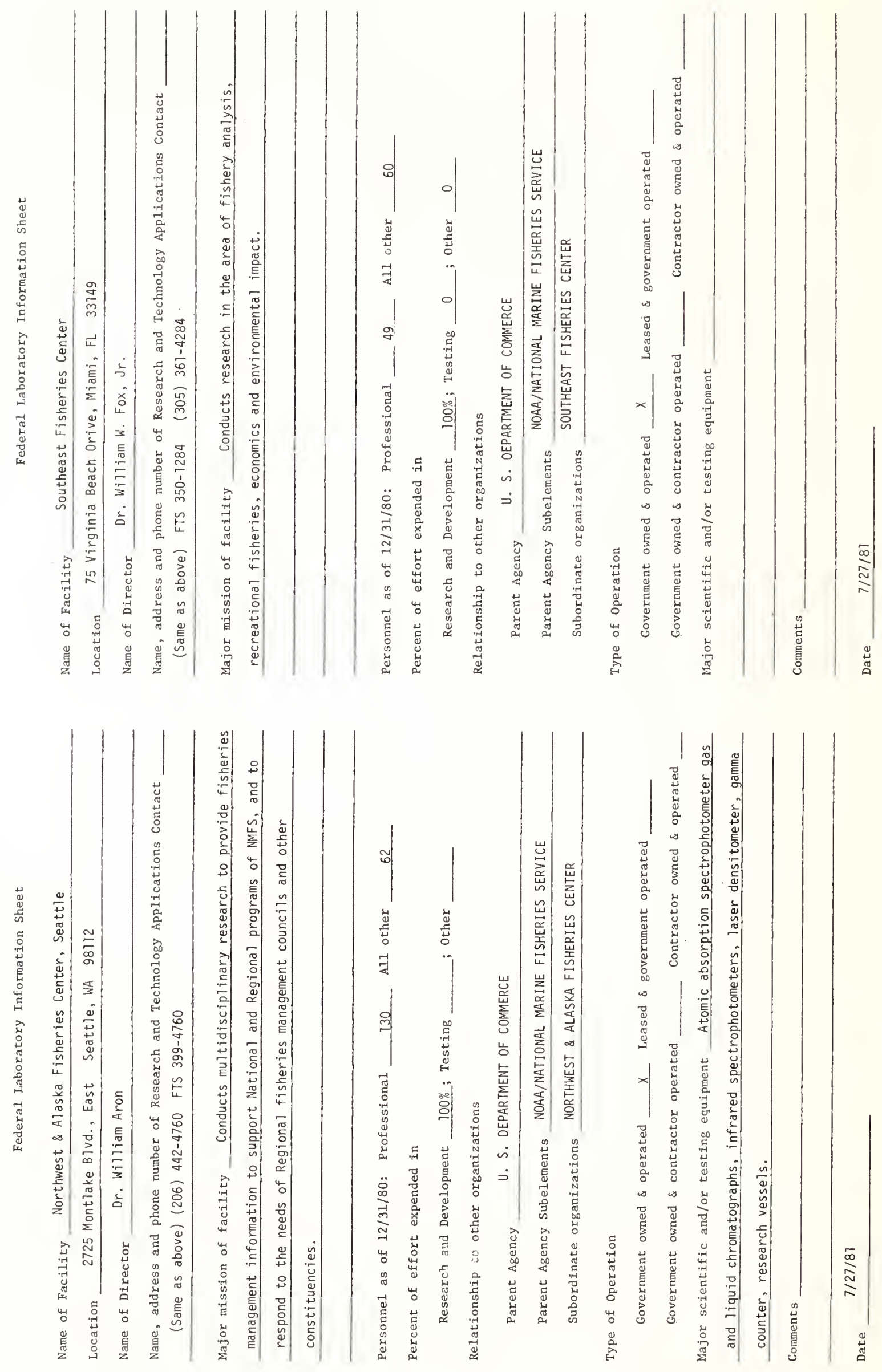

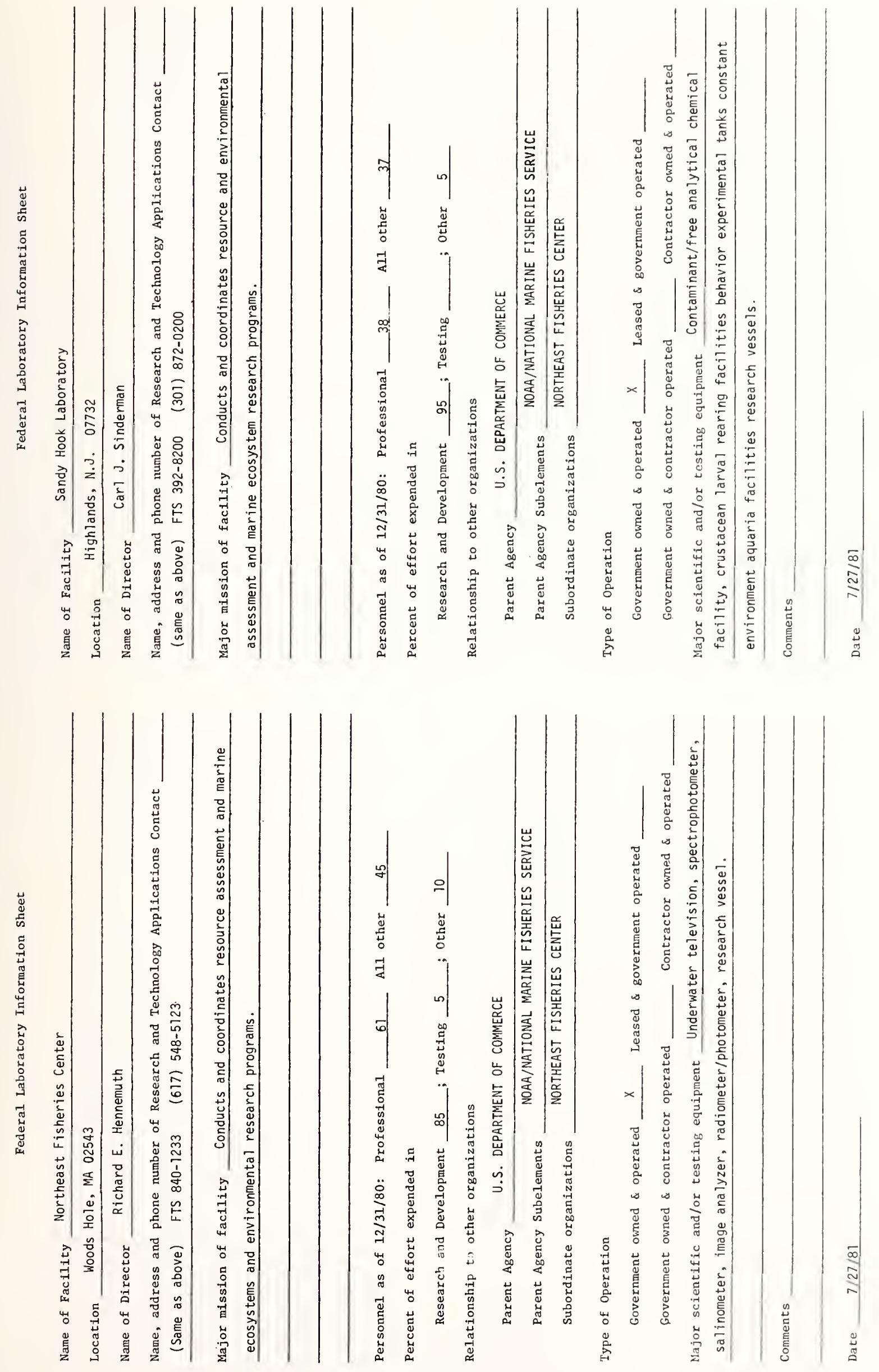

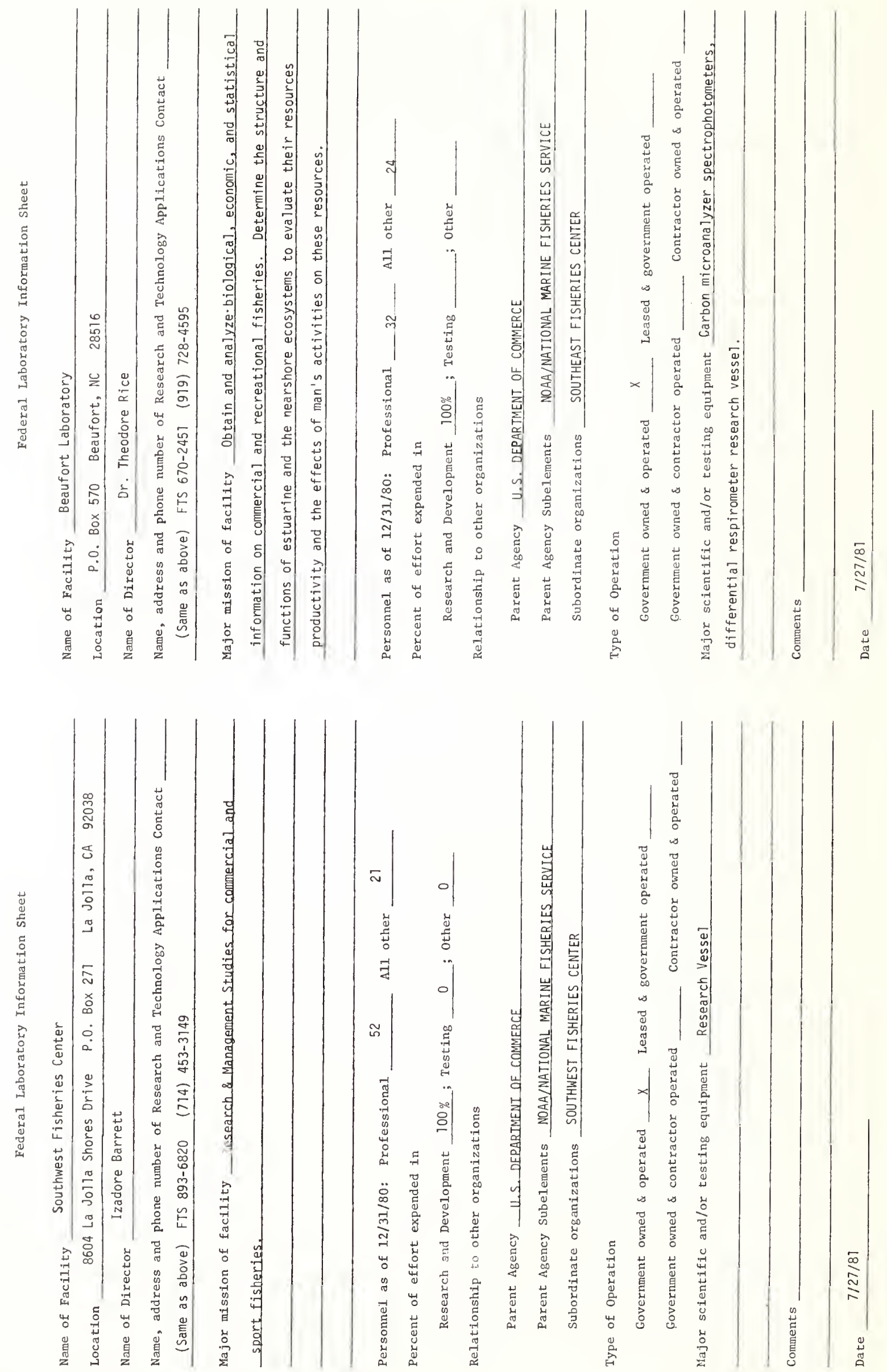

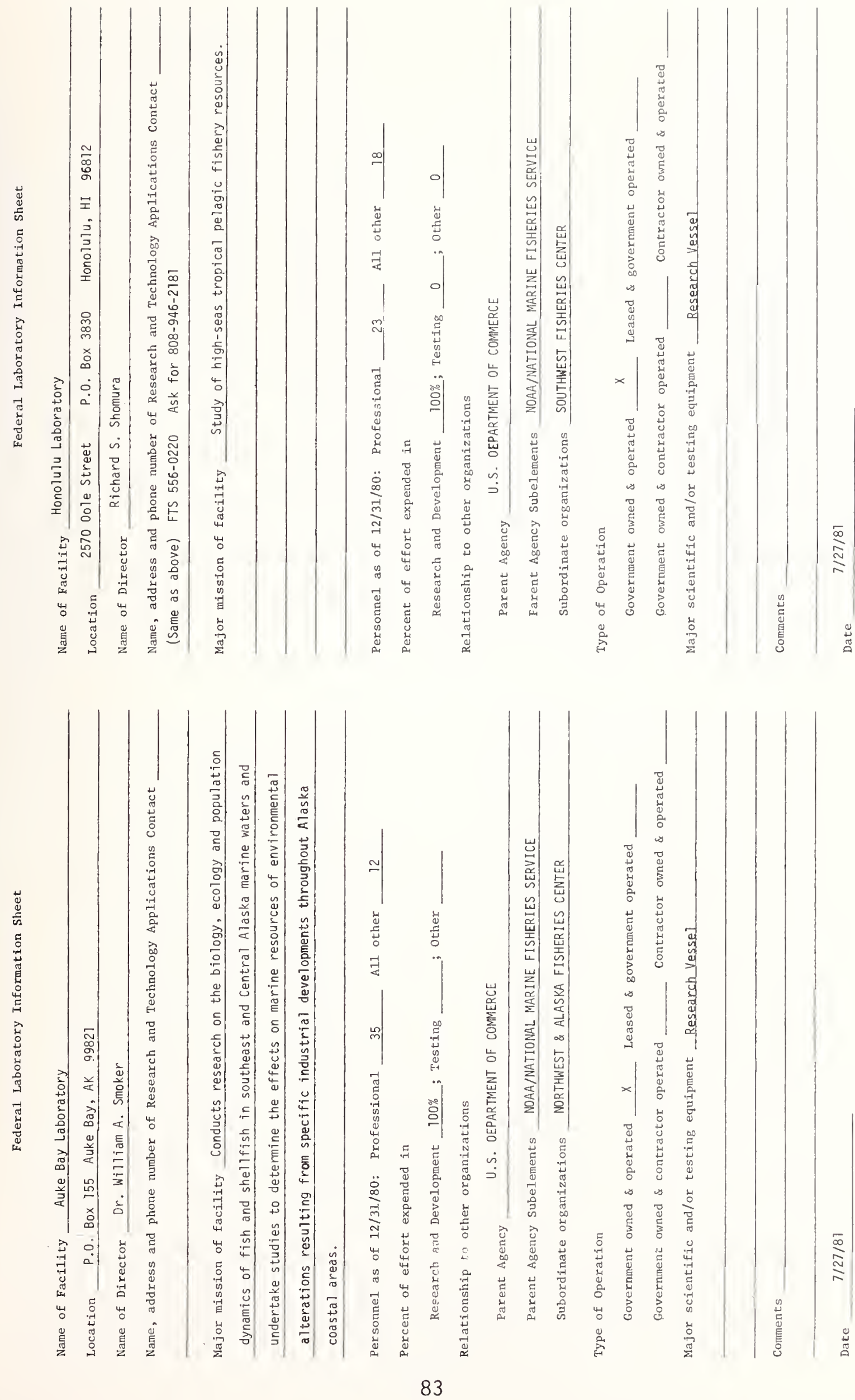

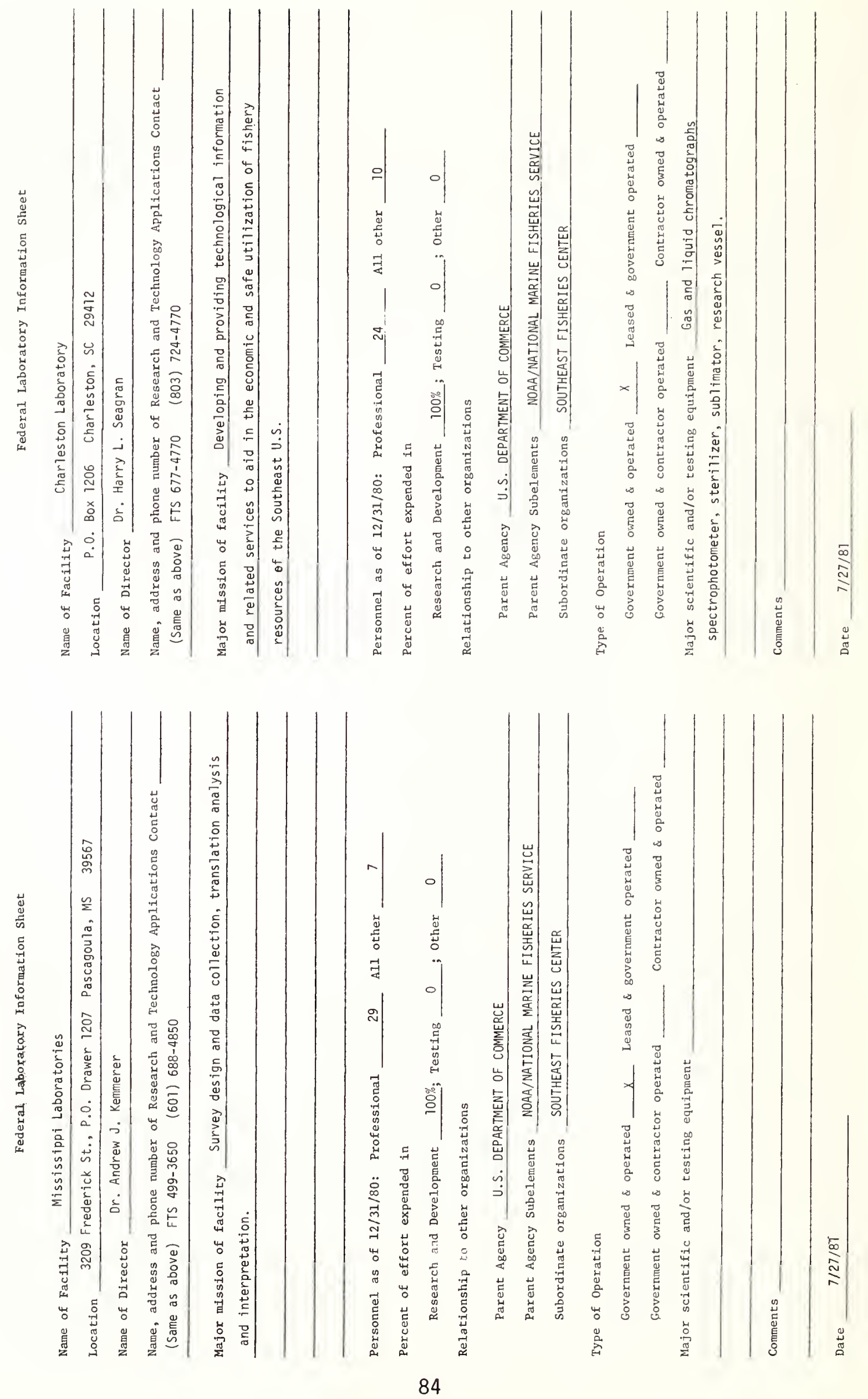

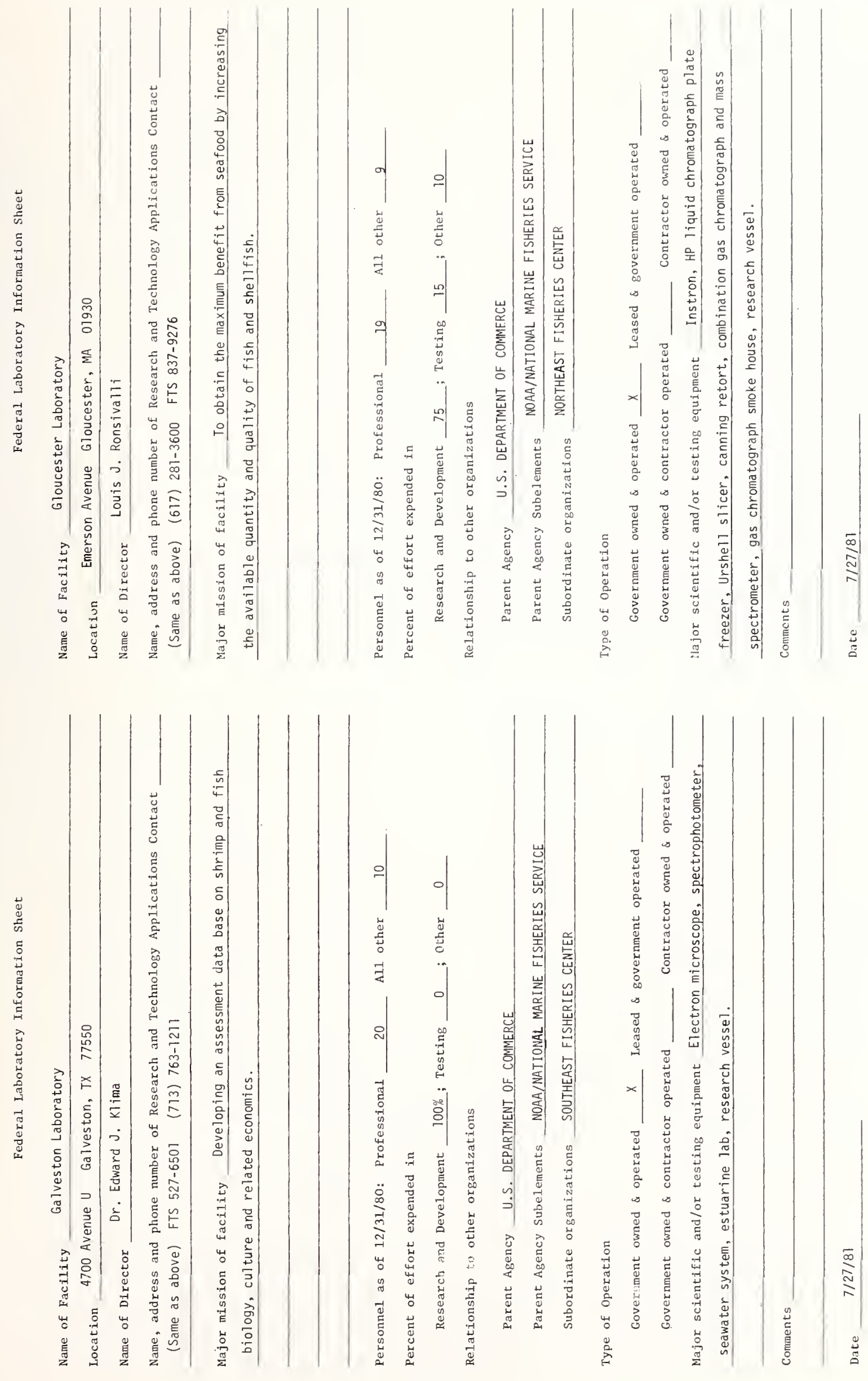

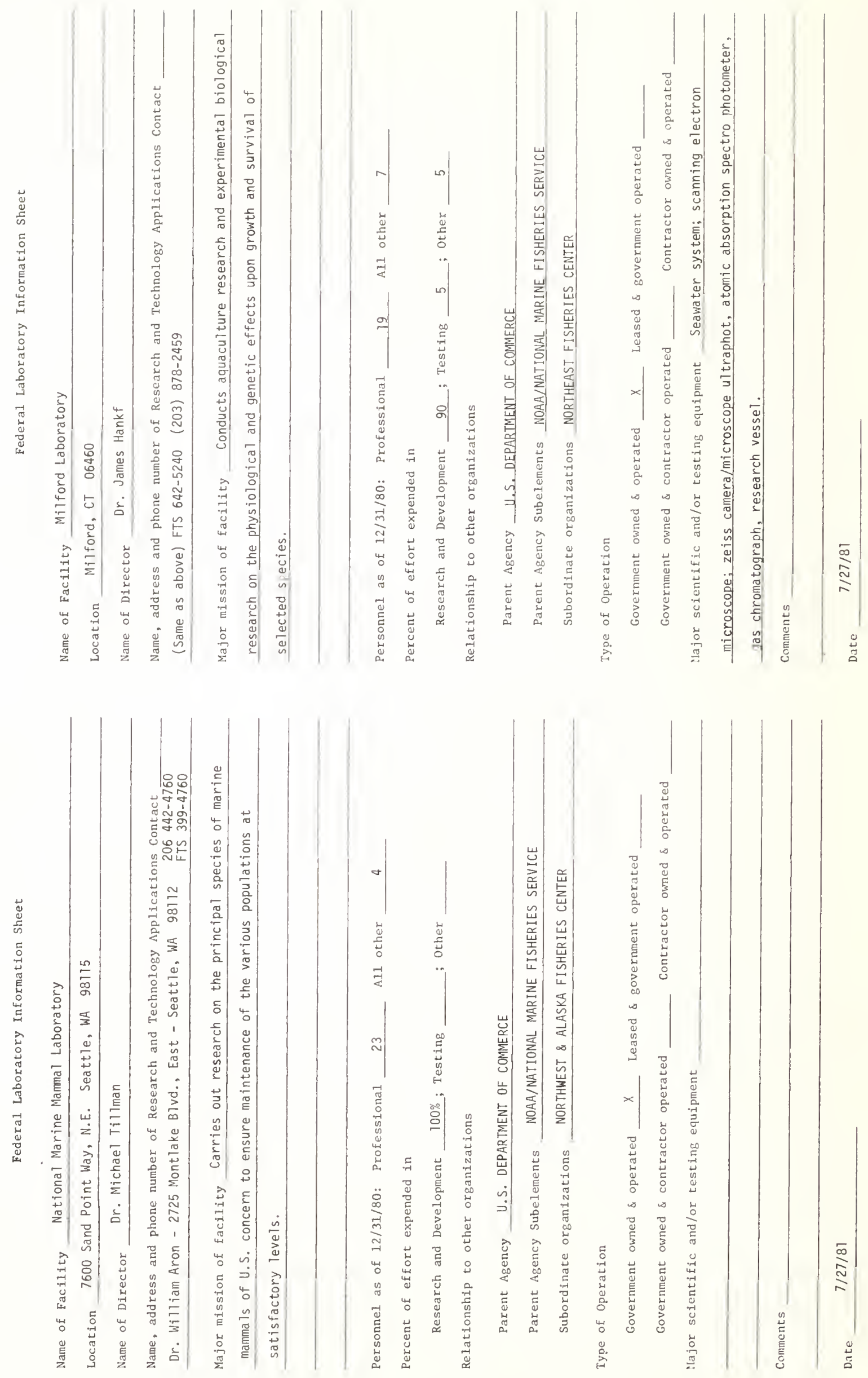

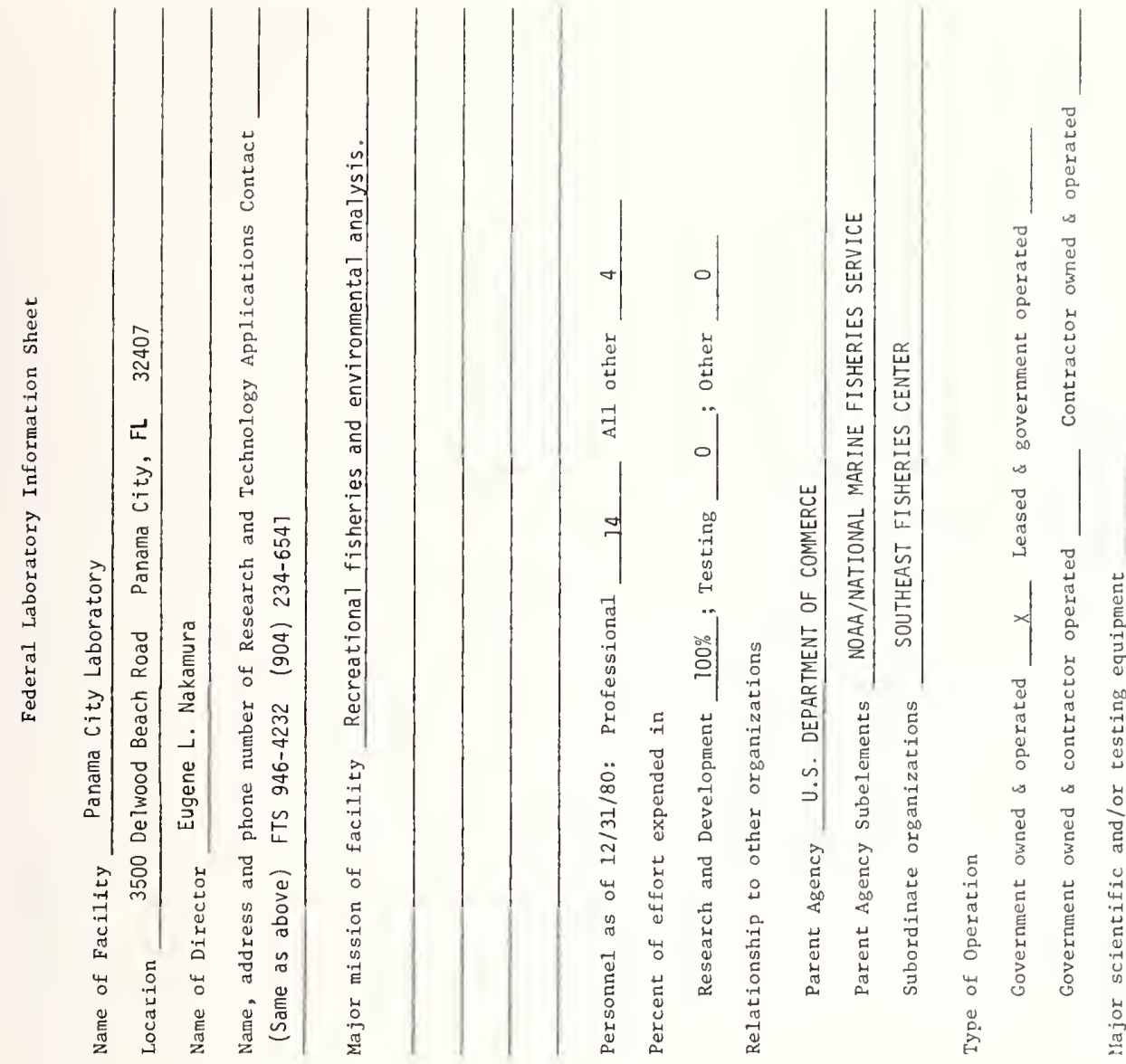

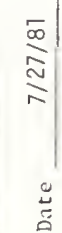

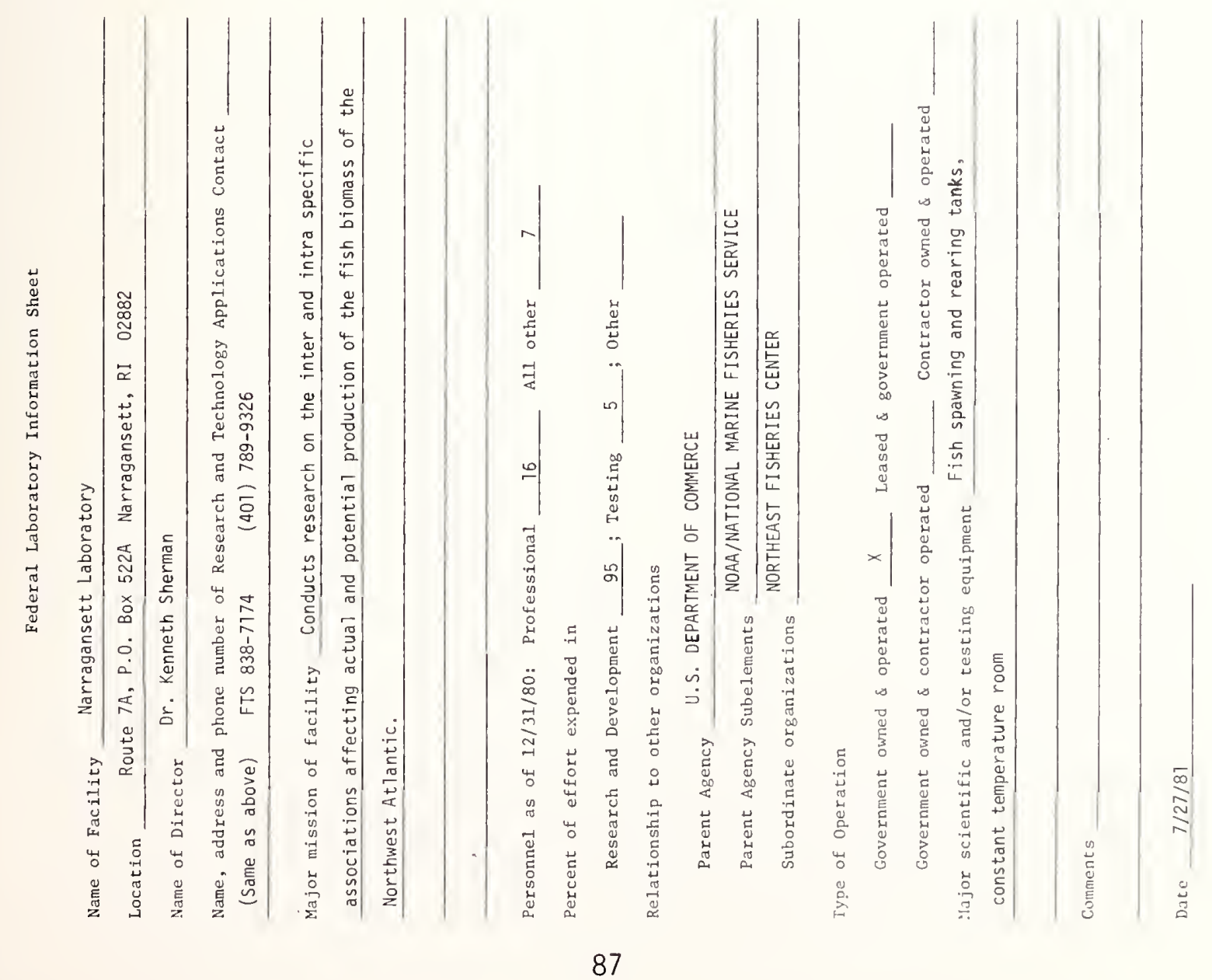



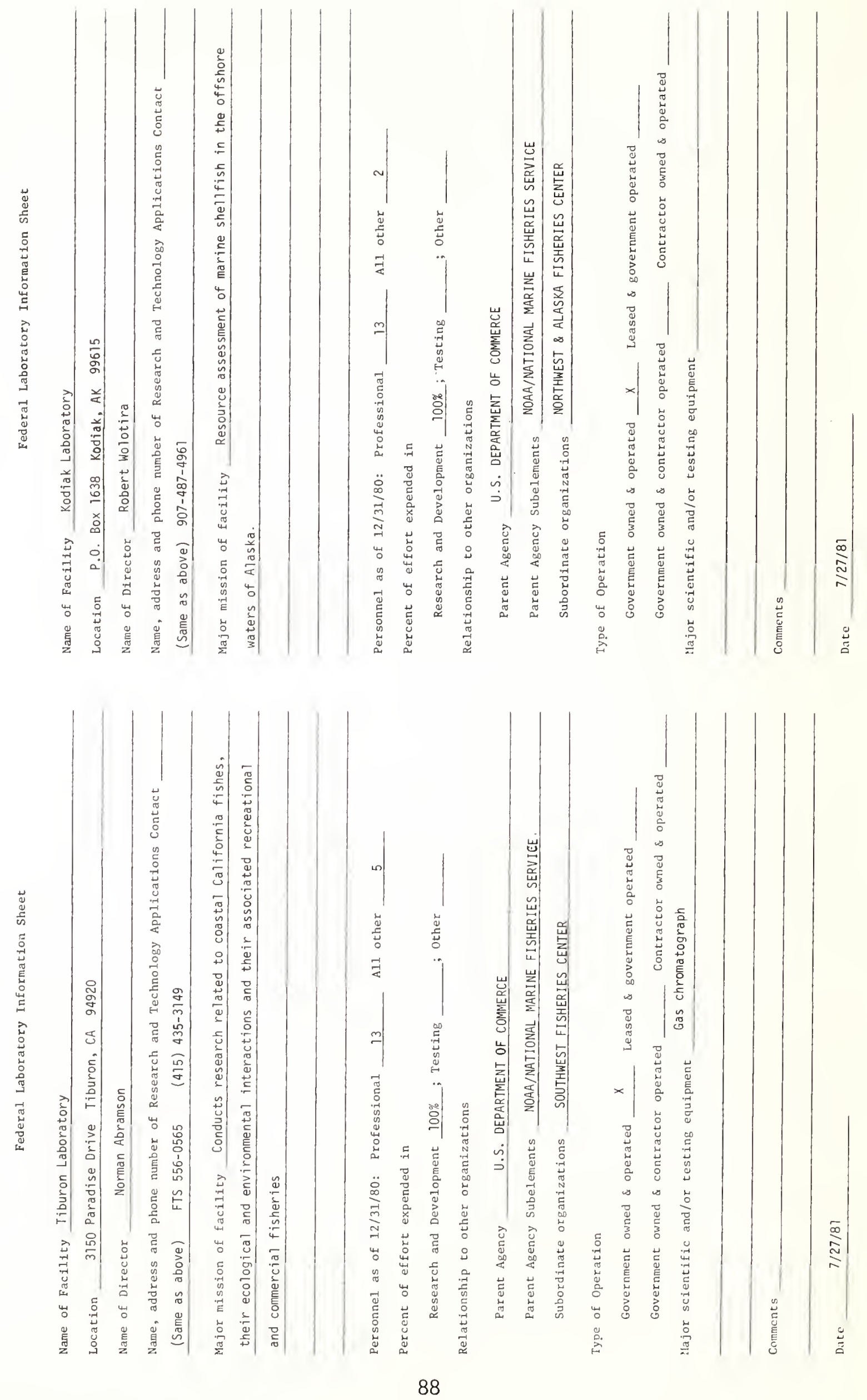

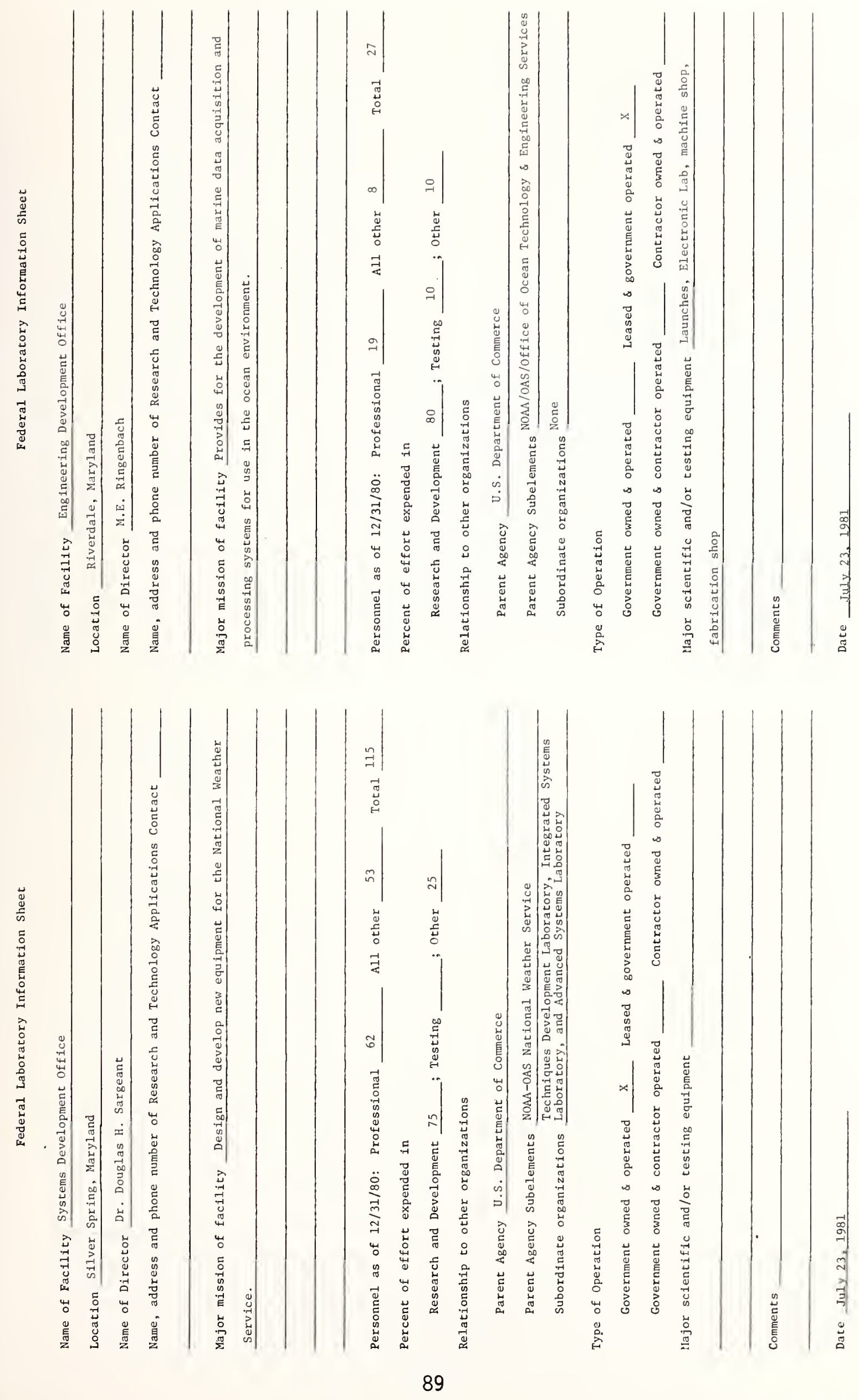

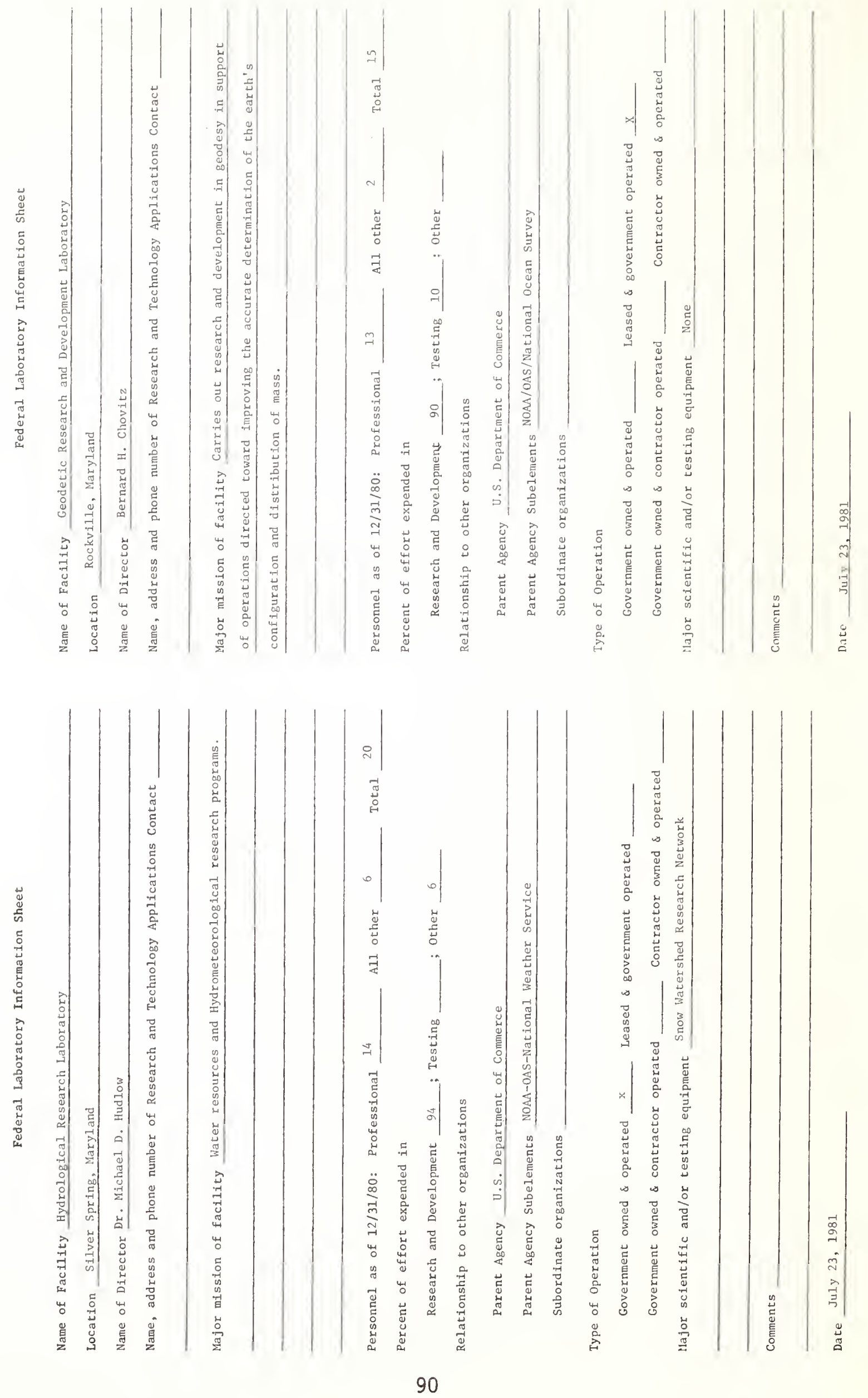


\section{DEPARTMENT OF DEFENSE}




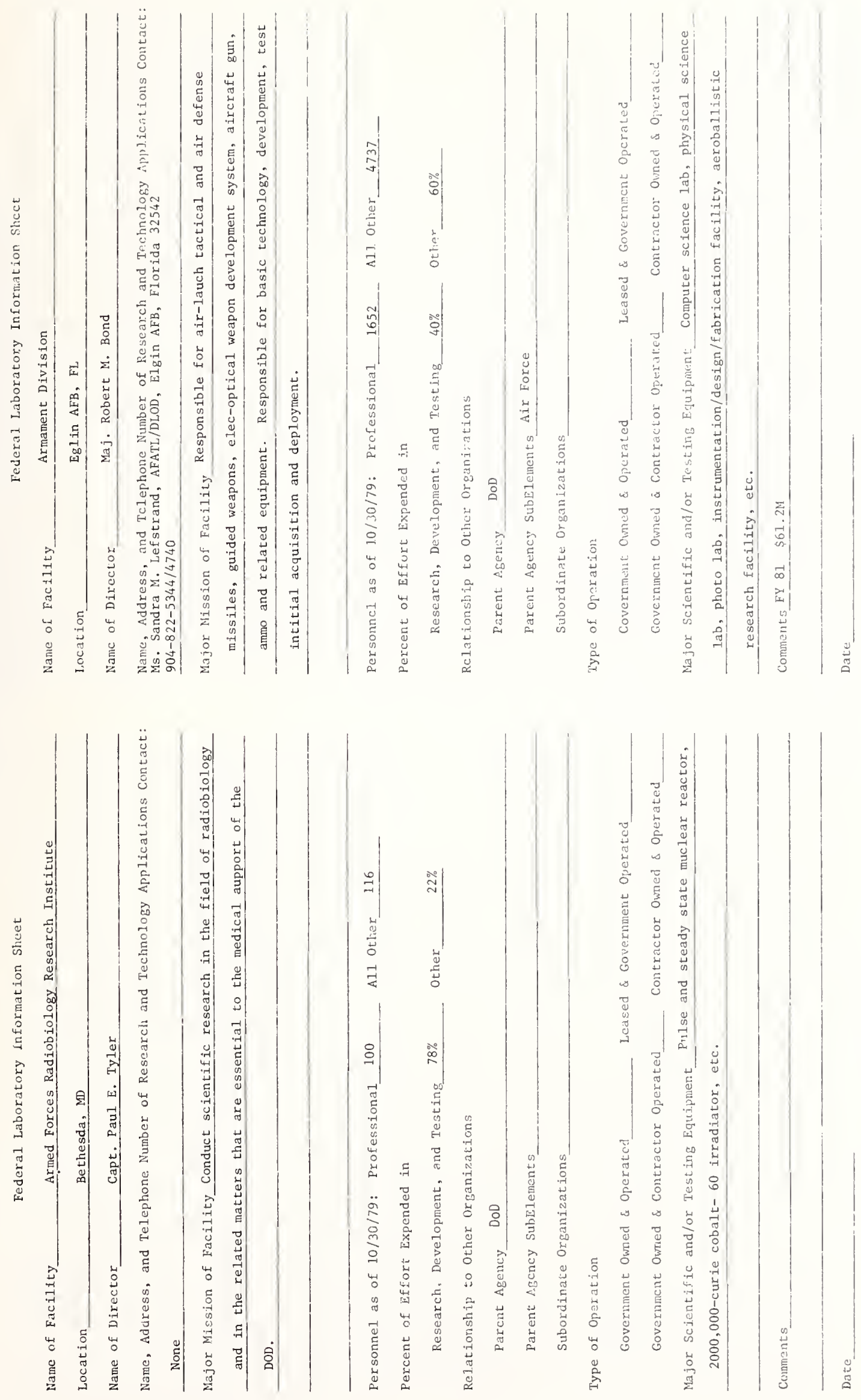

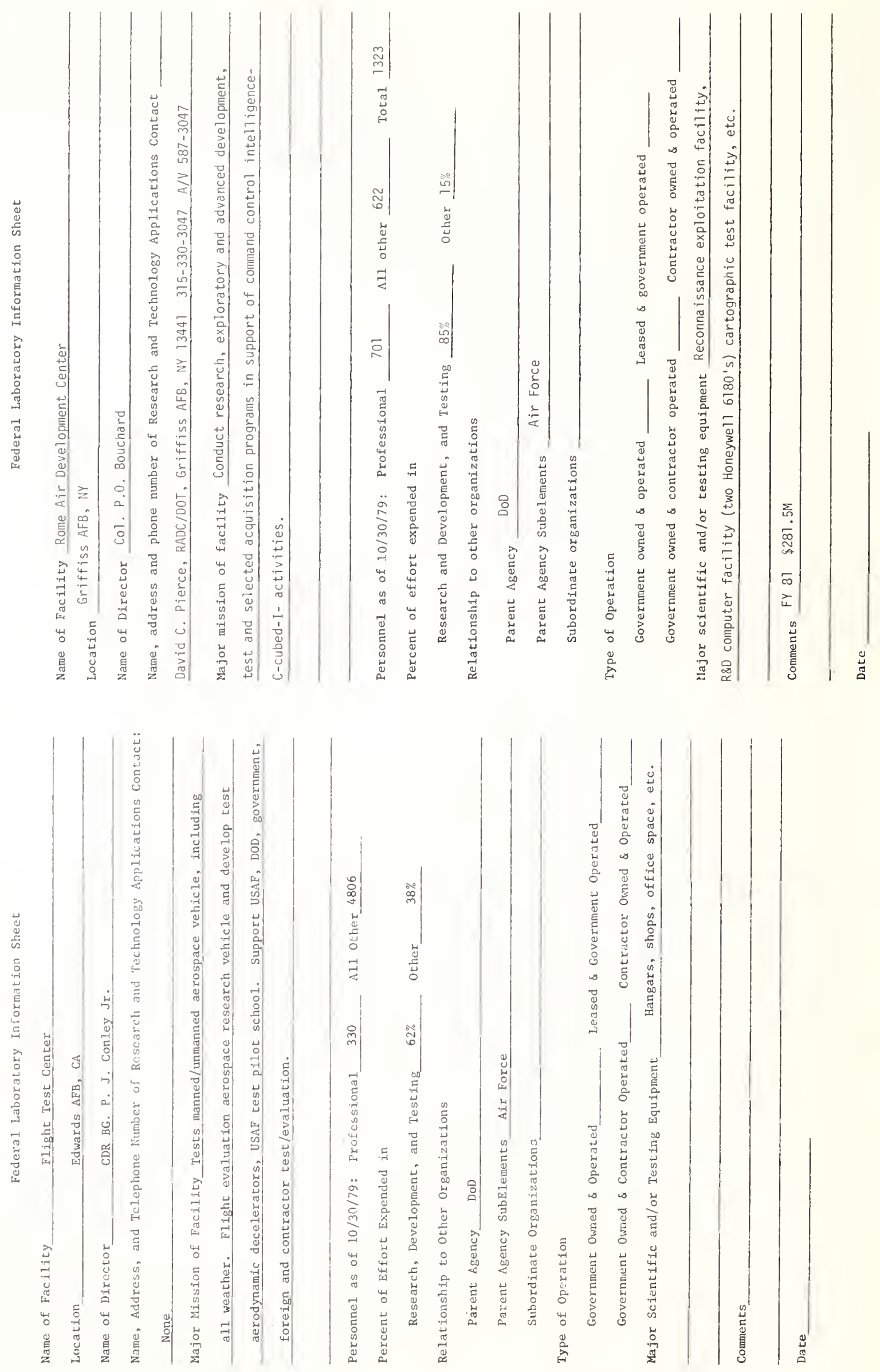

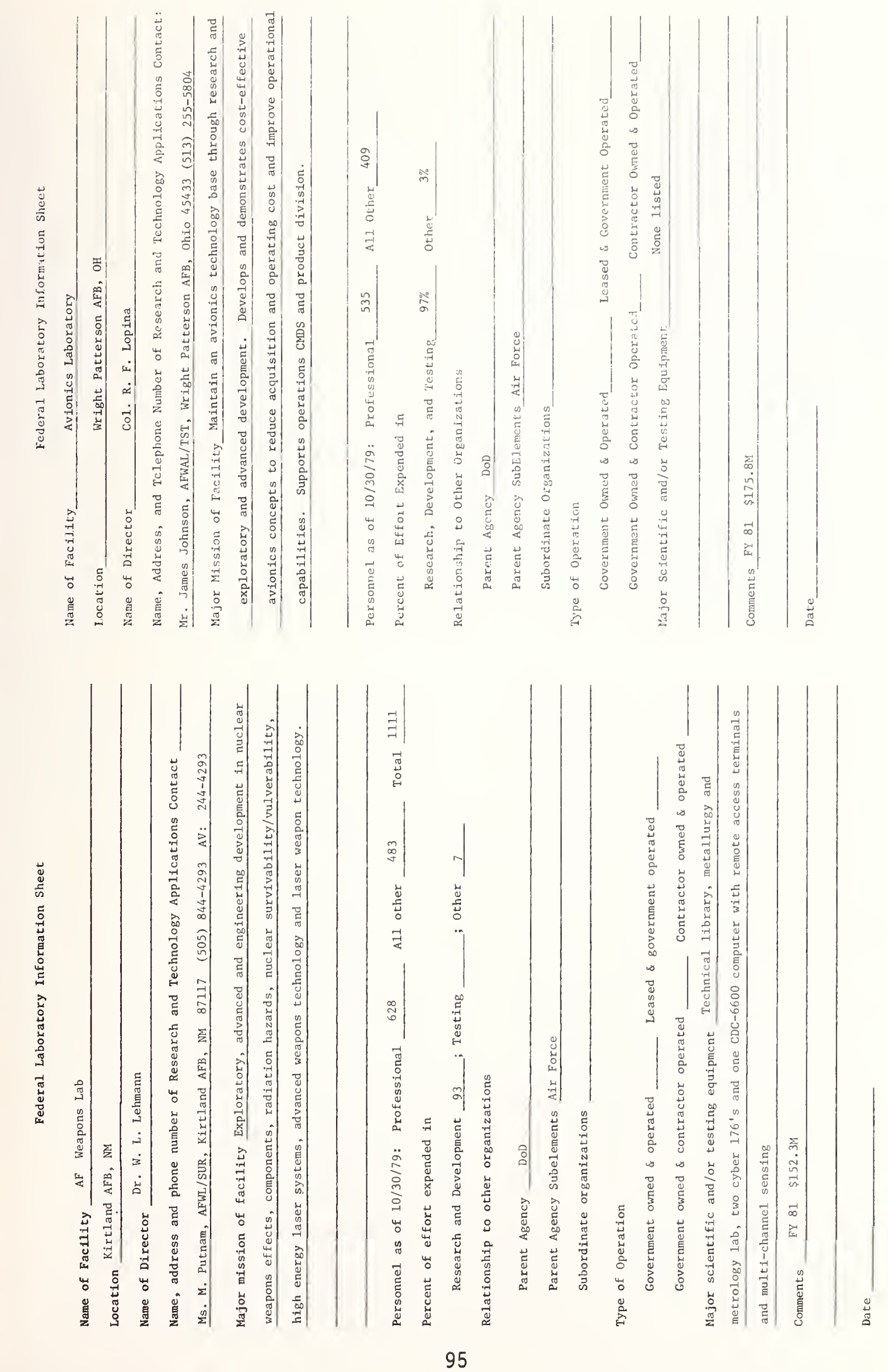

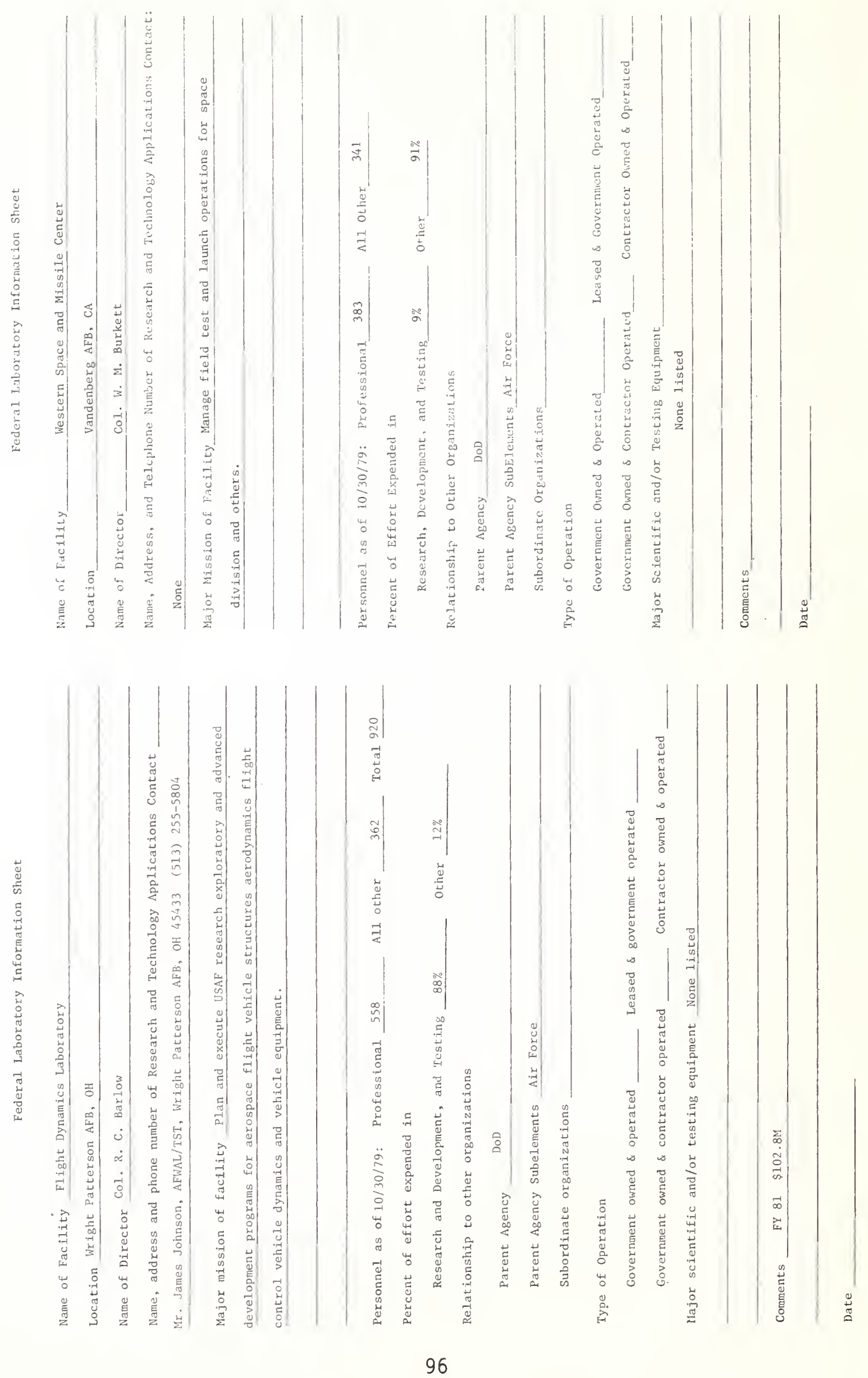

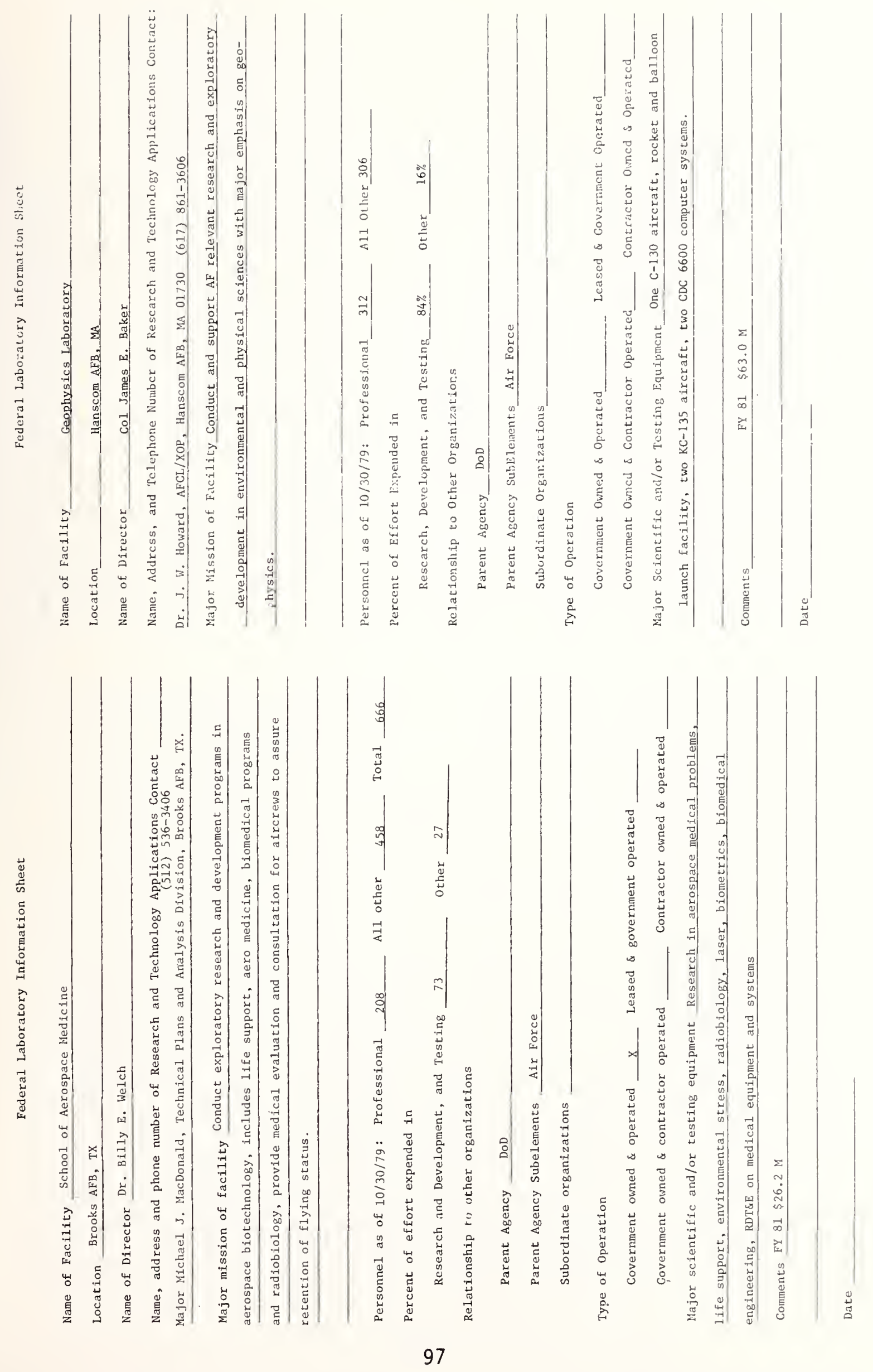

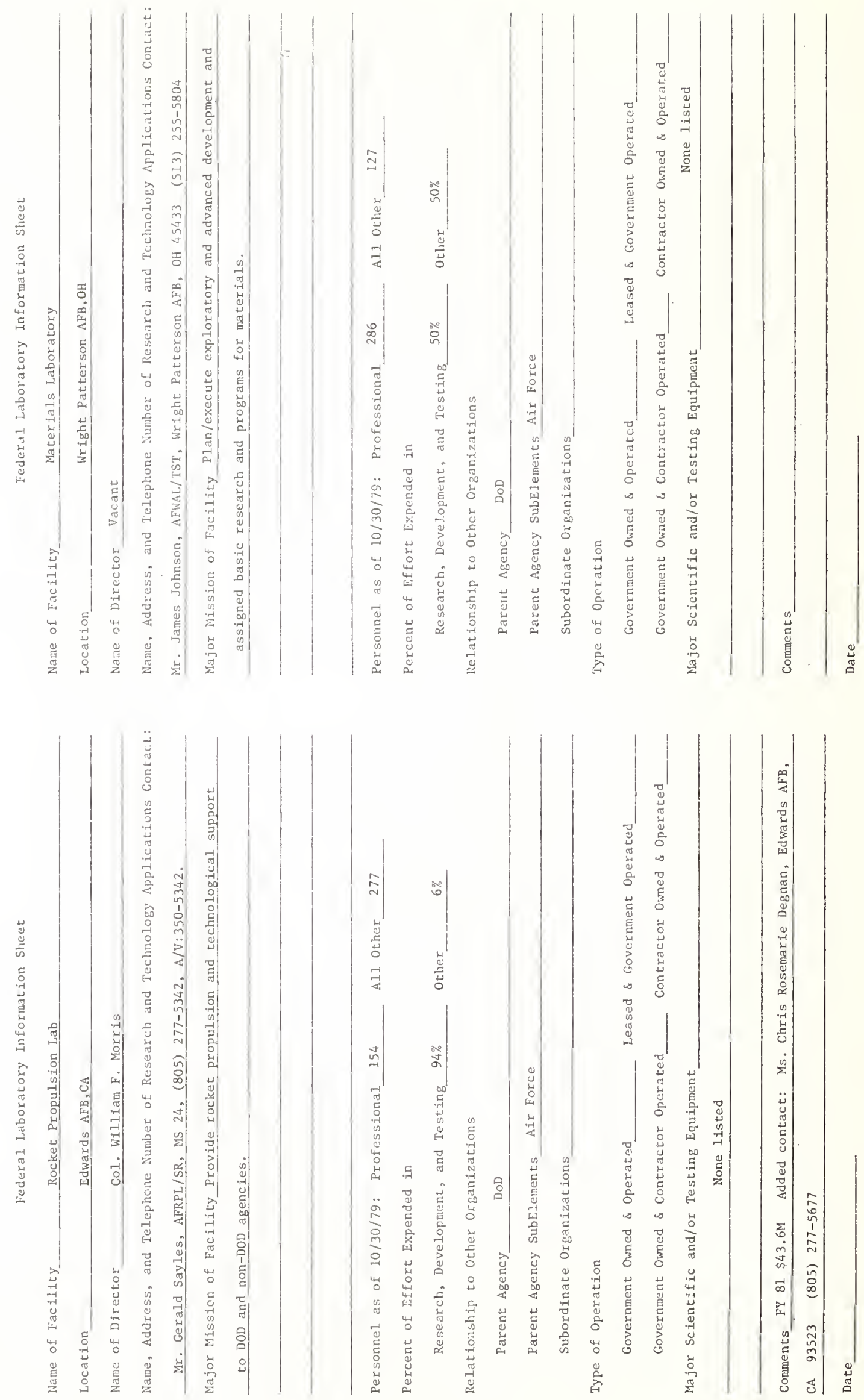

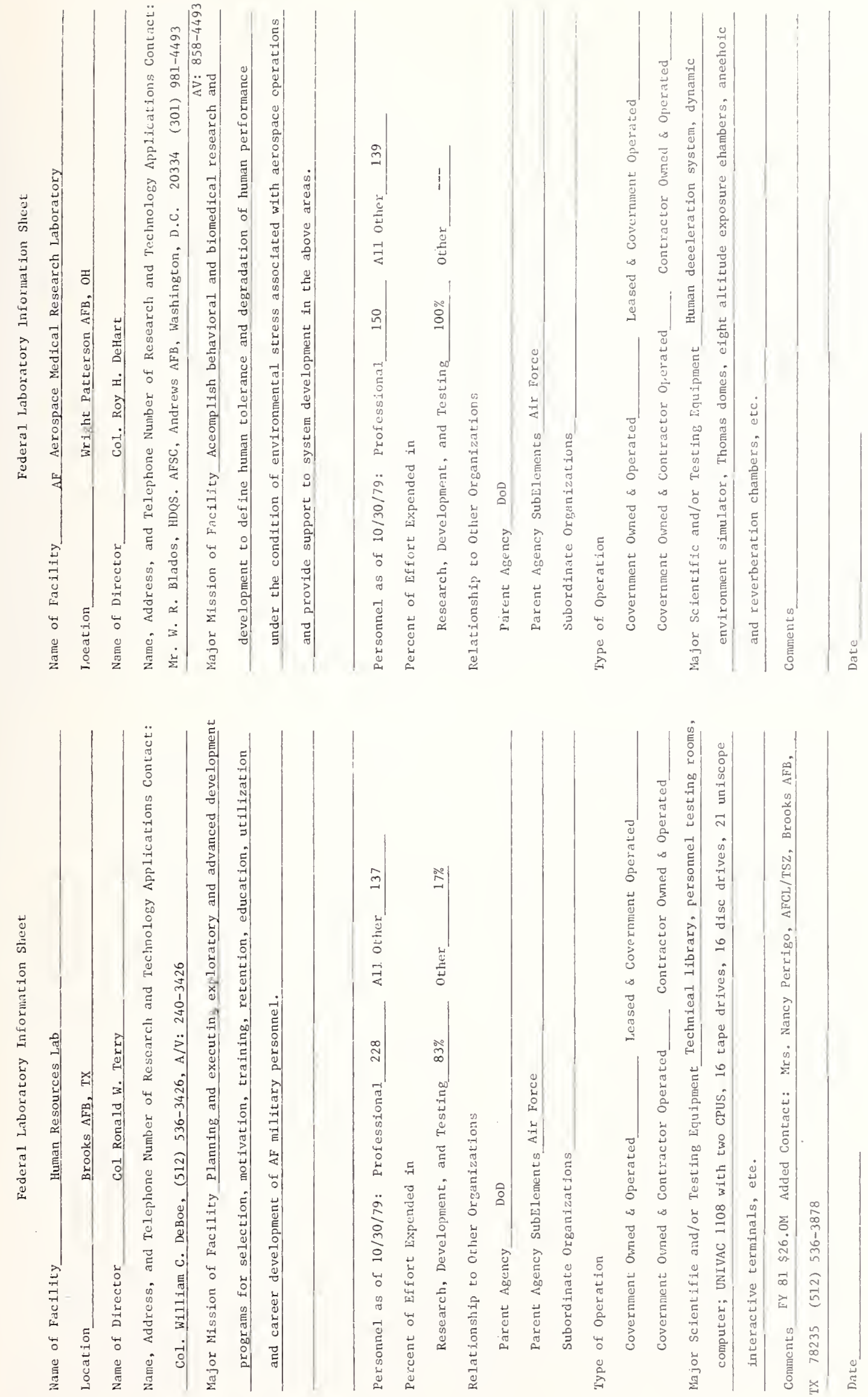

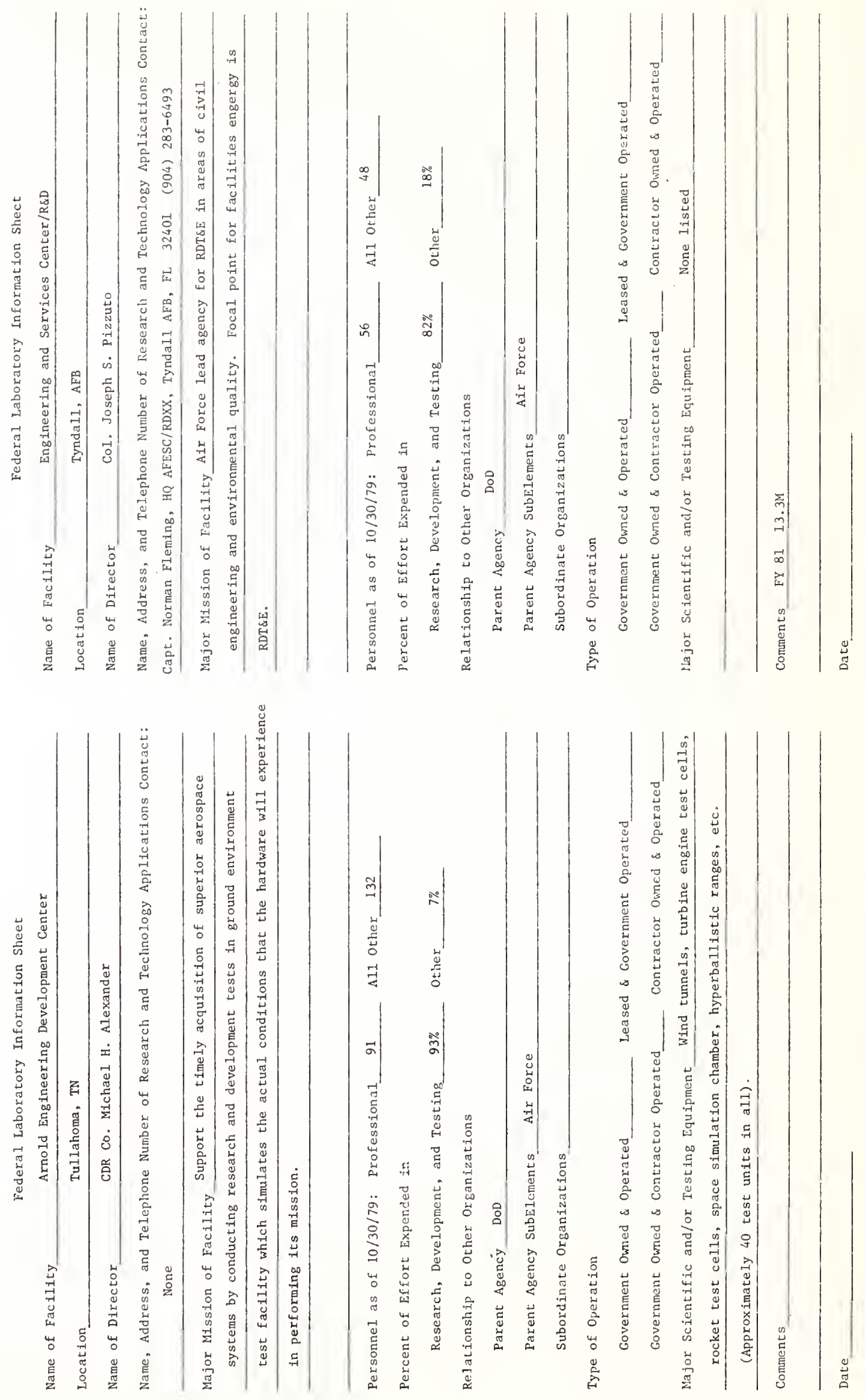

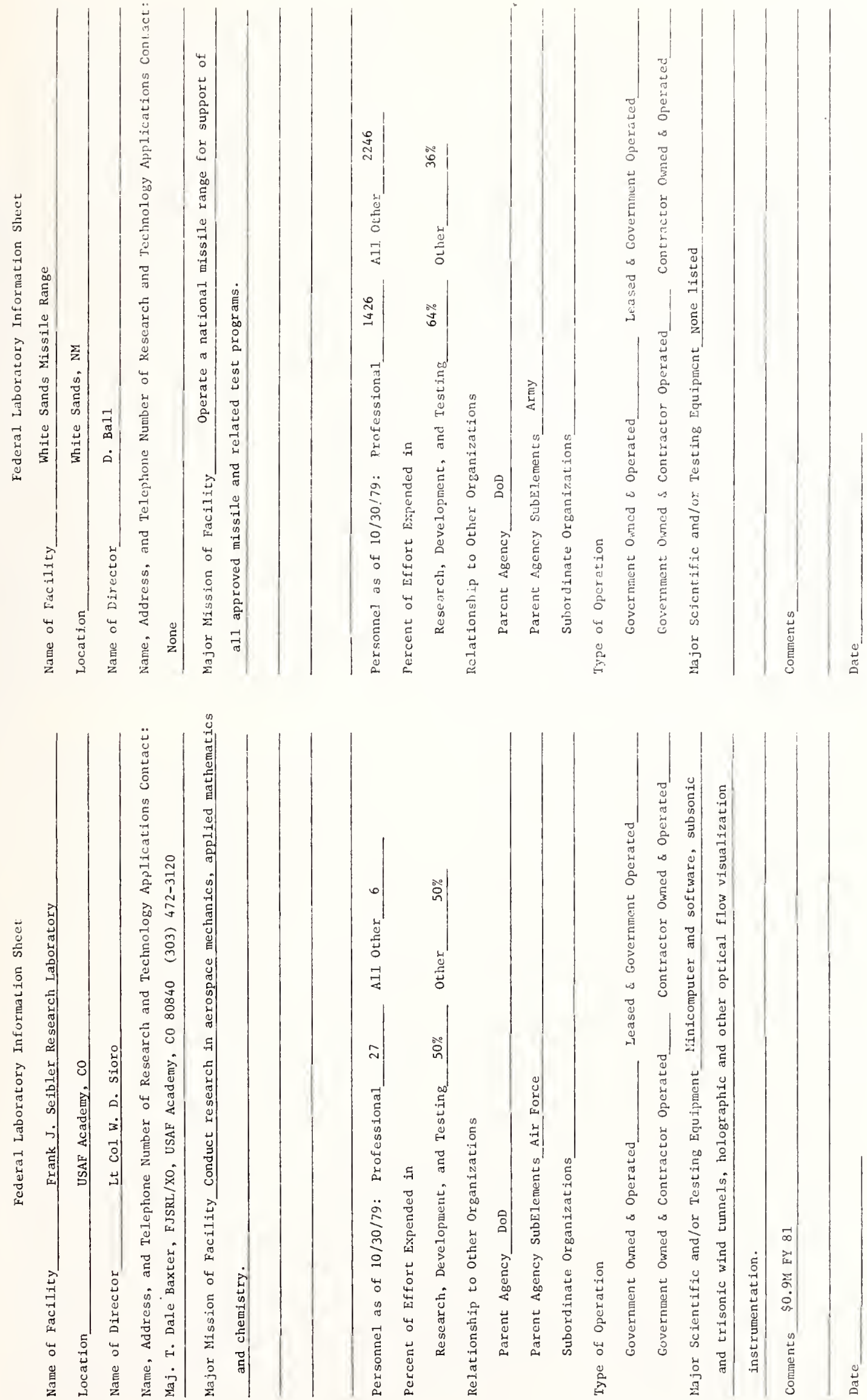

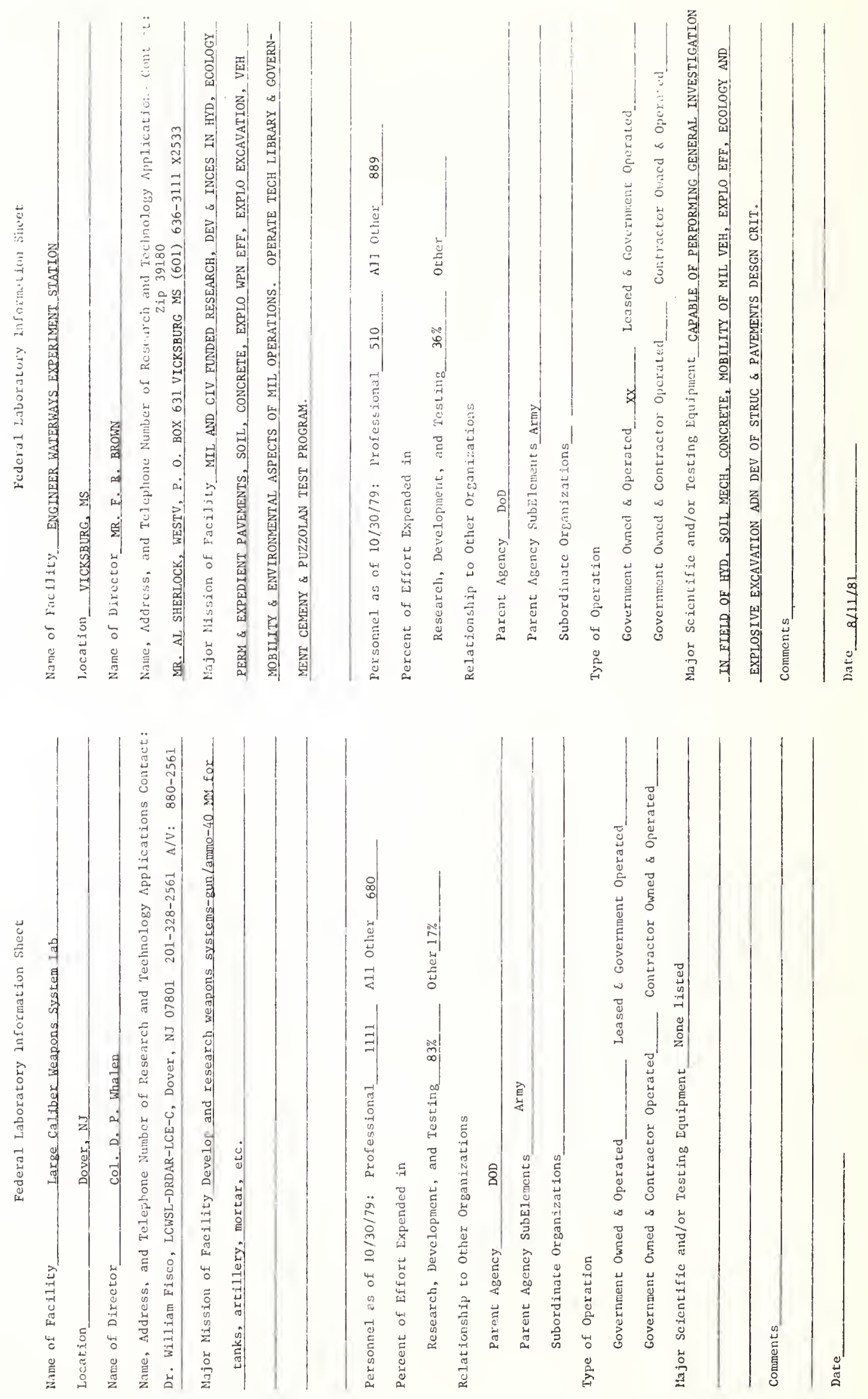

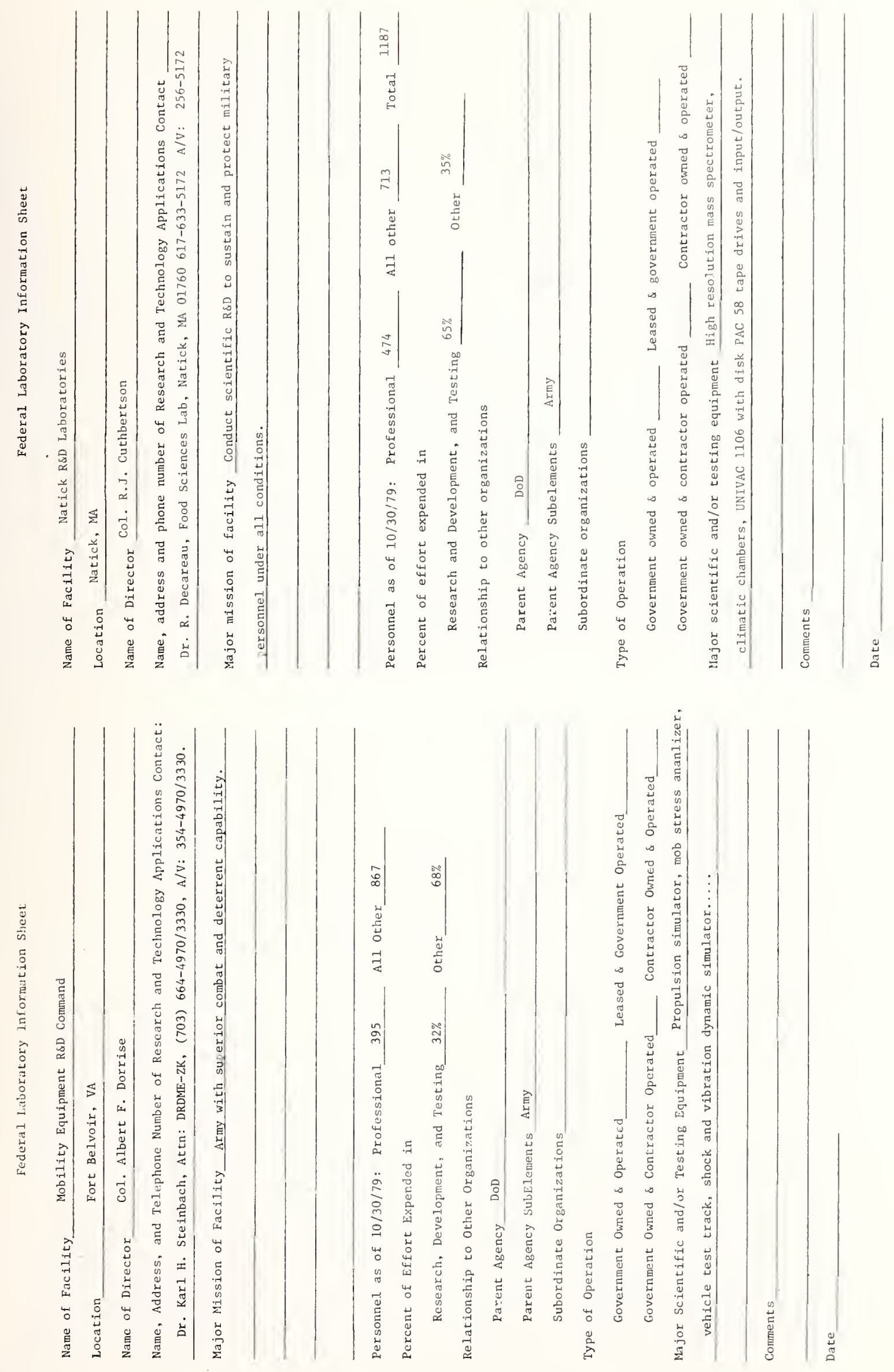

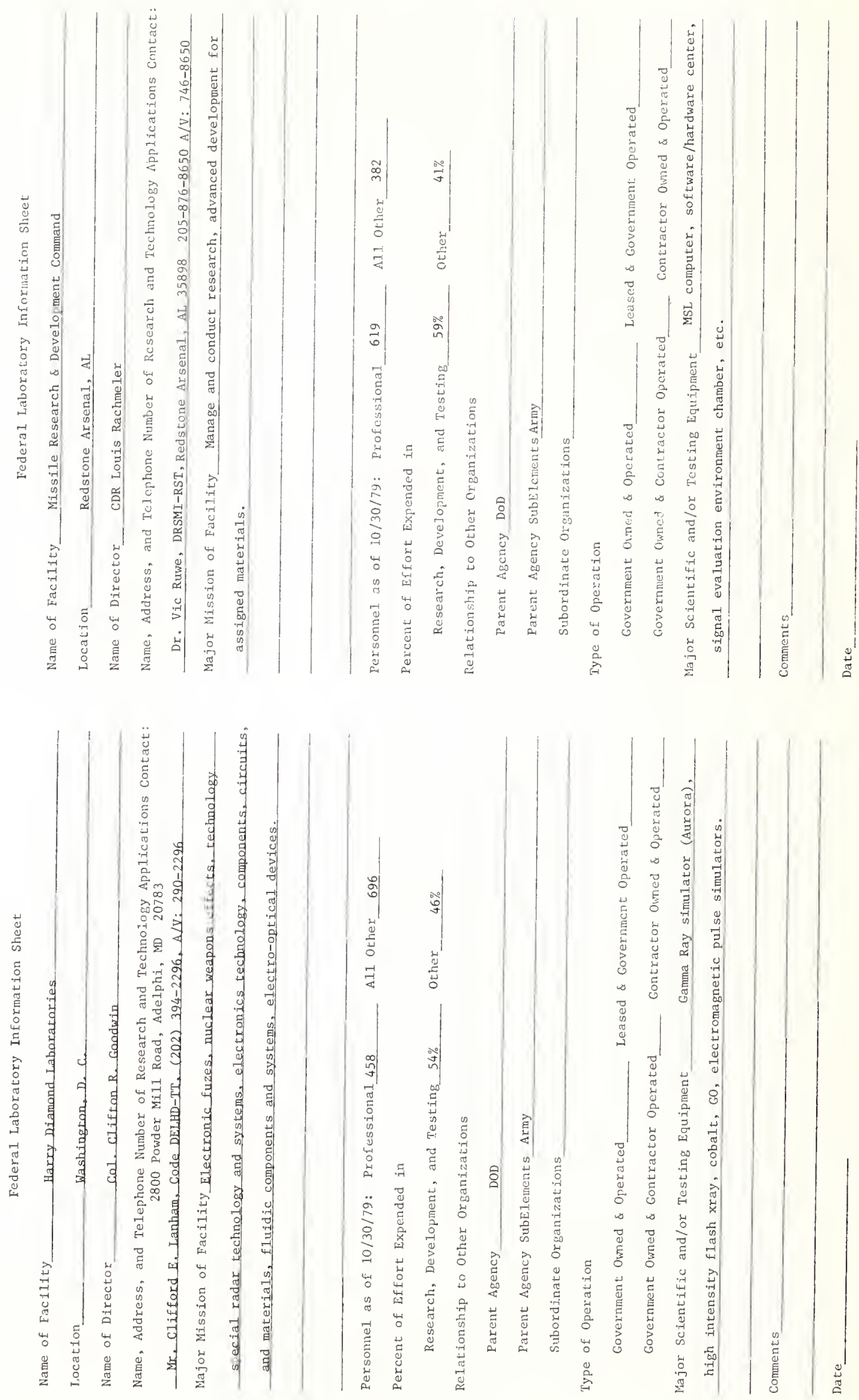

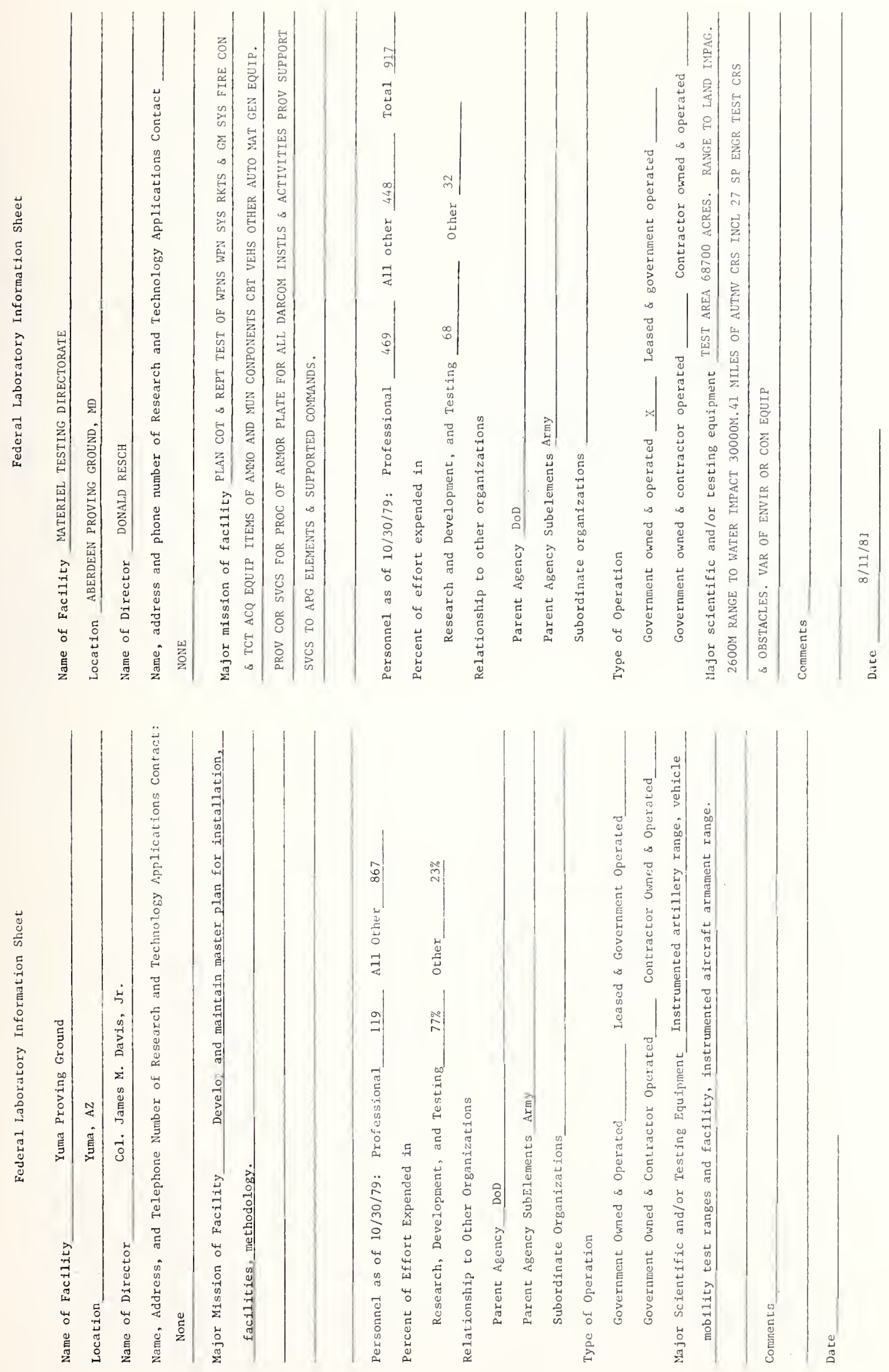

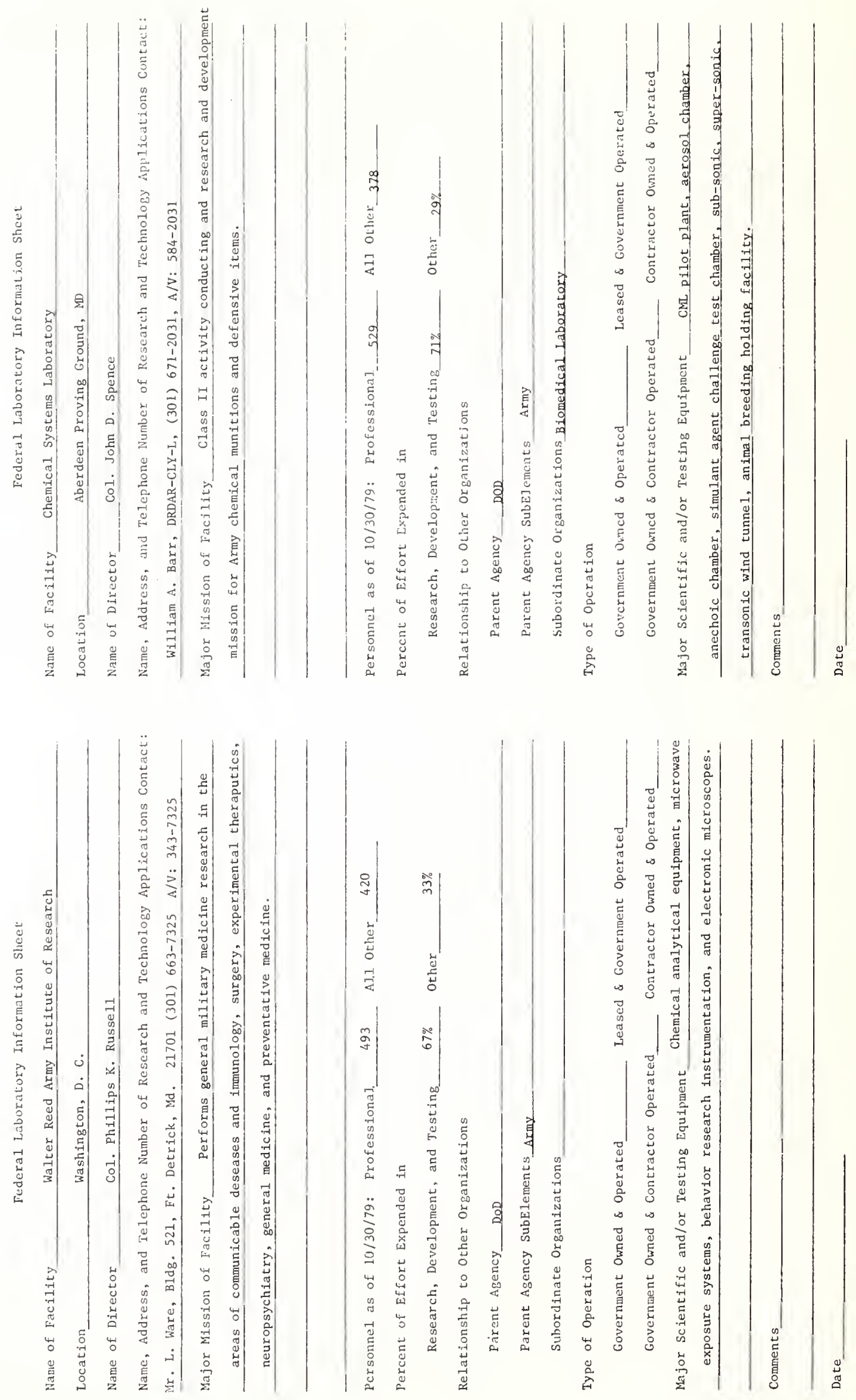

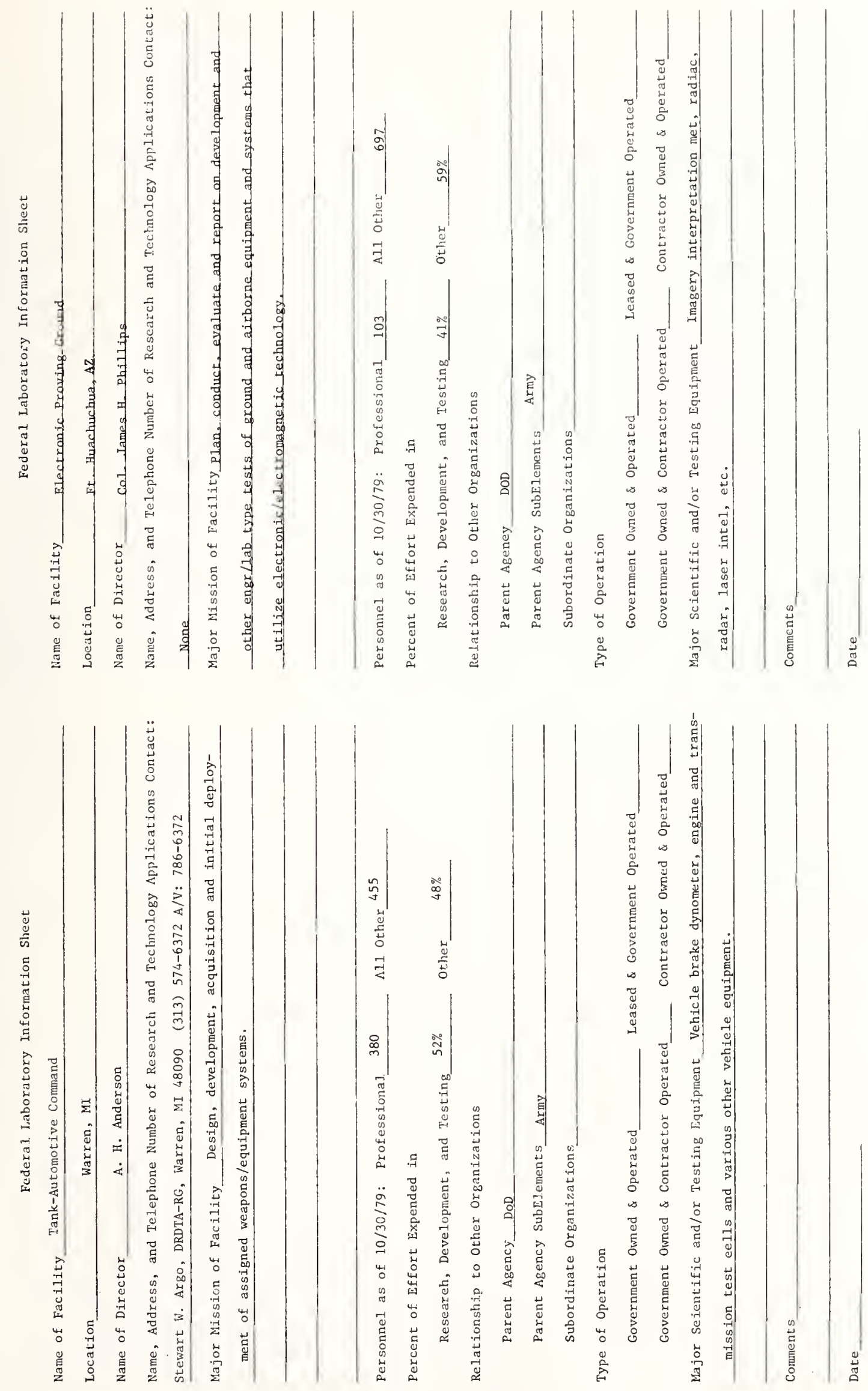

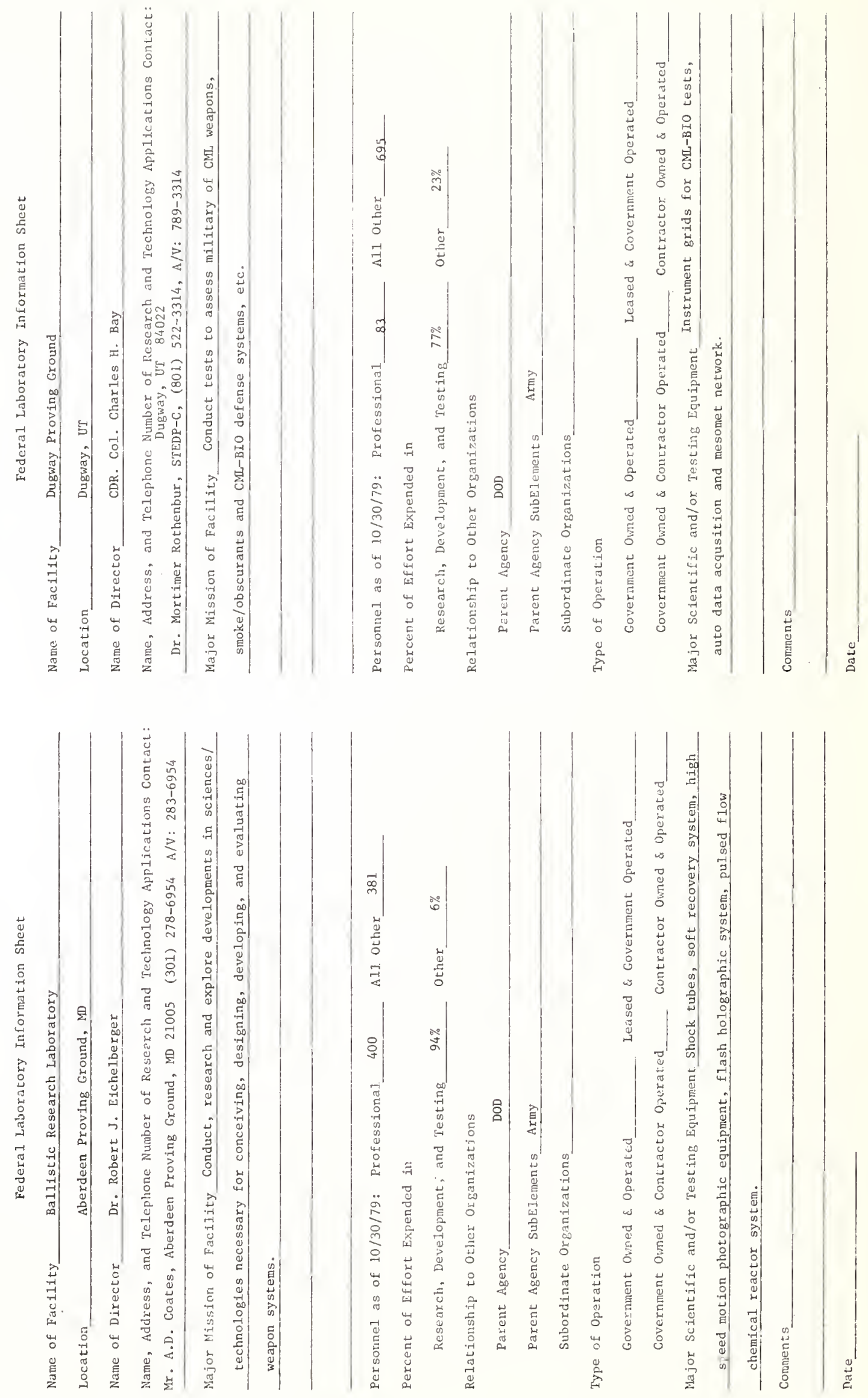

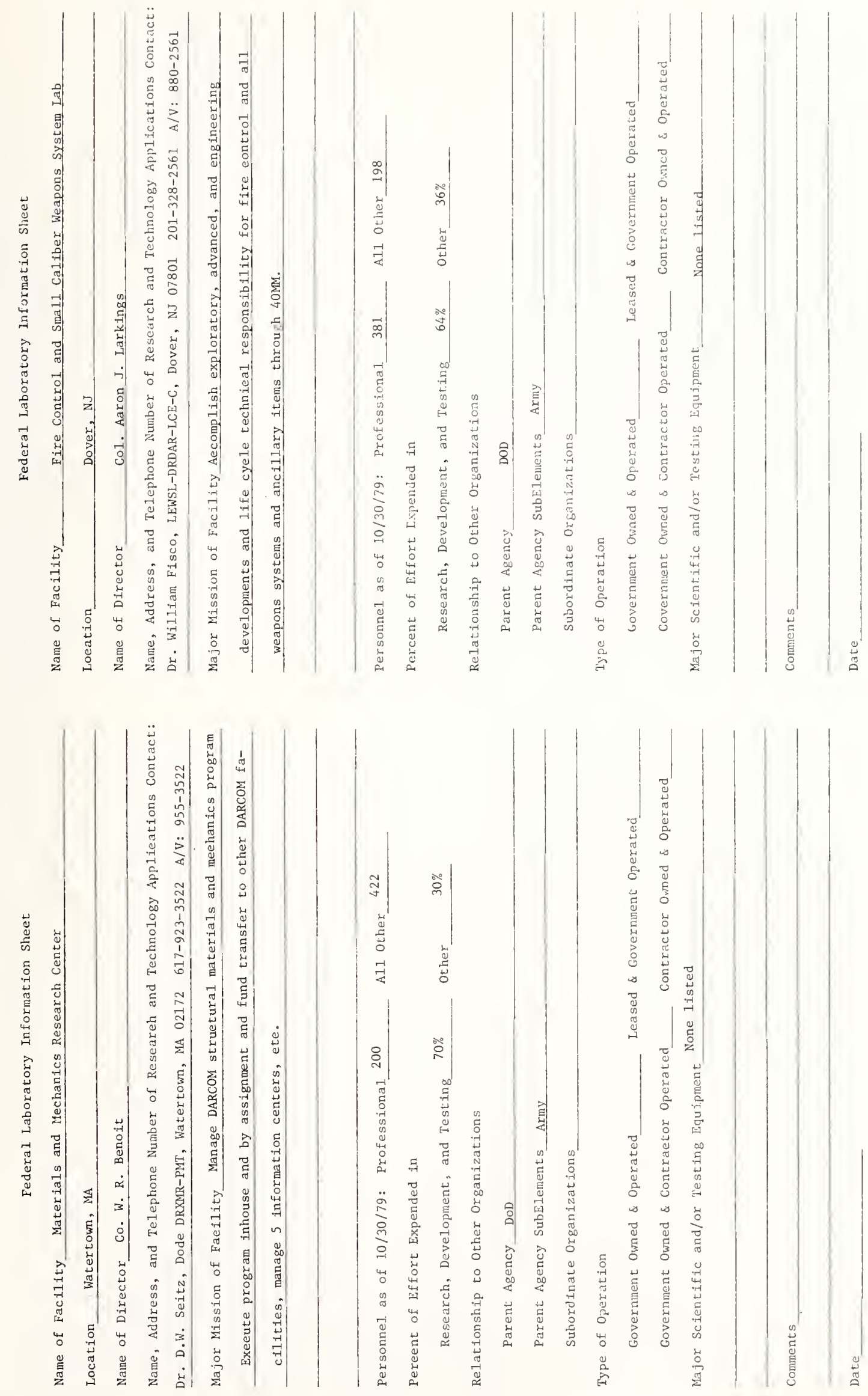

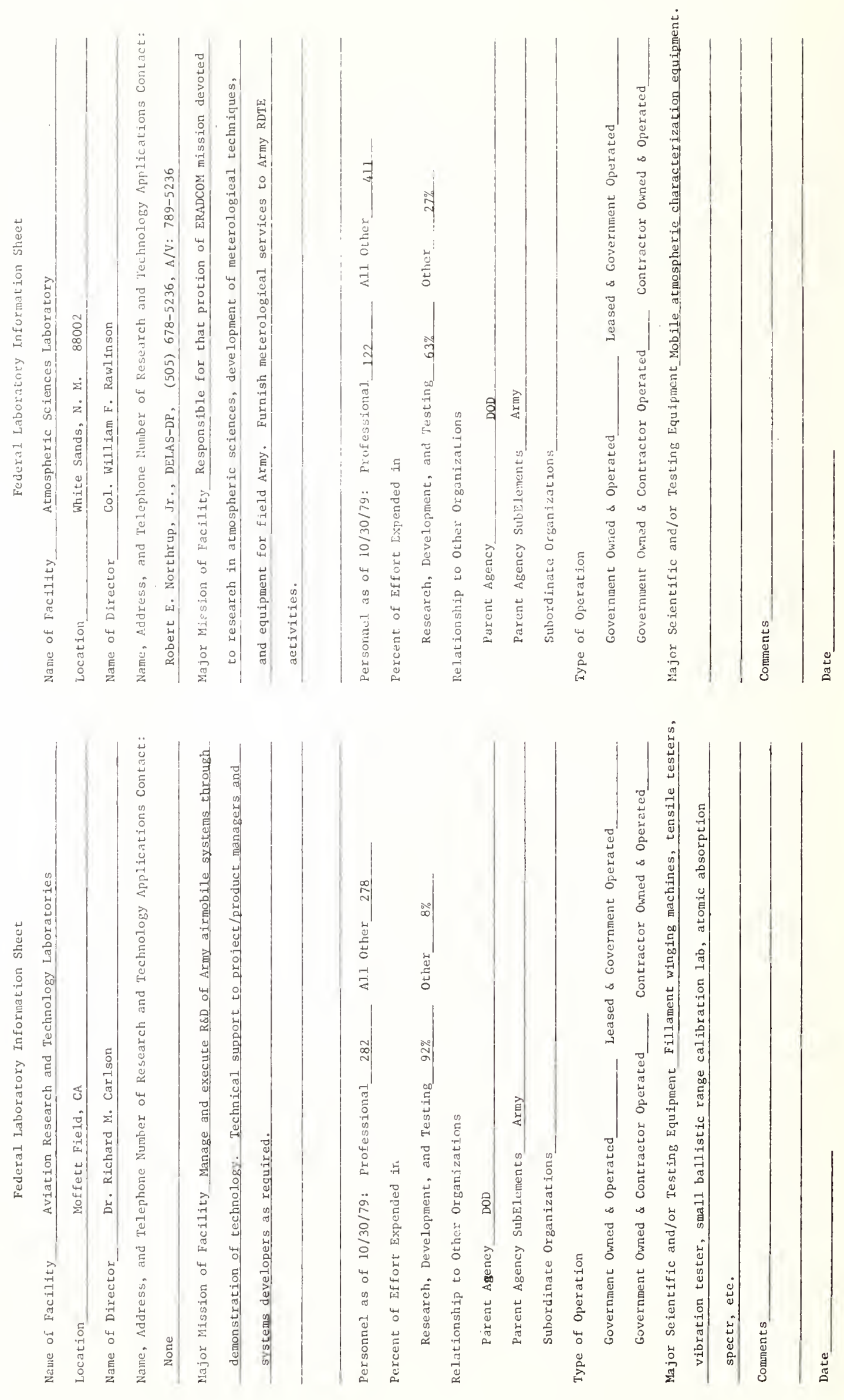

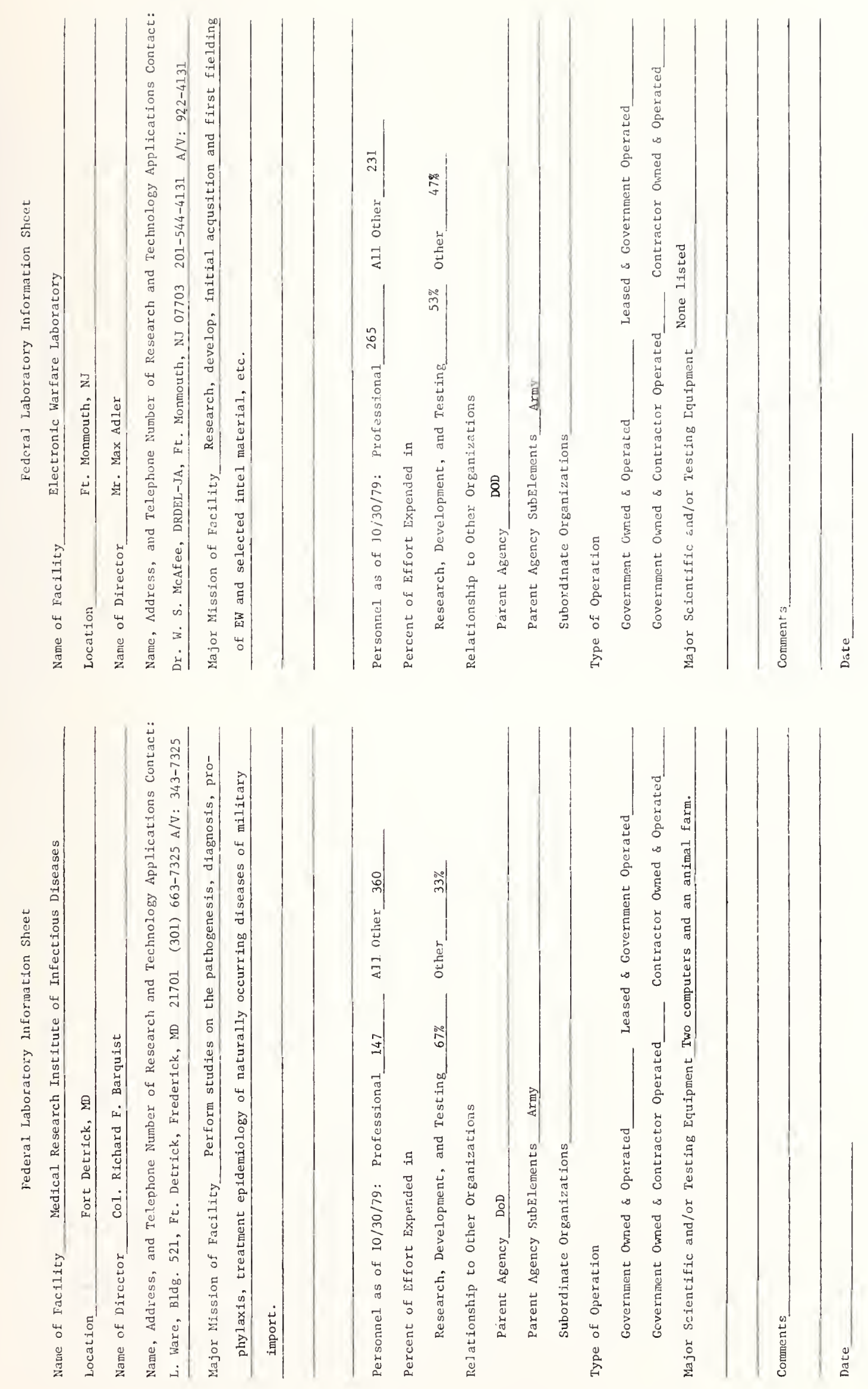

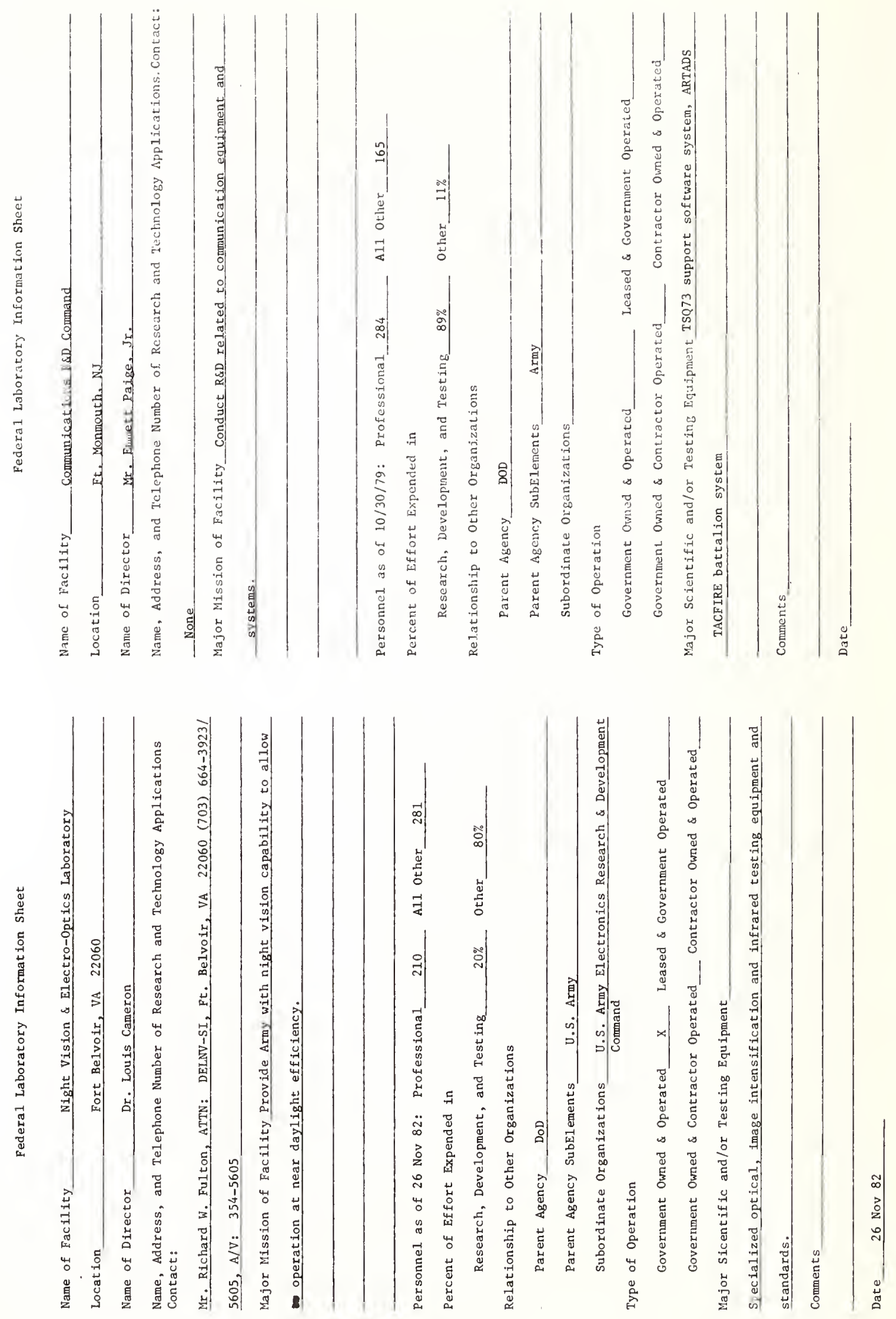

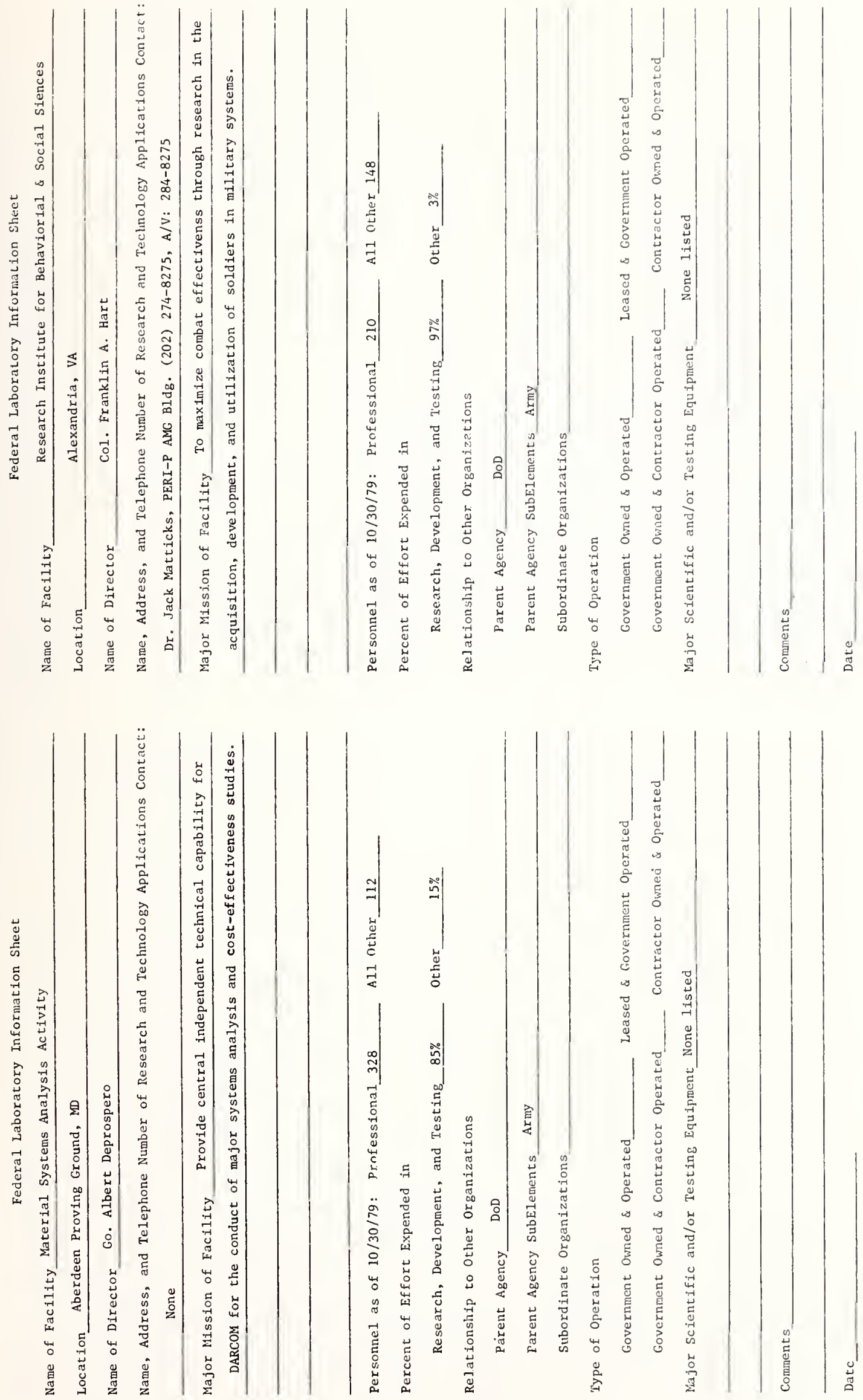

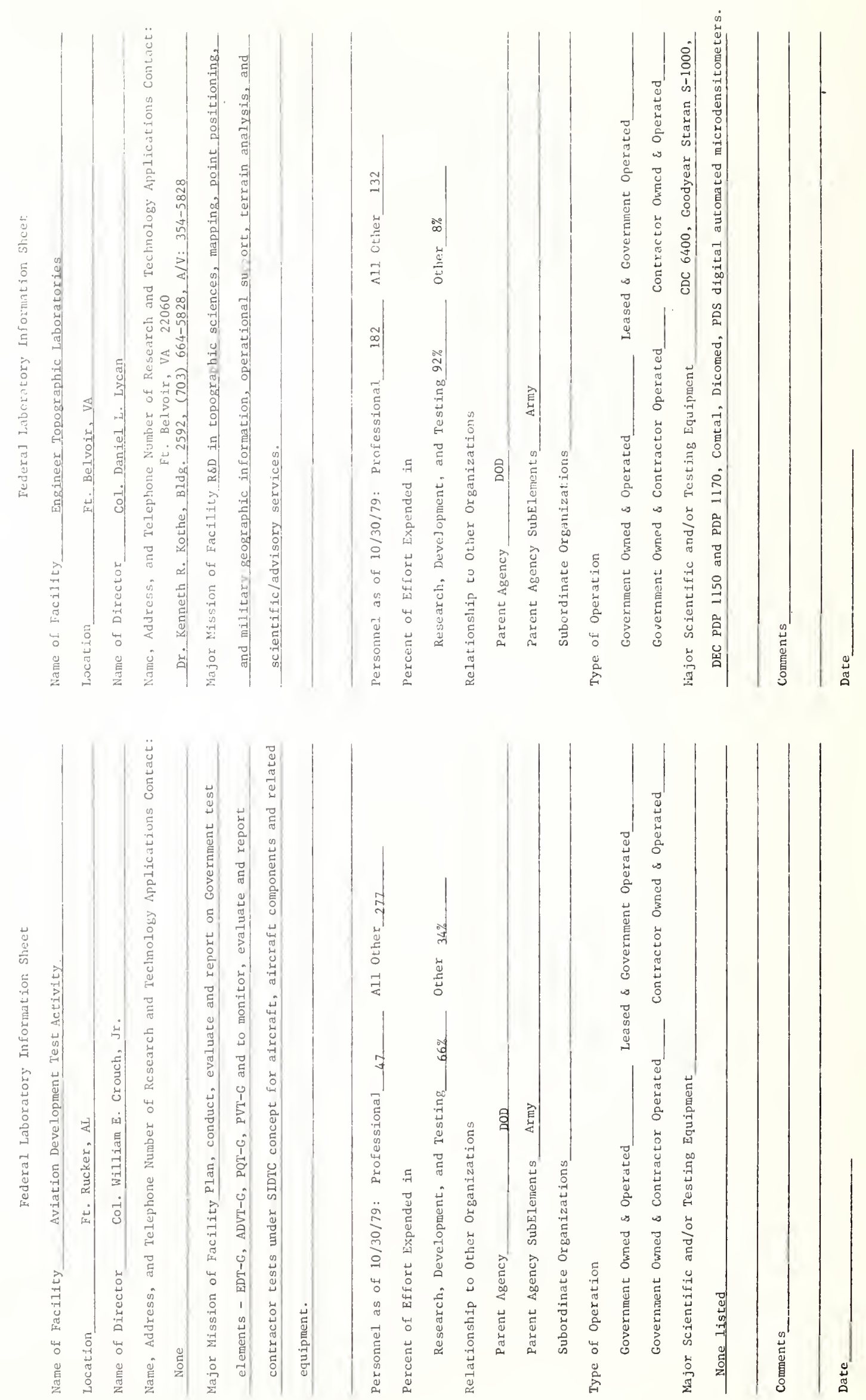

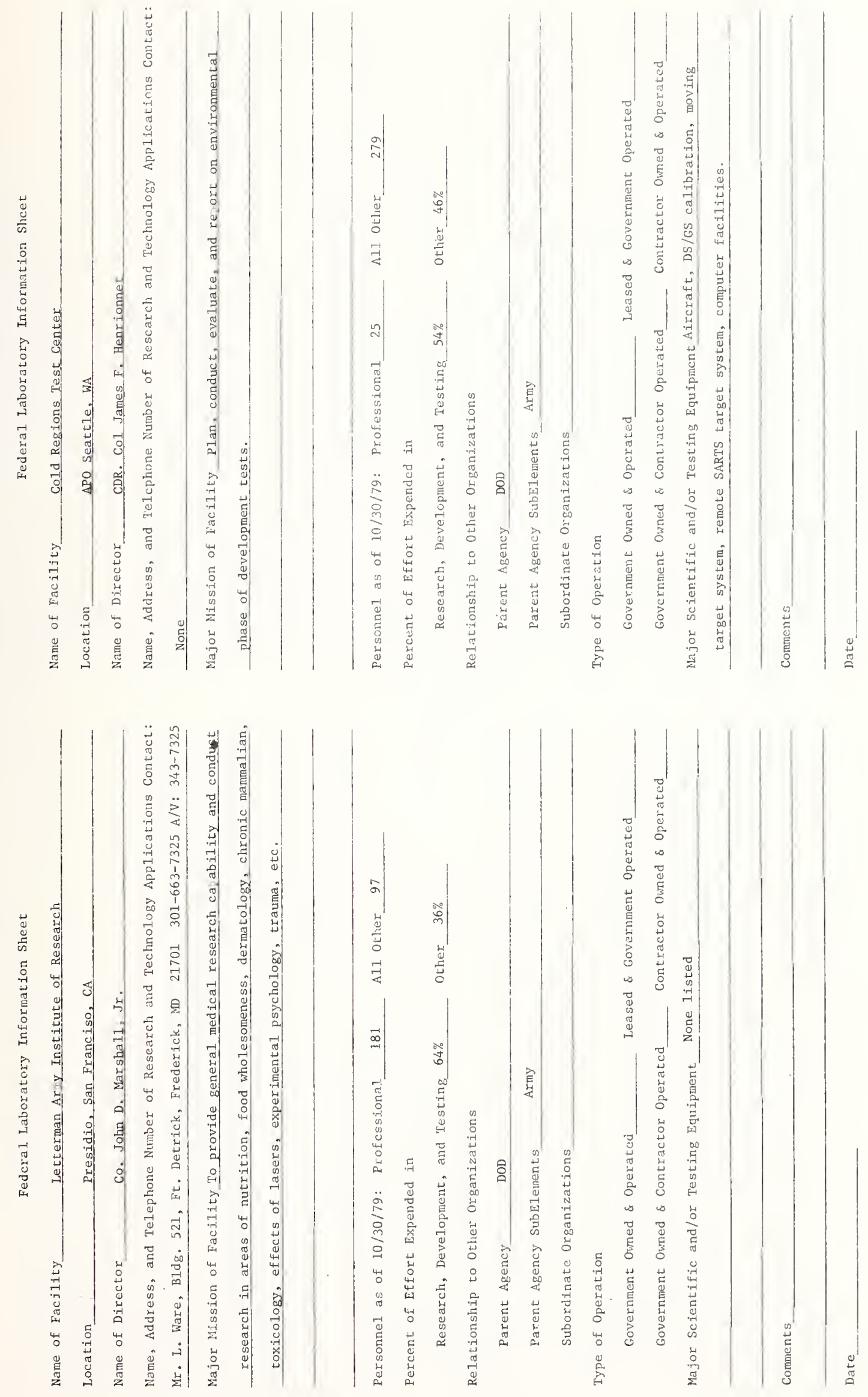

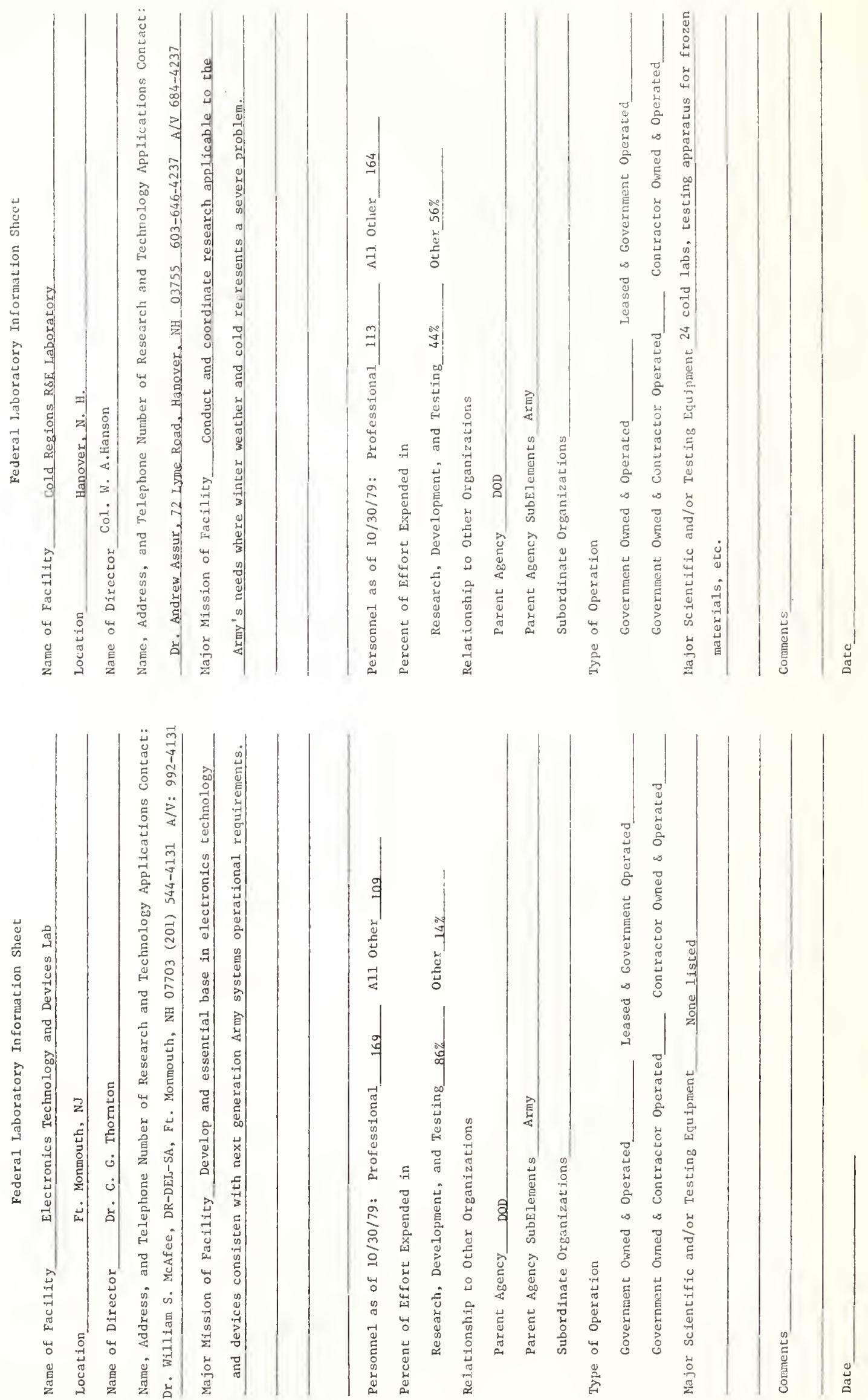

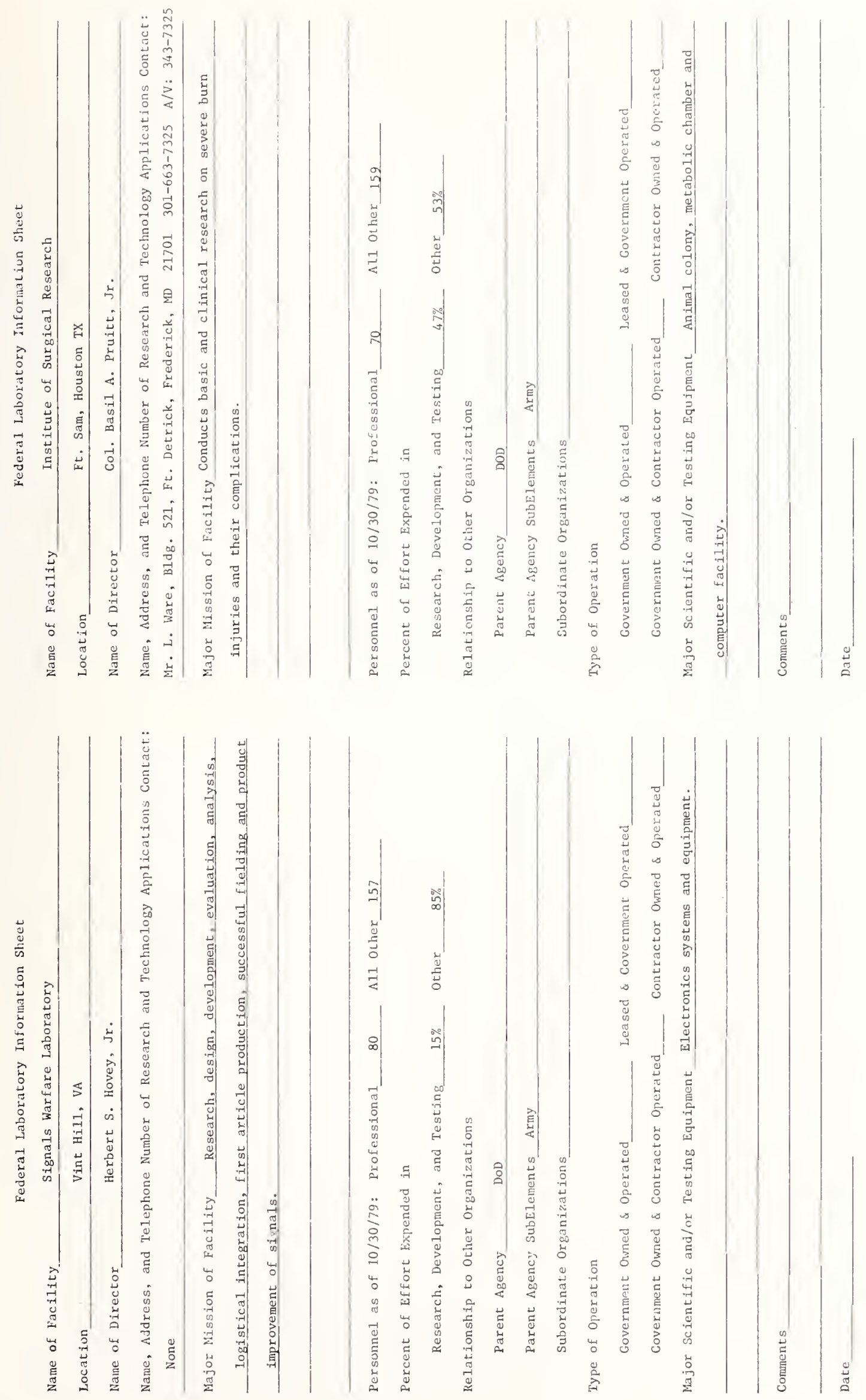

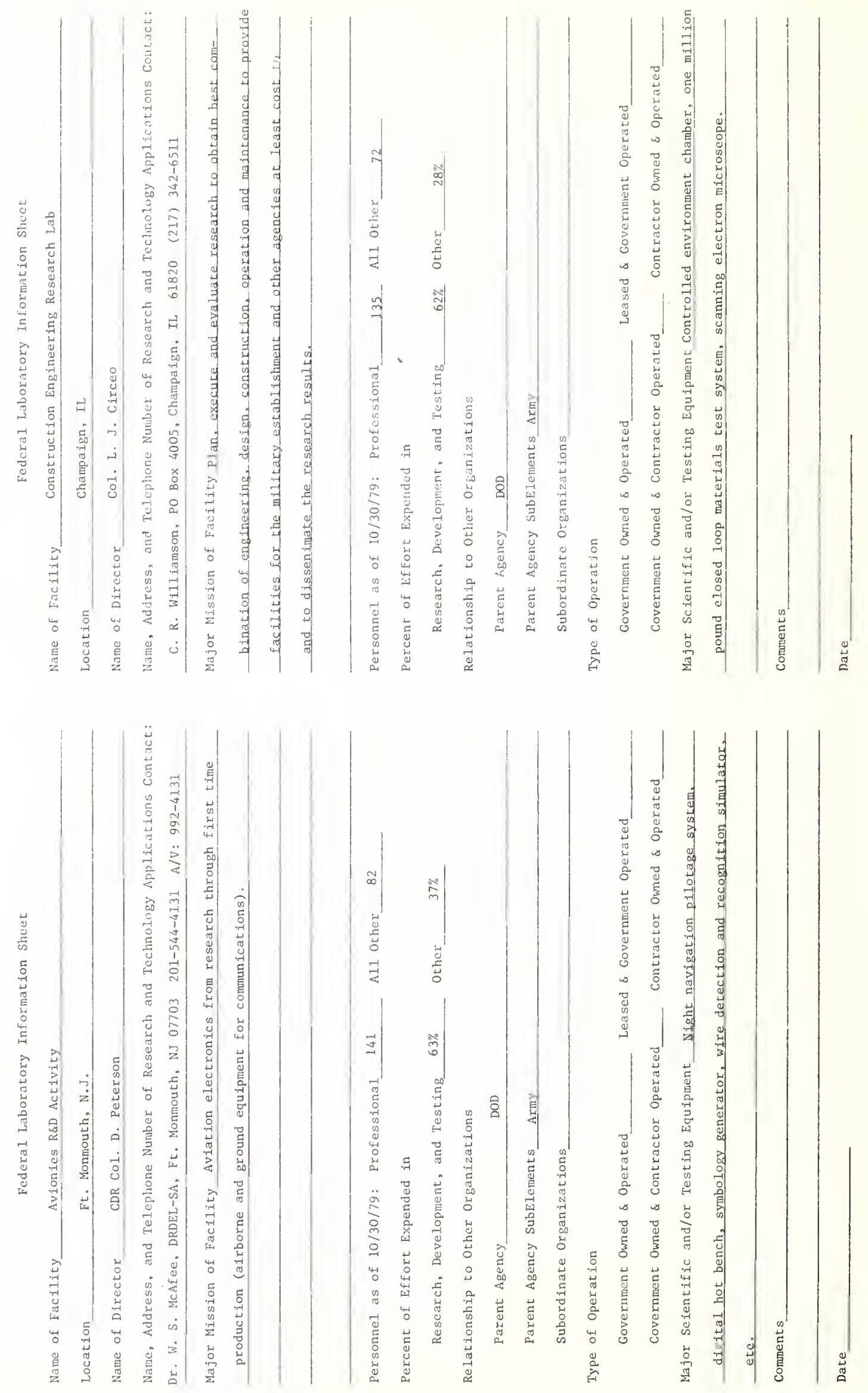

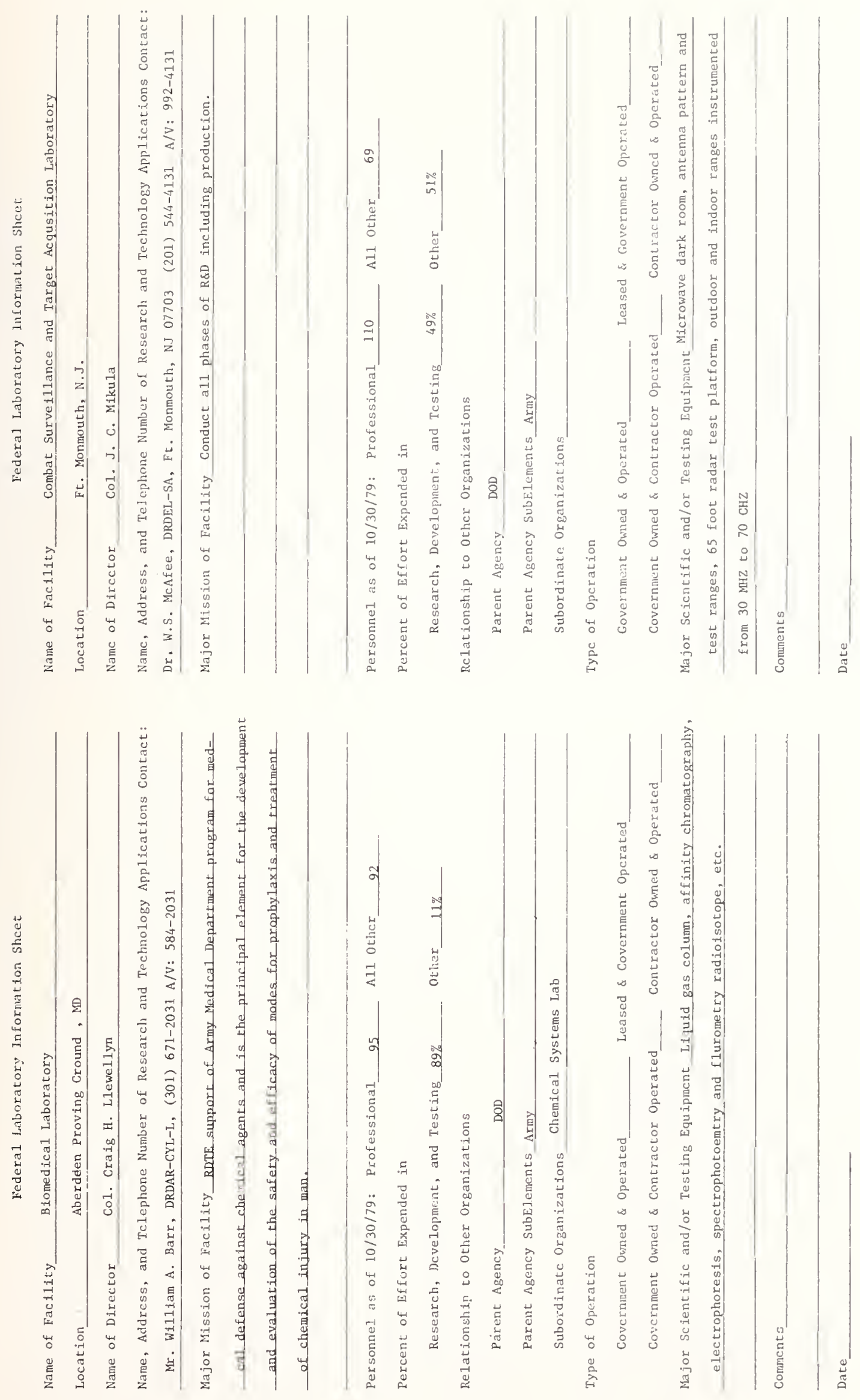

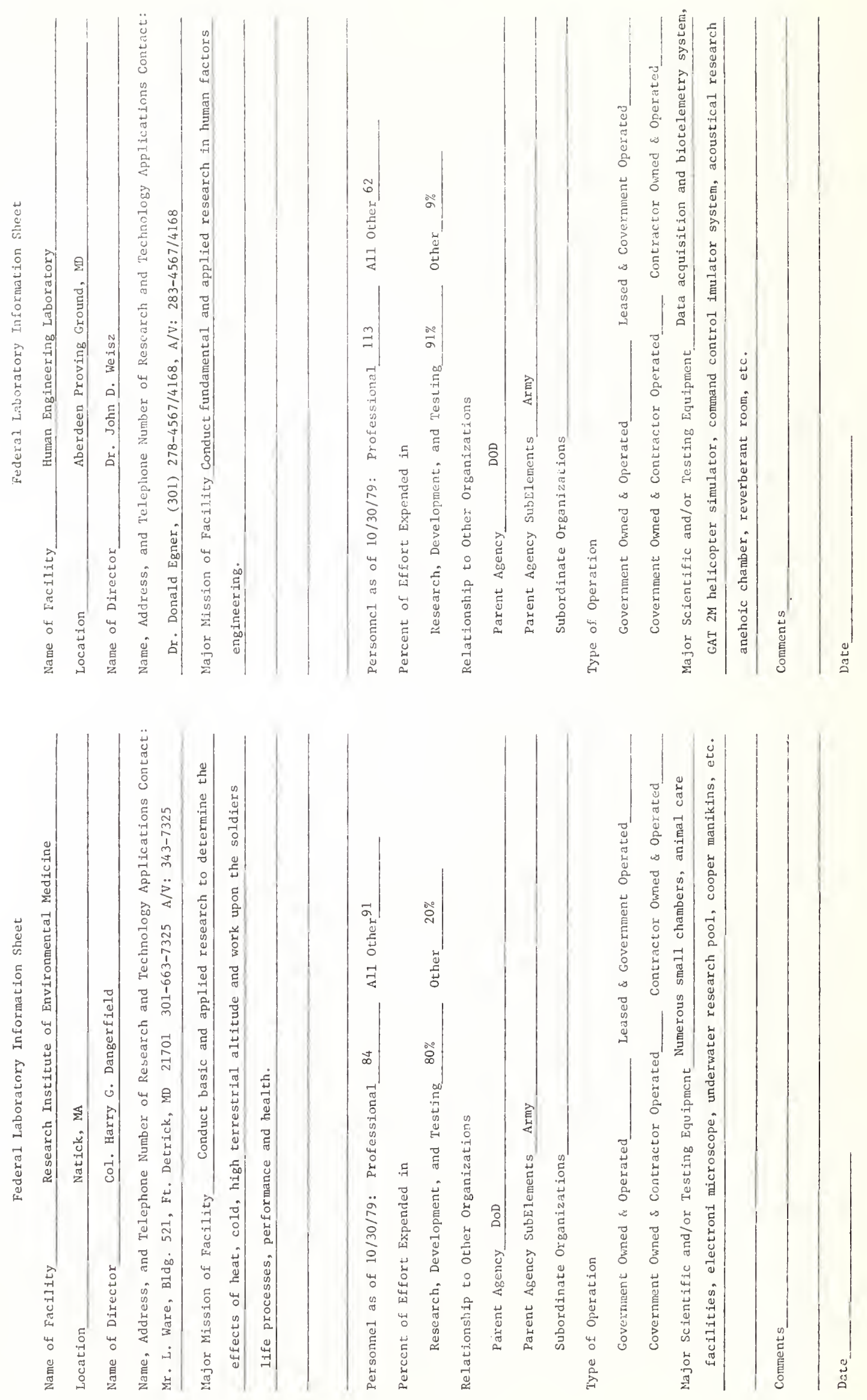

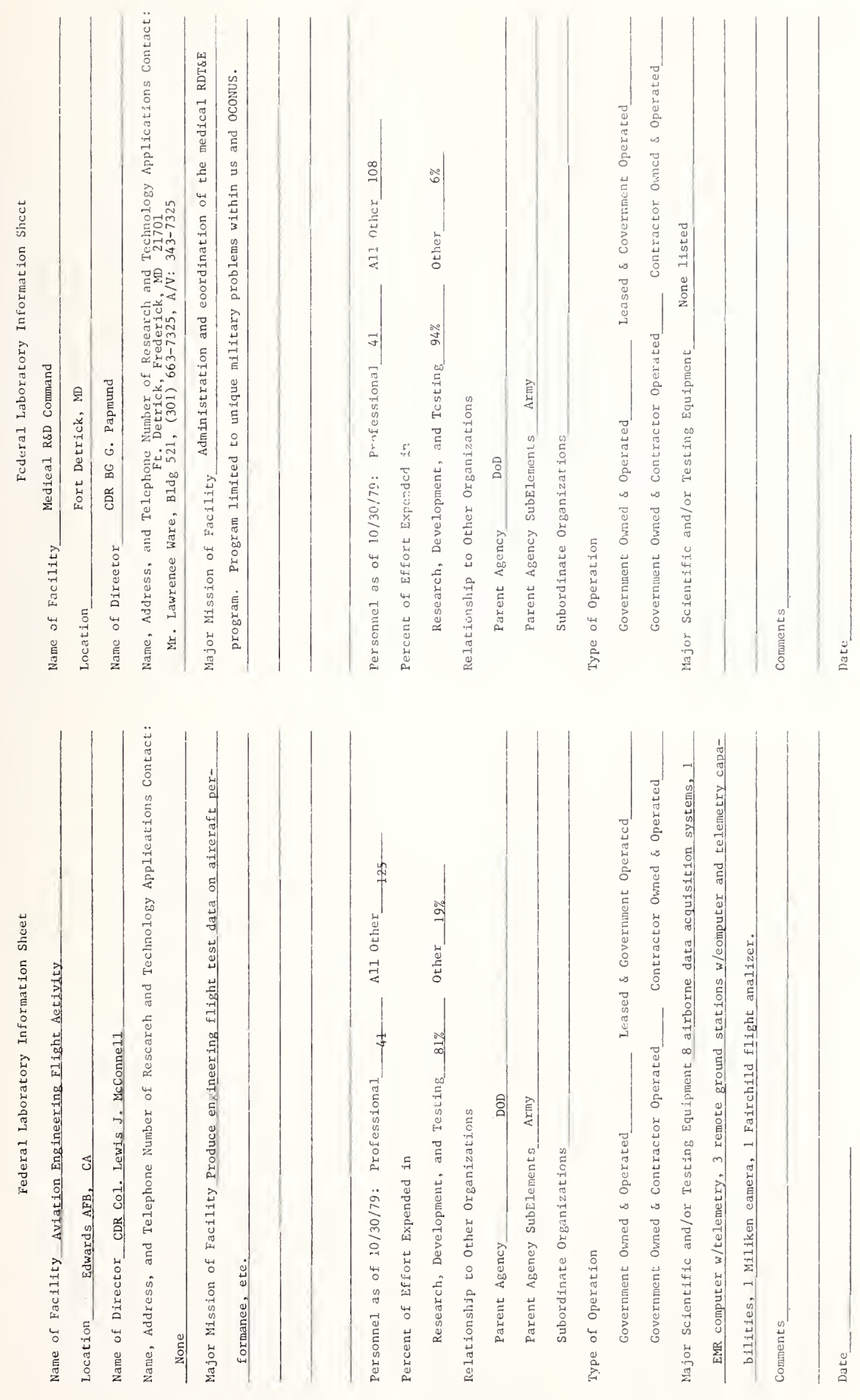

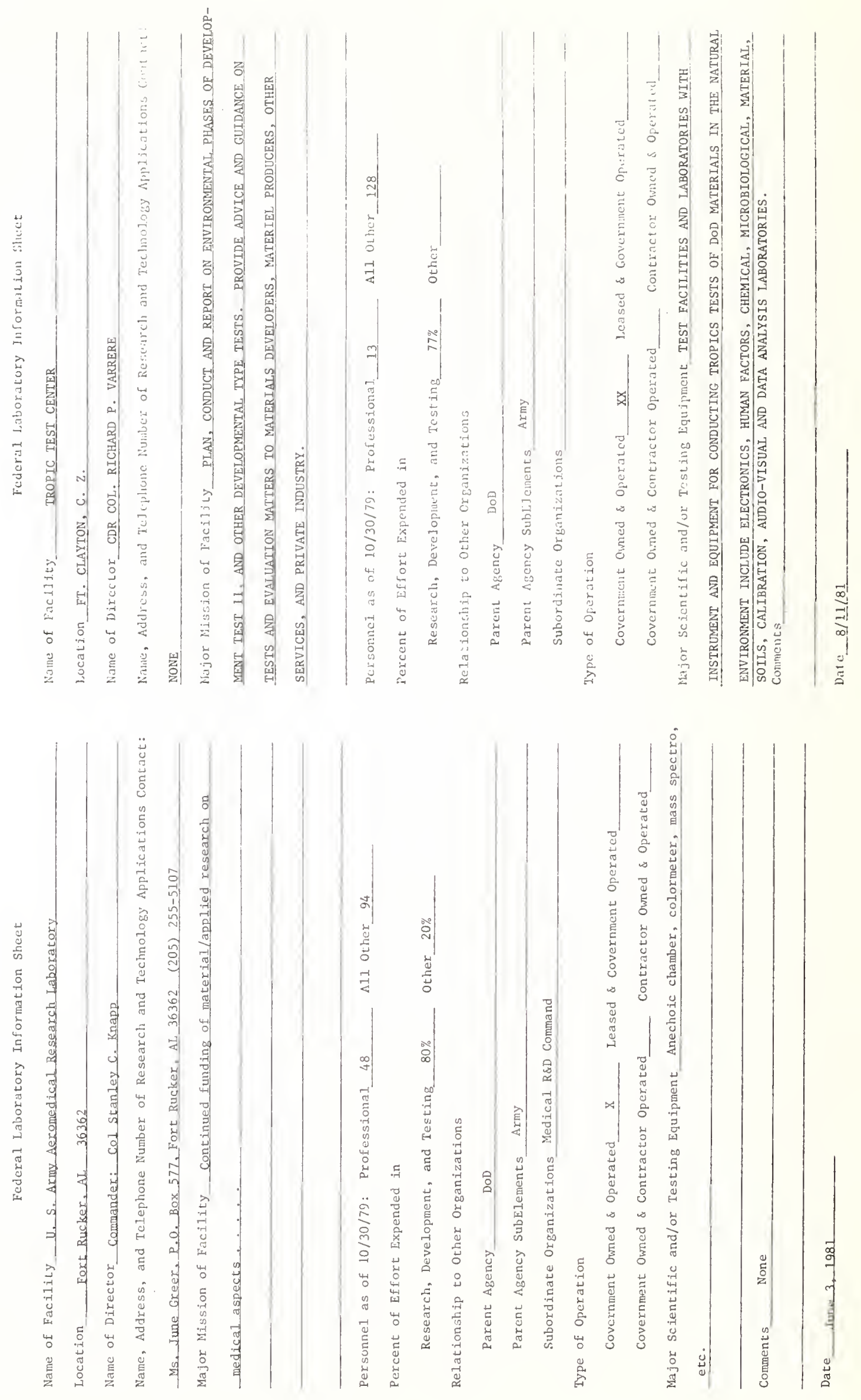

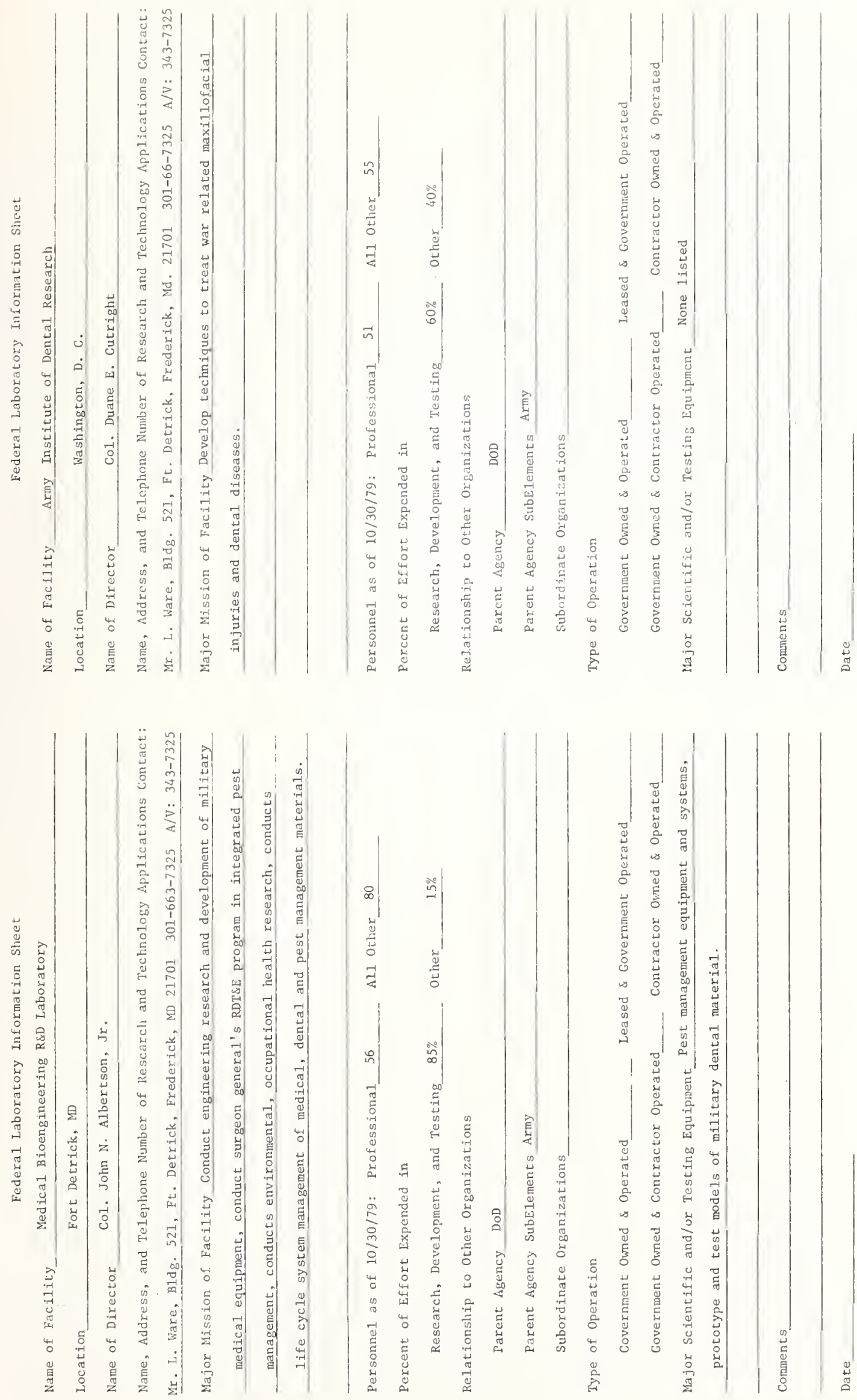

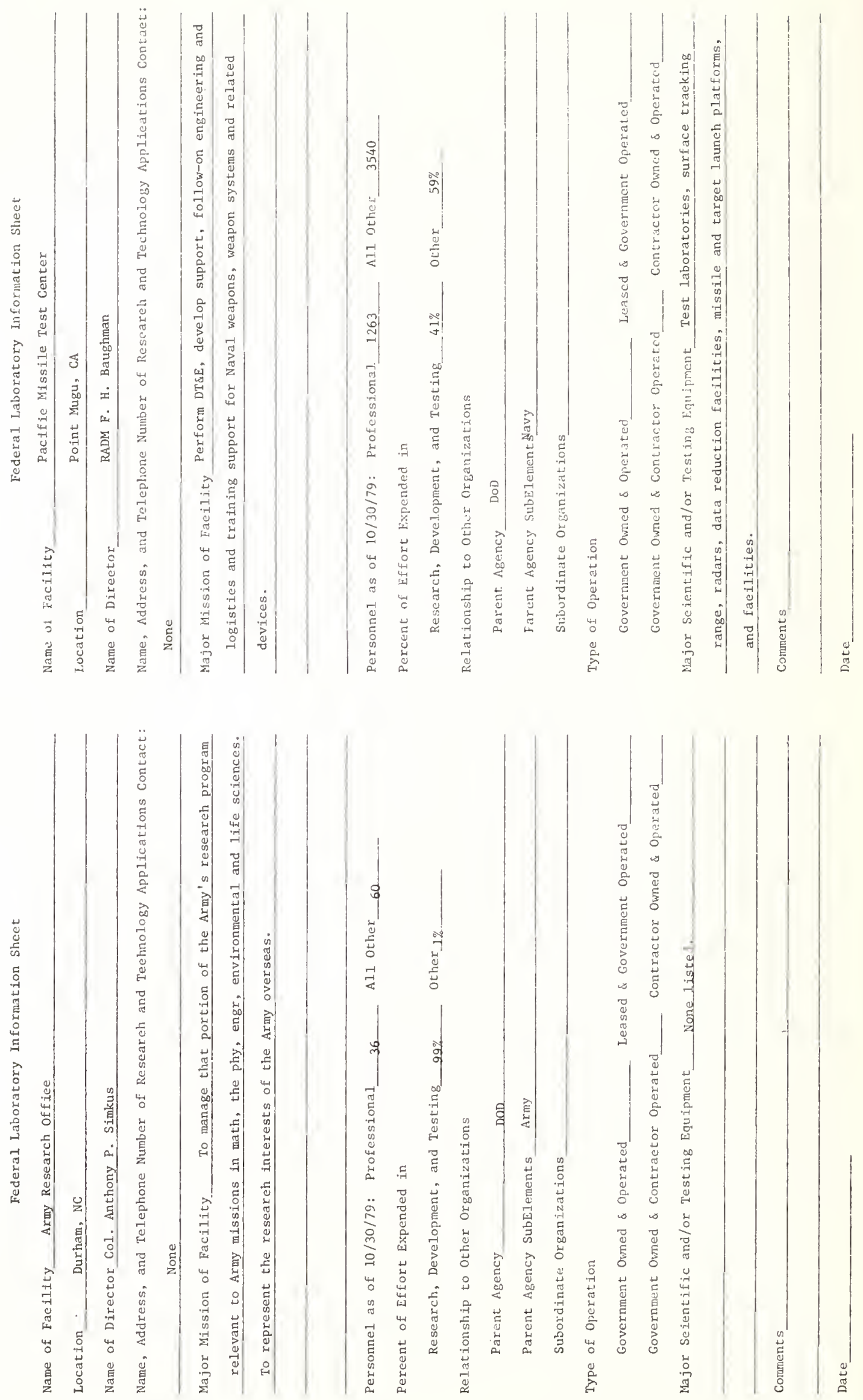

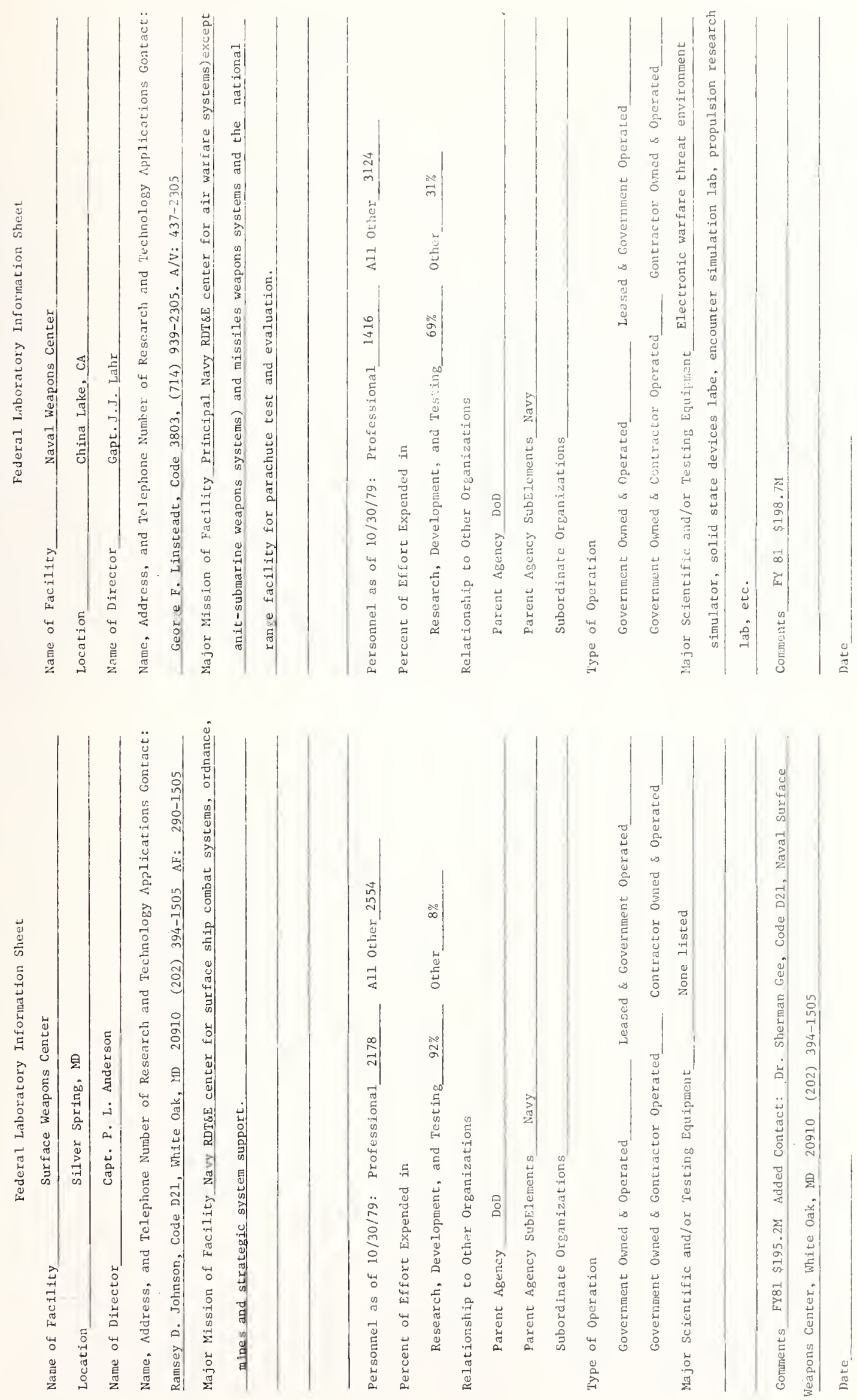

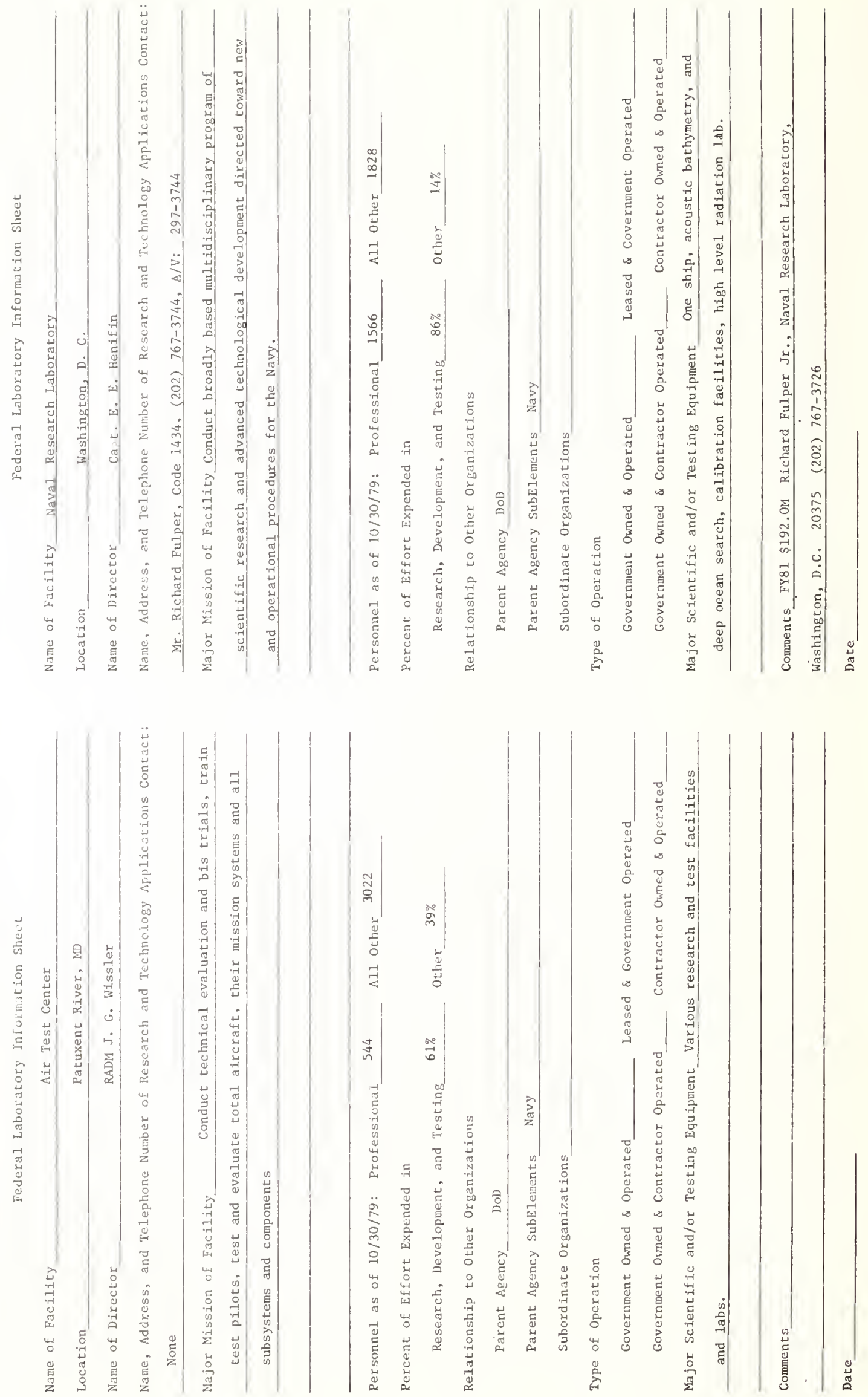

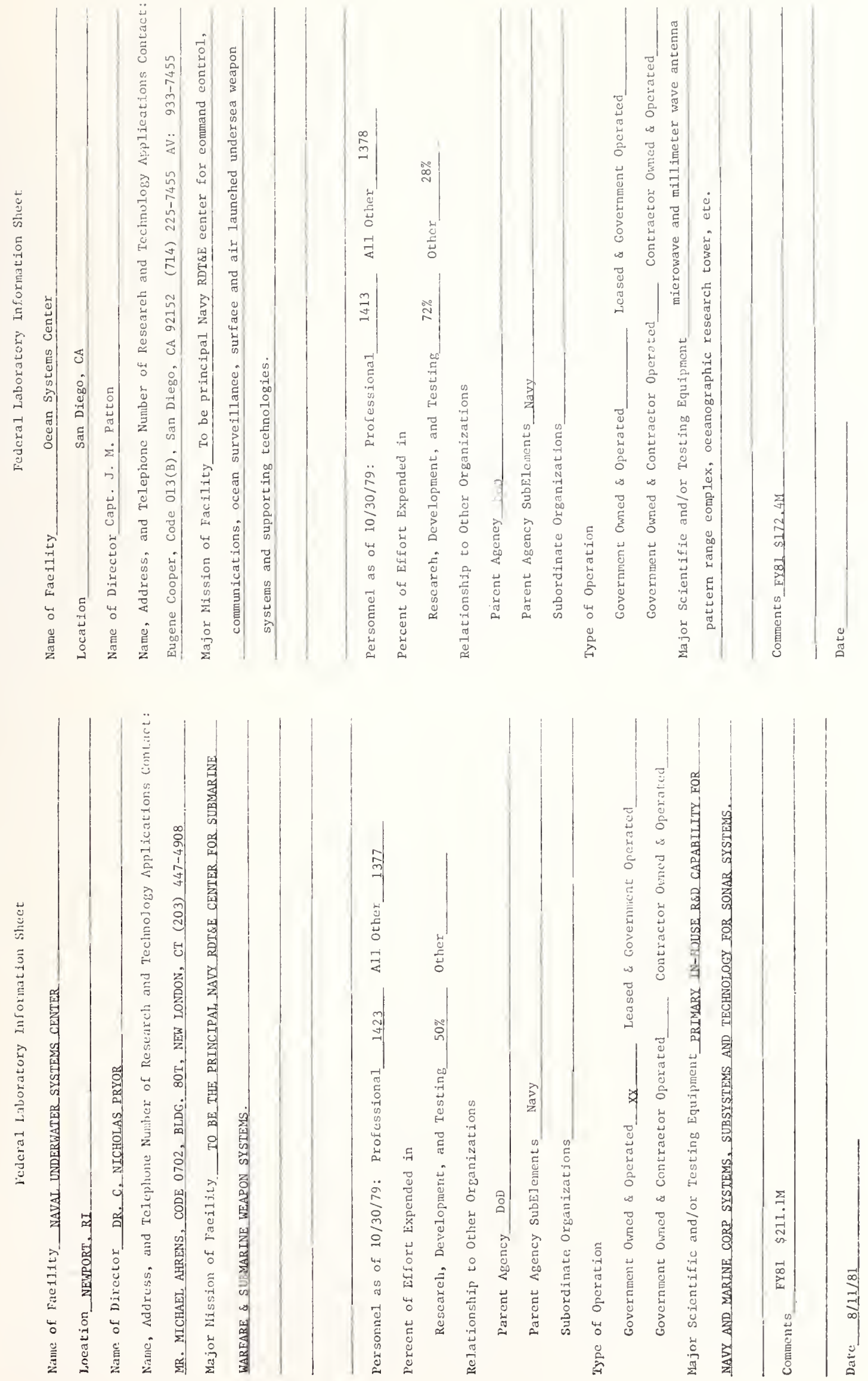

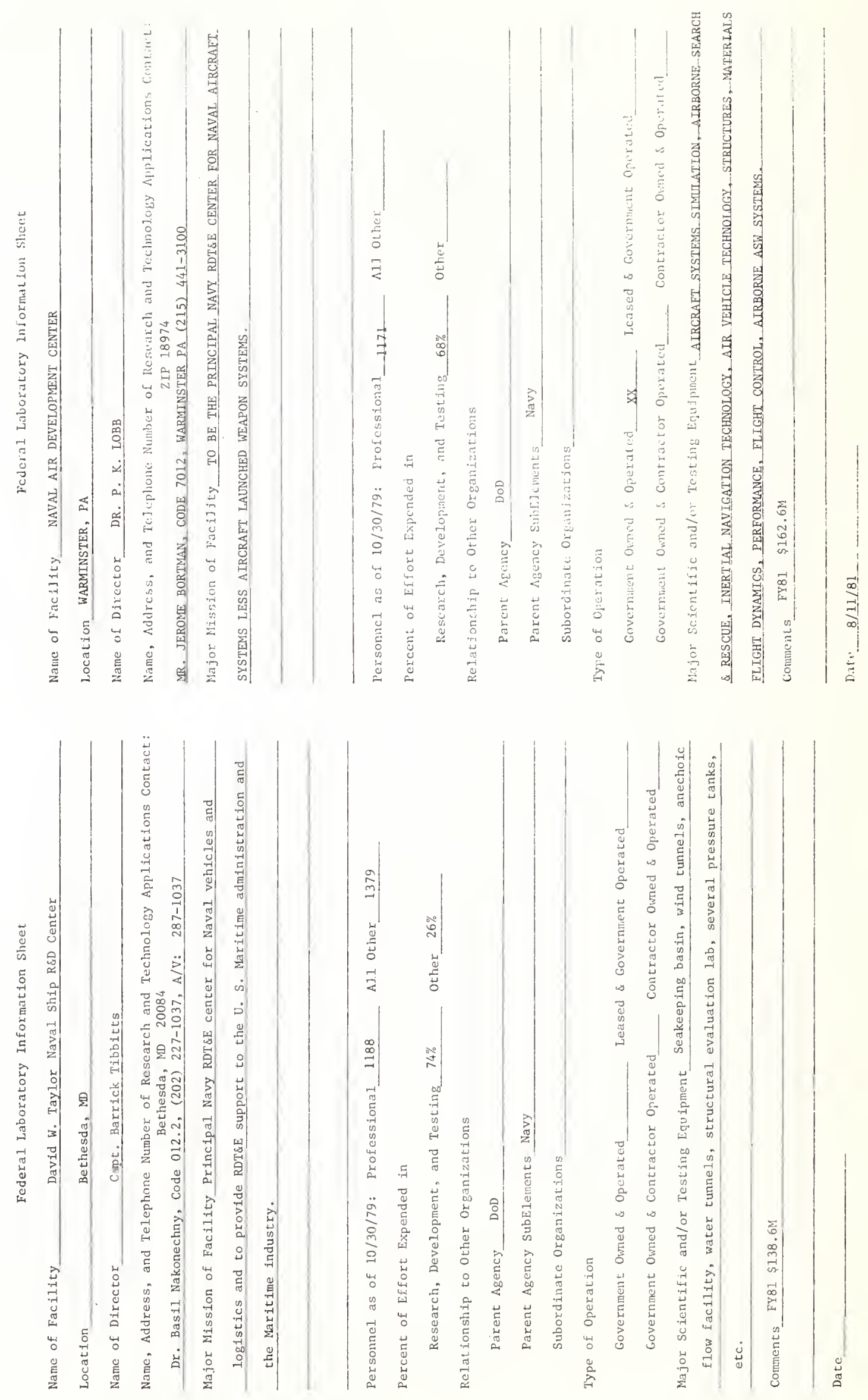

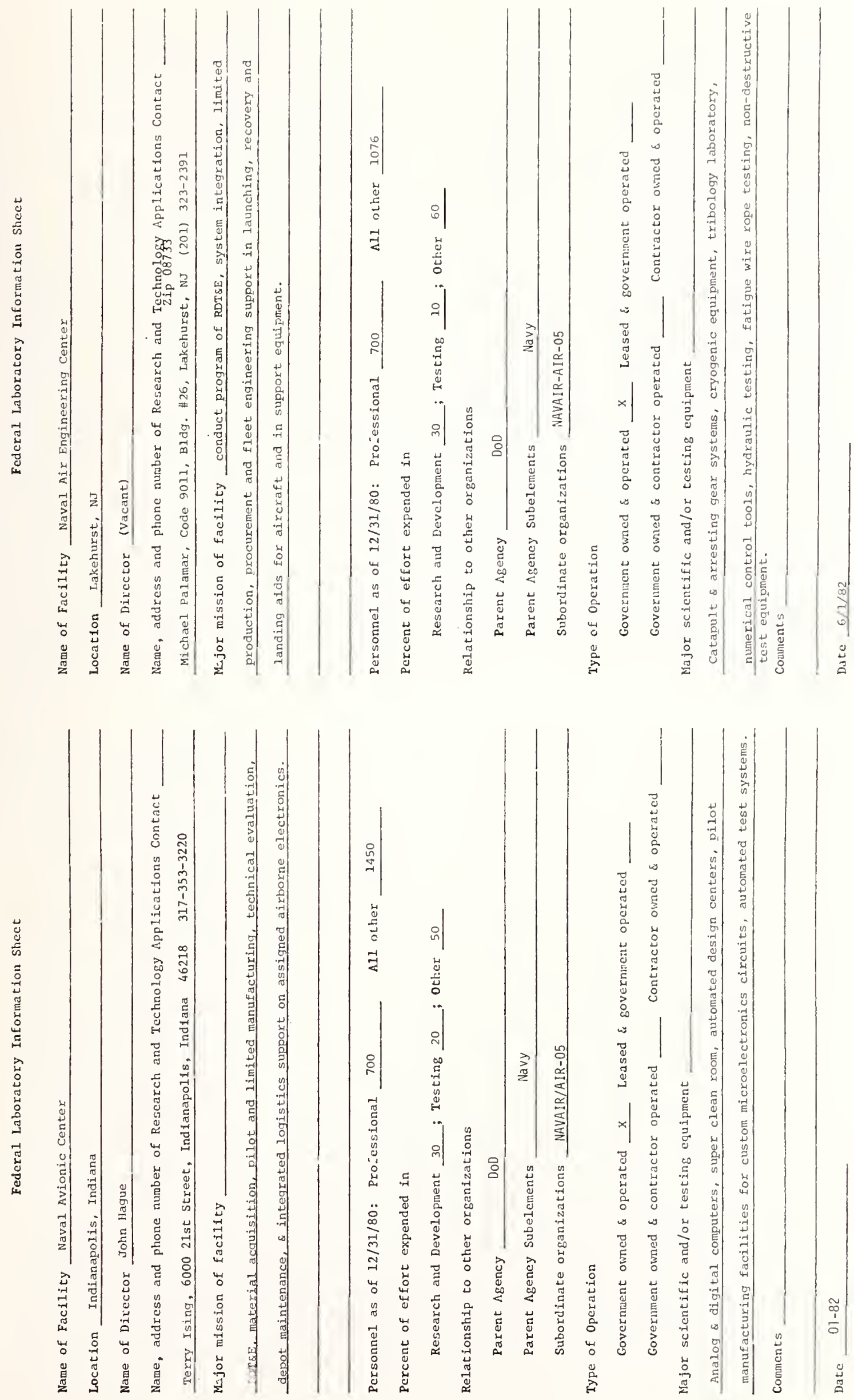

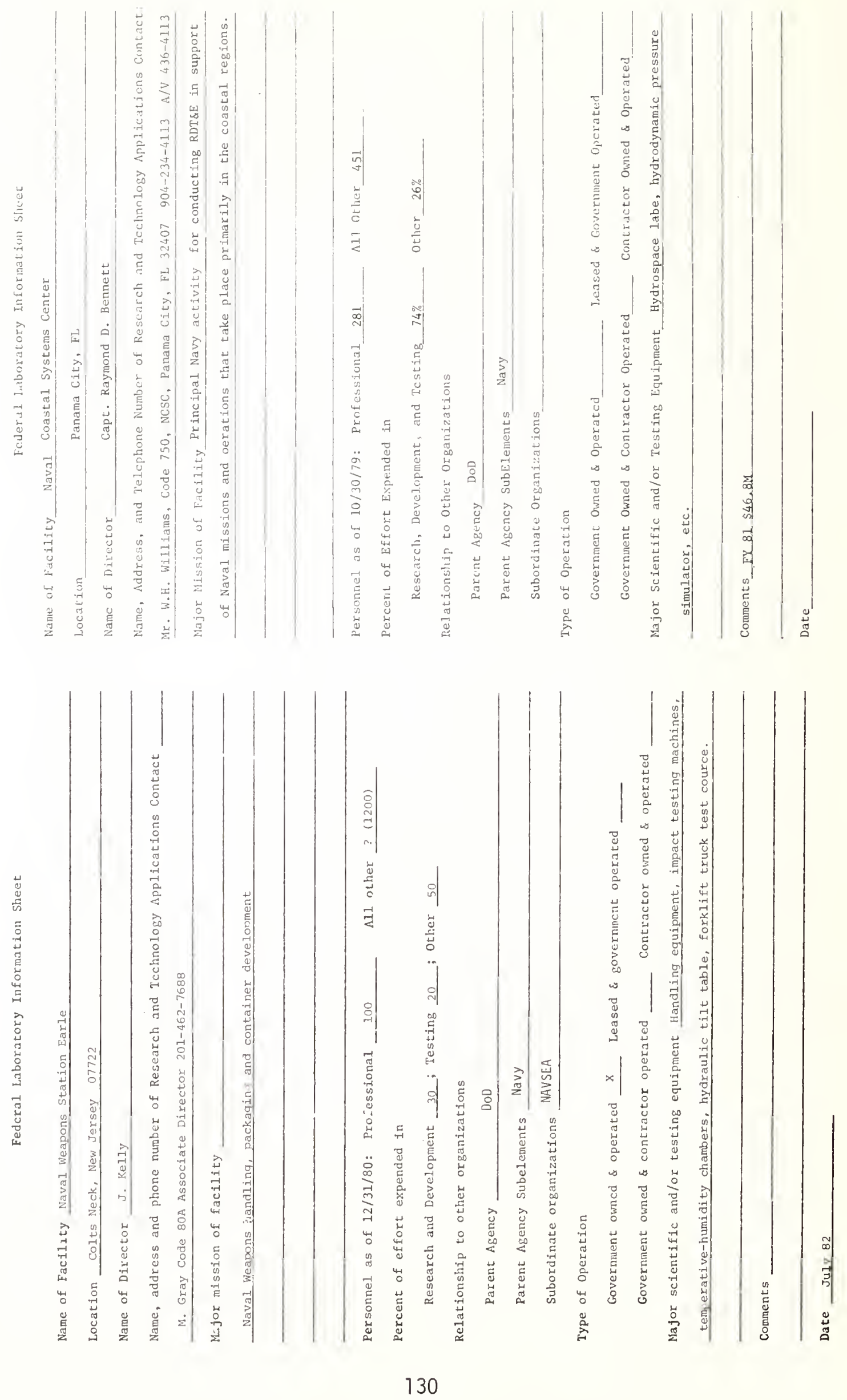

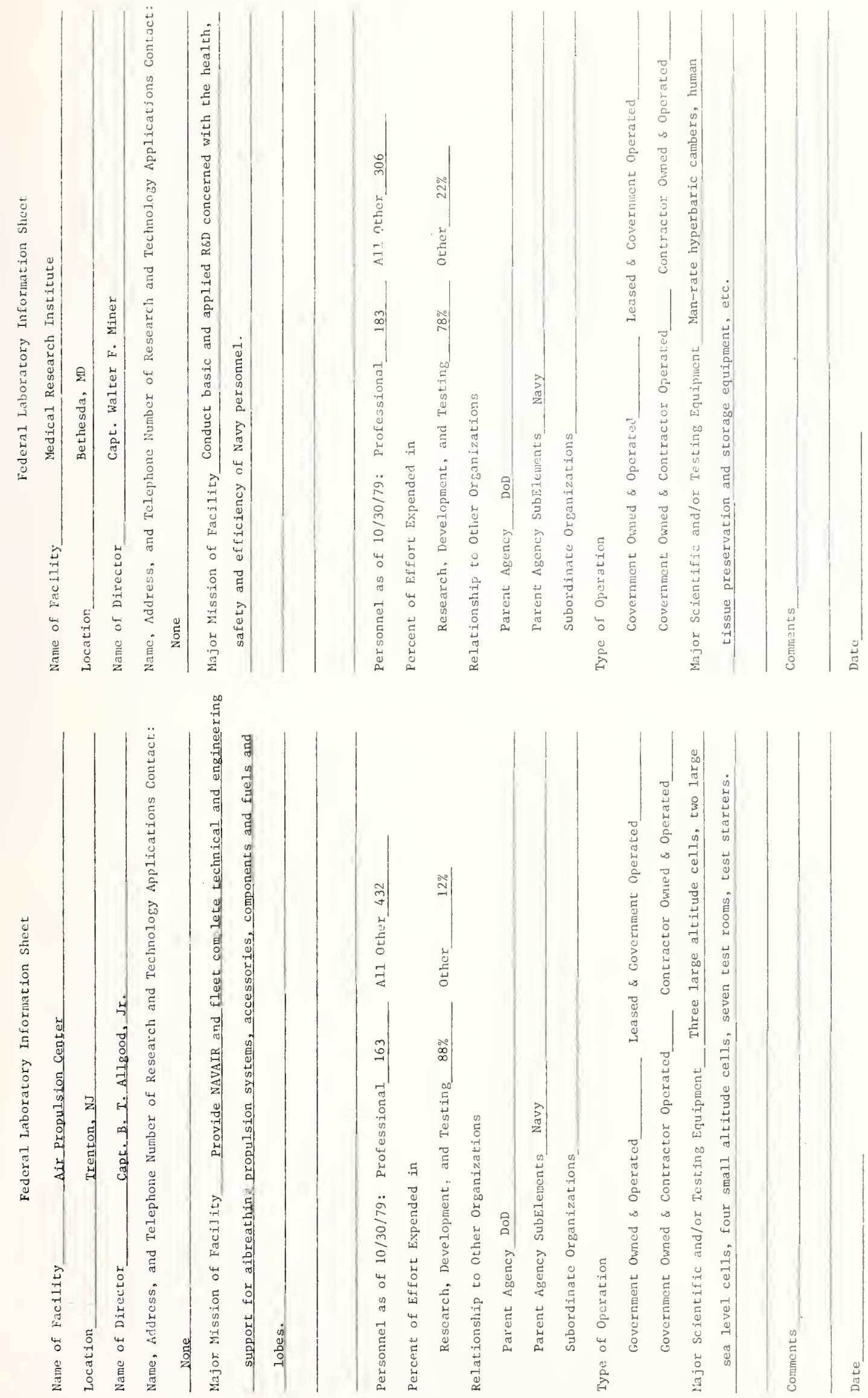

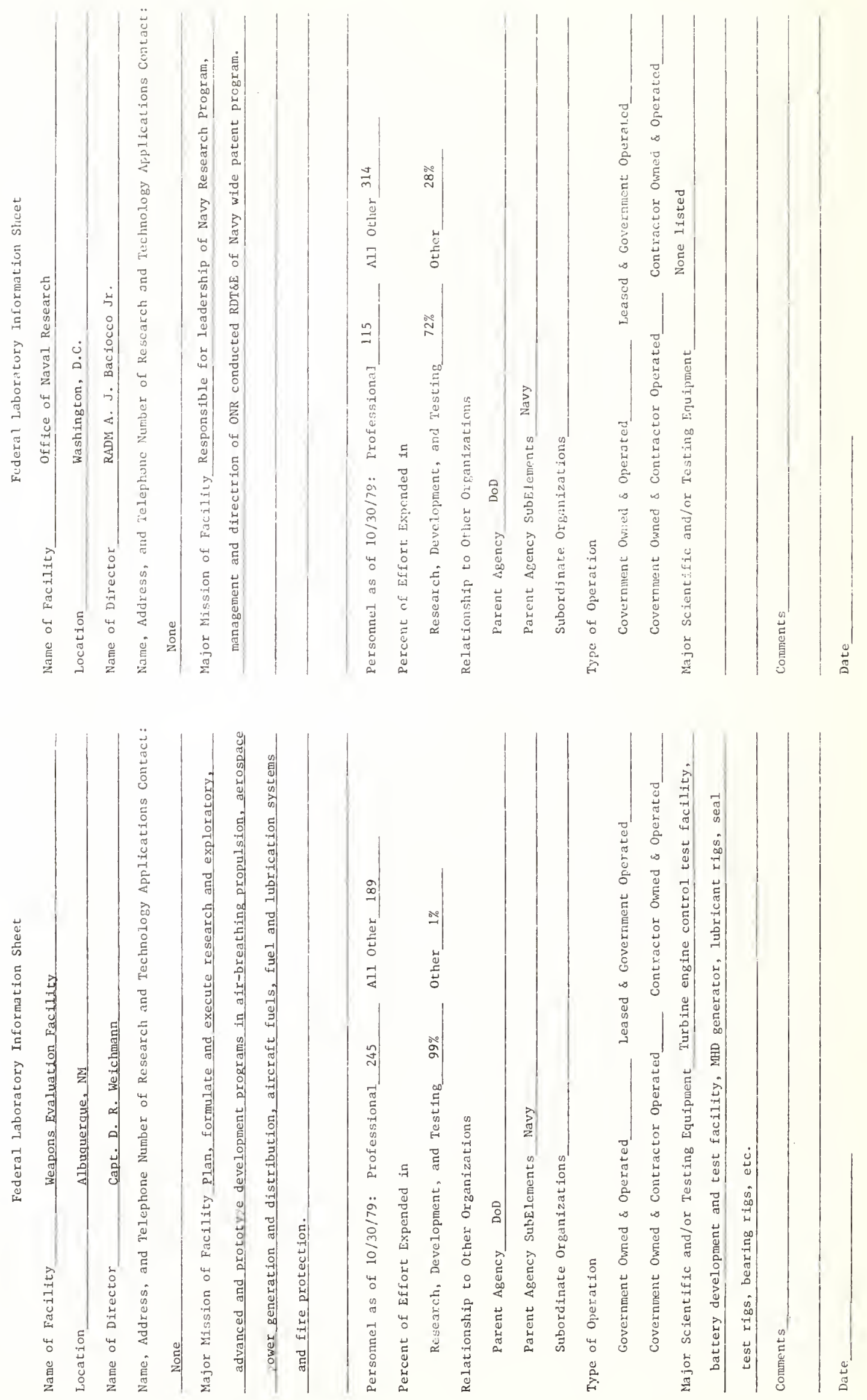

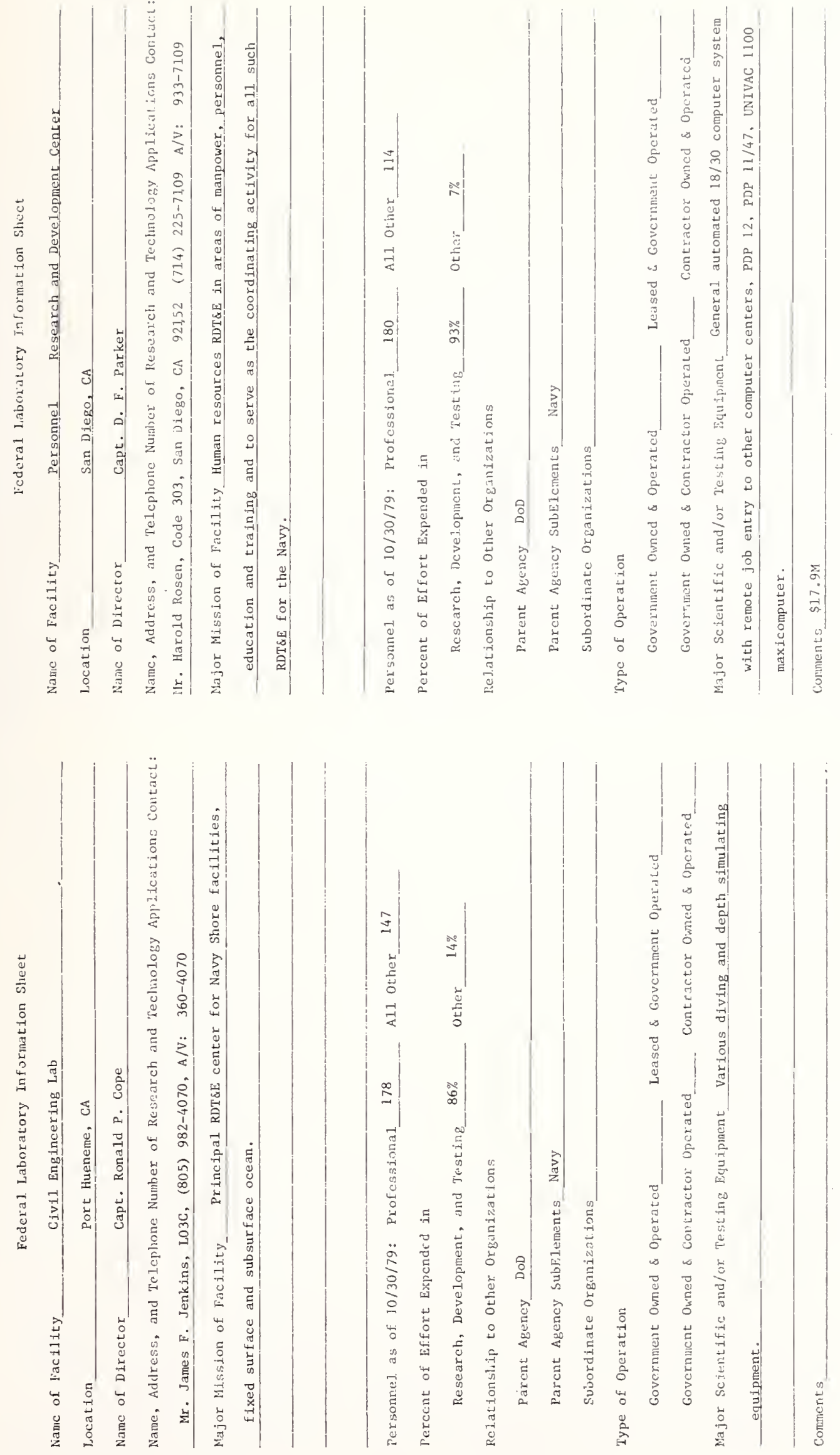

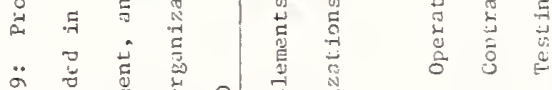

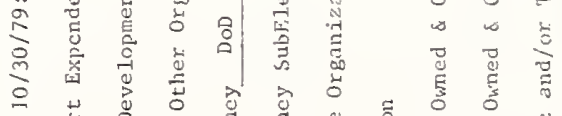

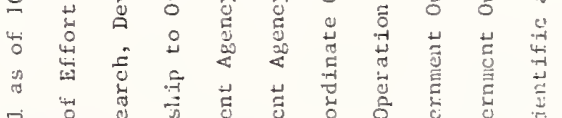

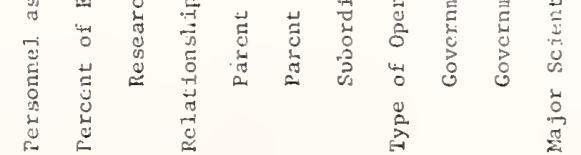



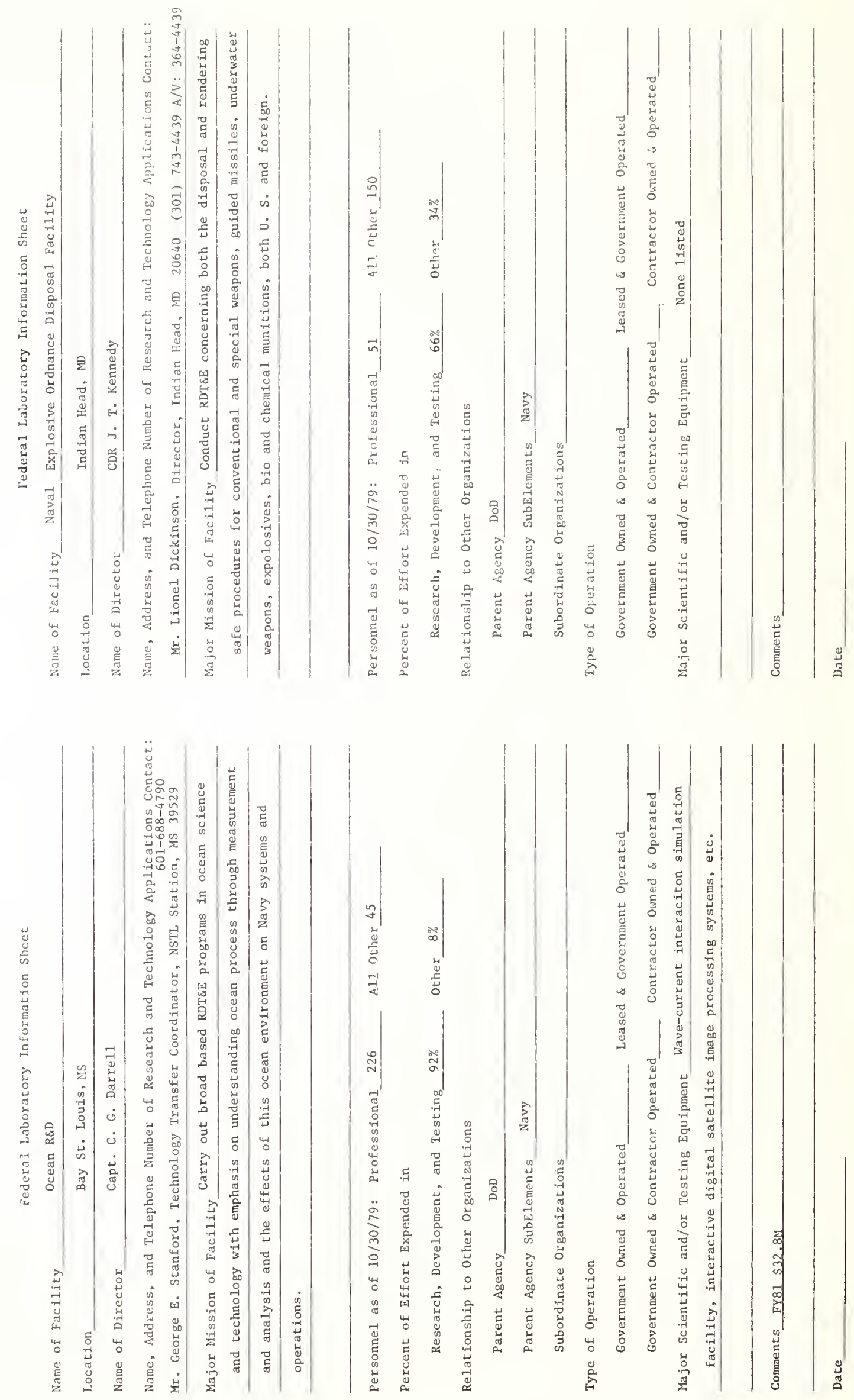

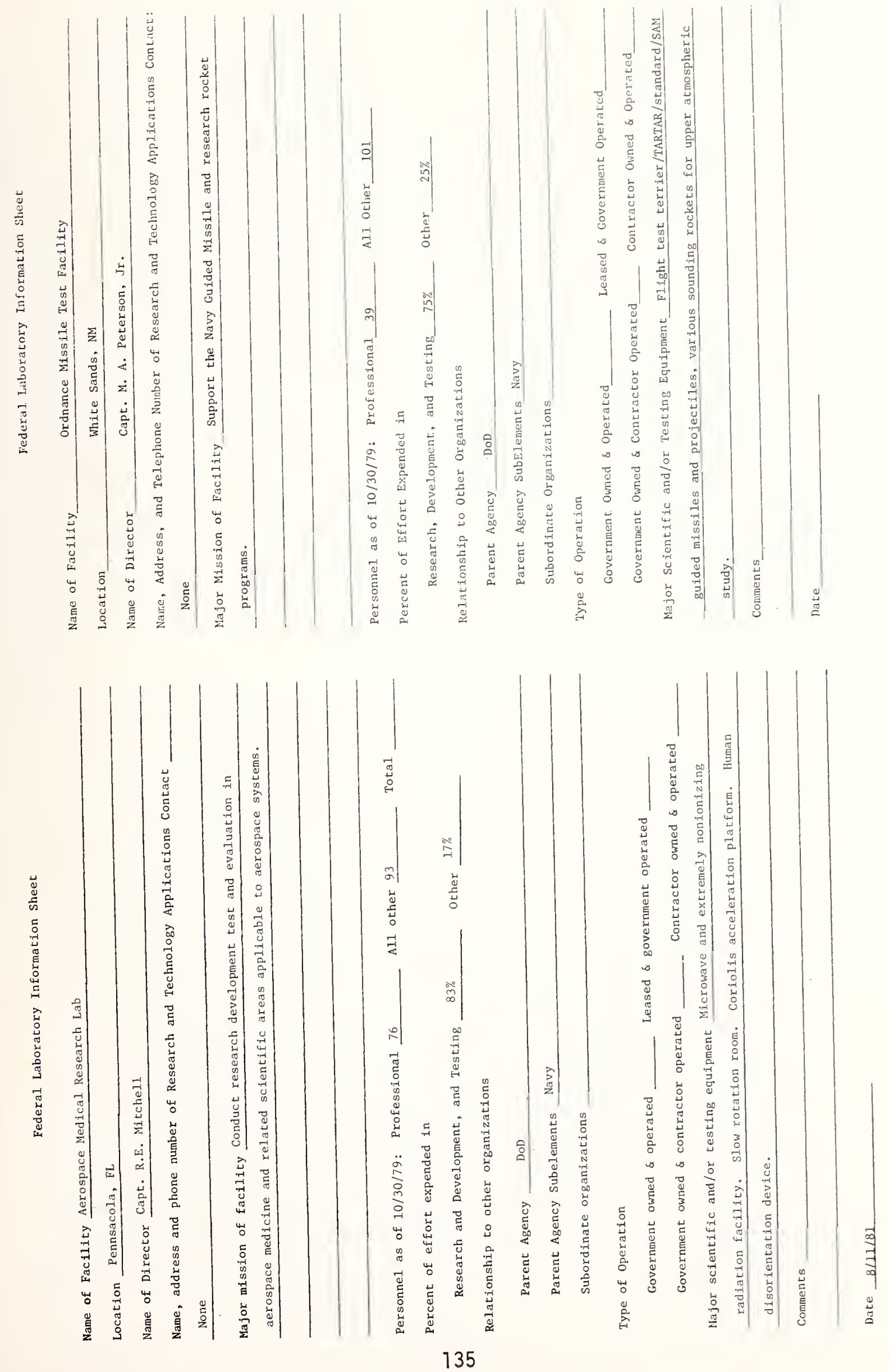

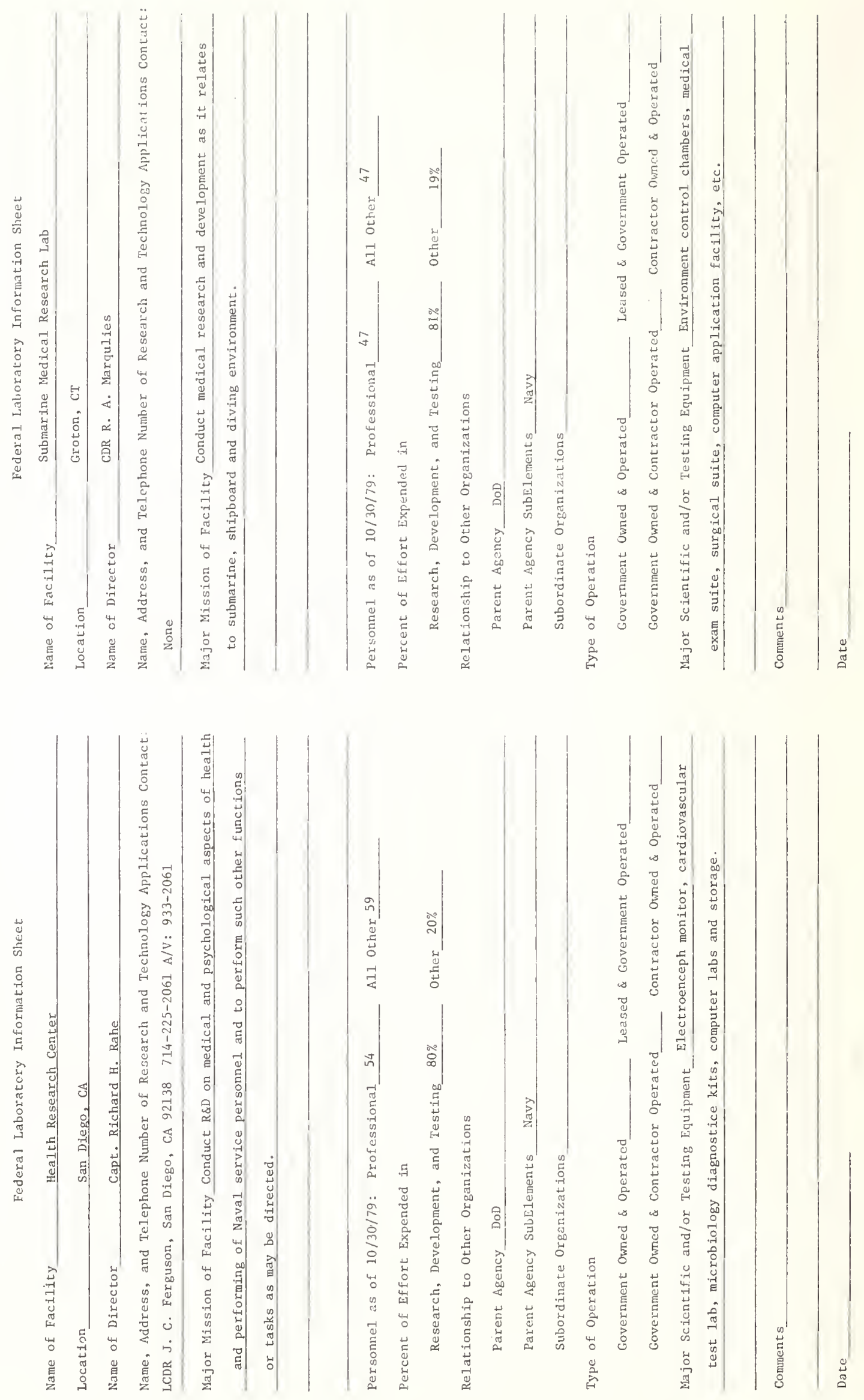

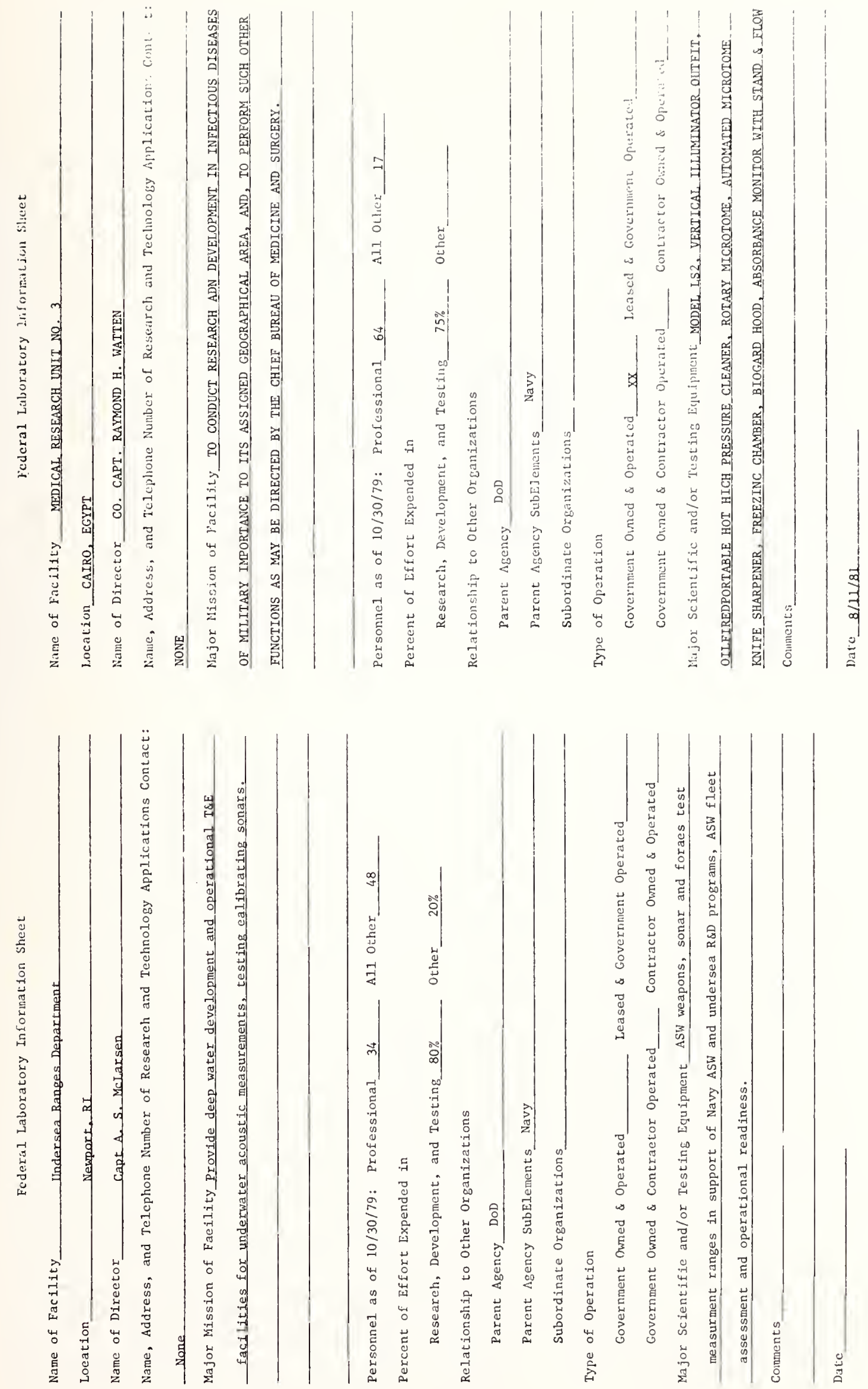

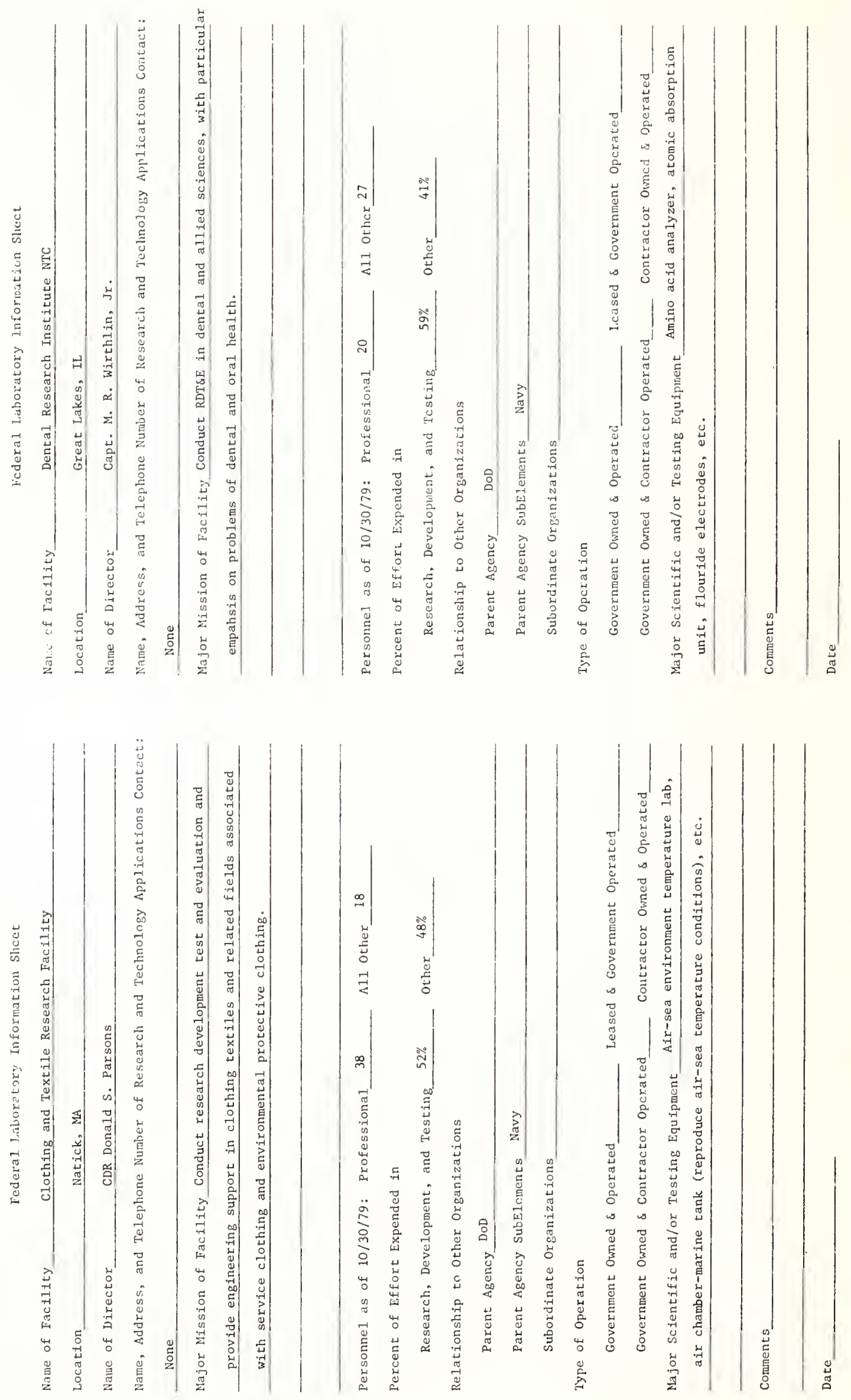
DEPARTMENT OF ENERGY 


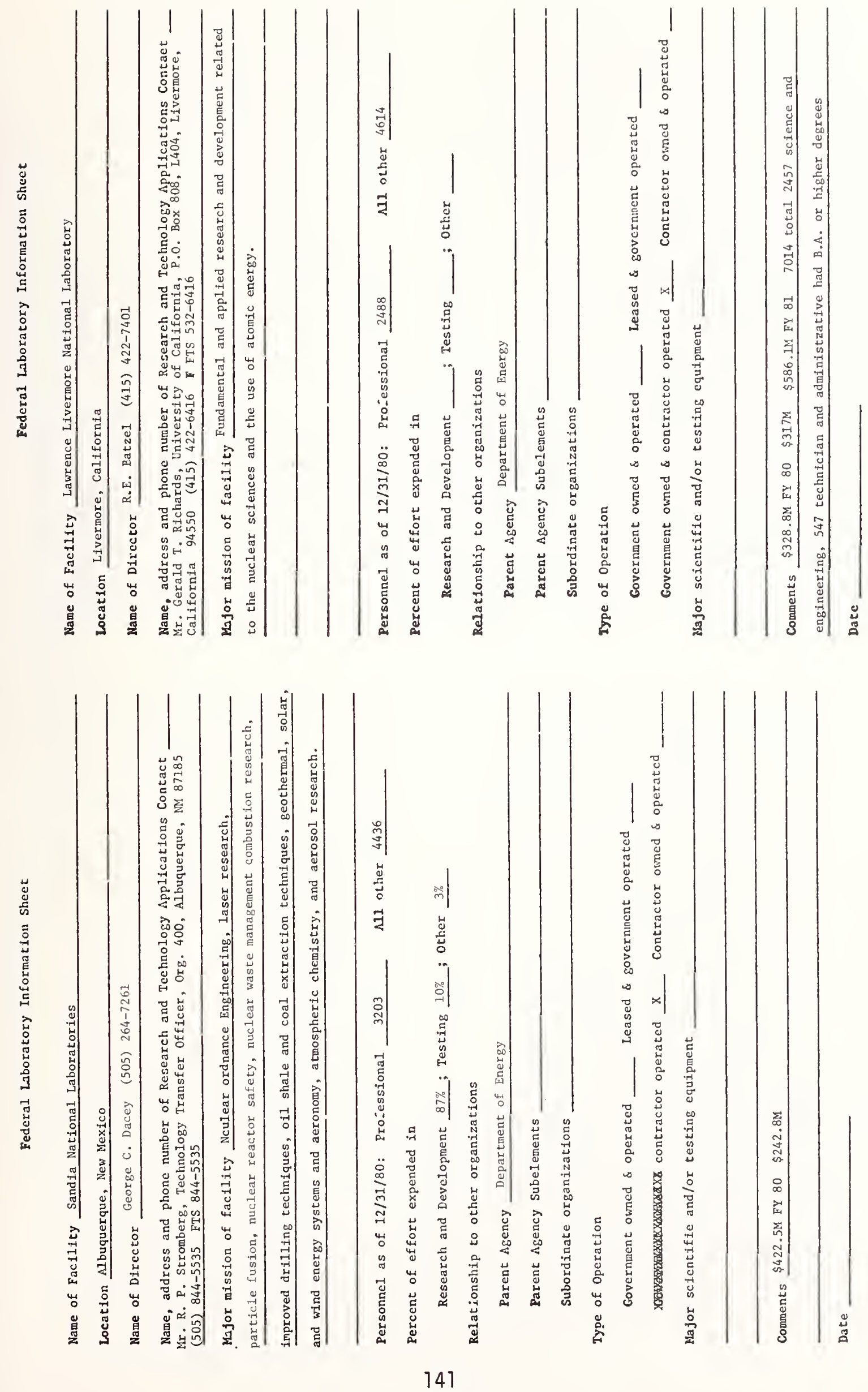

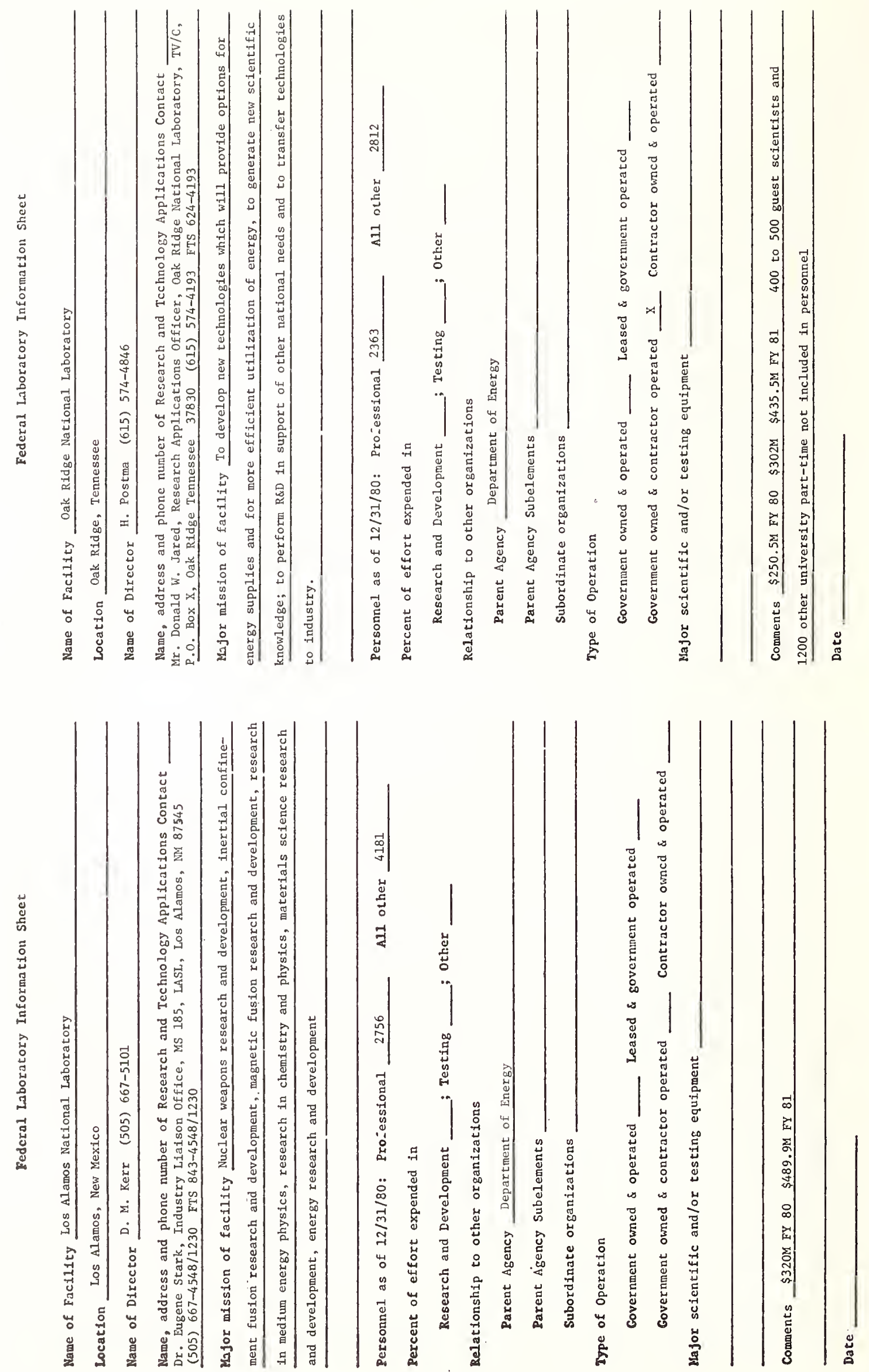


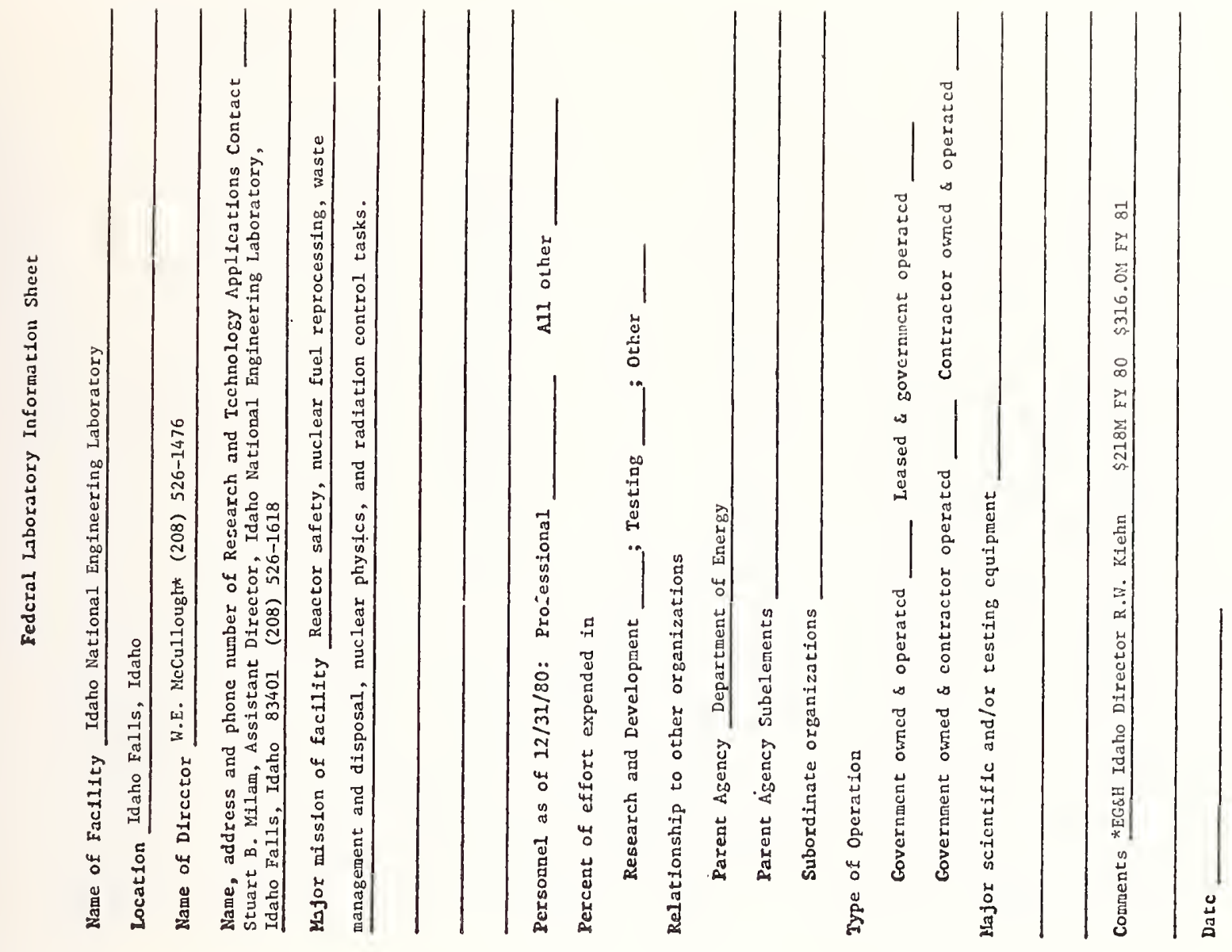

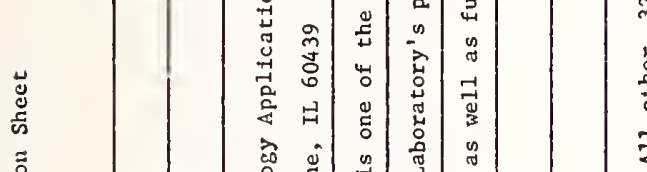



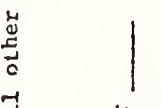

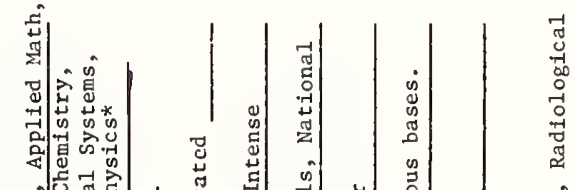

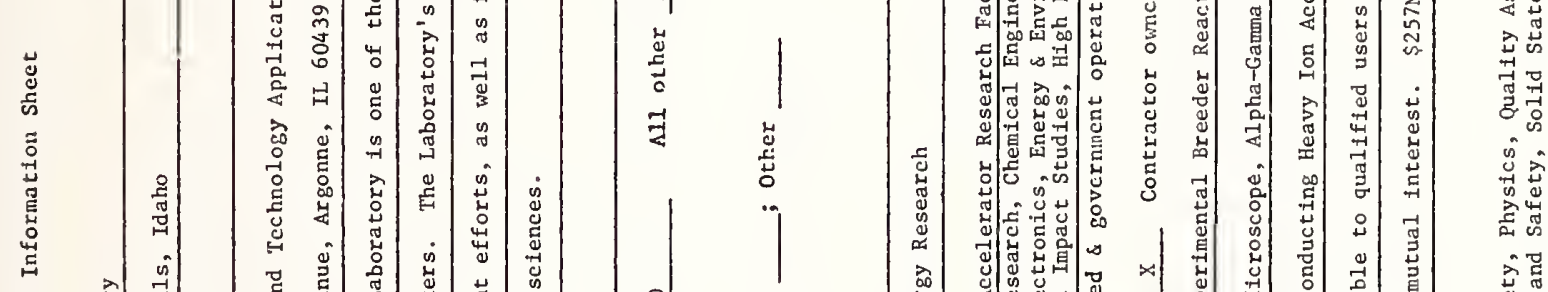

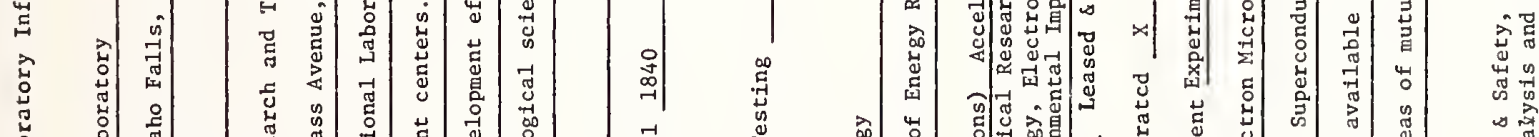

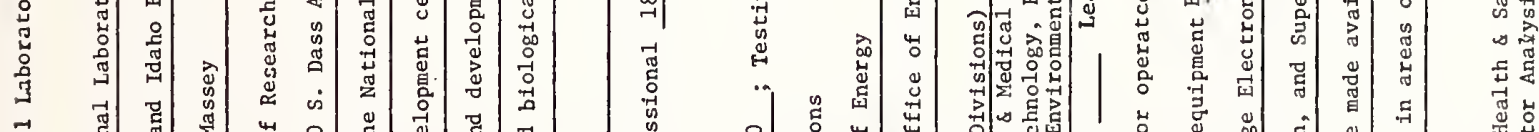

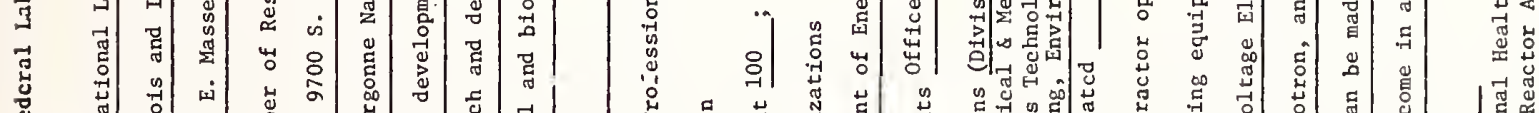

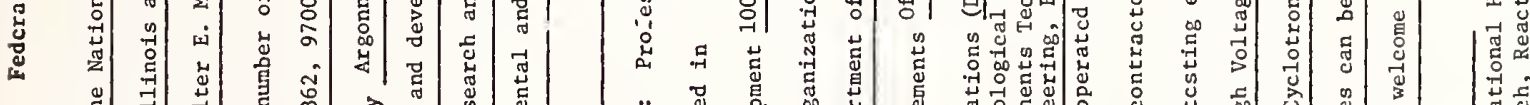

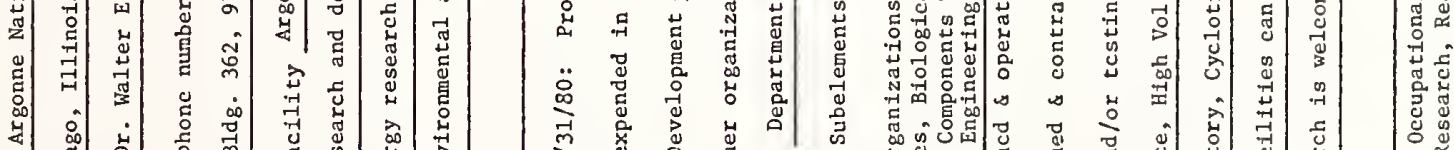

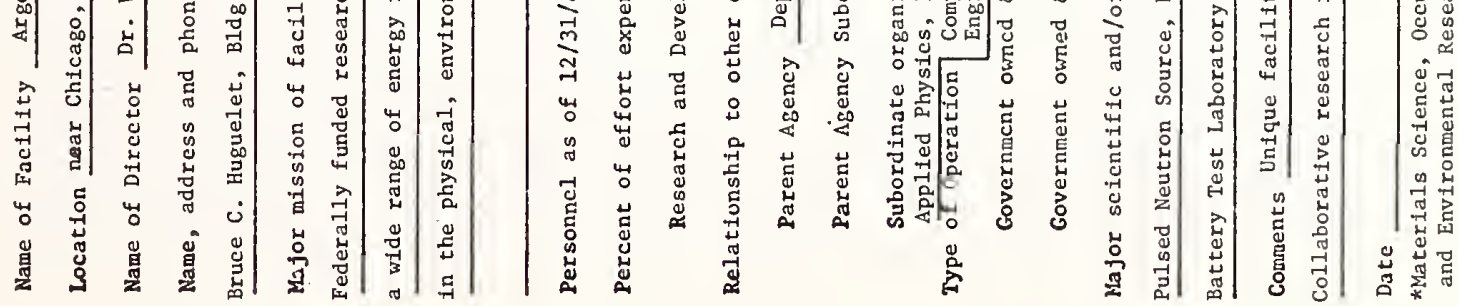



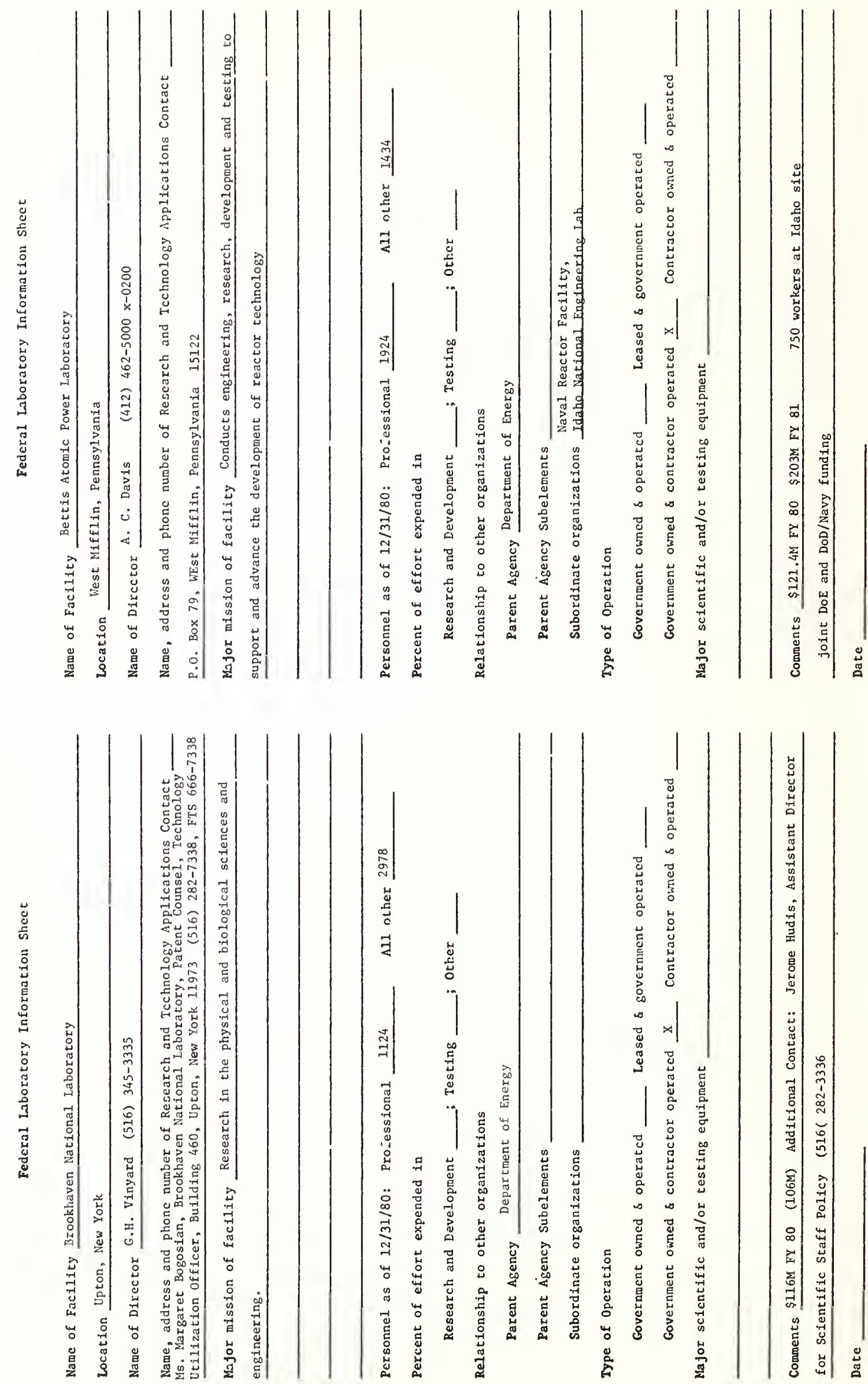


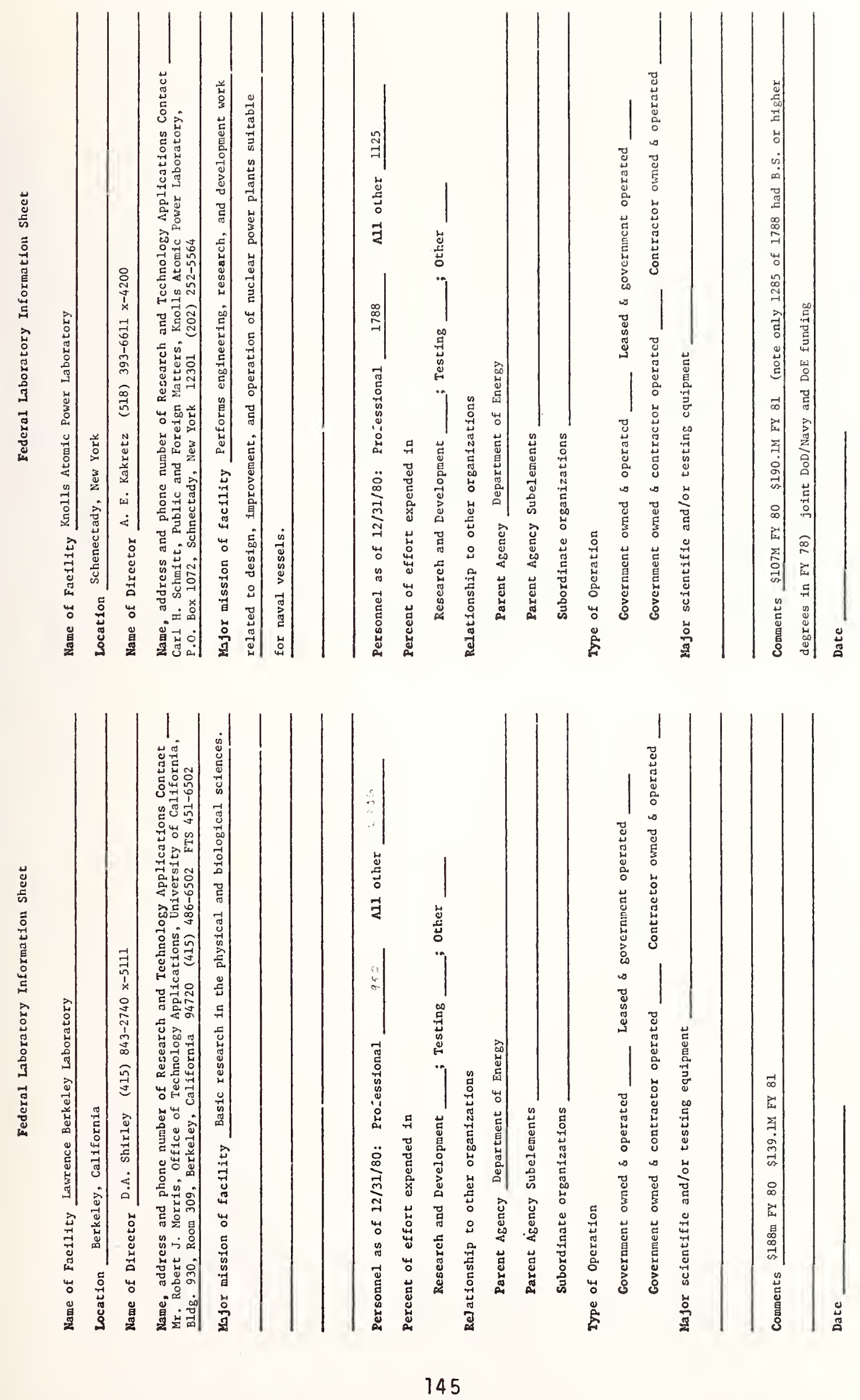



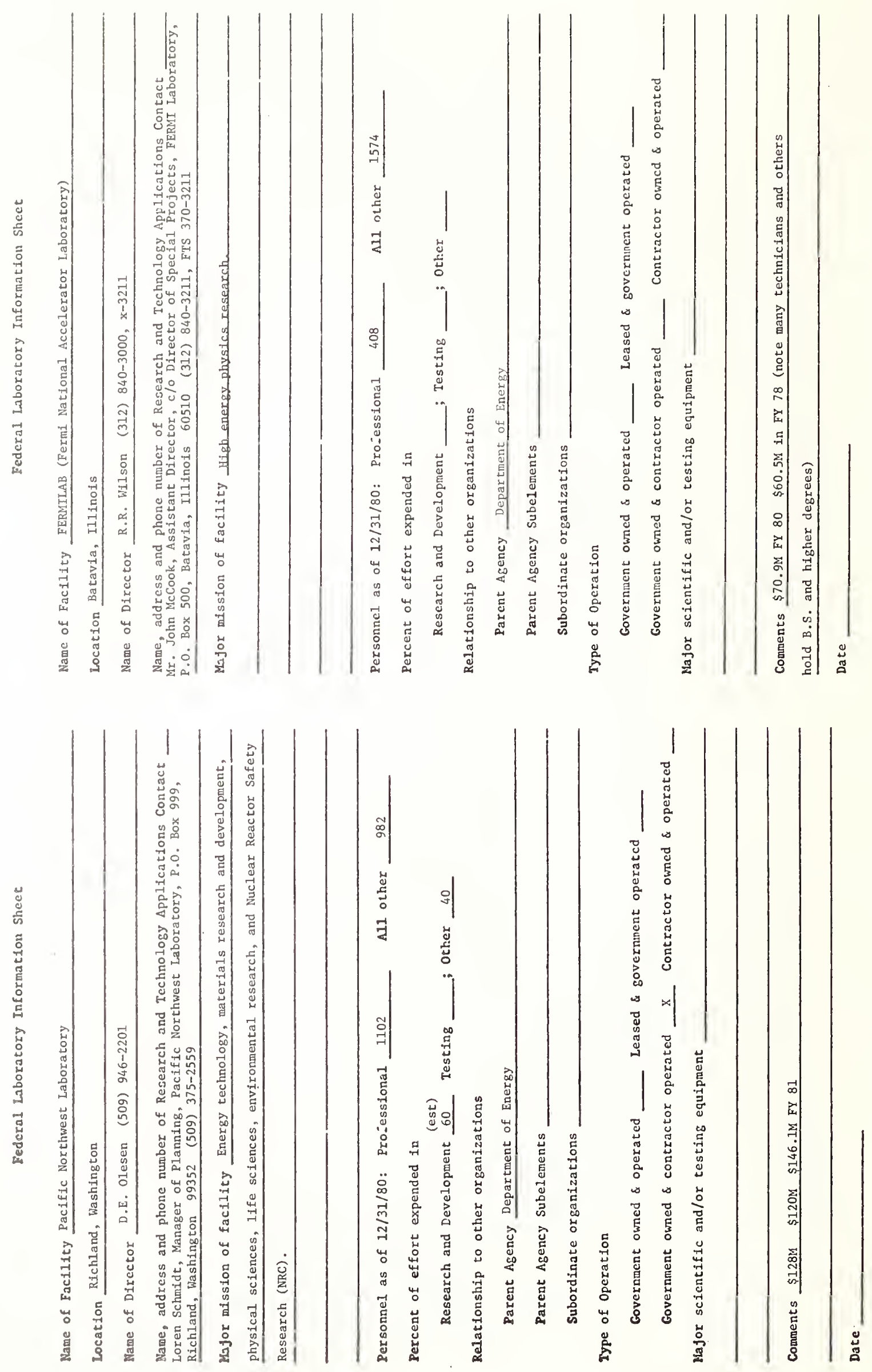

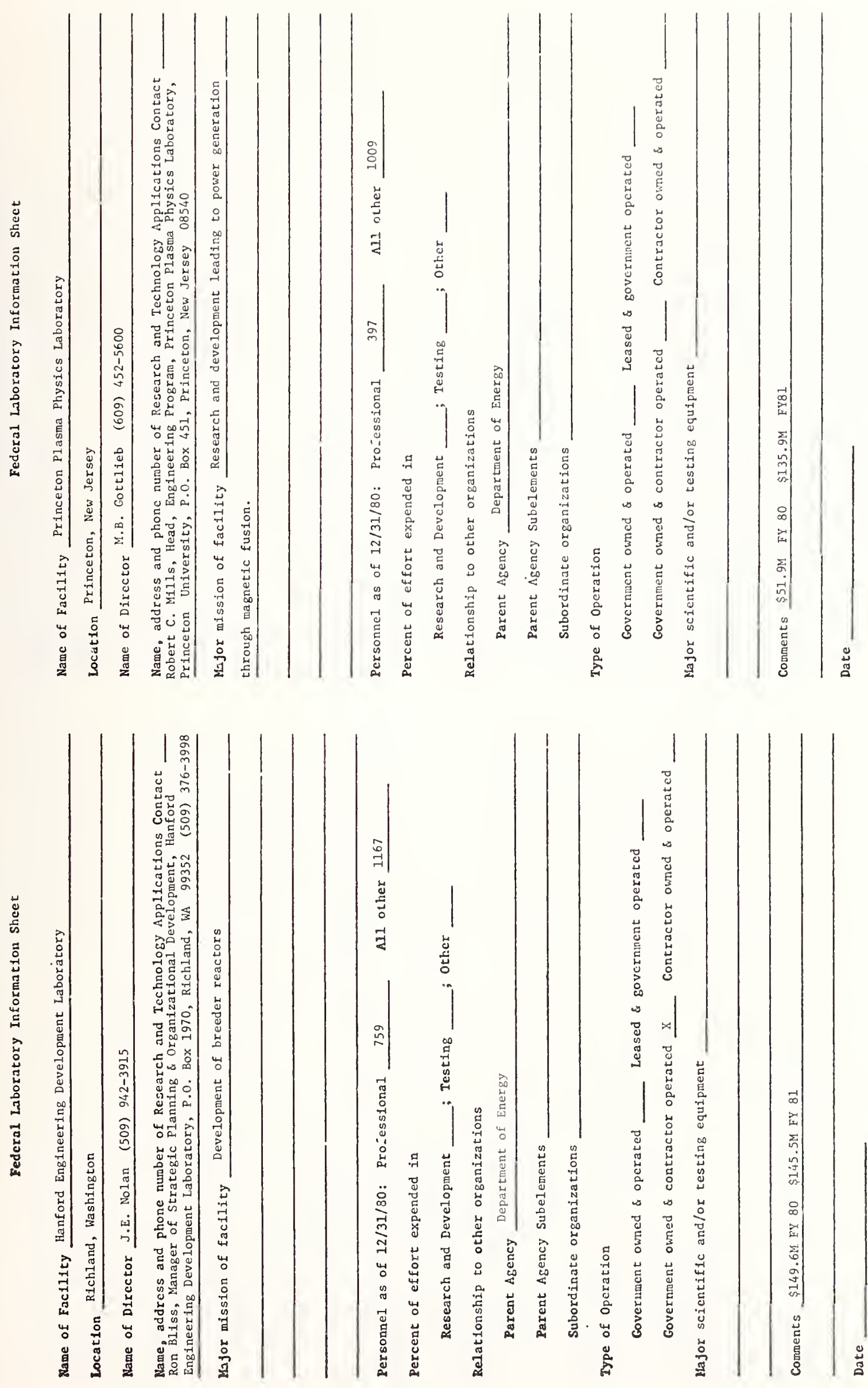

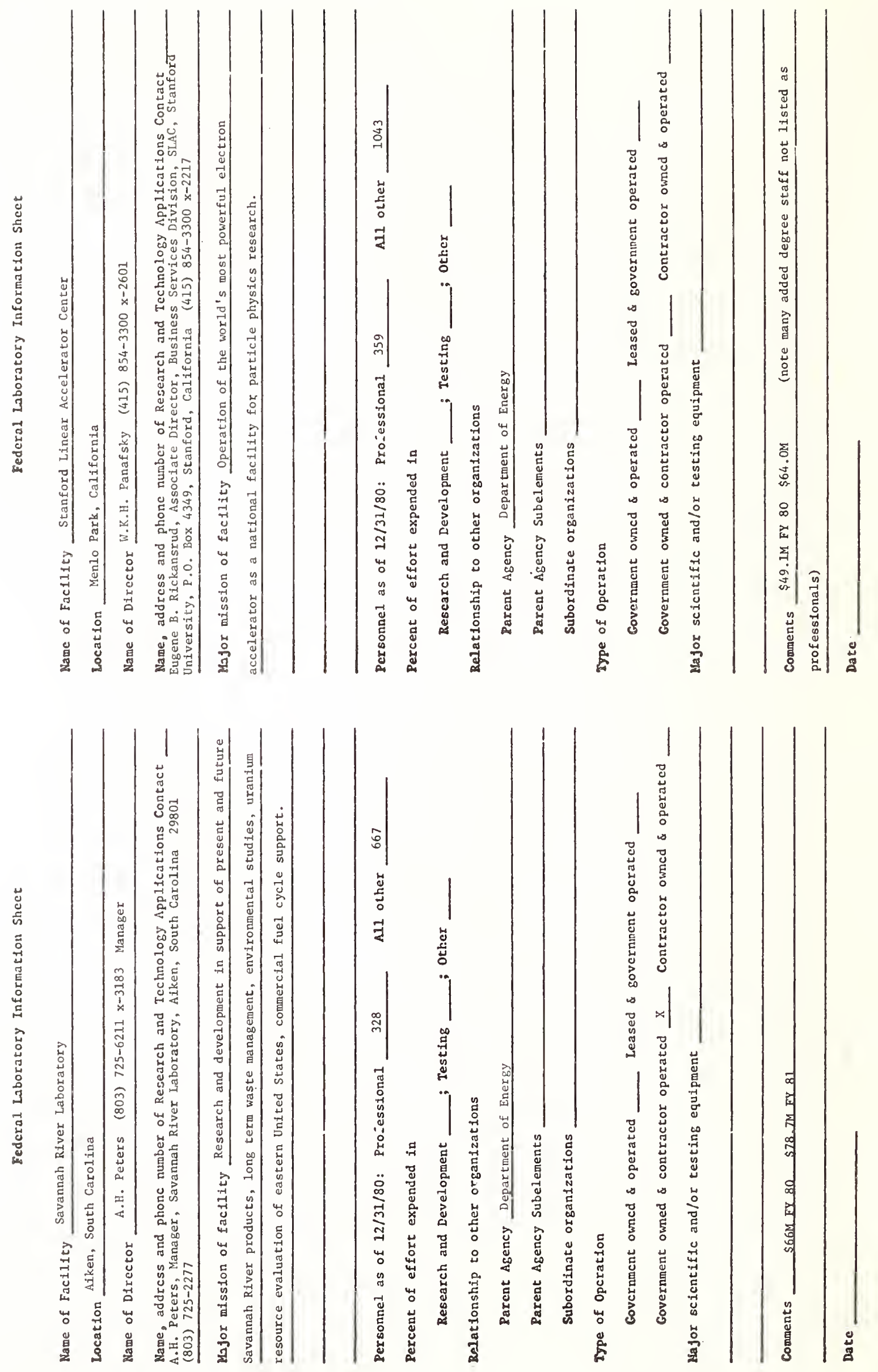

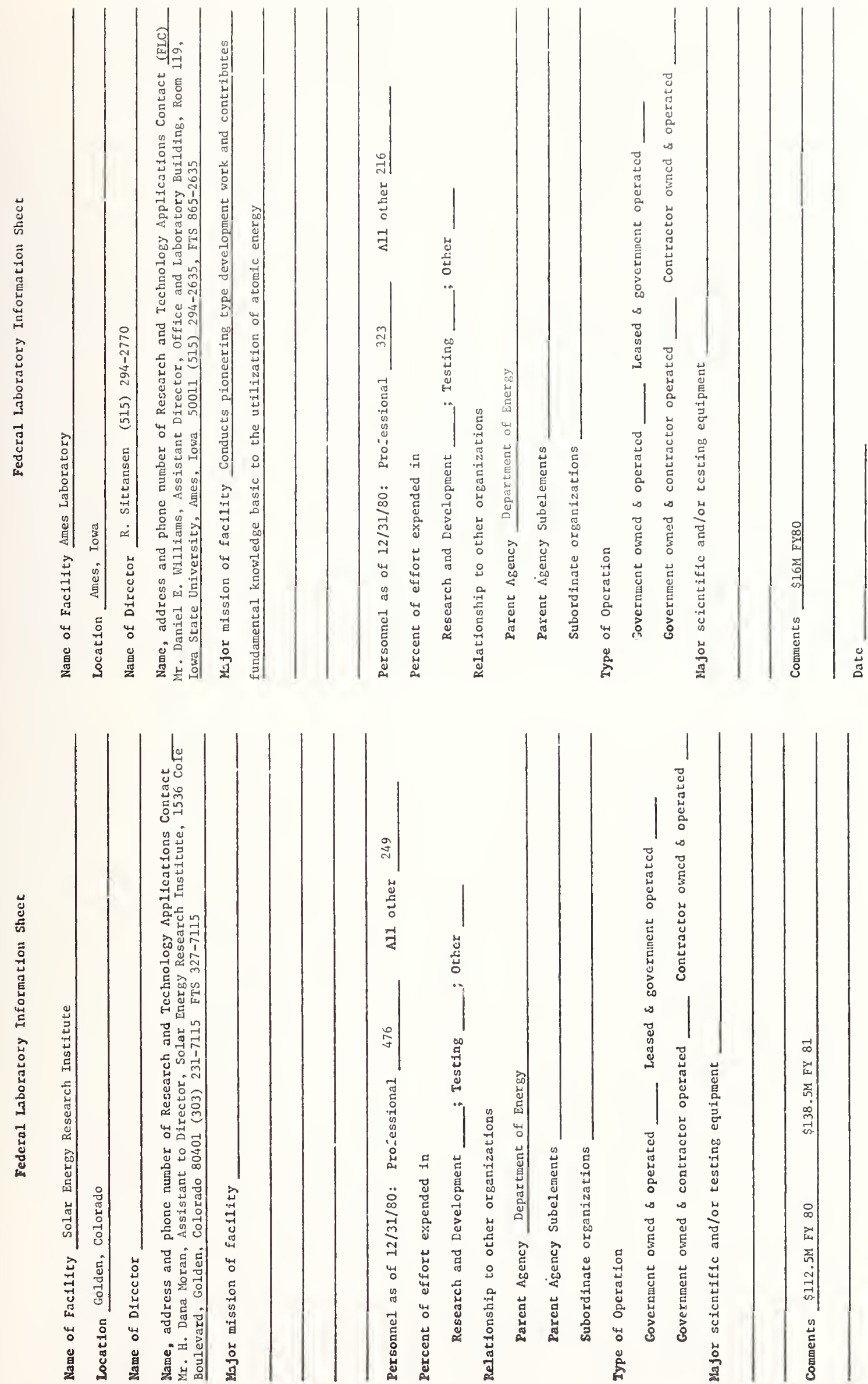

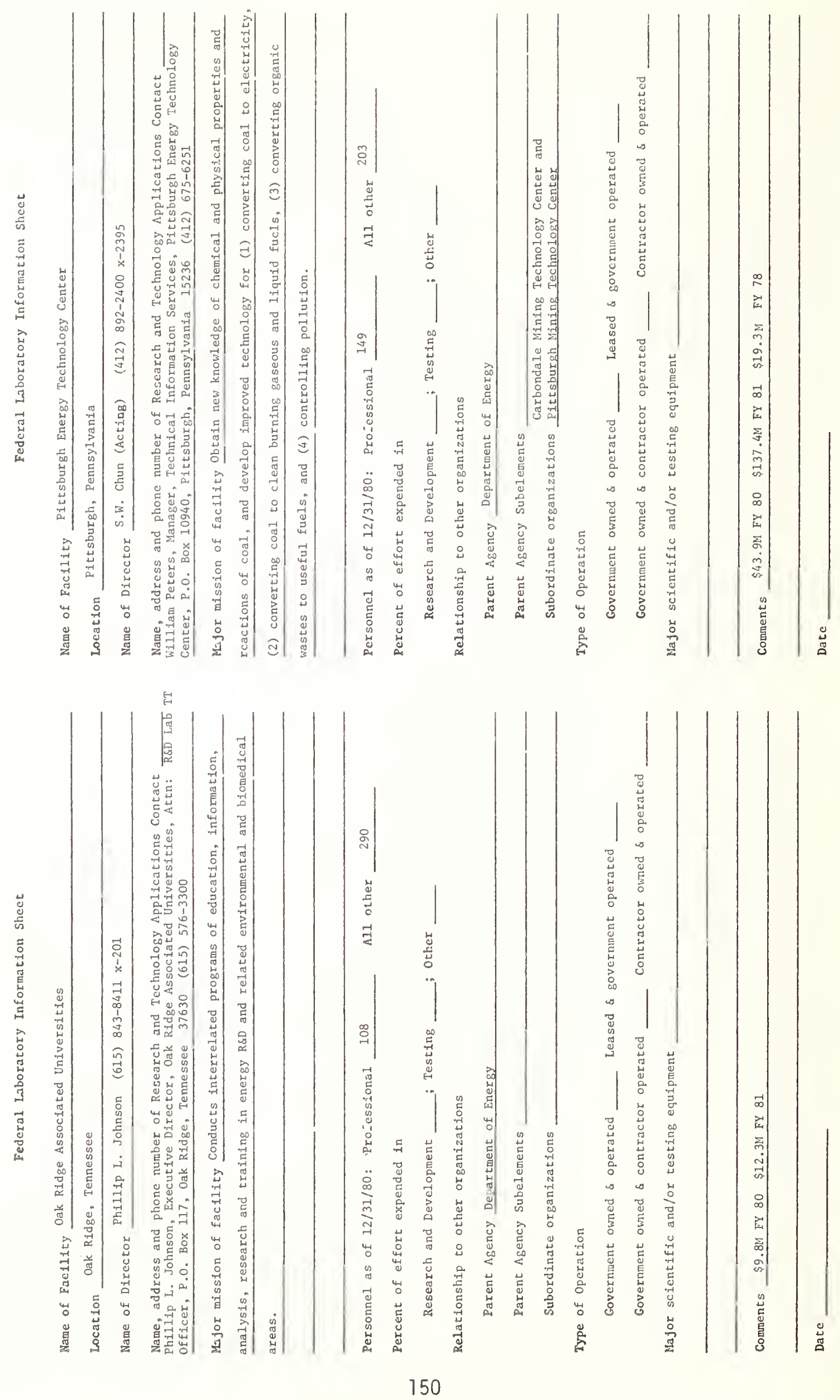

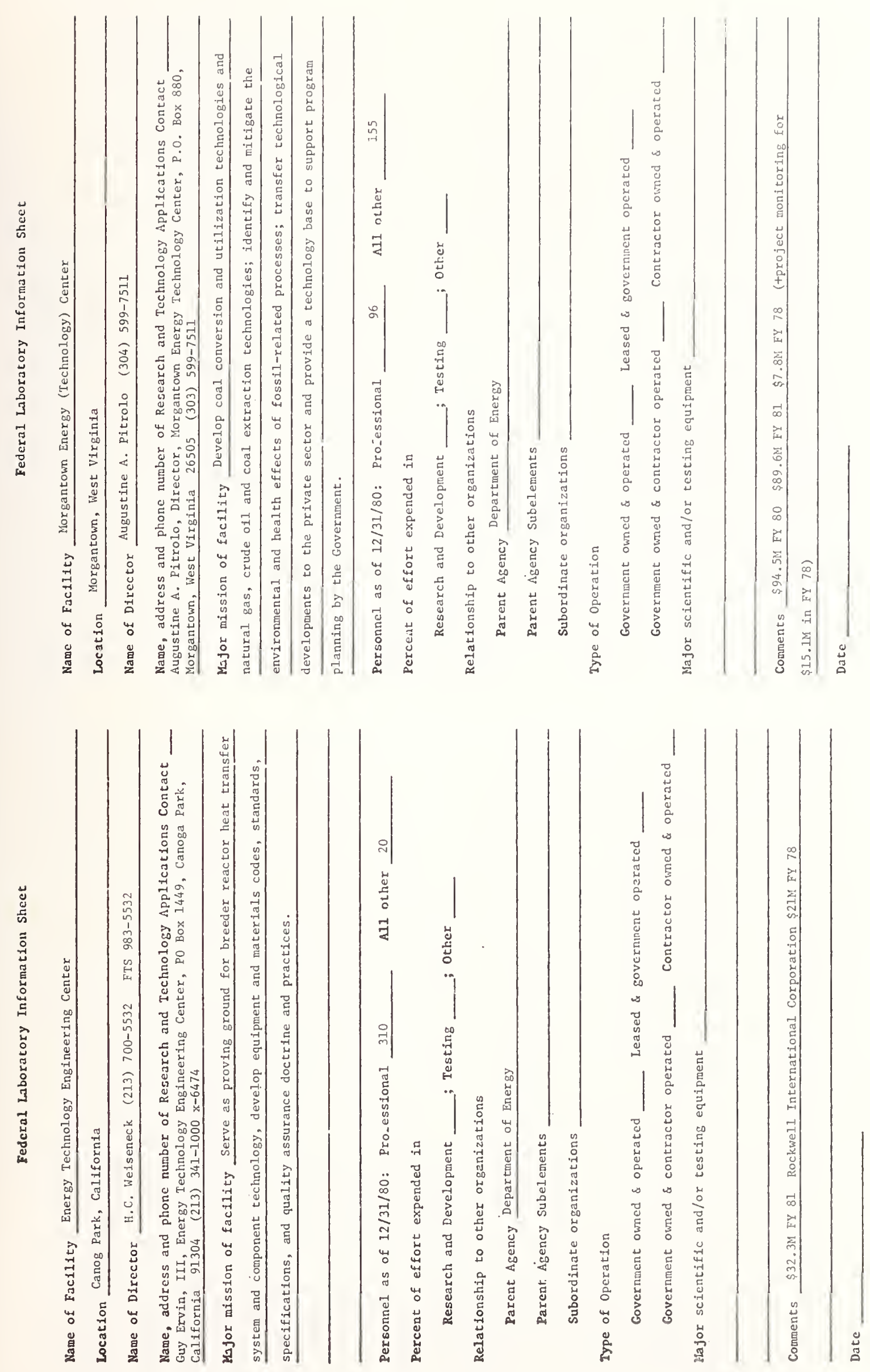

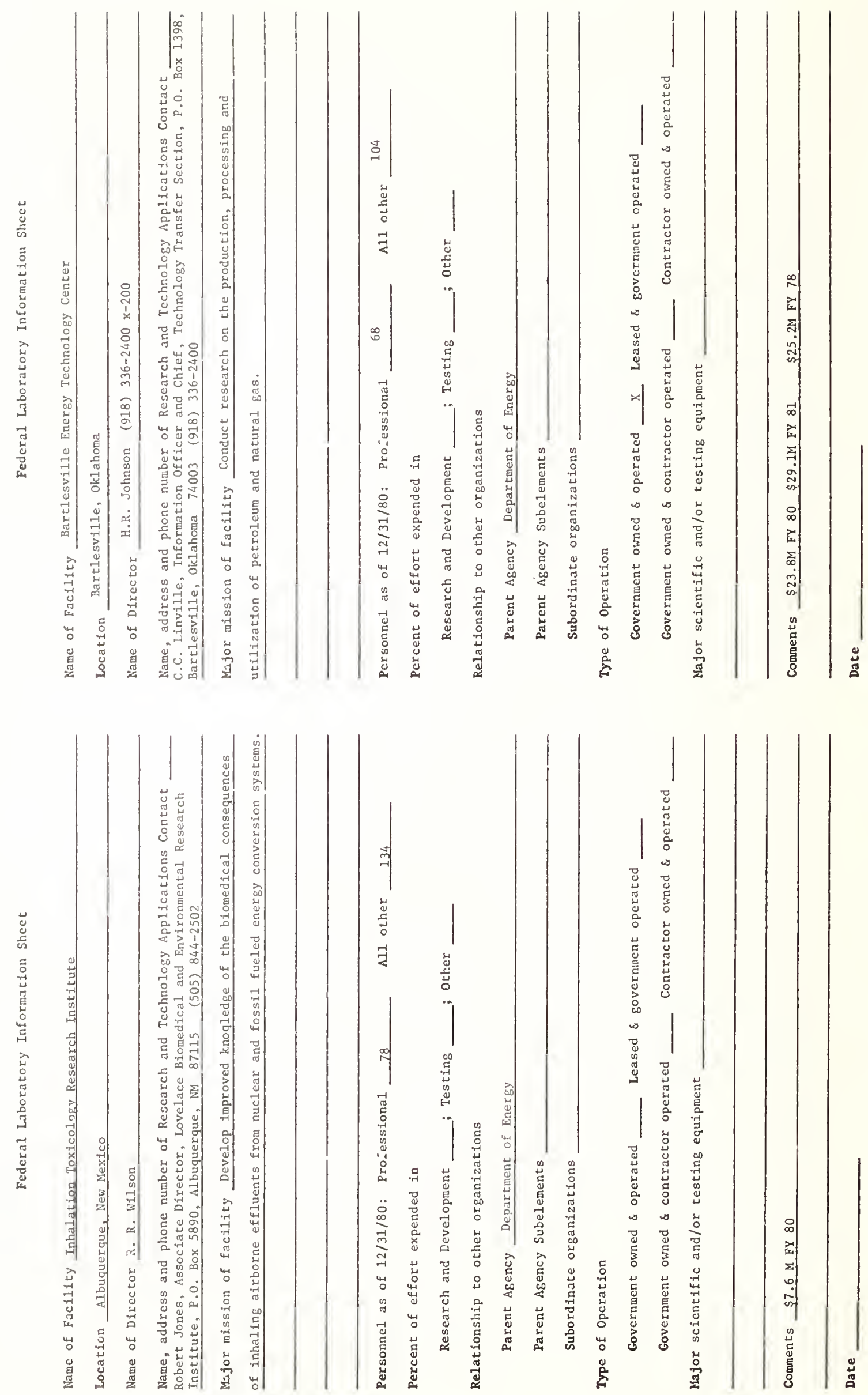

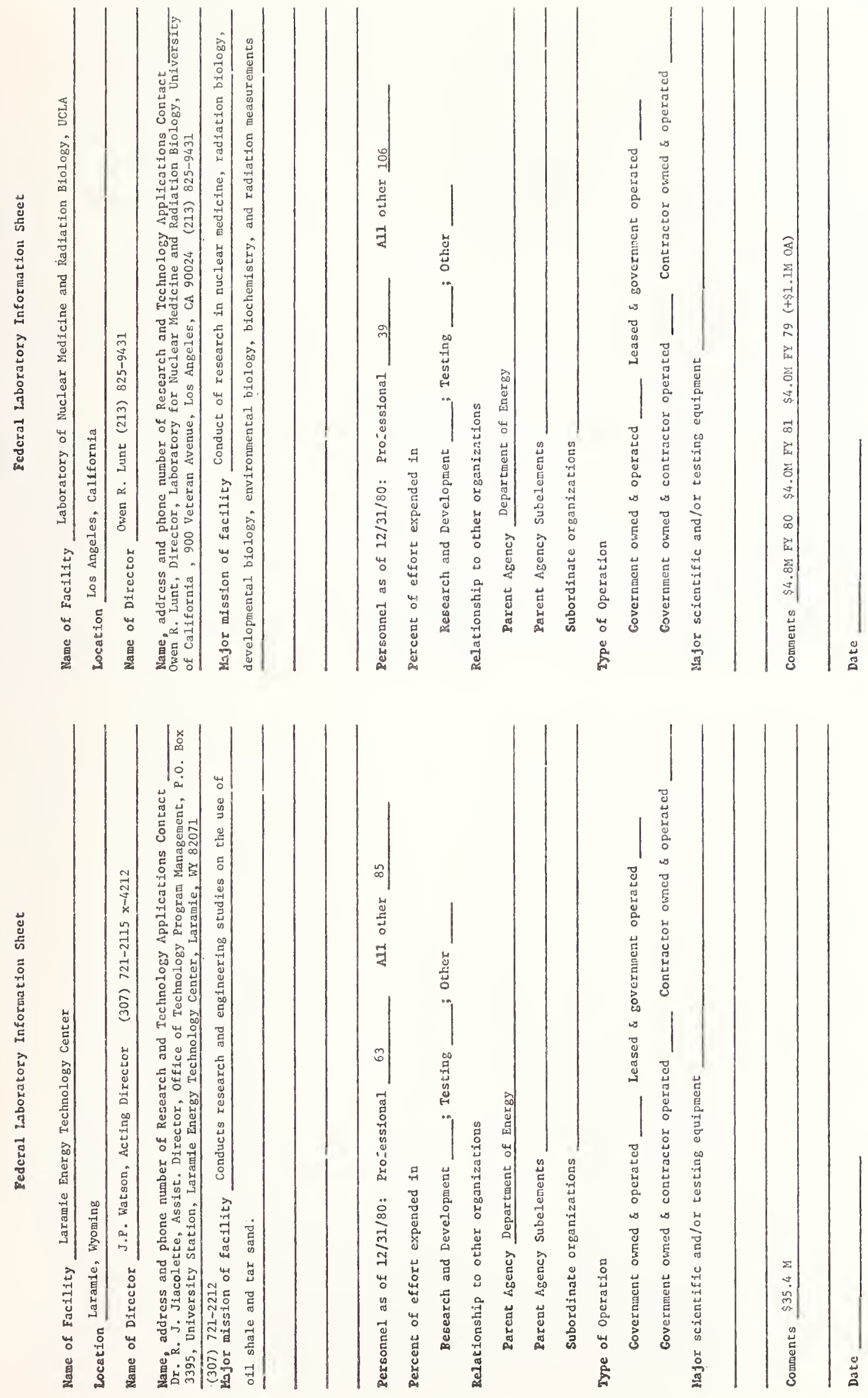

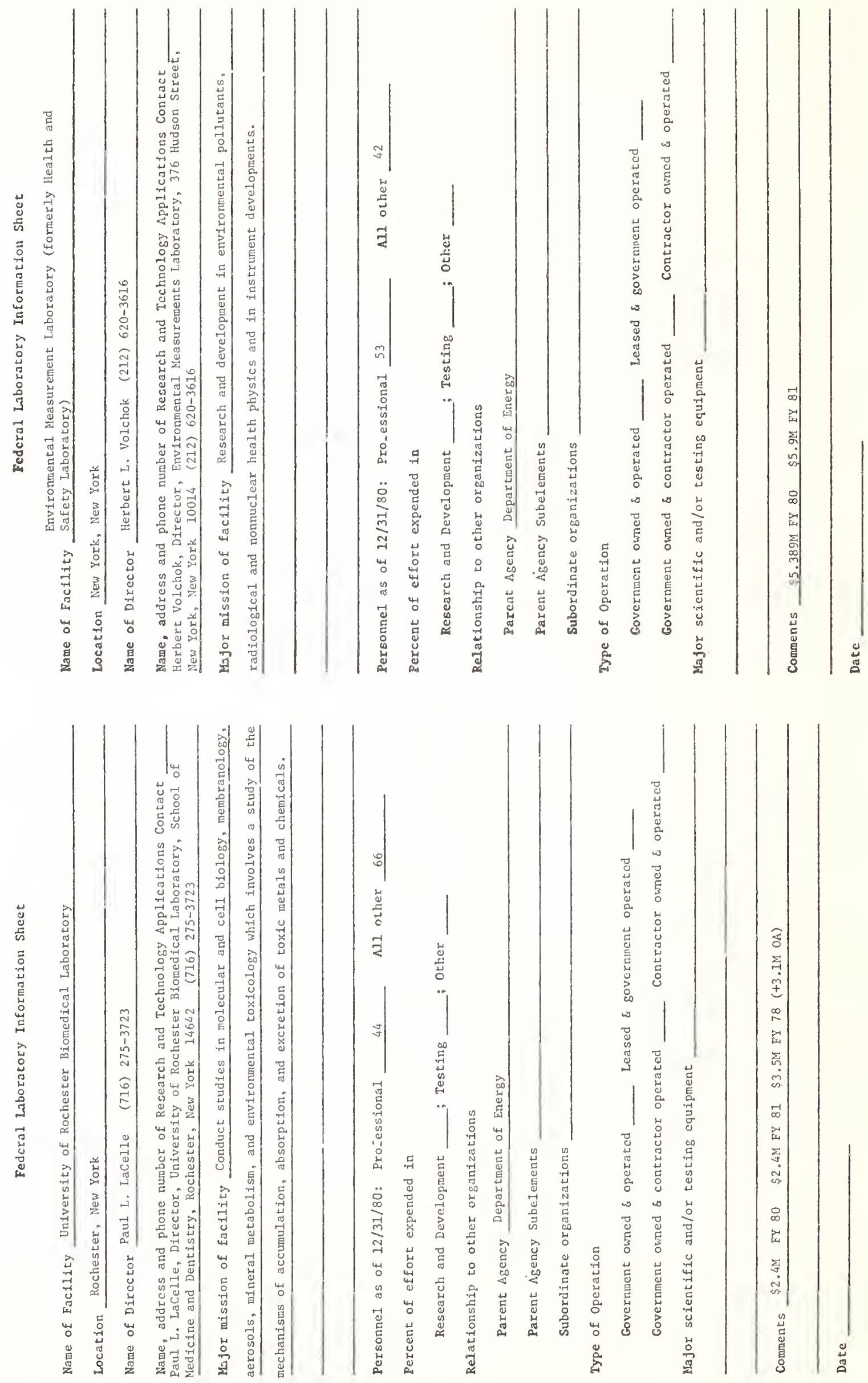

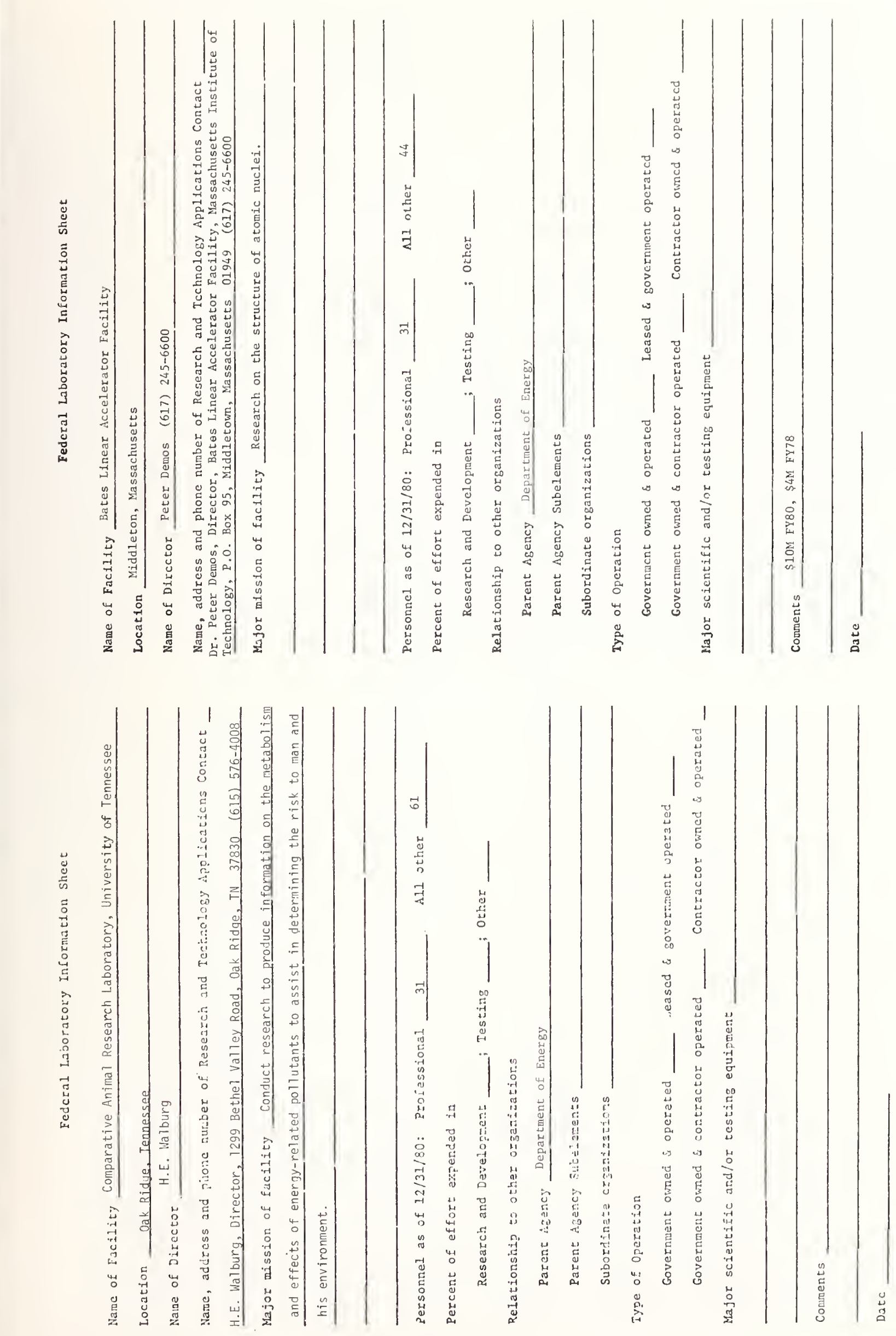

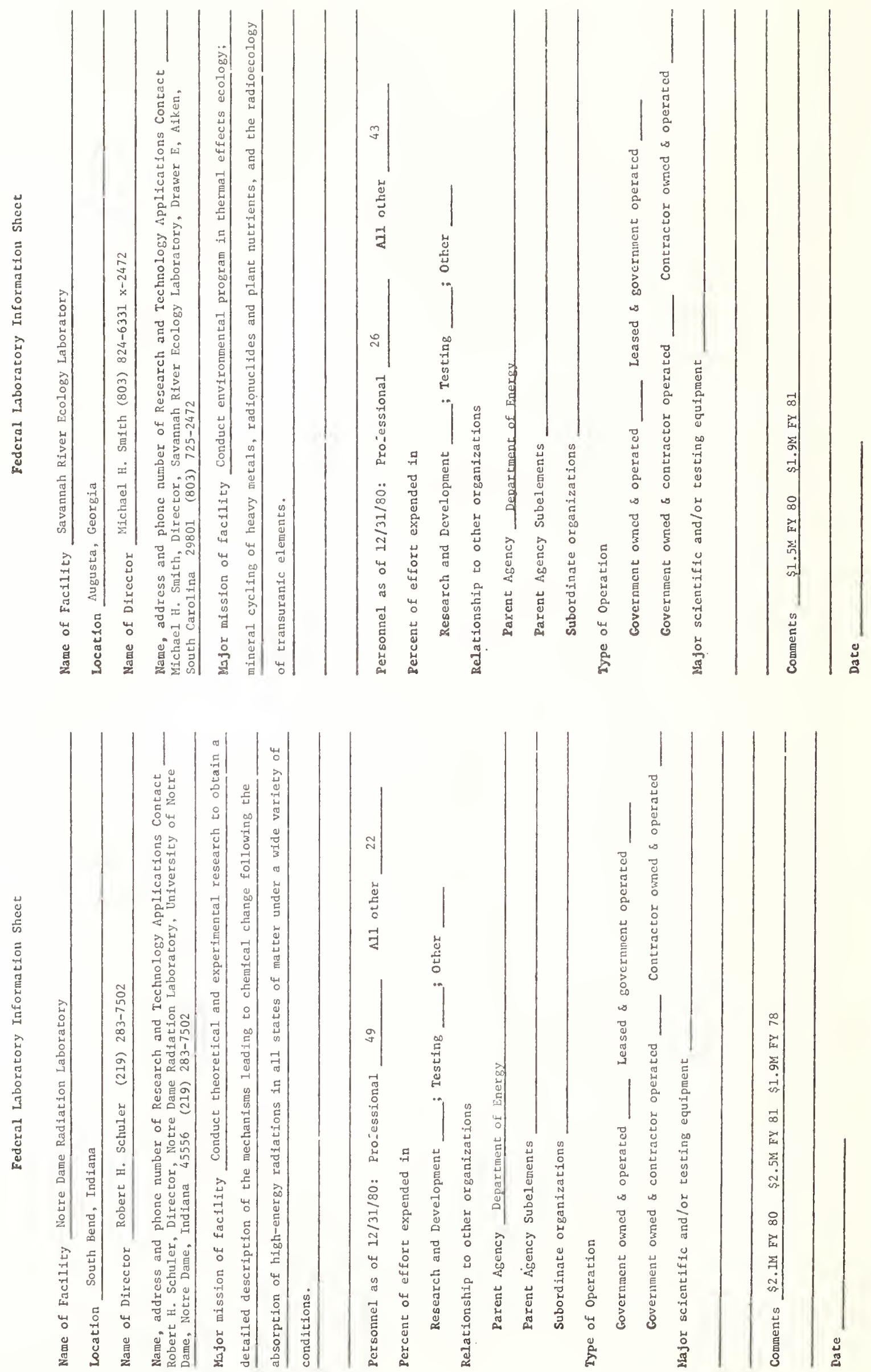

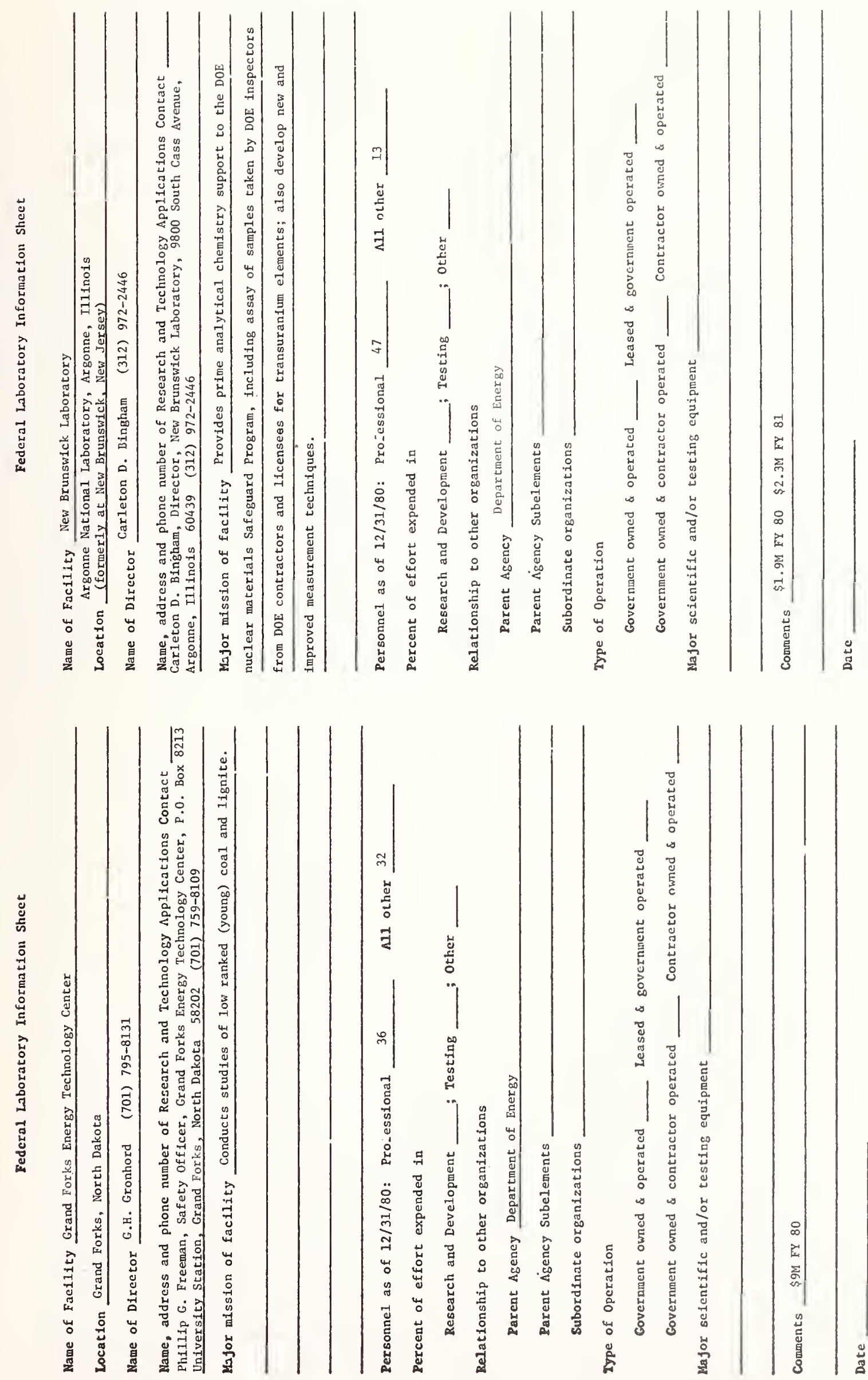

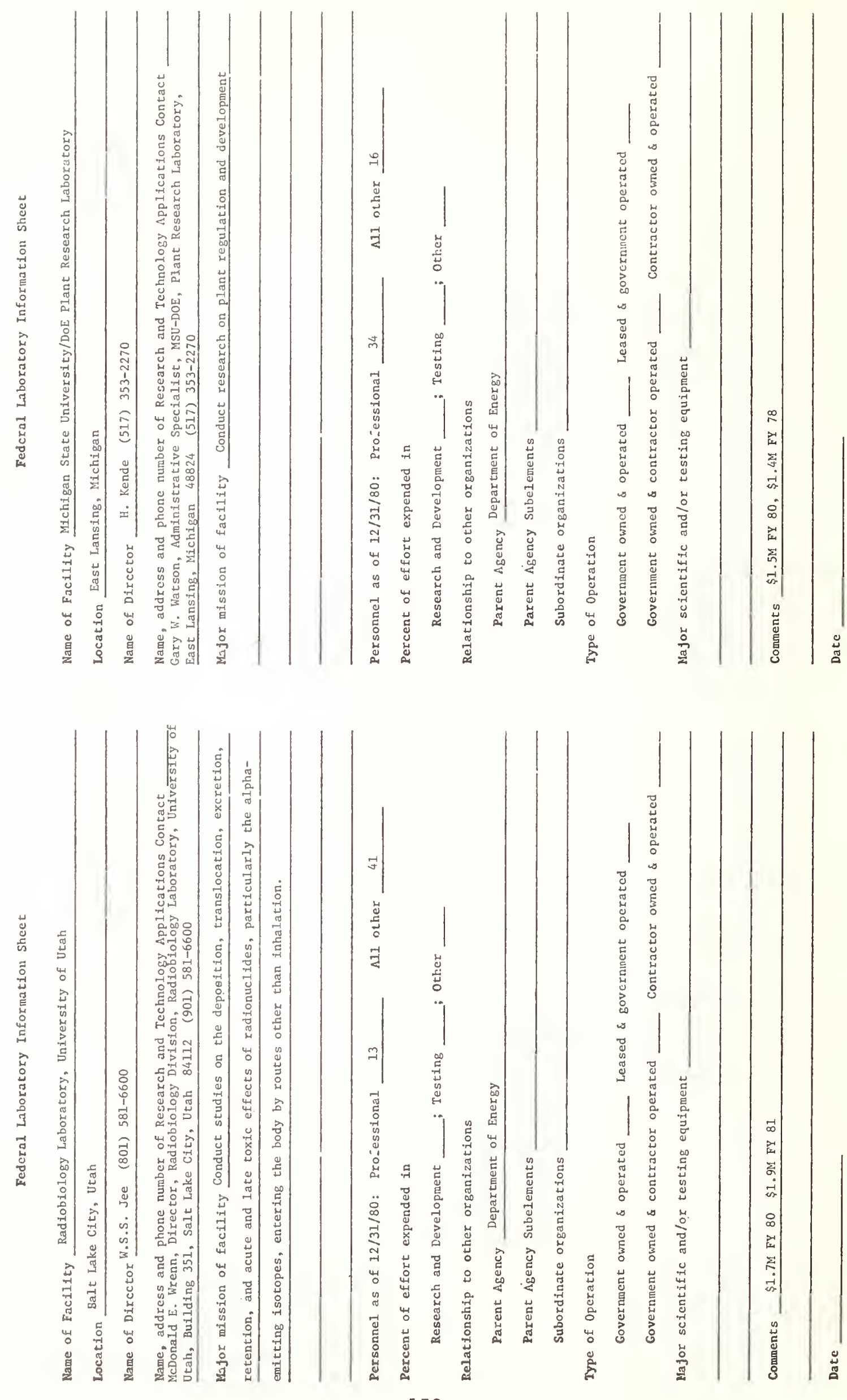

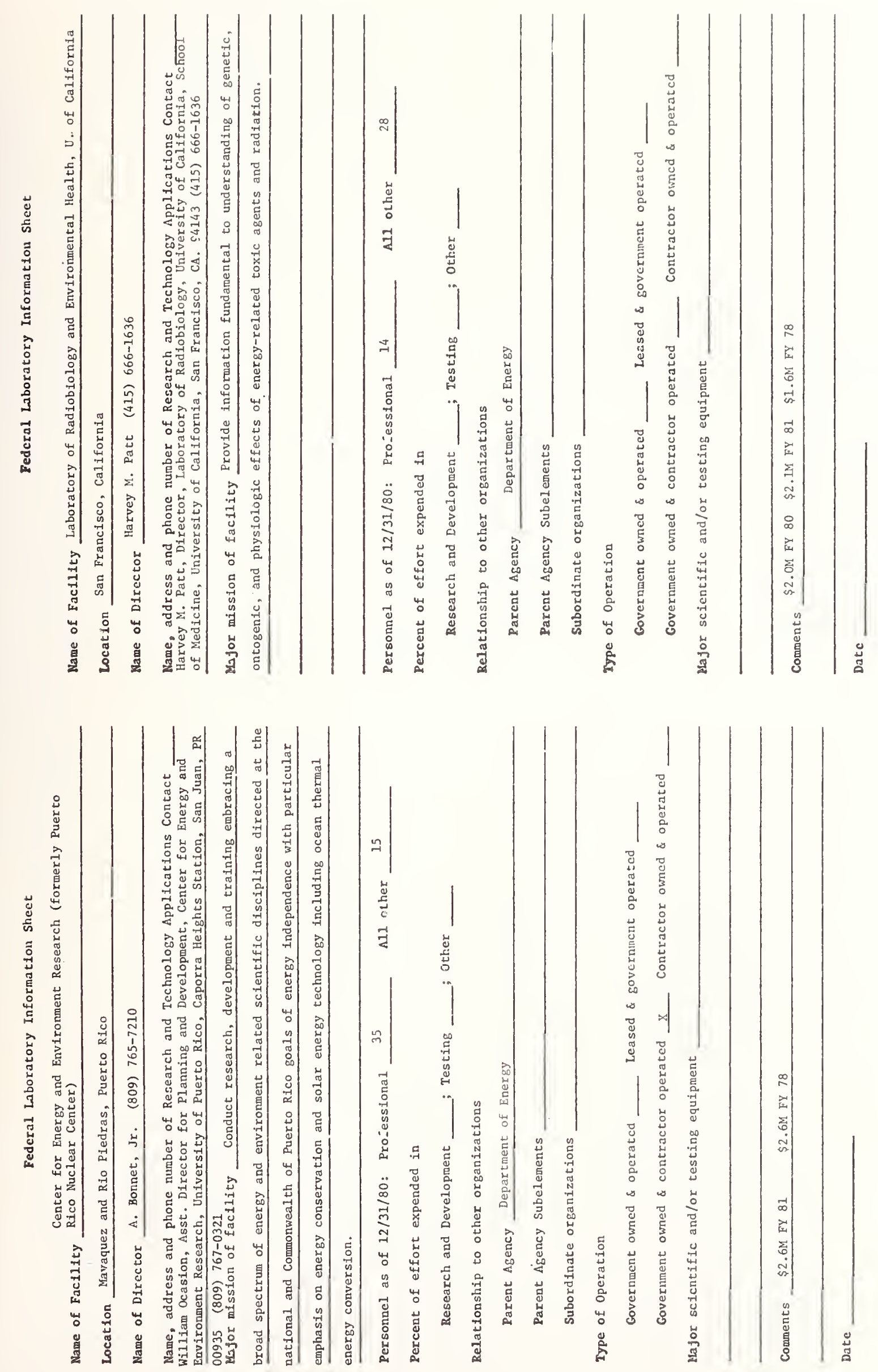


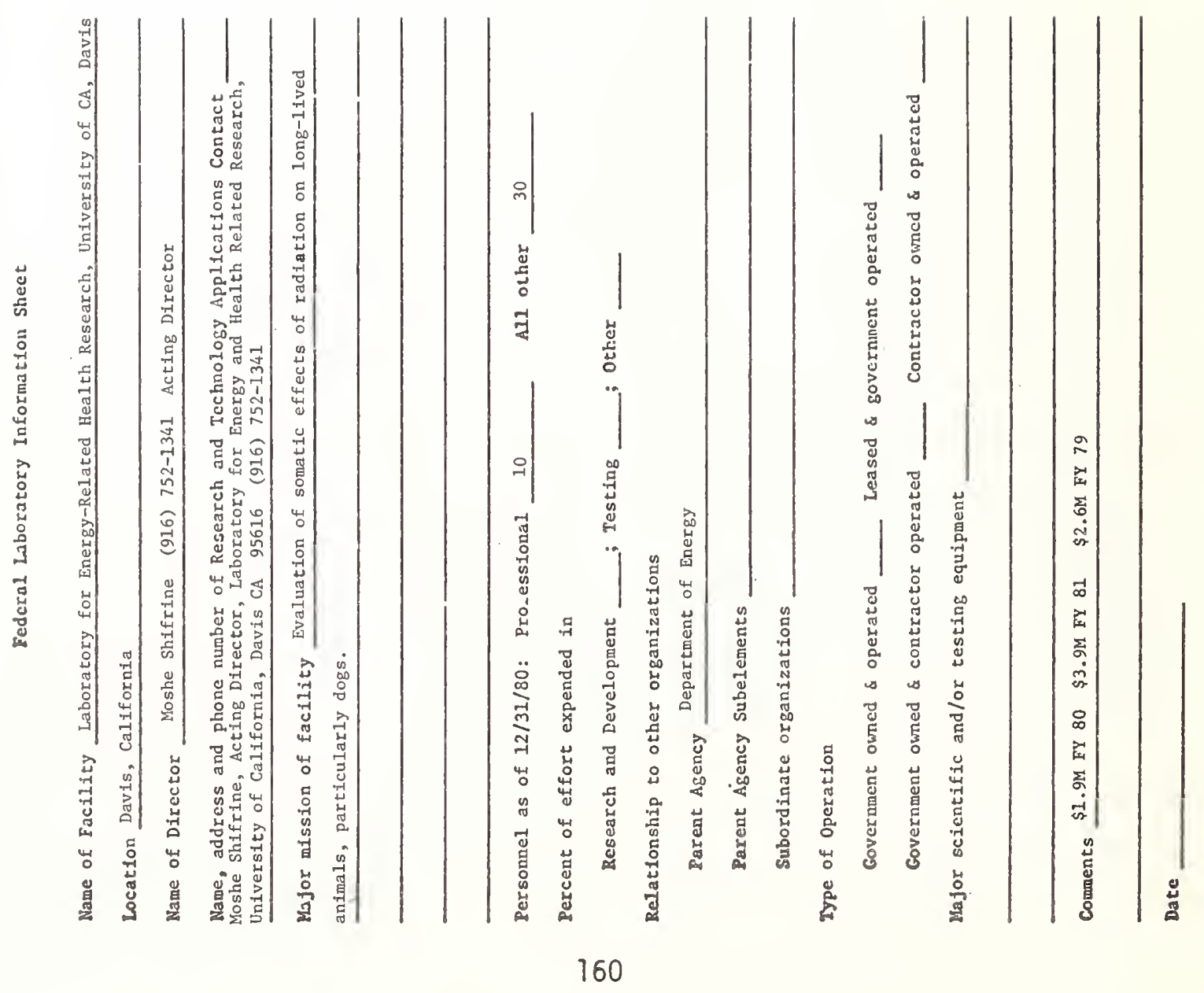


DEPARTMENT OF INTERIOR 


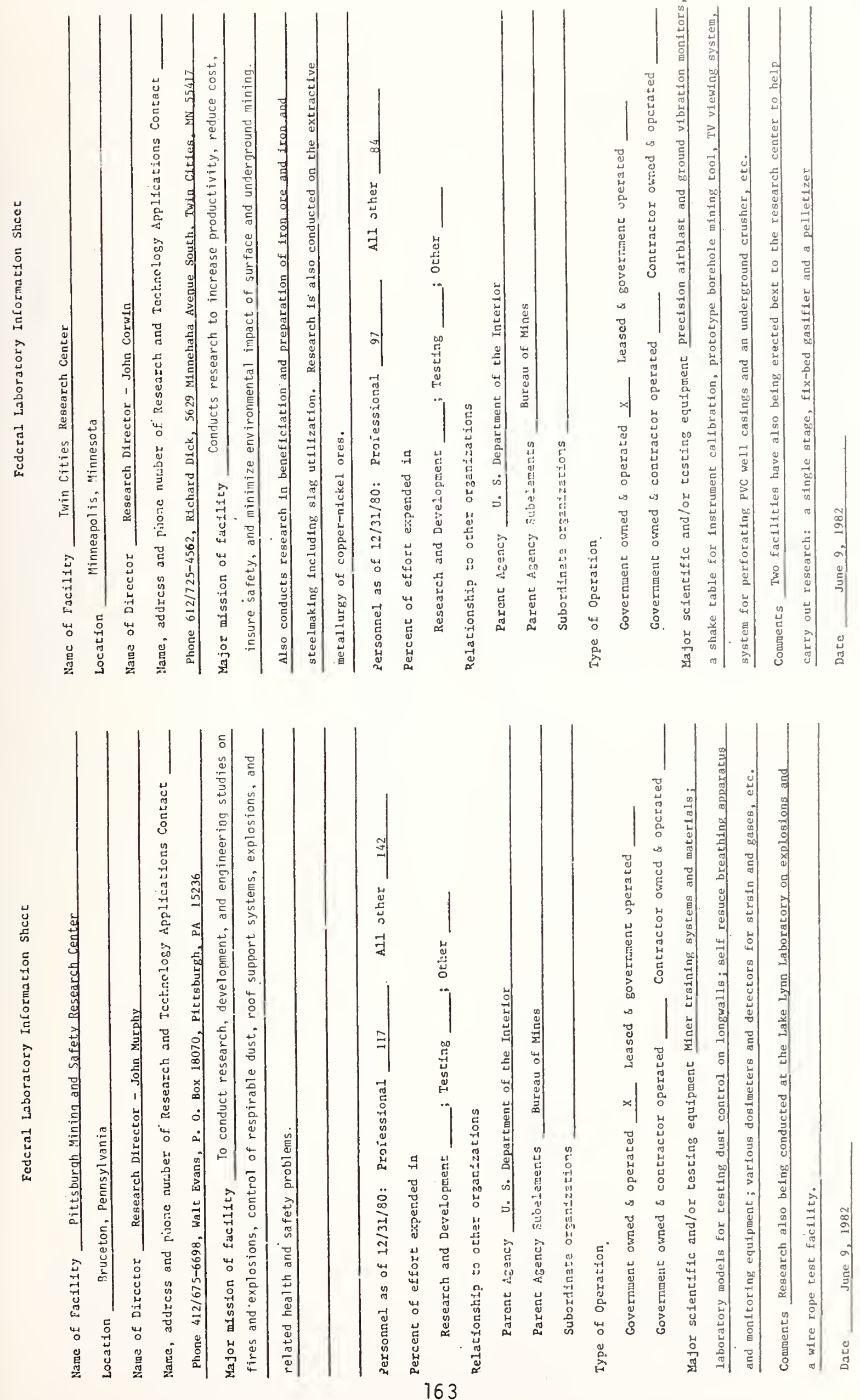

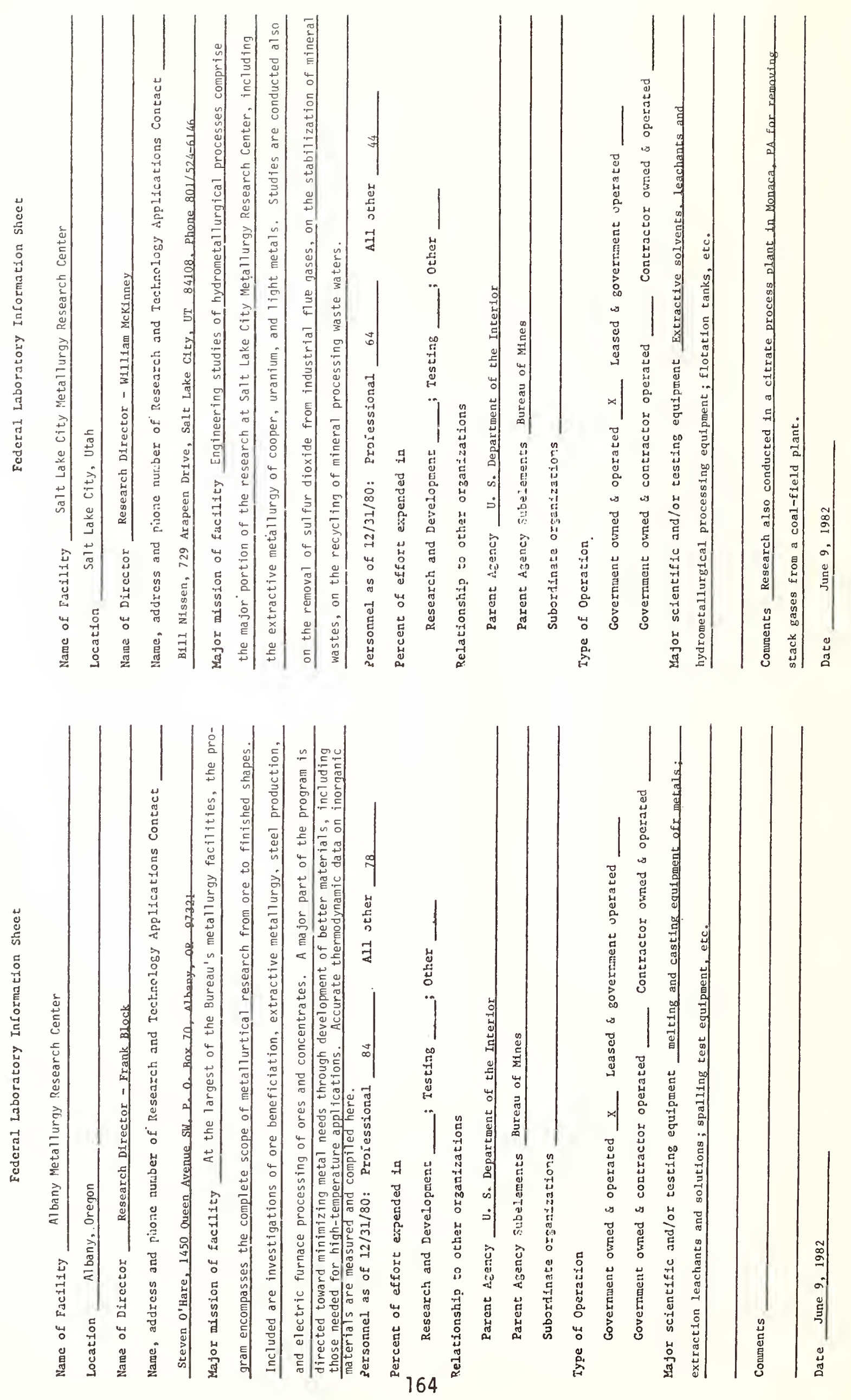

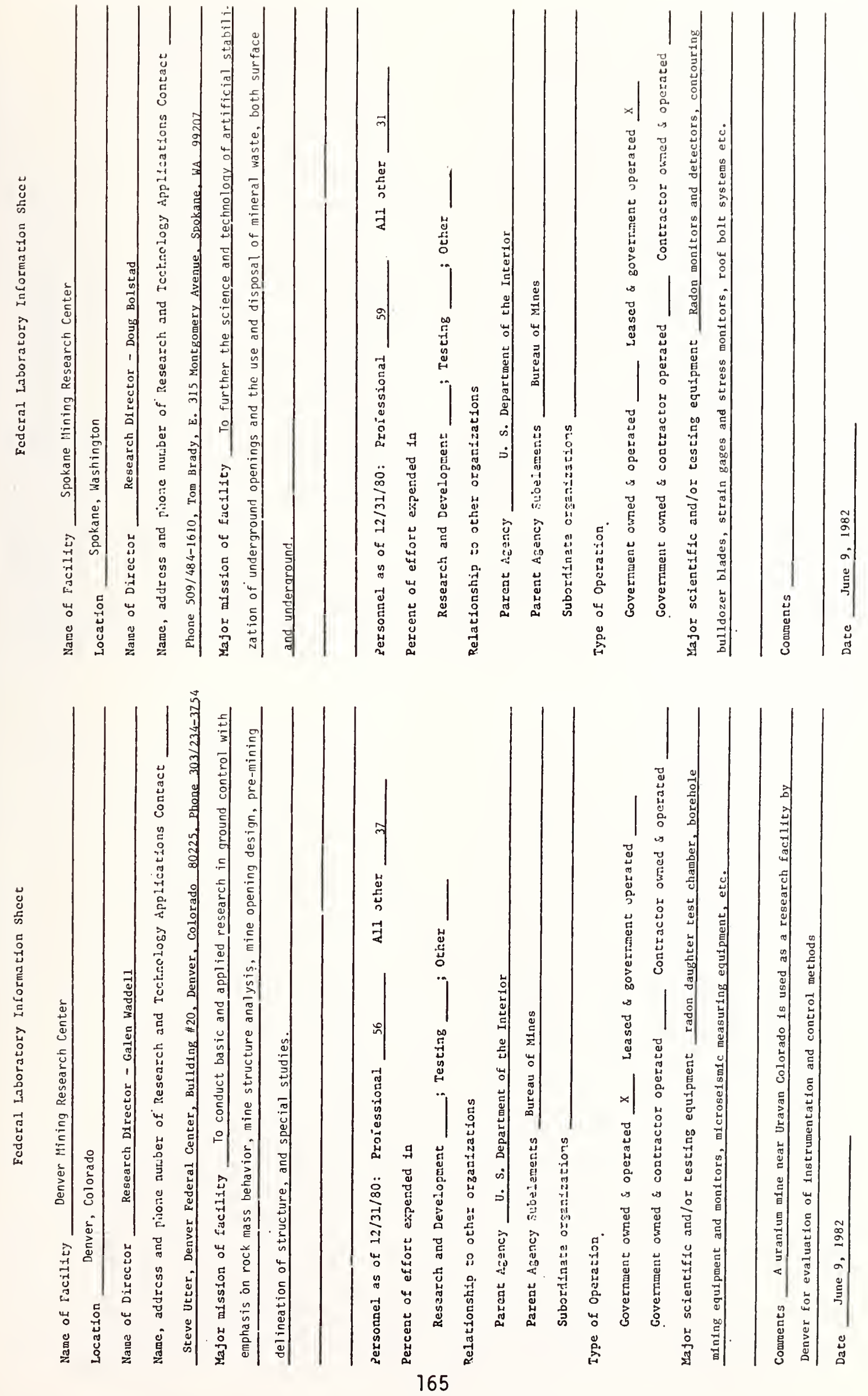

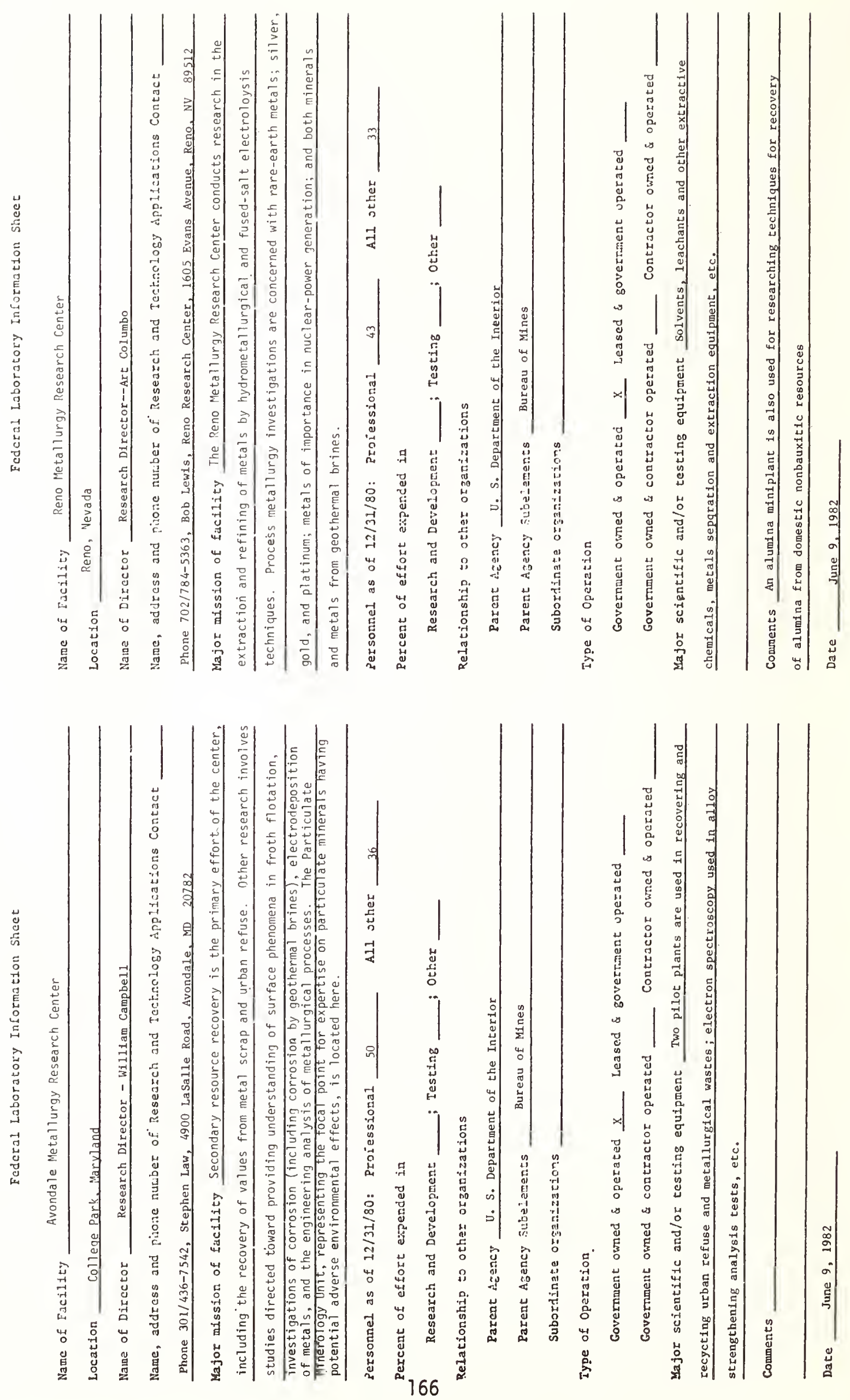

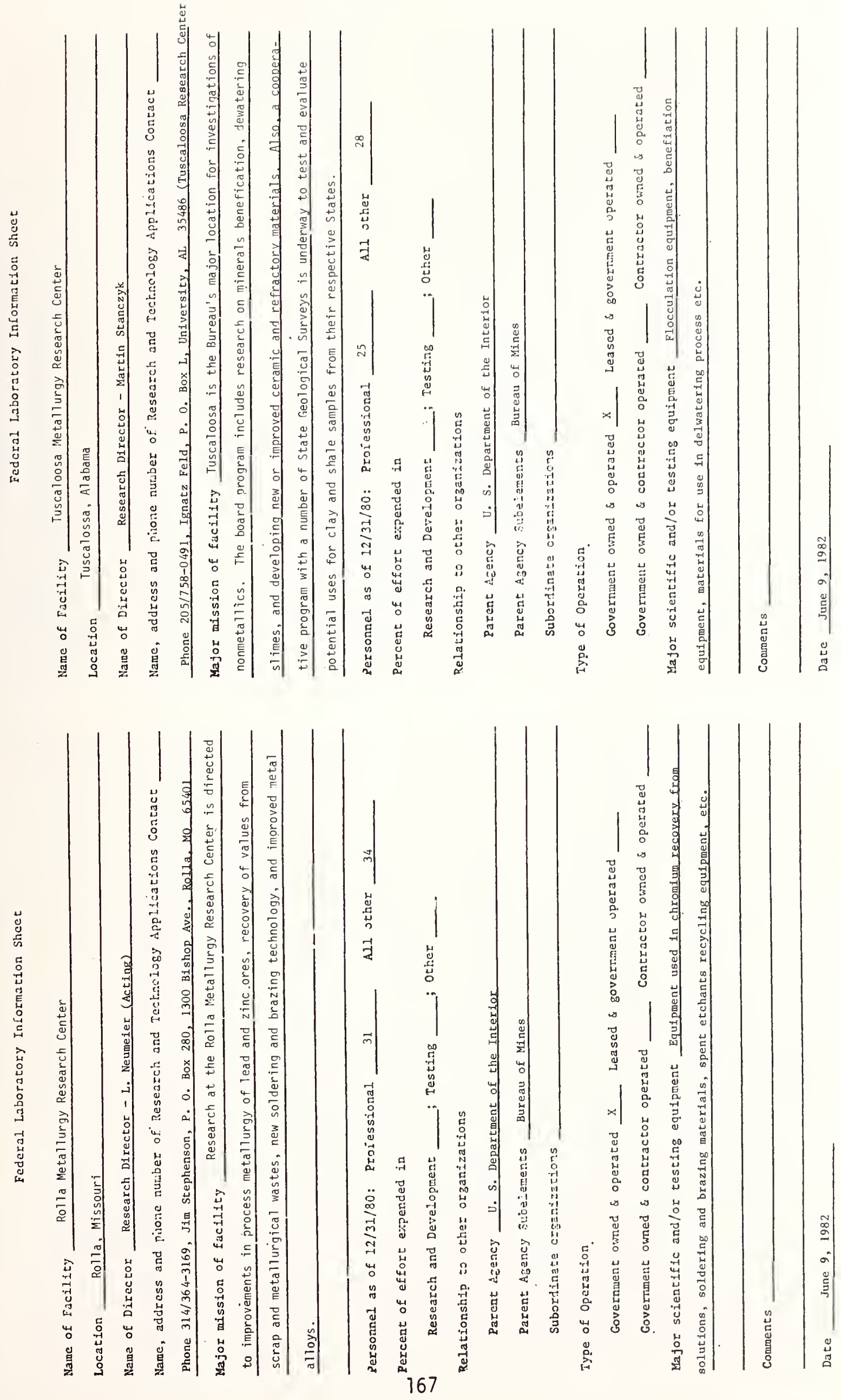

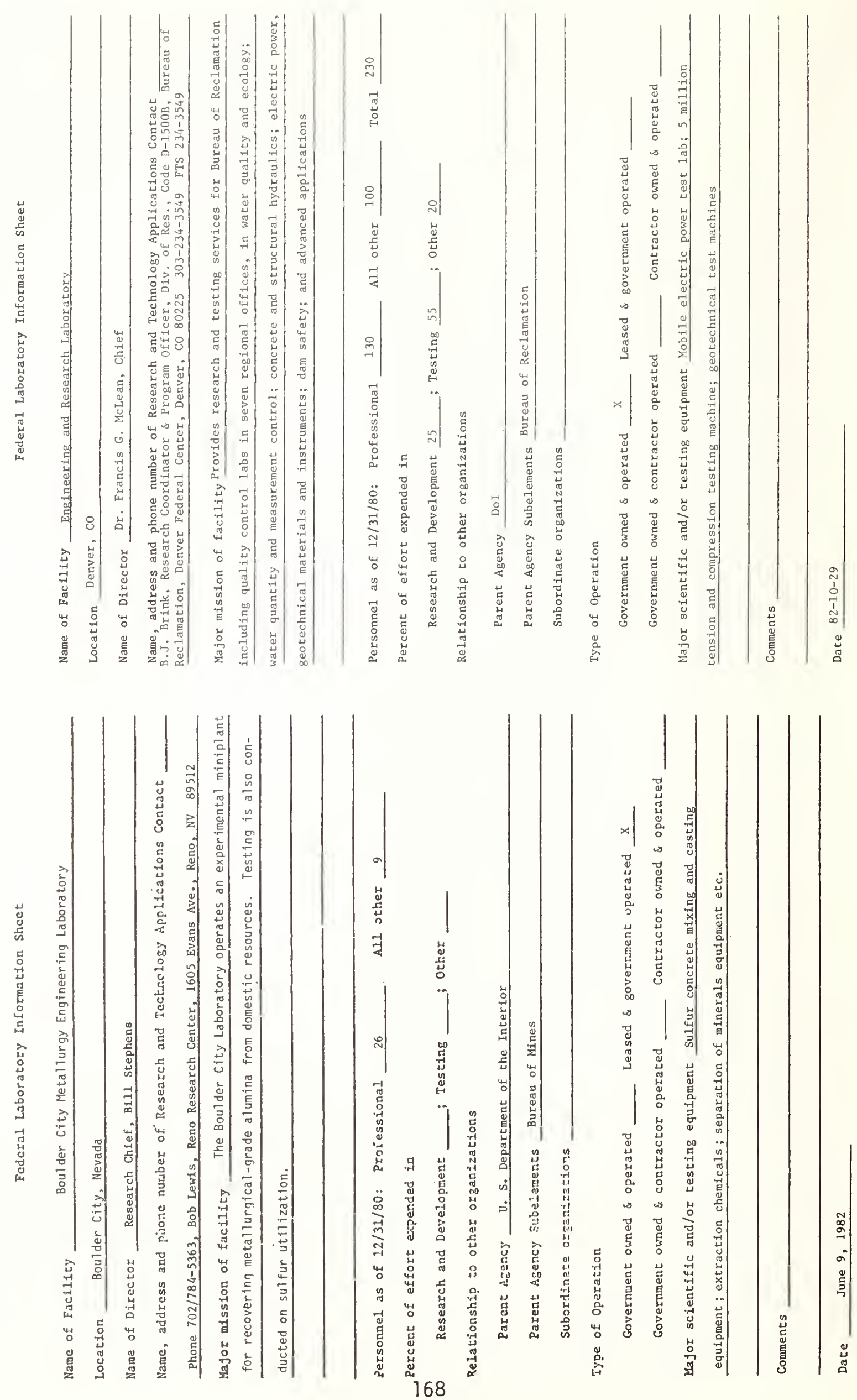

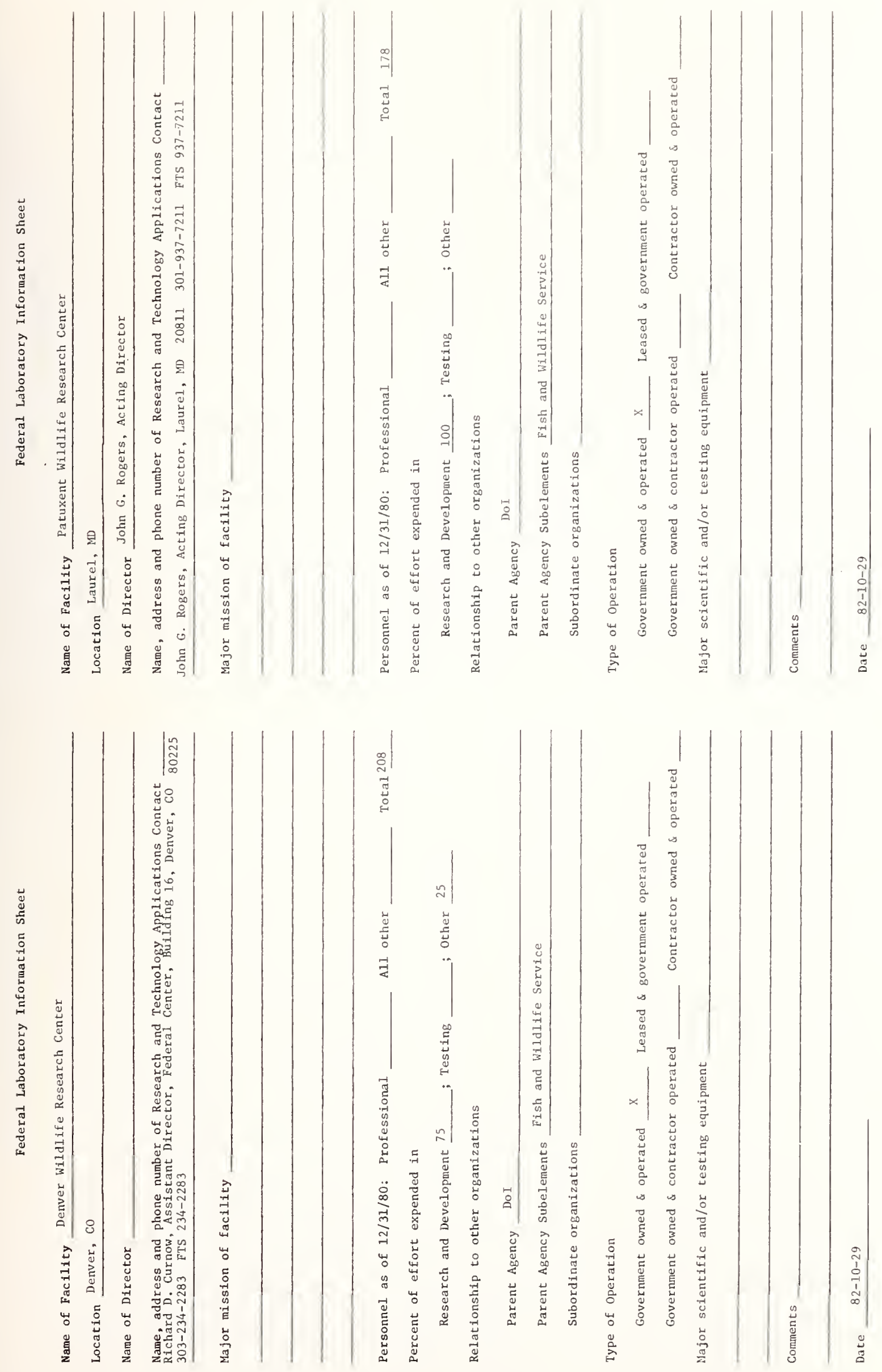

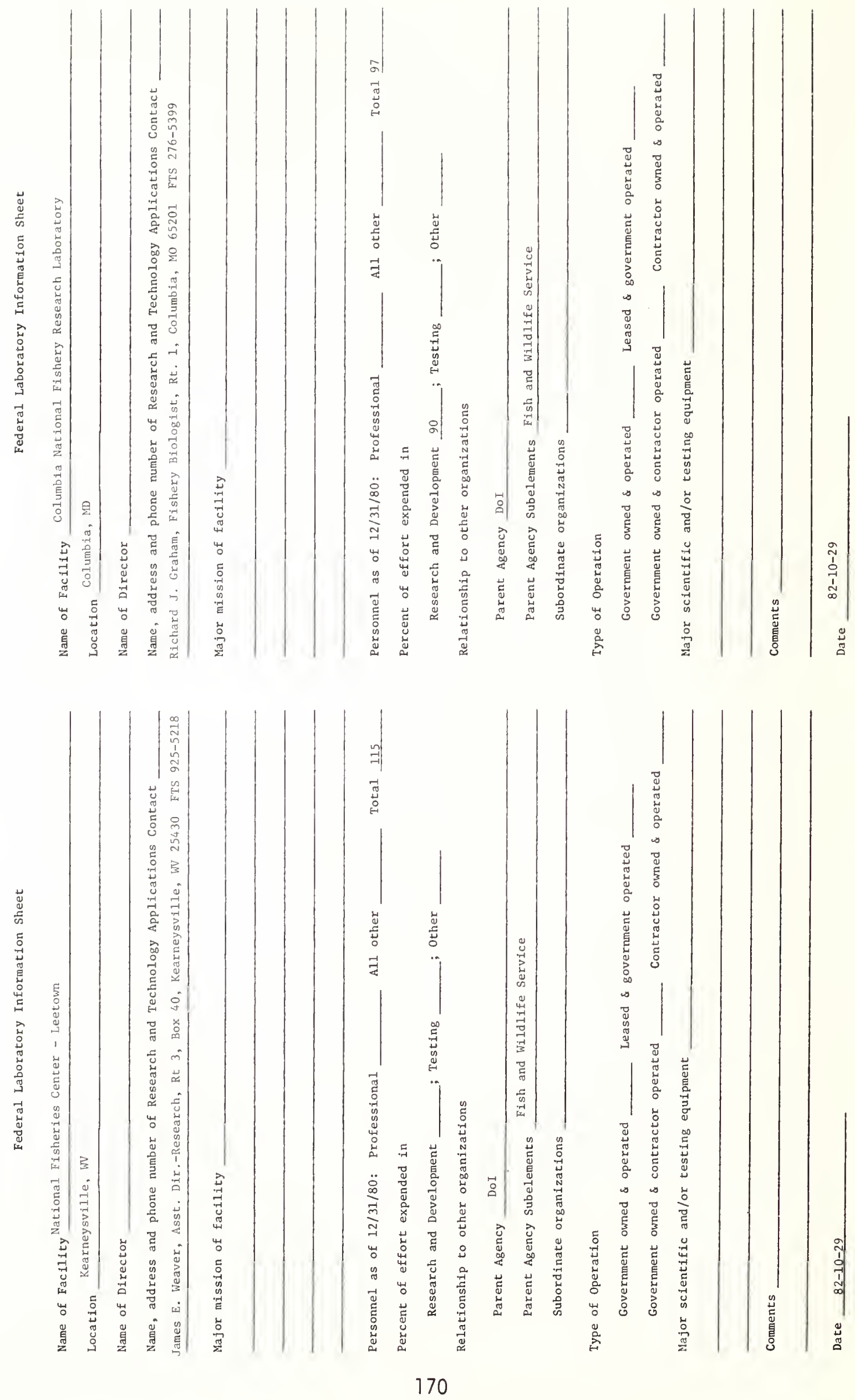

क्ञ 

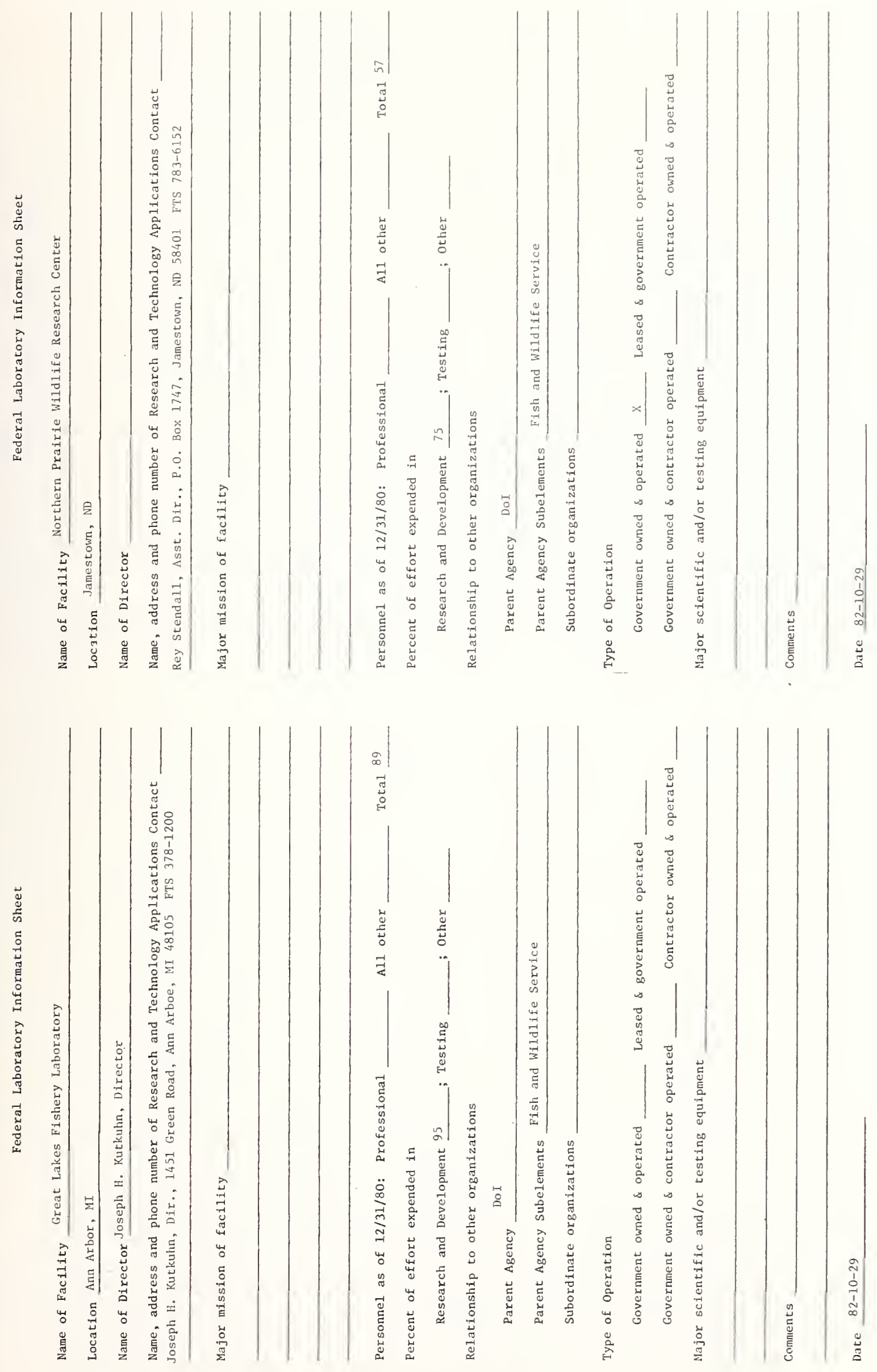

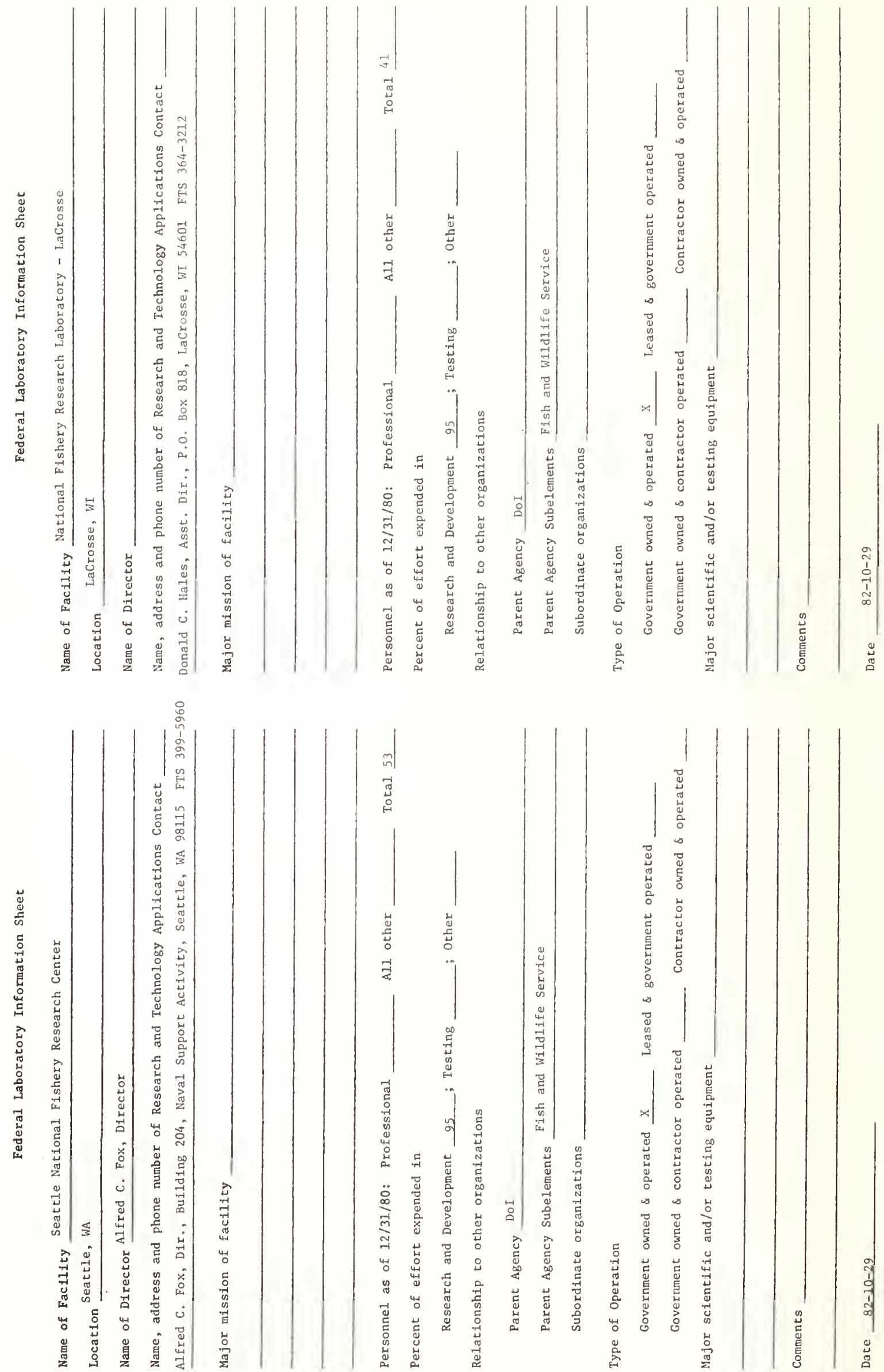

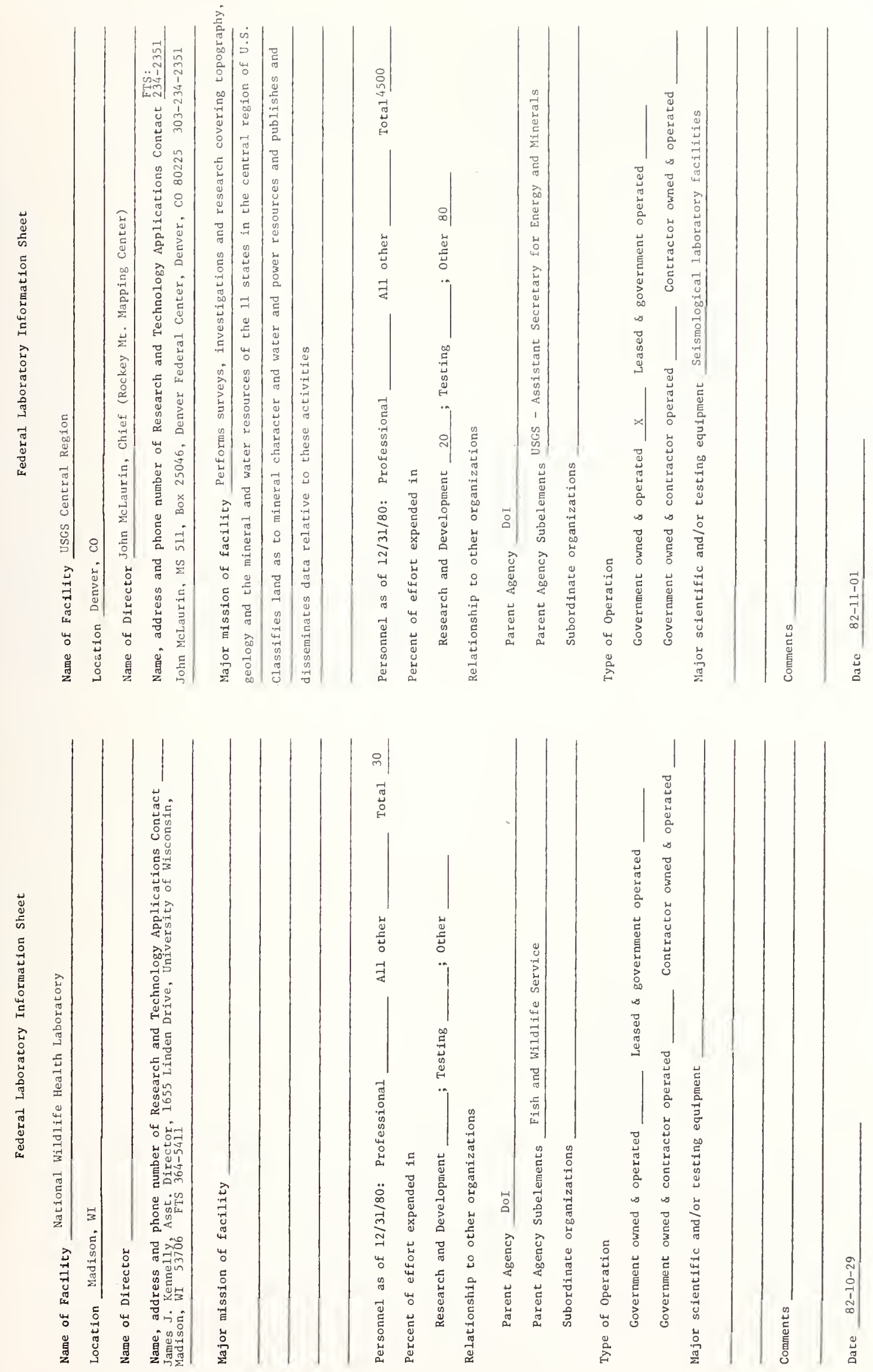

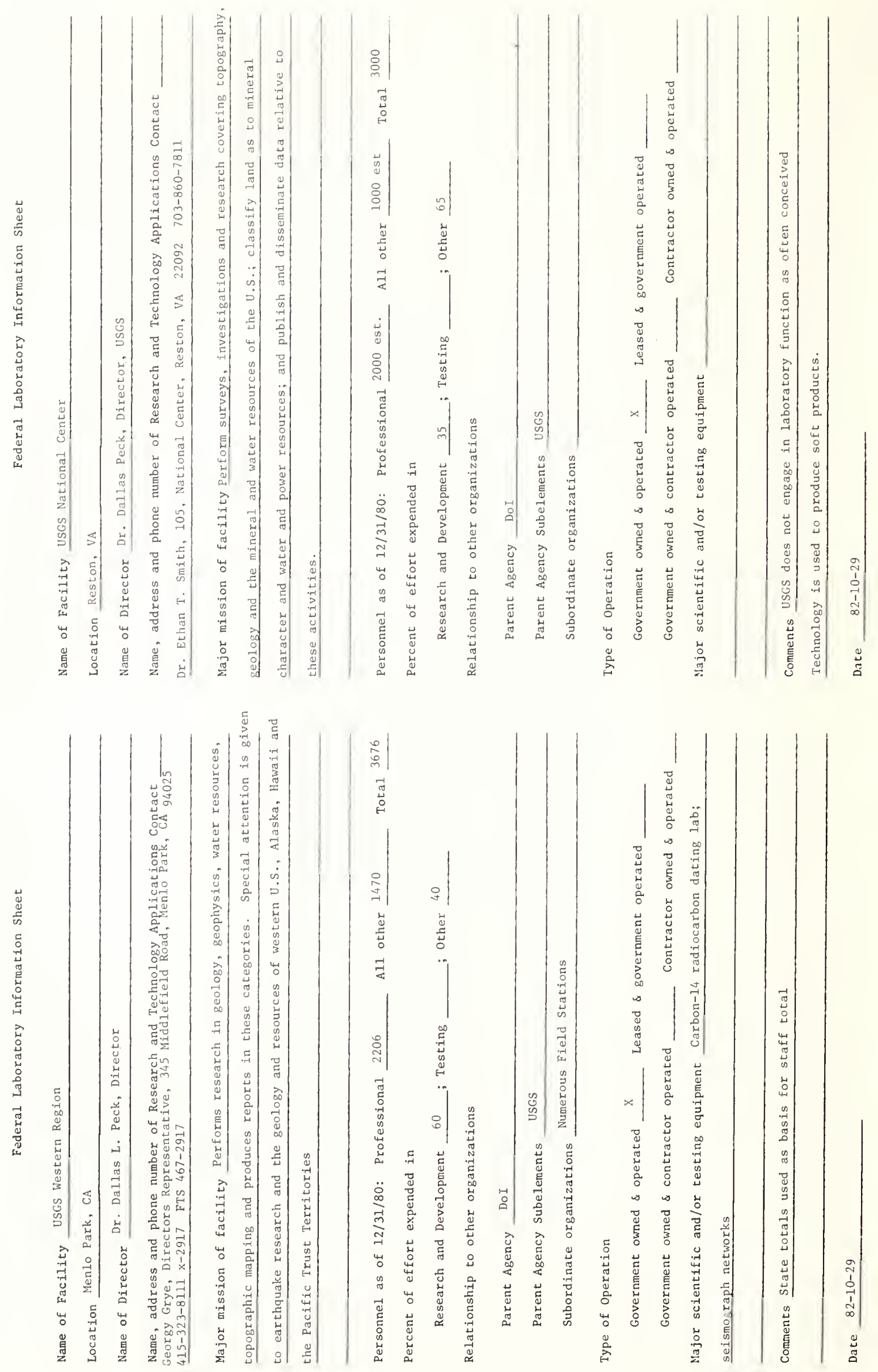
DEPARTMENT OF JUSTICE 



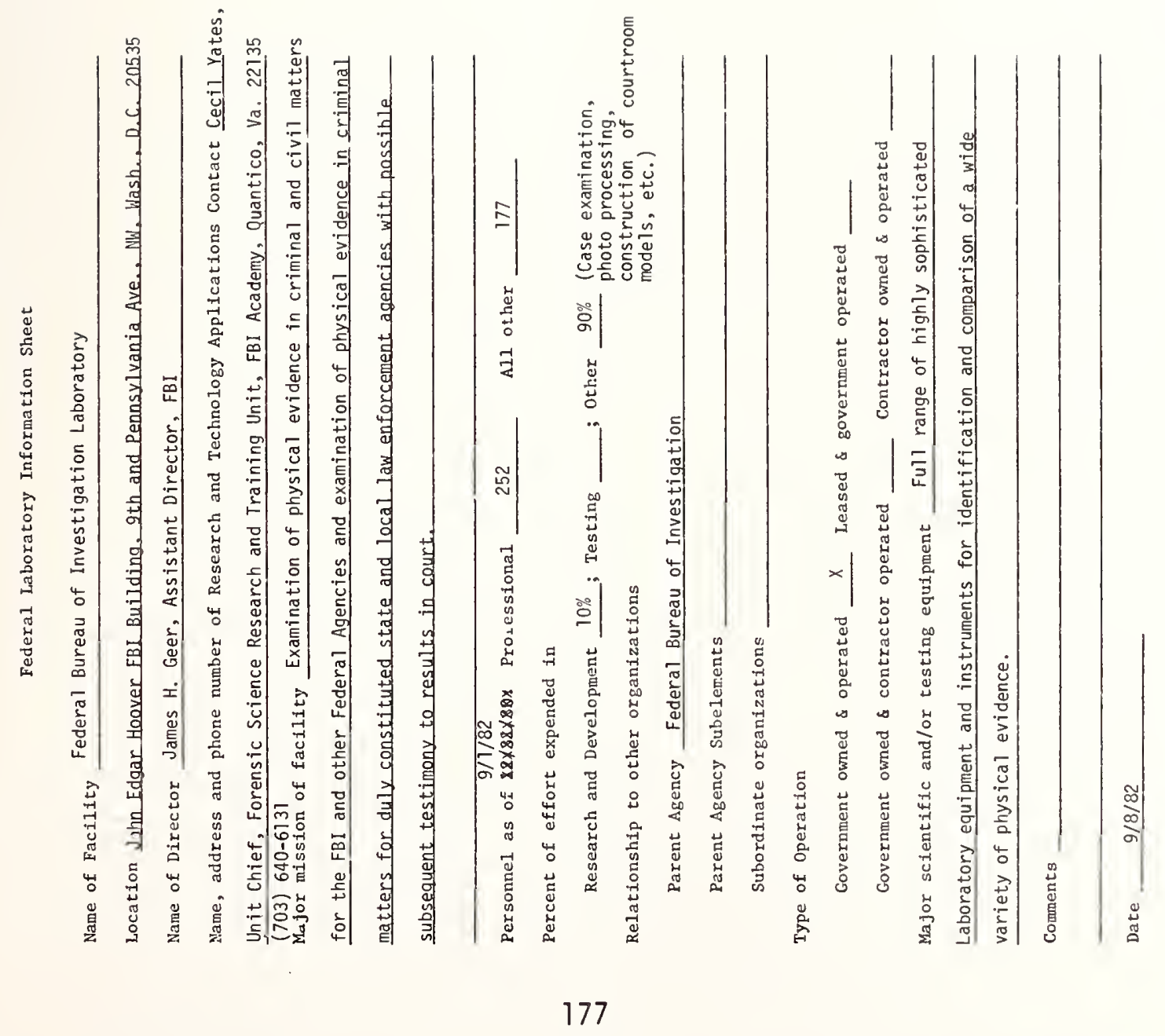



DEPARTMENT OF 


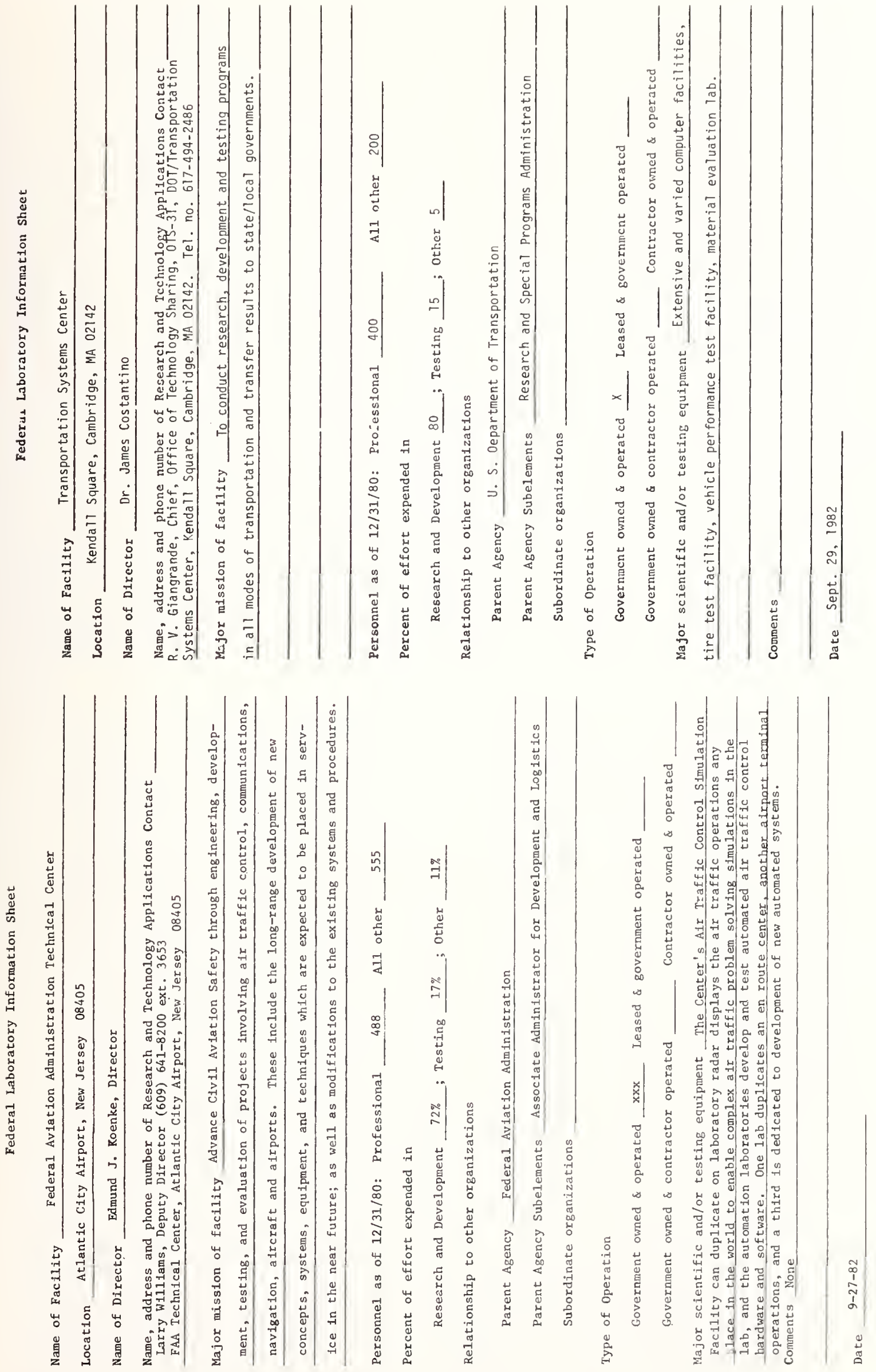

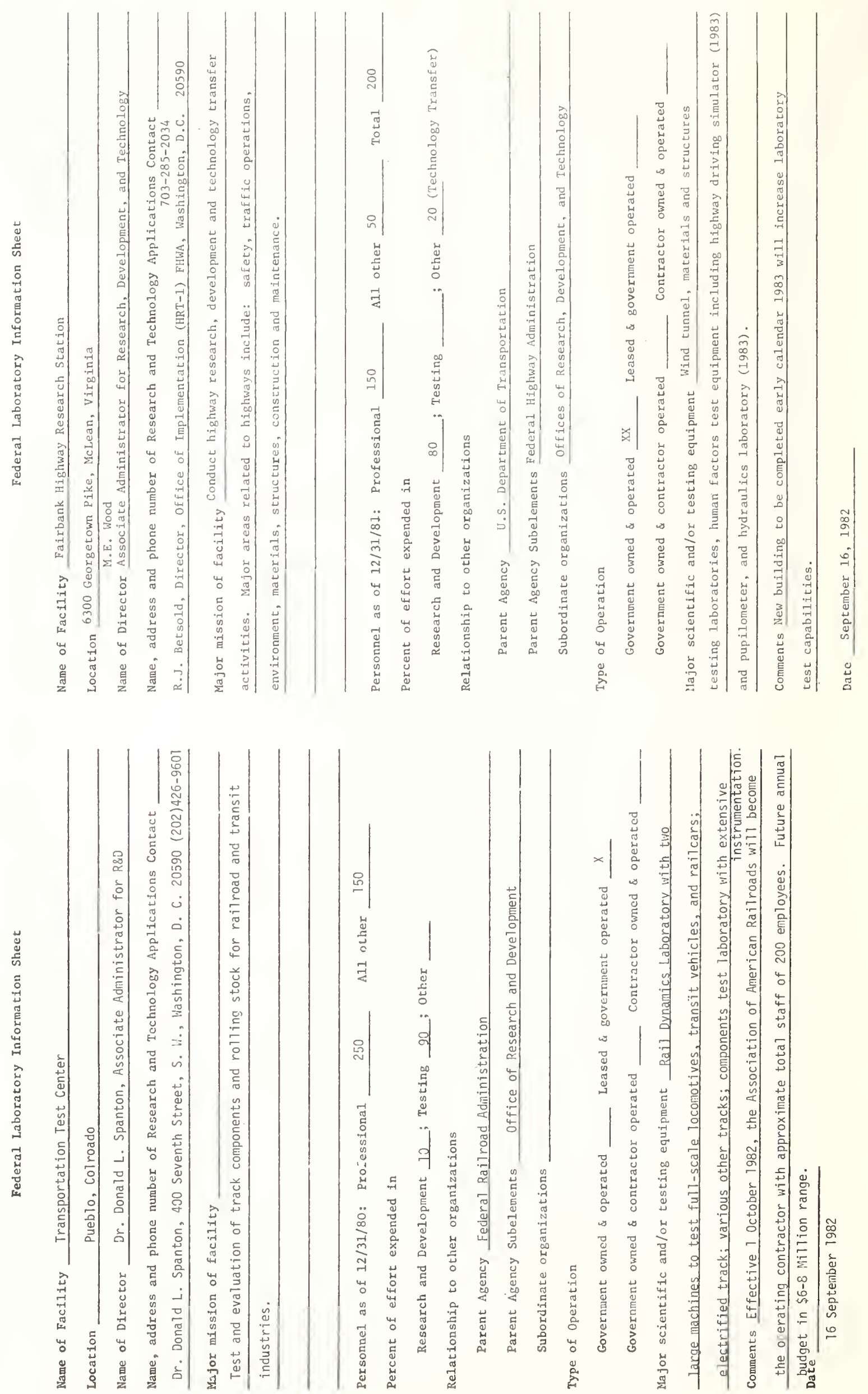

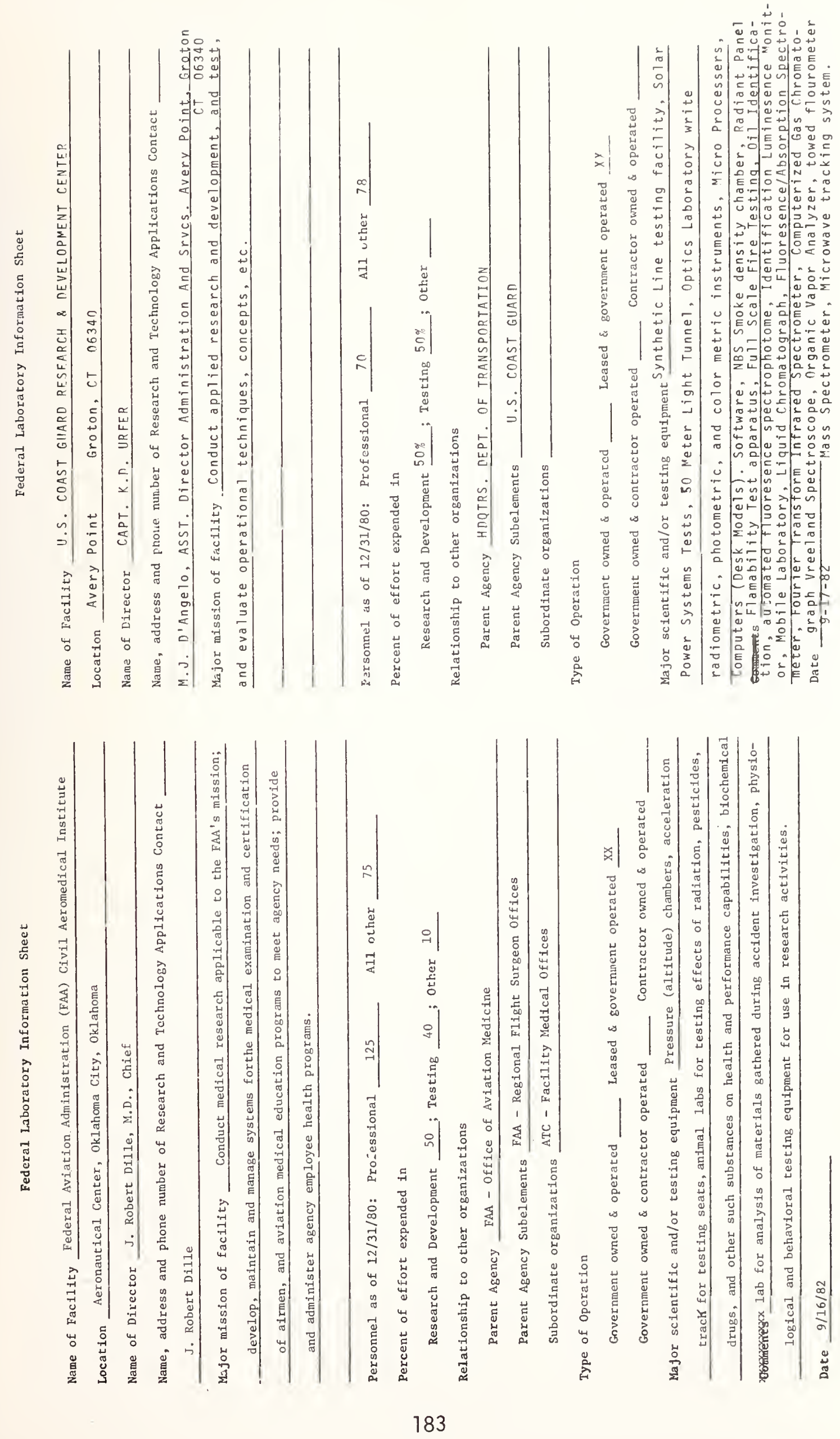


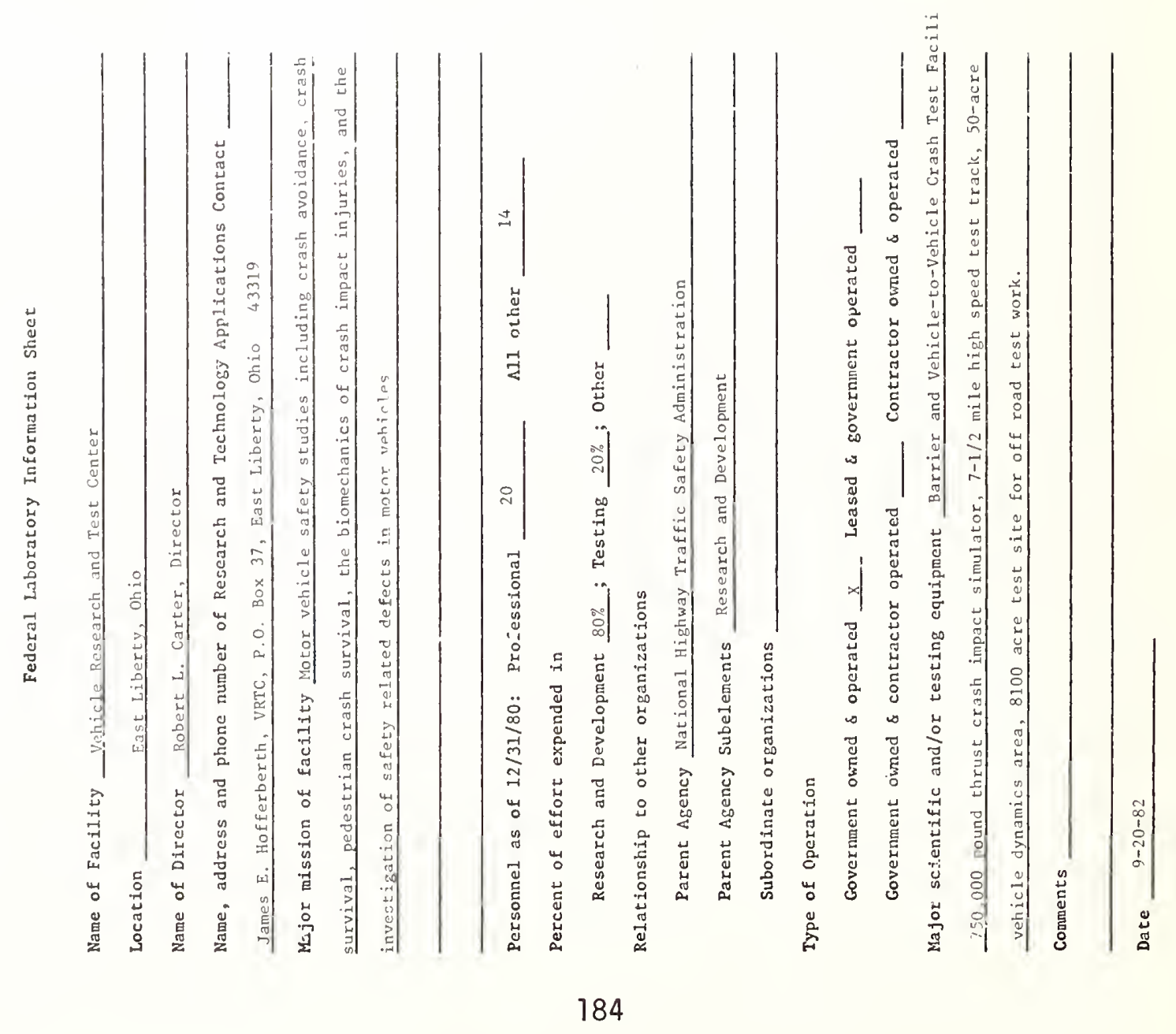




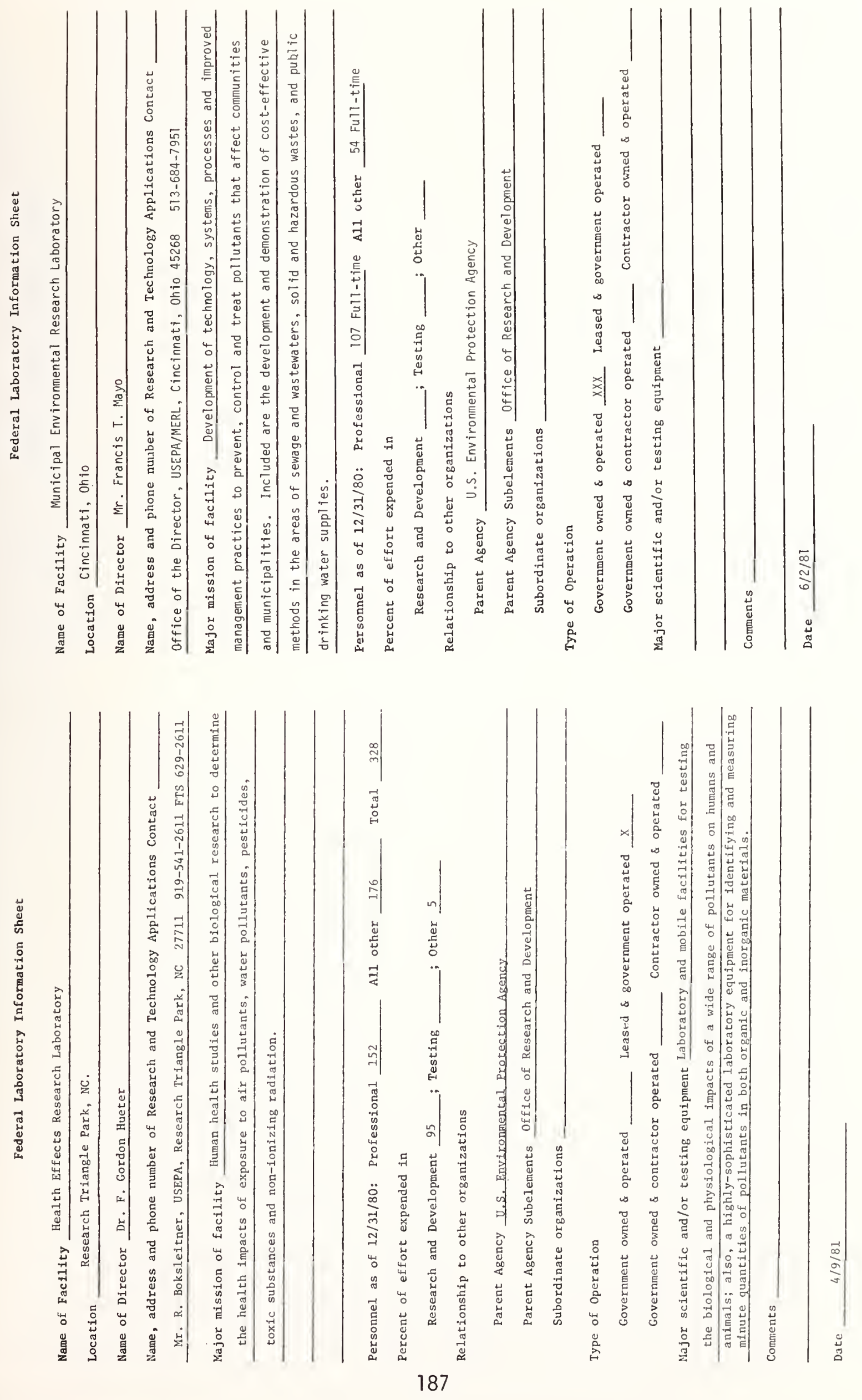

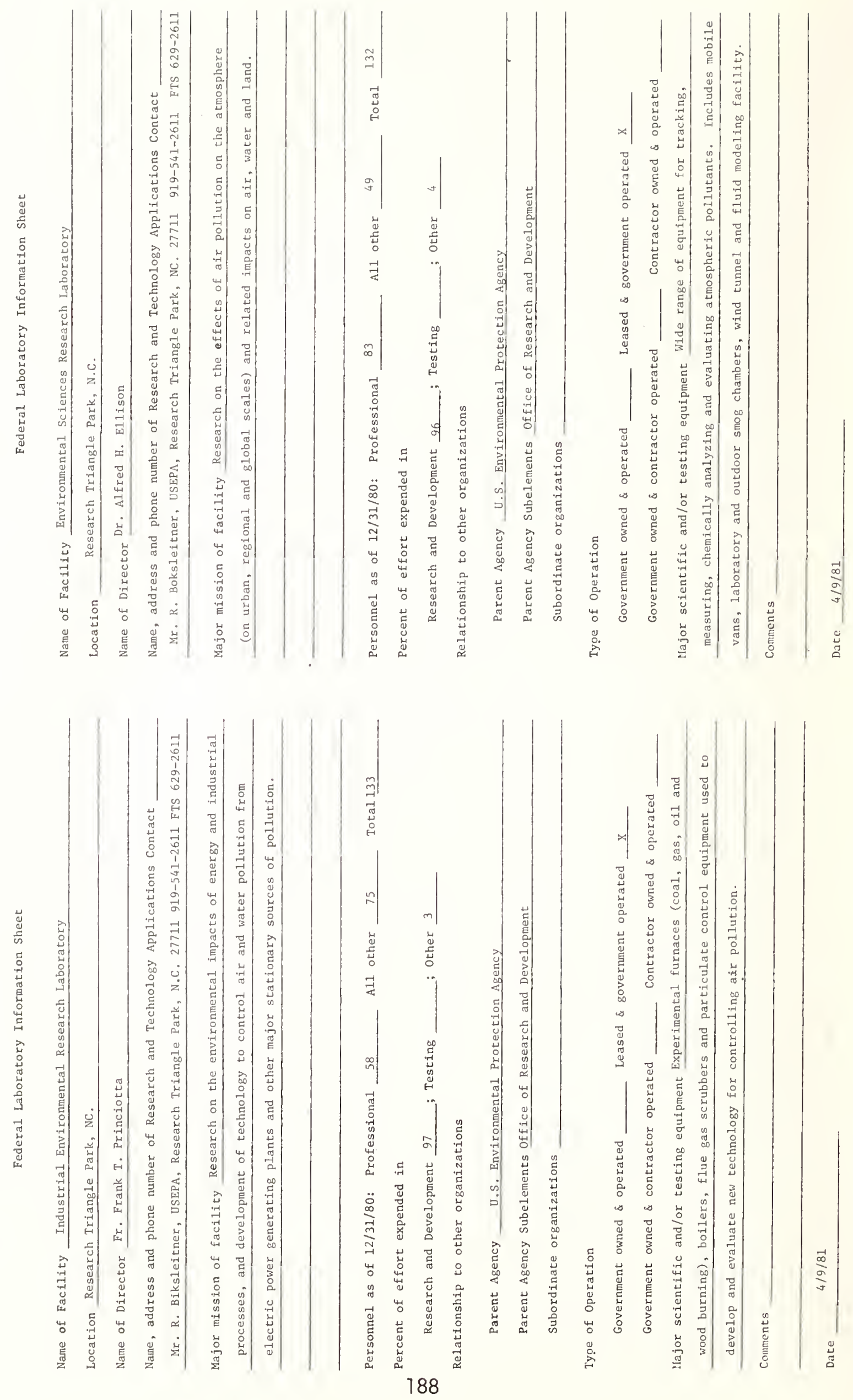

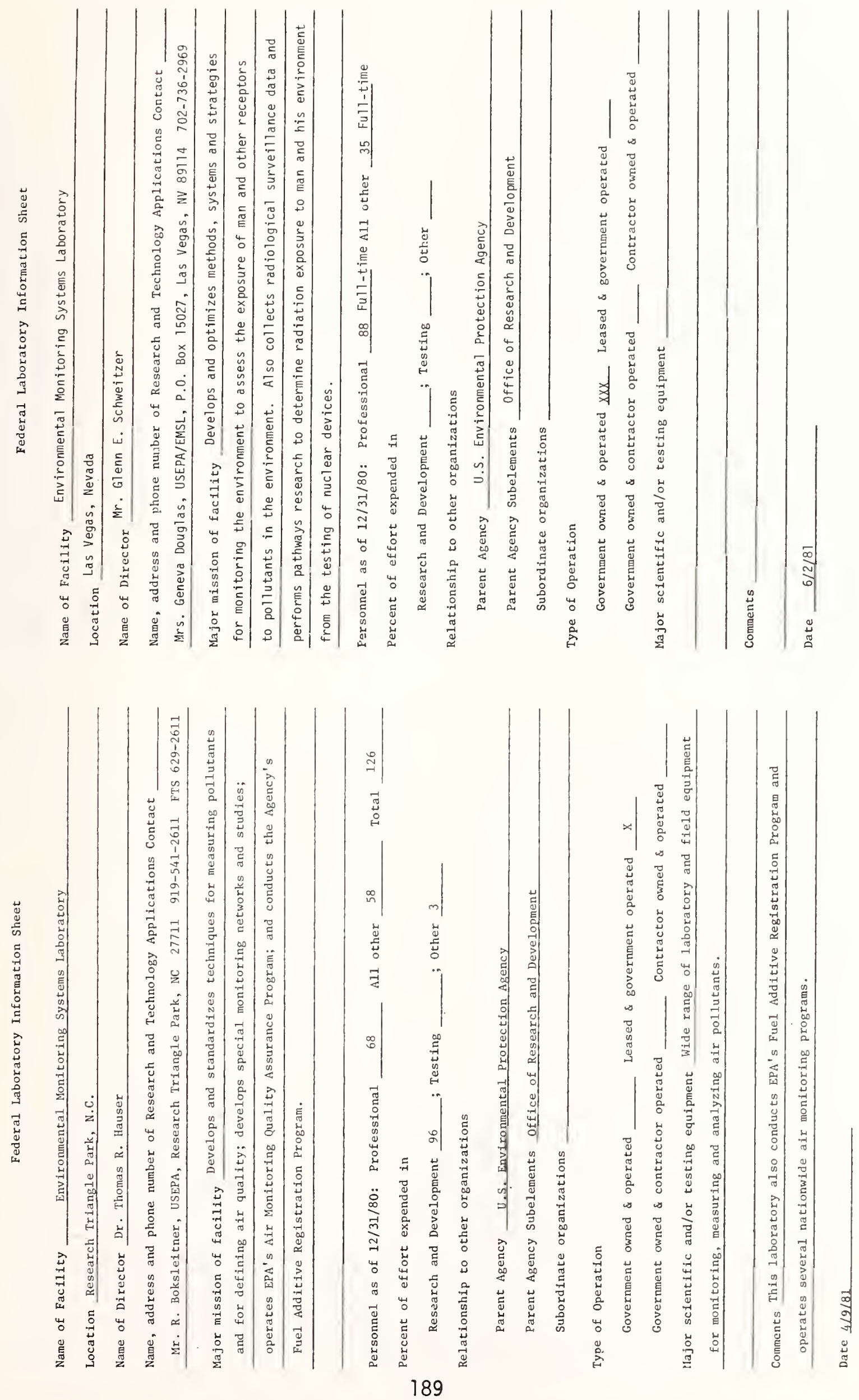

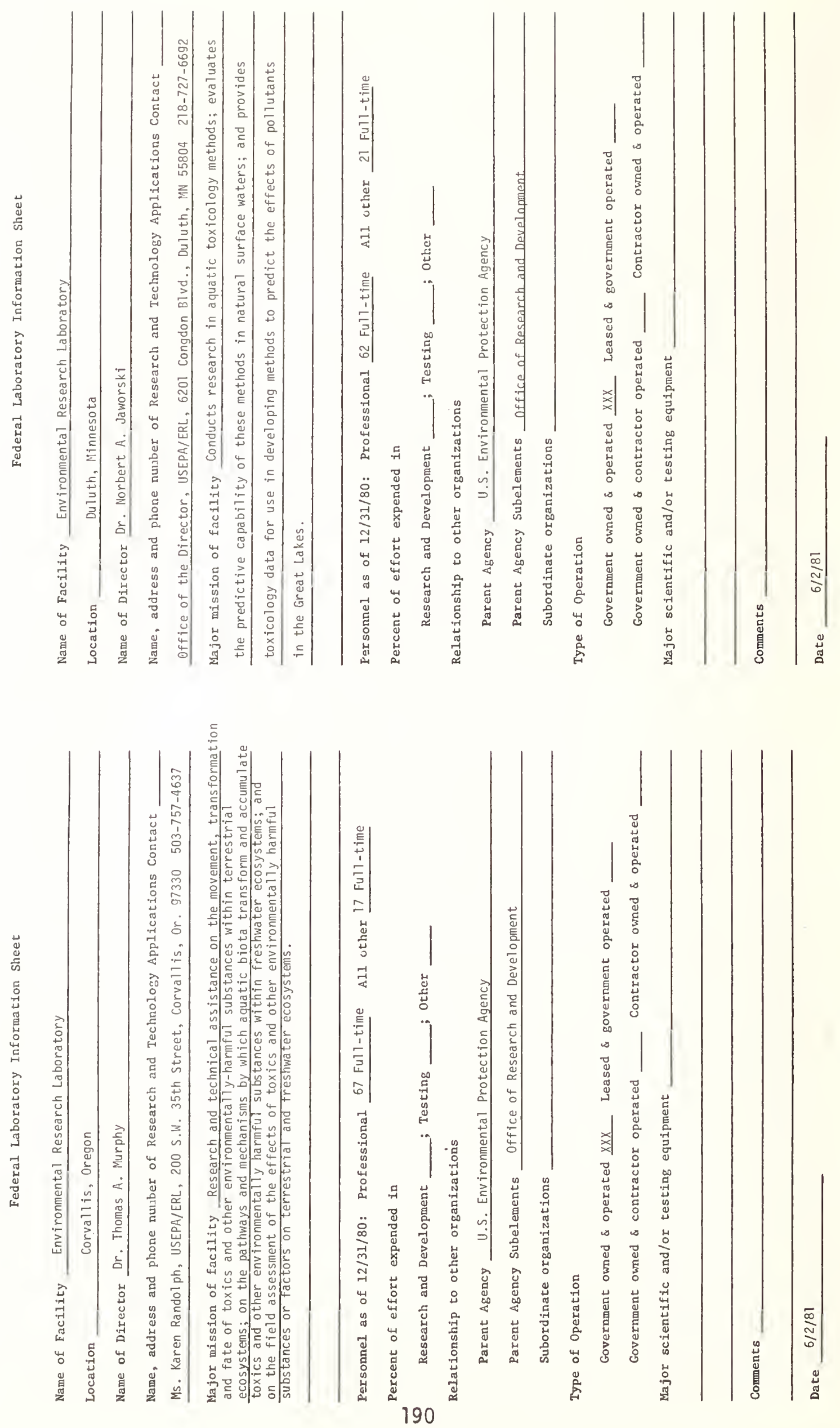

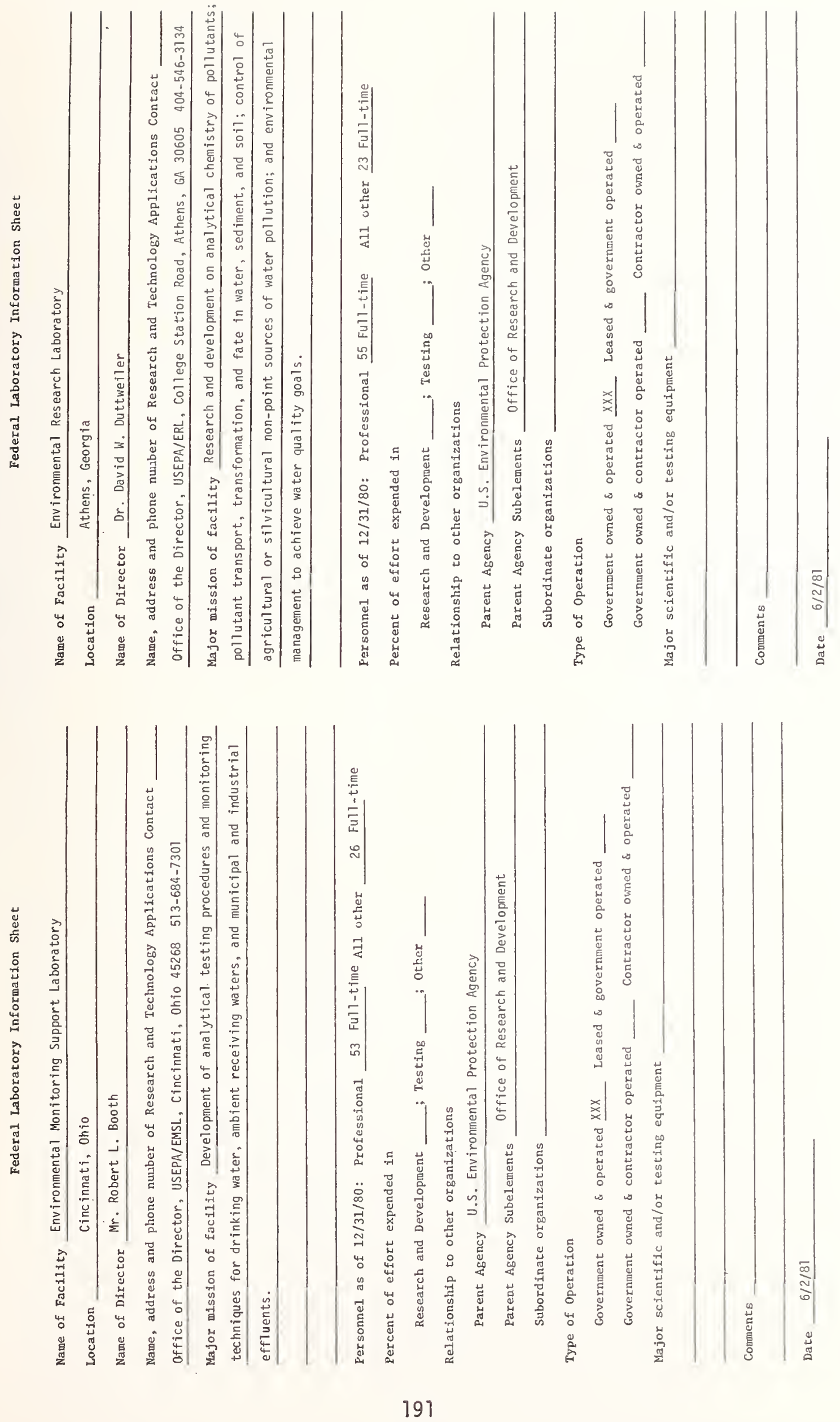

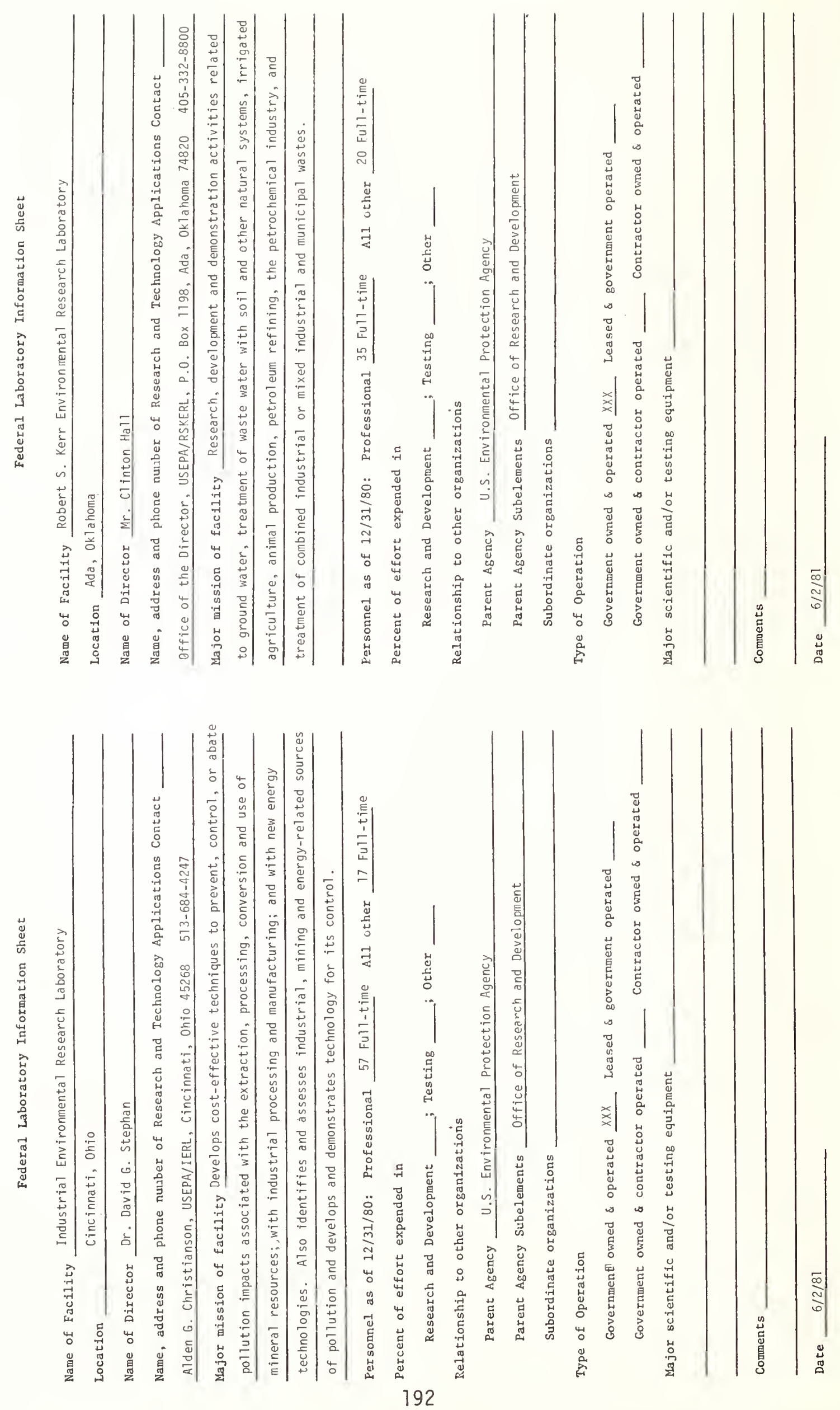

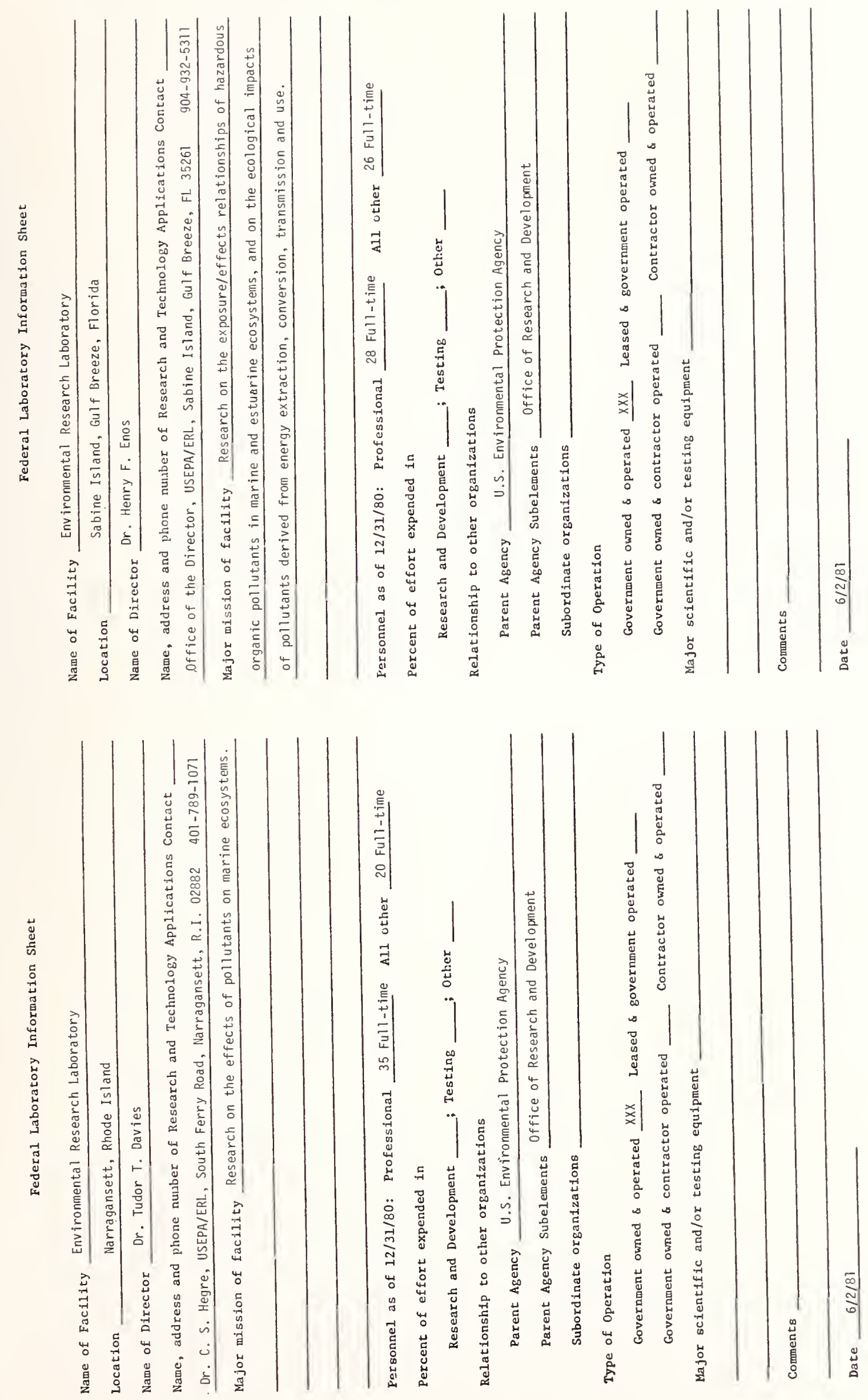

HEALTH AND

HUMAN SERVICES 


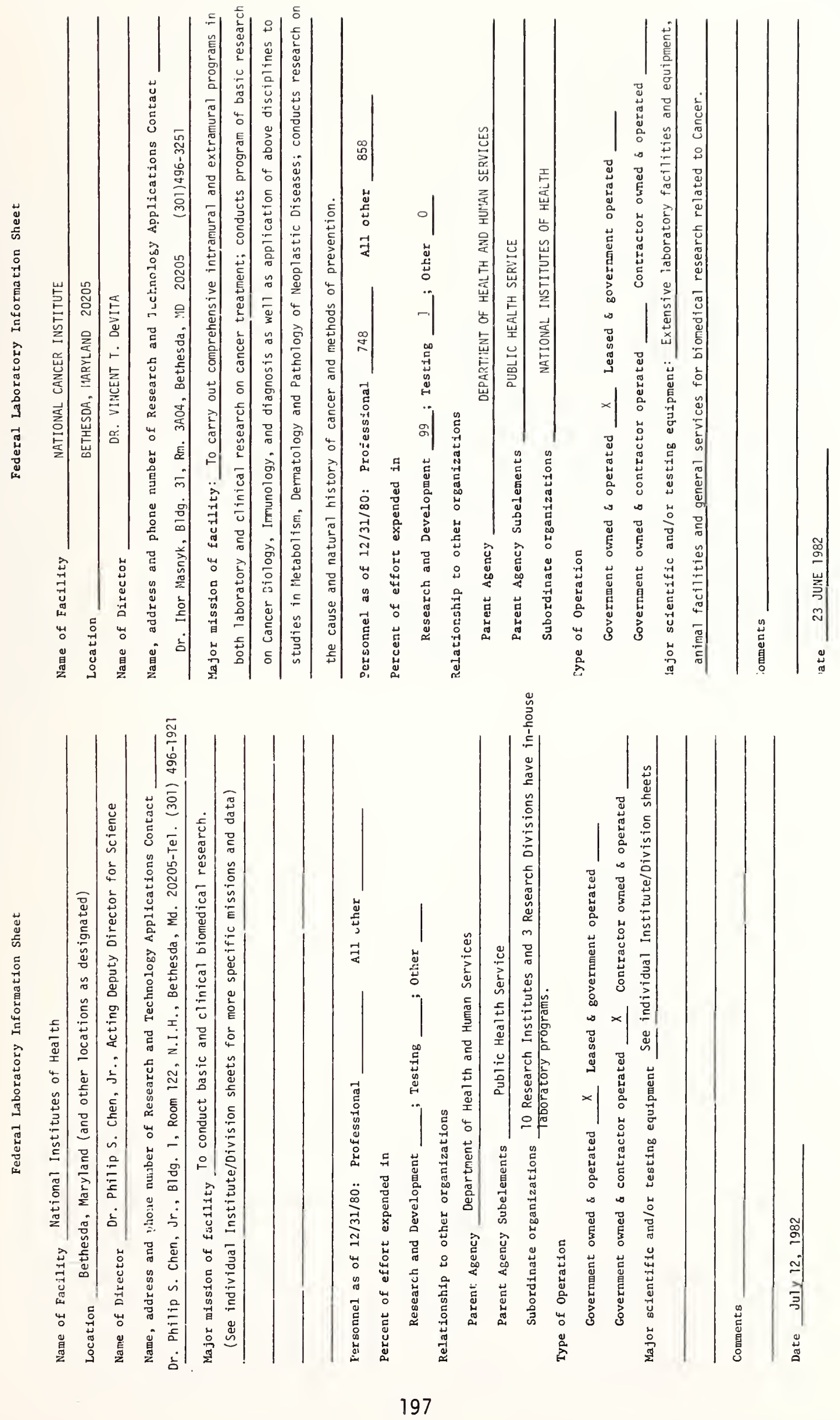

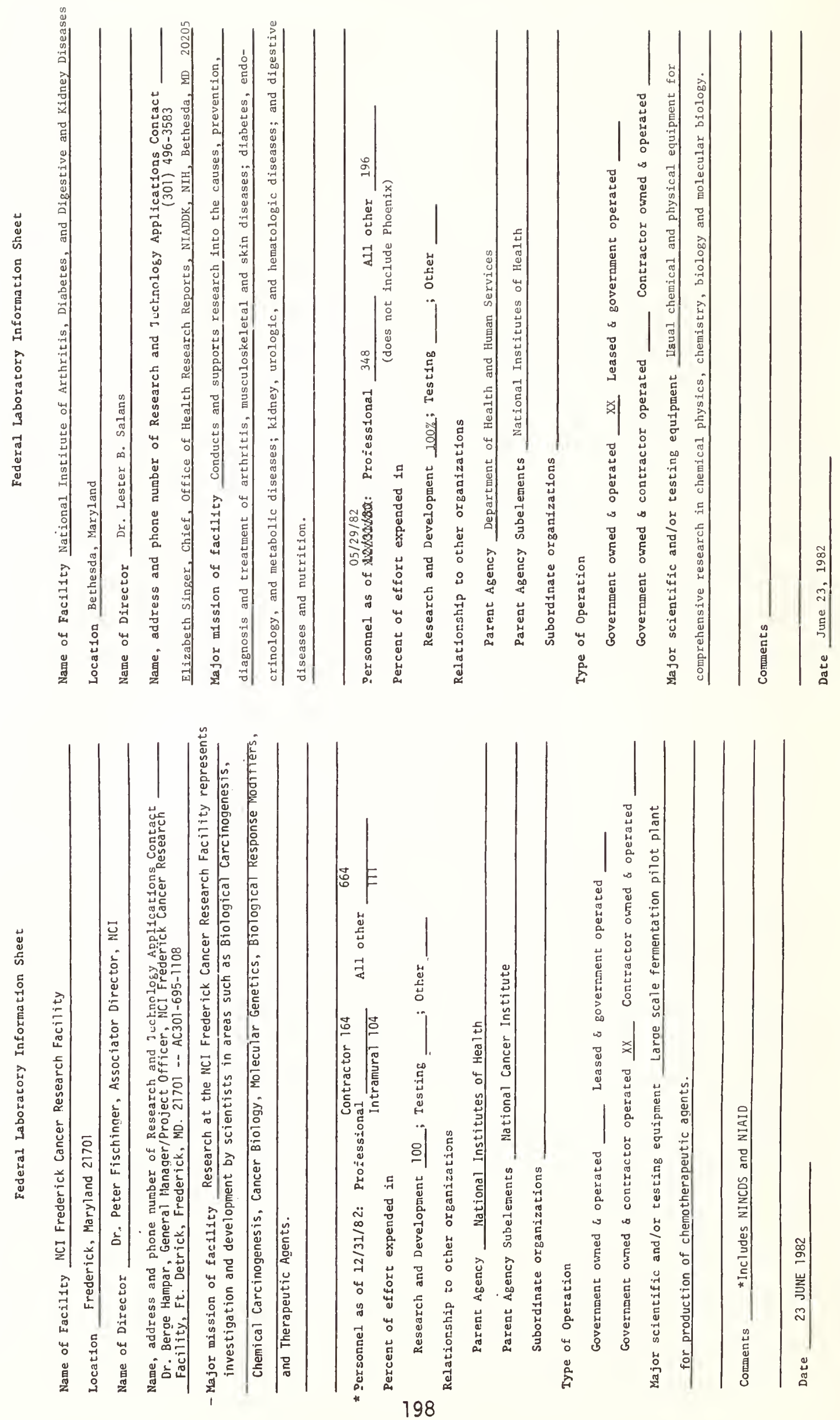

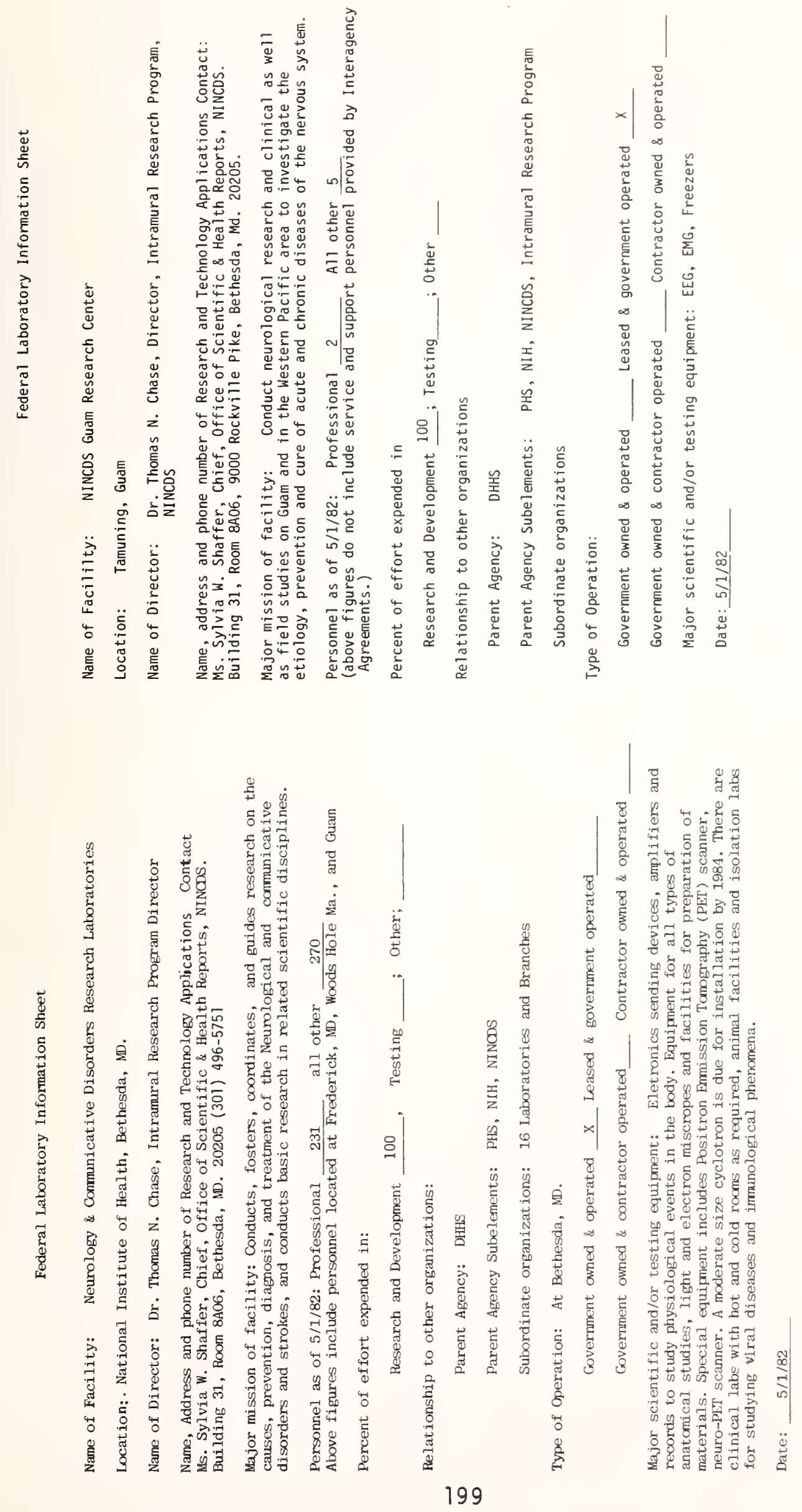

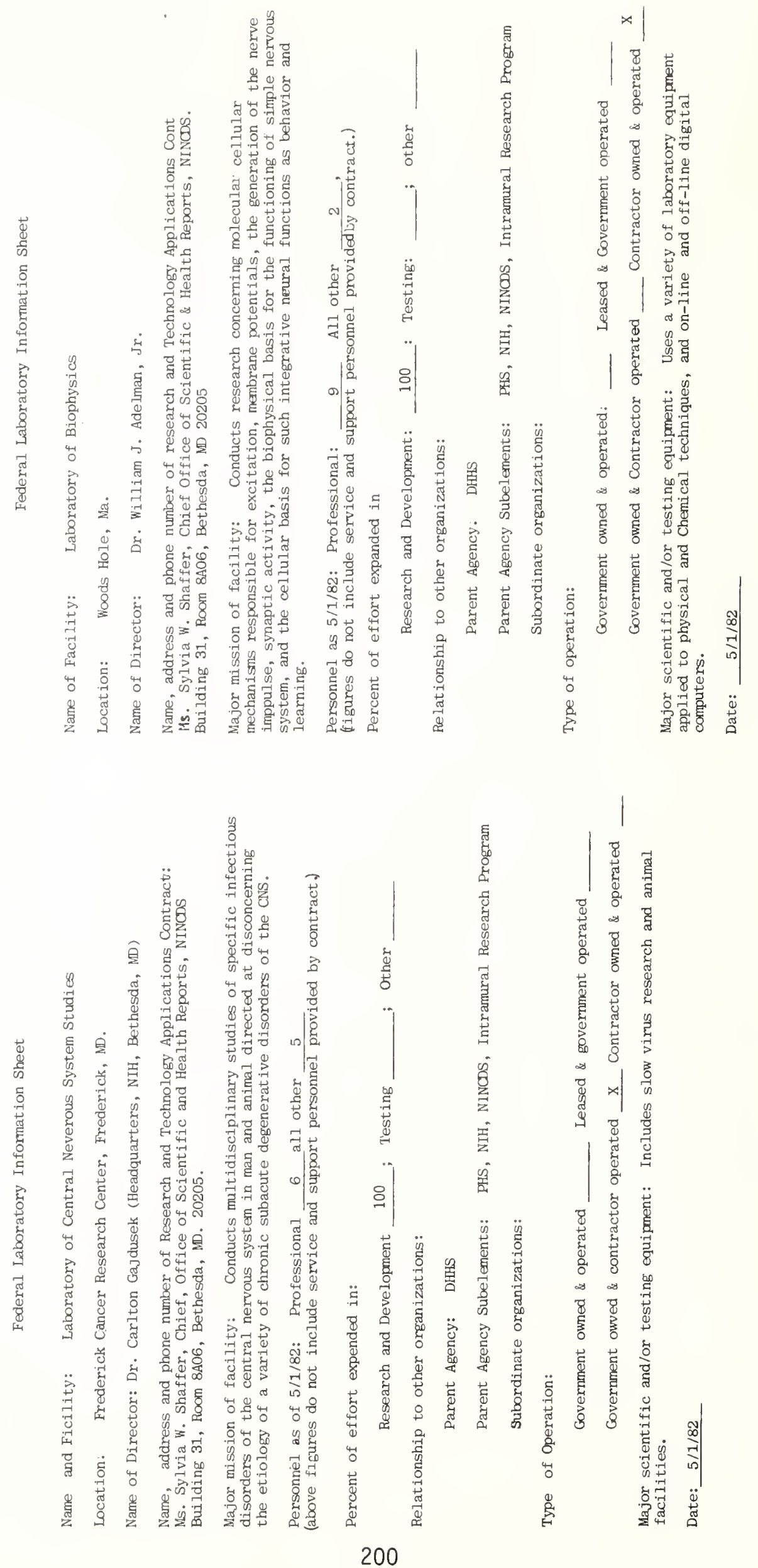

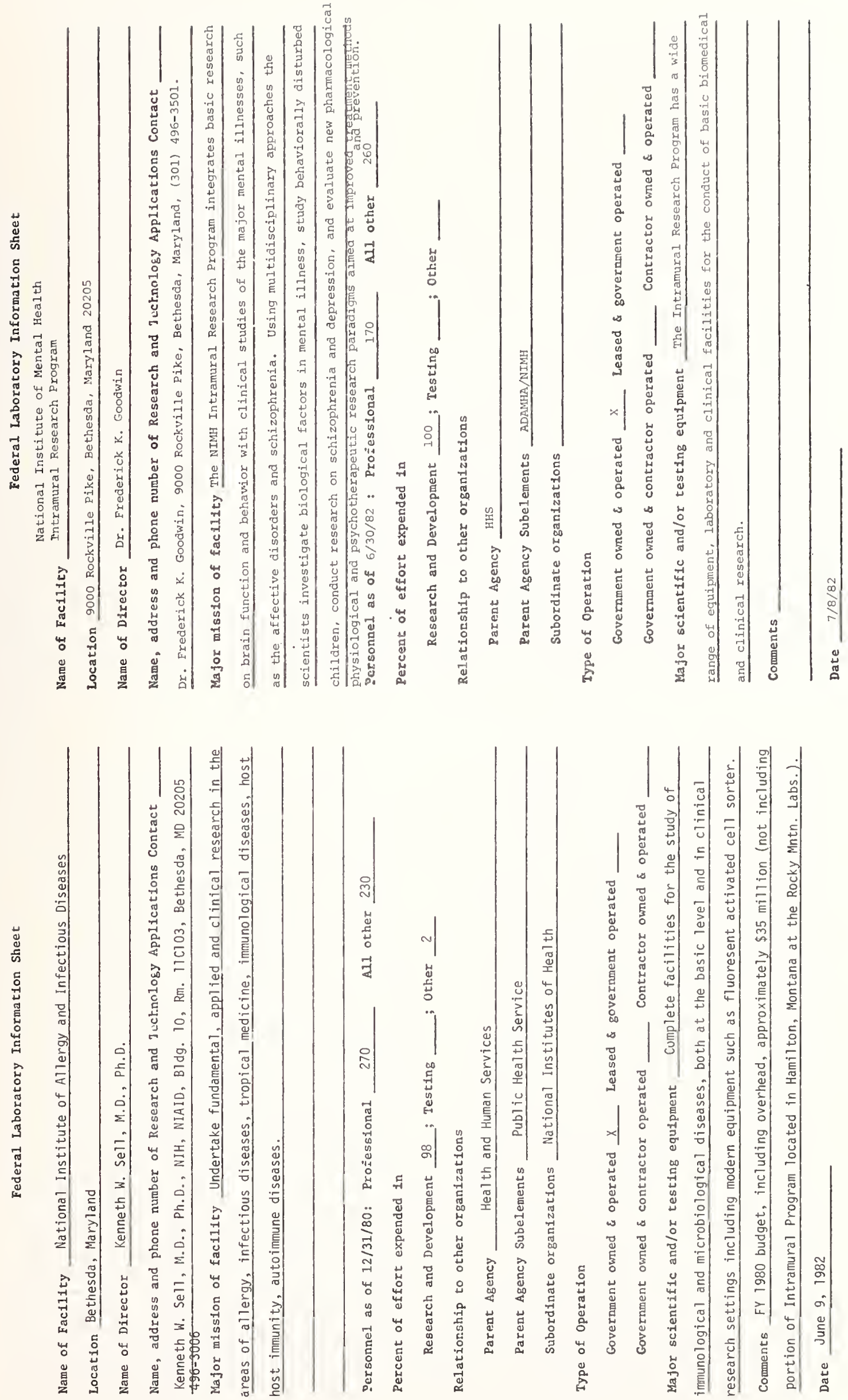

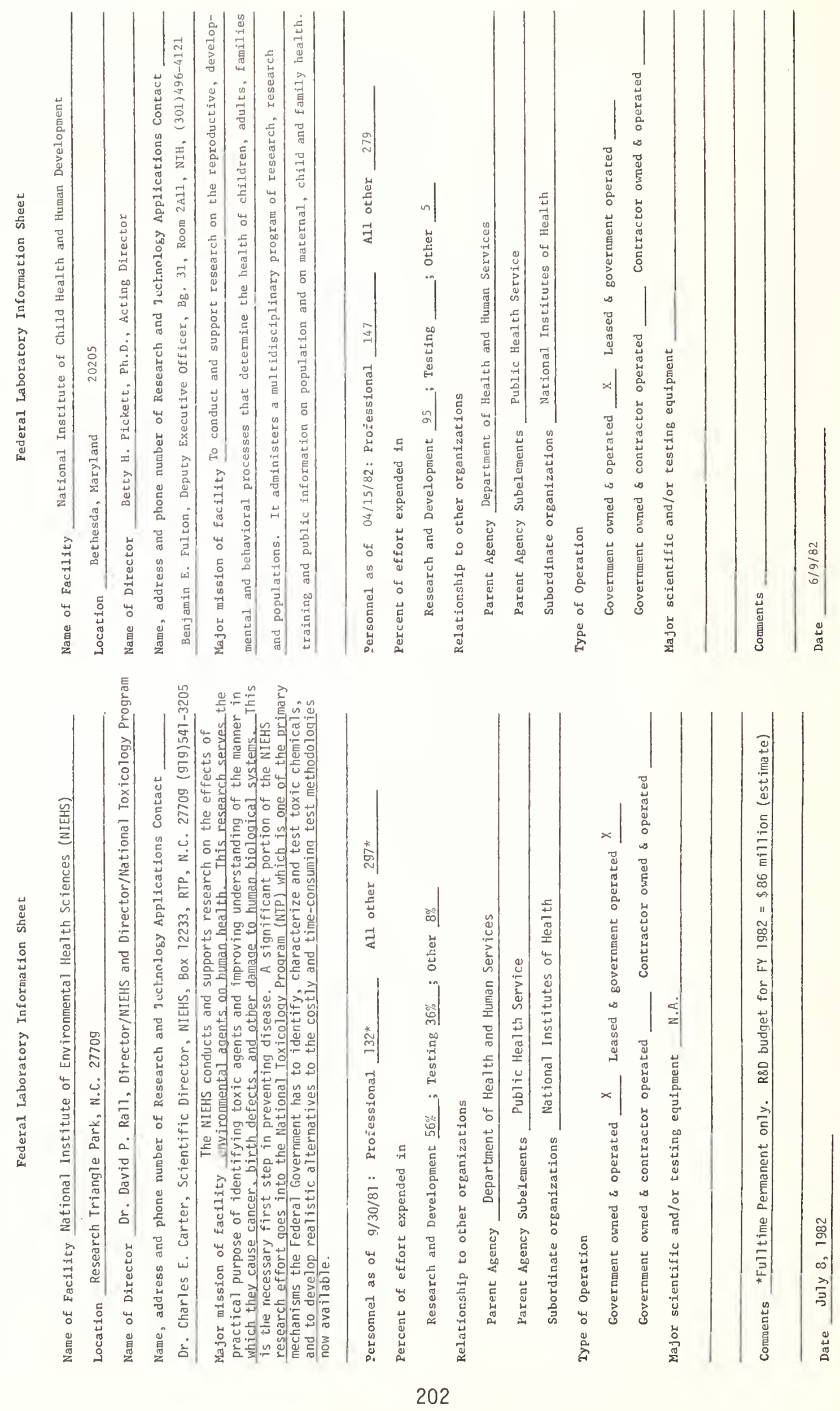

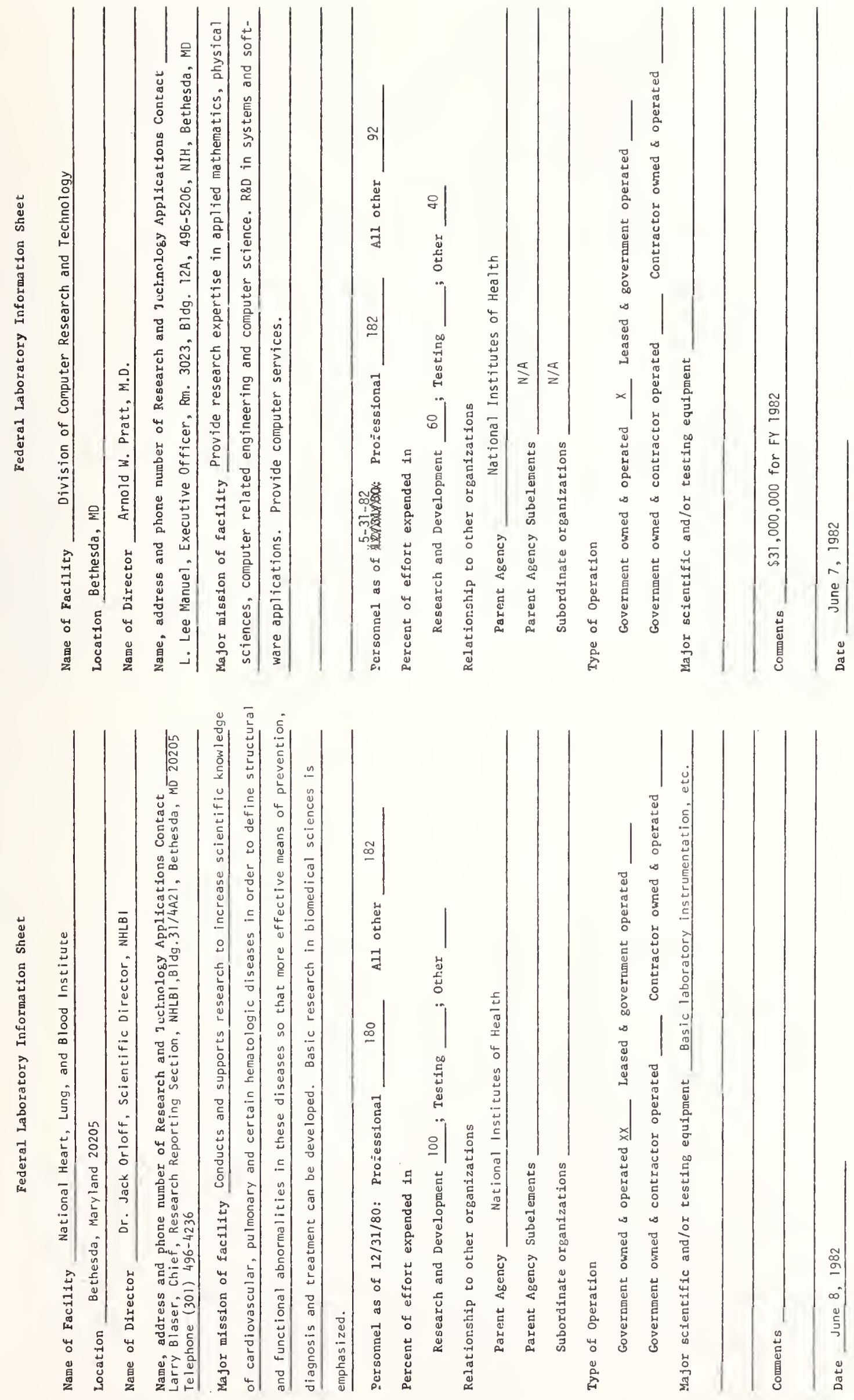

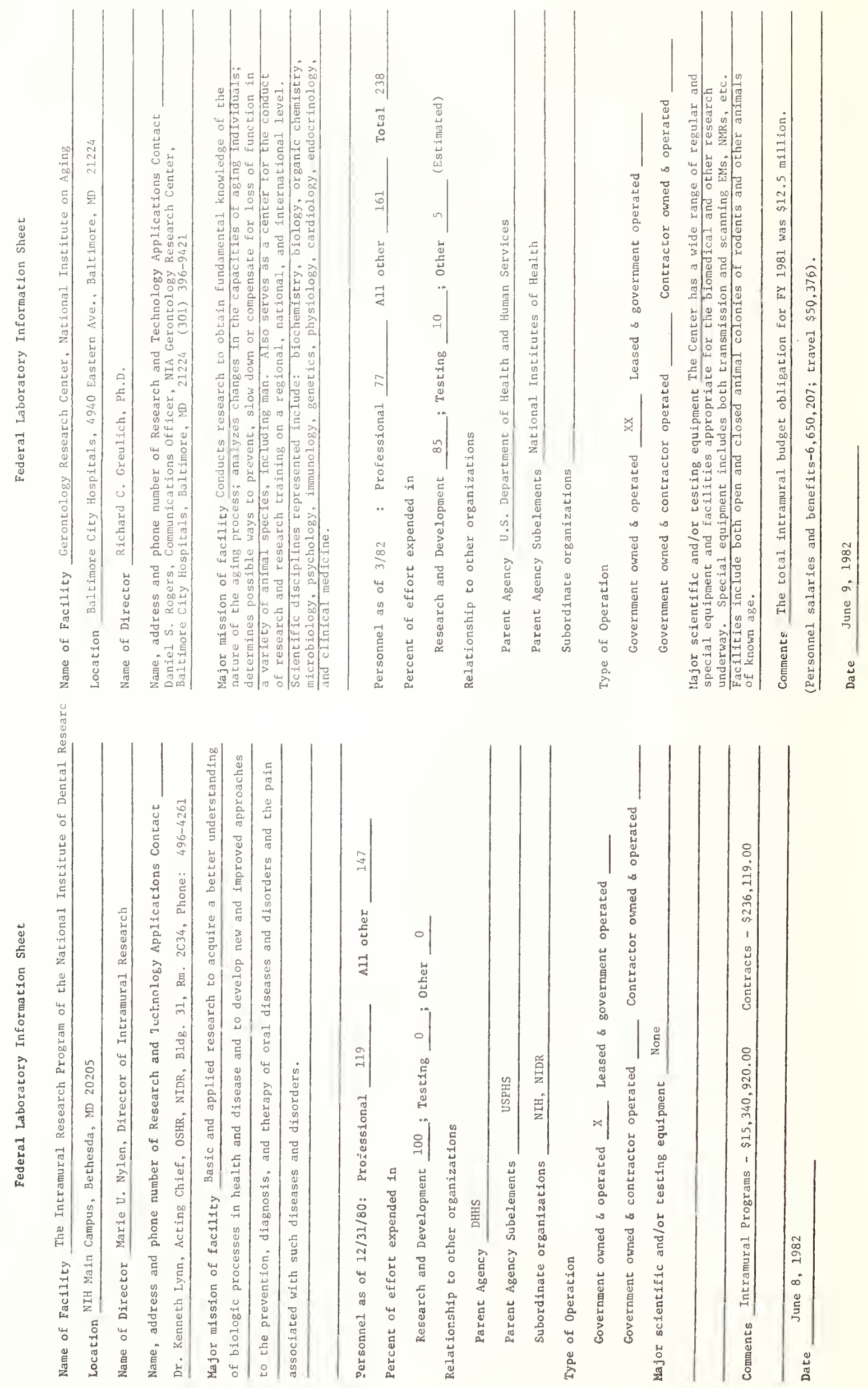

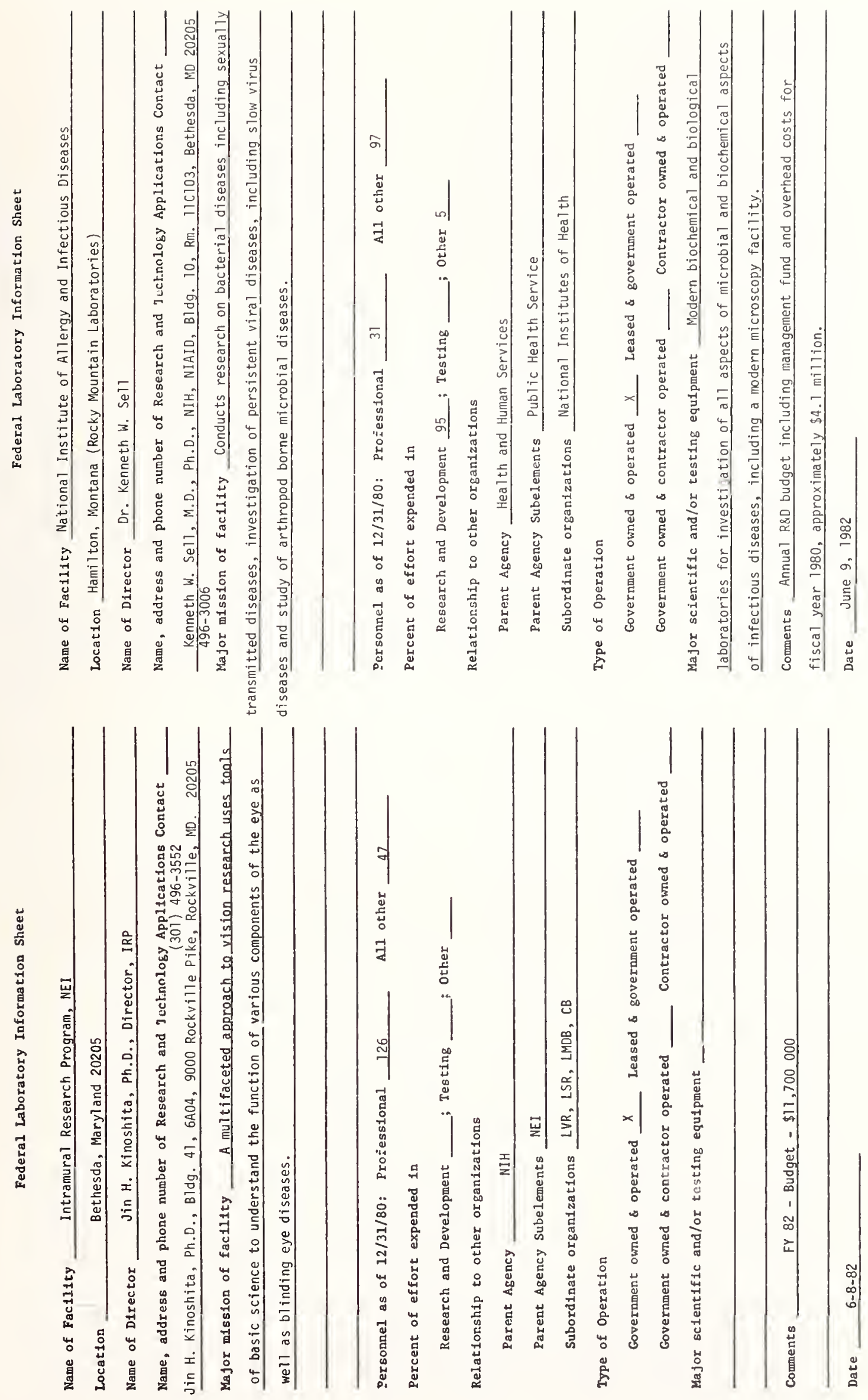

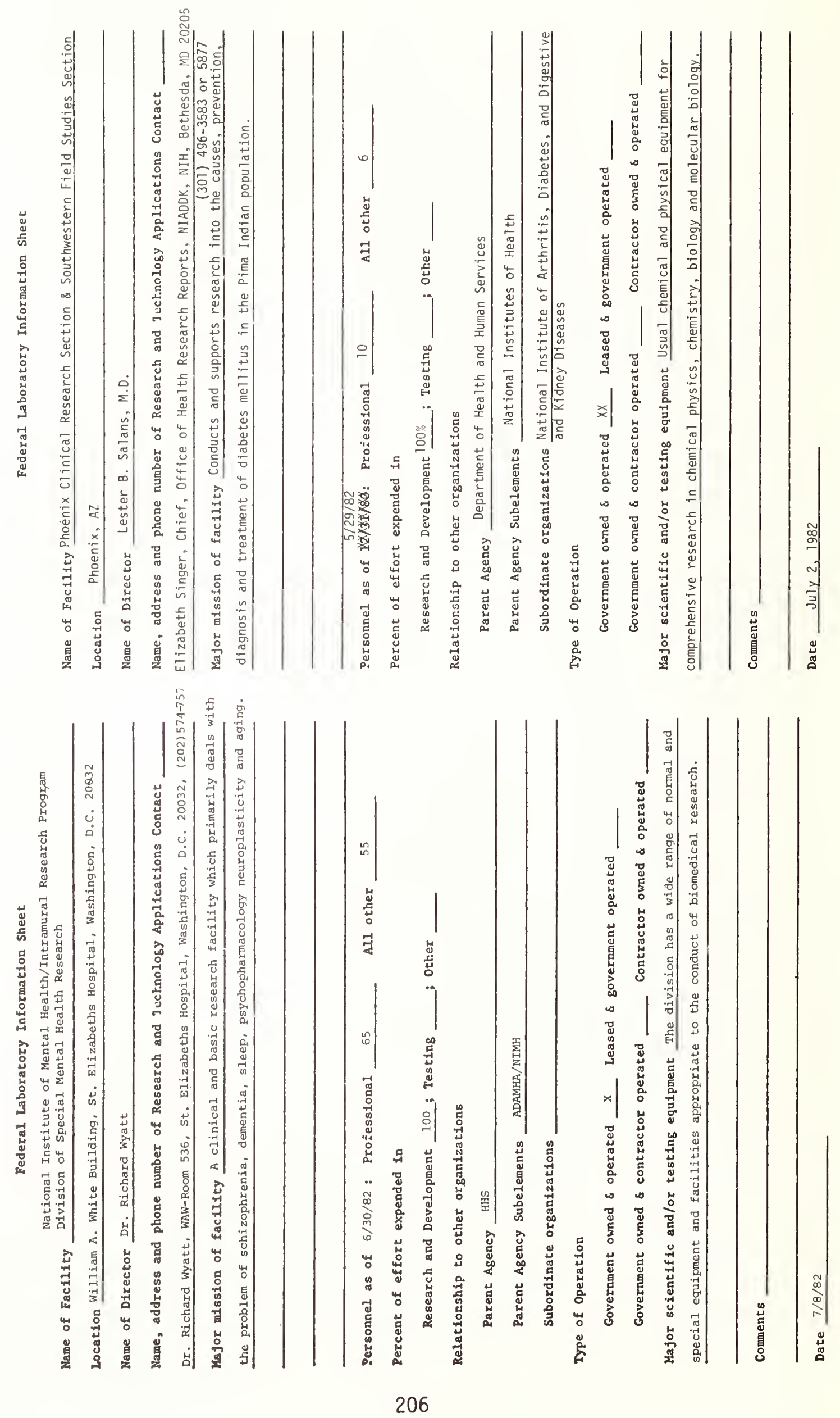

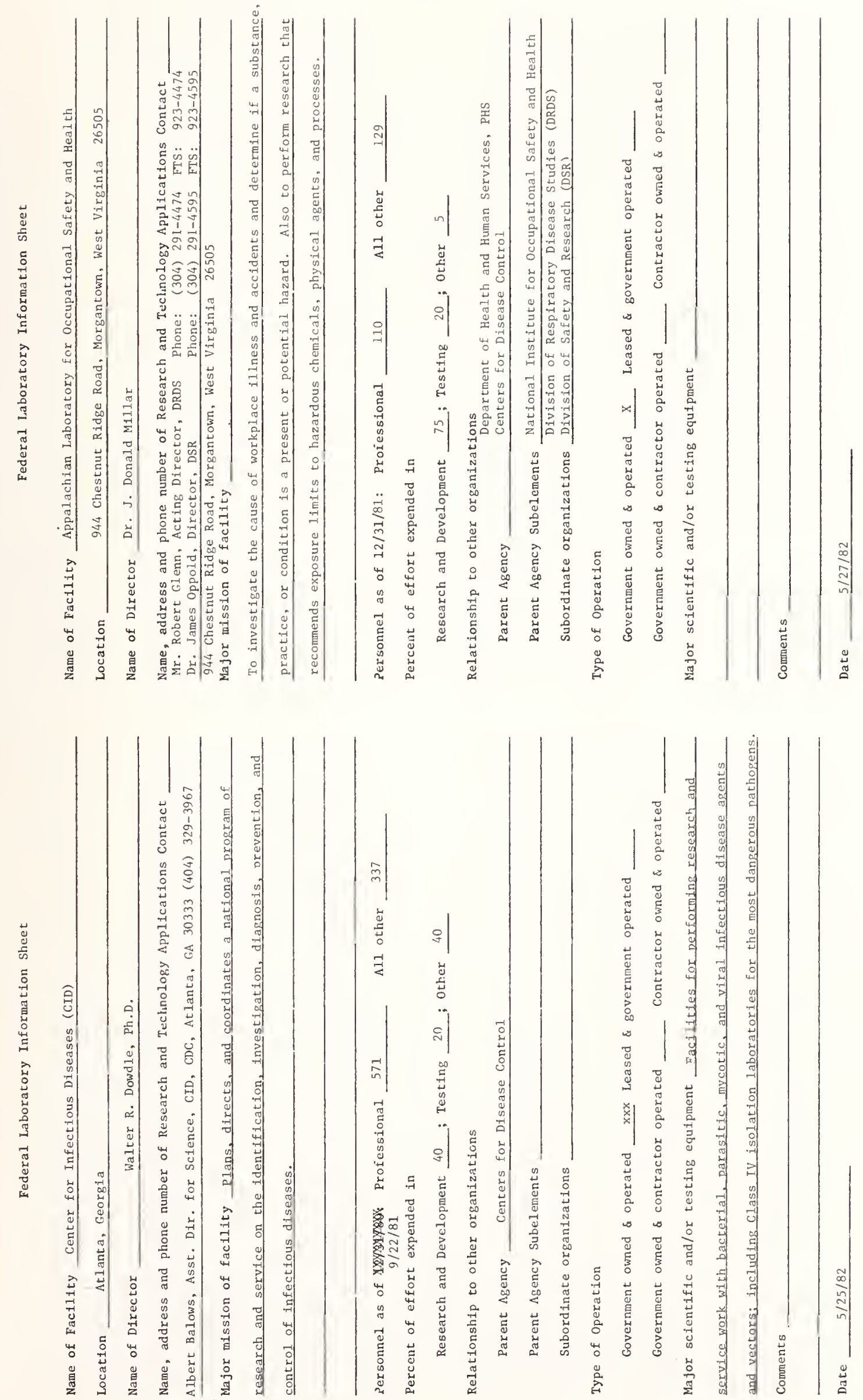

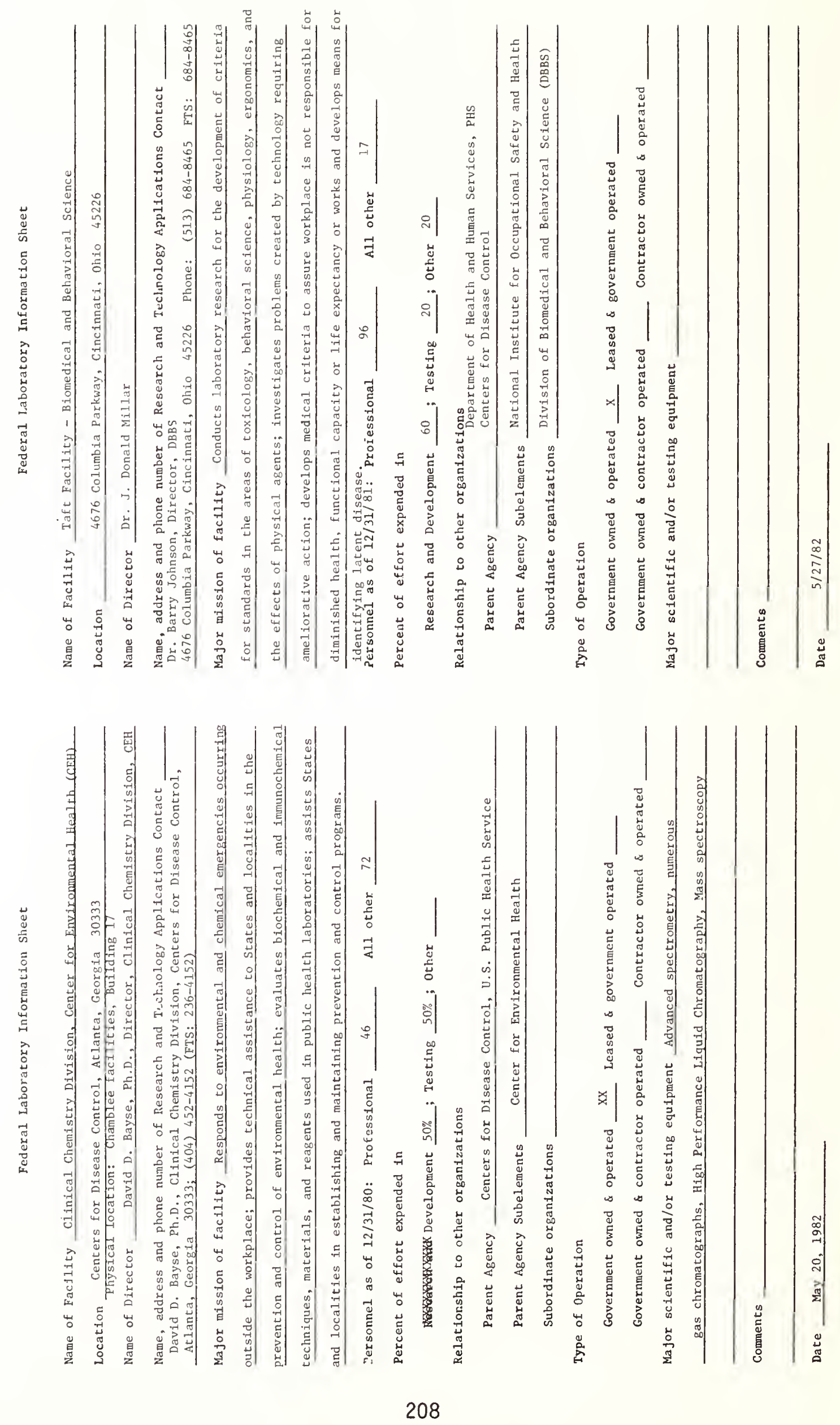


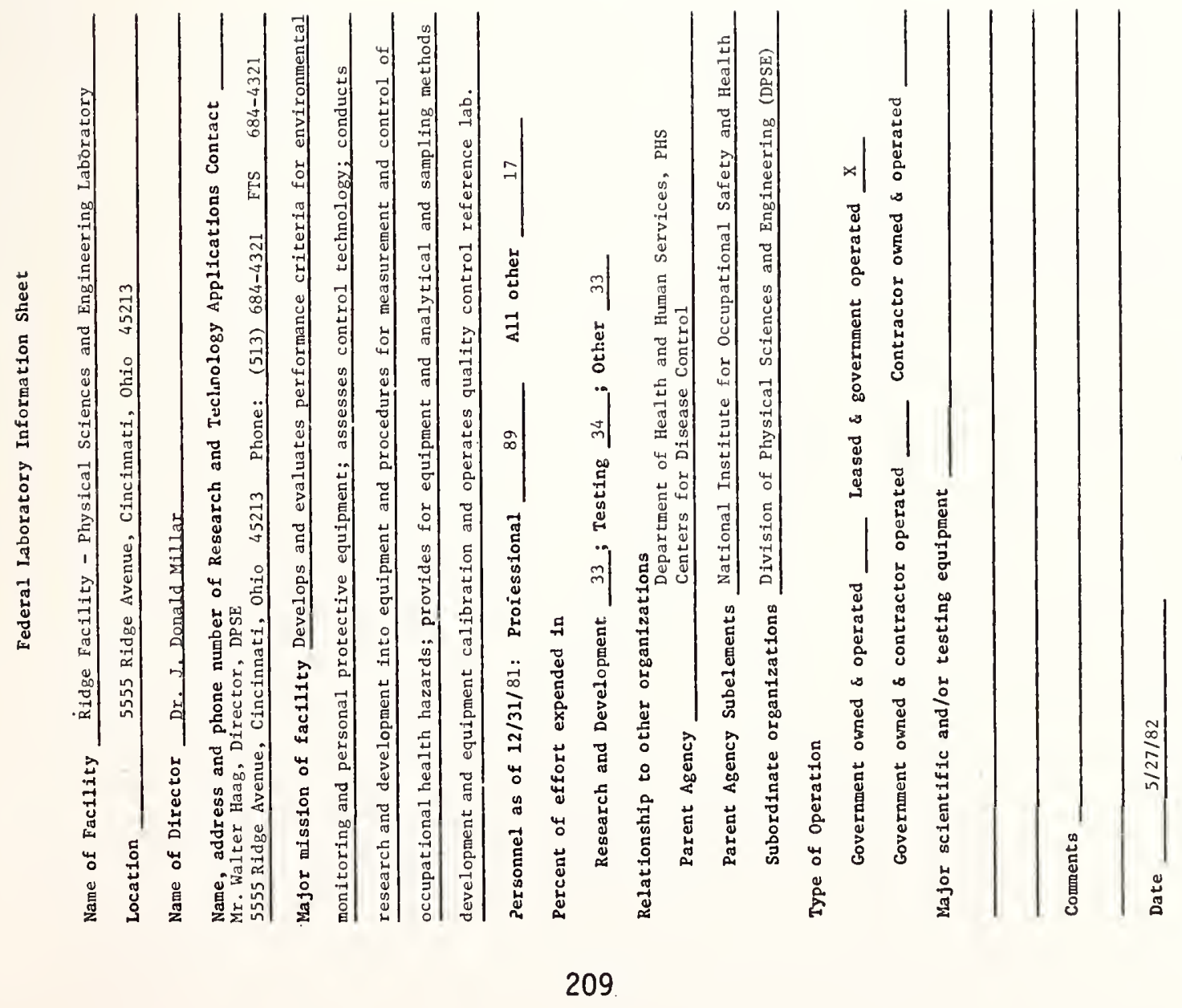



NATIONAL AERONAUTICS AND SPACE ADMINISTRATION 
. 


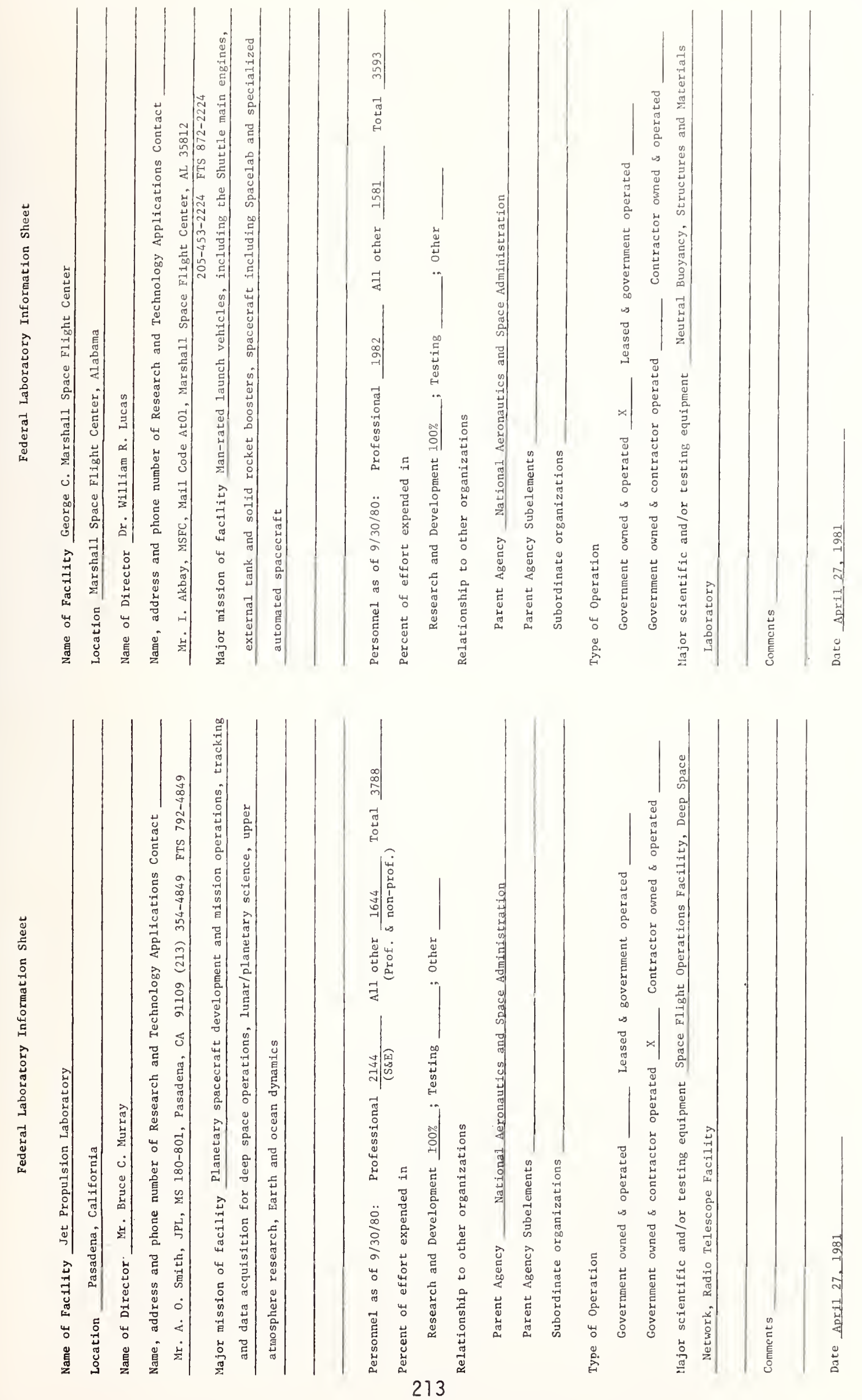



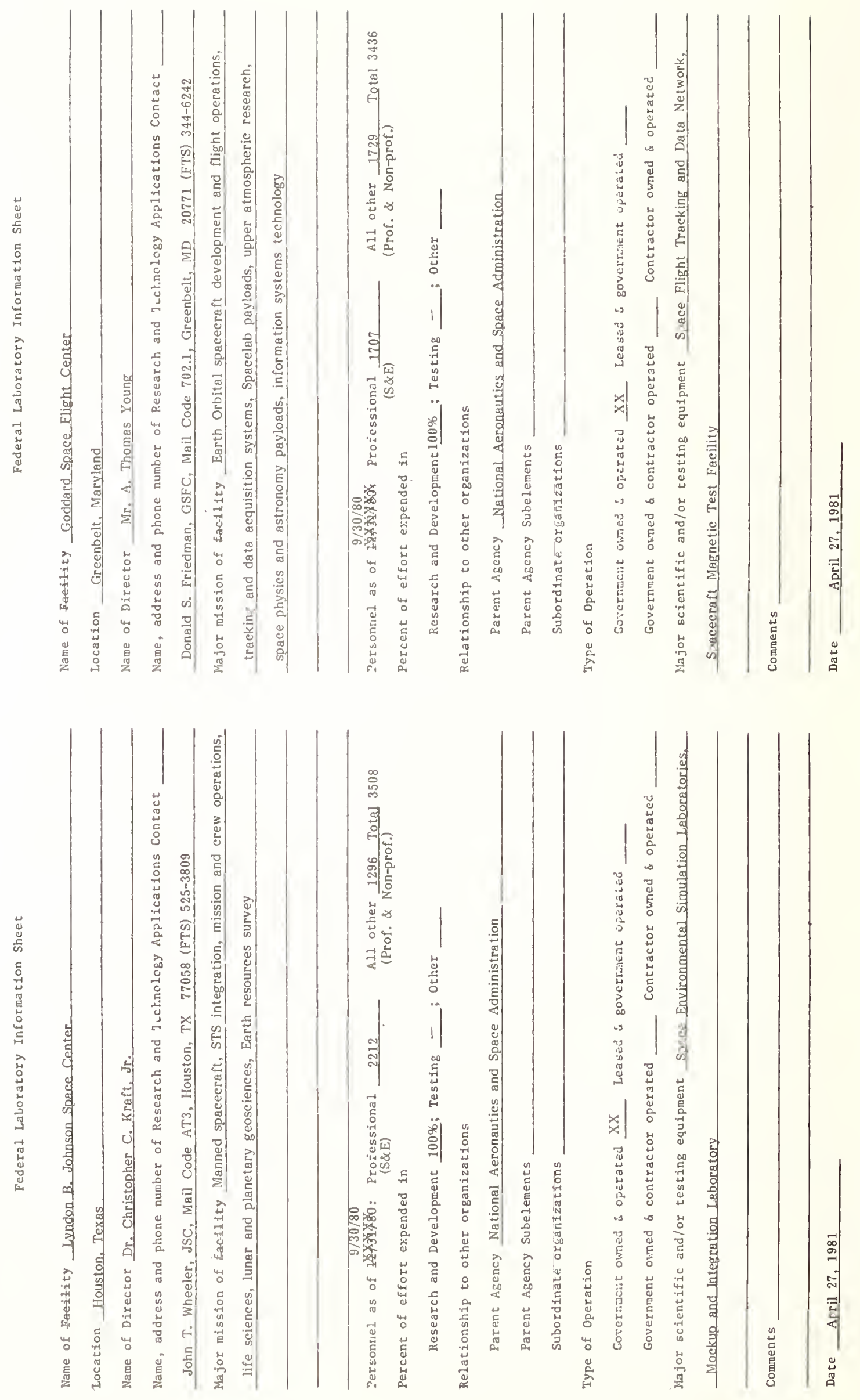

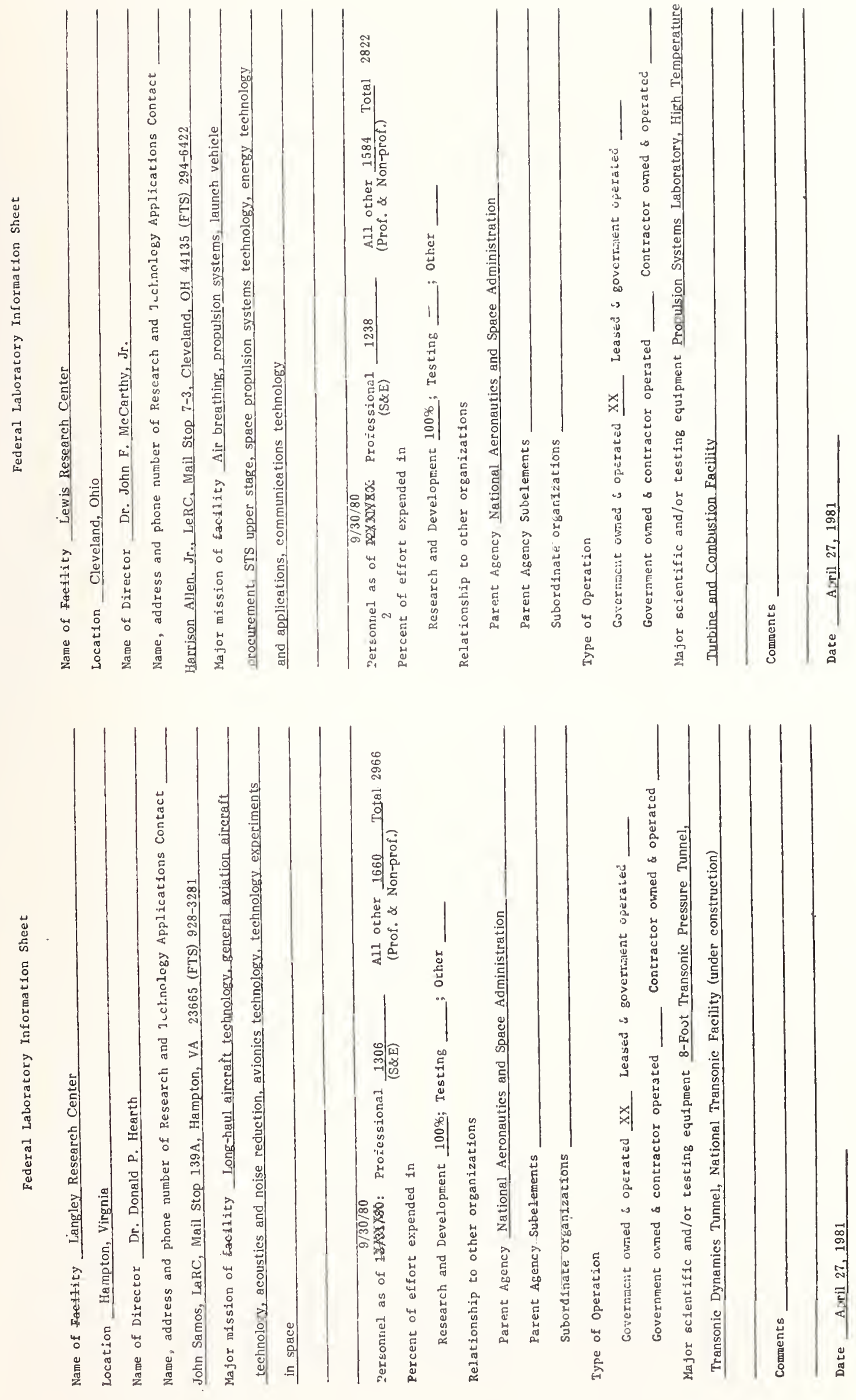

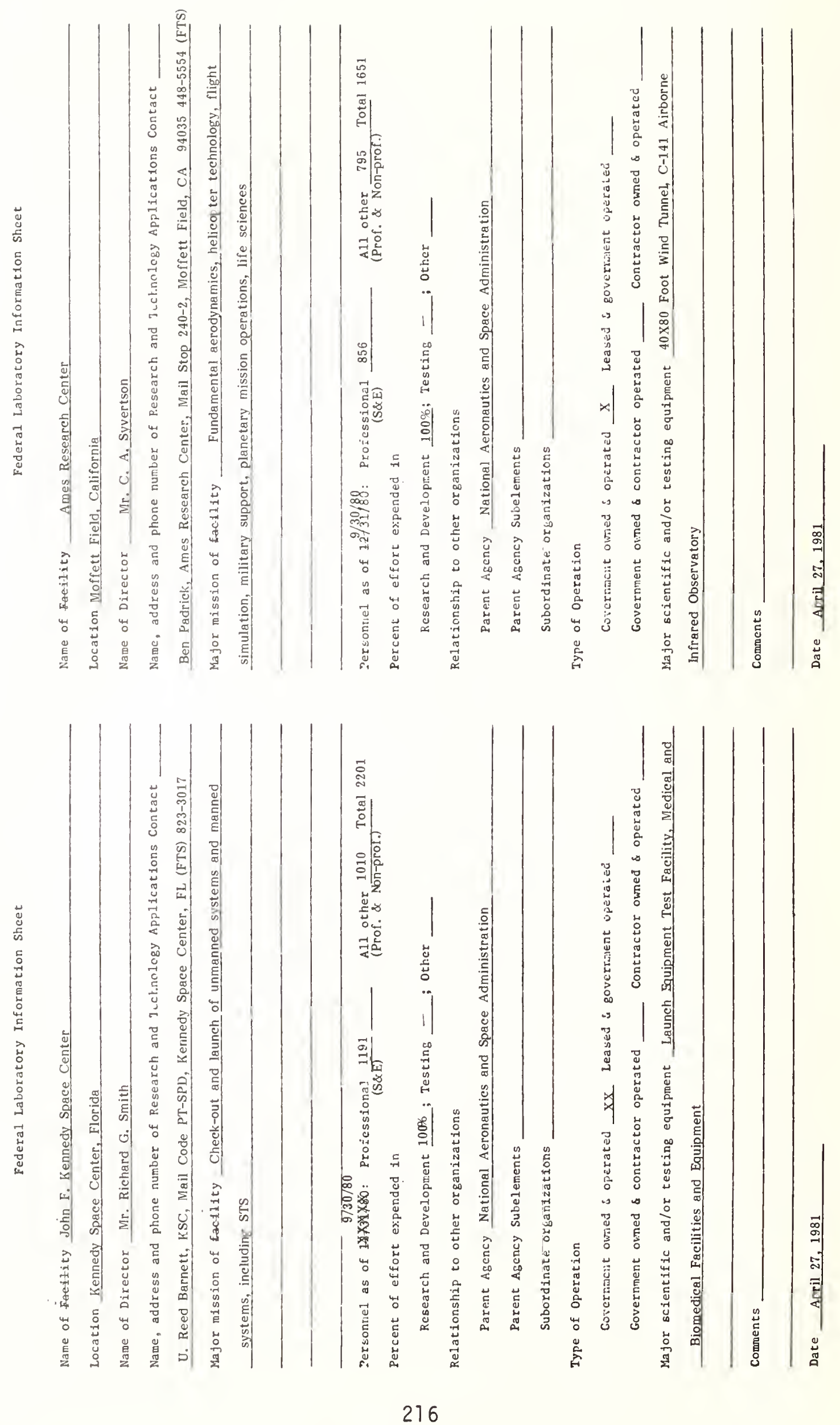

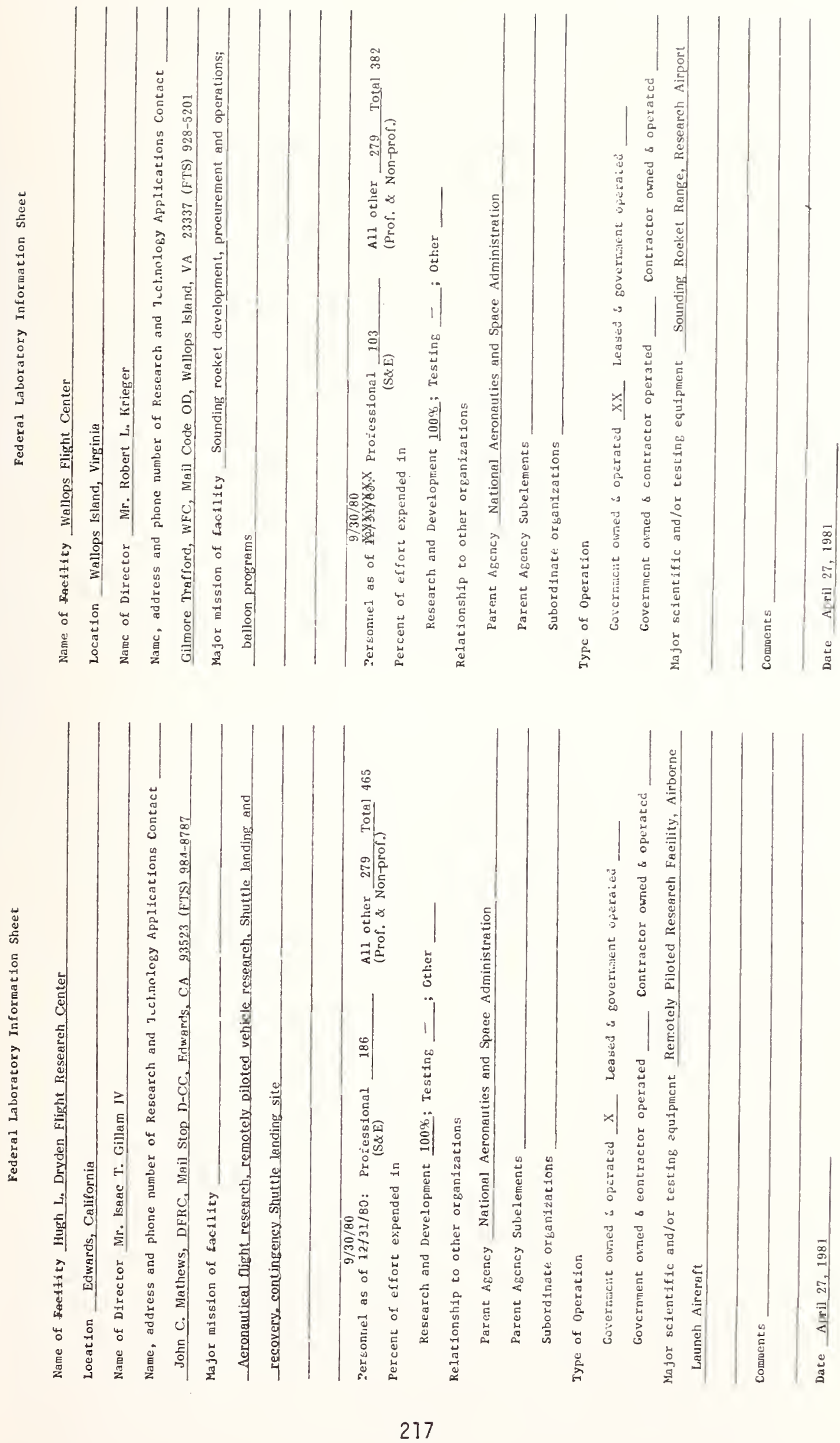

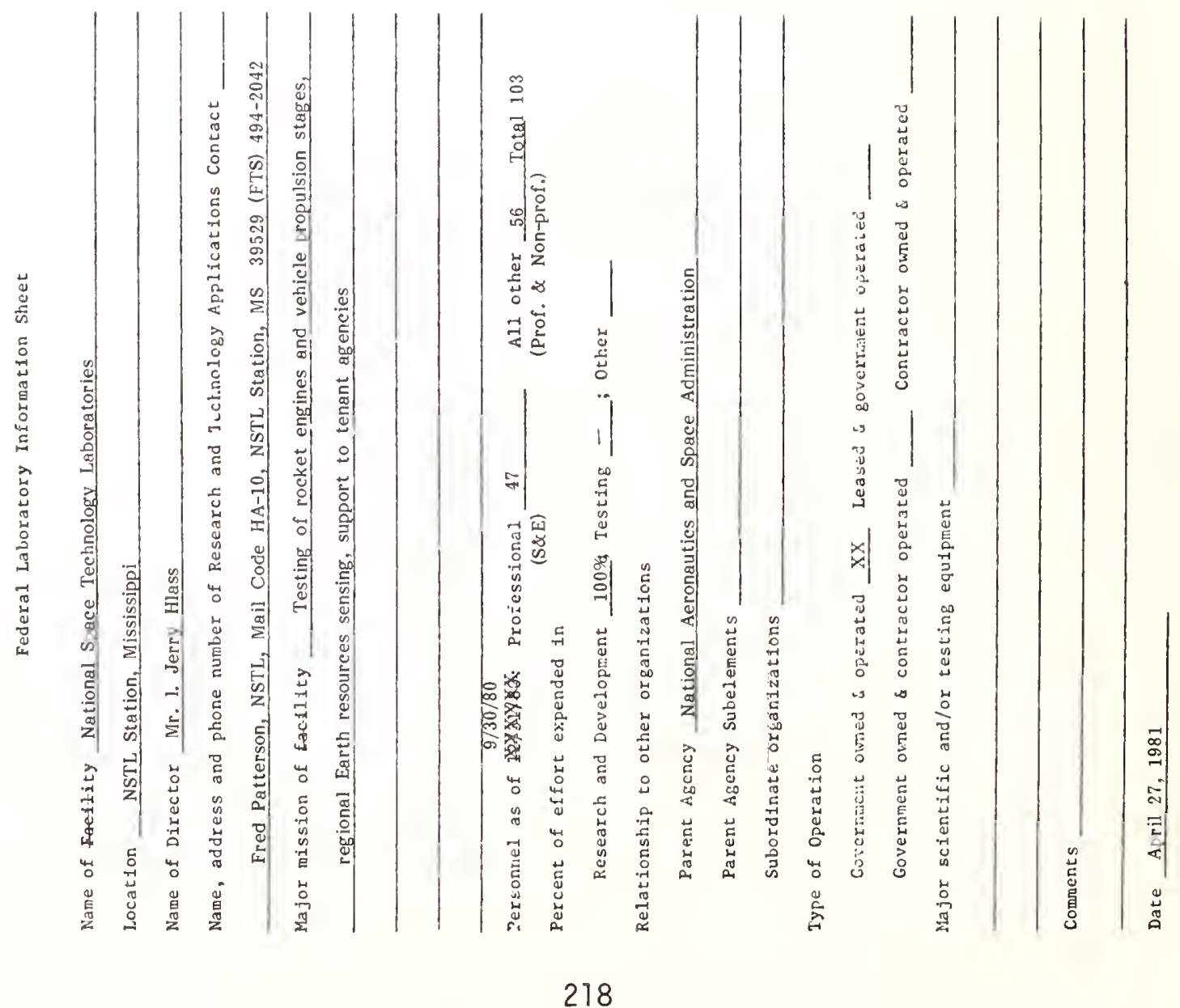
NATIONAL SCIENCE FOUNDATION 


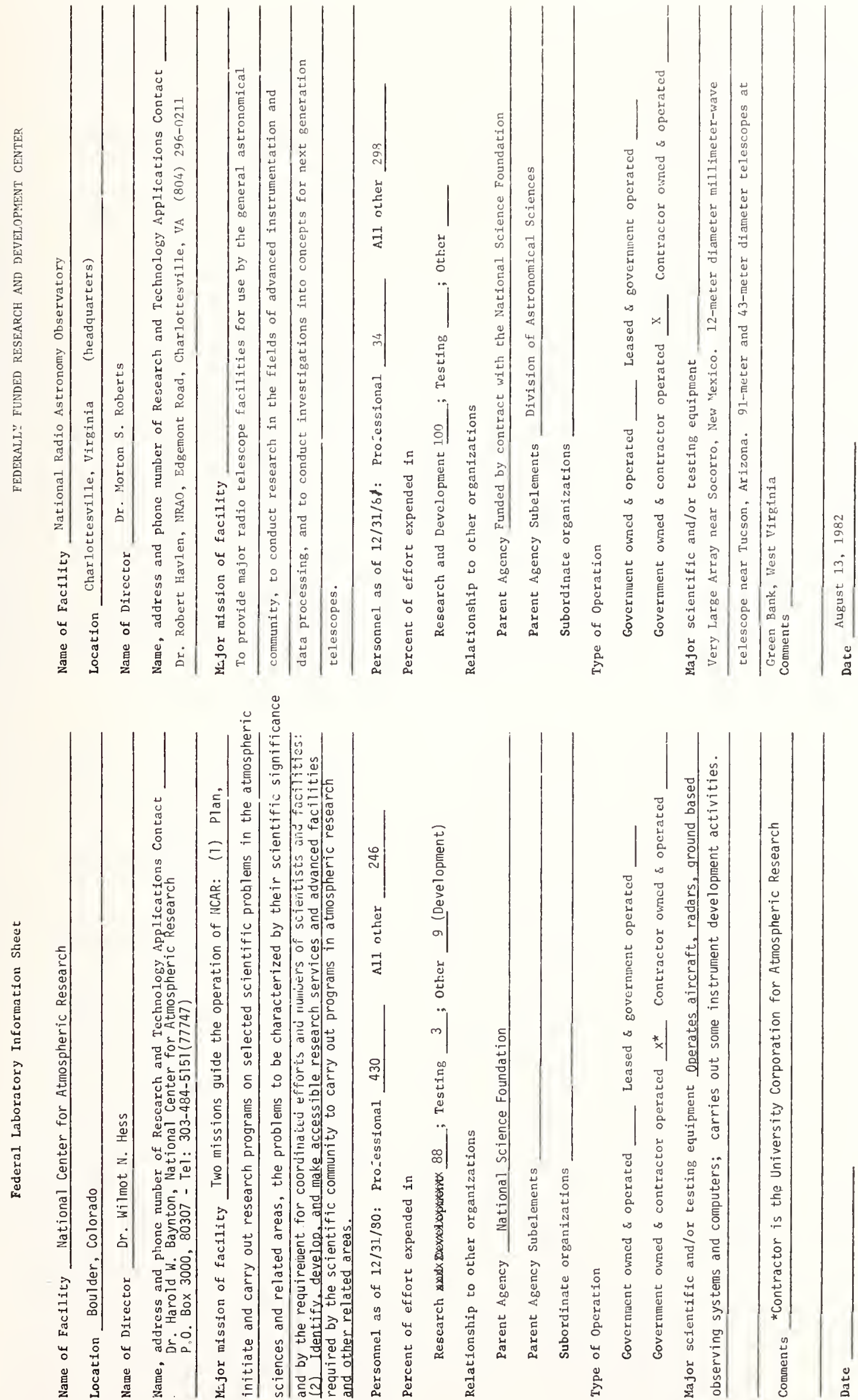

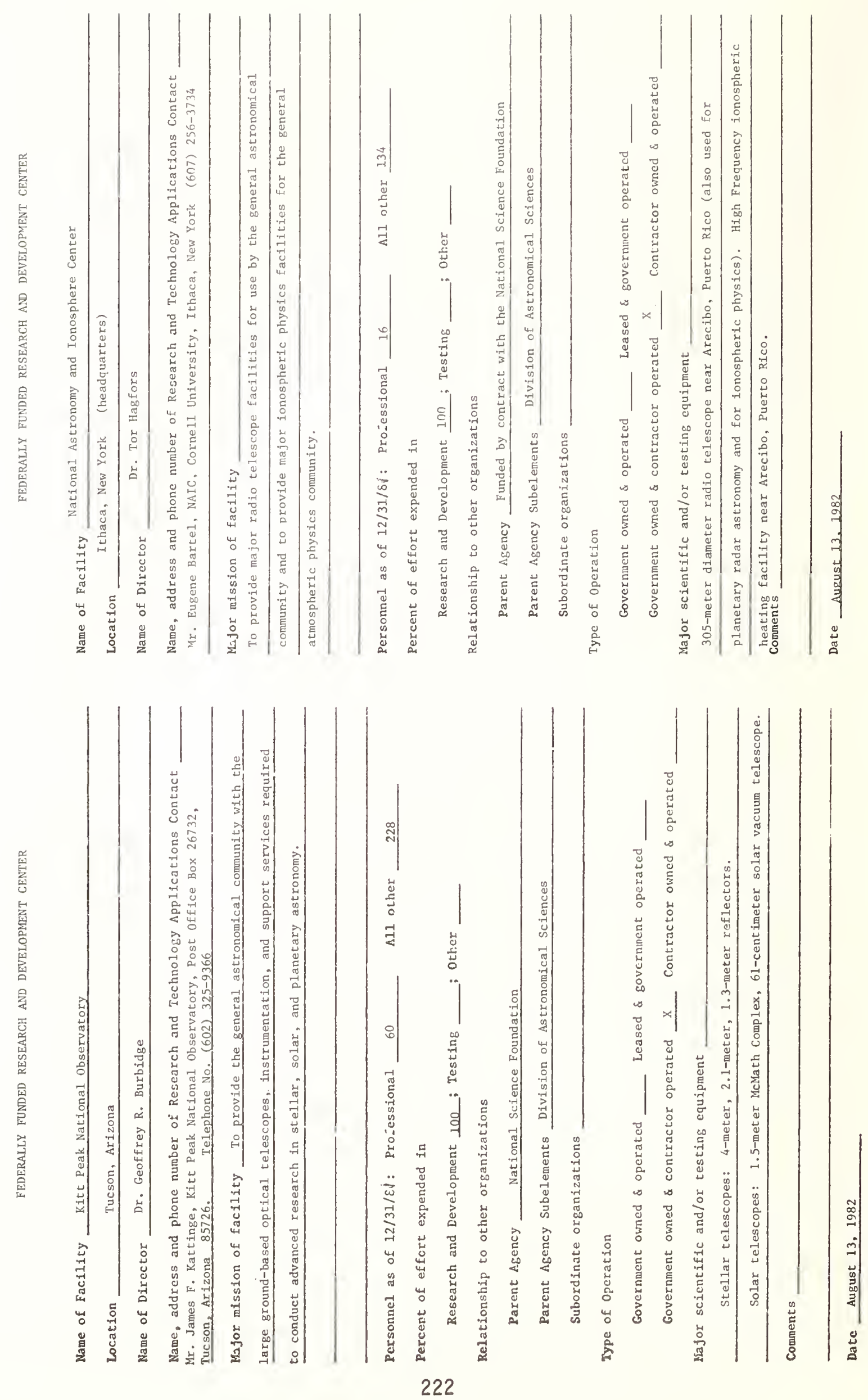

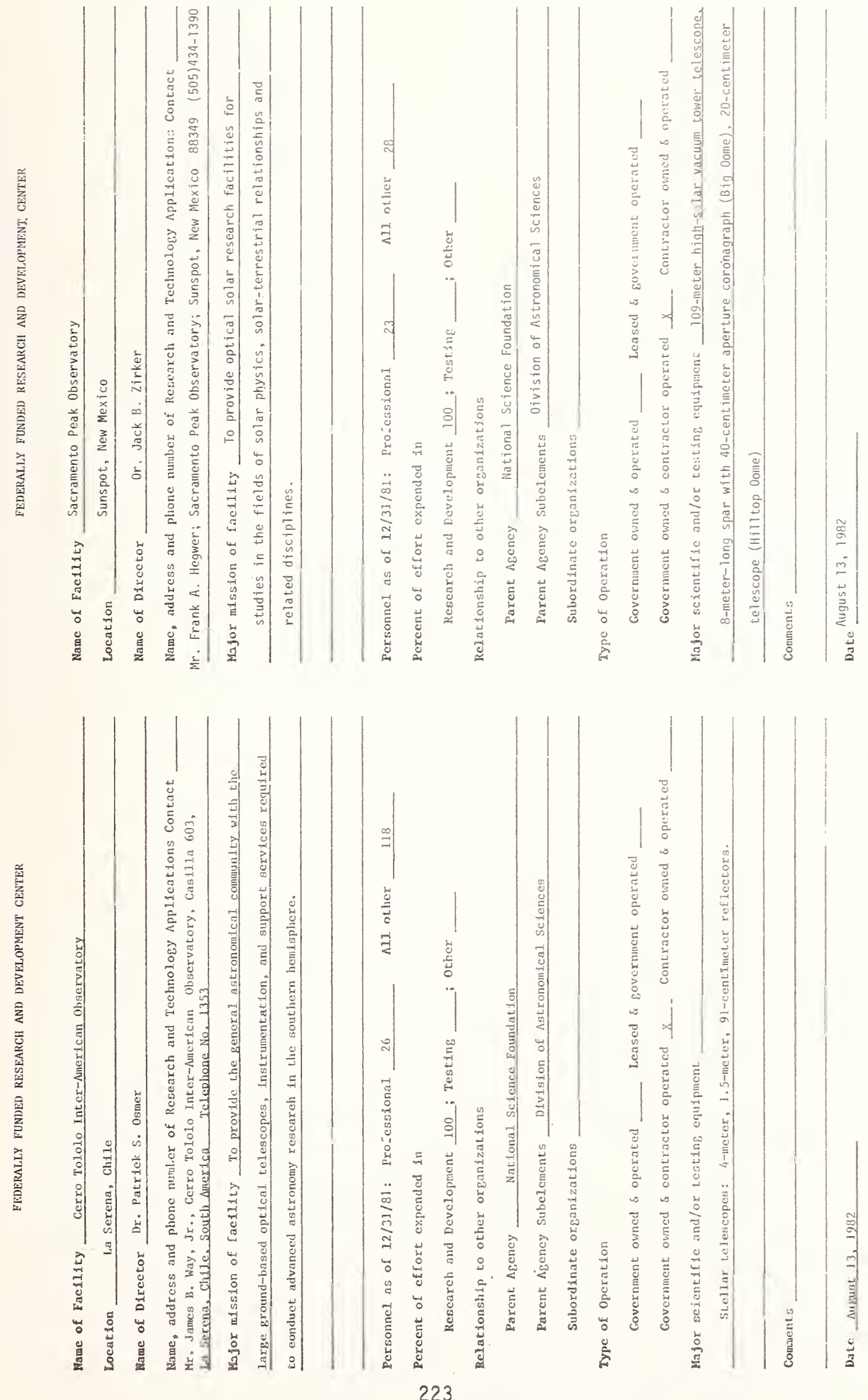



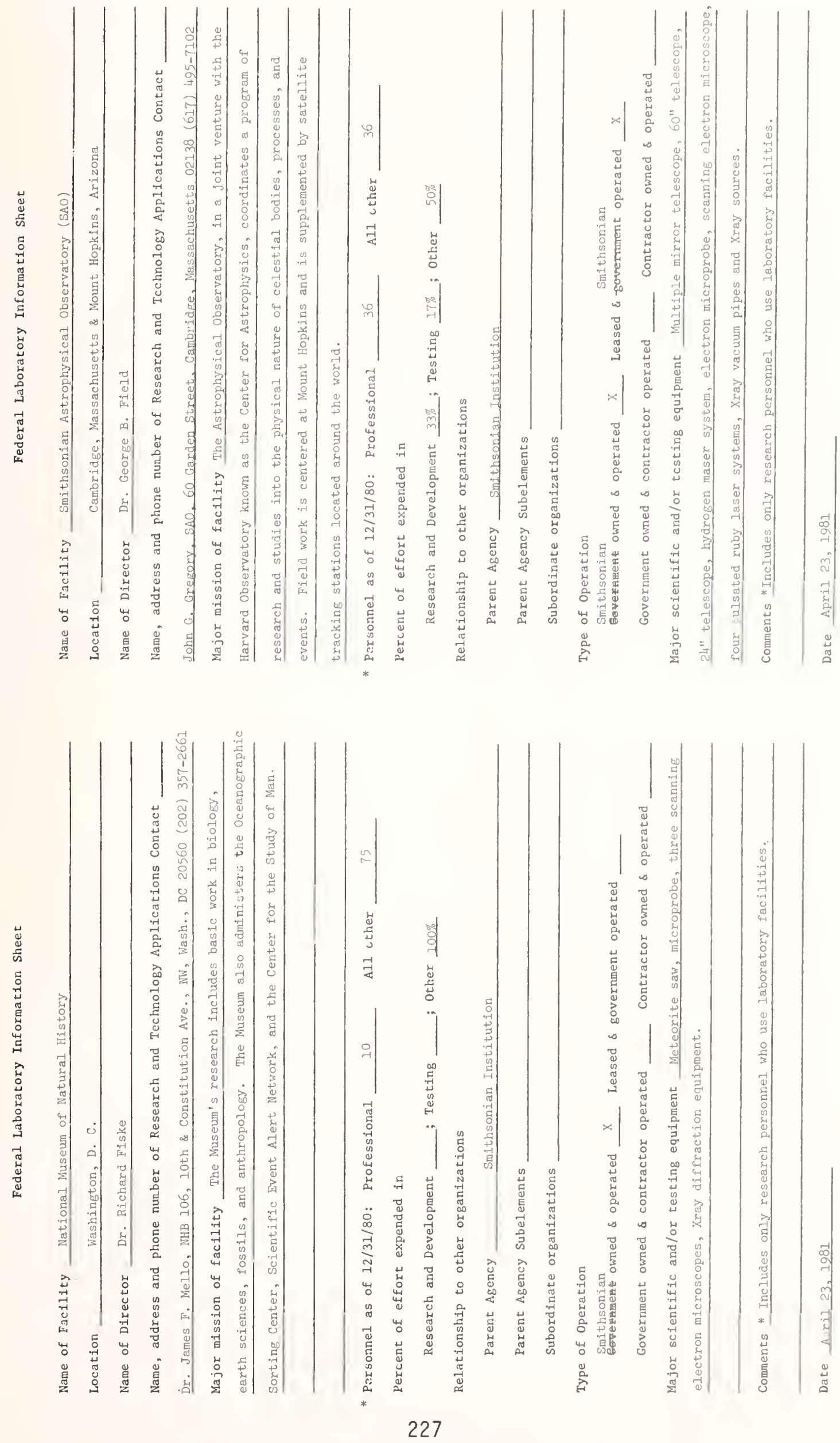

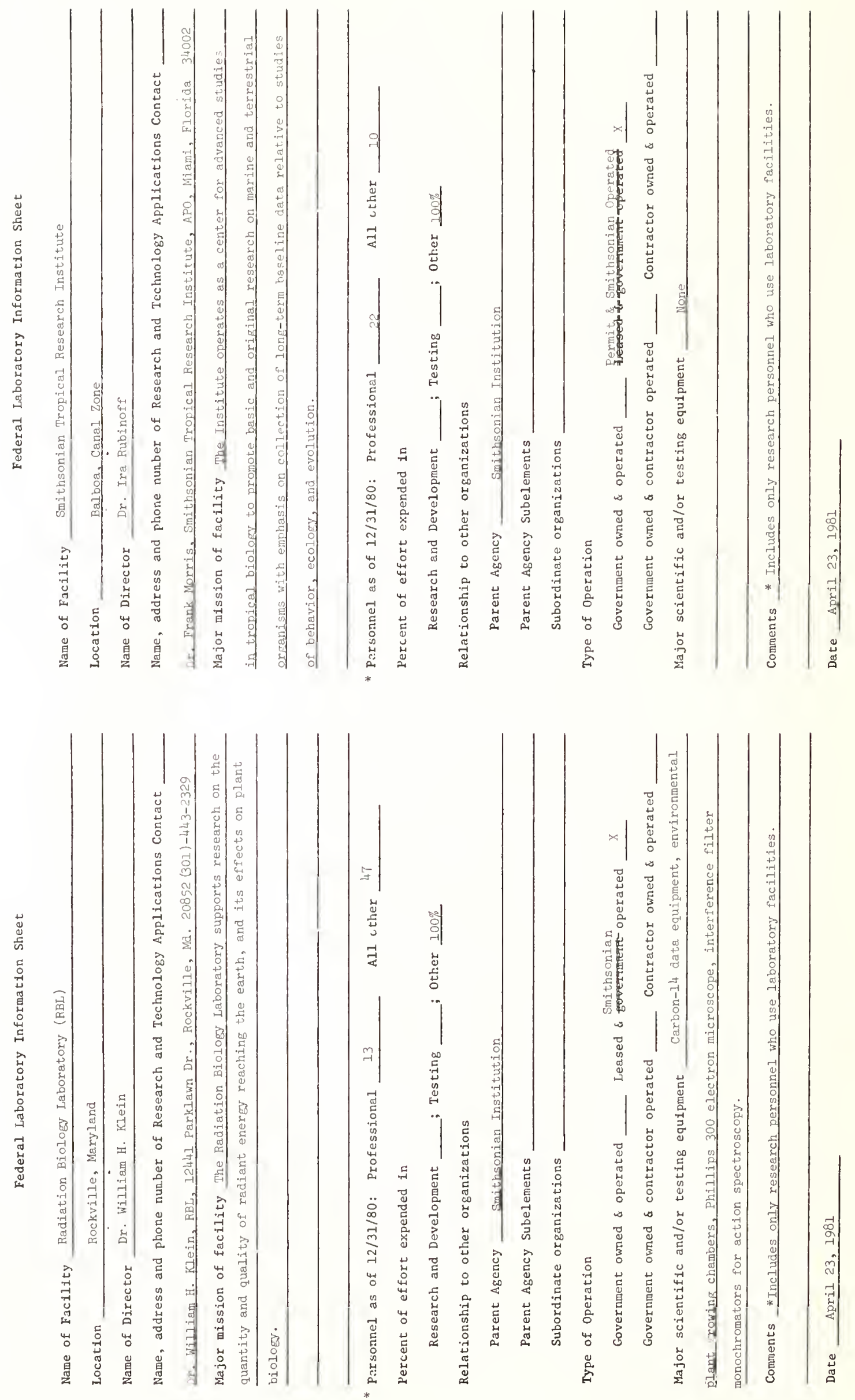

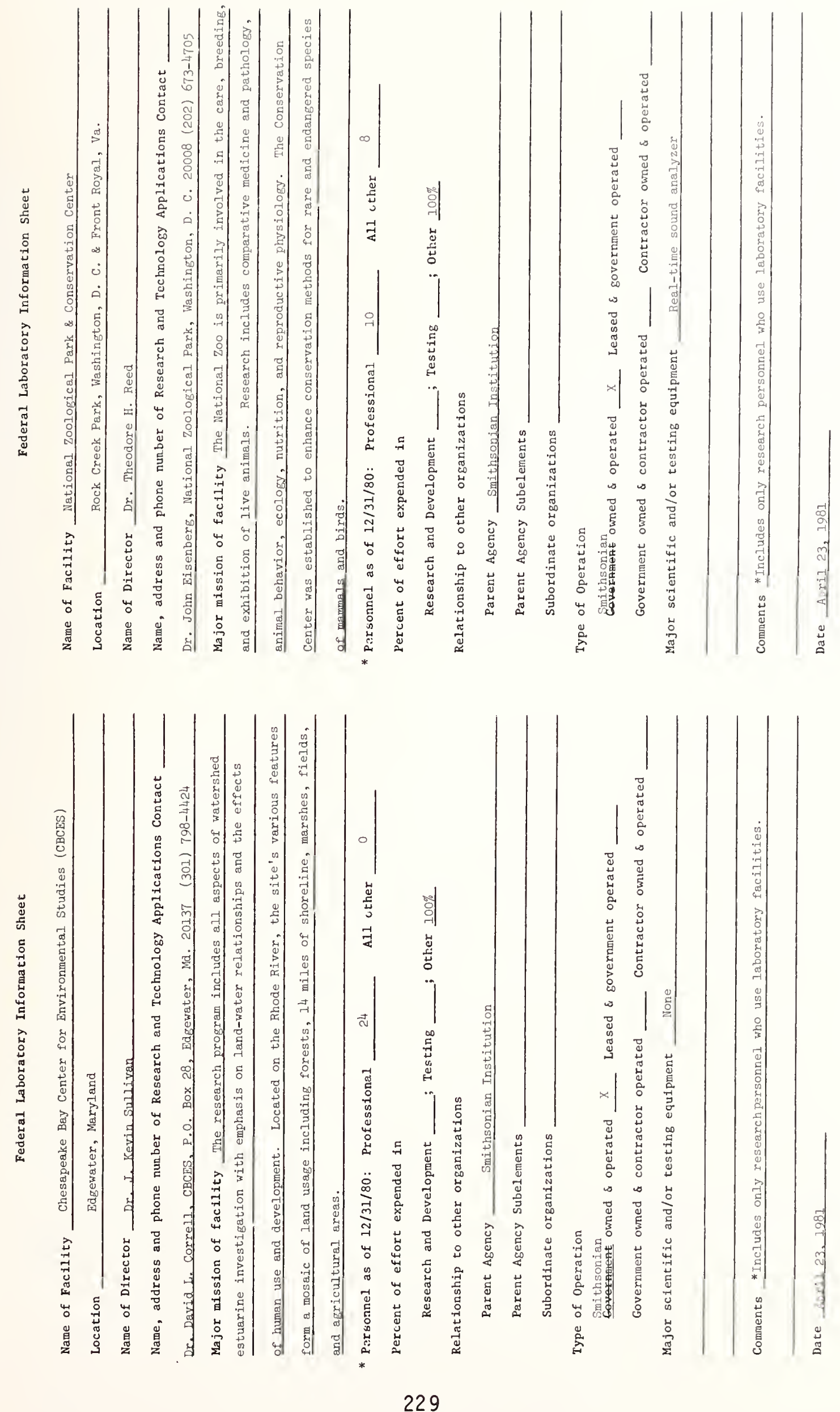

TENNESSEE VALLEY AUTHORITY 


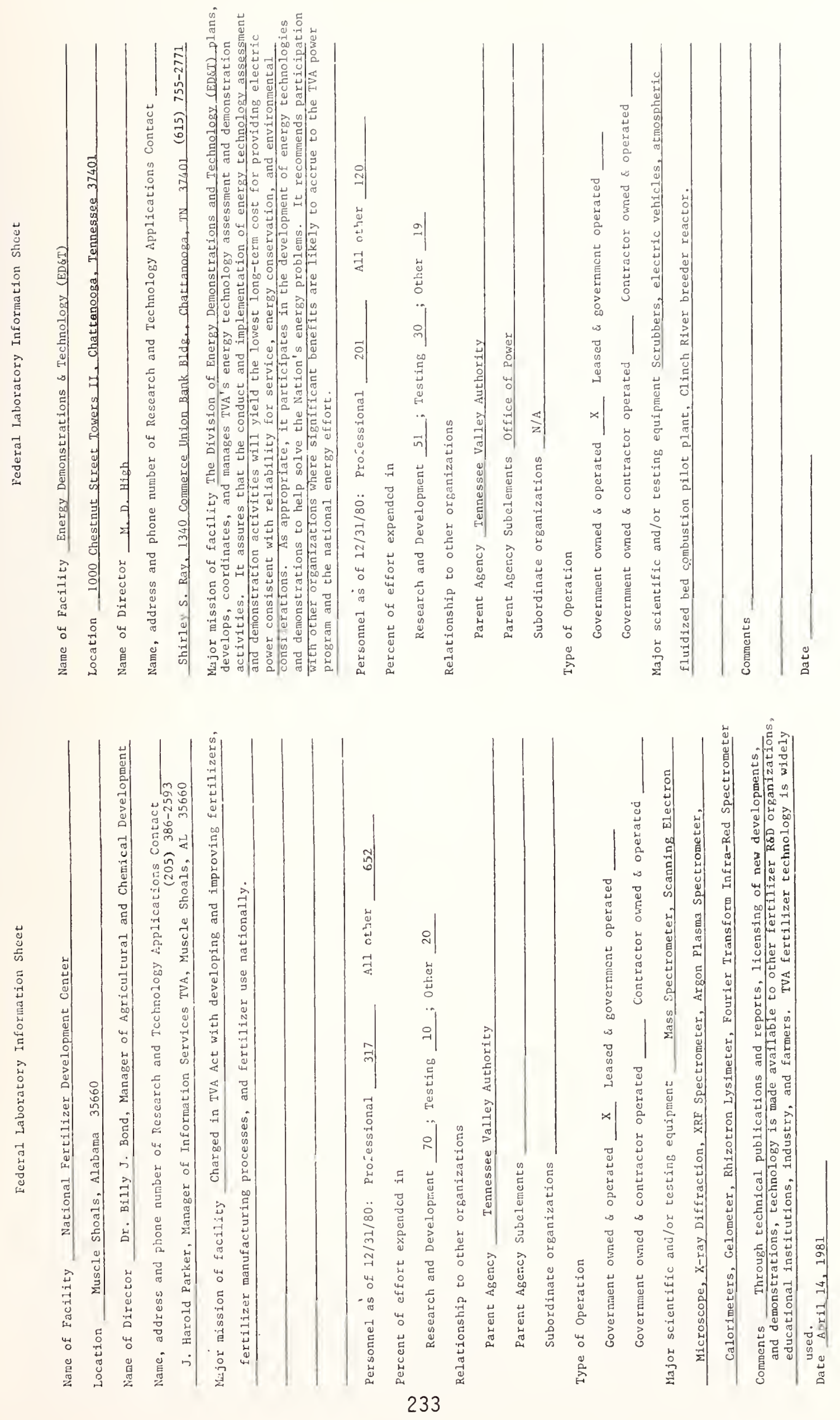

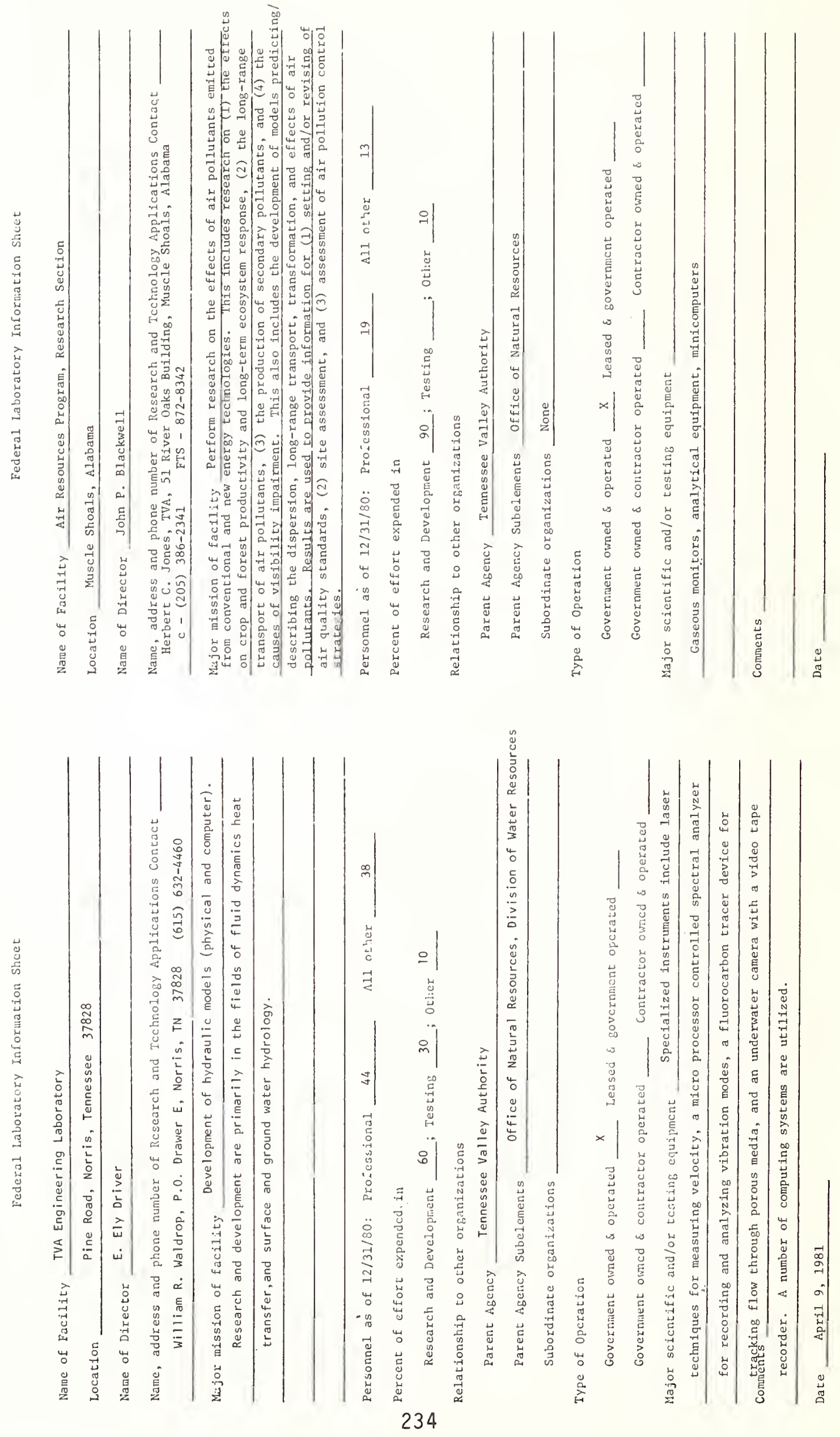


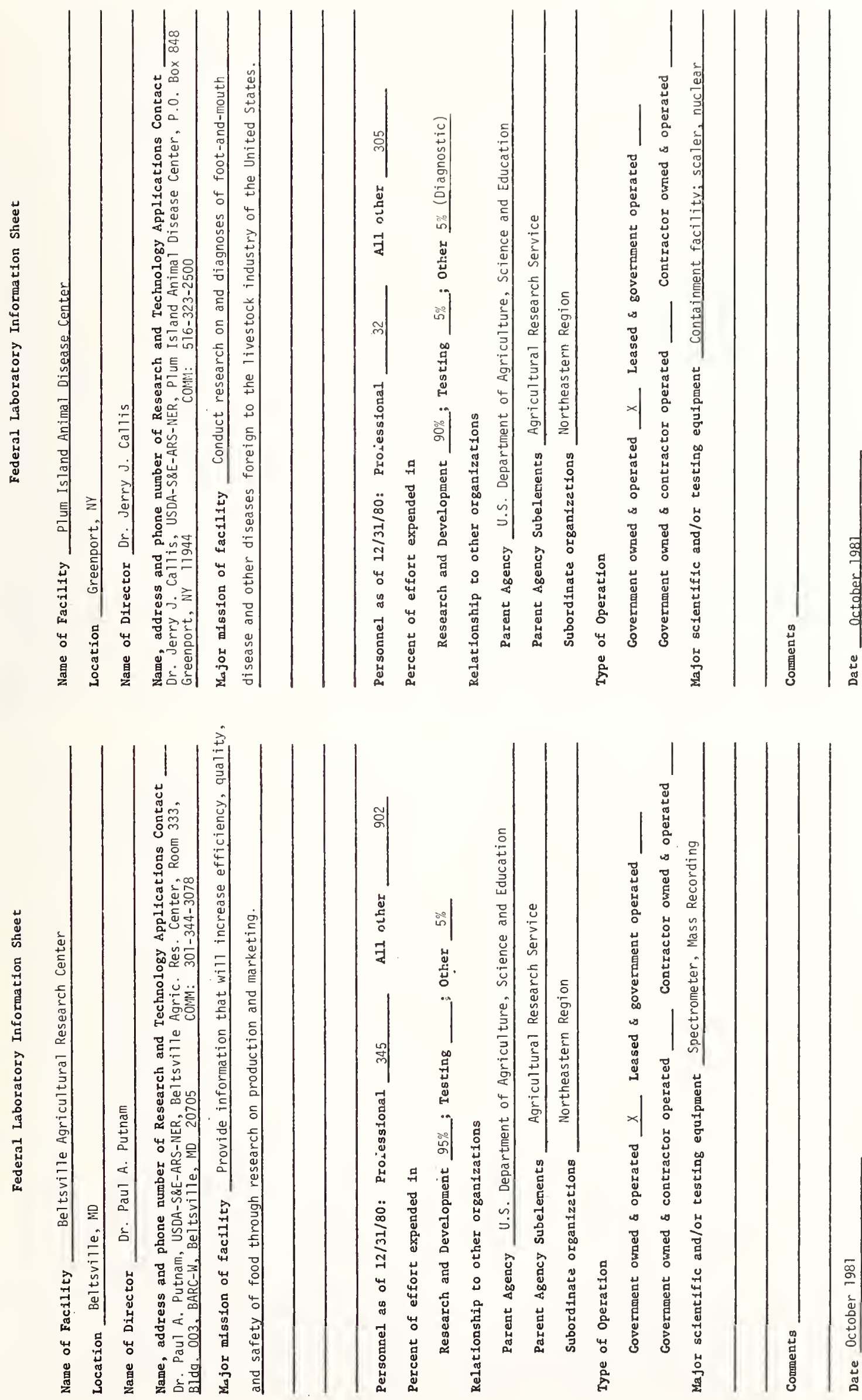

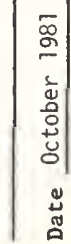



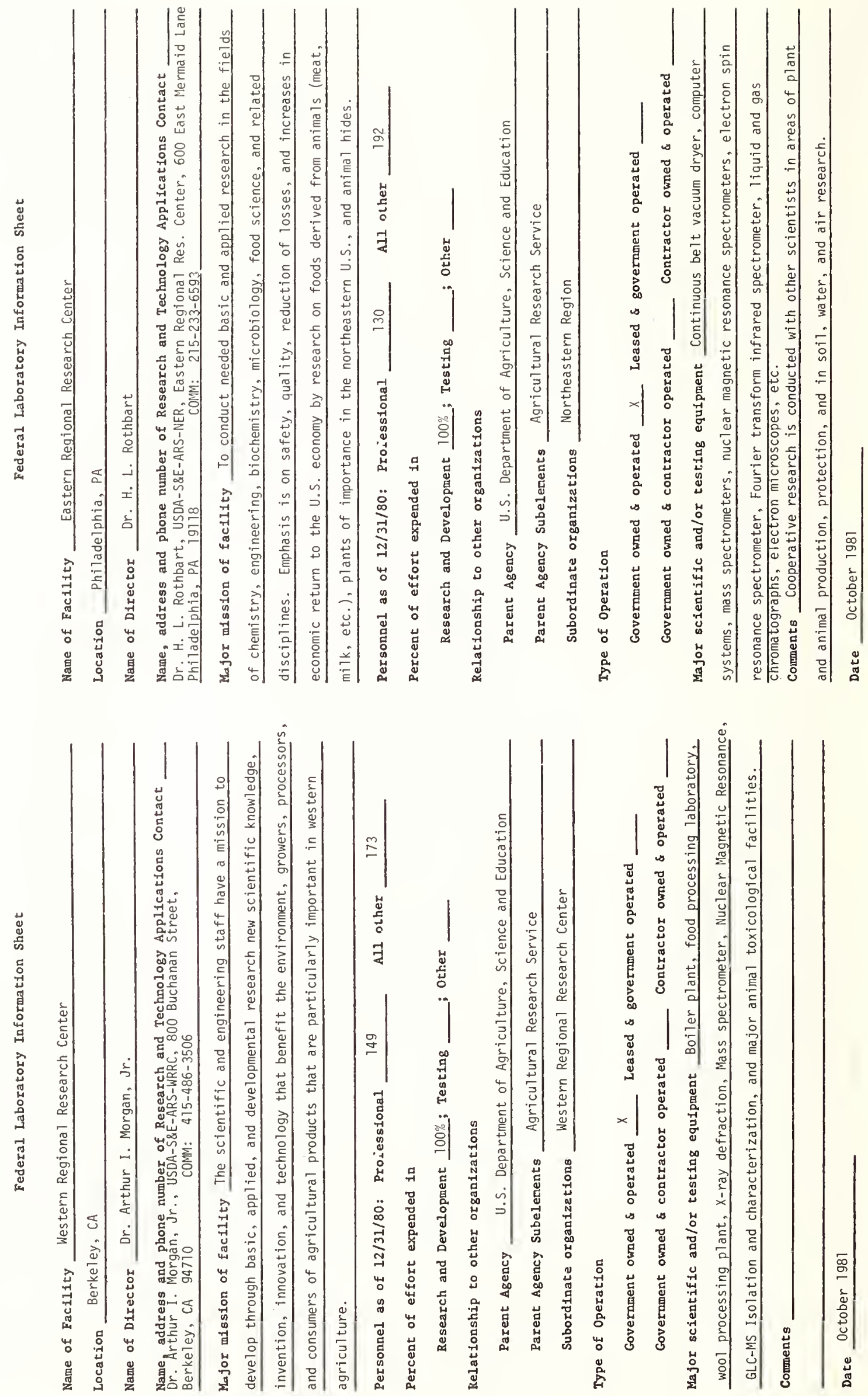

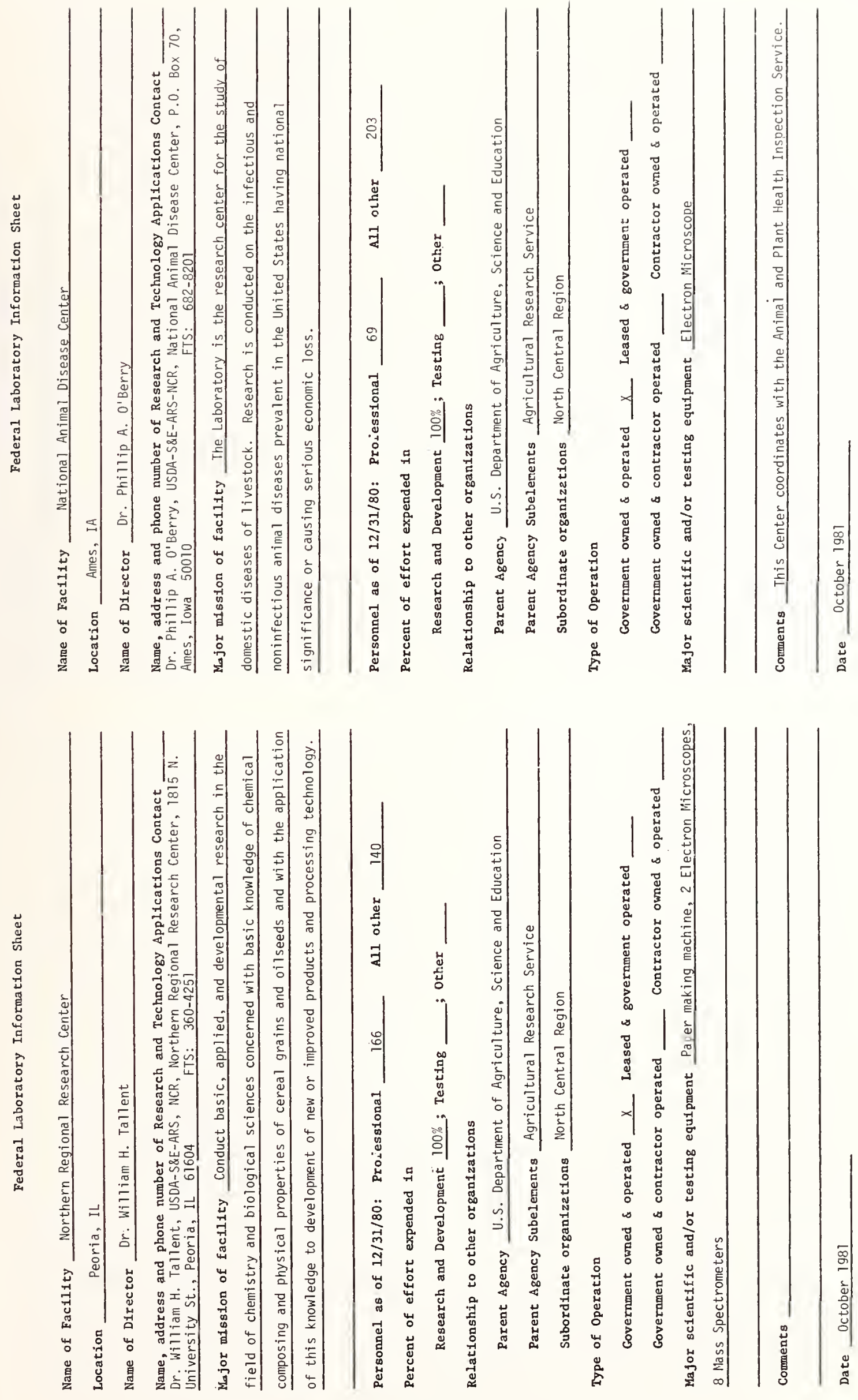

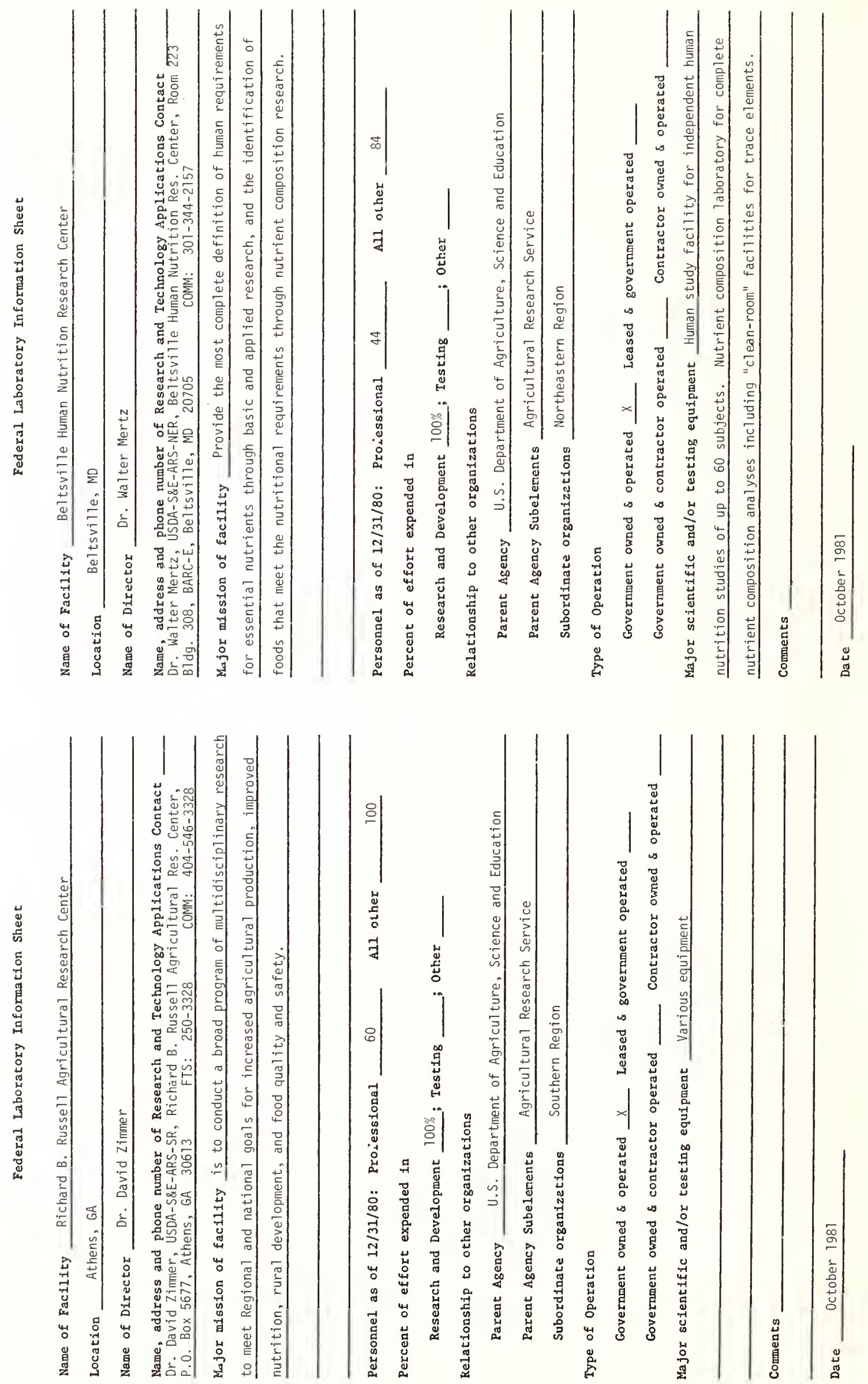

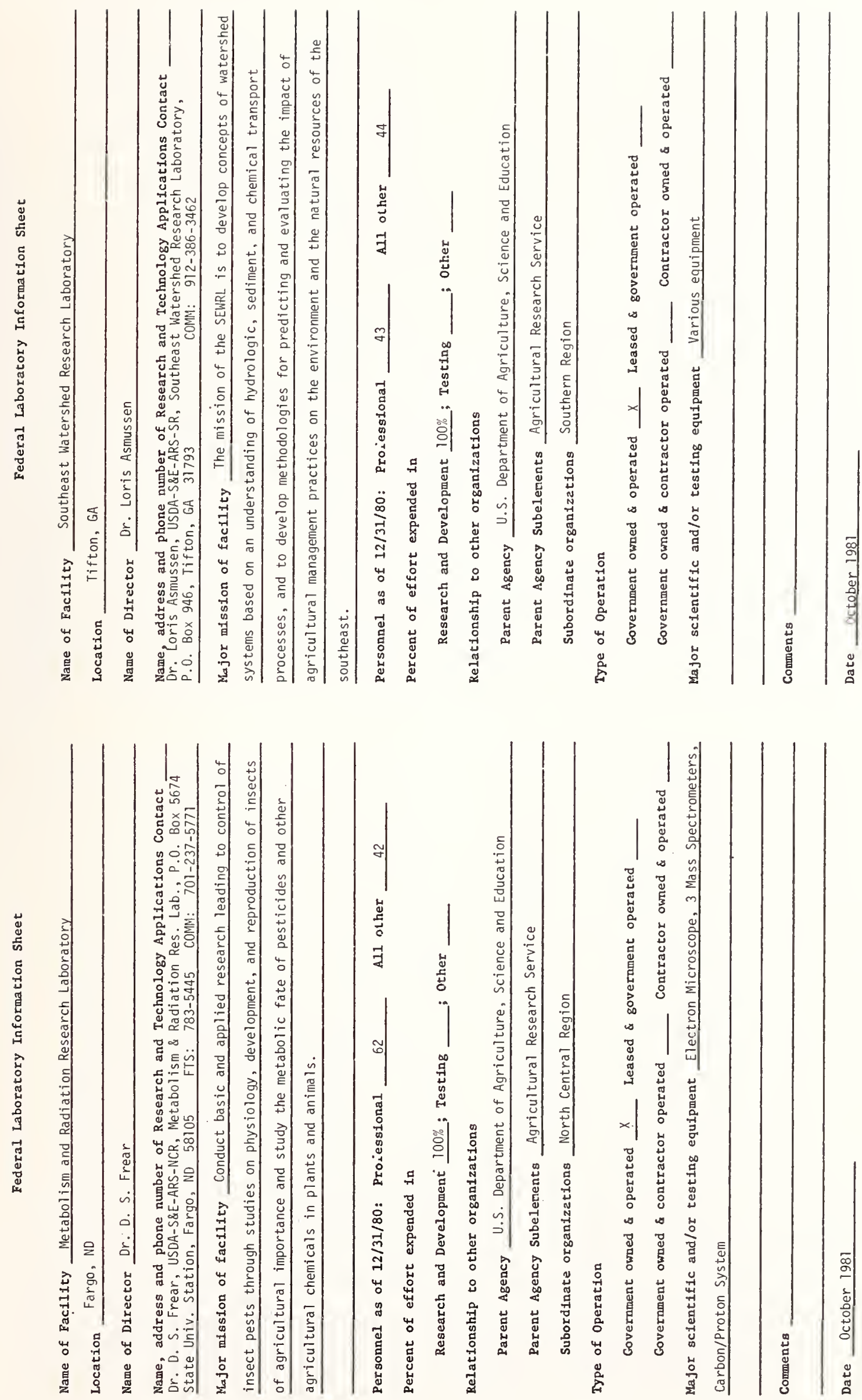

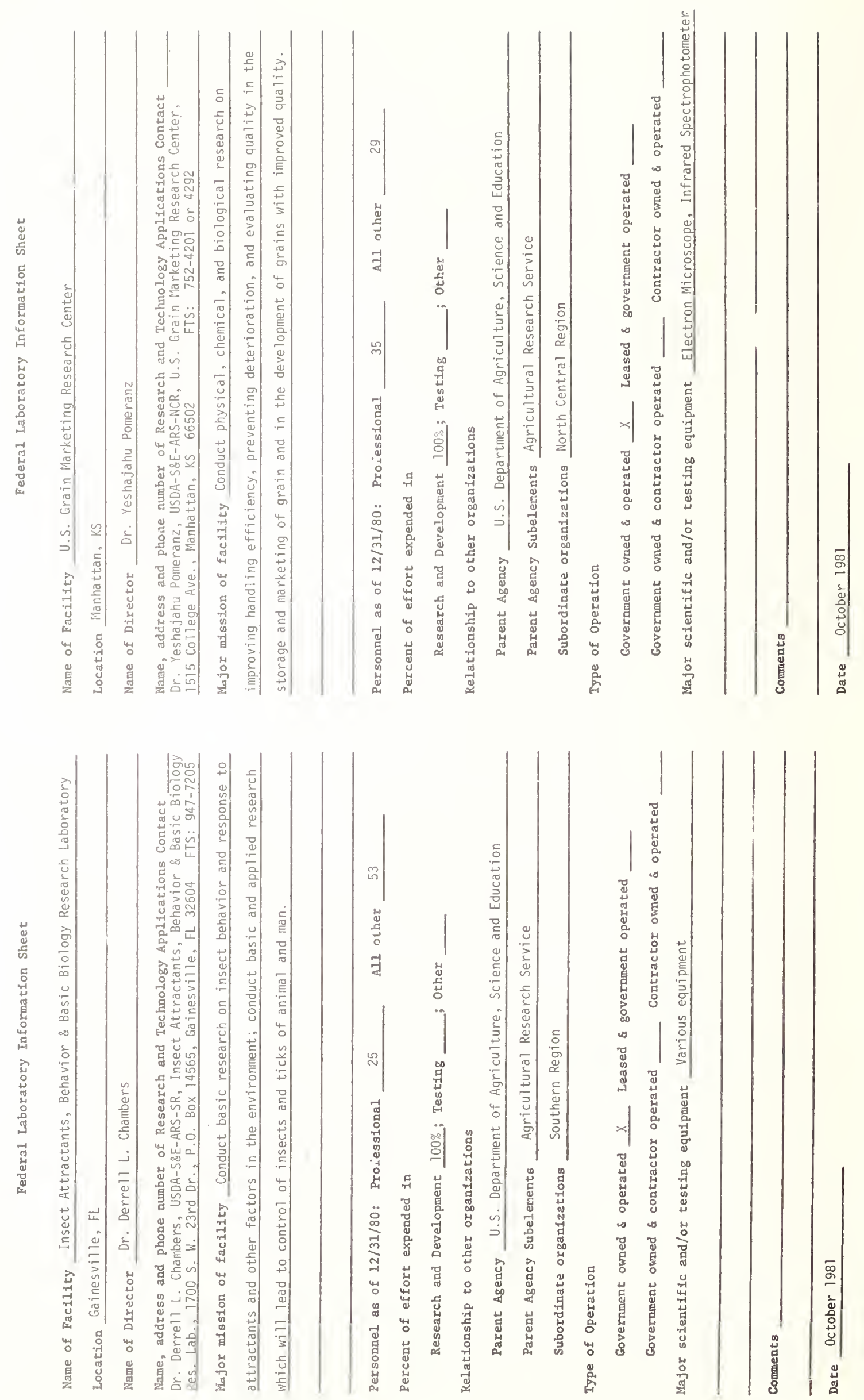

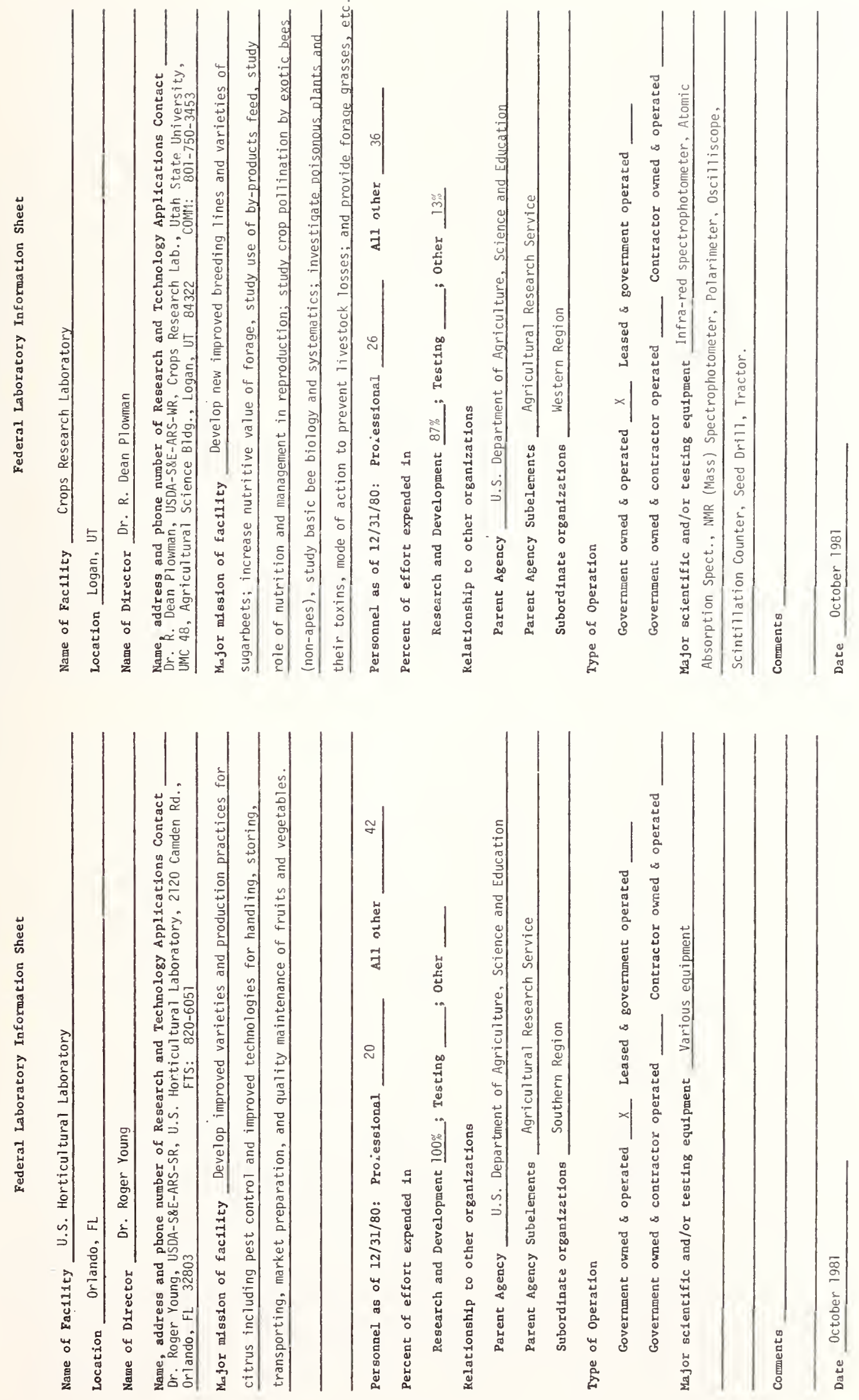

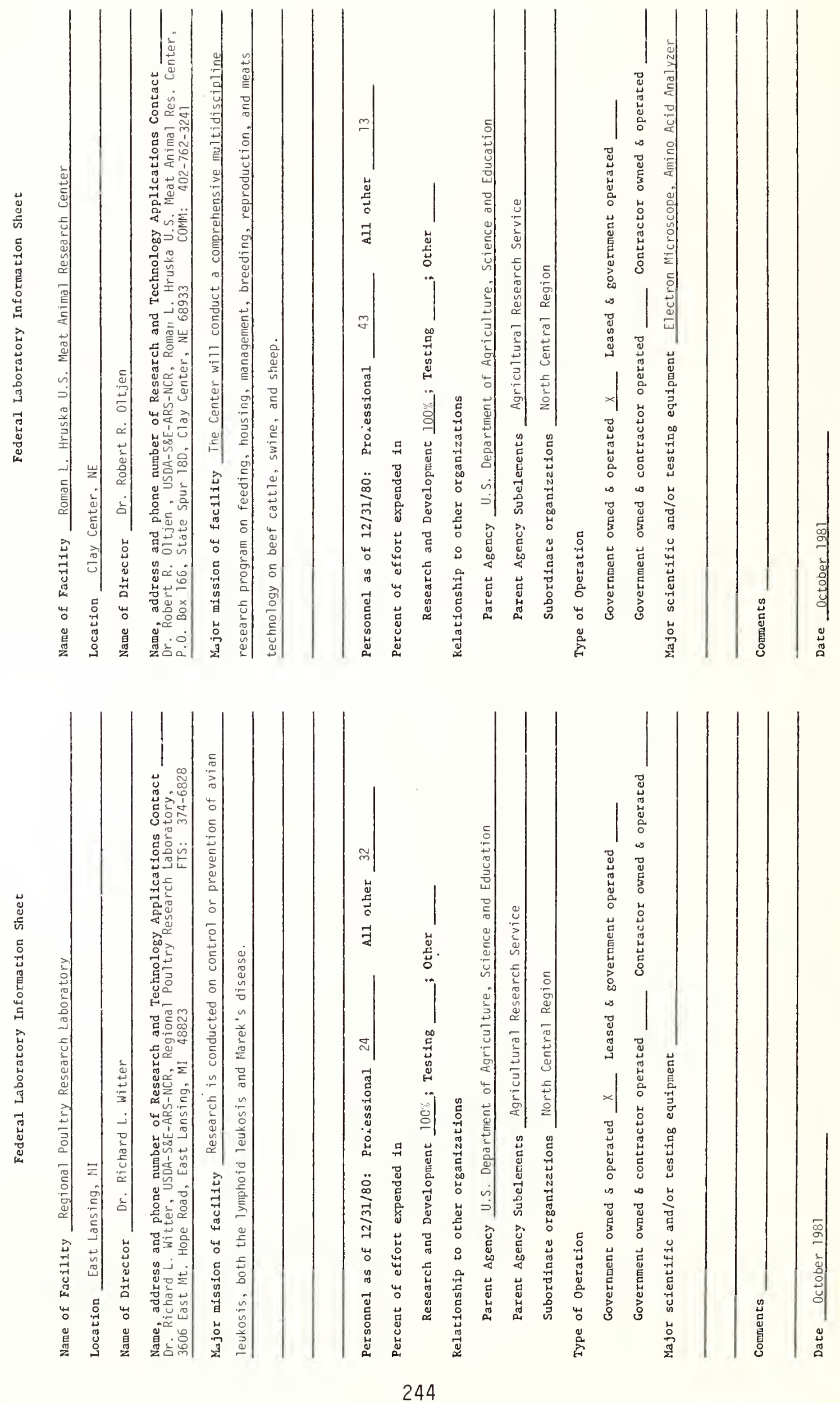

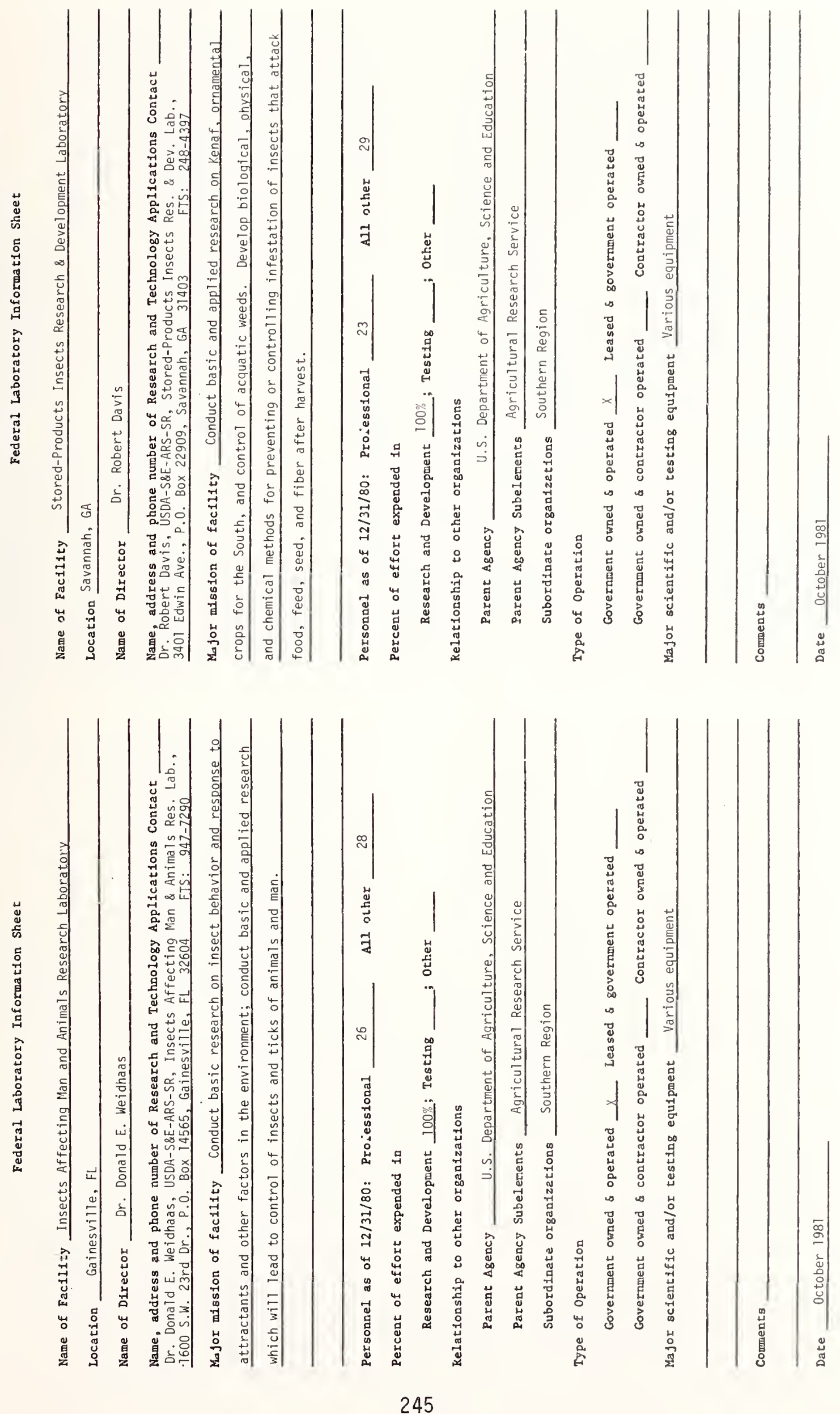

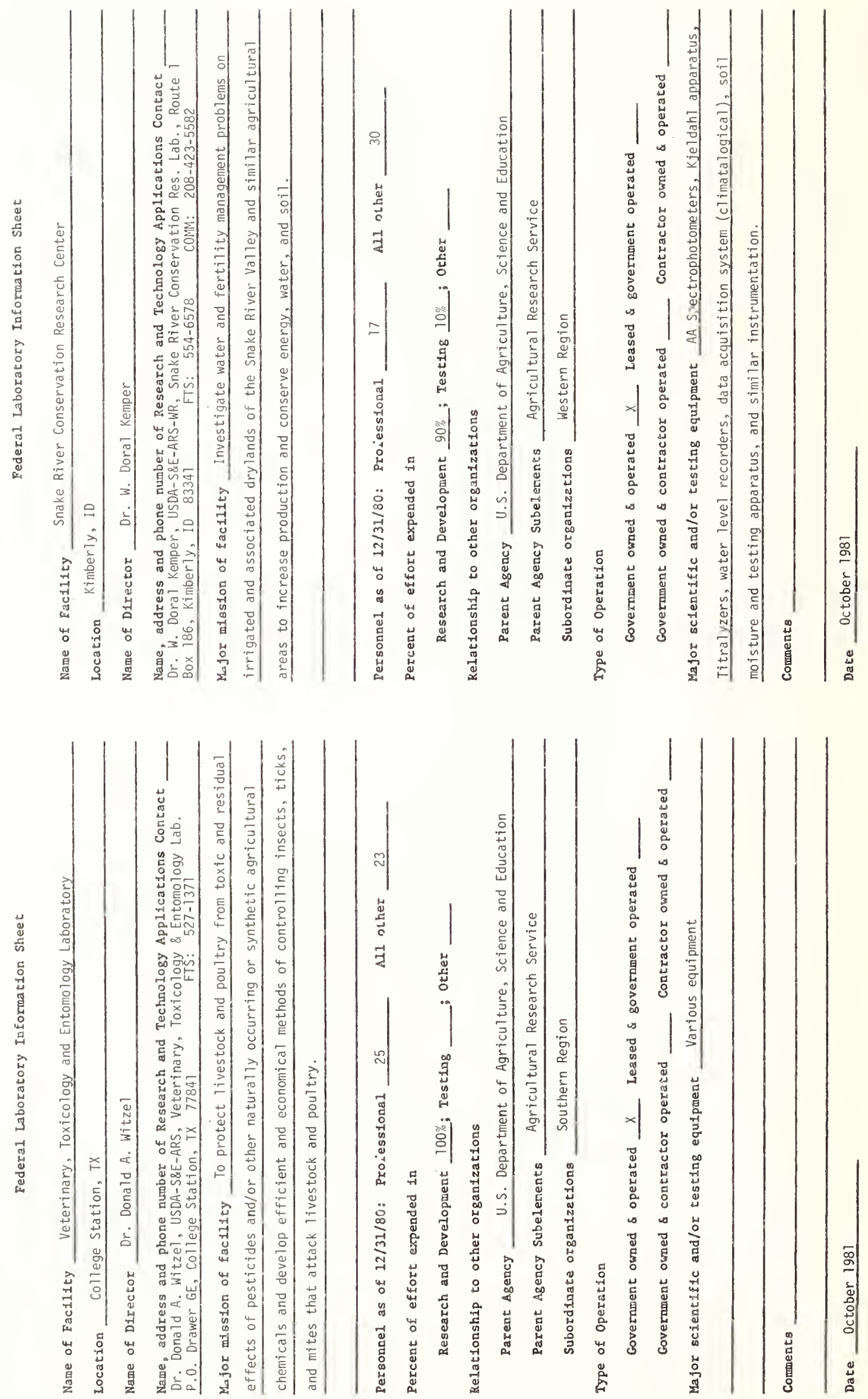

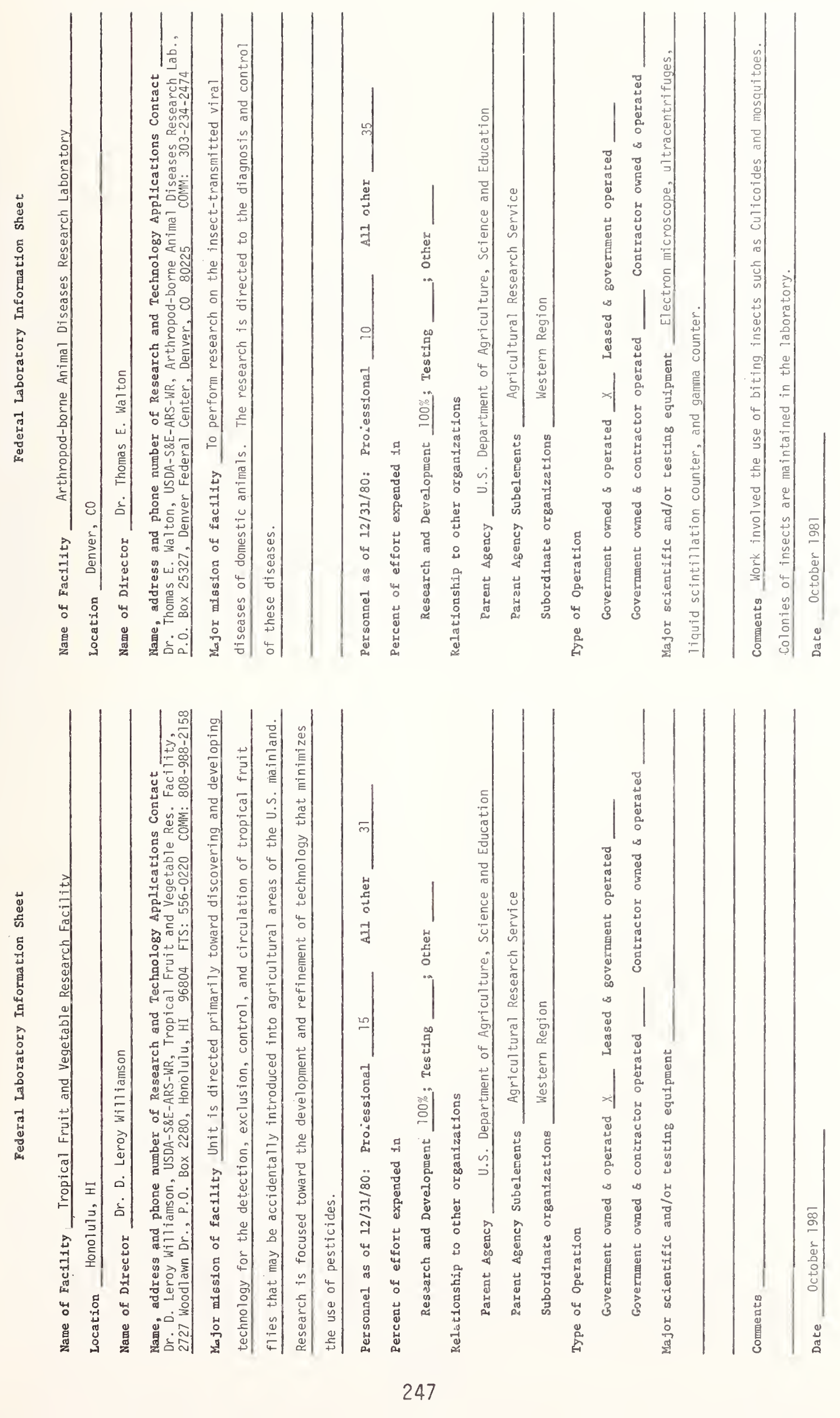

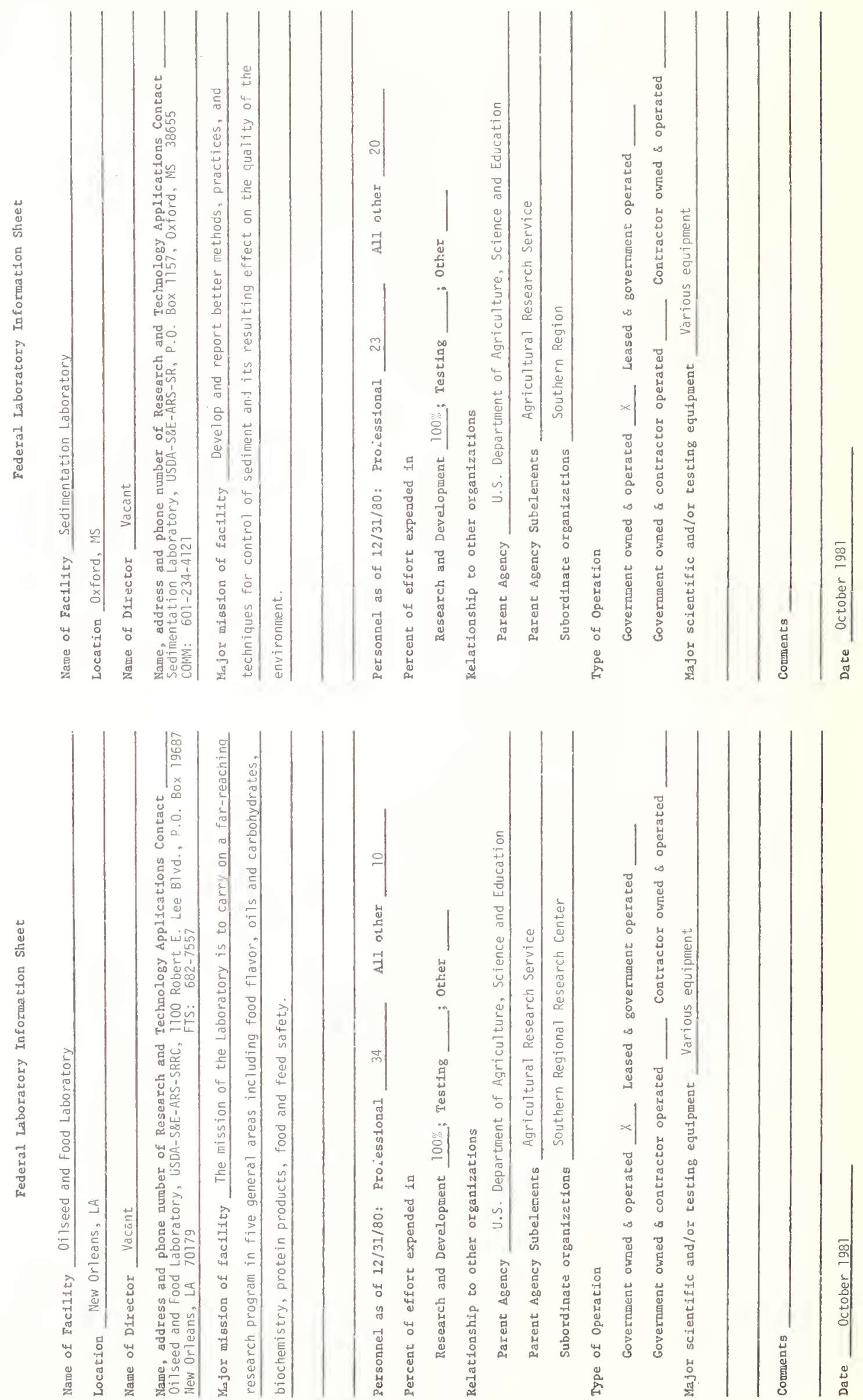

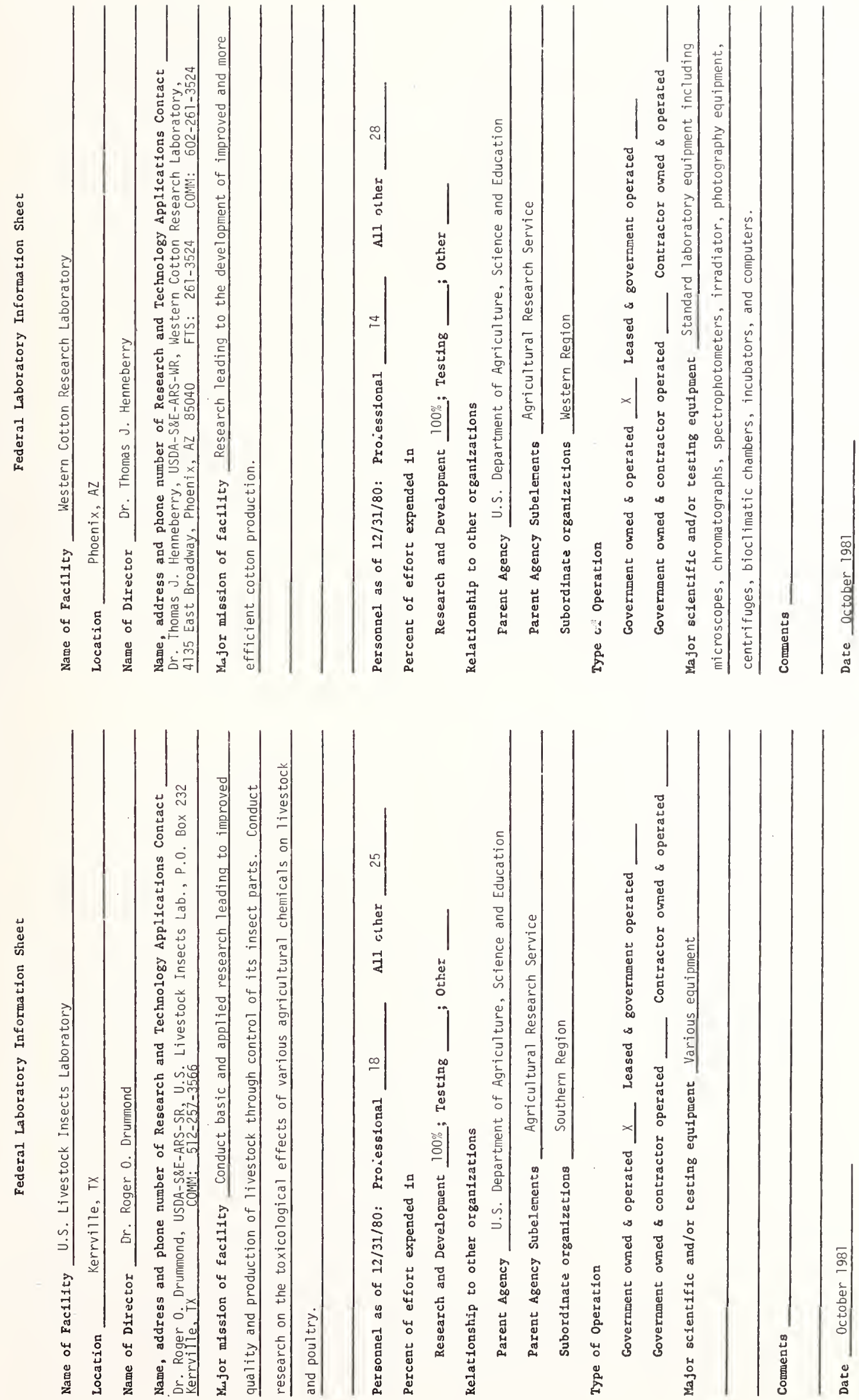

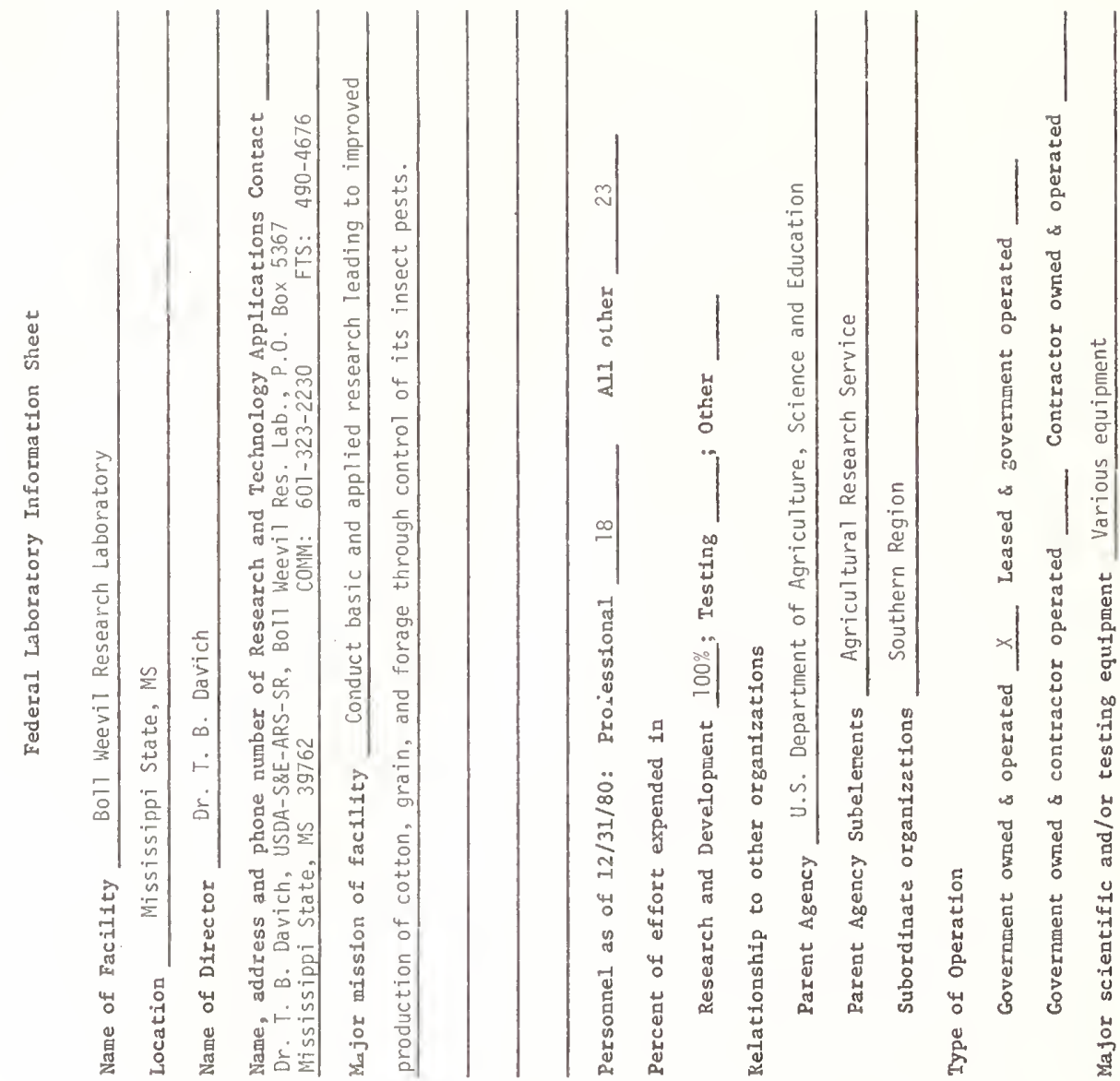

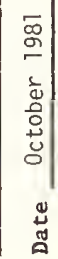

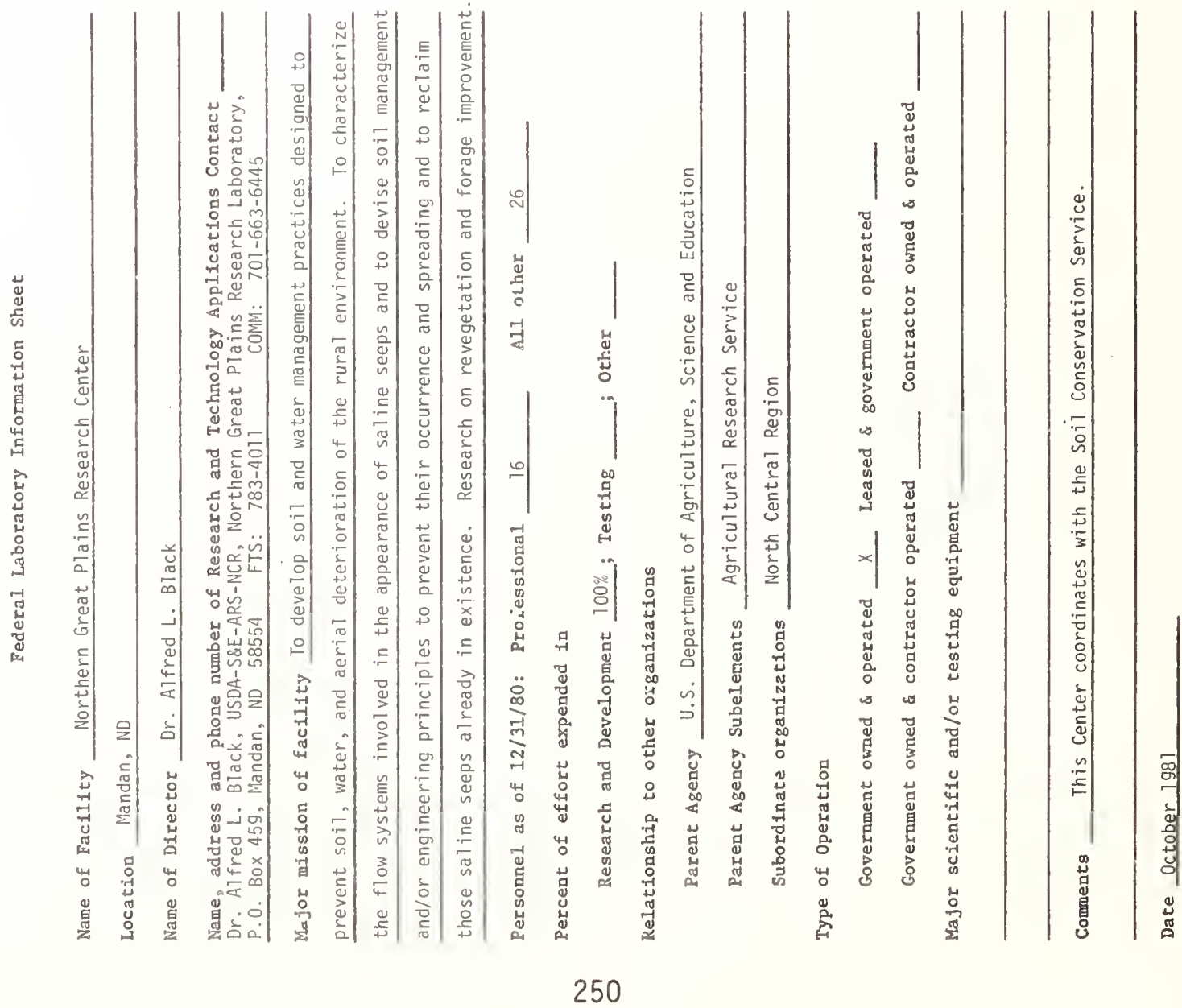



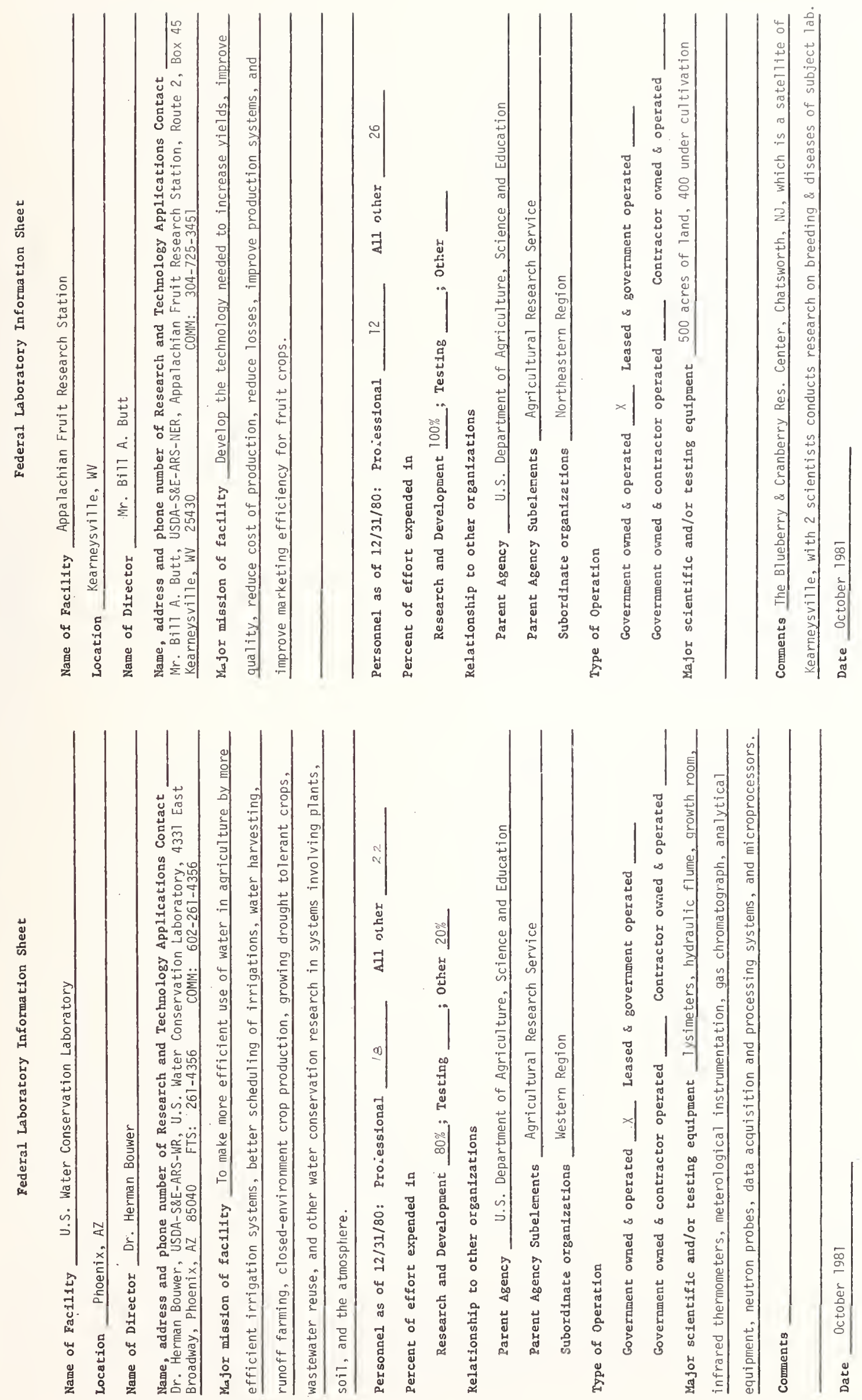

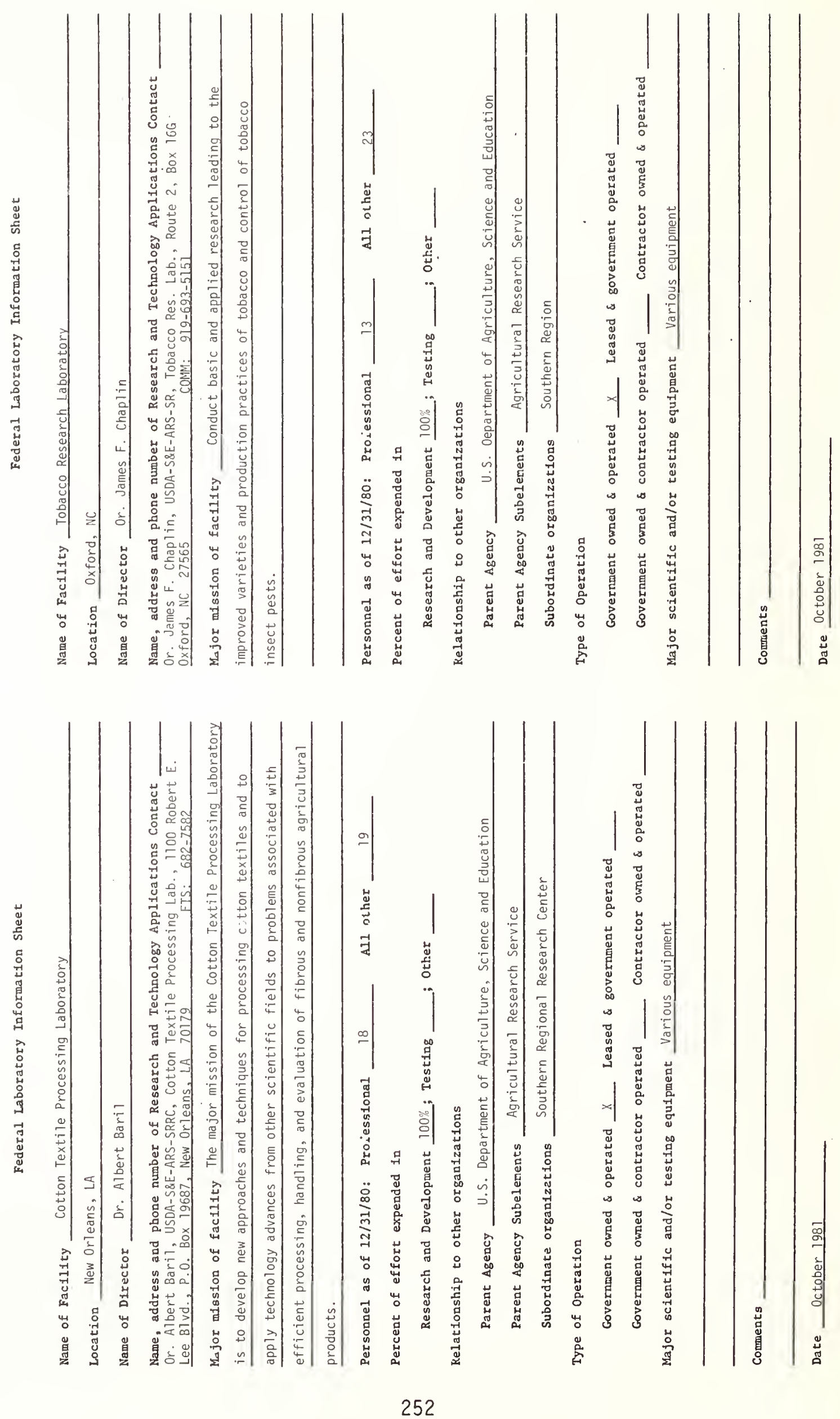

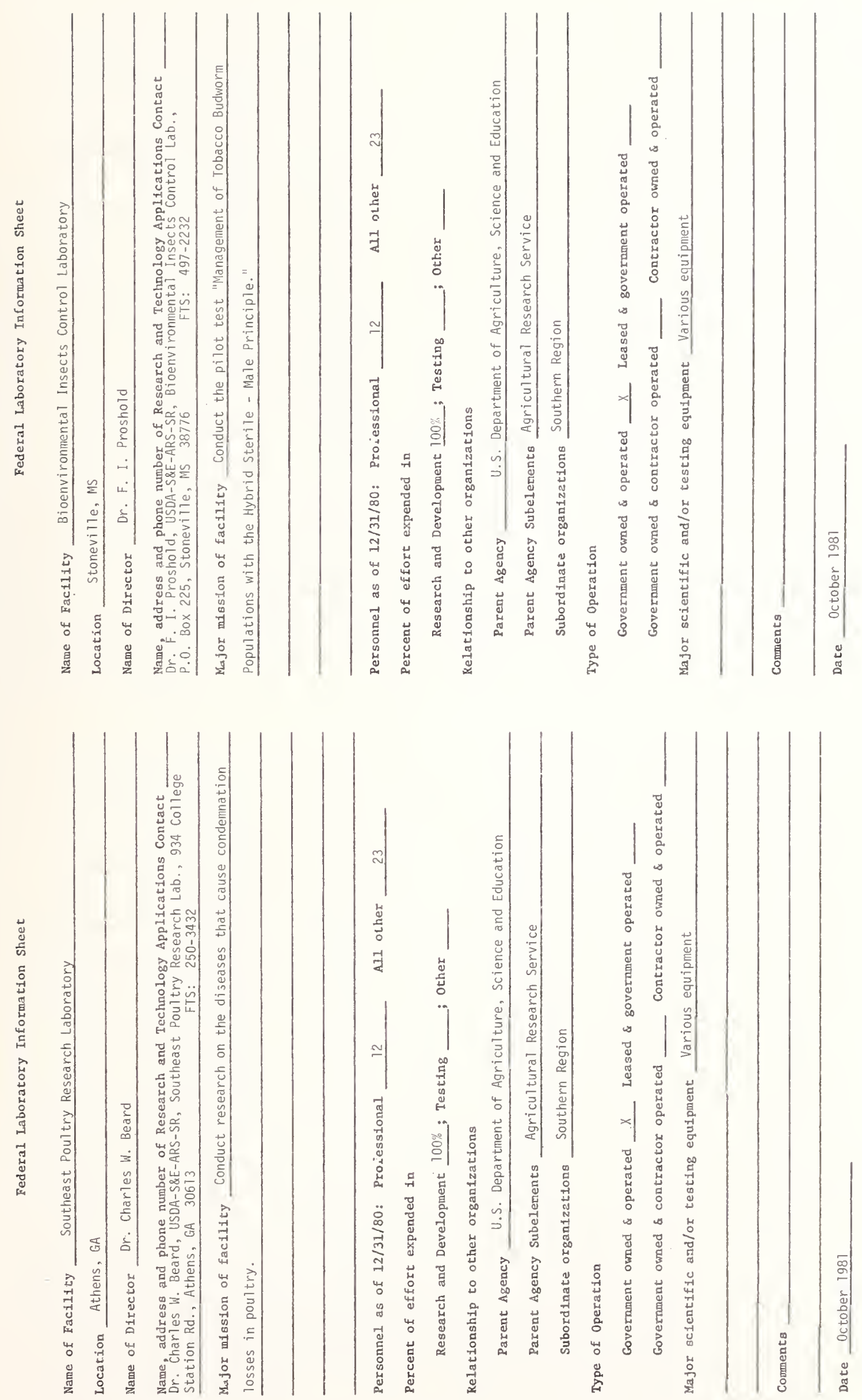

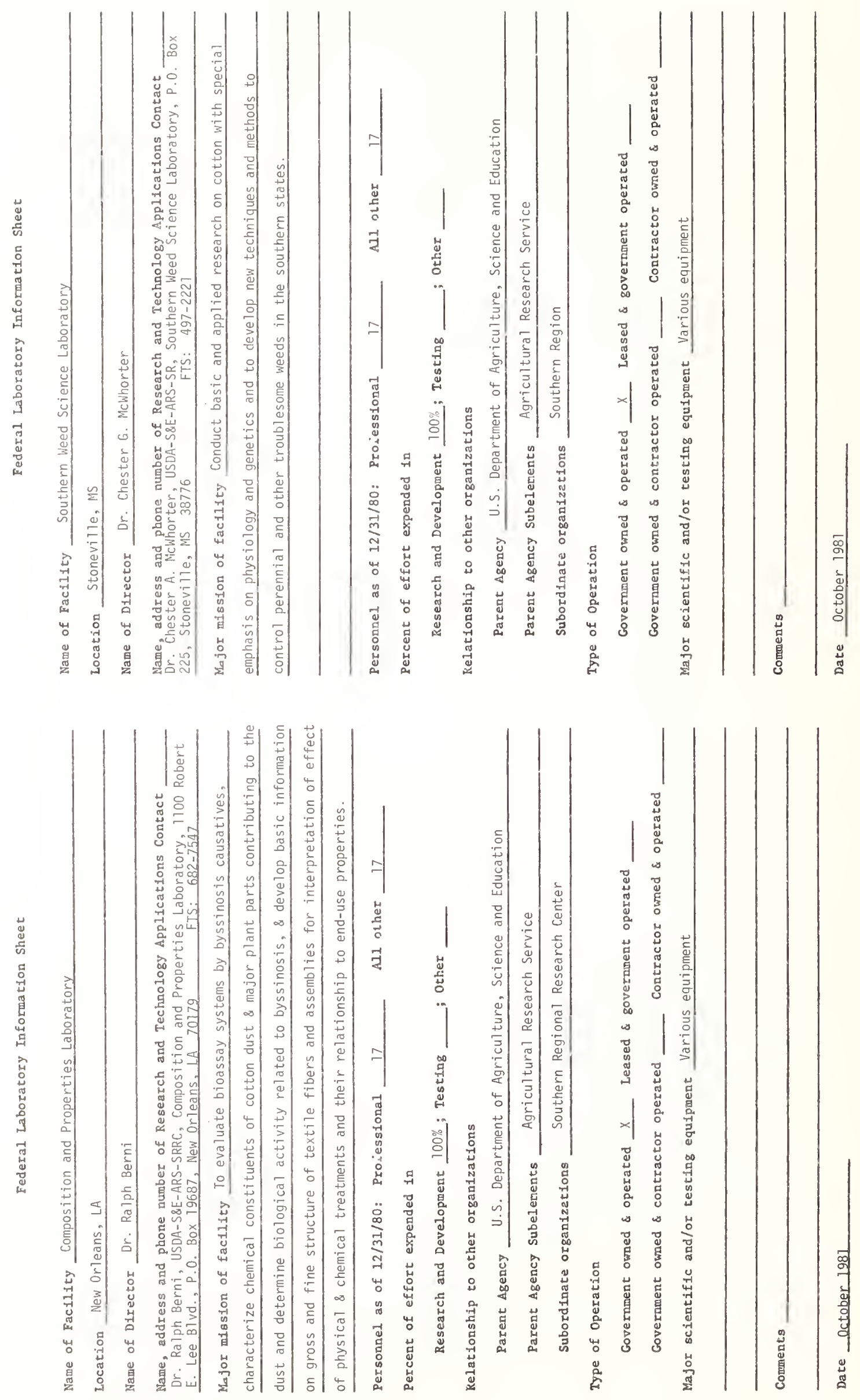

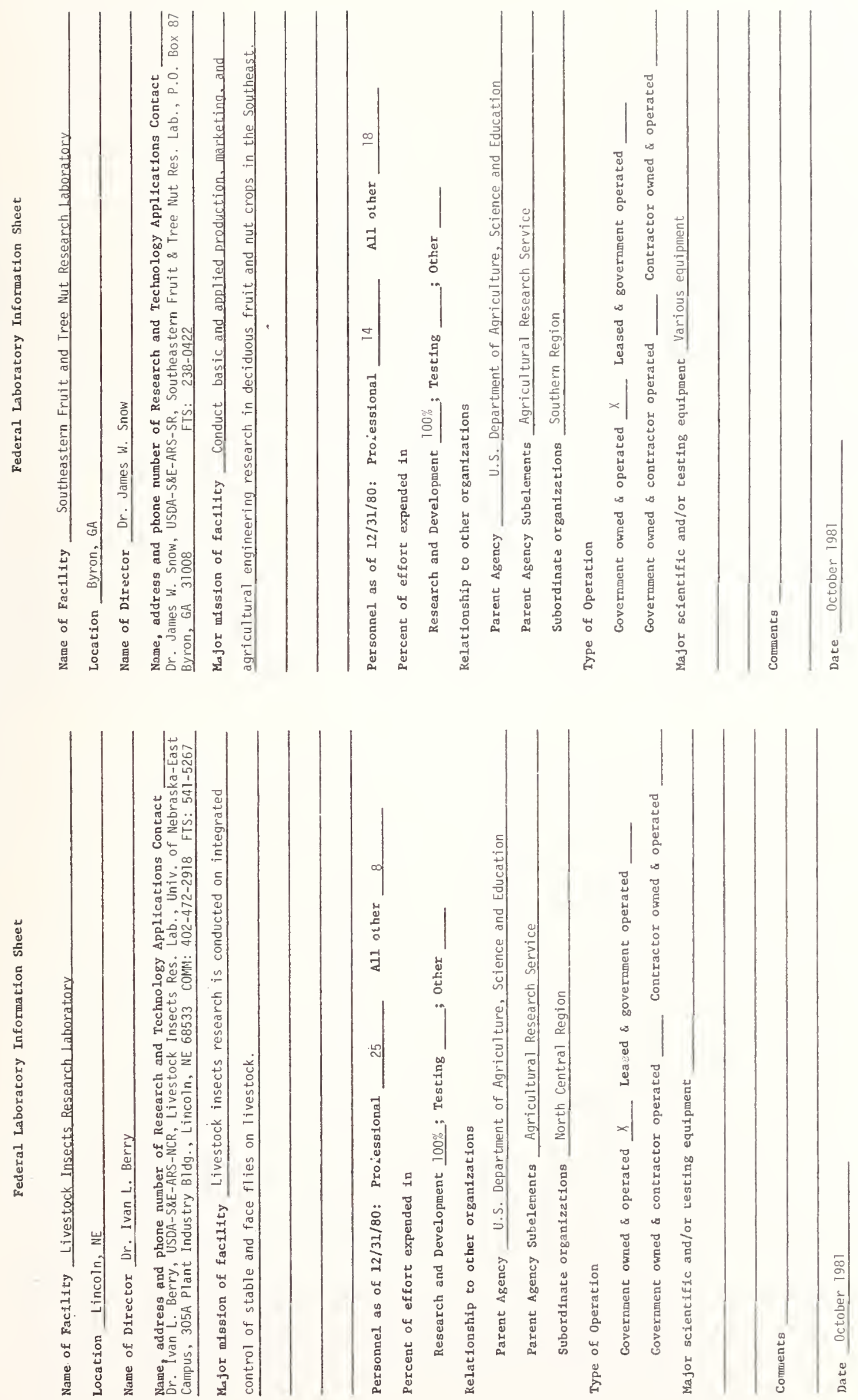

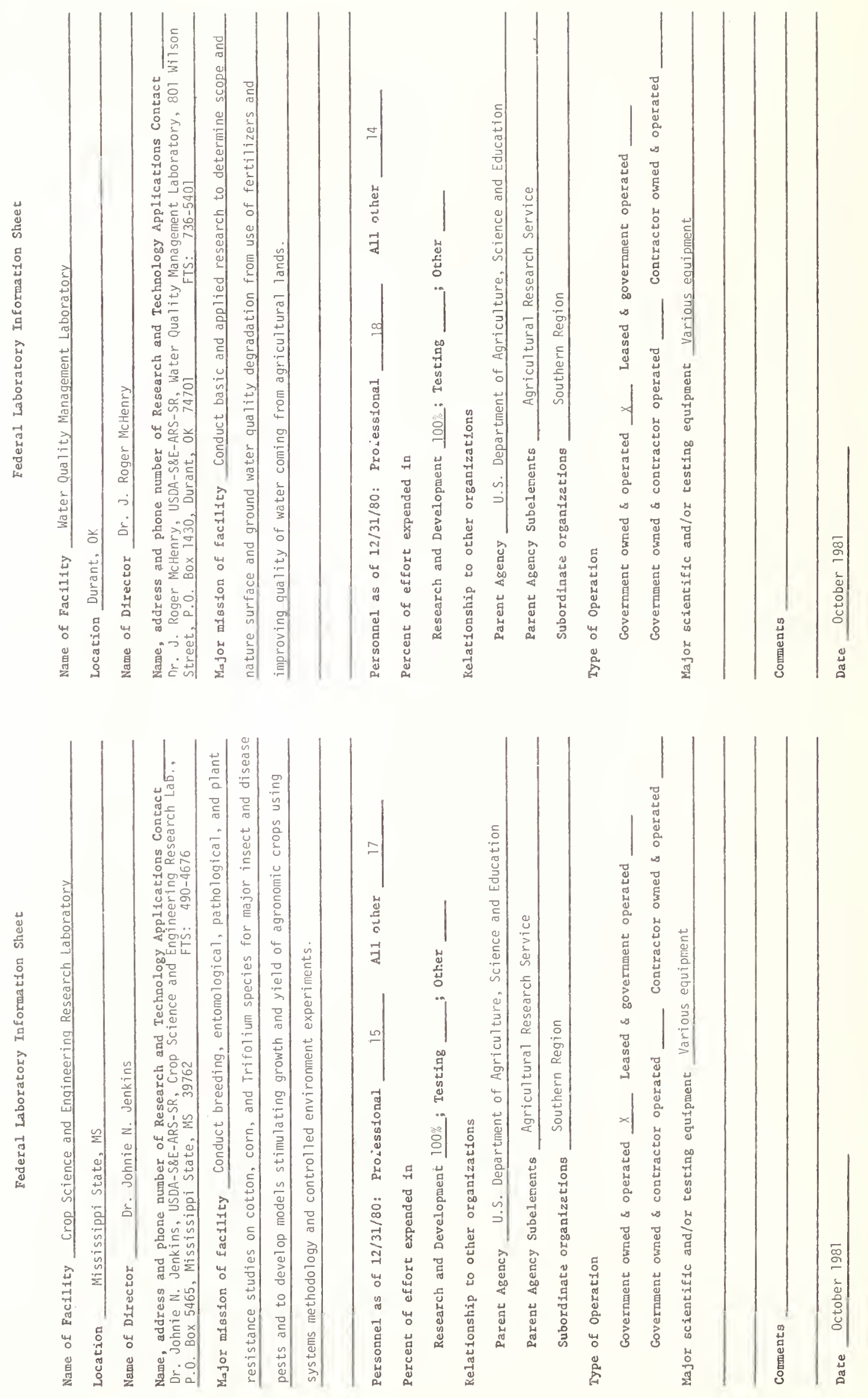

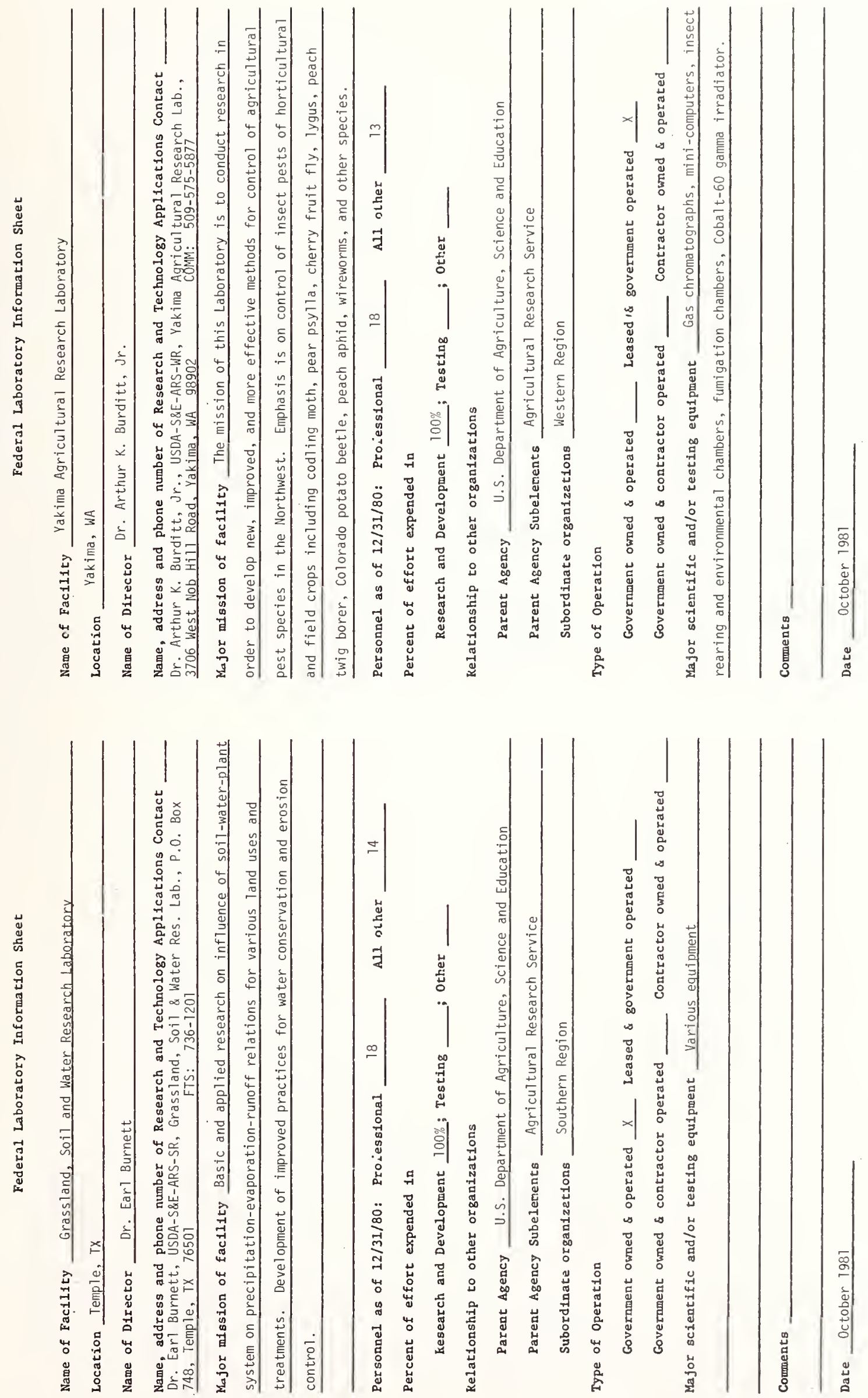

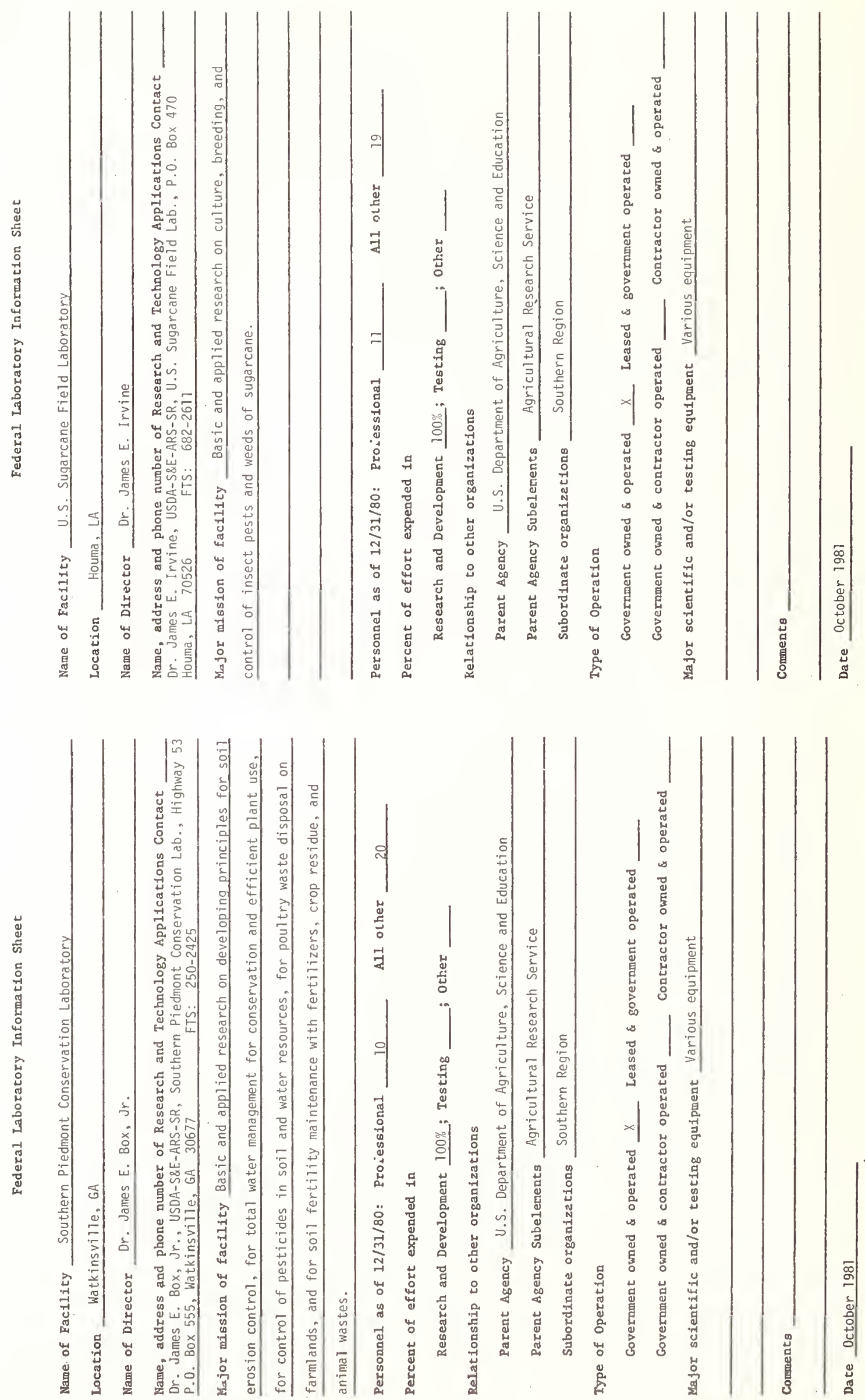

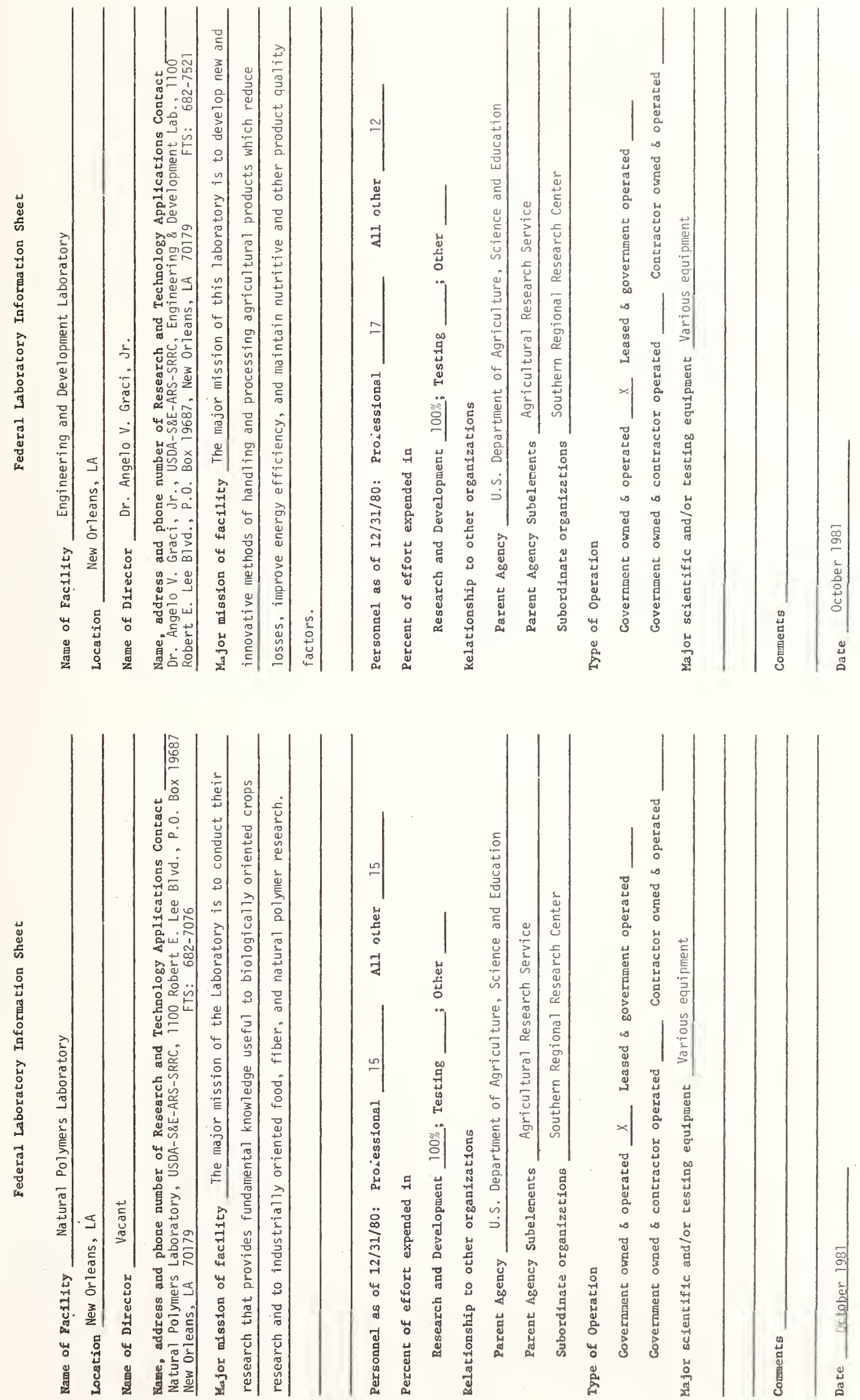

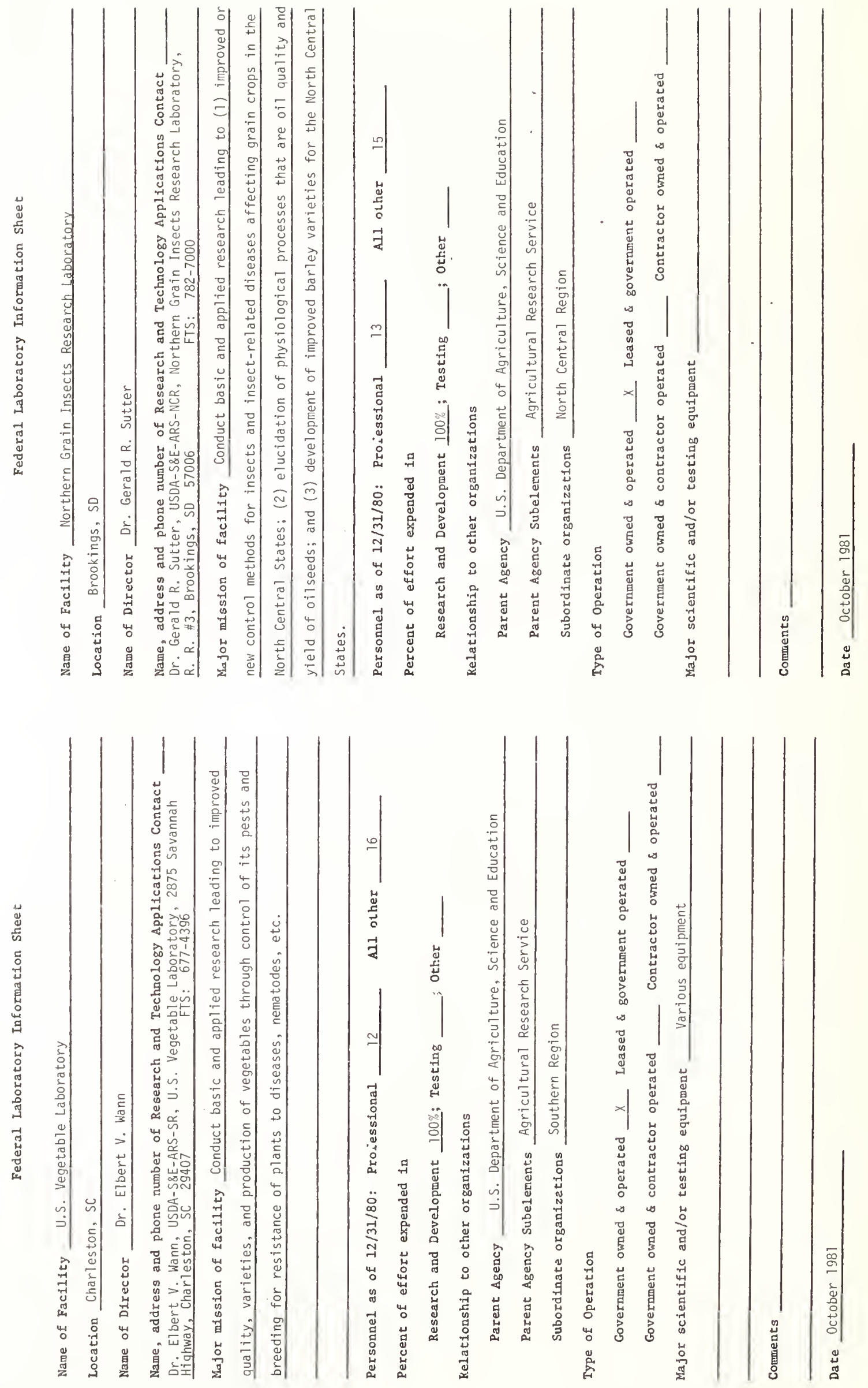

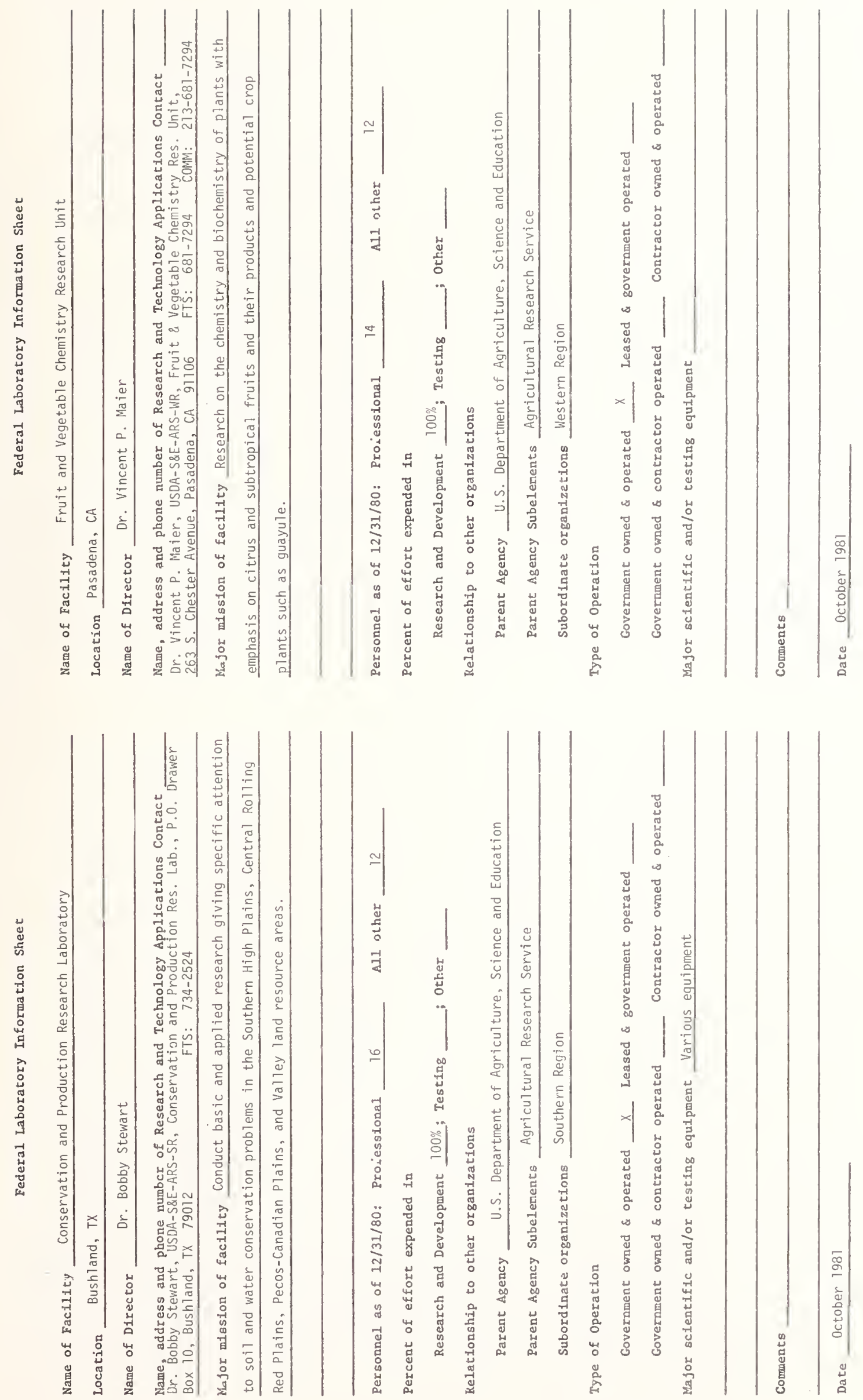

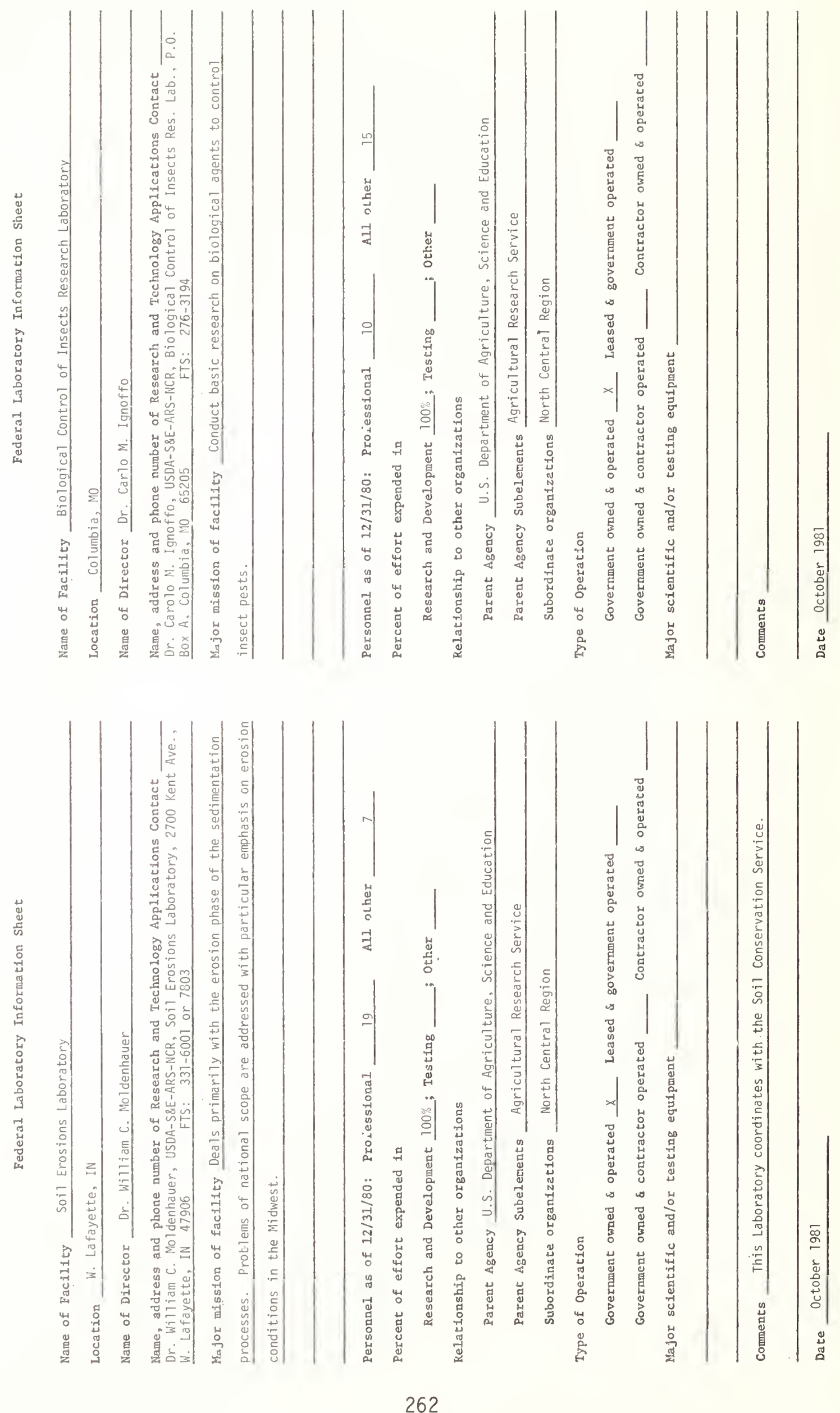

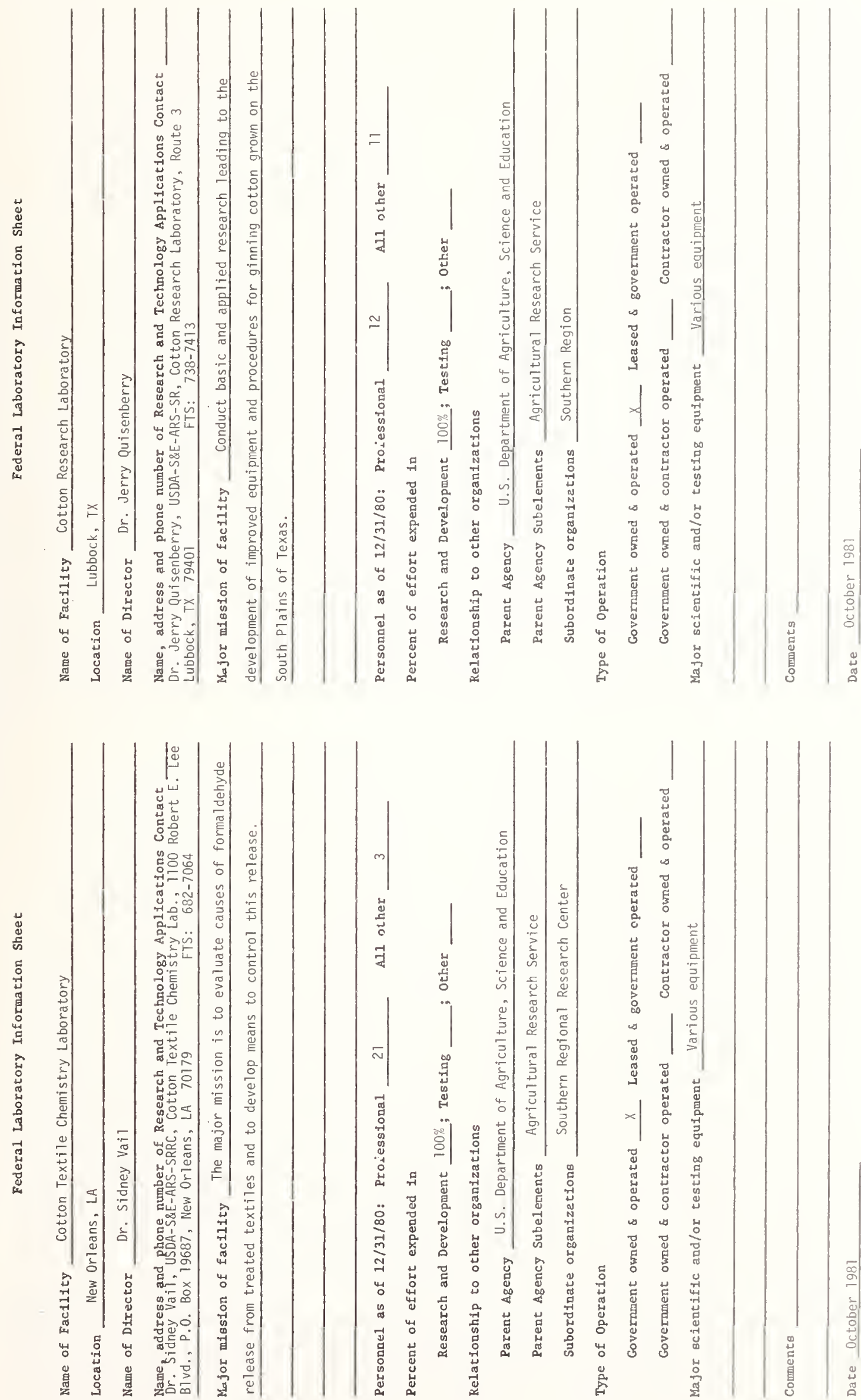

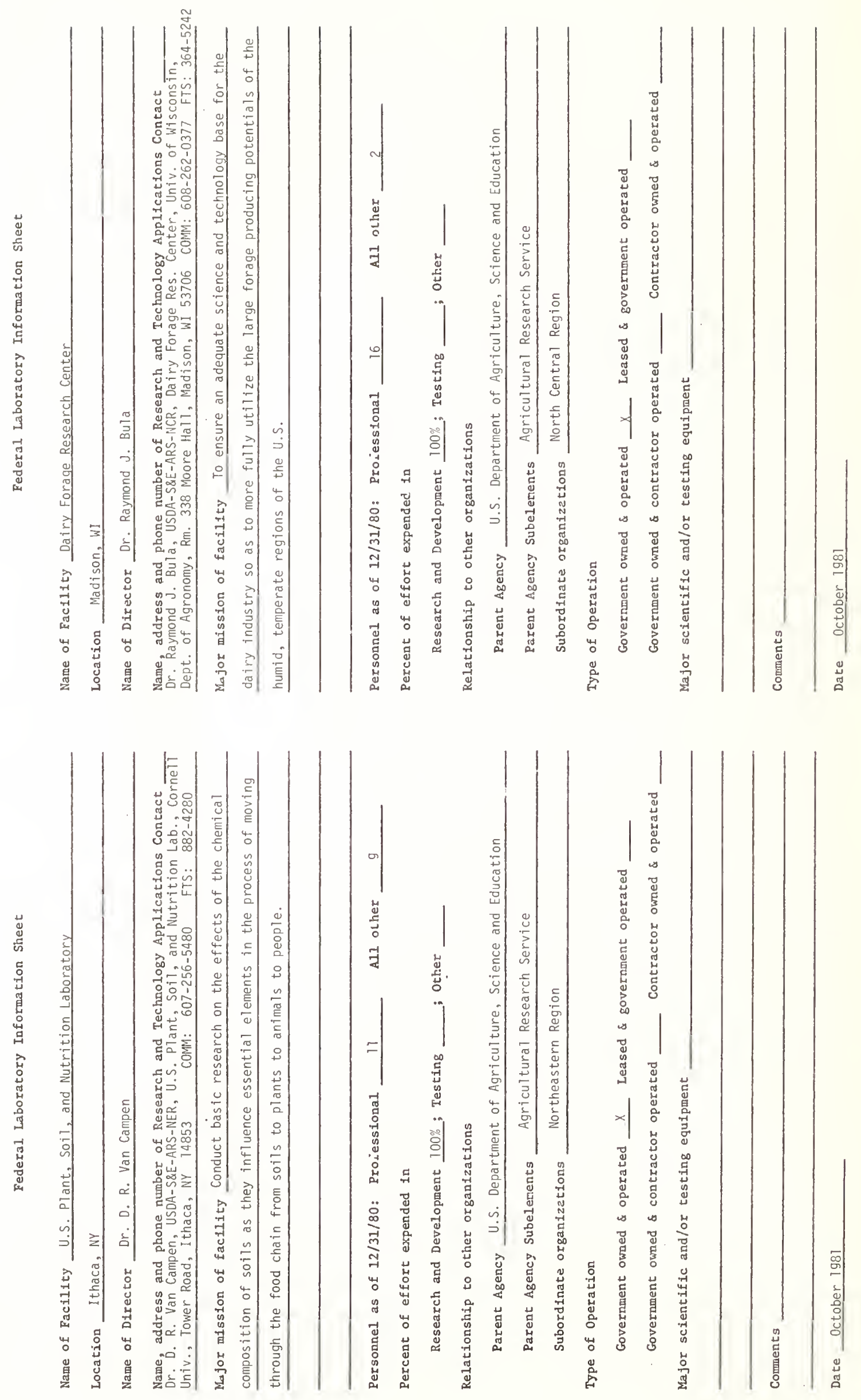

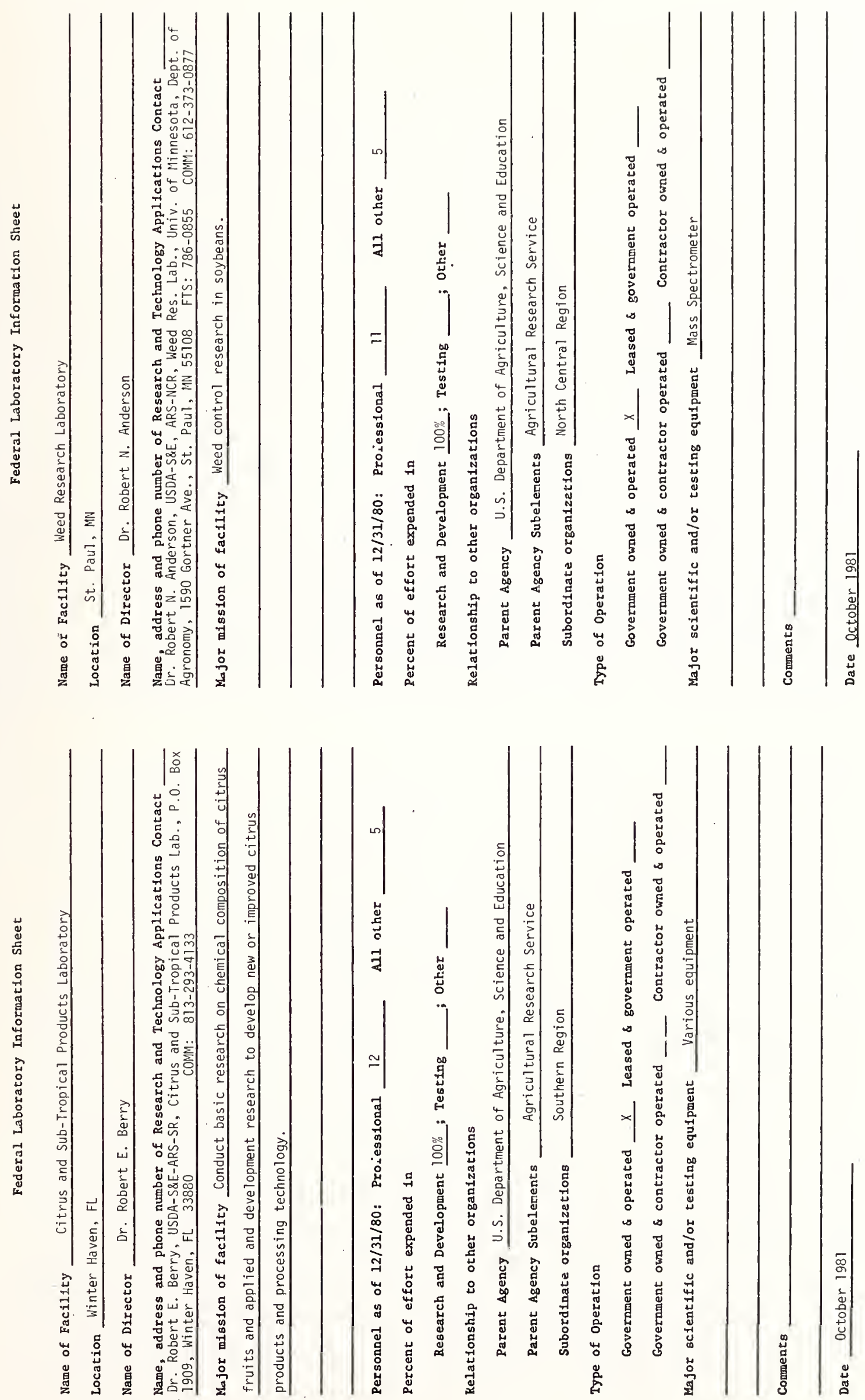

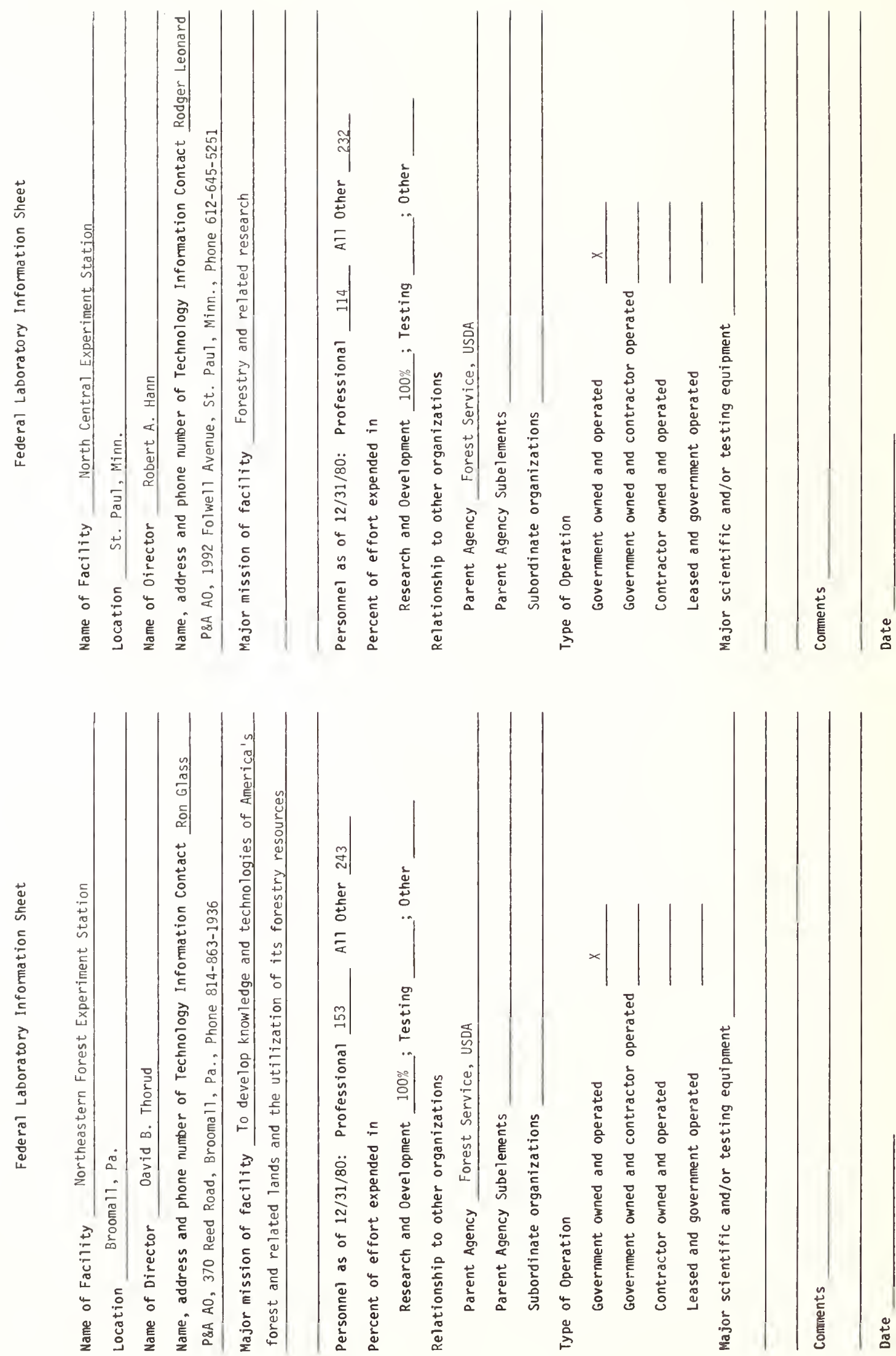

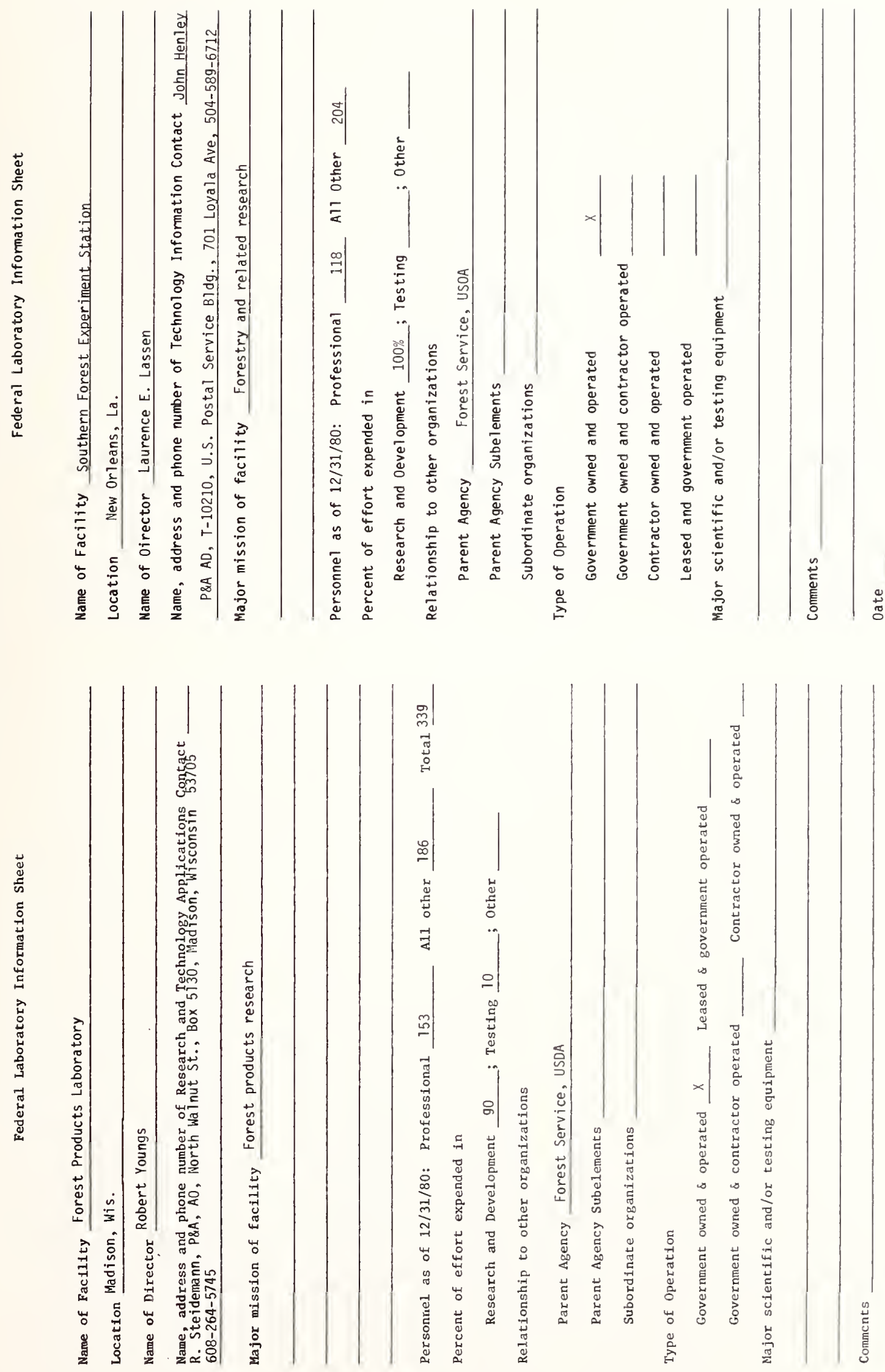

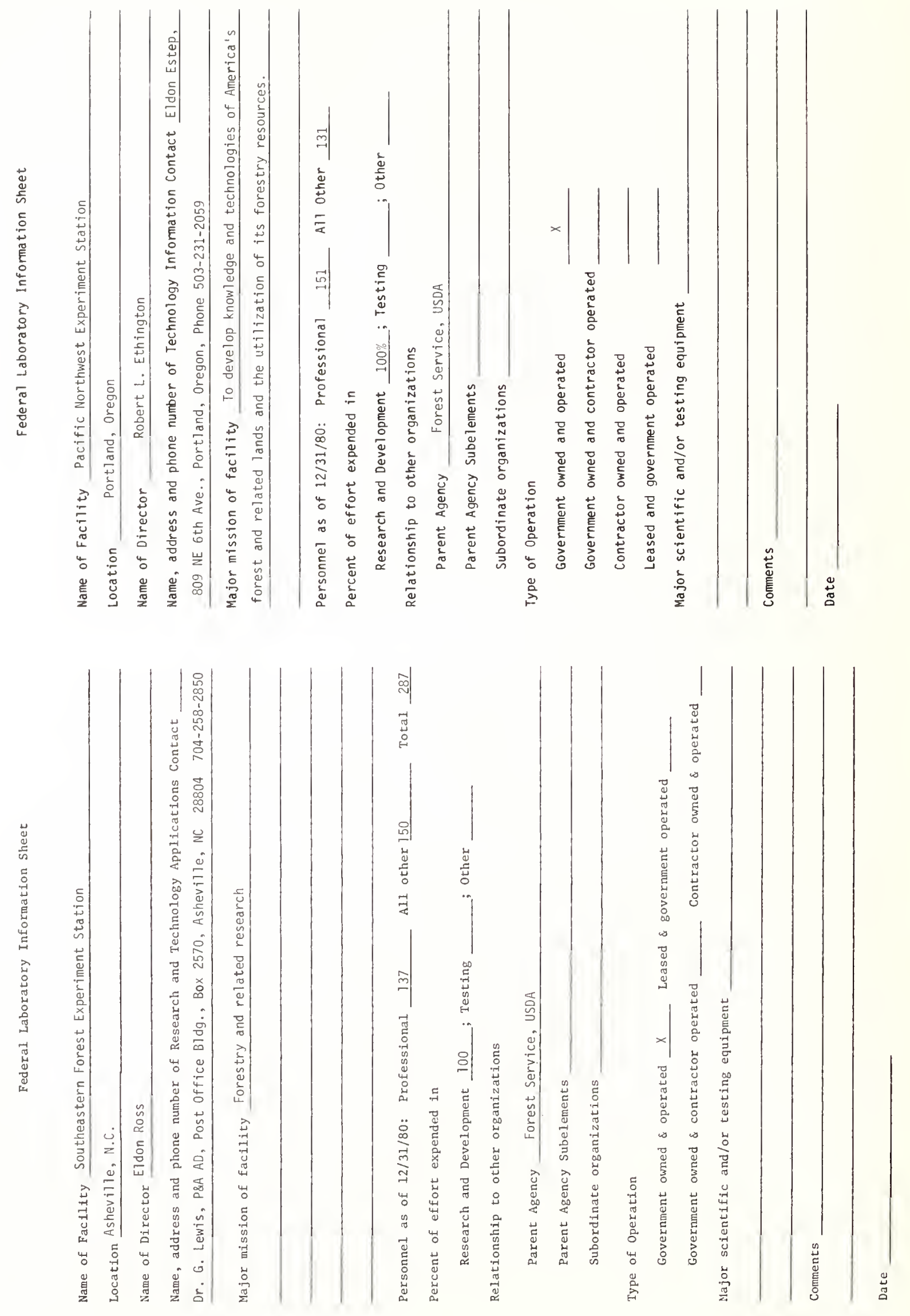

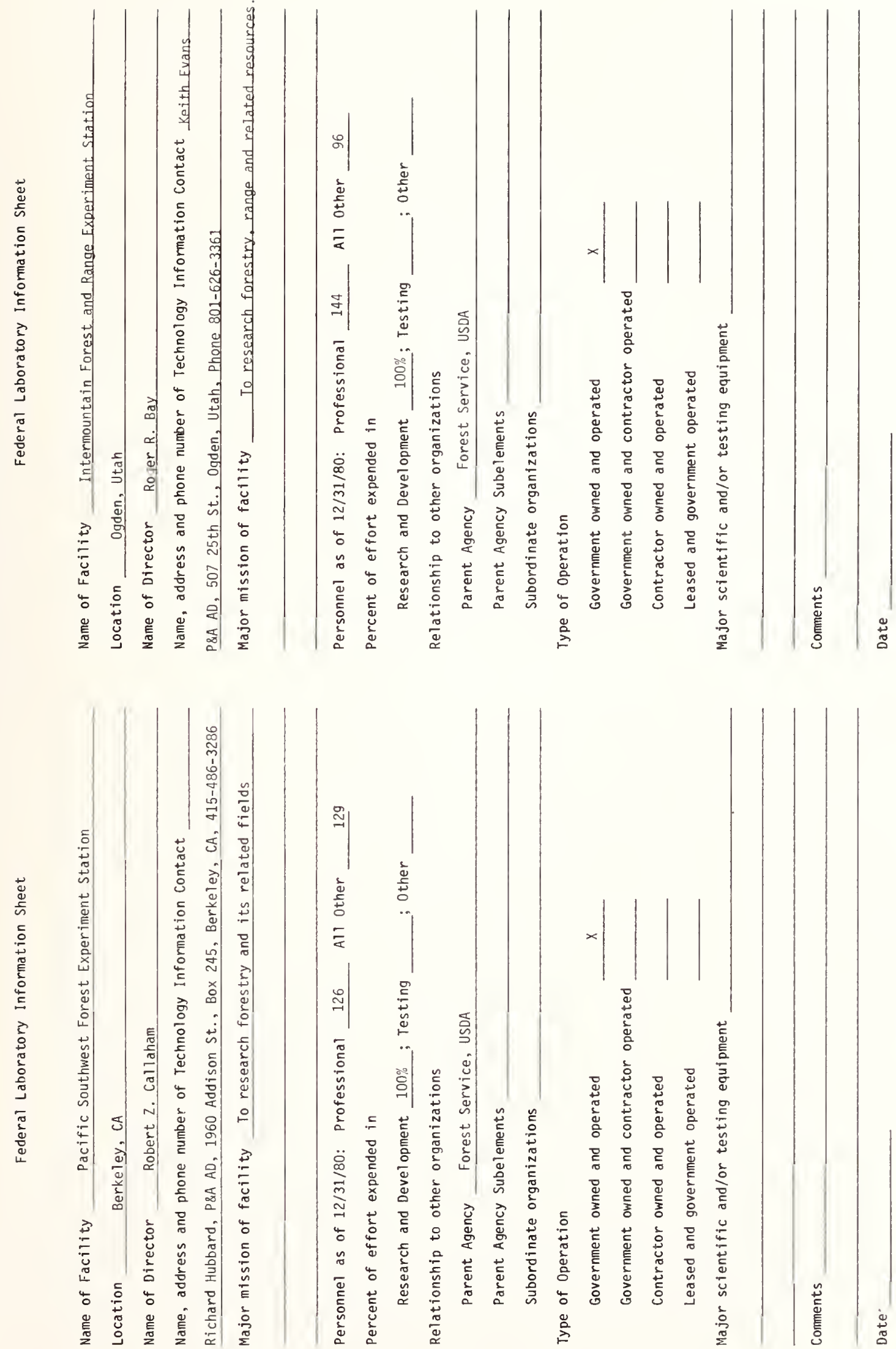


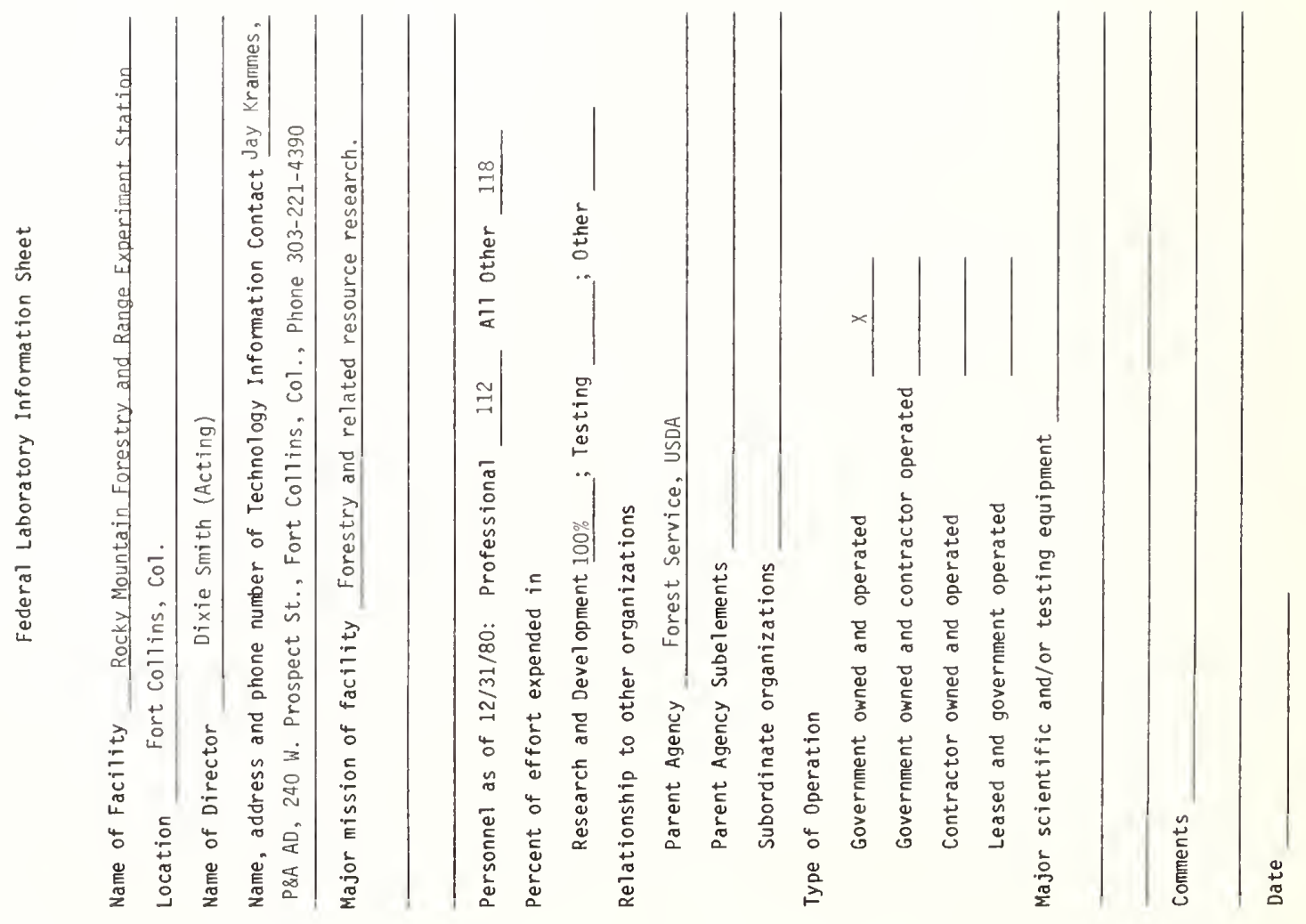




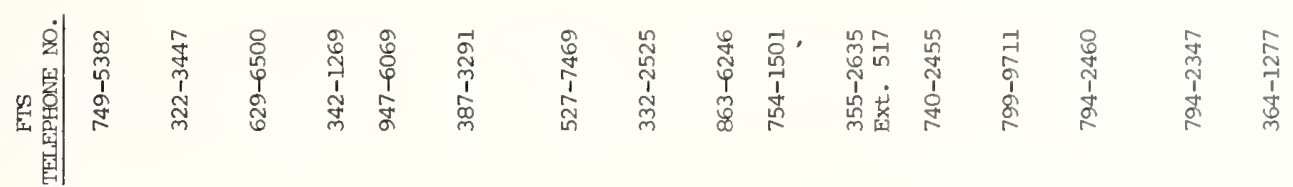

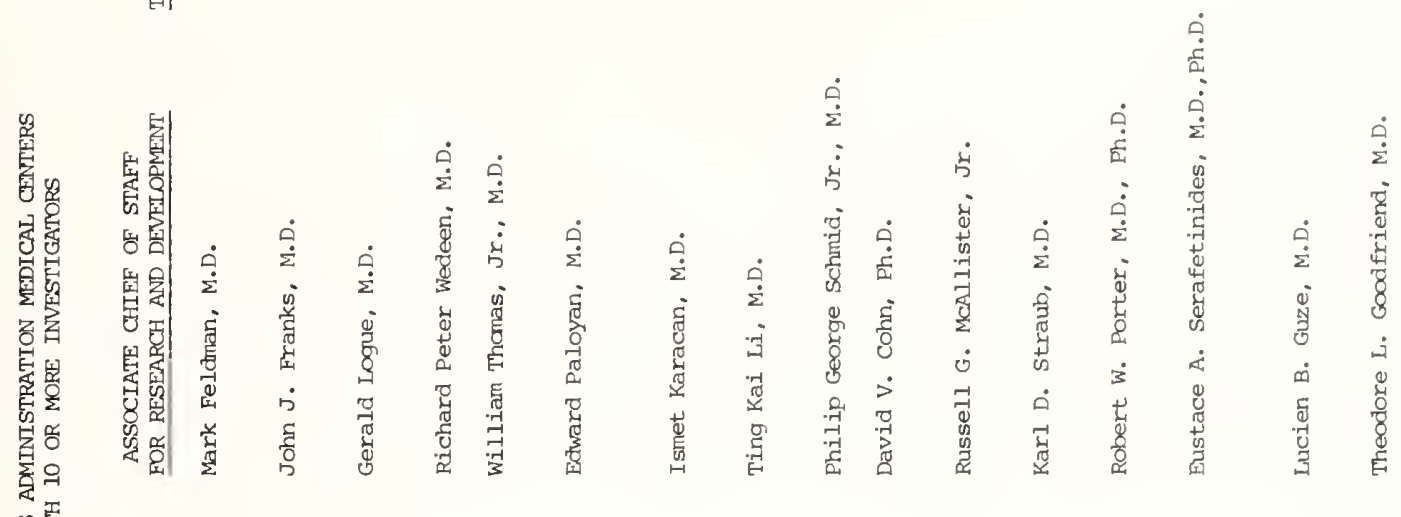
密

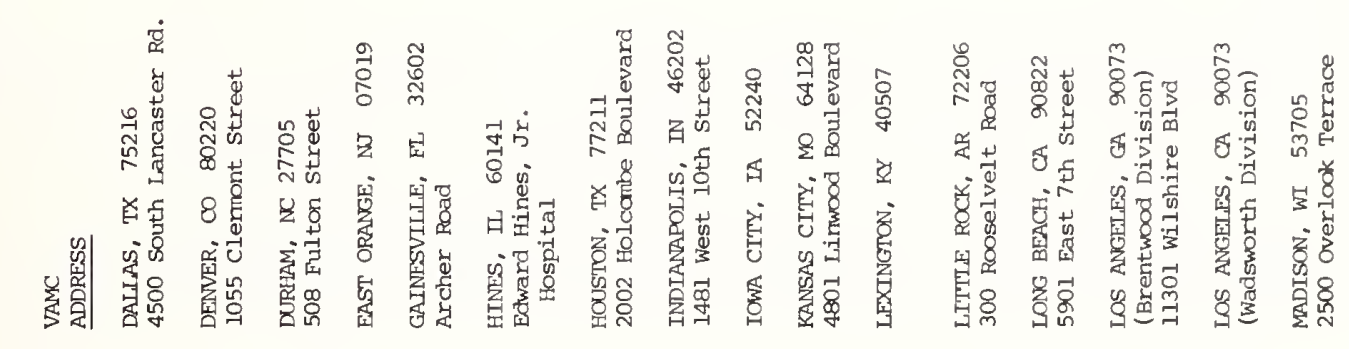

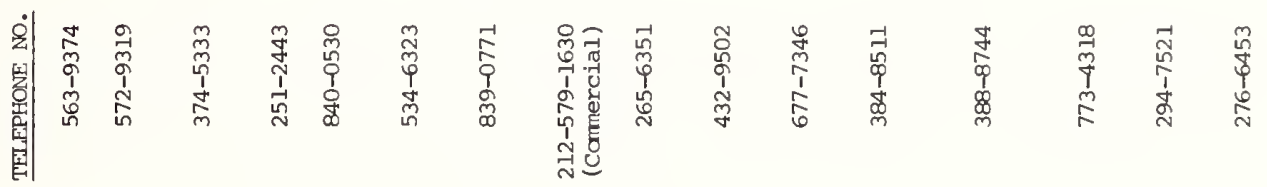

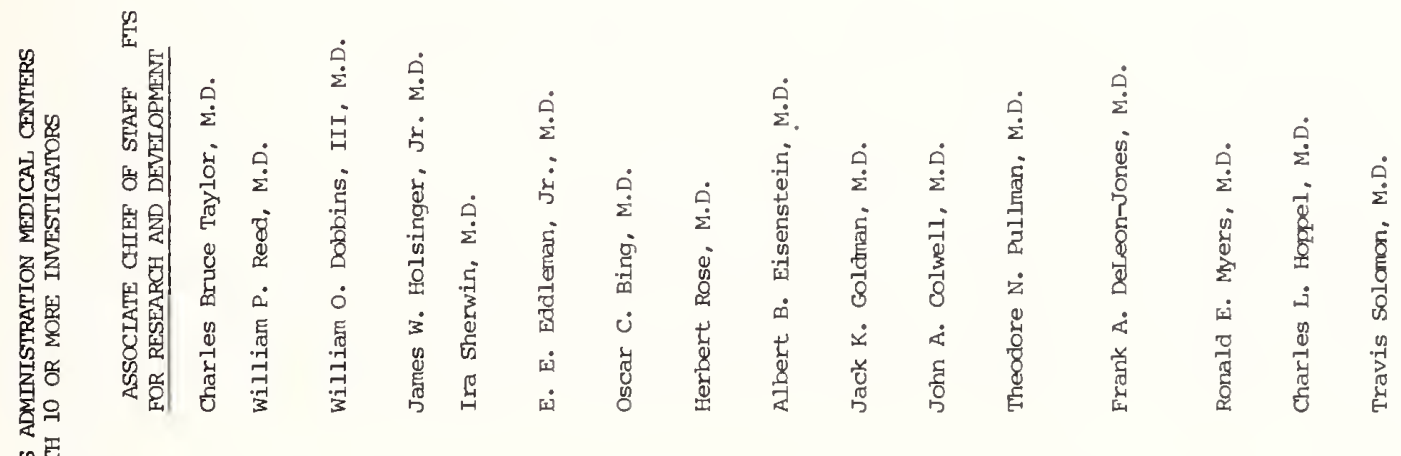

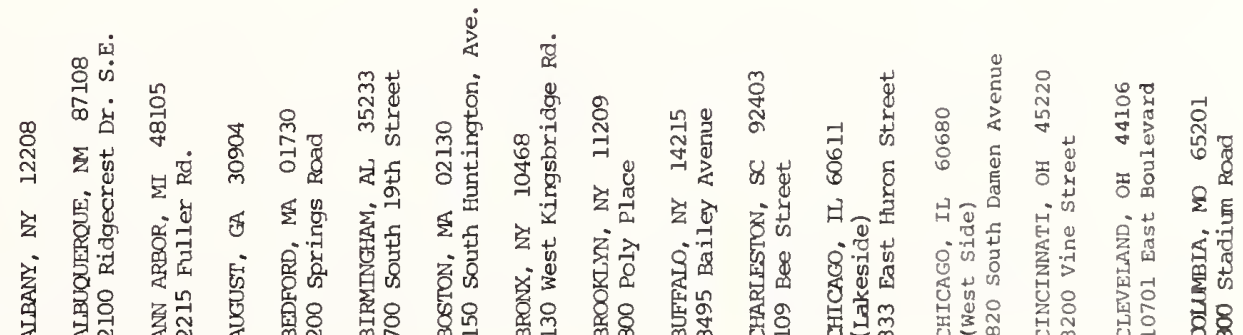




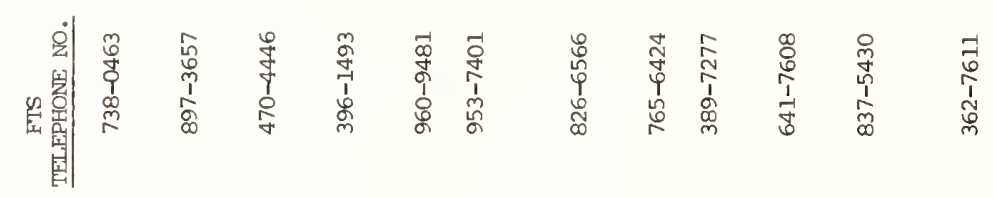

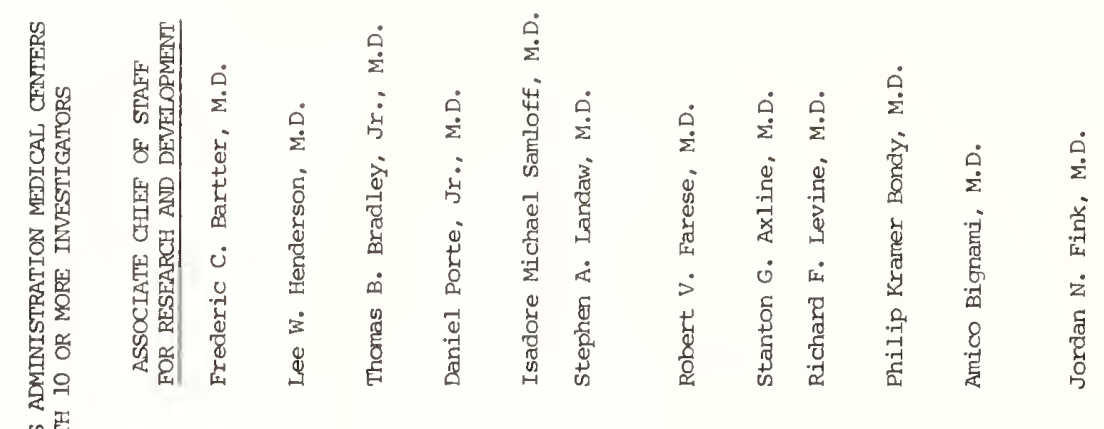

窟

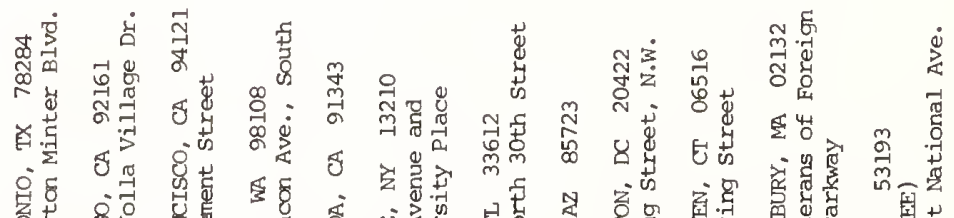

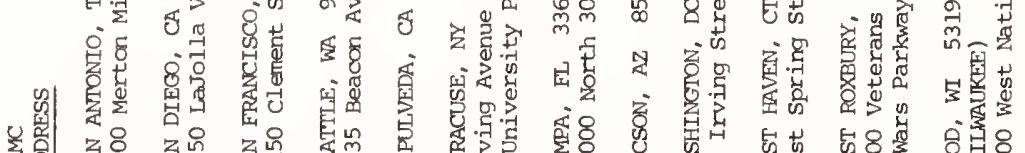

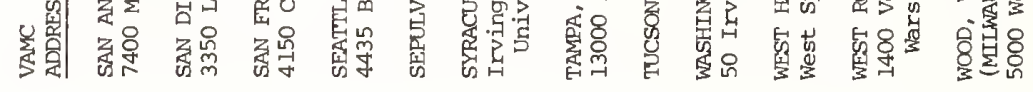

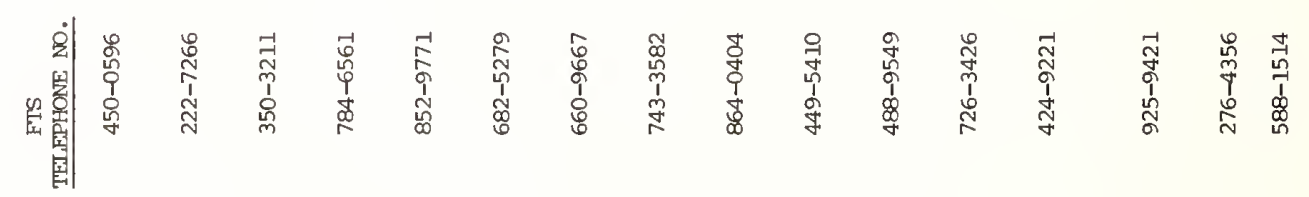

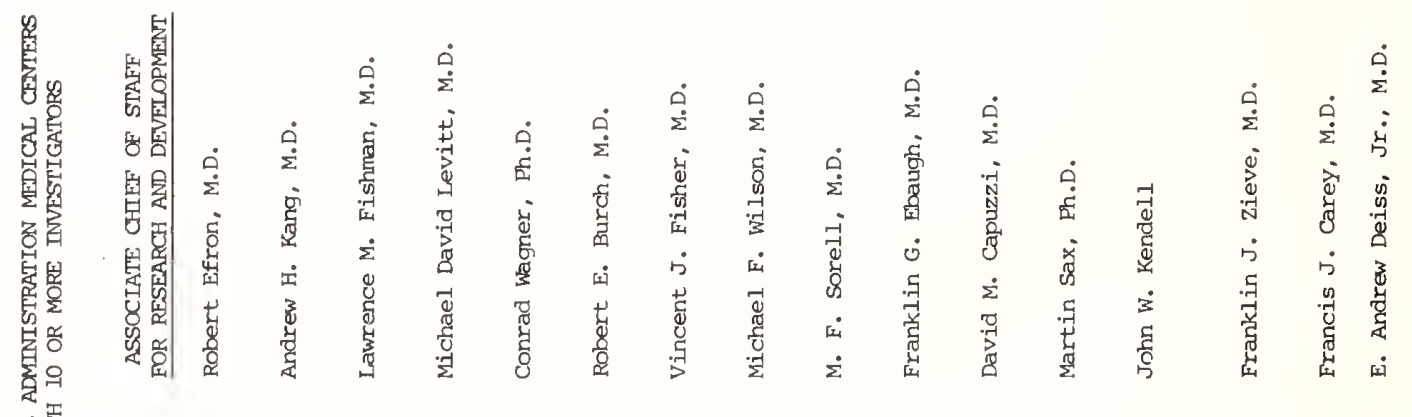

窟

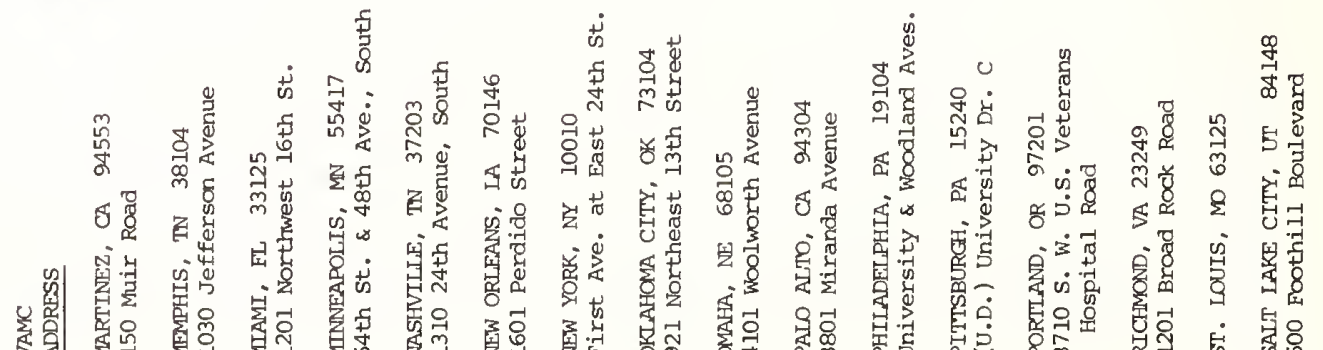




\author{
APR $\quad 81981$
}

Mr. James M. Wyckoff

Liaison Officer

State and Local Government Affairs

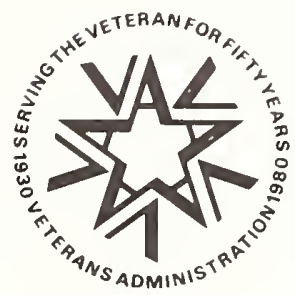

United States Department of Commerce

National Bureau of Standards

Washington, D.C. 20234

Dear Mr. Wyckoff:

This is in response to your letter dated March 24, 1981 requesting information about the Veterans Administration Research and Development program. We understand that this information is needed for preparing an updated reference document on Federal laboratories.

Biomedical, Health Services, and Rehabilitative Engineering research is conducted at approximately 129 VA medical centers and medical clinics. The appended listing identifies $60 \mathrm{VA}$ facilities which have 10 or more principal investigators conducting centrally reviewed and funded research programs. This listing identifies the address of each of these medical centers as well as the name and telephone number of the Associate Chief of Staff for Research and Development.

A brief summation of the VA's Research and Development program, highlighting its unique features, may help to clarify the difficulty we have in providing the requested data as outlined on your Information Sheet. Research and Development within the VA is chiefly an intramural program in which clinician-investigators at VA medical centers study problems arising during their care of veteran patients. Non-clinical scientists in VA medical centers work in close collaboration with clinicians and support their research efforts. The research projects are primarily investigator initiated. Consequently, research conducted at any one VA medical facility covers a broad range of program areas, e.g., cardiovascular, pulmonary, behavioral science. All VA studies are in concert with the overall mission of providing the best available health care for veteran patients.

Equipment and instrumentation needed for the conduct of research is provided as a common resource to be used by several investigators at a medical center or may be purchased for and used by only one investigator. Data on equipment and instrumentation purchased with an 
2.

acquisition cost of $\$ 2,000$ or more are available for each medical center. However, we evaluate that a listing for each of the 60 aforementioned VA medical centers, even if limited to equipment and instrumentation with an acquisition cost of over $\$ 10,000$, would not be practical for the purpose of your request.

Please contact me at 389-2616 for any questions you may have or further discussion.

Sincerely,

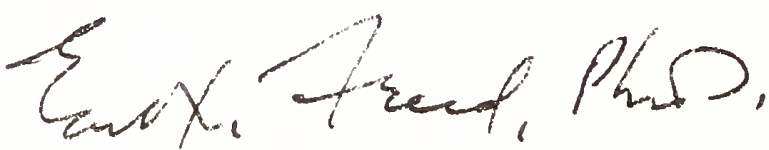

HOLLIS G. BOREN, M.D.

Assistant Chief Medical Director

for Research and Development

Enclosure 
NBS-114A (REV. 2-80)

U.S. DEPT. OF COMM.

BIBLIOGRAPHIC DATA

SHEET (See in structions)

1. PUBLICATION OR

REPORT NO.

NBS SP 646

February 1983

4. TITLE AND SUBTITLE

Federal Laboratory Directory 1982

5. AUTHOR(S)

James M. Wyckoff, Editor

6. PERFORMING ORGANIZATION (If joint or other than NBS, see instructions)

7. Contract Grant No.

NATIONAL BUREAU OF STANDARDS

DEPARTMENT OF COMMERCE

WASHINGTON, D.C. 20234

8. Type of Report \& Period Covered

Fina 1

9. SPOASOAHG ORGANZATION NAME AND COHFLETE A.ODRESS (Street, CiY, State, ZIF)

National Bureau of Standards

Department of Commerce

Washington, D.C. 20234

10. SUPPLEMENTARY NOTES

Prepared in cooperation with the Federal Laboratory Consortium for

Technology Transfer.

Library of Congress Catalog Card Number: 82-600663

$\square$ Document describes a computer program; SF-185, FIPS Software Summary, is attached.

11. ABSTRACT (A 200-word or less factual summary of most significant information. If document includes a significant bibliography or literature survey, mention it here)

This Directory provides limited information about some 388 Federal laboratories with ten or more full-time professionals engaged in research and development. Summary data arranged by Federal agency and by State provide a broad overview of the Federal laboratory system. Laboratory lists by staff size, by State and by agency provide a cross reference. For each laboratory, a contact for obtaining technical information is given by name, address, and phone number. Major mission and major scientific or testing equipment is listed for each laboratory.

12. KEY WORDS (Six to twelve entries: alphabetical order; capitalize only proper names: and separate key words by semicolons) contacts; directory; Federal laboratory; research and development;

technology transfer.

13. AVAILABILITY

X Unlimited

$\square$ For Official Distribution. Do Not Release to NTIS

$[X]$ Order From Superintendent of Documents, U.S. Government Printing Office, Washington, D.C. 20402.

[ Order From National Technical Information Service (NTIS), Springfield, VA. 2216I
14. NO, OF

PRINTED PAGES

263

15. Price

$\$ 800$ 


\section{NBS TECHNICAL PUBLICATIONS}

\section{PERIODICALS}

JOURNAL OF RESEARCH-The Journal of Research of the National Bureau of Standards reports NBS research and development in those disciplines of the physical and engineering sciences in which the Bureau is active. These include physics, chemistry, engineering, mathematics, and computer sciences. Papers cover a broad range of subjects, with major emphasis on measurement methodology and the basic technology underlying standardization. Also included from time to time are survey articles on topics closely related to the Bureau's technical and scientific programs. As a special service to subscribers each issue contains complete citations to all recent Bureau publications in both NBS and nonNBS media. Issued six times a year. Annual subscription: domestic \$18; foreign \$22.50. Single copy, $\$ 5.50$ domestic; $\$ 6.90$ foreign.

\section{NONPERIODICALS}

Monographs-Major contributions to the technical literature on various subjects related to the Bureau's scientific and technical activities.

Handbooks-Recommended codes of engineering and industrial practice (including safety codes) developed in cosperation with interested industries, professional organizations, and regulatory bodies.

Special Publications-Include proceedings of conferences sponsored by NBS, NBS annual reports, and other special publications appropriate to this grouping such as wall charts, pocket cards, and bibliographies.

Applied Mathematics Series-Mathematical tables, manuals, and studies of special interest to physicists, engineers, chemists, biologists, mathematicians, computer programmers, and others engaged in scientific and technical work.

National Standard Reference Data Series-Provides quantitative data on the physical and chemical properties of materials, compiled from the world's literature and critically evaluated. Developed under a worldwide program coordinated by NBS under the authority of the National Standard Data Act (Public Law 90-396).

NOTE: The principal publication outlet for the foregoing data is the Journal of Physical and Chemical Reference Data (JPCRD) published quarterly for NBS by the American Chemical Society (ACS) and the American Institute of Physics (AIP). Subscriptions, reprints, and supplements available from ACS, 1155 Sixteenth St., NW, Washington, DC 20056.
Building Science Series-Disseminates technical ir'ormation developed at the Bureau on building materials, components, systems, and whole structures. The series presents research results, test methods, and performance criteria related to the structural and environmental functions and the durability and safety characteristics of building elements and systems.

Technical Notes-Studies or reports which are complete in themselves but restrictive in their treatment of a subject. A nalogous to monographs but not so comprehensive in scope or definitive in treatment of the subject area. Often serve as a vehicle for final reports of work performed at NBS under the sponsorship of other government agencies

Voluntary Product Standards-Developed under procedures published by the Department of Commerce in Part 10. Title 15, of the Code of Federal Regulations. The standards establish nationally recognized requirements for products, and provide all concerned interests with a basis for common understanding of the characteristics of the products. NBS administers this program as a supplement to the activities of the private sector standardizing organizations.

Consumer Information Series-Practical information, based on NBS research and experience, covering areas of interest to the consumer. Easily understandable language and illustrations provide useful background knowledge for shopping in today's technological marketplace.

Order the above NBS publications from: Superintendent of Documients, Government Printing Office. Washington, DC 20402.

Order the following NBS publications-FIPS and NBSIR's-from the National Technical Information Service, Springfield, VA 22161.

Federal Information Processing Standards Publications (FIPS PUB)-Publications in this series collectively constitute the Federal Information Processing Standards Register. The Register serves as the official source of information in the Federal Government regarding standards issued by NBS pursuant to the Federal Property and Administrative Services Act of 1949 as amended, Public Law 89-306 (79 Stat. 1127), and as implemented by Executive Order 11717 (38 FR 12315, dated May 11, 1973) and Part 6 of Title 15 CFR (Code of Federal Regulations).

NBS Interagency Reports (NBSIR) - A special series of interim or final reports on work performed by NBS for outside sponsors (both government and non-government). In general, initial distribution is handled by the sponsor; public distribution is by the National Technical Information Service, Springfield, VA 22161, in paper copy or microfiche form. 
U.S. Department of Commerce National Bureau of Standards

Washington, D.C. 20234

Official Business

U.S.MAIL

Penaity for Private Use $\$ 300$

POSTAGE AND FEES PAID U.S DEPARTMENT OF COMMERCE COM-215

SPECIAL FOURTH-CLASS RATE BOOK 